\title{
ESTUDIO CONFORMACIONAL DE COMPUESTOS ORGANOFOSFORADOS Y SUS MECANISMOS DE ACCIÓN TÓXICA
}

Guido Enrique Mastrantonio Garrido

\author{
Trabajo de Tesis para optar al título \\ de Doctor en Ciencias Exactas - Área \\ Química, Facultad de Ciencias \\ Exactas de la Universidad Nacional \\ de La Plata. \\ Director: Carlos Omar Della Védova \\ Jurados: \\ Héctor Di Loreto (UNLP) \\ Adolfo Iribarren (UNQUI) \\ Gerardo D. Castro (UNSAM) \\ Presentado en La Plata, Argentina, a \\ los once días del mes de diciembre \\ del año 2003.
}


Este trabajo de tesis fue concebido, desarrollado y presentado en la Universidad Nacional de La Plata, a la fecha Universidad Pública, Libre y Gratuita. Con mucha satisfacción lo dedico a aquellos pocos profesores que, a lo largo de mi formación de grado y postgrado, tanto en Chile como en Argentina, intentaron enseñarme que la educación superior y la ciencia deben ser tareas reservadas para los privilegiados.

Estimados profesores: por lo menos en esto, fracasaron.

G.E.M.G. 


\section{Índice}

$\begin{array}{ll}\text { Introducción } & 7\end{array}$

Contexto histórico 8

$\begin{array}{ll}\text { Presupuestos de nuestro trabajo } & 15\end{array}$

Capítulo 1: Acerca de la estructura de los fosfatos de trimetilo y
compuestos relacionados

Alternativas conformacionales $\quad 28$

Momento dipolar $\quad 30$

Comportamiento espectroscópico vibracional 31

Espectroscopía de resonancia magnética nuclear $\quad 34$

$\begin{array}{ll}\text { Reactividad relativa } & 37\end{array}$

Estrategias para el estudio conformacional 38

Capítulo 2: Comportamiento conformacional del fosfato de trimetilo 39

Caracterización espectroscópica $\quad 46$

Correlación teórica $\quad 48$

Integración y justificación de resultados $\quad 54$

Capítulo 3: Comportamiento conformacional del fosfotioato de $\mathbf{0 , 0 , 0 -}$ $\begin{array}{ll}\text { trimetilo } & 57\end{array}$

$\begin{array}{ll}\text { Caracterización espectroscópica } & 61\end{array}$

$\begin{array}{ll}\text { Correlación teórica } & 62\end{array}$

Integración y justificación de resultados 66

Capítulo 4: Comportamiento conformacional del fosfoselenoato de O,O,O$\begin{array}{lr}\text { trimetilo } & 69\end{array}$

Caracterización espectroscópica $\quad 74$

$\begin{array}{ll}\text { Correlación teórica } & 74\end{array}$

$\begin{array}{ll}\text { Estudios espectroscópicos de alta resolución } & 76\end{array}$

$\begin{array}{ll}\text { Integración y justificación de resultados } & 80\end{array}$

Capítulo 5: Acerca de la estructura de las fosfamidatos de dialquilo 81

$\begin{array}{ll}\text { Espectroscopía vibracional } & 84\end{array}$

Espectroscopía de resonancia magnética nuclear $\quad 85$

$\begin{array}{lr}\text { Reactividad } & 87\end{array}$

$\begin{array}{ll}\text { Conformaciones preferenciales } & 88\end{array}$

Capítulo 6: La molécula de fosfamidato de dimetilo 91

Descripción teórica $\quad 94$

Caracterización espectroscópica $\quad 95$

Correlación de datos 95

Capítulo 7: La molécula de fosfamidotioato de dimetilo 97

$\begin{array}{lr}\text { Caracterización espectroscópica } & 98\end{array}$

$\begin{array}{lr}\text { Espectroscopía de alta resolución } & 103\end{array}$

$\begin{array}{ll}\text { Integración y justificación de resultados } & 105\end{array}$

Capítulo 8: Comportamiento conformacional: generalizaciones $\quad 107$

$\begin{array}{lr}\text { Análisis NBO } & 110\end{array}$

$\begin{array}{ll}\text { Sistemas con grandes restricciones } & 114\end{array}$

$\begin{array}{ll}\text { Conclusiones generales } & 116 \\ \text { Capitulo } & 119\end{array}$

Capítulo 9: Reseña de la toxicología de los compuestos organofosforados 119

$\begin{array}{ll}\text { Mecanismos de acción tóxica } & 119\end{array}$ 
Clínica de la intoxicación por organofosforados

Capítulo 10: Acetilcolinesterasa: Caracterización del sitio activo 129

$\begin{array}{ll}\text { Dinámica del sitio activo de la acetilcolinesterasa } & 130\end{array}$

$\begin{array}{ll}\text { Estructura del sitio activo de la acetilcolinesterasa } & 134\end{array}$

$\begin{array}{ll}\text { Comportamiento conformacional de la acetilcolina } & 140\end{array}$

Dinámica del sitio activo de la acetilcolinesterasa y sus aductos con organofosforados 142

Capítulo 11: Acetilcolinesterasa: Modelado del sitio activo y sus aductos con organofosforados

Constitución del modelo

Resultados

Conclusiones obtenidas a partir del modelo

Consideraciones respecto a la actividad anti colinesterasa de los organofosforados

Capítulo 12: Acetilcolinesterasa: cinética de la inhibición

Formalismo cinético

Evaluación de las constantes cinéticas para organofosforados modelo 168

Comportamiento anómalo del fosfotioato de trimetilo y fosfoselenoato de trimetilo $\quad 170$

Correspondencia del comportamiento cinético

Capítulo 13: Relaciones estructura función

Bioactivación de los organofosforados

Cuantificación de las relaciones estructura-actividad

Definiciones

Definición de descriptores moleculares

Análisis de las correlaciones encontradas

Análisis del poder predictivo de los QSAR ${ }^{\mathrm{C}}$

Análisis del poder interpretativo de los QSAR $^{\mathrm{C}}$

Capítulo 14: A modo de recapitulación...

Generalizaciones respecto del comportamiento conformacional de pequeños

organofosforados

Manifestaciones espectroscópicas de la variedad conformacional

Descripción de propiedades emergentes del sitio activo de la acetilcolinesterasa con relación a pequeños organofosforados

Papel de la restricción conformacional en la disminuida capacidad inhibitoria de tio y selenofosforados

Comportamiento conformacional del fosfato de trimetilo como justificación de su pobre poder inhibitorio

Activación in situ de pequeños tiofosforados

Aportes al diseño de variantes de la acetilcolinesterasa

Propiedades conformacionales como descriptores "4D" en la constitución de QSAR 


\section{Aclaración y agradecimientos}

Tanto en la discusión de los conceptos base de los temas desarrollados, como en el diseño, la ejecución y la interpretación de los trabajos experimentales, no es posible dejar de reconocer que todo lo presentado a lo largo de estas páginas es fruto de trabajo colectivo. El autor se considera entonces, en este caso, sólo un emergente de todos aquellos que directa o indirectamente entregaron tiempo y dedicación para la finalización de las tareas. Para todos mi profundo reconocimiento.

El Instituto madre de todo el trabajo fue el Laboratorio de Servicios a la Industria y al Sistema Científico (LaSeISiC - UNLP - CIC - CONICET), en el que se realizaron la mayoría de las síntesis, purificaciones, medidas espectroscópicas y cromatográficas, además de la mayor parte de las medidas cinéticas. Buena parte del período de trabajo fue financiado mediante distintas becas de la Universidad Nacional de La Plata asignadas mediante concursos abiertos.

La siguiente es una lista, seguramente no agotada, de los demás Centros, Institutos y Cátedras en las que desarrollan sus tareas las personas que de alguna manera aportaron a la culminación de esta tesis o que colaboraron con espacio, instrumental o material necesario. Comparto con ellos entonces el fruto del trabajo (y sus consecuencias):

(en orden alfabético)

- Anorganische Chemie, Universität GH Duisburg, Duisburg (Alemania), por las medidas de espectros infrarrojos en matrices a baja temperatura.

- Cátedra de Bioquímica de la Facultad de Ciencias Veterinarias - UNLP, La Plata (Argentina), por la cesión de material biológico para la obtención de extractos enzimáticos de rata y el equipamiento necesario para el procesado de los mismos.

- Cátedra de Farmacodinamia de la Facultad de Ciencias Exactas - UNLP, La Plata (Argentina), por la introducción en las metodologías de modelado molecular y las discusiones respecto de los primeros intentos de modelar organofosforados libres.

- Cátedra de Fisicoquímica II de la Facultad de Bioquímica, Química y Farmacia - UNT, San Miguel de Tucumán (Argentina), por el aporte en la implementación de las técnicas de medidas enzimáticas, además de la ayuda necesaria en los cálculos teóricos.

- Cátedra de Toxicología y Química Legal de la Facultad de Ciencias Exactas UNLP, La Plata, Buenos Aires (Argentina), por ceder parte de sus instalaciones durante los primeros pasos dados en las técnicas de medidas enzimáticas y de control biológico de exposición a organofosforados.

- Centre de Phytopharmacie - Université de Perpignan (Francia), por la bibliografía y la introducción a la temática de las aplicaciones de las enzimas recombinantes hipersensibles en el diseño de biosensores de organofosforados y carbamatos.

- Centro de Investigaciones de Pestes e Insecticidas (CIPEIN - CITEFA), por el aporte de patrones de fosforados y abundante bibliografía.

- Centro de Investigación y Desarrollo en Criotecnología de Alimentos (CIDCA UNLP - CIC - CONICET), por la cesión de equipos para realizar medidas cinéticas. 
- Centro de Investigaciones Toxicológicas (CEITOX - CITEFA), por el aporte de material bibliográfico y las discusiones acerca de los aspectos toxicológicos del trabajo.

- Centro de Química Inorgánica (CEQUINOR), Facultad de Ciencias Exactas UNLP, La Plata, Buenos Aires (Argentina), por la cesión de equipamiento para medidas de espectros infrarrojo y Raman, además del apoyo en los primeros pasos en la síntesis de compuestos organofosforados.

- Departamento de Artrópodos de la Facultad de Ciencias Naturales y Museo UNLP, La Plata, Buenos Aires (Argentina), por la facilitación de material necesario para la identificación y crianza de los insectos utilizados para obtención de extractos enzimáticos.

- Departamento de Química de la Facultad de Ciencias Exactas y Naturales UNLPam, Santa Rosa, La Pampa (Argentina), por la cesión de muestras purificadas de fosfatoiminas N-sulfóxido sustituidas.

- IPESA S.A., Buenos Aires (Argentina), por la provisión de los compuestos organofosforados necesarios para la mayoría de las síntesis y evaluaciones toxicológicas.

- Laboratorio de Bioquímica, Institut Químic de Sarrià, Universitat Ramon Llull, Barcelona (España), por las orientaciones respecto de las metodologías apropiadas para el modelado de grandes sistemas moleculares.

- Laboratorio de Compuestos Orgánicos (LADECOR), Facultad de Ciencias Exactas - UNLP, por el apoyo en la implementación de técnicas de purificación de compuestos organofosforados.

- Laboratoire de Synthèse et Physicochimie des Molécules d'Intérêt Biologique, Groupe de Biochimie des Protéines - Université Paul Sabatier, Toulouse (Francia), por la cesión de enzimas recombinantes y la introducción en la temática de la modificación dirigida de los sitios activos enzimáticos.

- Laboratorio de Toxicología y Química Legal, Dirección de Asesorías Periciales, Corte Suprema de Justicia de la Provincia de Buenos Aires (Argentina), por el aporte de abundante bibliografía y de material de interés legal para el análisis de aspectos de la temática de los compuestos organofosforados en el marco de aplicaciones forenses.

- Materials Chemistry Division, Indira Gandhi Centre for Atomic Research, Kalpakkam (India), por las interesantes discusiones acerca del carácter de los grados de degeneración de los confórmeros en organofosforados simétricos y la revisión de nuestros manuscritos al respecto. 


\section{Introducción}

Durante mucho tiempo los compuestos organofosforados (OPs) han despertado la curiosidad de especialistas y legos, motivados por el puro interés de saber y por la búsqueda de aplicaciones en diferentes áreas de la cultura humana.

Desde el descubrimiento de los OPs hasta el presente se ha acumulado una enorme cantidad de información respecto a sus propiedades químicas, biológicas y aplicaciones directas en distintas áreas tecnológicas. Se han resuelto muchos problemas, al tiempo que se han generado una cantidad mayor de interrogantes.

Sin embargo, en muchos aspectos no se ha logrado una integración de la información que permita tratar algunas problemáticas con un cierto orden. Esta afirmación es válida, por ejemplo, en lo concerniente a los efectos colaterales en la exposición a largo plazo de los compuestos OPs.

El presente trabajo intenta abordar una de las temáticas importantes aún no completamente resueltas que tiene que ver con los eventos íntimos de interacción de los OPs con su enzima diana más relevante: la acetilcolinesterasa (AChE).

Para esta tarea fue necesaria una recopilación y búsqueda de correspondencia de información en áreas muy distintas de las ciencias físicas, químicas y biológicas. Contando con ella, se intenta aportar algunas hipótesis que permitan unificar ciertos aspectos aún no bien comprendidos.

Al día de hoy existen muchos grupos que trabajan activamente en la mayor parte de los temas que son referidos a lo largo de estas páginas. Todos ellos se ven reflejados en las referencias bibliográficas utilizadas para basar las argumentaciones propuestas.

Sin embargo, es necesario advertir al lector que, entre la edición de este trabajo y su lectura, no tenemos dudas se generará una cantidad de información nueva que 
complementará datos, responderá interrogantes recién formuladas aquí o, incluso, podrá contradecir hipótesis basadas en datos existentes a la fecha de edición de estas páginas. Esta advertencia no hace más que transmitir la experiencia del autor durante los años dedicados a las tareas paralelas de recopilación de información y diseño de experiencias para la generación de datos inéditos.

Pero es a partir de esta experiencia que podemos afirmar, sin dudas, que el trabajo realizado en las temáticas relacionadas a los compuestos OPs constituye un quehacer apasionante, por saberse compartiendo un laborioso y extenso trabajo colectivo con numerosos científicos alrededor del mundo.

Uno de los aspectos relacionados con el tratamiento de los OPs es la profusión de nombres de uso común que comparten tribuna con la nomenclatura estándar, aún en la literatura científica de fuentes primarias. Para resolver esta dificultad, en las páginas siguientes se han usado los nombres comunes en la medida que ello no signifique ninguna confusión y además sean relevantes por el papel que juegan en la identidad del compuesto en cuestión. Pero en estos casos, estarán escritos en negrita.

Por el contrario, cuando el nombre de determinado compuesto OP se menciona en el texto sin marca distintiva, se entenderá que se está usando la nomenclatura estándar. ${ }^{(1)}$ En la medida de lo posible, en los respectivos Anexos se indicará el número del Chemical Abstract (CAS number) que individualiza a cada uno de los compuestos estudiados.

Antes de entrar en las temáticas específicas, creemos necesario mencionar el contexto en el que se aspira a realizar este esfuerzo de estudio de los compuestos OPs, máxime tomando en cuenta la variedad de disciplinas desde donde se pretende enfocar el problema.

\section{Contexto histórico}

Los compuestos OPs fueron descubiertos por Lassaigne a principios del siglo XIX, cuando éste verifica la

\footnotetext{
1 Editorial Report on Nomenclature. Apendix 3, Nomenclature of compounds containing one phosphorus atom, J.Chem.Soc. 5122-5131 (1952).
}<smiles>CCCCNP(=O)(OP(=O)(OCC)OP(=O)(OCC)OCC)OP(=O)(N(C)C)N(C)C</smiles>

Figura 0.1: Pirofosfatos orgánicos inhibidores de la AChE sintetizados durante los primeros desarrollos de estas sustancias. 
<smiles>CCO[PH](C)(=O)=O</smiles><smiles>CC(C)OP(C)(=O)F</smiles><smiles>CC(OP(C)(=O)F)C(C)(C)C</smiles>

Figura 0.2: Gases nerviosos del grupo de los "Agentes G". reacción de distintos alcoholes con ácido fosfórico. ${ }^{(2)} \mathrm{Sin}$ embargo, la primera descripción detallada de la síntesis de un OP, fue presentada por Phillipe de Clermont en la reunión de la Academia Francesa de Ciencias del año $1854 .{ }^{(3)}$ Se trató de la primera síntesis reportada del pirofosfato de tetraetilo (TEPP). Este mismo autor informa haber probado el compuesto (costumbre usual en esta época), y no sólo sobrevivió al ensayo, sino que murió muchos años después ¡a la edad de 90 años!. ${ }^{(4)}$ Este hecho y el no haber informado siquiera síntomas ni signos leves de intoxicación, sugiere que, de haber obtenido el compuesto, fue con un muy bajo rendimiento pues el TEPP, para un hombre adulto, es letal en dosis de unas pocas gotas. ${ }^{(5)}$

Durante el período que transcurre entre las dos guerras mundiales se descubre, en Alemania, el hecho que los ésteres del ácido fosfórico o pirofosfórico actúan como potentes neurotóxicos. ${ }^{(6)}$ El interés bélico por parte del régimen nazi durante la Segunda Guerra Mundial significó el inmediato aporte de muchísimos recursos humanos $\mathrm{y}$ materiales al desarrollo de potenciales gases de guerra y de paso se dio lugar a los primeros avances en el diseño de pesticidas OPs.

Entre los más brillantes científicos que participaron de esta etapa se encontraba Gerhard Schrader, químico empleado en la IG Farberindustrie ${ }^{(7)}$ a quién se lo considera actualmente como el padre de la química y toxicología moderna de los OPs. Schrader y su equipo sintetizaron y probaron más de un centenar de compuestos, entre los que se encuentra el ya mitológico Schradan o pirofosfato de tetra (dimetilamida), utilizado en su momento como insecticida.

\footnotetext{
2 Citado en Lange, W. and von Krüeger, G. Über ester der Monofluorphosphosaure. Ber. Dtsch. Chem. Ges. 65: 1598 - 1601 (1932).

${ }^{3}$ En detalle se menciona en Besser, R., Gutmann, L. Intoxication with organophosophorus compounds. In: Vinken, P.J., Bruyen, G.W. eds. Intoxication of the Nervous System. Amsterdam, the Nederlands: Elsevier Science Publishers; 151 - 181 (1989).

${ }^{4}$ Holmstedt, B. Structure activity relationships of the organophosphorus anticholinesterase agents. In: Koelle, G.B. ed. Cholinesterases and Anticholinesterases Agents. Berlin, Germany: Springer Publishing Co; 428 - 485 (1963).

${ }^{5}$ Khurana, D. and Prabhakar, S. Organophosphorus intoxication. Arch. Neurol. 57: 600 - 602 (2000).

${ }^{6}$ Fue en la Universidad de Berlín, sintetizados por Lange y su estudiante von Krüeger, quienes también documentaron sus efectos. Ver Lange $(1932)^{(2)}$.

Esta empresa fue la precursora del actual laboratorio Bayer AG, Leverkusen.
} 
Durante esta época en Alemania se sintetizan los potentes gases de guerra denominados "agentes G": tabún o GA (N,N-dimetilfosforamido-cianidato de O-etilo), sarín ${ }^{(8)}$ o GB (metilfosfonofluoridato de O-isopropilo), somán o GD (metilfosfonofluoridato de O-pinacolilo) y ciclosarín o GF (metilfosfonofluoridato de O-ciclohexilo).

Al mismo tiempo, norteamericanos y británicos desarrollan otros compuestos con potencial uso bélico, entre los que se encuentra el DF8 o fosfofluoridato de diisopropilo sintetizado en el año $1941 .^{(9)}$ Este compuesto se sigue utilizando en distintos modelos de inhibición enzimática in vivo e in vitro. ${ }^{(10)}$

Curiosamente, y aún existiendo un enorme desarrollo de estos gases de guerra durante toda la Segunda Guerra Mundial, no fueron usados en ningún evento bélico importante. Más aún, cuando el Ejército Rojo avanzó sobre Alemania, captura en Dührernfurth la factoría química más grande que disponía Hitler. En ella se encontraron intactos grandes depósitos que han sido estimados en 1200 toneladas de tabún, 600 toneladas de sarín y una cantidad no conocida de somán. ${ }^{(11)}$

Aún hoy es un misterio la razón por la cual tales armas no fueron utilizadas por los alemanes durante la invasión a Normandía por ejemplo; una de las hipótesis más aceptadas es la que indica que el régimen nazi recibió el rumor, difundido por la Oficina de Servicios Estratégicos de los Aliados, que debían esperar una brutal revancha en caso de emplear gases tóxicos, revancha que aparentemente no

\footnotetext{
${ }^{8}$ El compuesto Sarín debe su nombre a sus inventores: Schrader, Ambros, Radriger y van der Linde

${ }^{9}$ McCombie, H., Saunders, B.C. Report $N^{\circ} 2$ on fluorophosphonates to the Ministry of Supply. London, England. February 27 (1942).

${ }^{10}$ Por citar sólo algunos ejemplos de modelos biológicos: (a) Kamata, R. et al. Correlation of binding sites for diisopropyl phosphorofluoridate with cholinesterase and neuropathy target esterase in membrane and cytosol preparations from hen, Neurotoxicol. 22 (2): 203-214 (2001). (b) Petrikovics, I. et al. Long circulating liposomes encapsulating organophosphorus acid anhydrolase in diisopropylfluorophosphate antagonism, Toxicol.Sci. 57 (1): 16-21 (2000). (c) Dettbarn, W.D., Yang, Z.P. and Milatovic, D. Different role of carboxylesterases in toxicity and tolerance to paraoxon and DFP, Chem.Biol.Interact. 119-120: 445-454 (1999). (d) Gordon, C.J. Pharmacological analysis of diisopropyl fluorophosphate: effects on core temperature, heart rate, and motor activity in the unrestrined rats, Pharmacology Biochemistry \& Behavior. 55 (2): 185-194 (1996).

${ }^{11}$ Khurana, D. and Prabhakar, S. Organophosphorus Intoxication. Arch. Neurol. 57: 600-602 (2000).
}<smiles>CC(C)OP(=O)(F)OC(C)C</smiles>

Figura 0.3: El DF8 originalmente fue concebido como gas de guerra. Hoy se lo conoce como DFP y se utiliza frecuentemente como modelo de inhibidor enzimático. 
estaba preparada. ${ }^{(12)}$ Es necesario recordar que durante la Primera Guerra Mundial, ambos bandos utilizaron copiosamente armas químicas, sin grandes resultados en términos estratégicos.

En la inmediata posguerra, se continuó con la mayoría de las líneas de trabajo generadas durante la anterior etapa, promovidas por la migración de científicos alemanes a Norteamérica, el Reino Unido y a la Unión Soviética. Al mismo tiempo, la llamada "revolución verde" exigía grandes esfuerzos para el desarrollo de pesticidas cada vez más eficientes.

Un importante salto cualitativo en el desarrollo de nuevos compuestos OPs, aparece durante estas tempranas etapas con la incorporación de los primeros conceptos relacionados a la búsqueda de correlaciones cuantitativas entre la estructura química y la actividad biológica (Quantitative Structure Activity Relationships - QSAR) y, vinculado a esto, se incorporan los primeros planteos de diseño racional de estructuras químicas con una actividad determinada. ${ }^{(13)}$ De hecho, fueron los OPs el objeto de los estudios que sentaron las bases de los primeros presupuestos en los estudios QSAR, que aún hoy siguen vigentes en el diseño y estudio de pesticidas, fármacos o contaminantes orgánicos.

Durante estos años, científicos soviéticos predicen la toxicidad de los fosfonotionatos y fosfotionotos de S-2dialquilaminoetilo, también llamados "agentes V". Luego, en 1949, el británico Ranjit Ghosh logra la síntesis del metilfosfonotionato de O-etilo y S-2-diisopropilaminoetilo o agente VX. ${ }^{(14)}$ Los "agentes V" serán luego conocidos también como "gases soviéticos". A diferencia de los "agentes G", estas sustancias de uso bélico son líquidas a

\footnotetext{
12 Maselson, M. and Robison, J.P. Chemical warfare and chemical disarmament. Sci. Am. 242:38-47 (1980).

13 (a) Mackworth, J.F. and Webb, E.C. Biochem.J. 42: 91 (1948). (b) Aldridge, W.N. and Davison, A.N. Biochem.J. 51: 62 (1952). (c) Freedman, L.D. et al., The preparation of some organophosphorus compounds possessing anticholinesterase activity, J.Am.Chem.Soc. 75: 1379-1381 (1953). (d) Fukuto, T.R. and Metcalf, R.L. Structure and insecticidal activity of some diethyl subtituted phenil phosphates, J.Agric.Food.Chem. 4 (11): 930-935 (1956). (e) Fukuto, T.R. and Metcalf, R.L. The effect of structure on the reactivity of alquil phosphonate esters, J.Am.Chem.Soc. 81: 372-377 (1959).

${ }^{14}$ Paxman, J., Harris, R. A Higher Form of Killing: The Secret Story of Chemical and Biological Warfare, Hill and Wang, New York (1982).
} 
temperatura ambiente, presentan baja volatilidad y mayor persistencia ambiental.

Por su parte, Schrader continúa trabajando y desarrolla el paratión (1946), uno de los OPs más masivamente usados como pesticida en las décadas siguientes. Marcando hitos se sintetizaron luego el malatión (1952), el diazinón (1953) y el clorpirifós en 1965, año que marca el máximo en términos de aparición de nuevos productos OPs. ${ }^{(15)}$

En verdad, a partir de la enorme cantidad de esfuerzos dedicados al estudio de los compuestos OPs, y como consecuencia de ellos, la gran cantidad de compuestos sintetizados y probados, la variedad de modelos desarrollados, y la prolífica lista de fenómenos biológicos descritos referidos al metabolismo tisular, mecanismos de toxicidad y destoxicación, etcétera, se cuenta con una base científica que permite abordar casi cualquier nuevo problema asociado con abundantes elementos de partida.

Entre aquellos que han dedicado la mayor parte de su tarea científica al área de la química de los compuestos OPs podemos mencionar a Gennady Kosolapoff por sus vastos aportes a las metodologías de síntesis de estos compuestos, a R. A. Nyquist por sus trabajos en espectroscopía, a I. N. Nuredtinov y sus estudios de distintas propiedades moleculares, a Dimitrina Aslanian por su abordaje del problema conformacional tanto de la AChE como de su sustrato e inhibidores, a Jaak Järv y sus trabajos acerca de los OPs quirales y la relación con entornos asimétricos y a Oleg Kolodiazhnyi en el desarrollo de técnicas para la síntesis de OPs quirales.

En el área de la toxicología de los compuestos OPs se puede mencionar a T. Roy Fukuto por sus estudios en el área de las aplicaciones como plaguicidas y la búsqueda de relaciones entre la actividad y las estructuras químicas, a John Casida y Gary Quistad por sus trabajos acerca de la capacidad anticolinesterasa de cientos de compuestos, a Mohamed Abou-Donia por sus estudios de los mecanismos de toxicidad retardada, a Jiŕí Kassa y su aporte al desarrollo de antídotos para la intoxicación por OPs y a Joel Sussman e Israel Silman por la elucidación precisa de las estructuras de la AChE y los determinantes funcional del sitio activo.

\footnotetext{
${ }^{15}$ Casida, J.E. and Quistad, G.B. Golden age of insecticide research: past, present, or future? Annu.Rev.Entomol. 43:1-16 (1998).
}<smiles>COP(C)(=O)SCCN(C(C)C)C(C)C</smiles>

Figura 0.4: Estructura del agente VX, también llamado gas-V. 
En fin, la lista es vasta y seguramente este resumen es insuficiente, aunque hemos excluido intencionalmente a la mayor parte de los que aún siguen aportando en distintas áreas.

Pero no es posible dejar de mencionar a Norman Aldridge, ${ }^{(16)}$ fallecido en 1996, quien no sólo aportó al conocimiento de los fenómenos involucrado en la interacción íntima de estos compuestos con la $\mathrm{AChE}$, sino que replanteó los conceptos en la investigación moderna de las interacciones de las sustancias químicas con los organismos. Su conocido esquema sobre las cinco fases del proceso tóxico (ver Capítulo 10), es la idea central de su trabajo. Allí se indica que la investigación en toxicología se basa, no en describir alteraciones causadas por tóxicos, sino en diferenciar las alteraciones que están implicadas en el mecanismo de toxicidad de aquellas alteraciones que no conducen al efecto tóxico observado. ${ }^{(17)}$

A nuestro entender, desde el descubrimiento de las aplicaciones tecnológicas de los compuestos OPs hasta la actualidad, hay dos momentos relevantes en cuanto a avances cualitativos en el entendimiento de los mecanismos tóxicos de estas sustancias. Dichos momentos están representados respectivamente en la Conferencia Internacional en Alternativas Insecticidas para el Control de Vectores, realizada en Atlanta (EE.UU.) entre el 15 y el 19 de febrero de $1971^{(18)}$ y en la III Reunión Internacional sobre Reacción de Esterasas con Compuestos Organofosforados, realizada en Dubrovnik (Croacia) entre el 15 y 18 de abril de $1998^{(19)}$

Hacia principios de la década de los setenta, ya se habían sintetizado y probado prácticamente la mayor parte de las clases posibles de OPs, y se tenía un completo panorama de la naturaleza de su interacción como inhibidores sobre las esterasas, con una idea clara de los efectos tóxicos de estos compuestos sobre insectos y mamíferos. Luego, hacia fines

\footnotetext{
$16 \mathrm{Su}$ libro, en coautoría con Elsa Reiner (Enzymmes Inhibitors as Sustrates: Interaction of Esterases with Organophosphorus and Carbamic Acids), es considerado una obra maestra. Se encuentra agotado desde hace muchos años y, aunque es sólo conocido entre los especialistas en el campo, es uno de los mejores ejemplos de cómo afrontar los mecanismos de interaciones de xenobióticos con biomoléculas.

17 Toxicología de Postgrado. Repetto, M. ed. Área de Toxicología. Universidad de Sevilla. CD-ROM. Sevilla, 2003

${ }^{18}$ Las ponencias más importantes se encuentran en el volumen 44 (1971) del Bulletin World Health Organization.

${ }^{19}$ Este evento publicó sus proceedings en el volumen 119-120 (1999) de la revista Chemico-Biological Interactions.
} 
del siglo XX, con la elucidación de la arquitectura molecular del sitio activo de la AChE, se tenía la suficiente información acumulada para entender distintos fenómenos biológicos asociados al de la inhibición de la AChE, como los eventos del entorno del sitio activo accesorios a la fosforilación, los mecanismos enzimáticos de detoxicación, además de comenzar a avanzar racionalmente en el diseño de sitios biológicos modificados por ingeniería genética.

Al día de hoy, alrededor de 1,5 millones de toneladas de pesticidas se manufacturan cada año, generando un negocio de más de treinta mil millones de dólares. ${ }^{(20)}$ Los pesticidas OPs son los más ampliamente utilizados, superando su costo en más de un tercio respecto del gasto de la totalidad de los pesticidas en uso. ${ }^{(15)}$

En este marco, se produce una gran cantidad de intoxicaciones anuales por OPs, fundamentalmente por exposición laboral y en menor medida por accidentes o intención criminal (suicidios y homicidios). Las muertes por OPs pueden superar el diez por ciento de las intoxicaciones por los mismos ${ }^{(21)}$ o llegar, en el caso de zonas rurales de los países del tercer mundo, al cincuenta por ciento de las intoxicaciones totales. ${ }^{(2)}$ Una extrapolación desde datos muy limitados, estima en tres millones el número de intoxicaciones anuales por pesticidas en todo el mundo, resultando en alrededor de 220.000 muertes. ${ }^{(23)}$

Dichas cifras dan cuenta que, lejos de tener resueltos los problemas prácticos del uso de pesticidas OPs, existen muchas falencias que en su mayoría tienen relación con políticas de gestión y control del uso de estas sustancias.

Pero no menos importante es el hecho que, no teniendo el completo dominio del modo de acción de dichos compuestos, será difícil manejar adecuadamente las tecnologías de aplicación, prevención de intoxicaciones y tratamiento de las mismas.

\footnotetext{
${ }^{20}$ McKenzie, W. Agrochemical service: the world market in 2000. In: Annual review of the Crop Protection Association. Peterborough: Crop Protection Association (2001).

${ }^{21}$ Satoh, T. and Hosokawa, M. Organophosphates and their impact on the global environment, Neurotoxicology 21 (1-2): 223-228 (2000).

${ }^{22}$ Eddleston, M. et al. Primary Pesticide poisoning in the developing world-a minimum pesticides list. Lancet 360: 1163-1167. (2002).

${ }^{23}$ WHO in collaboration with UNEP. Public health impact of pesticides used in agriculture. Geneva: World Health Organization (1990).
}<smiles>COC(=O)CC(SP(=S)(OC)OC)C(=O)OP(=S)(OC)Oc1cc(C)nc(C(C)C(C)C)n1</smiles>

Figura 0.5: Compuestos tiofosforados utilizados como plaguicidas. 
Por otro lado, y aunque los Estados Unidos de América fabricaron grandes cantidades de sarín durante los años cincuenta y $\mathbf{V X}$ durante los sesenta ${ }^{(14)}$ además de haber antecedentes de la fabricación de gases neurotóxicos por parte de Franceses y Soviéticos, existe un solo reporte del uso de OPs en el campo de batalla, ${ }^{(24)}$ del que aún existen dudas. Sólo en forma muy aislada, grupos marginales han utilizado sarín con fines políticos. ${ }^{(21)}$

Sin embargo, actualmente hay una tendencia por parte de ciertos estados al uso de los gases neurotóxicos de guerra como armas disuasivas; el mismo papel que cumplía el armamento nuclear durante la Guerra Fría.

No es arriesgado suponer que es muy poco probable que los compuestos OPs lleguen a constituirse, en la práctica, como agentes de agresión bélica importantes, pero seguirán siendo objeto de desarrollo muchos de los múltiples aspectos involucrados en sus tecnologías de aplicación. De hecho, en la actualidad se dedican muchos recursos técnicos y científicos al desarrollo de metodologías de uso ${ }^{{ }^{(5)}}$ y a estrategias de defensa ${ }^{(25)}$ frente a los OPs en el contexto de la llamada guerra química. Esto, sin contar con todos los desarrollos en el área de los pesticidas.

\section{Presupuestos de nuestro trabajo}

Desde el punto de vista de su actividad tóxica, los ésteres del ácido fosfórico son inhibidores de la AChE, aunque bioquímicamente actúan como inhibidores de varias otras enzimas, ${ }^{(26)}$ en general esterasas serínicas. Los efectos

\footnotetext{
24 Durante la guerra Irán - Irak (1984-1988), las milicias separatistas kurdas habrían sufrido ataques a campo abierto con tabún.

25 (a) Le Jeune, K.E. and Russell, A.J. Biocatalytic nerve agent detoxification in fire fighting foams, Biotechnol.Bioeng. 62 (6): 659-665 (1999). (b) Broomfield, C.A., Lockridge, O. and Millard, C.B. Protein engineering of a human enzyme that hydrolyzes $V$ and $G$ nerve agents: design, construction and characterization, Chem.Biol.Interact. 119-120: 413-418 (1999). (c) Raushel, F. Bacterial detoxification of organophosphate nerve agents, Current Opinion in Microbiology 5 (3): 288-295 (2002).

26 (a) Hammock, B.D., Sparks, T.C. and Mumby, S.M. Selective inhibition of $\mathrm{JH}$ esterases from cockroach hemolymph, Pesticide Biochem. and Physiology 7 (6): 517-530 (1977). (b) Moscioni, A.D., Engel, J.L. and Casida, J.E. Kynurenine formamidase inhibition as a possible mechanism for certain teratogenic effects of organophosphorus and methylcarbamate insecticides in chicken embryos, Biochem.Pharmacol. 26 (23): 2251-2258 (1977). (c) Karnaukhova, T.B., Korobova, L.N. and Reikh, E.M. [Superoxide dismutase activity as an
} 
clínicos de la intoxicación por OPs se conocen ya desde los años treinta, ${ }^{(2)}$ y la AChE como enzima diana fue descrita en la década siguiente. ${ }^{(27)}$ Esta enzima está asociada a la transmisión sináptica de las terminales colinérgicas, presentes tanto en mamíferos ${ }^{(28)}$ e insectos, ${ }^{(29)}$ como en otras varias clases de organismos animales; ${ }^{(30)}$ de allí la actividad como neurotóxicos y las aplicaciones tecnológicas concurrentes.

Sin embargo, actualmente se asume que la interacción con la AChE no desencadena el único mecanismo tóxico de importancia. ${ }^{(31)}$ Entre los mecanismos alternativos propuestos, el mas relevante es de la neurotoxicidad retardada, correspondiente a un síndrome que se manifiesta algunas semanas después de la exposición a ciertos OPs. ${ }^{(32)}$

index of the toxic action of pesticides], Biol.Nauki. (5): 146-149 (1990). (d) Zhao, Q. et al. Enantioselective and reversible inhibition of trypsin and alpha- chymotrypsin by phosphonate esters, Biochem. 33 (26): 81288138 (1994). (e) Li, Q. et al., Organophosphorus pesticides markedly inhibit the activities of natural killer, cytotoxic $\mathrm{T}$ lymphocyte and lymphokine-activated killer: a proposed inhibiting mechanism via granzyme inhibition, Toxicology 172 (3): 181-190 (2002).

27 (a) Koelle, G.B. and Gillman, A. The relationship between cholinesterase inhibition and the pharmacological action of DFP. J.Pharmacol. 87:421-447 (1946). (b) Dubois, K.P., Doull, J. Salerno, P.R. and Coon, J.M. Studies on the toxicity and mechanism of action of pnitrophenyl diethyl thionophosphate (parathion). J.Pharmacol. Exp.Ther. 95:79-91 (1949).

${ }^{28}$ (a) Holmstedt, B. Distribution and determination of cholinesterases in mammals, Bull.World.Health.Organ. 44: 99-107 (1971). (b) Taylor, P. and Radic, Z. The cholinesterases: from genes to proteins, Ann.Rev.Pharmacol.Toxicol. 34: 281-320 (1994).

${ }^{29}$ (a) Booth, G.M. and Lee, A. Distribution of cholinesterases in insects, Bull.World.Health.Organ. 44: 91-98 (1971). (b) Byrne, F.J. and Devonshire, A.L. Kinetics of Insensitive Acetylcholinesterases in Organophosphate-Resistant Tobacco Whitefly, Bemisia tabaci (Gennadius) (Homoptera: Aleyrodidae), Pestic.Biochem.Physiol. 58 (2): 119-124 (1997).

${ }^{30}$ (a) Mora, P., Fournier, D. and Narbonne, J.F. Cholinesterases from the marine mussels Mytilus galloprovincialis Lmk. and M. edulis L. from the freshwater bivalve Corbicula fluminea Muller, Comp. Biochem. Physiol. C. Pharmacol. Toxicol. Endocrinol. 122 (3): 353-361 (1999). (b) Delmonte, C. et al. Evidence for the presence of a mammalian-like cholinesterase in Paramecium primaurelia (Protista, Ciliophora) developmental cycle, J.Exp.Zool. 283 (1): 102-105 (1999). (c) GimenezPardo, C. et al. Presence of cholinesterases in Echinococcus granulosus protoscolices, Parasite. 7 (1): 47-50 (2000).

${ }^{31}$ (a) Pope, C.N. Organophosphorus pesticides: do they all have the same mechanism of toxicity?, J.Toxicol.Environ.Health.B.Crit.Rev. 2 (2): 161181 (1999). (b) Richards, P.G. et al. Novel protein targets for organophosphorus compounds, Chem.Biol.Interact. 119-120: 503-511 (1999).

${ }^{32}$ (a) Aldridge, W.N. and Johnson, M.K. Side effects of organophosphorus compounds: delayed neurotoxicity, Bull. World. Health. Organ. 44: 259-263 (1971). (b) Abou-Donia, M.B. 
La enzima diana cuya inhibición desencadena este síndrome, se ha denominado NTE (Neuropathy Target Esterase). Esta enzima se encuentra presente tanto en el sistema nervioso ${ }^{(33)}$ como en otros tejidos, aunque no se conoce con exactitud su función. ${ }^{(34)}$ Se ha acumulado evidencia que indica que la inhibición de la NTE no es el único evento que desencadena este síndrome retardado. ${ }^{(35)}$

La AChE es notable por varias razones. La primera de ellas es que se está en presencia de una de las enzimas más eficientes que se conocen, en términos de sus propiedades como catalizador. En efecto, la velocidad de hidrólisis de la acetilcolina se encuentra limitada fundamentalmente por la difusión del sustrato ${ }^{(36)}$ desde el seno de la solución hasta el sitio activo de la $\mathrm{AChE}{ }^{(37)}$

Organophosphorus ester - induced delayed neurotoxicity, Ann. Rev. Pharmacol. Toxicol. 21: 511-548 (1981). (c) El Sebae, A.H. et al. Biochemical interaction of six OP delayed neurotoxicants with several neurotargets, J.Environ.Sci.Health.[B.]. 16 (4): 465-474 (1981).

33 (a) Johnson, M.K., Sensitivity and selectivity of compounds interacting with neuropathy target esterase. Further structure-activity studies, Biochem.Pharmacol. 37 (21): $4095-4104$ (1988). (b) Blasco, R. et al. In vitro modifications of rat NTE and other esterases by chemicals which induce delayed neurotoxicity in vivo, Vet.Hum.Toxicol. 32 (5): 435-439 (1990). (c) Lotti, M. et al. Interactions between neuropathy target esterase and its inhibitors and the development of polyneuropathy, Toxicol.Appl.Pharm.122 (2): 165-171 (1993). (d) Ehrich, M., Correll, L. and Veronesi, B. Acetylcholinesterase and neuropathy target esterase inhibitions in neuroblastoma cells to distinguish organophosphorus compounds causing acute and delayed neurotoxicity, Fundam. Appl. Toxicol. 38 (1): 55-63 (1997).

${ }^{34}$ (a) Lotti, M. and Moretto, A. The search for the physiological functions of NTE; is NTE a receptor?, Chem.Biol.Int. 87 (1-3): 407-416 (1993). (b) Vilanova, E., Barril, J. and Carrera, V. Biochemical properties and possible toxicological significance of various forms of NTE, Chem.Biol.Int. 87 (1-3): 369-381 (1993).

35 (a) Davis, S.L. et al. Organophosphorus-induced neurotoxicity in the absence of neuropathy target esterase inhibition: the effects of triphenyl phosphine in the European ferret, Toxicol.Sci. 49 (1): 78-85 (1999). (b) Hsieh, B.H. et al. Acetylcholinesterase inhibition and the extrapyramidal syndrome: a review of the neurotoxicity of organophosphate, Neurotoxicology 22 (4): 423-427 (2001).

${ }^{36}$ Harel, M. et al. The x-ray structure of a transition state analog complex reveals the molecular origins of the catalitic power and substrate specificity of the acetylcholinesterase, J.Am.Chem.Soc. 118: 2340-2346 (1996).

${ }^{37}$ (a) Ordentlich, A. et al., Dissection of the human acetylcholinesterase active center determinants of substrate specificity. Identification of residues constituting the anionic site, the hydrophobic site, and the acyl pocket, J.Biol.Chem. 268 (23): 17083-17095 (1993). (b) Axelsen, P. H. et al., Structure and dynamics of the active center gorge of acetylcholinesterase: Synergistic use of molecular dynamics simulation and X-ray crystallography, Protein.Sci. 3 (2): 188-197 (1994). (c) Hosea, N. A., Berman, H.A. and Taylor, P. Specificity and orientation of trigonal 
Por otro lado, la inhibición por parte de los OPs se describe en términos cinéticos como irreversible, a diferencia de otros inhibidores del enzima que actúan reversiblemente. $\mathrm{Se}$ reconoce que esta inhibición presenta una gran complejidad, determinada por las interacciones específicas del sitio activo de la enzima con las diferentes partes de la estructura molecular de los OPs. ${ }^{(38)}$

Aunque sigue siendo una simplificación, un modelo en dos etapas, con una interacción reversible seguida de una reacción de hidrólisis y formación de un enlace covalente con la enzima en una etapa irreversible, parece ser el que mejor ajusta a la mayoría de las condiciones experimentales de medida de la inhibición. ${ }^{(39)}$

Cuando hablamos de compuestos OPs con actividad tóxica, se asume la referencia a sustancias de síntesis. Sin embargo, existen en la naturaleza algunos OPs sencillos ${ }^{(40)}$ que en general no tienen las características estructurales necesarias para actuar como inhibidores enzimáticos, aunque en los últimos años se han descrito OPs de fuentes naturales

carboxyl esters and tetrahedral alkylphosphonyl esters in cholinesterases, Biochem. 34 (36): 11528-11536 (1995). (d) Szegletes, T. et al. Substrate binding to the peripheral site of acetylcholinesterase initiates enzymatic catalysis. Substrate inhibition arises as a secondary effect, Biochem. 38 (1): 122-133 (1999).

${ }^{38}$ (a) Segall, Y. et al., Direct observation and elucidation of the structures of aged and nonaged phosphorylated cholinesterases by ${ }^{31} \mathrm{P}$ NMR spectroscopy, Biochem. 32 (49): 13441-13450 (1993). (b) Hosea, N. A., Berman, H.A. and Taylor, P. Specificity and orientation of trigonal carboxyl esters and tetrahedral alkylphosphonyl esters in cholinesterases, Biochem. 34 (36): 11528-11536 (1995). (c) Taylor, P. et al. Determining ligand orientation and transphosphonylation mechanisms on acetylcholinesterase by $\mathrm{Rp}, \mathrm{Sp}$ enantiomer selectivity and site-specific mutagenesis, Enantiomer. 2 (3-4): 249-260 (1997). (d) Bernard, P.P. et al. 3D Model of the Acetylcholinesterase Catalytic Cavity Probed by Stereospecific Organophosphorous Inhibitors, J.Mol.Model. 4: 323-334 (1998). (e) Hong, S.B. and Raushel, F.M. Stereochemical constraints on the substrate specificity of phosphotriesterase, Biochem. 38 (4): 11591165 (1999).

${ }^{39}$ (a) Aldridge, W. N., The nature of the reaction of organophosphorus compounds and carbamates with esterases, Bull.World.Health.Organ. 44 (1): 25-30 (1971). (b) Raevsky, O. A. et al., [Formation of models of the interaction between organophosphate compound structure and their ability to inhibit cholinesterase], Bioorg.Khim. 16 (11): 1509-1522 (1990). (c) Worek, F., Eyer, P. and Szinicz, L. Inhibition, reactivation and aging kinetics of cyclohexylmethyl phosphonofluoridate - inhibited human cholinesterases, Arch.Tox. 72 (9): $580-587$ (1998). (d) Worek, F., Diepold, C. and Eyer, P. Dimethylphosphoryl - inhibited human cholinesterases: inhibition, reactivation, and aging kinetics, Arch.Tox. 73 (1): 7-14 (1999).

40 Umezawa, S. et al. New microbial metabolite phosphoramidon. (Isolation and structure), Tetrahedron Lett. 1: 97-100 (1972).

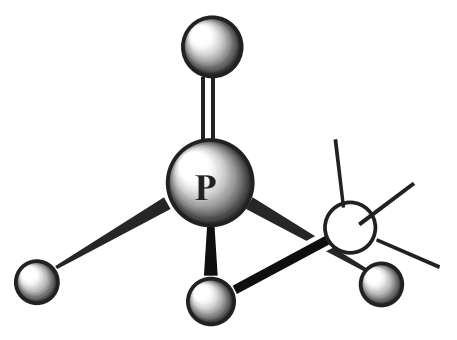

Figura 0.7: Disposición gauche de los derivados éster del ácido fosfórico. 


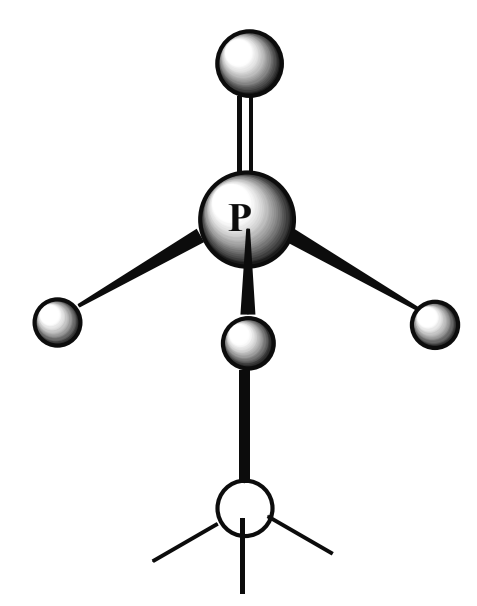

Figura 0.8: Disposición trans de los derivados éster del ácido fosfórico. con capacidad neurotóxica, ${ }^{(41)}$ estando en estos casos en presencia de los pocos compuestos xenobióticos naturales con actividad antiacetilcolinesterasa.

Al referirnos a la interacción de los OPs con la AChE es importante indicar que estos compuestos poseen una flexibilidad conformacional notable. Esta flexibilidad estaría determinada por las alternativas posiciones preferenciales que adoptan sus restos alquilo por la rotación alrededor de cada uno de los enlaces éster fosfórico $(\mathrm{C}-\mathrm{O})-(\mathrm{P}=\mathrm{X})$. En esta variación conformacional, ciertas posiciones resultarían favorecidas energéticamente respecto a otras. $^{(42)}$

Este hecho es fuertemente sustentado por una gran cantidad de datos obtenidos durante el estudio de un vasto número de OPs pentavalentes tetracoordinados. En ese sentido, en los trabajos de Durig, realizados a partir de los años sesenta y durante más de treinta años, se acumuló abundante evidencia espectroscópica y teórica. ${ }^{(43)}$ Incluso en presencia de esta gran cantidad de información, las causas de esta preferencia permanecen sin ser establecidas con claridad.

\footnotetext{
${ }^{41}$ Monserrat, J.M., Yunes, J.S. and Bianchini, A. Effects of Anabaena spiroides (Cyanobacteria) aqueous extracts on the acetylcholinesterase activity of aquatic species, Environ.Toxicol.Chem. 20 (6): 1228-1235 (2001).

${ }^{42}$ Hay mucha bibliografía al respecto. En forma seleccionada se puede mencionar: (a) Marsault - Herail, F., Vibrational spectra of organophosphorus compounds at $293 \mathrm{~K}-15 \mathrm{~K}$. Molecular conformations, J. Chim. Phys. Physicochim. Biol. 68 (2): 274-286 (1971). (b) Ishmaeva, E.A. et al., Dipole moments of organophosphorus compounds. XII. Conformational isomerism of acyclic derivatives of phosphorus dithio acids, Zh.Obshch.Khim. 44 (12): 2625-2628 (1974). (c) Guan, Y., Wurrey, C.J. and Thomas, G.J. Vibrational analysis of nucleic acids. I. The phosphodiester group in dimethyl phosphate model compounds: $\left(\mathrm{CH}_{3} \mathrm{O}\right)_{2} \mathrm{PO}_{2}^{-},\left(\mathrm{CD}_{3} \mathrm{O}\right)_{2} \mathrm{PO}_{2}^{-}$, and $\left({ }^{13} \mathrm{CH}_{3} \mathrm{O}\right)_{2} \mathrm{PO}_{2}^{-}$, Biophys.J. 66 (1): $225-$ 235 (1994).

${ }^{43}$ Ver, por ejemplo: (a) Durig, J.R. and Diyorio, J.S. Vibrational spectra and structure of organophosphorus compounds. VII. IR and Raman spectra of $\left(\mathrm{CH}_{3}\right)_{3} \mathrm{P}=\mathrm{S}$, J. Mol. Struct. 3: 179-190 (1969). (b) Durig, J.R. et al. Spectra and structure of organophosphorus compounds - XXXVII. Infrared and Raman spectra, conformation stability, vibrational assignment and normal coordinate analysis of ethyldichloro phosphine $-\mathrm{d}_{\mathrm{o}}$ and $-\mathrm{d}_{5}$, Spectrochim.Acta.Part.A. 44 (9): 911-928 (1988). (c) Durig, J.R., Hizer, T.J. and Harlan, R.J. Spectra and structure of organophosphorus compounds. XLIV. Conformational stability, barriers to internal rotation, vibrational assignment, and $a b$ initio calculations for ethylphosphonic difluoride, J.Phys.Chem. 96 (2): $541-548$ (1992). (d) Durig, J.R. and Gounev, T.K. Spectra and structure of organophosphorus compounds. LIII. Infrared and Raman spectra, conformational stability, vibrational assignment and $a b$ initio calculations of n-propyldichlorophosphine, $J$. Mol. Struct. 350 (1): 19-42 (1995).
} 
El mismo Durig invoca el "efecto gauche" (44) que, en principio, se aplicaría como regla a este tipo de estructuras. Sobre esta cuestión profundizaremos en el Capítulo 8.

A partir de la década del veinte durante el siglo XX, el desarrollo de la Física Cuántica promovió la idea que los sistemas moleculares, en esencia, son resueltos completamente a partir de la descripción de sus funciones de onda (Figura 0.9). Con el perfeccionamiento de las metodologías de la Química Computacional durante la década del setenta, el notable progreso de los métodos $a b$ initio durante la década del ochenta y el espectacular avance de la tecnología de cálculo durante las dos últimas décadas del siglo pasado, se arroja bastante más luz sobre la cuestión estructural, entregándose herramientas para una interpretación más sutil de los datos experimentales, además de poder representarse con modelos más precisos las entidades moleculares aisladas o en interacción con distintos entornos. $^{(46)}$

En forma ilustrativa, se puede hacer un interesante seguimiento revisando los antecedentes en los estudios acerca de los calcogenofosfatos de trimetilo, tratados en detalle en los Capítulos 2, 3 y 4.

44 Durig, J.R., Structures and conformations of organophosphorus molecules, J. Mol. Struct. 113: 127-140 (1984).

${ }^{45}$ Imagen obtenida desde el Institut für Theoretische Physik, Johann Wolfgang Goethe - Universität Frankfurt am Main [http:// www.th.physik.uni-frankfurt.de/]. Último acceso Junio del 2003.

${ }^{46}$ Se dispone de muchísimos trabajos en donde se invocan métodos teóricos para la interpretación de datos experimentales de OPs. En forma seleccionada mencionamos: (a) Perahia, D. and Pullman, B. Molecular orbital calculations on the conformation of phosphodiesters. An extended correlation between the geometry and the conformation of the phosphate group, Biochim.Biophys.Acta 435 (3): 282-289 (1976). (b) Gorenstein, D.G., Luxon, B.A. and Findlay, J.B. The torsional potential for phosphate diesters. The effect of geometry optimization in CNDO and $a b$ initio molecular orbital calculations, Biochim.Biophys.Acta 475 (1): 184-190 (1977). (c) LeBreton, P.R. et al. UV photoelectron spectroscopy and $a b$ initio characterization of valence orbital structures and conformations of neutral phosphate esters, J.Biomol.Struct.Dyn. 6 (1): 199-222 (1988). (d) Sablinskas, V., Horn, A. and Klaeboe, P. Conformational stability of trimethyl phosphate studied by vibrational spectroscopy and ab initio calculations, J. Mol. Struct. 349: 157-160 (1995). (e) Feshin, V.P. and Feshina, E.V. Peculiarities of atomic interaction in molecules $\mathrm{ClP}(\mathrm{M})\left(\mathrm{OCH}_{3}\right)_{2}(\mathrm{M}=\mathrm{O}$ and $\mathrm{S})$ using ab initio calculations, J. Mol. Struct. 508: 1-4 (1999). (f) Sankaran, K., Vidya, V. and Viswanathan, K.S. Trimethyl phosphate - water interaction: a matrix/isolation infrared and $a b$ initio study, J.Phys.Chem.A. 102: 2944-2953 (1998). ( $g$ ) Sundararajan, K. et al. Trimethyl phosphate-acetylene interaction: a matrix-isolation infrared and ab initio study, Spectrochim.Acta 56A (10): 1855-1867 (2000).

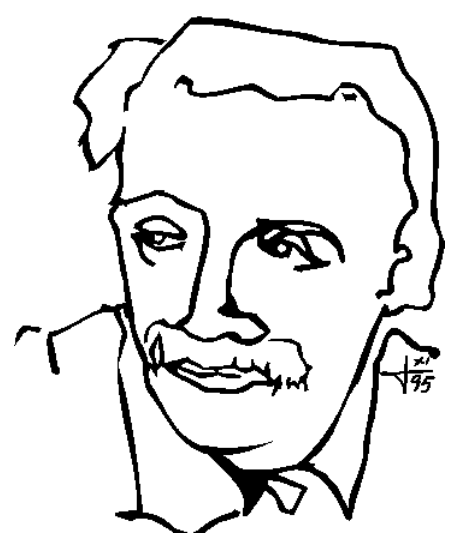

Figura $\mathbf{0 . 9}{ }^{(45)}$ : “Las leyes físicas subyacentes necesarias para la teoría matemática de una gran parte de la física y la totalidad de la química se conocen completamente y la única dificultad, es que la aplicación exacta de estas leyes conduce a ecuaciones demasiado complejas para ser solubles" (Paul Andrien Maurice Dirac, 1902-1984). 
Mortimer, en los años cincuenta, asume una primera interpretación de la coexistencia de confórmeros en el espectro del fosfato de trimetilo y el fosfotioato de $\mathrm{O}, \mathrm{O}, \mathrm{O}-$ trimetilo. ${ }^{(47)}$ Estos espectros son reinterpretados por los franceses, treinta años después, asignando señales también al fosfoselenoato de $\mathrm{O}, \mathrm{O}, \mathrm{O}$-trimetilo utilizando espectros infrarrojos de líquidos y sólidos. ${ }^{(42 a)}$ Recién a fines del siglo pasado se logra una asignación conformacional definitiva mediante el auxilio de la espectroscopía en matrices y la química computacional de alto nivel.

Se describen tres confórmeros teóricos estables, siendo el más estable de simetría $\mathrm{C}_{3}$, con los tres restos alquilos en posición gauche. Los dos confórmeros que le siguen en energía son de simetría $\mathrm{C}_{1} \mathrm{y} \mathrm{Cs}$, cada uno con dos restos alquilo en posición gauche y sólo un resto en posición trans. ${ }^{(48)}$ En esta publicación sólo se entrega evidencia espectroscópica para los dos confórmeros más estables y son nuestros trabajos los que completan la evidencia experimental con la publicación de señales asignadas al confórmero de simetría Cs en el espectro infrarrojo en matrices del fosfoselenoato de O,O,O-trimetilo. ${ }^{(49)}$

Entonces, la flexibilidad conformacional así descrita, se encuentra restringida por ciertos factores estéricos y electrónicos. A grandes rasgos, uno de estos factores correspondería a la naturaleza del calcógeno unido al fósforo mediante un doble enlace. Esto se sustenta en las evidencias que sugieren que la flexibilidad conformacional va decreciendo en el orden $\mathrm{O}=\mathrm{P}\left(\mathrm{OCH}_{3}\right)_{3}>\mathrm{S}=\mathrm{P}\left(\mathrm{OCH}_{3}\right)_{3}>$ $\mathrm{Se}=\mathrm{P}\left(\mathrm{OCH}_{3}\right)_{3}{ }^{(50)}$ para la serie de calcogenofosfatos de trimetilo.

Aunque no es el único factor determinante, la potencia inhibitoria de los OPs es mayor para los oxofosfatos

\footnotetext{
${ }^{47}$ Mortimer, F.S. Vibrational assignment and rotational isomerism in some simple organic phosphates, Spectrochim.Acta. 9: 270-281 (1957). 48 George, L., Viswanathan, K.S. and Singh, S. Ab initio study of trimethyl phosphate: conformational analysis, dipole moments, vibrational frecuencies, and barriers for conformer interconversion, J.Phys.Chem.A. 101 (13): 2459-2464 (1997).

49 Mastrantonio, G. and Della Vedova, C.O. On the structural characteristics of the O,O,O trimethyl selenophosphate molecule, J. Mol. Struct. 645: 1-8 (2003).

50 Mastrantonio, G. and Della Vedova, C.O. Spectroscopic and conformational comparative study of trimethyl chalcogenphosphates, $J$. Mol. Struct. 561 (1-3): 161-174 (2001).
} 
y decrece en el sentido $\mathrm{O}=\mathrm{P} \equiv>>>\mathrm{S}=\mathrm{P} \equiv .^{(51)}$ Esta serie se puede hacer extensiva a los compuestos del tipo $\mathrm{Se}=\mathrm{P} \equiv$ dada la sucesión de comportamientos de reactividad química de estos compuestos.

Este comportamiento se verifica en un sinnúmero de ejemplos de sustancias utilizadas como pesticidas o en modelos experimentales, aunque hay algunas excepciones para la toxicidad relativa de fosfoselenoatos y fosfotioatos. ${ }^{(52)}$ La generalización es válida entonces para los oxofosfatos respecto a los fosfotioatos, pero no está verificada para los fosfoselenoatos, respecto a los fosfotioatos.

Según la bibliografía, ${ }^{(53)}$ la acetilcolina (ACh) el sustrato natural de la AChE, adopta al menos dos conformaciones. Una de ellas prevalece en solución y en estado sólido, pero es otra la que aparece como preferencial en el sitio activo de la AChE. ${ }^{(54)}$ Es decir, hay una inversión de las energías relativas cuando existe interacción con los grupos químicos que conforman este sitio activo, de manera que la conformación de mayor energía en solución, por interacción con el entorno proteico del sitio activo, es estabilizada hasta el punto de corresponder a la conformación más estable.

En nuestros estudios preliminares de interacción de OPs con la AChE, estos adoptan conformaciones que no son las preferenciales respecto a las medidas en solución. ${ }^{(55)}$

\footnotetext{
${ }^{51}$ Maxwell, D.M. and Brecht, K.M. Quantitative structure-activity analysis of acetylcholinesterase inhibition by oxono and thiono analogues of organophosphorus compounds, Chem.Res.Toxicol. 5 (1): 66-71 (1992).

${ }^{52}$ Åkerfeldt, S. and Fagerlind, L. J. Med. Chem. 10:115-116 (1967).

${ }^{53}$ (a) Cushley, R.J. and Mautner, H.G. NMR studies on the conformation of acetylcholine isologues, Tetrahedron 26: 2151-2159 (1970). (b) Aslanian, D. Vibrational spectroscopic approach to the study of acetylcholine and related compounds, Life.Sci. 32 (25): 2809-2825 (1983). (c) Edvardsen, O. and Dahl, S.G. Molecular structure and dynamics of acetylcholine, J.Neural.Transm.Gen.Sect. 83 (3): 157-170 (1991). (d) Frydenvang, K. and Jensen, B. Conformational analysis of acetylcholine and related choline esters, Acta.Crystallogr. 52 ( Pt 1) (B): 184-193 (1996).

${ }^{54}$ Enyedy, I.J., Kovach, I.M. and Bencsura, A. Molecular dynamics study of active-site interactions with tetracoordinate transients in acetylcholinesterase and its mutants, Biochem.J. 353 (Pt 3): 645-653 (2001).

${ }^{55}$ Mastrantonio, G., Della Vedova, C.O., Giannuzzi,L. Cálculo de un modelo teórico para estudios cinéticos de la inhibición de la acetilcolinesterasa por OPs. Comunicación Libre en Póster al XI Congreso Argentino de Toxicología y XIX Jornadas Interdisciplinarias de Toxicología. La Plata, Argentina (1999).
}<smiles>CC(=O)OCC[N+](C)(C)C</smiles>

Figura 0.10: Estructura de la acetilcolina, neurotransmisor involucrado en los mecanismos de acción tóxica de los OPs. 
Otros estudios verifican hechos similares. ${ }^{(56)}$ De esta manera, los inhibidores por un lado y los sustratos xenobióticos por otro lado, reproducirían el mismo fenómeno que se observa en la interacción de la AChE con el sustrato natural, la ACh. Uno de los objetivos generales de este trabajo, es intentar entregar elementos para sustentar la regla general que indicaría que en el sitio activo de la $\mathrm{AChE}$ es preferencial una conformación del OP, y otros inhibidores, que no lo es en solución.

Se estaría en presencia de un fenómeno general, en donde la interacción de moléculas con flexibilidad conformacional, ya sean sustratos o inhibidores, da lugar a la estabilización en el sitio activo de estructuras que en solución o en el vacío corresponden a conformaciones de alta energía. Este fenómeno, más que un comportamiento de sustratos o inhibidores, correspondería entonces a una propiedad intrínseca del sitio activo de la $\mathrm{AChE}$.

Nuestras estrategias para abordar el problema son, en primer lugar, la búsqueda de series de OPs que presenten diferencias en sus flexibilidades conformacionales, pero que a su vez no tengan importantes diferencias en sus limitaciones estéricas. En este sentido están planteadas las series sobre las que se realiza este estudio: calcogenofosfatos de trimetilo (en sus variantes con oxígeno, azufre y selenio) y dimetil calcogenofosfamidatos de dimetilo (en sus variantes con oxígeno, azufre y selenio).

Por otro lado, se llevaron a cabo diversas medidas espectroscópicas (FT-IR, IR en matrices, Raman, NMR ${ }^{1} \mathrm{H}$ y ${ }^{13} \mathrm{C}$ ) y cálculos teóricos (ab initio y DFT) para el estudio de las propiedades conformacionales de cada uno de los miembros de estas series.

El estudio teórico de la interacción de estos OPs con la AChE se realiza mediante el desarrollo de un modelo molecular teórico. Este modelo de interacción se construye a partir de un polipéptido que reproduce el entorno del sitio

\footnotetext{
56 (a) Hosea, N.A., Berman, H.A. and Taylor, P. Specificity and orientation of trigonal carboxyl esters and tetrahedral alkylphosphonyl esters in cholinesterases, Biochem. 34 (36): 11528-11536 (1995). (b) Bernard, P.P. et al. 3D Model of the Acetylcholinesterase Catalytic Cavity Probed by Stereospecific Organophosphorous Inhibitors, J.Mol.Model. 4: 323-334 (1998). (c) Hirashima, A., Kuwano, E. and Eto, M. Docking study of enantiomeric fonofos oxon bound to the active site of Torpedo californica acetylcholinesterase, Bioorg.Med.Chem. 8 (3): 653-656 (2000).
} 
activo de la $\mathrm{AChE}$, cuyos parámetros son obtenidos desde medidas publicadas de difracción de RX. ${ }^{(57)}$

Nos interesa determinar las características conformacionales y energéticas de esta interacción, además de la interpretación de los fenómenos en función de los comportamientos geométricos de las moléculas. No es objeto del modelo estudiar el curso de la reacción de hidrólisis subsiguiente, sino sólo obtener una idea de las relaciones dinámicas de las variantes conformacionales de los inhibidores en este entorno.

Las medidas de inhibición de AChE de cada uno de las moléculas estudiadas aportan los datos experimentales de interacción. Estos valores nos permitirán la búsqueda de correlaciones entre determinados parámetros estructurales surgidos de los modelos utilizados y la actividad biológica correspondiente.

Finalmente, el acceso a variantes de la $\mathrm{AChE}$ hipersensibles a la inhibición por OPs, ${ }^{(58)}$ plantea la posibilidad de nuevos sistemas que puedan medir con cierta precisión las diferencias buscadas, además de permitir una mayor generalización de las correlaciones estructura-función hallados.

En este contexto, el hecho que exista una menor libertad torsional en fosfotioatos y fosfoselenoatos, es decir, una barrera energética superior en ellos respecto a los ésteres fosfóricos oxigenados, la mayor dificultad de los mismos para "invertir" sus preferencias conformacionales, sería un factor de importancia que ayudaría a entender el fenómeno integral de la inhibición de la AChE por parte de los OPs. Y este es, básicamente, el estímulo conceptual del presente trabajo.

\footnotetext{
57 Sussman, J.L. et al. Atomic structure of acetylcholinesterase from Torpedo californica: a prototypic acetylcholine - binding protein, Science 253 (5022): 872-879 (1991).

${ }^{58}$ Durante los años 2001 y 2002 hemos entablado una relación con el grupo del Dr. Didie Fournier de la Universidad de Toulousse (Francia), quién ha cedido amablemente especímenes de enzimas recombinantes purificadas que fueron utilizadas en este trabajo, como se describirá en el Capítulo 12: (a) Estrada-Mondaca, S. and Fournier, D. Stabilization of Recombinant Drosophila Acetylcholinesterase, Prot.Exp.Pur. 12 (2): 166172 (1998). (b) Villatte, F. et al. Engineering sensitive acetyl cholinesterase for detection of organophosphate and carbamate insecticides, Biosens.Bioelectron. 13 (2): 157-164 (1998).
} 
Primera PARTE: ESTUdios CONFORMACIONALES DE MOLÉCULAS LIBRES

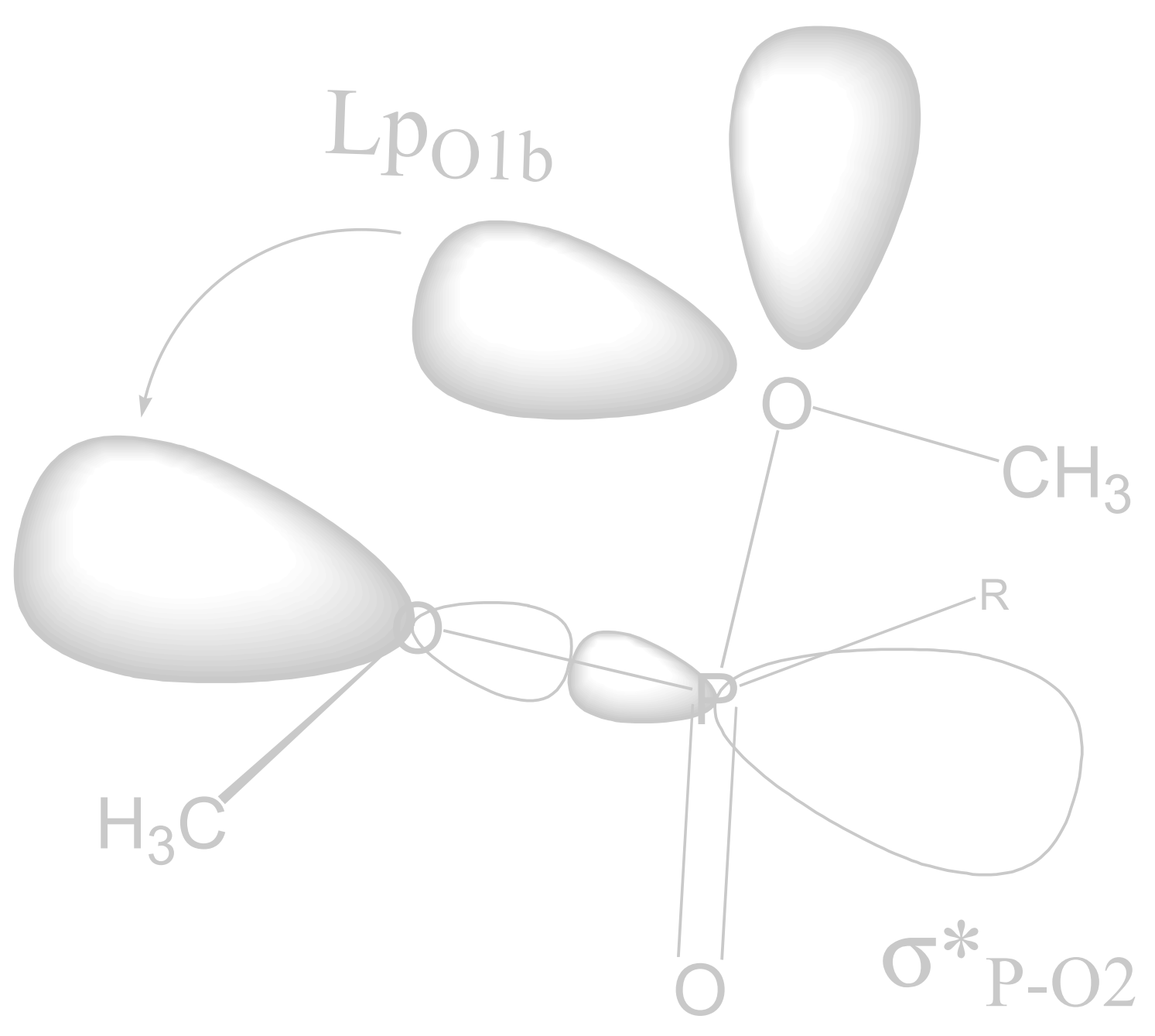





\section{Acerca de la estructura de los fosfatos de trimetilo y compuestos relacionados}

En los compuestos OPs se encuentran representados una variedad de interesantes modelos para estudios estructurales. Además el estudio de la estructura y la dinámica conformacional de estos compuestos, está muy relacionado al entendimiento de distintos sistemas biológicos, al formar parte componente, por ejemplo, de ácidos nucleicos y fosfolípidos. La bioquímica y fisiología de las membranas celulares y del material genético (ADN y ARN), dependen en forma crítica del comportamiento de los ésteres de fosfato. ${ }^{(1)}$ Menos esfuerzos se han dedicado también al estudio conformacional de los fosfatos en su papel como xenobióticos, ${ }^{(2)}$ en donde su actividad como inhibidores enzimáticos tal vez sea el aspecto más relevante.

Se cuenta con muchas publicaciones que entregan una gran cantidad de datos experimentales y teóricos a partir de los cuales se intenta una descripción aproximada de características estructurales y espectroscópicas en distintos contextos. Son muy interesantes los trabajos que en este

${ }^{1}$ (a) Guan, Y., Wurrey, C.J. and Thomas, G.J. Vibrational analysis of nucleic acids. I. The phosphodiester group in dimethyl phosphate model compounds: $\left(\mathrm{CH}_{3} \mathrm{O}\right)_{2} \mathrm{PO}_{2}^{-},\left(\mathrm{CD}_{3} \mathrm{O}\right)_{2} \mathrm{PO}_{2}^{-}$, and $\left({ }^{13} \mathrm{CH}_{3} \mathrm{O}\right)_{2} \mathrm{PO}_{2}^{-}$, Biophys.J. 66 (1): 225-235 (1994). (b) Guan, Y. and Thomas, G.J. Vibrational analysis of nucleic acids. V. Force field and conformation-dependent modes of the phosphodiester backbone modeled by diethyl phosphate, Biophys.J. 71 (5): 2802-2814 (1996). (c) Yuhasz, S.C., Ts'o, P.O.P. and Kan, L.S. NMR studies on ${ }^{31} \mathrm{P}$ relaxation mechanisms in trimethyl phosphate as a model for nucleic acid phosphodiester linkage, Bull. Inst. Chem., .Acad.Sin. 39: 91-101 (1992). (d) Rasadkina, E.N., Predvoditelev, D.A. and Nifant'ev, E.E. Amidophosphate glycolic phospholipids, Bioorg.Khim.18 (2): 302-304 (1992).

2 (a) Hosea, N.A., Berman, H.A. and Taylor, P. Specificity and orientation of trigonal carboxyl esters and tetrahedral alkylphosphonyl esters in cholinesterases, Biochem. 34 (36): 11528-11536 (1995). (b) Bernard, P.P. et al. 3D Model of the Acetylcholinesterase Catalytic Cavity Probed by Stereospecific Organophosphorous Inhibitors, J.Mol.Model. 4: 323-334 (1998). (c) Bernard, P.P. et al. A CoMFA Study of Enantiomeric Organophosphorus Inhibitors of Acetylcholinesterase. J.Mol.Model. 6: 618-629. (2000). (d) Hirashima, A., Kuwano, E. and Eto, M. Docking study of enantiomeric fonofos oxon bound to the active site of Torpedo californica acetylcholinesterase, Bioorg.Med.Chem. 8(3): 653-656 (2000). 
sentido y a lo largo de más de dos décadas llevó adelante David Gorenstein ${ }^{(3)}$ quién, mediante el auxilio de cálculos teóricos y desarrollando medidas de espectroscopía de resonancia magnética nuclear, estudió el comportamiento del los ésteres fosfóricos como componente estructural de los nucleótidos y ácidos polinucleicos.

El abordaje más sencillo para el estudio de las características conformacionales fundamentales, implica considerar pequeñas moléculas modelo de cuyo análisis puedan desprenderse conclusiones lo suficientemente generalizables. En este sentido, los calcogenofosfatos de dialquilo y calcogenofosfatos de trialquilo, con sustituyentes metilo y etilo, son los modelos más estudiados. En este trabajo utilizaremos como modelo a los calcogenofosfatos de trimetilo: fosfato de trimetilo (TMP), fosfotioato de O,O,Otrimetilo (TMSP) y fosfoselenoato de O,O,O-trimetilo (TMSeP). Estas moléculas se caracterizan por poseer un alto grado de simetría, propiedad deseable para comenzar con un análisis del comportamiento conformacional.

\section{Alternativas conformacionales}

La nomenclatura usualmente utilizada para la descripción ángulos diedros que determinan las características geométricas de los calcogenofosfatos de trimetilo se describe en la Figura 1.1. Esta nomenclatura es la que se aplicará en todos los compuestos examinados en este trabajo. El valor de la mayoría de los parámetros

\footnotetext{
${ }^{3}$ (a) Gorenstein, D.G. and Kar, D. ${ }^{31} \mathrm{P}$ chemical shifts in phosphate diester monoanions. Bond angle and torsional angle effects, Biochem. Biophys. Res. Commun. 65 (3): 1073-1080 (1975). (b) Gorenstein, D.G. Dependence of ${ }^{31} \mathrm{P}$ chemical shifts on oxygen-phosphorus oxygen bond angles in phosphate esters, J.Am.Chem.Soc. 97(4): 898-900 (1975). (c) Gorenstein, D.G. et al. Conformational study of cyclic and acyclic phosphate esters. CNDO/2 calculations of angle strain and torsional strain, J.Am.Chem.Soc. 98(7): 1668-1673 (1976). (d) Gorenstein, D.G. et al. Temperature dependence of the ${ }^{31} \mathrm{P}$ chemical shifts of nucleic acids. A probe of phosphate ester torsional conformations, Biochem. 15 (17): 3796-3803 (1976). (e) Gorenstein, D.G., Luxon, B.A. and Findlay, J.B. The torsional potential for phosphate diesters. The effect of geometry optimization in CNDO and ab initio molecular orbital calculations, Biochim.Biophys.Acta 475 (1): 184-190 (1977). (f) Gorenstein, D.G. and Kar, D. Effect of bond angle distortion of torsional potentials. Ab initio and $\mathrm{CNDO} / 2$ calculations on dimethoxymethane and dimethyl phosphate, J.Am.Chem.Soc. 99 (3): 672-677 (1977). (g) Gorenstein, D.G. ${ }^{31}$ P NMR of enzyme complexes: bound ligand structure, dynamics, and environment, Methods.Enzymol. 177: 295-316 (1989). (h) Gorenstein, D.G. Conformation and dynamics of DNA and protein-DNA, Chem.Rev. 94 (5): 1315-1338 (1994).
}

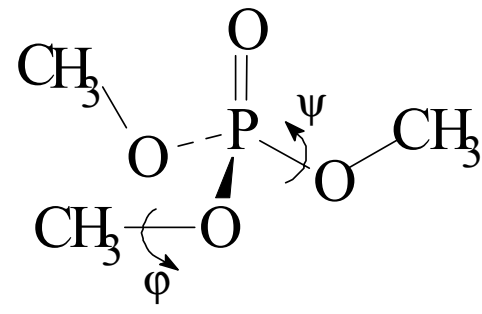

Figura 1.1 : Ángulos diedros correspondientes a la rotación de enlaces $\mathrm{P}-\mathrm{O}$ y O-C. 


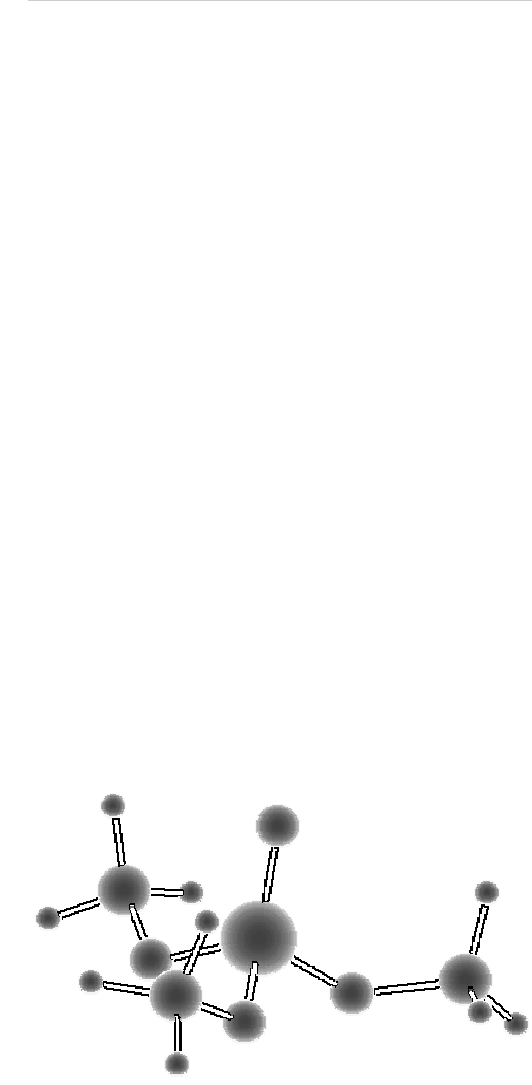

geométricos varía dentro de rangos muy estrechos, conservando formas concordantes con el tipo de hibridación orbital para cada uno de los enlaces, de manera que no son relevantes en el momento de describir la geometría global de cada confórmero. La excepción corresponde a los valores posibles de $\varphi$, que variando en un amplio rango, no determinan modificaciones energéticas importantes.

Sin embargo, las distintas conformaciones de los trimetil calcogenofosfatos dependen de la rotación independiente de los ángulos diedros $\psi$. Se ha reportado la preferencia de las posiciones gauche por sobre las trans, en un gran número de compuestos $\mathrm{OPs}$ con grupos $\mathrm{P}=\mathrm{O}, \mathrm{P}=\mathrm{S}$ y $\mathrm{P}=\mathrm{Se}^{(4)}$ Cada uno de los confórmeros se describen según la simetría que presentan. Aquellos de más relevancia, desde el punto de vista de su estabilidad energética, tal como se describirá más adelante, son:

Confórmero $\mathbf{C}_{3}$ : con valores $\psi 1, \psi 2, \psi 3$ entre 10 y $90^{\circ}$. Corresponde a posiciones gauche de los tres metilos respecto al fosforilo (Figura 1.2). Desde el punto de vista energético es el más estable. Presenta degeneración energética con el

Figura 1.2: Confórmero de geometría $\mathrm{C}_{3}$. confórmero con valores $\psi 1, \psi 2, \psi 3$ de entre $-10 \mathrm{y}-90^{\circ}$.

Confórmero Cs: con valores $\psi 1, \psi 2$ entre 10 y $90^{\circ}$ y $\psi 3$ de $180^{\circ}$. Corresponde a posiciones gauche, gauche, trans (Figura 1.3).

Confórmero $\mathbf{C}_{1}$ : con valores $\psi 1$ entre 10 y $90^{\circ}, \psi 2$ de entre -10 y $-90^{\circ}$ y $\psi 3$ de $180^{\circ}$. Corresponde a posiciones gauche, -gauche, trans (Figura 1.4). Las posiciones de los metilos correspondientes a $\psi 1$ y $\psi 2$, permiten una minimización de los impedimentos estéricos, pues significan un alejamiento simétrico de estos metilos respecto del eje $\mathrm{P}=\mathrm{X}$ (X:calcógeno).

Confórmero $\mathbf{C}_{1}{ }^{\prime}$ : los valores de $\psi 1$ se encuentran entre -10 y $-90^{\circ}$ y de $\psi 2$ están entre 10 y $90^{\circ}$. Corresponde a posiciones -gauche, gauche, trans (Figura 1.4), para las cuales habría gran impedimento estérico, por la cercanía de los hidrógenos metílicos.

Confórmeros $\mathbf{C}_{3 \mathbf{v}}$ : esta simetría conformacional está representada por dos confórmeros: aquel con posiciones trans para todos los ángulos $\psi 1$ y el que dispone todos sus metilos en posición cis (Figura 1.5). Ambas son conformaciones de

\footnotetext{
${ }^{4}$ Shagidullin, R.R., Vandyukova, I.I., Nuretdinov, I.A. and Poltev, V.I. Conformation of some thio and selenophosphoryl compounds. (Mezhmol. Vzaimodeistvie Konform. Mol., Tezisy Dokl. Vses. Simp., $3^{\text {rd }}$ ), 52-53. 1976. Akad. Nauk SSSR, Nauchn. Tsentr Biol. Issled., Pushchino, USSR.
} 
fuerte impedimento estérico, aunque han sido descritos como confórmeros estables.

\section{Momento dipolar}

El momento dipolar es uno de los parámetros fisicoquímicos que se encuentra más directamente relacionado con el comportamiento conformacional de las moléculas. Su valor corresponde al vector resultante de la suma de todos los vectores locales que representan la distribución de carga de cada uno de los enlaces interatómicos de una molécula. Este parámetro ha sido frecuente estudiado en los compuestos OPs. ${ }^{(5)}$ Una interesante serie de estudios al respecto se desarrollaron en la Unión Soviética durante la década del setenta. ${ }^{(6)}$

Cuando existe flexibilidad conformacional, el momento dipolar de una molécula neutra corresponderá a una resultante proporcional de la polaridad de los confórmeros teóricamente coexistentes ponderados según su abundancia relativa. Esto es expresado en la Ecuación 1.a, en donde $\mu_{\exp }$ corresponde al valor neto resultante de la ponderación de los

5 (a) George, L., Viswanathan, K.S. and Singh, S. Ab initio study of trimethyl phosphate: conformational analysis, dipole moments, vibrational frecuencies, and barriers for conformer interconversion, J.Phys.Chem.A. 101 (13): 2459-2464 (1997). (b) Durig, J.R. et al. Spectra and structure of organophosphorus compounds. XL. microwave, infrared, and raman spectra, dipole moment, $\mathrm{r}_{0}$ structure, conformational stability and vibrational assignment of isopropylisofluorophosphine, J.Phys.Chem. 93 (9): 3492-3503 (1989). (c) Chatterjee, K.K. and Durig, J.R. Spectra and structure of organophosphorus compounds: XLIX. Microwave, infrared, and Raman spectra, electric dipole moment, molecular structure, and $a b$ initio calculations of dimethylphosphonothioic fluoride, Struct.Chem. 5 (4): 239-253 (1994). (d) Becker, U. et al. Dielectric relaxation of trimethylphosphate in its pure liquid state and in mesitylene solution, Z.Phys.Chem.(Munich.). 191 (2): 251-258 (1995).

${ }^{6}$ (a) Matrosov, E.I., Petov, G.M. and Kabachnik, M.I. Determination of the dipole moment of the free electron pair of trivalent phosphorus and analysis of its dependence on the geometry of the molecule, J.Struct.Chem. (transl. Zhurnal Struktornoi Khimii) 15 (2): 229-233 (1974). (b) Ishmaeva, E.A. et al. Dipole moments of organophosphorus compounds. XII. Conformational isomerism of acyclic derivatives of phosphorus dithio acids, Zh.Obshch.Khim. 44(12): 2625-2628 (1974). (c) Katolichenko, V.I. et al. Dipole moments and IR spectra of some compounds with a phosphorus-sulfur double bond, Vopr.Stereokhim. 4: 98-107 (1974). (d) Ishmaeva, E.A. et al. Conformational states of some derivatives of phosphorus dithio acids, Primen. Konform. Anal. Sint. Nov. Org. Veshchestv., Mater. Resp. Konf. Din. Stereokhim. Konform. Anal. Meeting Date 1973, 79-88 (1975). (e) Shagidullin, R.R., Vandyukova, I.I. and Nuretdinov, I.A. Dipole moments of a series of selenophosphoryl compounds, Izv.Akad.Nauk.SSSR., Ser.Khim. 6: 1407-1409 (1978).

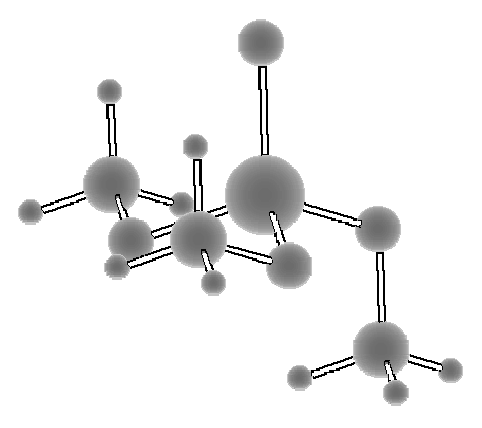

Figura 1.3 : Confórmero de geometría Cs. 


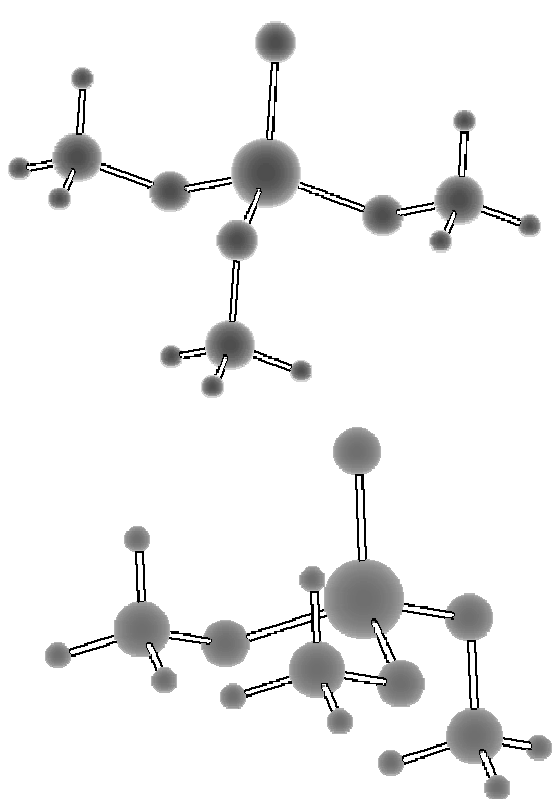

Figura 1.4 : Confórmeros de geometría $\mathrm{C}_{1}$ y $\mathrm{C}_{1}{ }^{\prime}$. momentos dipolares individuales $\mu_{i}$ de cada uno de los respectivos confórmeros coexistentes en una proporción $\mathrm{P}_{\mathrm{i}} \cdot{ }^{(7)}$

Esta ecuación puede utilizarse en ambos sentidos, es decir, por un lado es posible estimar el momento dipolar neto a partir de los momentos dipolares individuales teóricos de cada uno de todos los confórmeros predichos, pesados por las proporciones de abundancia. Las abundancias proporcionales $\mathrm{P}_{\mathrm{i}}$, se establecerán a una temperatura determinada $(\boldsymbol{T})$ mediante el uso de la ecuación de Boltzmann (Ecuación 1.b), si se conoce la energía relativa (Ei) de cada uno de los confórmeros y el grado de degeneración de cada uno de ellos $\left(A_{i}\right)$

Según el procedimiento inverso, se podrá estimar la proporción de confórmeros propuestos, a partir del valor experimental del momento dipolar. En calcogenofosfatos de trialquilo de distinta naturaleza, se ha observado que el comportamiento conformacional evidenciado en el momento dipolar resultante, no depende de la longitud de la cadena alcohólica que esterifica al fósforo. ${ }^{(6 b)}$

En el caso de los calcogenofosfatos de trimetilo, el vector dipolar para el enlace carbono - oxígeno se encuentra en dirección $\mathrm{C} \rightarrow \mathrm{O}$. Por otro lado, en el enlace fósforo calcógeno $(\mathrm{X})$ tiene una dirección $\mathrm{P} \rightarrow \mathrm{X}$. Por ello, la presencia de metilos en posiciones gauche tienden en general a disminuir el momento dipolar de la molécula, mientras que los metilos en posiciones trans, tienen el efecto contrario.

\section{Comportamiento espectroscópico vibracional}

$$
\mu_{\exp }=\left(\sum_{\text {Ecuación 1.a }} \mu_{i}^{2} P_{i}\right)^{1 / 2}
$$

La espectroscopía vibracional infrarroja, durante mucho tiempo fue la técnica más poderosa para identificar coexistencia de confórmeros en moléculas particulares. Por esta razón, se han acumulado gran cantidad de espectros vibracionales de compuestos OPs. Este hecho permite desarrollar generalizaciones para los distintos modos vibracionales de estos compuestos, ${ }^{(8)}$ para de esta manera

\footnotetext{
7 Raevsky, O.A. Conformational analysis of organophosphorus compounds by means of IR spectroscopy and dipole moments methods, J.Mol.Struc. 19: 275-287 (1973).

${ }^{8}$ (a) Chittenden, R.A. and Thomas, L.C. Characteristic infrared absortion frecuencies of organophosphorus compounds: I, Spectrochim.Acta. 20: 467-487 (1964). (b) II, Spectrochim.Acta. 20: 489-502 (1964). (c) III, Spectrochim.Acta. 20: 1679-1696 (1964). (d) IV, Spectrochim.Acta. 21: 861-876 (1965).
} 
identificar fácilmente ciertas características del comportamiento espectroscópico vibracional de nuevas moléculas, con manifestaciones de coexistencia conformacional.

Tempranamente se identifican en más de un centenar de compuestos OPs la presencia de dobletes, que se asocian a la coexistencia de confórmeros en la banda correspondiente al modo de estiramiento $\mathrm{P}=\mathrm{O}$. Dicho doblete se caracteriza por una diferencia de $50 \mathrm{~cm}^{-1} \mathrm{o}$ menos en oxofosforados.

En fosfotioatos se identifica un doblete análogo asociado al estiramiento $\mathrm{P}=\mathrm{S} .{ }^{(9)}$ Este modo vibracional se encontró que depende linealmente de la suma de las electronegatividades de los sustituyentes del fósforo. ${ }^{(6 c)}$ Por otro lado, es posible derivar la frecuencia de estiramiento $\mathrm{P}=\mathrm{X}$ para compuestos en donde $\mathrm{X}=\mathrm{O}$ y $\mathrm{S}$, a partir de la estructura molecular y electrónica de los compuestos, con un buen índice predictivo. ${ }^{(10)}$

Se argumenta que la banda de vibración asignada al estiramiento $\mathrm{P}=\mathrm{O}$ no corresponde en forma pura a ese modo vibracional sino también, aunque en menor medida, a estiramiento de enlaces $\mathrm{P}-\mathrm{O}$, como así también a la deformación de los ángulos $\mathrm{O}-\mathrm{P}=\mathrm{O}, \mathrm{O}-\mathrm{P}-\mathrm{O}$ y $\mathrm{P}-\mathrm{O}-\mathrm{C},{ }^{(11)} \mathrm{de}$ manera que las variantes conformacionales que involucren a estos parámetros geométricos se manifestarán en la banda asignada al estiramiento $\mathrm{P}=\mathrm{O}$. El mismo argumento se puede aplicar para el desdoblamiento de la banda de estiramiento en fosfotioatos y fosfoselenoatos.

La menor complejidad que en general se manifiesta en los espectros Raman de los compuestos OPs, permite que esta técnica resulte muy adecuada para complementar la interpretación de los espectros infrarrojos ${ }^{(12)}$ y obtener información vibracional que no es posible resolver mediante

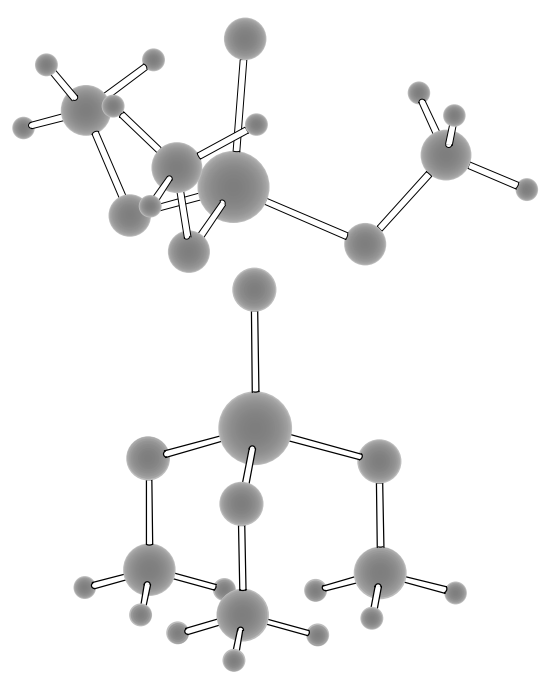

Figura 1.5: Confórmeros de geometría $\mathrm{C}_{3 \mathrm{v}}$.

\footnotetext{
${ }^{9}$ Wan, J. and Zhan, C.G. Maximum bond order hybrid orbital calculations of the $\mathrm{P}=\mathrm{S}$ stretching frequencies for organophosphorus compounds. Spectrochim. Acta (51A(10)):1665-1669 (1995).

${ }^{10}$ Wan, J. and Zhan, C.G. Generalized correlation of $\mathrm{P}=\mathrm{O}$ and $\mathrm{P}=\mathrm{S}$ bond stretching vibrational frequencies with electronic structure in organophosphorus compounds, Phosphorus, Sulfur and Silicon.and the Related.Elements. 157: 67-85 (2000).

${ }^{11}$ Popov, E.M., Kabachnik, M.I. and Mayants, L.S. Vibrational spectra of organophosphorus compounds. Russ. Chem. Revs. 30 (7): 362-377 (1961).

12 Baudler,M. Über dir Ramanspektren von einiegen Estern der phosphoriden Säure und der phosphosäure, Z.Elektrochem. 59 (3): 173184 (1955).
}

Ecuación 1.b

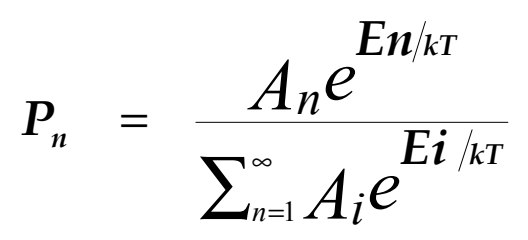


un espectro vibracional infrarrojo de baja resolución. Por esta razón se ha utilizado con éxito en el análisis cualitativo en matrices complejas de compuestos OPs. ${ }^{(13)}$ En particular, en los trabajos de Durig se ha acumulado mucha información correlacionada de espectros Raman de compuestos OPs pentavalentes y de estados de oxidación menores. ${ }^{(14)}$

${ }^{13}$ Alak, A.M. and Vo-Dinh, T. Surface-enhanced Raman spectrometry of organophosphorus chemical agents, Anal.Chem. 59 (17): 2149-2153 (1987).

${ }^{14}$ Entre los mas importantes: (a) Durig, J.R. and Diyorio, J.S. Vibrational spectra and structure of organophosphorus compounds. VII. IR and Raman spectra of $\left(\mathrm{CH}_{3}\right)_{3} \mathrm{P}=\mathrm{S}$, J.Mol.Struc.3: 179-190 (1969). (b) Durig, J.R. et al. Spectra and structure of organophosphorus compounds. XXII. Microwave, infrared, and raman spectra, structure, vibrational assignment, and normal-coordinate analysis of methylphosphonothioic difluoride, Inorg.Chem. 22 (26): 4134-4141 (1983). (c) Durig, J.R. et al. Spectra and structure of organophosphorus compounds - XXXVII. Infrared and Raman spectra, conformation stability, vibrational assignment and normal coordinate analysis of ethyldichlorophosphine -do and -d5, Spectrochim.Acta.Part.A. 44 (9): 911-928 (1988). (d) Durig, J.R. et al. Spectra and structure of organophosphorus compounds. XL. microwave, infrared, and raman spectra, dipole moment, r0 structure, conformational stability and vibrational assignment of isopropyliso fluorophosphine, J.Phys.Chem. 93 (9): $3492-3503$ (1989). (e) Durig, J.R. and Chatterjee, K.K. Spectra and structure of organophosphorus molecules. XXXIX. Microwave, Raman, and infrared spectra of trimethyl phosphine sulfide and three deuteriated species, Inorg.Chem. 28: 298-304 (1989). (f) Durig, J.R. et al. Spectra and structure of organophosphorus compounds. XLVIII. Raman and infrared spectra, conformational stability, vibrational assignments and ab initio calculations of dimethyl (methylthio) phosphine, J.Raman.Spectrosc. 23 (2): 107-124 (1992). (g) Durig, J.R., Hizer, T.J., and Harlan, R.J. Spectra and structure of organophosphorus compounds. XLIV. Conformational stability, barriers to internal rotation, vibrational assignment, and ab initio calculations for ethylphosphonic difluoride, J.Phys.Chem. 96 (2): $541-548$ (1992). (h) Chatterjee, K.K. and Durig, J.R. Spectra and structure of organophosphorus compounds: XLIX. Microwave, infrared, and Raman spectra, electric dipole moment, molecular structure, and $a b$ initio calculations of dimethylphosphonothioic fluoride, Struct.Chem. 5 (4): 239-253 (1994). (i) Durig, J.R., Daeyaert, F.F.D. and van der Veken, B.J. Spectra and structure of organophosphorus compounds. L. Raman and infrared spectra, conformational stability, $a b$ initio calculations and vibrational assignment of fluoromethylphosphonic dichloride, J. Raman. Spectrosc. 25 (11): 869-886 (1994). (j) Durig, J.R., Robb, J.B.I. and Little, T.S. Spectra and structure of organophosphorus compounds. LII. Raman and infrared spectra, conformational stability, and $a b$ initio calculations for methoxydichlorophosphine, J.Mol.Struc. 328: 37-54 (1994). (k) Durig, J.R. et al. Spectra and structure of organophosphorus compounds. LI. IR and Raman spectra, conformational stability, barriers to internal rotation, vibrational assignment, and $a b$ initio calculations of n- propylphosphine, J.Mol.Struc. 327 (1): 23-53 (1994). (l) Durig, J.R. and Gounev, T.K. Spectra and structure of organophosphorus compounds. LIII. Infrared and Raman spectra, conformational stability, vibrational assignment and $a b$ initio calculations of n-propyldichlorophosphine, J.Mol.Struc. 350 (1): 19-42 (1995). (m) Durig, J.R. et al. Spectra and structure of organophosphorus compounds. LVI. Vibrational spectra, 


\section{Espectroscopía de resonancia magnética nuclear}

La espectroscopía RMN constituye una poderosa metodología, adecuada para el estudio de características de una molécula en términos de las posiciones relativas de los átomos dentro de la misma. En la medida que los equipos fueron adquiriendo mayor resolución, fue posible la descripción de nuevos fenómenos y correlaciones entre los parámetros de resonancia magnética $\mathrm{y}$ otras variables fisicoquímicas. Existe una acumulación importante de información espectroscópica de RMN. Ésta da cuenta de una gran variedad de propiedades fisicoquímicas, como la estructura molecular, ${ }^{(15)}$ el comportamiento en soluciones, ${ }^{(16)}$ la reactividad química ${ }^{(17)}$ y el comportamiento en interfases y

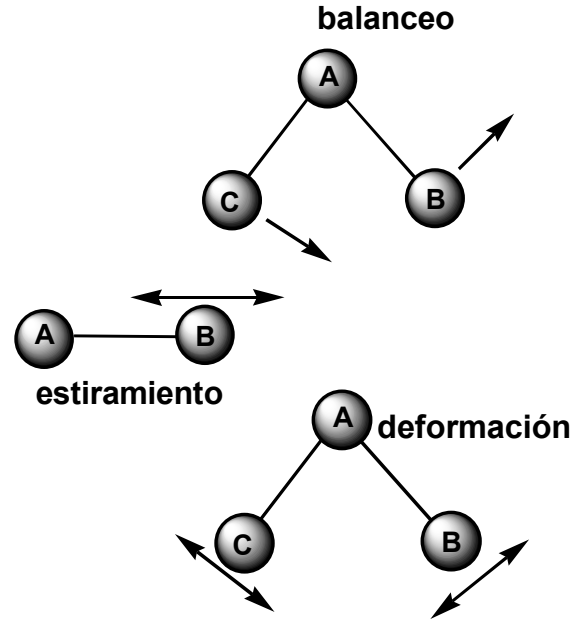

Figura 1.6a: Modos normales de vibración.

conformational stability, ab initio calculations, and vibrational assignment of fluoromethyl phosphonic difluoride, Chem.Phys. 201: 363-380 (1995). (n) Durig, J.R. and Robb, J.B. Spectra and structure of organophosphorus compounds. LVII. Raman and infrared spectra, conformational stability, and ab initio calculations for methoxydifluorophosphine, J.Mol.Struc. 375 (1-2): 53-66 (1996).

${ }^{15}$ (a) Muller, N., Lauterbur, P.C. and Goldenson, J. NMR spectra of phosphorus compounds, J.Am.Chem.Soc. 78: 3557-3561 (1956). (b) Jones, R.A. and Katritsky, A.R. Kermagnetische Resonanzspektroskopie des Phosphors, Angew.Chem.(Internat.). 1 (2): 60-68 (1962). (c) Attou, M., Moriyama, H. and Azzouz, A. NMR study of some organophosphorus compounds, Bul. Inst. Politeh. Iasi., Sect.2.: Chim. Ing. Chim. 41 (1-4): 71-77 (1995).

${ }^{16}$ (a) Skvortsov, N.K. et al. Study of the protonation of phosphoryl and thiophosphoryl compounds by a NMR method, Zh.Obshch.Khim. 46 (3): 521-531 (1976). (b) Paasivirta, J. et al. Three nuclei NMR spectroscopy of dimethoate compounds. A large solvent effect on the phosphorus-31sulfur-carbon-proton, Org.Magn.Reson. 9 (12): 708-711 (1977). (c) Dean, P.A.W. NMR studies of the solvation of phosphorus(V) selenides, 1,2-bis (diphenylphosphino) ethane, and tris (dimethylamino) phosphine telluride by sulfur dioxide, Can.J.Chem. 57 (7): 754-761 (1979). (d) Eaton, G. et al. Infrared and NMR spectroscopic studies on the solvation of trimethyl phosphate and dimethyl methylphosphonate, J.Chem.Soc.,Faraday.Trans. 88 (24): 3527-3531 (1992). (e) Streck, R. and Barnes, A.J. Solvent effects on infrared, ${ }^{13} \mathrm{C}$ and ${ }^{31} \mathrm{P}$ NMR spectra of trimethyl phosphate. Part 1. Single solvent systems, Spectrochim.Acta 55A (5): 1049-1057 (1999). (f) Streck, R. and Barnes, A.J. Solvent effects on infrared, ${ }^{13} \mathrm{C}$ and ${ }^{31} \mathrm{P}$ NMR spectra of trimethyl phosphate part 2. Binary solvent systems, Spectrochim. Acta 55A (5): 1059-1076 (1999).

${ }^{17}$ (a) Muller, N. and Goldenson, J. Rapid analysis of reaction mixtures by NMR spectroscopy, J.Am.Chem.Soc. 78: $5182-5183$ (1956). (b) Fluck, E. and Binder, H. NMR of phosphorus compounds. XVI. Reactions of phosphorus (III) compounds with sulfur dioxide, Z. Anorg. Allg. Chem. 354 (3-4): 139-148 (1967). (c) Pudovik, A.N. and Gareev, R.D., Stereochemistry of phosphazines and their reaction products, Zh. Obshch. Khim. 46 (5): 945-953 (1976). (d) Vandenborre, M.T., Maquet, J. and Livage, J. ${ }^{31} \mathrm{P}$ NMR study of phosphoric ester hydrolysis, J.Chim.Phys. 91 (3): 329-339 (1992). 
de formación de aductos ${ }^{(18)}$ de compuestos OPs respecto de los núcleos ${ }^{1} \mathrm{H},{ }^{13} \mathrm{C}$ y ${ }^{31} \mathrm{P}$, suficiente para intentar generalizaciones.

Desde el punto de vista de las aplicaciones de la espectroscopía RMN, se ha utilizado esta técnica para el análisis cualitativo de compuestos OPs en matrices complejas con fines tecnológicos ${ }^{(19)}$ y de investigación bioquímica. ${ }^{(20)}$ Pero las medidas de RMN se vuelven ineficaces en el momento de estudiar el comportamiento conformacional, puesto que los fenómenos que fundamentan la medida, ocurren en tiempos algunos órdenes de magnitud por encima de los tiempos de interconversión conformacional. Es decir, la técnica es demasiado "lenta" para la detección de formas conformacionales que se interconvierten "rápido".

Uno de los valores de utilidad en el estudio cualitativo de las estructuras variables de los compuestos de fósforo, es la constante de acoplamiento espín-espín fósforohidrógeno, de segundo $\left({ }^{2} J_{\mathrm{P}-\mathrm{H}}\right)$ y tercer orden $\left({ }^{3} J_{\mathrm{P}-\mathrm{H}}\right)$, para fosfonatos $\mathrm{y}$ fosfatos respectivamente. Considerando series

${ }^{18}$ (a) Screttas, C.G. et al., ${ }^{31} \mathrm{P}$ contact shifts as a measure of weak ligand affinities. Interaction between alkali metal fluorenone radical anions and certain phosphorus(III or V) ligands, Magn.Reson.Chem. 36 (9): 656-662 (1998). (b) Harris, R.K. et al. ${ }^{31} \mathrm{P}$ NMR studies of adsorption onto activated carbon, Carbon. 37 (9): 1425-1430 (1999).

${ }^{19}$ (a) Keith, L.H., Garrison, A.W. Alford, A.L. The high resolution NMR spectra of insecticides I. Organophosphorus pesticides, J. Assoc. Off. Anal. Chem. 51: 1063-1094 (1968). (b) Keith, L.H., Alford, A.L. Supplementary interpretations of the NMR spectra of phosphorus pesticides, Anal.Chim.Acta. 44 (2): 447-448 (1969). (c) Greenhalgh, R., Shoolery, J.N. The use of ${ }^{31} \mathrm{P}$ NMR for the rapid analysis of fenitrothion formulations and technical products, Publ.Environ.Secr., Natl. Res. Counc. Can. (1977). (d) Greenhalgh, R. et al. ${ }^{31} \mathrm{P}$ NMR analysis of technical organophosphorus insecticides for toxic contaminants, J.Agric.Food Chem. 31 (4): 710-713 (1983). (e) Mortimer, R.D. and Dawson, B.A. A study to determine the feasibility of using ${ }^{31} \mathrm{P}$ NMR for the analysis of organophosphorus insecticide residues in cole crops, J.Agric.Food Chem. 39 (5): 911-916 (1991). (f) Li, H., Miao, Z. and Ruan, J. Application of ${ }^{1} \mathrm{H}$ NMR spectroscopy to direct detection of traces of drugs, toxins and their metabolites in biological samples, Fenxi Ceshi Xuebao 13 (2): 14-18 (1994).

${ }^{20}$ (a) Salama, A.K. et al., Studies on the metabolism of methamidophos and acephate: synthesis and identification by proton NMR and mass spectrometry of ${ }^{14} \mathrm{C}$-acephate and some metabolites, J. Occup. Med. Toxicol. 2 (4): 399-408 (1993). (b) Segall, Y. et al. Direct observation and elucidation of the structures of aged and nonaged phosphorylated cholinesterases by ${ }^{31} \mathrm{P}$ NMR spectroscopy, Biochem. 32 (49): 1344113450 (1993). (c) Lai, K., Stolowich, N.J. and Wild, J.R. Characterization of P-S bond hydrolysis in organophosphorothioate pesticides by organophosphorus hydrolase, Arch.Biochem.Biophys. 318 (1): 59-64 (1995). 
del tipo $\mathrm{CH}_{3} \mathrm{P}(\mathrm{X}) \mathrm{Cl}_{2}$, donde $\mathrm{X}$ corresponde a un calcógeno, el valor de ${ }^{2} J_{\mathrm{P}-\mathrm{H}}$ se encuentra variando linealmente desde el $\mathrm{O}$ al $\mathrm{S}$ y al Se, ${ }^{(21)}$ sin encontrarse en este caso correlaciones adecuadas con los números de onda vibracionales.

Los valores de ${ }^{3} J_{\mathrm{P}-\mathrm{H}}$, permiten distinguir adecuadamente el fósforo trivalente del pentavalente, ${ }^{(22)}$ reportándose valores de entre 10,2 y 11,4 Hz para moléculas con fósforo pentavalente. ${ }^{(23)}$ Se han correlacionado con cierto éxito con variables fisicoquímicas menos sensibles como la polaridad de enlace, el ángulo $\Psi$ y las conformaciones preferenciales de distintos fosfatos, fosfotioatos, fosfamidatos y fosfamidotioatos. ${ }^{(24)}$ En particular, la dependencia de ${ }^{3} J_{\mathrm{P}-\mathrm{H}}$ con el ángulo diedro $\Psi$ se ha descrito según la Ecuación 1.c, en donde $\mathrm{A}, \mathrm{B}$ y $\mathrm{C}$ corresponden a constantes definidas para cada ambiente molecular. ${ }^{(23)}$ En sistemas con restricciones estructurales, incluso es posible observar una dependencia de acoplamientos espín-espín de cuarto orden $\left({ }^{4} J_{\mathrm{P}-\mathrm{H}}\right)$ respecto de los ángulos de torsión. ${ }^{(25)}$

Finalmente, se han hecho intentos para la estimación teórica de estos valores. ${ }^{(26)}$ En ésteres de fosfato con restricciones en la rotación del ángulo diedro $\Psi$ se observa una fuerte dependencia del corrimiento químico del ${ }^{31} \mathrm{P}$ respecto del ángulo $\mathrm{O}-\mathrm{P}-\mathrm{O},{ }^{(27)}$ utilizándose como parámetro de conformación de ésteres de fosfatos,

\footnotetext{
${ }^{21}$ Freymann, R. NMR and infrared spectroscopy, Nucl. Magn. Reson. Chem., Proc.Symp. Meeting Date 1964, 13-34 (1965).

${ }^{22}$ Shaw, R.A., Woods, M. Phosphorus-sulfur compounds. II. Synthesis and some properties of trialkyl and triaryl trithiophosphites. Comparison of ${ }^{3} J_{\mathrm{P}-\mathrm{H}}$ values in trialkylthio derivatives of phosphorus(III) and phosphorus(V), and of their trialkoxy analog, Phosphorus 1 (4): 191-197 (1972).

${ }^{23}$ Gorenstein, D.G. Phosphorus-31 NMR. Principles and Applications, Academic Press, New York (1984).

${ }^{24}$ Wang, Z. et al. Phosphorus-proton spin-spin coupling constants ${ }^{3} J_{\mathrm{P}-\mathrm{H}}$ of organophosphorus compounds, Huaxue Tongbao. 11: 647-650 (1981).

${ }^{25}$ Hassner, A. and Galle, J.E. Stereochemistry. LXIX. Ring opening of aziridine phosphonates. Correlation of structure, NMR spectra, and reactivity, J.Org.Chem. 41 (13): 2273-2276 (1976).

${ }^{26}$ (a) Safiullin, R.K., Aminova, R.M. and Samitov, Y.Y. LCAO MO calculation of the ${ }^{1} J,{ }^{2} J$ and ${ }^{3} J$ spin-spin coupling constants in the $\mathrm{CH}_{3} \mathrm{PH}_{2}$ molecule, J.Struct.Chem. (transl.Zhurnal Struktornoi Khimii) 15 (5): 798801 (1974). (b) Safiullin, R.K., Aminova, R.M. and Samitov, Y.Y. Stereochemistry of organophosphorus compounds V. MO LCAO calculation of spin-spin interaction constants in $\left(\mathrm{CH}_{3}\right)_{3} \mathrm{PO}$, $\left(\mathrm{CH}_{3}\right)_{3} \mathrm{P}(\mathrm{O}) \mathrm{OH}, \mathrm{CH}_{3} \mathrm{P}(\mathrm{O}) \mathrm{Cl}_{2}, \mathrm{CH}_{3} \mathrm{PH}_{2}$, and $\left(\mathrm{CH}_{3}\right) \mathrm{P}$ molecules, J.Struct. Chem. (transl.Zhurnal Struktornoi Khimii) 16 (1): 36-41 (1975).

${ }^{27}$ Gorenstein, D.G. Dependence of ${ }^{31} \mathrm{P}$ chemical shifts on oxygen phosphorus - oxygen bond angles in phosphate esters, J.Am.Chem.Soc. 97 (4): 898-900 (1975).
}

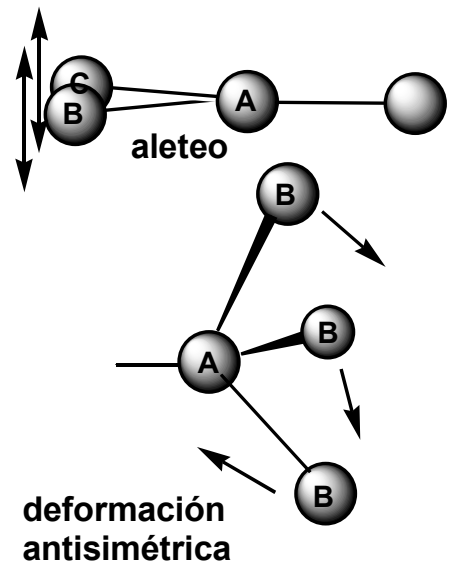

Figura 1.6b: Modos normales de vibración. 


\section{${ }^{3} J_{\mathrm{P}-\mathrm{H}}=\mathrm{A} \cos ^{2} \Psi+\mathrm{B} \cos \Psi+\mathrm{C}$}

\section{Ecuación 1.c}

entendiéndose que las conformaciones gauche dan lugar a corrimientos químicos menores para el ${ }^{31} \mathrm{P} .{ }^{(28)}$ Tomando en cuenta las variables involucradas, se han descrito reglas empíricas que dan cuenta de la magnitud prevista del corrimiento químico del ${ }^{31} \mathrm{P}{ }^{(29)}$ además de aproximar estimaciones teóricas en función de las estructuras electrónicas, ${ }^{(30)}$ encontrándose buenas correlaciones entre estos datos y los parámetros de resonancia magnética nuclear y los espectros vibracionales. ${ }^{(31)}$

\section{Comparando compuestos oxofosforados}

fosfotioatos, se verifica que los corrimientos químicos del ${ }^{31} \stackrel{\mathrm{y}}{\mathrm{P}}$ son función fundamentalmente de los sitios de protonación de la molécula y el número de átomos de oxígeno y azufre unidos directamente al fósforo. ${ }^{(32)}$ Por otro lado en fosfotioatos se han identificado efectos isotópicos respecto del ${ }^{34} \mathrm{~S}$ en los corrimientos químicos del ${ }^{31} \mathrm{P} .{ }^{(33)}$

\section{Reactividad relativa}

Mediante medidas calorimétricas, complementadas por espectroscopía RMN, se ha mostrado que, dentro de la serie de calcogenofosfatos de trimetilo, y en presencia de $\mathrm{BF}_{3}$, se produce la isomerización al correspondiente

\footnotetext{
${ }^{28}$ Gorenstein, D.G. et al. Temperature dependence of the ${ }^{31} \mathrm{P}$ chemical shifts of nucleic acids. A probe of phosphate ester torsional conformations, Biochem. 15 (17): 3796-3803 (1976).

${ }_{29}$ (a) Hu, W. ${ }^{31} \mathrm{P}$ NMR and its principle for organophosphorus compounds. I. Novel empirical rules and principle of ${ }^{31} \mathrm{P}$ NMR chemical shifts of organophosphorus compounds, Junshi. Yixue. Kexuеyuan. Yuankan. 17 (1): 1-6 (1993).

${ }^{30}$ (a) Da Silva, C. et al. Electronic structure and ${ }^{31} \mathrm{P}$ NMR chemical shift of substituted triaryl, diaryl methyl and dimethyl aryl phosphates - a semiempirical molecular orbital approach, Phosphorus, Sulfur Silicon. Relat. Elem. 131: 97-105 (1997). (b) Zhan, C.G. and Iwata, S. Theoretical prediction of the ${ }^{31} \mathrm{P}$ NMR chemical shift of the hexa-coordinate phosphorus intermediate for the phosphoryl ester exchange and $\mathrm{N} \rightarrow \mathrm{O}$ migration reactions of dimethyloxyphosphoryl-threonine, Chem.Lett. 1: 34 (1997). (c) Zhan, C.G. Theoretical prediction of ${ }^{31} \mathrm{P}$ NMR chemical shifts of intermediates in phosphoryl ester exchange and $\mathrm{N} \rightarrow \mathrm{O}$ migration reactions of dialkyloxyphosphoryl amino acid, Phosphorus, Sulfur Silicon.Relat.Elem. 144-146: 655-658 (1999).

${ }^{31}$ Gregan, F. et al. Correlation analysis of IR, ${ }^{31} \mathrm{P}$ NMR and theoretical data of derivates of phosphoric and thiophosphoric acids, Chem.Pap. 52 (4): 230-234 (1998).

32 Olah, G.A. and McFarland, C.W. Organophosphorus compounds. XIII. Protonation, cleavage, and alkylation of thiophosphates and thiophosphites, J.Org.Chem. 40 (18): 2582-2587 (1975).

${ }^{33}$ Bjorholm, T. and Larsson, F.C.V. ${ }^{34} \mathrm{~S}$ isotope effects on ${ }^{31} \mathrm{P}$ chemical shifts in thiophosphate esters, Mag.Res.Chem. 27 (2): 184-186 (1989).
} 
compuesto de adición $\left(\mathrm{CH}_{3} \mathrm{O}\right)_{2}\left(\mathrm{CH}_{3} \mathrm{X}\right) \mathrm{P}=\mathrm{O} \cdot \mathrm{BF}_{3}$. Estas isomerizaciones ocurren rápidamente, alcanzando el 30 al $40 \%$ del total en 6 horas y se completan en algunos días. Las entalpías de isomerización fueron medidas en $-5,5 \mathrm{kcal} / \mathrm{mol}$ para el compuesto azufrado (medido a $25^{\circ} \mathrm{C}$ ) y de $-5,0$ $\mathrm{kcal} / \mathrm{mol}$ para el compuesto de selenio (medido a $35^{\circ} \mathrm{C}$ ). ${ }^{(34)}$

Por otro lado, la hidrólisis alcalina de la serie de calcogenofosfatos de trimetilo, medida a distintas temperaturas se verifica decreciendo en el orden $\mathrm{PSe}>\mathrm{PO}>$ PS, cuando la reacción se verifica en solventes acuosos, pero decrece en el orden PSe $>$ PS $>$ PO cuando la experiencia se realiza en solventes apróticos. Este fenómeno se ha explicado indicando que un dador de protones, capaz de solvatar al grupo $\mathrm{P}=\mathrm{X}$, puede acelerar la reacción, por lo que el orden relativo de la hidrólisis en medio acuoso es proporcional a la capacidad de los compuestos de ser aceptores de protones. ${ }^{(35)}$

\section{Estrategias para el estudio conformacional}

El análisis conformacional que se presenta se basa en el trabajo realizado previamente, contextualizado o no en sistemas biológicos. Aunque se entregan resultados que son válidos para las moléculas en el vacío o en la fase líquida pura y en principio hay conclusiones extensibles a otros entornos, el objeto fundamental de este estudio es la posterior interpretación en el contexto del papel de los OPs como inhibidores enzimáticos.

Para ello se tomarán en cuenta los distintos aspectos que guardan relación con las propiedades fisicoquímicas dependientes del comportamiento conformacional, fundamentalmente propiedades energéticas, momentos dipolares, distribución espacial de orbitales y tamaños moleculares. Esto es lo que se desarrollará en los Capítulos posteriores.

\footnotetext{
${ }^{34}$ Elegant, L., Gal, J.F. and Azzaro, M. Enthalpies of isomerization of trimethyl thiophosphate and trimethyl selenophosphate in the presence of $\mathrm{BF}_{3}$, Bull.Soc.Chim.Fr. 12: 4273-4274 (1969).

35 Bel'skii,V.E. et al. Kinetics of the alkaline hydrolysis of some organoselenophosphorus compounds, Zh.Obshch.Khim. 43 (6): 12551257 (1973).
} 


\section{Comportamiento conformacional del fosfato de trimetilo}

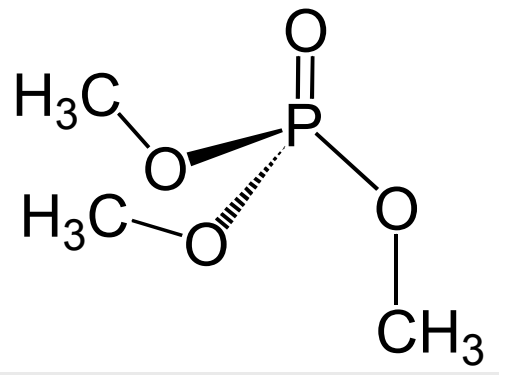

Figura 2.1: Estructura del fostato de trimetilo (TMP).
El fosfato de trimetilo (TMP) es una molécula interesante, pues corresponde al triéster fosfórico más pequeño presentando además un alto grado de simetría.

Desde el punto de vista de su toxicidad, se han desarrollado algunas experiencias en distintos modelos animales. Aunque no se ha demostrado carcinogenicidad, ${ }^{(1)}$ por exposición crónica se han descrito efectos a nivel del sistema nervioso ${ }^{(2)}$ y los tejidos hemático y muscular, ${ }^{(1)}$ aunque el efecto más notable se relaciona con una fuerte incidencia en la espermatogénesis. ${ }^{(3)}$ Organismos de regulación lo consideran teratogénico para humanos. ${ }^{(4)}$ En Argentina es impureza de declaración obligatoria en dos insecticidas. $^{(5)}$

De esta sustancia se dispone abundante información fisicoquímica. Aporta a ello su simplicidad y el hecho de servir de modelo adecuado para el estudio de sistemas químicos y biológicos más complejos. ${ }^{(6)}$ Por esta razón se cuenta con una gran cantidad de estudios respecto de sus propiedades físicas y químicas. Ellos dan cuenta de su

\footnotetext{
1 Bomhard, E.M. et al. Trimethylphosphate: a 30-month chronic toxicity/carcinogenicity study in Wistar rats with administration in drinking water, Fundam.Appl.Toxicol. 40 (1): 75-89 (1997).

2 Schaeppi, U., Krinke, G.J. and Kobel, W. Prolonged exposure to trimethylphosphate induces sensory motor neuropathy in the dog, Neurobehav.Toxicol.Teratol. 6 (1): 39-50 (1984).

3 (a) Hanna, P.J. Sterilizing effects of trimethylphosphate in Drosophila melanogaster, Aust.J.Biol.Sci. 33 (5): $563-573$ (1980). (b) Toth, G.P. et al. Effects of three male reproductive toxicants on rat cauda epididymal sperm motion, Reprod.Toxicol. 6 (6): $507-515$ (1992). (c) Cho, N.H., Park, C. Effects of dimethyl methylphosphonate (DMMP) and trimethylphosphate (TMP) on spermatogenesis of rat testis, Yonsei.Med.J. 35 (2): 198-208 (1994).

4 NIOSH, National Institute for Occupational Safety and Health; International Program on Chemical Safety and the Commission of the European Communities; ICSC \#0686 (1998).

${ }^{5}$ Para el monocrotofós y el DDVP; Resoluciones del IASCAV (actual SENASA) $\mathrm{N}^{\circ} 229 / 95 \mathrm{y} \mathrm{N}^{\circ} 230 / 95$ respectivamente.

${ }^{6}$ Yuhasz, S.C., Ts'o, P.O. and Kan, L.S.NMR studies on ${ }^{31} \mathrm{P}$ relaxation mechanisms in trimethylphosphate as a model for nucleic acid phosphodiester linkage, Bull.Inst.Chem.,.Acad.Sin. 39: 91-101 (1992).
} 
reactividad, ${ }^{(7)}$ de las características polares de la molécula, ${ }^{(8)}$ de su comportamiento en solución o en sistemas binarios, ${ }^{(9)}$ de su comportamiento en condiciones de alta energía ${ }^{(10)}$ y en interfases. ${ }^{(1)}$ Por otro lado, en diversas ocasiones se ha

7 Entre otras: (a) Ruostesuo, P., Salminen, U., and Liias, R. Thermodynamic and spectroscopic properties of phosphorus compoundscomplex formation between phosphorus compounds and various $\mathrm{OH}$ and NH proton donors, Spectrochim.Acta.Part.A. 43 (9): 1135-1140 (1987). (b) LeBreton, P.R. et al. UV photoelectron spectroscopy and ab initio characterization of valence orbital structures and conformations of neutral phosphate esters. J. Biomol. Struct. Dyn. 6 (1):199-222 (1988). (c) Lum, R.C. and Grabowski, J.J. Trimethyl phosphate: the intrinsic reactivity of carbon versus phosphorus sites with anionic nucleophiles. J. Am. Chem. Soc. 114 (22):8619-8627 (1992). (d) Bayada, A., Lawrance, G.A., Maeder, M. and Molloy, K.J. ATR-IR spectroscopy for the investigation of solution reaction kinetics: hydrolysis of trimethyl phosphate. Appl. Spectrosc. 49 (12):1789-1792 (1995). (e) Nqvist, J. et al. Mechanistic alternatives in phosphate monoester hydrolysis: what conclusions can be drawn from available experimental data? [Review article], Chemistry and.Biology. 6 (3): R71-R80 (1999).

${ }^{8}$ Las descripciones más completas se pueden encontrar en: (a) Daasch, L.W. and Smith, D.C. Infrared spectra of phosphorus compounds. Anal. Chem. 23 (6):853-868 (1951). (b) Becker, U., Wessels, V., Turky, G., Ghoneim, A. and Stockhausen, M. Dielectric relaxation of trimethyl phosphate in its pure liquid state and in mesitylene solution. Z. Phys. Chem. (Munich) 191 (2): 251-258 (1995). (c) Raevsky, O.A. Conformational analysis of organophosphorus compounds by means of IR spectroscopy and dipole moments methods. J. Mol. Struct. 19: 275287 (1973).

9 (a) Sagert, N.H. and Lee, W. The distribution of trialkylphosphates between dodecane and water, Can.J.Chem. 58 (14): 1463-1467 (1980). (b) Comor, J.J. and Kopecni, M.M. Excess and Partial Excess Molar Volumes of Mixing Trimethyl- and Triethylphosphate with Water, J.Solution.Chem. 20 (9): 945-953 (1991). (c) Abou-Hamdan, A. et al. Variable-pressure ${ }^{1} \mathrm{H}$ NMR studies of hexamethylphosphoramide and trimethyl phosphate exchange on dioxouranium(VI), Inorg.Chim.Acta 207 (1): 27-30 (1993).

${ }^{10}$ (a) Santoro, E. Fragmentation of some alkyl thiophosphate esters by electron-impact, Org.Mass Spectrom. 7 (5): $589-599$ (1973). (b) Korobeinichev, O. et al. Molecular beam mass-spectrometric study of $\mathrm{H}_{2} / \mathrm{O}_{2} / \mathrm{Ar}$ flame doped with phosphorus compounds, Appl.Free-Jet., Mol.Beam., Mass Spectrom.Sampling., Proc.: 92-97 (1994). (c) Korobeinichev, O.P. et al. The destruction chemistry of organophosphorus compounds in flames-II: structure of a hydrogen-oxygen flame doped with trimethyl phosphate, Combust.Flame 118 (4): 727-732 (1999). (d) Korobeinichev, O.P. et al. The chemistry of the destruction of organophosphorus compounds in flames. III. Destruction of dimethyl methyl phosphonate and trimethylphosphate in a flame of $\mathrm{H}_{2}$ and $\mathrm{O}_{2}$, Combustion and Flame 121: 593-609 (2000). (e) Korobeinichev, O.P. et al. Inhibition and promotion of combustion by organophosphorus compounds added to flames of $\mathrm{CH}_{4}$ or $\mathrm{H}_{2}$ in $\mathrm{O}_{2}$ and $\mathrm{Ar}$, Combustion and Flame 125: 744-751 (2001).

${ }^{11}$ (a) Harris, R.K. et al. ${ }^{31} \mathrm{P}$ NMR studies of adsorption onto activated carbon, Carbon. 37 (9): 1425-1430 (1999). (b) Kanan, S.M. and Tripp, C.P. An infrared study of adsorbed organophosphonates on silica: a 
abordado el problema de las distintas alternativas respecto de los confórmeros más estables, planteándose diversas soluciones a este problema. ${ }^{(12)}$

Se han utilizado con cierta frecuencia las medidas de resonancia magnética nuclear en el estudio de las características estructurales del TMP. En particular, las medidas de ${ }^{31} \mathrm{P}$ RMN dan cuenta de los acoplamientos ${ }^{3} J_{\mathrm{P}-\mathrm{H}}$ en valores de $5 \mathrm{~Hz}$ para compuestos del tipo $\mathrm{P}=\mathrm{O}$ en $\mathrm{OPs}$ pentavalentes. ${ }^{(13)}$ De igual manera se han utilizado las medidas ${ }^{13} \mathrm{C}$ RMN como herramienta comparativa entre organofosfatos de trialquilo y de triarilo. ${ }^{(14)} \mathrm{La}$ información entregada por las medidas de RMN se utilizó también como complemento de las medidas de espectroscopía vibracional. ${ }^{(12 a)}$ Por otro lado, se realizó el estudio del TMP en solución mediante la obtención de espectros ${ }^{13} \mathrm{C}$ RMN y ${ }^{31} \mathrm{P}$ RMN, encontrándose una correlación lineal entre los corrimientos químicos y los números de onda para un amplio rango de constantes dieléctricas del solvente utilizado, ${ }^{(15)}$ no

prefiltering strategy for the detection of nerve agents on metal oxide sensors, Langmuir. 17 (7): 2213-2218 (2001).

${ }^{12}$ El desarrollo histórico del problema fue descripto en la introducción de este trabajo. En este sentido es interesante revisar los siguientes trabajos: (a) Eaton, G., Harris, L., Patel, K. and Symons, M.C. Infrared and NMR spectroscopic studies on the solvation of trimethyl phosphate and dimethyl methylphosphonate. J. Chem. Soc. , Faraday. Trans. 88 (24): 3527-3531 (1992). (b) George, L., Viswanathan, K.S. and Singh, S. $A b$ initio study of trimethyl phosphate: conformational analysis, dipole moments, vibrational frecuencies, and barriers for conformer interconversion. J. Phys. Chem. 101A (13): 2459-2464 (1997). (c) Khetrapal, C.L., Govil, G. and Yeh, H.J. The preferred conformation(s) of trimethylphosphate as derived from RMN spectra of partially oriented molecules and potential energy calculations. J. Mol. Struct. 116: 303-311 (1984). (d) Sablinskas, V., Horn, A. and Klaeboe, P. Conformational stability of trimethyl phosphate studied by vibrational spectroscopy and ab initio calculations. J. Mol. Struct. 349: 157-160 (1995). (e) Streck, R., Barnes, A.J., Herrebout, W.A. and van der Veken, B.J. Conformational behavior of trimethyl phosphate studied by infrared spectroscopy. J. Mol. Struct. 376: 277-287 (1996). (f) Taga, K., Hirabayashi, N., Yoshida, T. and Okabayashi, H. Raman spectra and rotational isomerism of dimethyl phosphoro chloridate and trimethyl phosphate. J. Mol. Struct. 212: 157168 (1989).

${ }^{13}$ Jones, R.A. and Katritsky, A.R.Kermagnetische Resonanzspektroskopie des Phosphors, Angew.Chem.(Internat.). 1 (2): 60-68 (1962). Ver también la referencia ${ }^{(6)}$

14 Attou, M., Moriyama, H. and Azzouz, A. NMR study of some organophosphorus compounds, Bul. Inst. Politeh. Iasi., Sect.2.: Chim.Ing.Chim. 41 (1-4): 71-77 (1995).

${ }^{15}$ Streck, R. and Barnes, A.J. Solvent effects on infrared, ${ }^{13} \mathrm{C}$ and ${ }^{31} \mathrm{P}$ NMR spectra of trimethyl phosphate. Part 1. Single solvent systems, Spectrochim. Acta 55A (5): 1049-1057 (1999). 
así para sistemas binarios, donde predominan efectos asociados a la formación de puentes de hidrógeno. ${ }^{(16)}$

En la investigación conformacional del TMP, son interesantes las medidas realizadas en moléculas parcialmente orientadas. ${ }^{(12 c)}$ Por otro lado, se utilizaron medidas de ${ }^{1} \mathrm{H}$ RMN para el estudio de la interacción del TMP con actínidos. ${ }^{(9 c)}$

En uno de los primeros estudios de espectroscopía vibracional del TMP, ${ }^{(17)}$ donde se describe en detalle el espectro infrarrojo y se intenta una primera asignación completa de los modos de vibración a la mayor parte de las bandas del espectro. En esta descripción se informa la aparición de dobletes en las regiones ubicadas entre 1290 1275 y $740-755 \mathrm{~cm}^{-1}$ (Tabla 2.I) en fase líquida pura y en distintas soluciones. Sin embargo, en fase sólida en estas mismas regiones aparecen bandas únicas, de lo que se deduce que en fase líquida se manifiesta el fenómeno de polimorfismo simple, ${ }^{(12 f)}$ es decir, habría coexistencia de confórmeros.

Se reporta que la relación de intensidades relativas de los máximos de las correspondientes bandas de absorción de estos dobletes varía con la polaridad del solvente ${ }^{(18)}$ y con la temperatura de la medida ${ }^{(19)}$ planteándose la posibilidad de coexistencia de al menos dos confórmeros de energías comparables en estado líquido.

Como ya se explicó en el Capítulo anterior, dentro de las bandas más conspicuas en términos de comanifestación de confórmeros, corresponde al modo de estiramiento $\mathrm{P}=\mathrm{O}$, entre otras razones por su carácter mezcla con otros modos vibracionales, fuertemente influidos por el valor de cada uno de los ángulos diedros $\psi$. $^{(20)}$

\footnotetext{
${ }^{16}$ Streck, R. and Barnes, A.J. Solvent effects on infrared, ${ }^{13} \mathrm{C}$ and ${ }^{31} \mathrm{P}$ NMR spectra of trimethyl phosphate part 2. Binary solvent systems, Spectrochim. Acta 55A (5): 1059-1076 (1999).

${ }_{17}$ Mortimer, F.S. Vibrational assignment and rotational isomerism in some simple organic phosphates. Spectrochim. Acta 9: 270-281 (1957). Este es el primer trabajo en donde se aborda el problema de la coexistencia de confórmeros para trimetilcalcógenofosfatos.

${ }^{18}$ Los trabajos de Streck ${ }^{(12 e)}$ y Mortimer ${ }^{(17)}$ son los que aportan datos experimentales definitivos en este sentido.

19 Vidya, V., Sankaran, K. and Viswanathan, K.S. Matrix isolationsupersonic jet infrared spectroscopy: conformational cooling in trimethyl phosphate. Chem. Phys. Lett. 258: 113-117 (1996).

${ }^{20}$ Wan, J. and Zhan, C.G. Maximum bond order hybrid orbital calculations of the P:S stretching frequencies for organophosphorus compounds. Spectrochim. Acta 51A (10):1665-1669 (1995).
} 
Ya tempranamente se asignó a las absorciones que se presentan a $\sim 1290 \mathrm{~cm}^{-1}$ y $\sim 735 \mathrm{~cm}^{-1}$ al confórmero más estable y las que se presentan a $\sim 1275 \mathrm{~cm}^{-1} \mathrm{y} \sim 755 \mathrm{~cm}^{-1}$ a un confórmero de menor estabilidad (Tabla 2.I). ${ }^{(17)}$ Más adelante, en espectros obtenidos en matrices de $\mathrm{N}_{2}$ a bajas temperaturas, se resuelve la banda a mayores números de onda de $\sim 1300 \mathrm{~cm}^{-1}$ en dos bandas a $1301,7 \mathrm{~cm}^{-1}$ y 1306,5 $\mathrm{cm}^{-1}$, asumiéndose que estas corresponden a un sólo confórmero de menor estabilidad, respecto al que se manifiesta en la banda a $1287 \mathrm{~cm}^{-1}$. 19 )

En otro estudio, y mediante el uso de cálculos

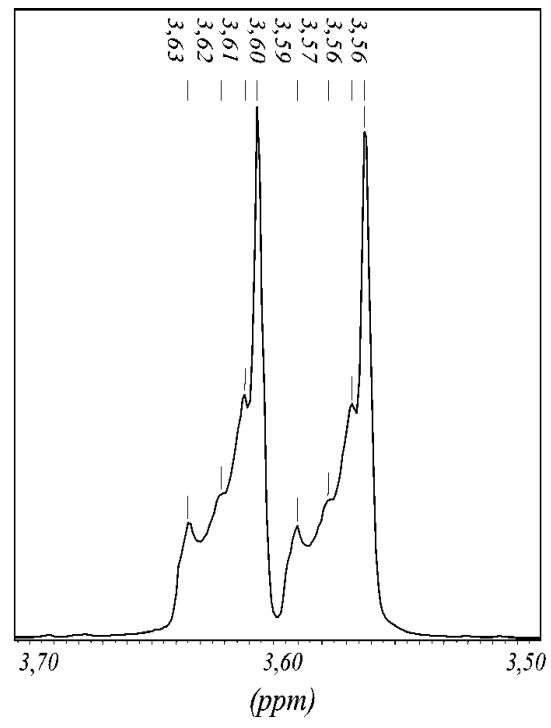

Figura 2.2: Doblete metílico ${ }^{1} \mathrm{H}$ RMN del TMP.

clásicos de los modos normales de vibración ${ }^{(21)}$ que no consideraron confórmeros de simetría $\mathrm{C}_{1}$, se asignó el doblete 734 - $751 \mathrm{~cm}^{-1}$ al modo de estiramiento $(\mathrm{P}-\mathrm{O})_{3}$ correspondiente a los confórmeros $\mathrm{C}_{3}$ y $\mathrm{C}_{3 \mathrm{v}}$ respectivamente. También se reporta la manifestación de la coexistencia de confórmeros en la región de alrededor de $\sim 1040 \mathrm{~cm}^{-1}$ correspondiente al estiramiento (P)-O-C. En medidas obtenidas en matrices de $\mathrm{N}_{2}$ a bajas temperaturas, se resuelve un pico doblete a $1041,0 \mathrm{~cm}^{-1}$ y $1045,6 \mathrm{~cm}^{-1}$ asignándose la primera de ellas como correspondiente a un confórmero menos estable. ${ }^{(19)}$

Finalmente se reporta la manifestación de la coexistencia de confórmeros en la región de alrededor de $\sim 855 \mathrm{~cm}^{-1}$ correspondiente al estiramiento $(\mathrm{P}-\mathrm{O})_{3}$. En medidas obtenidas en matrices de $\mathrm{N}_{2}$ a bajas temperaturas, se resuelven tres bandas a $850,5 \mathrm{~cm}^{-1}, 862,2 \mathrm{~cm}^{-1}$ y $865,8 \mathrm{~cm}^{-1}$ asignándose la primera de ellas a un confórmero energéticamente más estable. ${ }^{(19)}$

Respecto al comportamiento en solución en solventes de distinta polaridad, las intensidades de las bandas asignadas teóricamente a un confórmero de simetría $C_{1}$ incrementaron su intensidad respecto a aquellas asignadas a un confórmero de simetría $\mathrm{C}_{3}$ al aumentar la polaridad del solvente. $^{(12 d)}$ Considerando la Ecuación 1.a, para el TMP, ${ }^{(22)}$ con un momento dipolar medido ${ }^{(23)}$ de $3 \mathrm{D}$, se estimó una relación de 0,7/0,3 para los confórmeros de simetrías $\mathrm{C}_{3} / \mathrm{C}_{1}$. Para cálculos a nivel $\mathrm{HF} / 4-31 \mathrm{G}^{*}$ se reporta

\footnotetext{
${ }^{21}$ Maiants, L.S., Popov, E.M. and Kabachnik, M.I. Calculations of the caracteristics vibrations of phosphorus compounds. II. Characteristics vibrations of the $\left(\mathrm{CH}_{3} \mathrm{O}\right)_{3} \mathrm{PO}$ and $\left(\mathrm{CH}_{3} \mathrm{O}\right)_{3} \mathrm{PS}$ molecules. Opt. Spectrosc. 7 (2):108-113 (1959).

${ }^{22}$ Este tratamiento teórico a los datos experimentales fue por primera vez aplicado al TMP por Raevsky ${ }^{(8 c)}$.

${ }^{23}$ Ver Waeschke, H. and Mitzner, R.Z. Chem. 19: 379-380(1979), además del trabajo de Raevsky ${ }^{(8 c)}$.
} 
un momento dipolar teórico ponderado según la expresión indicada, de $3,52 \mathrm{D}^{(24)}$

En la dilucidación de las geometrías que describieran más adecuadamente los confórmeros estables del TMP, se ha recurrido a distintas metodologías tanto experimentales ${ }^{(25)}$ como teóricas. ${ }^{(26)}$ Aunque posteriormente otros autores reconsideraron esta posibilidad, ${ }^{(27)}$ ya tempranamente se descarta la estabilidad de confórmeros con simetría $\mathrm{C}_{3 \mathrm{v}},{ }^{(28)}$ por la evaluación del número de bandas Raman que se manifiestan entre 367 y $524 \mathrm{~cm}^{-1}$.

Algunos autores han restringido la búsqueda
conformacional, considerando solamente aquellos
confórmeros con iguales ángulos diedros de rotación del
grupo metilo $(\psi 1=\psi 2=\psi 3)$, es decir, sólo a las posibles

${ }^{24}$ Van Wazer, J.R. and Ewig, C.S. Ab initio structures of phosphorus acids and esters. II. Methyl Phosphinate, dimethyl phosphonate, and trimethyl phosphate. J. Am. Chem. Soc. 108: 4354-4360 (1986).

${ }^{25}$ Oberhammer, H.Z. Naturforsch. 28A: 1140(1973), además de Le Breton $^{(7 b)}$, Streck ${ }^{(12 e)}$ y George ${ }^{(12 b)}$.

${ }^{26}$ Los primeros trabajos son de Gorenstein, quién utiliza metodologías de cálculo aún muy elementales: ( $a$ ) Gorenstein, D.G. and Kar, D. Effect of bond angle distortion of torsional potentials. $A b$ initio and $\mathrm{CNDO} / 2$ calculations on dimethoxymethane and dimethyl phosphate. J. Am. Chem. Soc. 99, (3):672-677 (1977a) (b) Gorenstein, D.G., Luxon, B.A. and Findlay, J.B. The torsional potential for phosphate diesters. The effect of geometry optimization in CNDO and ab initio molecular orbital calculations. Biochim. Biophys. Acta. 475 (1): 184-190 (1977).Metodologías de más poder se utilizaron luego: LeBreton ${ }^{(7 b)}$, George ${ }^{(12 b)}$, Sablisnskas ${ }^{(12 d)}$ y Van Wazer ${ }^{(24)}$. Hay un interesante trabajo de correlación teórica con las características electrónicas del TMP: (c) Okhota, B.V., Alekseiko L.N. and Vovna, V.I. [Effect of rotational isomerism on the electronic structure and photoelectron spectrum of trimethyl phosphate.], Zh.Obshch.Khim. 61 (7): 1566-1572 (1991).

${ }^{27}$ En tres trabajos se discute la posibilidad de que el confórmero $\mathrm{C}_{3} v$ tenga cierta estabilidad a tempratura ambiente, aunque alguno de estos autores en trabajos posteriores también descartan esta opción: (a) Marsault-Herail, F. Spectres de vibration de composes organophosphores de 293 K a 15K conformations moleculaires. J. Chim. Phys. 68 (39):274286 (1971) (b) George, L., Sankaran, K., Viswanathan, K.S. and Mathews, C.K. Matrix-isolation infrared spectroscopy of organic phosphates. Appl. Spectrosc. 48 (1):7-12 (1994) (c) Alexeiko, L.N. Shchka, O.L. SCF-X $\alpha$-SW and X-ray emission study of the electronic structure of some phosphorus compounds. J. Elec.Spec.Rel.Phenom., 77: 291-305 (1996).

28 Baudler, M. Über dir Ramanspektren von einiegen Estern der phosphoriden Säure und der phosphosäure. Z. Elektrochem. 59 (3):173184 (1955). Este trabajo es frecuentemente referenciado en la mayoría de los estudios posteriores que consideraron las distintas estructuras conformacionales del TMP.

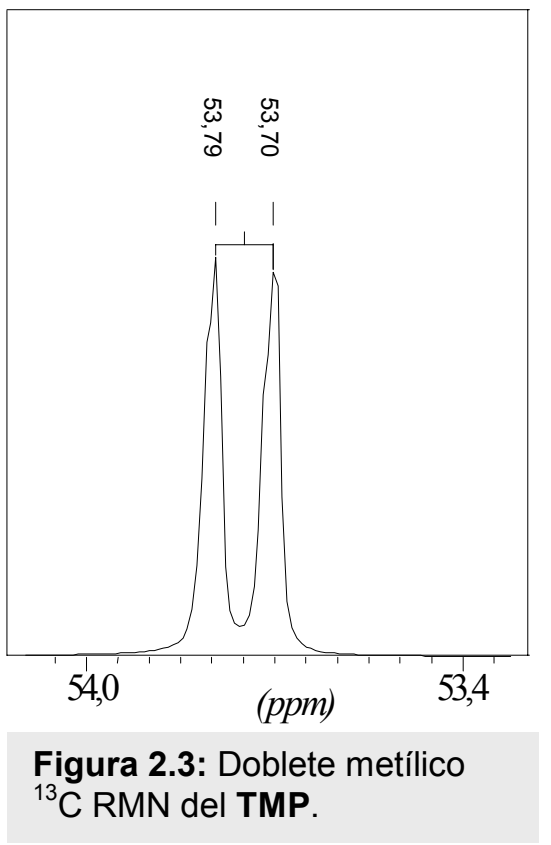

Figura 2.3: Doblete metílico ${ }^{3} \mathrm{C}$ RMN del TMP. 


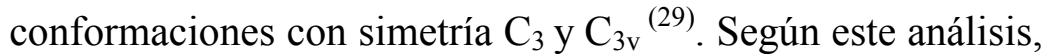
en todos los casos los ángulos diedros preferentes de rotación corresponden a las posiciones gauche en rangos de $53^{\circ}-60^{\circ}$ y $141^{\circ}-155^{\circ}$.

Otros estudios abordaron la posibilidad de la rotación independiente de los tres ángulos $\psi$, de manera de investigar los distintos confórmeros posibles con simetría $\mathrm{C}_{1}$ y Cs. Para cálculos a nivel $\mathrm{HF} / 4-31 \mathrm{G}^{*}$ se informan dos confórmeros $\mathrm{Cs}$ y $\mathrm{C}_{3}$, de menor y mayor energía respectivamente. ${ }^{(24)}$

En otros cálculos realizados a un nivel HF/6-31G* se reportan tres mínimos de energía correspondientes a confórmeros de simetrías $\mathrm{C}_{3}, \mathrm{C}_{1} \mathrm{y}_{\mathrm{s}}$, en ese orden de estabilidad relativa. ${ }^{(12 e)}$ En el reporte más reciente de cálculos teóricos a nivel HF-MP2/6-31G* y HF-MP2/6-31G** realizados sobre el TMP, ${ }^{(12 b)}$ se informa el mismo resultado. En este mismo estudio se correlacionan los confórmeros $\mathrm{C}_{3} \mathrm{y}$ $\mathrm{C}_{1}$, con bandas IR resueltas en espectros obtenidos en matrices de $\mathrm{N}_{2}$, asignándose a los modos de estiramiento $\mathrm{P}=\mathrm{O}, \mathrm{P}-(\mathrm{O}-\mathrm{C})$ y $(\mathrm{P}-\mathrm{O})-\mathrm{C}$, contradiciendo las primeras asignaciones realizadas. Todos estos resultados son coherentes con experiencias de relajación dieléctrica del TMP realizadas en soluciones diluidas. ${ }^{(8 b)}$

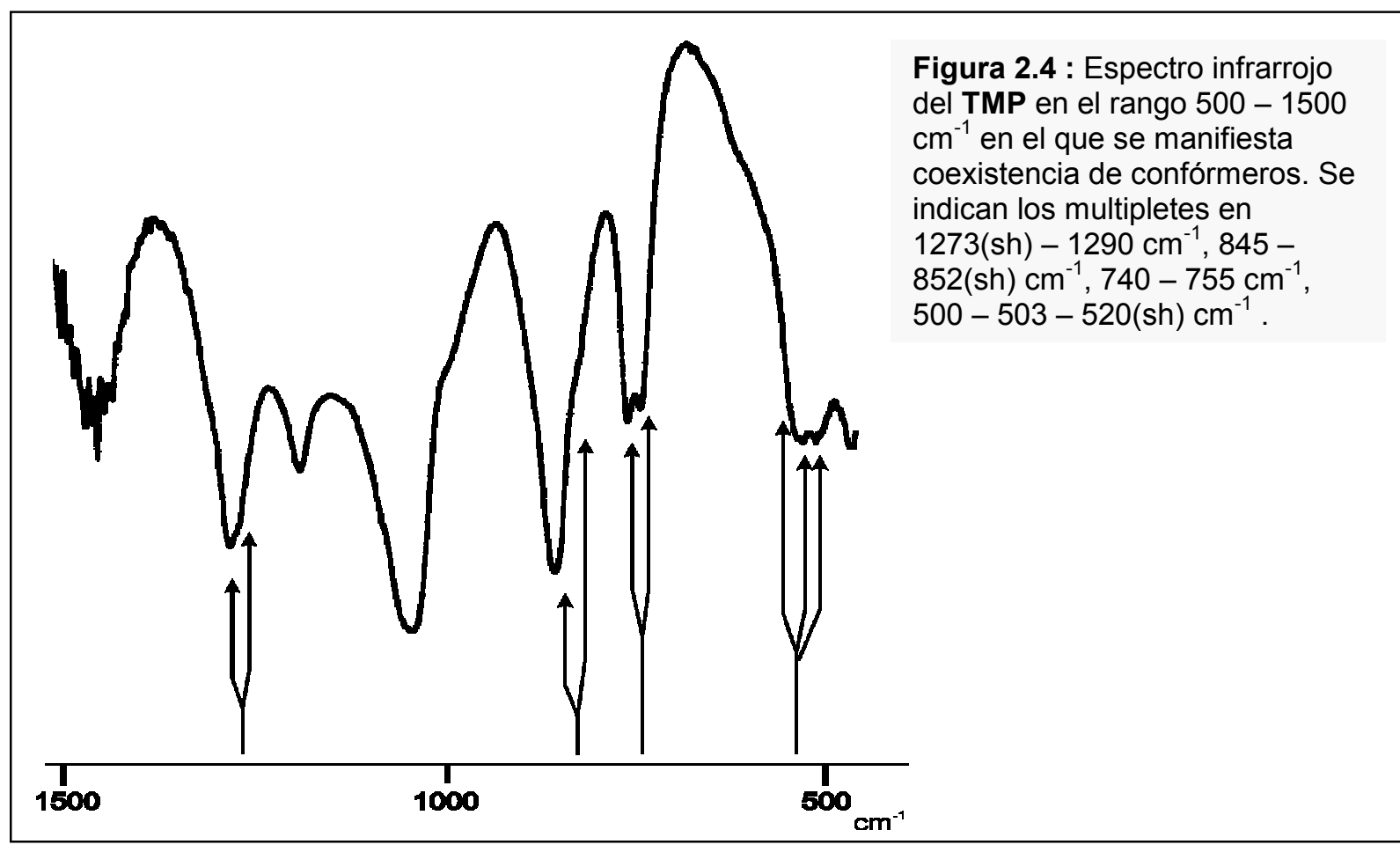

${ }^{29}$ Este tipo de análisis se puede ver en Oberhammer ${ }^{(25)}$, Khetrapal ${ }^{(12 c)}$ y LeBreton ${ }^{(7 b)}$. Aunque con grandes limitaciones, esta simplificación 
En todos los estudios expuestos se describieron estructuras de confórmeros estables con al menos uno de los ángulos $\psi$ en posición gauche entre $30^{\circ}$ y $50^{\circ}$. Experimentalmente se han reportado barreras de interconversión $\mathrm{C}_{1} \rightarrow \mathrm{C}_{3}$ superiores a $2,4 \mathrm{kcal} / \mathrm{mol},{ }^{(12 d)}$ y para las interconversiones $\mathrm{C}_{1} \rightarrow \mathrm{C}_{3}$ y $\mathrm{C}_{3} \rightarrow \mathrm{C}_{1}$ se calcularon barreras energéticas, a un nivel MP2/6-31G**, de 2,20 y 2,76 $\mathrm{kcal} / \mathrm{mol}$ respectivamente. ${ }^{(12 b)}$

\section{Caracterización espectroscópica}

Nuestras medidas de ${ }^{1} \mathrm{H}$ RMN realizadas sobre el TMP sugieren que existe más de un entorno de grupos metilos que se manifiesta en una estructura fina compleja de las señales de $\mathrm{H}$ metílico (Figura 2.2). ${ }^{(30)}$ Se observa un desdoblamiento en, al menos, cuatro tipos de hidrógenos distintos presentados en doblete.

Es interesante especular acerca de manifestaciones de coexistencia conformacional mediante una técnica que, como lo es la espectroscopía de resonancia magnética nuclear, se fundamenta en fenómenos que son medidos en tiempos que sobrepasan en varios órdenes de magnitud los tiempos de interconversión de estos confórmeros. Es decir, por esta razón por ejemplo, no es posible detectar cada uno de los tres hidrógenos de cada metilo en forma independiente. Entonces, es necesario justificar la interpretación de la detección de metilos que presentan un entorno molecular lo suficientemente diferenciado, en términos de la técnica aplicada.

La presencia de al menos cuatro tipos de hidrógeno presentes en el espectro ${ }^{1} \mathrm{H}$ RMN del TMP se podría relacionar con la manifestación de hidrógenos metílicos en trans y gauche para los distintos valores de los ángulos diedros $\mathrm{C}-\mathrm{O}-\mathrm{P}=\mathrm{O}$. Las diferencias de entorno se relacionarían con las grandes diferencias en distancia de los metilos en trans o en gauche con el oxígeno del grupo fosforilo. Entonces, el hecho que puedan manifestarse señales diferenciadas para de los metilos en ${ }^{1} \mathrm{H}$ RMN debería estar asociada a un cierto impedimento energético, que pudiese dar lugar a un desdoblamiento de la señal única prevista.

\footnotetext{
permite tener una primera aproximación teórica al problema.

${ }^{30}$ Mastrantonio,G. Della Védova, C.O. Spectroscopic and conformational comparative study of trimethyl chalcogenphosphates. J.Mol.Struc. 561:161-174 (2001).
} 
Tabla 2.I: Valores experimentales, reportados previamente y calculados del espectro vibracional IR y Raman en fase líquida para el TMP. Se muestran sólo las bandas que evidencian coexistencia de confórmeros.

\begin{tabular}{|c|c|c|c|c|c|}
\hline \multirow[t]{2}{*}{ Asignación } & \multirow[t]{2}{*}{$I R$} & \multirow[t]{2}{*}{ Raman } & \multicolumn{3}{|c|}{ Calculado(HF/6-31G**) } \\
\hline & & & $\mathrm{C}_{3}$ & $\mathrm{C}_{1}$ & Cs \\
\hline$v(\mathrm{P}=\mathrm{O}) \mathrm{C}_{1}, \mathrm{C}_{\mathrm{s}}$ & $\begin{array}{l}1290^{(17)} \\
1282(, 73)^{(30)} \\
1282^{(27 a)(12 e)}\end{array}$ & $\begin{array}{l}1285^{(2 / a)} \\
1276(, 36)^{(12 f)(30)} \\
1275^{(17)} \\
1272^{(28)}\end{array}$ & & 1285 & 1282 \\
\hline$v(\mathrm{P}=\mathrm{O}) \mathrm{C}_{3}$ & $\begin{array}{l}1276^{(27 b)} \\
1273^{(27 a)(17)} \\
1272(, 69)^{(30)} \\
1269^{(122)}\end{array}$ & $1267^{(12 f)(30)}$ & 1261 & & \\
\hline va $(\mathrm{C}-\mathrm{O})_{3} \mathrm{C}_{1}, \mathrm{C}_{\mathrm{s}}$ & $\begin{array}{l}1080^{(17)} \\
1079(, 68)^{(30)} \\
1076^{(122)} \\
1050^{(27 a)}\end{array}$ & $\begin{array}{l}1080^{(27 a)} \\
1079^{(17)} \\
1076^{(12 f)}\end{array}$ & & 1079 & 1079 \\
\hline $\mathrm{va}(\mathrm{C}-\mathrm{O})_{3}$ & $\begin{array}{l}1045^{(17)} \\
1044^{(12 e)} \\
1040(1,00)^{(30)} \\
1038^{(276)}\end{array}$ & $\begin{array}{l}1050^{(27 a)} \\
1043^{(12 f)} \\
1042(28)^{(30)} \\
1037^{(17)}\end{array}$ & $1067(2)$ & 1061 & 1050 \\
\hline va $(\mathrm{P}-\mathrm{O})_{3} \mathrm{C}_{3}, \mathrm{C}_{1}$ & $\begin{array}{l}852^{(17)} \\
850^{(12 e)(27 a)}(, 80)^{(30)}\end{array}$ & $\begin{array}{l}861^{(27 a)} \\
853^{(12 f)} \\
852(25)^{(30)} \\
848^{(17)(28)}\end{array}$ & $842(2)$ & 839 & \\
\hline $\mathrm{va}(\mathrm{P}-\mathrm{O})_{3} \mathrm{C}_{1}, \mathrm{C}_{\mathrm{s}}$ & $\begin{array}{l}846(44)^{(30)} \\
845^{(17)} \\
842^{(12 e)}\end{array}$ & $843^{(12 f)}$ & & 825 & $\begin{array}{l}832 \\
827\end{array}$ \\
\hline vs $(\mathrm{P}-\mathrm{O})_{3} \mathrm{C}_{3}$ & $\begin{array}{l}755^{(17)} \\
753^{(12 e)}(, 47)^{(30)} \\
752^{(27 a)}\end{array}$ & $\begin{array}{l}755^{(27 a)} \\
754(, 49)^{(30)} \\
753^{(12 f)} \\
751^{(28)} \\
750^{(17)}\end{array}$ & 741 & & \\
\hline vs $(\mathrm{P}-\mathrm{O})_{3} \mathrm{C}_{1}, \mathrm{C}_{\mathrm{s}}$ & $\begin{array}{l}740^{(17)} \\
737^{(12 e)(27 a)}(, 45)^{(30)}\end{array}$ & $\begin{array}{l}745^{(27 a)} \\
739^{(12 f)} \\
736(163)^{(30)} \\
734^{(17)(28)}\end{array}$ & & 723 & 726 \\
\hline$\delta \mathrm{a}(\mathrm{P}-\mathrm{O})-\mathrm{C} \mathrm{C}_{\mathrm{s}}$ & $\begin{array}{l}529^{(12 e)(27 a)} \\
527(, 50)^{(30)} \\
526^{(17)}\end{array}$ & & & & 522 \\
\hline$\delta \mathrm{a}(\mathrm{P}-\mathrm{O})-\mathrm{C} \mathrm{C}_{1}$ & $\begin{array}{l}521^{(12 e)} \\
520(, 50)^{(30)} \\
518^{(27 a)}\end{array}$ & $\begin{array}{l}524^{(28)} \\
523^{(17)(27 a)} \\
517(, 19)^{(30)}\end{array}$ & & 511 & \\
\hline$\delta \mathrm{a}(\mathrm{P}-\mathrm{O})-\mathrm{C} \mathrm{C}_{3}, \mathrm{C}_{\mathrm{s}}$ & $504(, 51)^{(30)}$ & $\begin{array}{l}518^{(12 f)} \\
515^{(27 a)} \\
509(, 18)^{(30)}\end{array}$ & $506(2)$ & & 504 \\
\hline$\delta \mathrm{a}(\mathrm{P}-\mathrm{O})-\mathrm{CC}_{1}$ & $\begin{array}{l}500^{(17)} \\
499^{(12 e)} \\
498^{(27 a)} \\
496(, 50)^{(30)}\end{array}$ & $\begin{array}{l}501^{(122 f)(28)} \\
498^{(17)}(, 18)^{(30)}\end{array}$ & & 495 & \\
\hline$\delta a \mathrm{P}-(\mathrm{O}-\mathrm{C})_{3} \mathrm{C}_{1}$ & & $\begin{array}{l}377^{(27 a)} \\
372^{(12 f)} \\
368(, 17)^{(30)} \\
368^{(17)} \\
367^{(28)}\end{array}$ & & 361 & \\
\hline$\delta \mathrm{a}-(\mathrm{O}-\mathrm{C})_{3} \mathrm{C}_{3}, \mathrm{C}_{\mathrm{s}}$ & & $\begin{array}{l}362(, 16)^{(30)} \\
360^{(12 f)}\end{array}$ & $\begin{array}{l}350(2) \\
350\end{array}$ & & 350 \\
\hline$\delta a \mathrm{P}-(\mathrm{O}-\mathrm{C})_{3} \mathrm{C}_{1}, \mathrm{C}_{\mathrm{s}}$ & $243^{(12 \theta)}$ & $\begin{array}{l}245(, 14)^{(30)} \\
244^{(1217} \\
241^{(17)} \\
239^{(28)}\end{array}$ & & 344 & 346 \\
\hline $\operatorname{\rho r}(\mathrm{P}-\mathrm{O})_{3}$ & & $\begin{array}{l}232(, 15)^{(30)} \\
230^{(27 a)} \\
229^{(12 f)}\end{array}$ & 227 & 231 & 230 \\
\hline $\operatorname{pra}(\mathrm{P}-\mathrm{O})_{3} \mathrm{C}_{1}, \mathrm{C}_{\mathrm{s}}$ & $205^{(12 e)}$ & $205^{(27 a)}$ & & 207 & 205 \\
\hline $\operatorname{\rho ra}(\mathrm{P}-\mathrm{O})_{3} \mathrm{C}_{3}$ & & $\begin{array}{l}197^{(12 f)} \\
195(, 14)^{(30)} \\
188^{(17)} \\
184^{(28)}\end{array}$ & $\begin{array}{l}190 \\
190\end{array}$ & & \\
\hline
\end{tabular}

v: vibración de estiramiento; vs: vibración de estiramiento simétrica; va: vibración de estiramiento

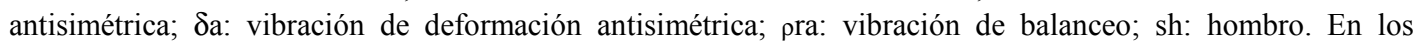
valores experimentales, entre paréntesis se encuentra la intensidad de cada banda. En los valores teóricos, entre paréntesis se indica el grado de degeneración energética, si existe. 
Capítulo 2: Comportamiento conformacional del TMP

Tabla 2.II: Corrimientos químicos y constantes de acoplamiento en los espectros $\mathrm{RMN}$ de ${ }^{1} \mathrm{H}$ y ${ }^{13} \mathrm{C}$ para el TMP. Entre paréntesis se indican las intensidades relativas de los picos.

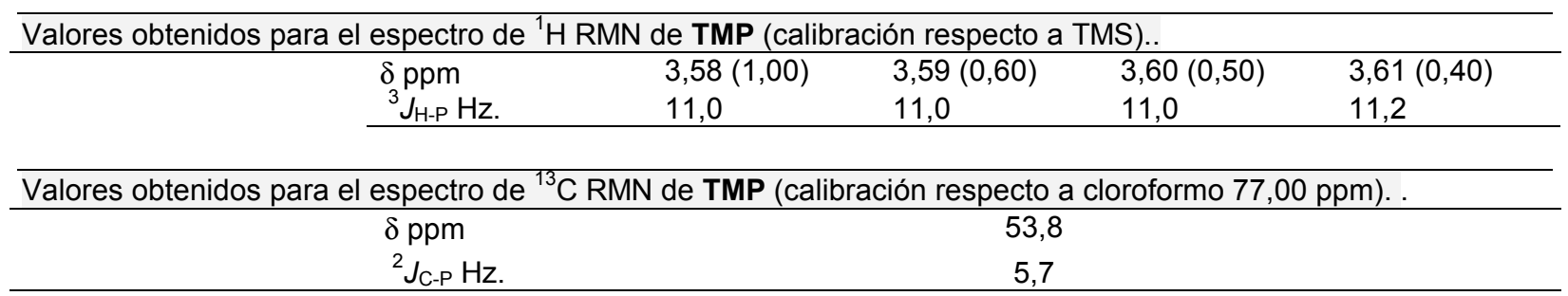

En el espectro obtenido por ${ }^{13} \mathrm{C}$ RMN, se observa un doblete ubicado en 53,7 ppm (Figura 2.3) con una constante de acoplamiento ${ }^{2} J_{\mathrm{C}-\mathrm{P}}$ igual a $5,7 \mathrm{~Hz}$. (Tabla 2.II). Es interesante observar un hombro en cada una de las dos señales que, de corroborar la hipótesis aplicada a la interpretación de las cuatro señales en el espectro ${ }^{1} \mathrm{H}$ RMN, mediante una medida de ${ }^{13} \mathrm{C} \mathrm{RMN}$ de mayor resolución sería posible observar un segundo doblete de menor intensidad.

En la Tabla 2.I se resumen los valores de los números de onda de absorción IR y Raman para el TMP, experimentales y teóricas, en los rangos de números de onda en las que se manifestaría la coexistencia de confórmeros, con las asignaciones propuestas.

Es interesante el doblete que aparece en el modo normal de vibración asignado al estiramiento $\mathrm{P}=\mathrm{O}$, que aunque se encuentra oculto en el espectro vibracional infrarrojo, lo hemos observado claramente en Raman cuando la obtención del espectro se realiza mediante irradiación láser de 459 nm (Figura 2.5).

Este modo vibracional, en virtud de la naturaleza de las posiciones de los metilos que se ubicarían a una cierta distancia de interacción con el grupo fosforilo para posiciones gauche y a distancias de no interacción para las posiciones trans, aparece claramente afectado. Sobre este punto, otros autores ${ }^{(21)}$ han argumentado en el mismo sentido.

\section{Correlación teórica}

Los datos espectroscópicos experimentales se correlacionan e interpretan mediante el contraste con los cálculos teóricos de modelado molecular. En la Tabla 2.I, se presentan ambos tipos de datos, experimentales y teóricos, usando un nivel de cálculo HF/6-31G** (ab initio). Las asignaciones de los modos vibracionales se realizan

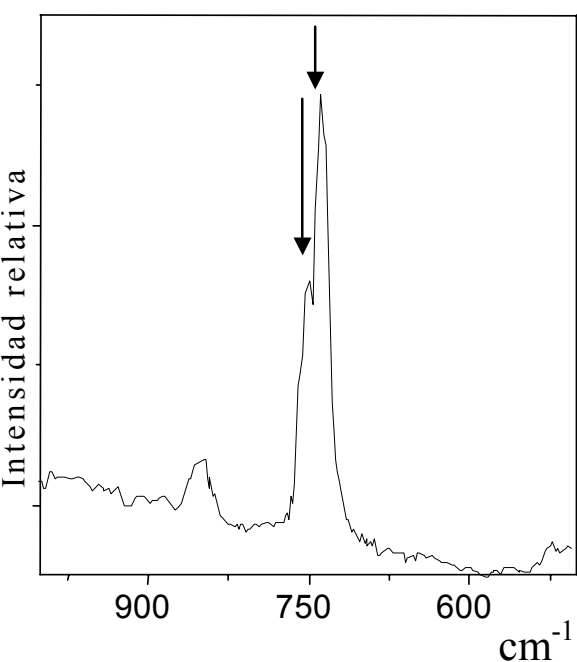

Figura 2.5: Doblete $\mathrm{P}=\mathrm{O}$ en el espectro Raman del TMP ubicado en $736 \mathrm{~cm}^{-1}$ y $750 \mathrm{~cm}^{-1}$. 


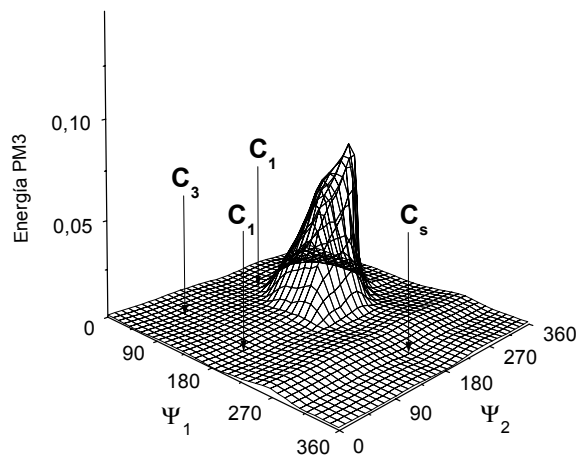

Figura 2.6: Superficie potencial energética del TMP, obtenida por rotación alrededor de dos ángulos $\psi$ y manteniendo el otro fijo en $42^{\circ}$ calculadas a nivel HF/PM3. cálculos. En la Tabla 2.III se presentan los demás parámetros fisicoquímicos obtenidos teóricamente para el TMP.

En otro tipo de correlación teórica, en la Figura 2.6 se ilustra, según nuestros cálculos realizados a un nivel HF/PM3 (método semiempírico), la libertad conformacional de los ángulos alrededor de los enlaces del éster para el TMP. Manteniéndose uno de los ángulos $\psi$ fijo en $42^{\circ}$, los ejes horizontales representan la variación de los otros dos entre $0^{\circ}$ y $360^{\circ}$. En el eje vertical se representa la energía potencial relativa. Este tipo de cálculo permite una interpretación de la facilidad de interconversión de un confórmero en otro y los posibles caminos seguidos para ello.

Se observa una extensa superficie aproximadamente plana, con varios mínimos relativos conformacionales fácilmente accesibles unos desde otros lo que, mirado en forma retrospectiva, explicaría en parte las dificultades de los distintos investigadores para discernir aquellas conformaciones que corresponden a mínimos relativos de energía. En esta superficie de energía potencial, el confórmero con mayor dificultad energética para acceder es de simetría $\mathrm{C}_{1}$ con ambos valores de $\psi$ en $\sim 180^{\circ}$.

Se desarrolla también un cálculo semiempírico HF/PM3 para la rotación simultánea de los tres ángulos diedros $\psi 1, \psi 2$ y $\psi 3$. En la Figura 2.7, se presentan las superficies de energía potencial con una graduación de grises. Las energías arrojadas por el cálculo semiempírico fueron reescaladas tomando en consideración las diferencias de energía de los mínimos conformacionales obtenidas a nivel MP2/6-31G**. Desde negro a gris, están representadas las energías desde 0 a $30 \mathrm{kcal} / \mathrm{mol}$. El blanco representa las regiones con energía superior a $30 \mathrm{kcal} / \mathrm{mol}$.

Se aprecian con cierta definición la presencia de los confórmeros $\mathrm{C}_{3}, \mathrm{C}_{1}$ y $\mathrm{Cs}$ calculados. Además aparece la posibilidad de estabilización de un confórmero Cs' de mayor energía que el correspondiente Cs calculado previamente. Con los cálculos $a b$ initio, no fue posible encontrar este confórmero. De existir, no sería relevante por la energía relativa elevada y la abundancia despreciable en un rango de temperaturas muy amplio, según la estimación del cálculo semiempírico.

En la representación alternativa de los mismos datos presentada en la Figura 2.8, en el espacio dado por los ejes $\mathrm{x}, \mathrm{y}, \mathrm{z}$ que representan los valores de cada uno de los tres 
Figura 2.7: Representación de planos sucesivos respecto del ángulo diedro $\psi_{1}$ de las energías conformacionales del TMP en función de la rotación de ángulos $\psi_{2}$ y $\psi_{3}$.
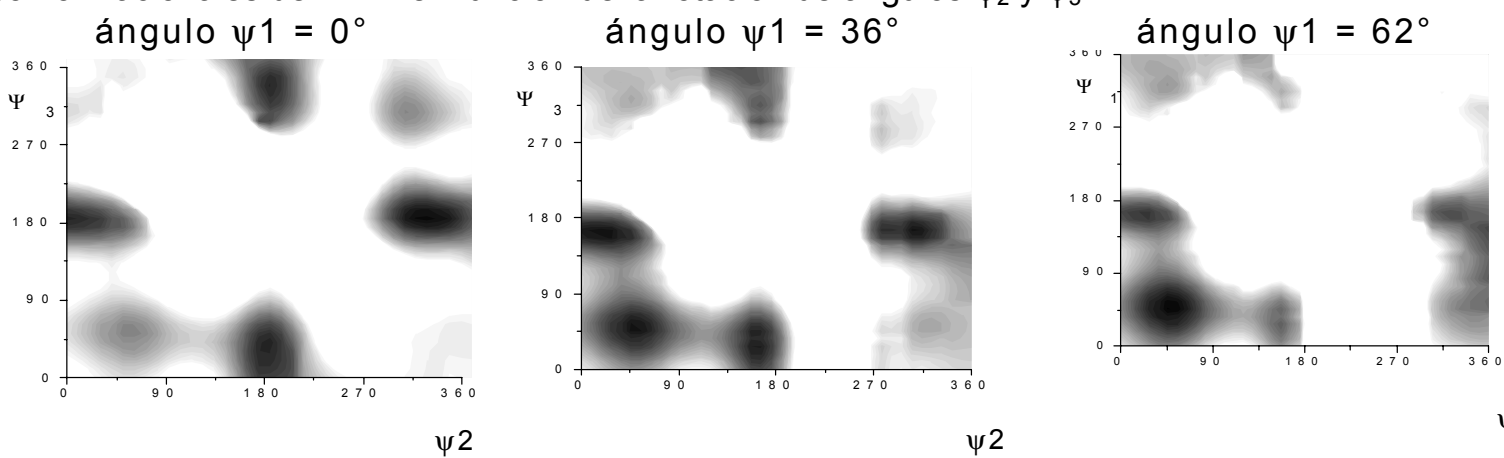

ángulo $\psi 1=90^{\circ}$

2

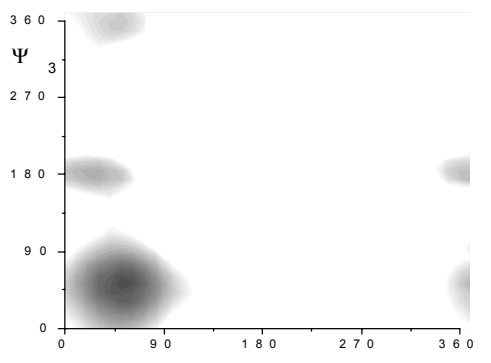

ángulo $\psi 1=117^{\circ}$

$\psi 2$

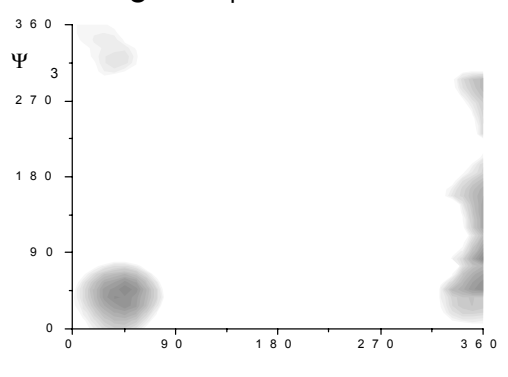

ángulo $\psi 1=162^{\circ}$

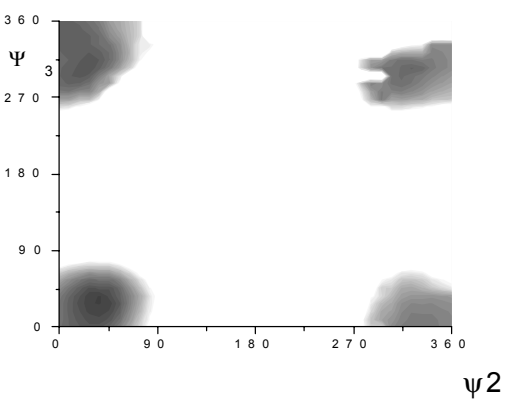

ángulo $\psi 1=189^{\circ}$

$\psi 2$

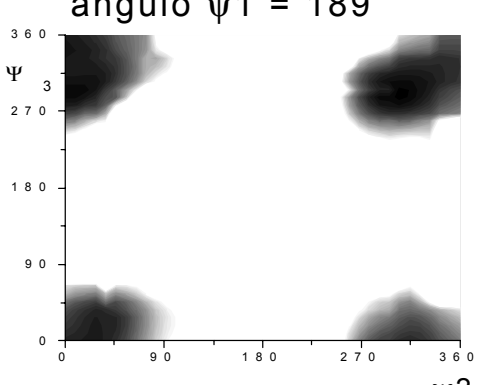

ángulo $\psi 1=216^{\circ}$

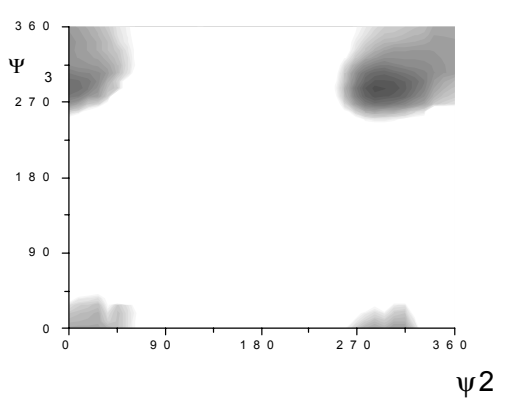

ángulo $\psi 1=243^{\circ}$

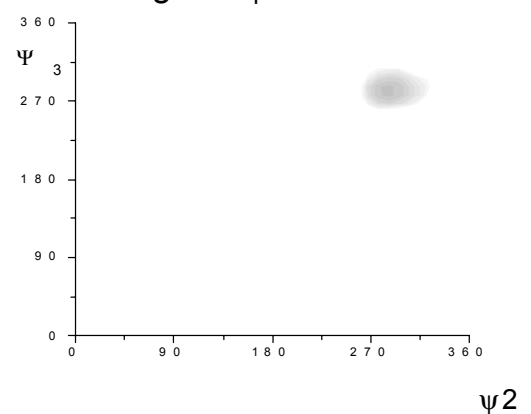

ángulo $\psi 1=270^{\circ}$

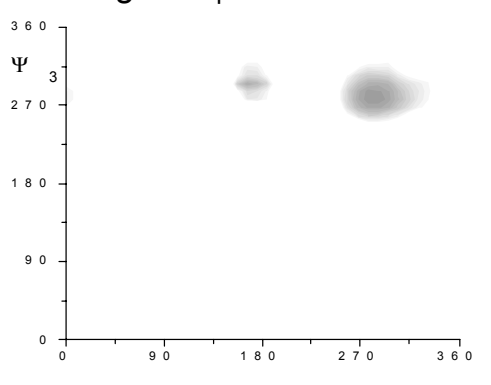

ángulo $\psi 1=300^{\circ}$

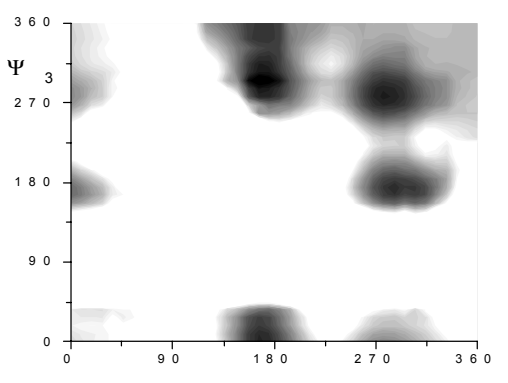

ángulo $\psi 1=330^{\circ}$

ángulos diedros $\psi 1, \psi 2$ y $\psi 3$, se grafican los puntos correspondientes a las energías equivalentes a $10 \mathrm{kcal} / \mathrm{mol}$.

Estos puntos se agrupan en superficies envolventes de las regiones que encierran los puntos de mínima energía en una relación de degeneración conformacional 2:6:3, es 
decir, corresponde a un espacio conformacional asimétrico. Aparece además el cuarto confórmero accesible teóricamente, con simetría Cs' y degeneración 3 .

Estas superficies cerradas definen el alcance de la libertad conformacional para cada uno de los mínimos reportados. Por otro lado, se evidencia una sobreestimación de energías de estas superficies, puesto que las barreras de interconversión no superan los $3 \mathrm{kcal} / \mathrm{mol}$, según los resultados mostrados más adelante.

En el cálculo del momento dipolar ponderado para los calcogenofosfatos de trimetilo, existe un aspecto que no es resuelto en las publicaciones que tratan al respecto, y es que existen tres opciones de asignación de pesos relativos para cada uno de los tres confórmeros que se describen como mínimos energéticos. Cada una de ellas implica distintos grados de degeneración energética para cada confórmero.

La primera de las asignaciones realizada para el TMP, no propone argumentos que la fundamenten ${ }^{(31)} \mathrm{y}$

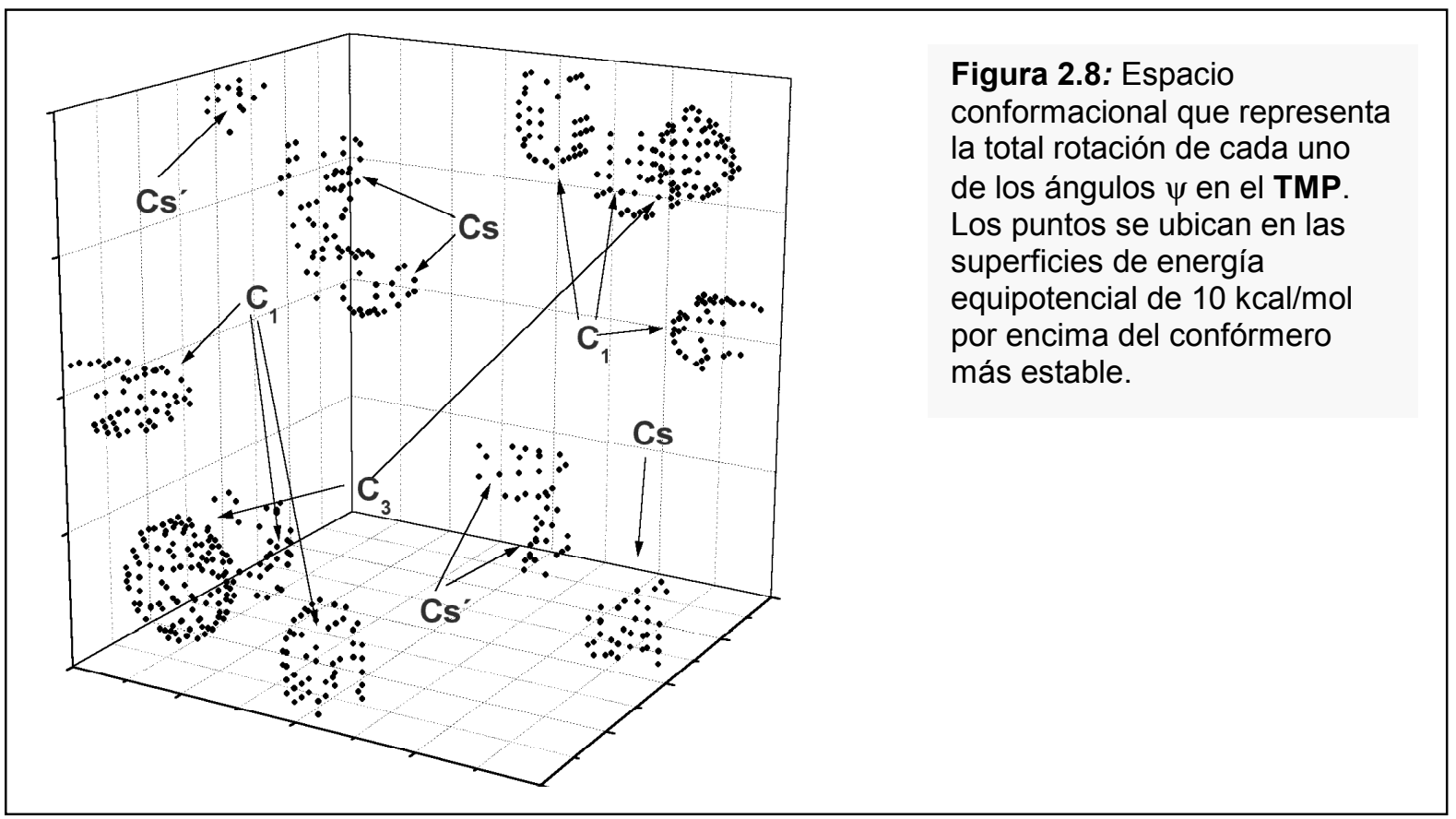

31 Ver George ${ }^{(12 b)}$. Durante un tiempo mantuvimos un intercambio epistolar con el Dr. Viswanathan, coautor mencionado y Director de dicho grupo de trabajo, discutiendo acerca de los grados de degeneración para cada uno de los confórmeros del TMP, válidos también para el TMSP y TMSeP. Finalmente no existió coincidencia y aunque sus publicaciones y las nuestras reportan las mismas diferencias energéticas para los confórmeros del TMP, comunican abundancias relativas diferentes, pues se basan en considerar diferentes grados de degeneración energética para cada uno de los tres confórmeros más estables reportados. 
asume una asignación de peso 1 para el confórmero $\mathrm{C}_{3}$, un peso de 6 para el confórmero $\mathrm{C}_{1} \mathrm{y}$ un peso de 3 para el confórmero Cs. Esta relación 1:6:3 implica una sobre valoración en la abundancia de la forma $\mathrm{C}_{1}$, a tal punto que predomina respecto al confórmero de menor energía $\mathrm{C}_{3}$.

La segunda de estas asignaciones se realiza en el supuesto de un entorno asimétrico que permita la distinción espacial de cada uno de los tres metilos, de manera que se asigna un peso de 2 para el confórmero $\mathrm{C}_{3}$, un peso de 6 para el confórmero $\mathrm{C}_{1} \mathrm{y}$ un peso de 3 para el confórmero Cs. Esta asignación de pesos queda representada en la Figura 2.8, en donde se asume la independencia de las rotaciones de los ángulos $\psi$ entre sí.

Un entorno asimétrico que se adecuaría a una asignación de pesos relativos 2:6:3 correspondería a un modelo de solvente que involucre un entorno asimétrico de moléculas de solvatación ${ }^{(32)}$ o a un entorno biológico como el sitio activo de una enzima. Esta asignación sólo tiene sentido en la medida que la asimetría del entorno sea fundamental para el fenómeno que se pretende describir, punto sobre el que volveremos en el Capítulo 14.

La tercera de estas asignaciones es realizada en supuesto que los tres metilos sean indistinguibles entre sí, asumiendo un peso de 2 para el confórmero $\mathrm{C}_{3}$, un peso de 2 para el confórmero $\mathrm{C}_{1} \mathrm{y}$ un peso de 1 para el confórmero $\mathrm{Cs}$.

Como las correlaciones teóricas de los fenómenos enumerados en éste Capítulo se basan en cálculos en las cuales se modela con un entorno o medioambiente homogéneo (simétrico), la discusión de los resultados se basa en la última de las asignaciones de pesos relativos descritas, aunque en la Tabla 2.III se muestran los resultados obtenidos para las tres opciones de asignación. Este modelo es válido en la medida que se contrasten con resultados experimentales en los que la asimetría del entorno no sea crítica, por ejemplo, medidas en fase gaseosa, sustancias en solución con solvatación débil o en matrices a baja temperatura.

Aplicando la Ecuación 1.a a los datos reportados a un nivel HF/6-31G** y MP2/6-31G**, ${ }^{(12 b)}$ tomando en cuenta una degeneración de energías conformacionales de $2: 2: 1$, se obtiene un valor teórico ponderado para el momento

\footnotetext{
${ }^{32}$ Sankaran, K., Vidya, V. and Viswanathan, K.S. Trimethyl phosphate water interaction: a matrix/isolation infrared and ab initio study. J. Phys. Chem. 102A, 2944-2953 (1998).
} 
dipolar del TMP de 1,85 D y 2,30 D respectivamente, como se muestra en la Tabla 2.III. Estos valores subestiman notablemente el valor experimental del momento dipolar, que fue medido en valores ubicado levemente por encima de $3 \mathrm{D}$. El hecho que el cálculo del momento dipolar ponderado mediante la Ecuación 1.a arroje resultados sensiblemente inferiores al valor experimental sugiere que, de la forma en que es aplicada, pierde validez para el caso del TMP.

Tabla 2.III: Parámetros geométricos, energéticos y fisicoquímicos de los confórmeros correspondientes a mínimos de energía del TMP $^{(33)}$.

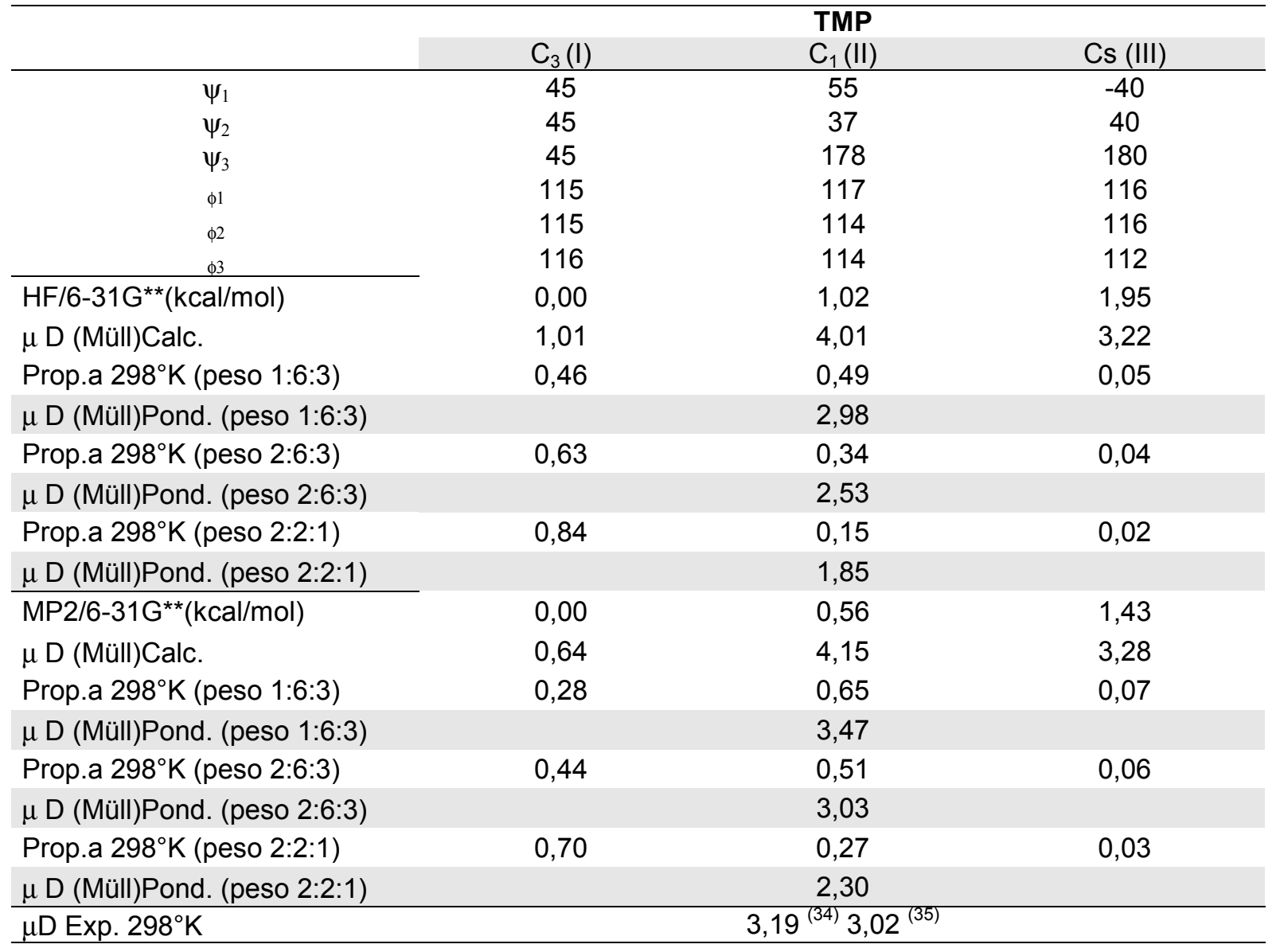

Una hipótesis plausible es que en la aplicación de la Ecuación 1.a se está considerando solamente la contribución de los confórmeros de mínima energía encontrados por los cálculos $a b$ initio, despreciando otros, en la práctica

\footnotetext{
${ }^{33}$ Los valores de $\psi$, energías HF y MP2, momento dipolar de cada confórmero y momento dipolar ponderado (1:6:3) fueron obtenidos de George, L. ${ }^{(12 b)}$.

${ }^{34} \mathrm{La}$ referencia es mencionada en varios trabajos posteriores, pero la original corresponde a Waeschke,H. and Mitzner,R. Z.Chem. 19: 379-380 (1979).

${ }^{35}$ La medida en $\mathrm{CCl}_{4}$ está reportada en Raevsky ${ }^{(8 c)}$.
} 
accesibles a la temperatura de la medida. Se puede conjeturar entonces, que en la superficie potencial representada en la Figura 2.6, podrían estar incluidos otros mínimos de energía conformacional, que se manifestarían sólo mediante cálculos de mayor resolución.

De ser cierta esta hipótesis, si pudiera ser posible valorar el aporte de cada uno de estos confórmeros despreciados, para ser aplicados en la Ecuación 1.a, se podría estimar un valor teórico más cercano al valor experimental; de la misma manera se puede indicar que, aquellos confórmeros no considerados deberían tener un momento dipolar alto, por lo que deberán prevalecer en ellos las posiciones trans por sobre las gauche.

En la búsqueda de las estructuras de transición entre los distintos confórmeros de energía mínima se utiliza un cálculo con base orbital MP2/6-31G**. Se encuentran dos confórmeros de transición. Uno de ellos corresponde a las transiciones $\mathrm{Cs} \leftrightarrow \mathrm{C}_{1}$ de menor energía y el otro corresponde a las transiciones $\mathrm{C}_{3} \rightarrow \mathrm{Cx}{ }^{(36)}$ con mayor energía, en donde $\mathrm{Cx}$ corresponde a cualquiera de los dos confórmeros de alta energía (Tabla 2.IV).

La metodología para el cálculo de estas barreras conformacionales consistió en el cálculo de la energía conformacional para cada uno de los confórmeros resultantes de la rotación de uno de los ángulos diedros correspondientes a las geometrías intermedias entre los confórmeros estables estudiados, con lo cual se encuentra un confórmero de alta energía con $\psi=130^{\circ}$ que será el punto de partida para el cálculo de una estructura del estado de transición.

Respecto a los resultados obtenidos en las medidas de ${ }^{1} \mathrm{H}$ RMN, se puede evaluar cuantitativamente la hipótesis sustentada en cuanto a la asignación de la estructura fina de los picos. Considerando la proporción de hidrógenos de metilos en una $\mathrm{u}$ otra posición, y la altura relativa de los picos, hacia corrimientos menores se ubicarían los hidrógenos metílicos en posición gauche, mientras que hacia corrimientos mayores se ubicarían los hidrógenos metílicos en posición trans.

Dado que en el confórmero $\mathrm{C}_{3}$ se manifestarán tres metilos en gauche; en el confórmero $\mathrm{C}_{1}$ se manifestarán dos

\footnotetext{
${ }^{36}$ El estado de transición $\mathrm{C}_{3} \rightarrow$ Cs ya fue descrito previamente por George $^{(12 b)}$. Es el que se muestra en la Tabla 2.III.
} 
Tabla 2.IV: Parámetros energéticos y geométricos de las formas correspondientes a estados de transición entre confórmeros estables.

\begin{tabular}{|c|c|c|c|}
\hline 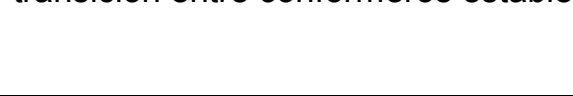 & $\begin{array}{c}\text { Transición } \\
\mathrm{C}_{3} \rightarrow \mathrm{Cs}\end{array}$ & $\begin{array}{c}\text { Transición } \\
\mathrm{C}_{3} \rightarrow \mathrm{Cx}\end{array}$ & $\begin{array}{c}\text { Transición } \\
\mathrm{Cs} \leftrightarrow \mathrm{C}_{1}\end{array}$ \\
\hline MP2/6-31G** $(\mathrm{kcal} / \mathrm{mol})$ & 2,76 & 2,78 & 1,88 \\
\hline$\mu$ (Debyes) & & & 2,82 \\
\hline$\psi 1$ & 49 & 46 & -40 \\
\hline$\psi 2$ & 38 & 38 & 9 \\
\hline$\psi 3$ & 129 & 120 & 184 \\
\hline
\end{tabular}

hidrógenos en gauche y uno en trans; finalmente para el confórmero Cs se manifestarán dos gauche, la relación teórica de picos, dadas las abundancias relativas obtenidas por los cálculos $a b$ initio a nivel MP2/6-31G** para los tres confórmeros considerados, sería aproximadamente de 20:6:3:1, que dada la incertidumbre experimental de la medida ${ }^{1} \mathrm{H}$ RMN, es una aproximación adecuada a los valores experimentales.

\section{Integración y justificación de resultados}

Se verifica en distintos sistemas y mediante distintas técnicas que en el TMP existe abundancia conformacional y que aquellas conformaciones energéticamente accesibles, se estabilizan en la medida que los metilos se aproximan a los pares libres del grupo fosforilo.

En los espectros vibracionales, las descripciones de bandas múltiples atribuidos a la coexistencia de confórmeros, se encuentran suficientemente justificadas por evidencia experimental y teórica. Para explicar la estabilidad relativa de los distintos confórmeros, se ha recurrido a argumentos que otorgan importancia a los impedimentos estéricos, además de a las interacciones entre los hidrógenos de los grupos metilo y los pares de electrones libres del oxígeno del grupo fosforilo ${ }^{(37)}$.

Entre los aspectos interesantes de dichas argumentaciones es el hecho que se reconoce la importancia de la capacidad del TMP como donor de protones, hecho que se ha cuantificado en solventes apróticos; como valor comparativo, la entalpía de formación del complejo por puentes de hidrógeno, entre el TMP como donor y la

${ }^{37}$ Éste es el argumento fundamental en los trabajos de George ${ }^{(12 b)} \mathrm{y}^{(27 b)}$. 
tiopropionamida como aceptor, se ha cuantificado en un valor $\mathrm{de}-12,8 \mathrm{~kJ} / \mathrm{mol}$ en tetracloruro de carbono. ${ }^{(38)}$

Estas interacciones estarían reforzadas por el fuerte carácter polar del enlace fósforo-oxígeno, a tal punto que algunos autores se inclinaron por representar dicho enlace como $\mathrm{P}^{+}-\mathrm{O}^{-}$en lugar de $\mathrm{P}=\mathrm{O}^{(39)}$ aún a expensas de minimizar la participación de las interacciones $\pi$.

De esta manera, las diferencias en los valores de números de onda manifestadas por los distintos confórmeros, se corresponderían con una variación en las constantes de fuerza del enlace $\mathrm{P}=\mathrm{O}$ por diferencias en las interacciones entre los grupos metilo y los pares libres del oxígeno ${ }^{(40)}$. Un argumento concurrente indica que la tendencia observada en los ésteres neutros de oxofosfatos en relación con la estabilización de aquellos confórmeros con momentos dipolares bajos, se explica por la alineación de momentos dipolares locales en cada parte de la molécula. ${ }^{(41)}$

Finalmente se puede indicar que existe abundante evidencia y fundamento teórico para afirmar la coexistencia de confórmeros en un amplio rango de temperaturas y en distintos medios solventes.

\footnotetext{
$38 \mathrm{Ju}$, S.A. et al. Near infrared spectroscopic studies on the hydrogen bonding ability of thiopropionamide, J.Korean.Chem.Soc. 39 (11): 837 841 (1995).

${ }^{39}$ Este punto de vista es adoptado por Daasch ${ }^{(8 a)}$ y recogido nuevamente por Eaton ${ }^{(12 a)}$.

${ }^{40}$ En el trabajo temprano de Maiants ${ }^{(21)}$, ya se opta claramente por esta explicación para la presencia de dobletes por coexistencia de confórmeros en los espectros vibracionales.

${ }^{41}$ Vidya, V., Sankaran, K., Sundararajaran, K. and Viswanathan, K.S. Conformations of triethylphosphate: a supersonic jet-matrix isolatio and semi-empirical (AM1) study. J. Mol. Struct. 476, 97-104 (1999).
} 


\section{Comportamiento conformacional del fosfotioato de $\mathrm{O}, \mathrm{O}, \mathrm{O}$-trimetilo}

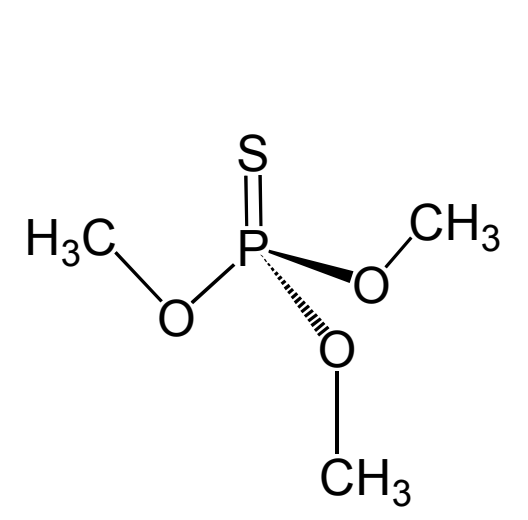

Figura 3.1: Estructura del fosfotioato de trimetilo (TMSP).
El fosfotioato de O,O,O-trimetilo (TMSP) suele encontrarse como impureza en muchos OPs utilizados como plaguicidas o sus materias primas de grado técnico, ${ }^{(1)}$ como contaminante en efluentes de establecimientos de elaboración de OPs ${ }^{(2)}$ y en aguas abiertas. ${ }^{(3)}$ De allí su importancia de su estudio como tóxico.

Se ha determinado que el TMSP presenta una cierta capacidad alquilante ${ }^{(4)}$ además de ser un tóxico pulmonar, ${ }^{(5)}$ probablemente actuando como inhibidor de ciertas isoformas de los citocromos $\mathrm{P}_{450}{ }^{(6)}$ aunque también actúa como agente protector frente a la toxicidad retardada de otros fosfotioatos. (1b)

1 (a) Dulak, K. Analysis of technical-grade O,O-dimethyl chloro thiophosphate by infrared spectrophotometry, Chem.Prum. 28 (12): 630-632 (1978). (b) Umetsu, N. et al. Toxicological properties of phosphorothioate and related esters present as impurities in technical organophosphorus insecticides, J.Toxicol.Environ.Health 7 (3-4): $481-497$ (1981). (c) Bor Fuh, C. and Wu, M.L. Impurity analysis of methamidophos with chromatographic methods, Anal.Chim.Acta. 395 (3): 257-263 (1999).

${ }^{2}$ (a) Kuan, Y.W. et al. Studies of organophosphorus pollutants in the well water near a dimethoate plant. II. Gas chromatographic method for the determination of $\mathrm{O}, \mathrm{O}, \mathrm{S}$-trimethyldithiophosphate and O,O,O-trimethyl thiophosphate, Pei.-ching.Ta.Hsueh.Hsueh.Pao., Tzu.Jan.K.'o.Hsueh.Pan. 3: 75-83 (1980). (b) Kuan, Y.W. et al. Studies of organophosphorus pollutants in well water near a dimethoate plant. III. Hydrolysis rates of $\mathrm{O}, \mathrm{O}, \mathrm{S}$-trimethyl dithiophosphate, O,O,O-trimethyl thiophosphate, DDVP, dimethoate and its ester, Huan.Ching.K.'o.Hsueh. 2: 4-9 (1979).

3 (a) Gatermann, R. et al. The distribution of O,O,Otrimethylthiophosphate and O,O,S-trimethyldithiophosphate in the North Sea, Chemosphere 32 (10): 1907-1918 (1996). (b) Gotz, R. et al. Organic trace compounds in the water of the River Elbe near Hamburg. Part II, Chemosphere 36 (9): 2103-2118 (1998).

${ }^{4}$ Imamura, T. and Talcott, R.E. Mutagenic and alkylating activities of organophosphate impurities of commercial malathion, Mutat.Res. 155 (1-2): 1-6 (1985).

${ }^{5}$ Verschoyle, R.D. and Cabral, J.R.P. Investigation of the acute toxicity of some trimethyl and triethyl phosphorothioates with particular reference to those causing lung damage, Arch.Toxicol. 51 (3): 221-231 (1982).

${ }^{6}$ Verschoyle, R.D., Dinsdale, D. and Wolf, C.R. Inhibition and induction of cytochrome P 450 isoenzymes in rat lung, J.Pharmacol.Exp.Ther. 265 (1): 386-391 (1993). 
A grandes rasgos, desde el punto de vista estructural el comportamiento conformacional del TMSP, es análogo al del TMP, lo que simplifica la interpretación de las características estructurales y espectroscópicas de esta molécula. Su alto grado de simetría permite realizar una interpretación de la coexistencia de confórmeros con un número de estados conformacionales degenerados energéticamente, tal como en el TMP, con el adicional de encontrarnos frente a un enlace $\mathrm{P}=\mathrm{S}$ que aporta características particulares respecto a estas diferencias energéticas, como veremos más adelante.

Desde el punto de vista del comportamiento en los espectros vibracionales, para los fosfotioatos en general se describen dos bandas características del espectro vibracional muy bien separadas, asociadas al enlace $\mathrm{P}=\mathrm{S}$. Estas bandas se encuentran en los rangos $862-685 \mathrm{~cm}^{-1}$ y $730-550 \mathrm{~cm}^{-1}$. $^{(7)}$ Se han descrito compuestos del tipo $\mathrm{P}=\mathrm{S}$ en los cuales no es posible identificar una banda en la región $600-700 \mathrm{~cm}^{-1}$ que pueda asociarse en forma inequívoca al estiramiento $\mathrm{P}=\mathrm{S} .{ }^{(8)}$

De esta manera, la ausencia de esta banda no implica la inexistencia del enlace $\mathrm{P}=\mathrm{S}$ y se puede deducir por ello que corresponde a un modo de vibración no puro para el cual la componente de estiramiento $\mathrm{P}=\mathrm{S}$ es la más importante. Este fenómeno también se sugiere para el modo vibracional asignado al estiramiento $\mathrm{P}=\mathrm{O}$ en el TMP.

Se ha calculado que la constante de fuerza del enlace $\mathrm{P}=\mathrm{S}$ decrece en un $10 \%$ respecto a la del enlace $\mathrm{P}=\mathrm{O}{ }^{(9)}$ y se argumenta que, solamente sobre la base de las diferencias de electronegatividad entre ambos calcógenos, la contribución de la forma canónica $\mathrm{P}^{+}-\mathrm{S}^{-}$es de menor importancia que en el caso del grupo $\mathrm{P}=\mathrm{O}$. $^{(10)}$

El carácter no puro de estiramiento $\mathrm{P}=\mathrm{X}$, siendo $\mathrm{X}$ oxígeno o azufre, ayudaría a explicar su desdoblamiento como manifestación de coexistencia de confórmeros, pues

\footnotetext{
${ }^{7}$ Wan, J. and Zhan, C.G. Maximum bond order hybrid orbital calculations of the $\mathrm{P}=\mathrm{S}$ stretching frequencies for organophosphorus compounds. Spectrochim. Acta 51A(10):1665-1669 (1995).

${ }^{8}$ Una buena compilación de datos se recoge en: Popov, E.M., Kabachnik, M.I. and Mayants, L.S. Vibrational spectra of organophosphorus compounds. Russ. Chem. Revs. 30, (7):362-377 (1961).

${ }^{9}$ Wafa, O.A., Lentz, A. and Goubeau, J. Vibrational spectra and force constants of the transition series $\mathrm{OP}\left(\mathrm{OCH}_{3}\right)_{3}-\mathrm{OP}\left(\mathrm{SCH}_{3}\right)_{3}$ and $\mathrm{SP}\left(\mathrm{OCH}_{3}\right)_{3}$ $\mathrm{SP}\left(\mathrm{SCH}_{3}\right)_{3}$, Z.Anorg.Allg.Chem. 380 (2): 128-136 (1971).

${ }^{10}$ Grim, S.O., Walton, E.D. Unsymmetrical Bis-phosphorus ligands. 12. Synthesis and nuclear magnetic resonance studies of some derivatives of bis(diphenilphosphino)methane, Inorg. Chem. 19:1982-1987 (1980).
}

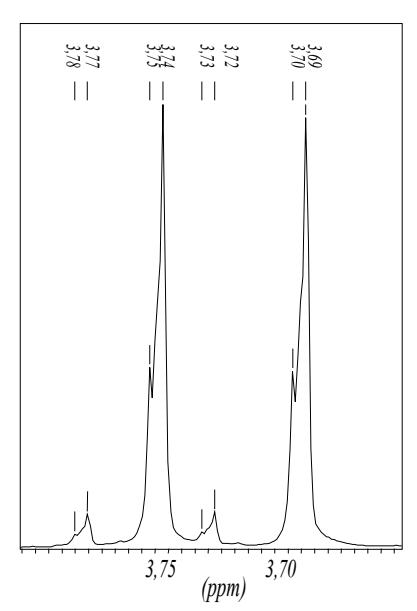

Figura 3.2: Doblete metílico ${ }^{1} \mathrm{H}$ RMN del TMSP. 
$v_{\mathrm{P}=\mathrm{S}}=-85,5 \mathrm{Q}_{\mathrm{S}}+527,7 \mathrm{Q}_{\mathrm{P}}+878$

Ecuación 3.a distinguen variaciones geométricas del entorno cercano al enlace $\mathrm{P}=\mathrm{X}$.

Aún así, es posible derivar el número de onda de estiramiento $\mathrm{P}=\mathrm{S}$ a partir de la estructura electrónica $\mathrm{y}$ molecular de los fosfotioatos con un buen índice predictivo ${ }^{(7)}$, disponiéndose además de una relación empírica simplificada (Ecuación 3.a) que asocia la carga neta del fósforo $\left(Q_{\mathrm{P}}\right)$ y del azufre $\left(Q_{\mathrm{S}}\right)$ con la frecuencia de estiramiento $\mathrm{P}=\mathrm{S}$.

En el TMSP, la molécula más pequeña a considerar entre los triésteres alquílicos, las bandas de balanceo y de estiramiento del enlace $\mathrm{P}=\mathrm{S}$ aparecen en $826 \mathrm{~cm}^{-1}$ y en un doblete 618 y $598 \mathrm{~cm}^{-1}$. Una importante evidencia de la relación con el modo $v_{\mathrm{P}=\mathrm{S}}$ es el corrimiento isotópico de las estas bandas hacia números de onda más bajos en compuestos de ${ }^{34} \mathrm{~S}$, comparados con los correspondientes a ${ }^{32} \mathrm{~S}$, que en el TMSP se manifiestan a valores de $613 \mathrm{~cm}^{-1}$ y $591 \mathrm{~cm}^{-1}$ respectivamente. ${ }^{(11)}$

Tabla 3.I: Corrimientos químicos y constantes de acoplamiento en los espectros de ${ }^{1} \mathrm{H}$ RMN (calibración respecto a TMS) y ${ }^{13} \mathrm{C}$ RMN (calibración respecto a cloroformo 77,00 ppm) del TMSP. Para las bandas en ${ }^{1} \mathrm{H}$ RMN, se indican entre paréntesis las alturas relativas.

\begin{tabular}{cc}
\hline \multicolumn{1}{l}{$\mathrm{H}$ NMR } \\
\hline$\delta$ ppm & ${ }^{3} J_{\mathrm{H}-\mathrm{P}} \mathrm{Hz}$ \\
\hline $3,71(1,00)$ & 13,4 \\
$3,71(0,34)$ & 12,0 \\
$3,74(0,07)$ & \\
$3,75(0,03)$ & \\
\hline${ }^{13} \mathrm{C} \mathrm{NMR}$ & ${ }^{2} \mathrm{~J}_{\mathrm{C}-\mathrm{P}} \mathrm{Hz}$ \\
\hline $5 \mathrm{ppm}$ & 5,3 \\
\hline
\end{tabular}

Basados en la existencia del doblete de números de onda $618-598 \mathrm{~cm}^{-1}$ en IR ${ }^{(13)}$ y $616-594 \mathrm{~cm}^{-1}$ en Raman ${ }^{(12)}$ se sugiere la coexistencia de a lo menos dos isómeros rotacionales en fase líquida, reducido a uno con simetría $\mathrm{C}_{3}$ en fase sólida. Este confórmero correspondería al número de onda de estiramiento a $618 \mathrm{~cm}^{-1}$.

La persistencia de esta banda en fase sólida indica que la misma corresponde a una manifestación del confórmero más estable. ${ }^{(13)}$ Dada la relación de intensidades de las bandas en fase líquida, existe una relación de

${ }^{11}$ Miller, M.P. Infrared spectra of ${ }^{34} \mathrm{~S}$ thionophosphate esters. Appl. Spectrosc. 48, 1553(1994).

12 Baudler, M. Über dir Ramanspektren von einiegen Estern der phosphoriden Säure und der phosphosäure, Z.Elektrochem. 59 (3): 173-184 (1955). 
abundancia de 1,38 entre este y el/los confórmeros menos estable/s. ${ }^{(11)}$

En el espectro Raman en fase líquida, se identifican además otro par de dobletes que se relacionan con la manifestación de confórmeros en $1085-1065 \mathrm{~cm}^{-1}$, asignados al estiramiento $(\mathrm{C}-\mathrm{O})_{3}$ y en $453-425 \mathrm{~cm}^{-1}$ en el espectro Raman, cuya contraparte en el espectro infrarrojo aparece en $448-422 \mathrm{~cm}^{-1}$, asignados a la deformación asimétrica $\mathrm{P}-\mathrm{O}_{3} .{ }^{(14)}$

En estas asignaciones reportadas, se utiliza la posibilidad de existencia de dos isómeros conformacionales de simetrías $\mathrm{C}_{3}$ y $\mathrm{C}_{3 v}$, de menor y mayor energías respectivamente, aunque otros autores (15) proponen la coexistencia de tres confórmeros con simetrías $\mathrm{C}_{3}, \mathrm{C}_{3 v}$ trans $\mathrm{y}$ $\mathrm{C}_{3 v}$ cis. Sobre esta cuestión volveremos más adelante.

En un reporte reciente de resultados teóricos hechos sobre el TMSP se informan cuatro mínimos, cuando el cálculo se realiza a un nivel HF/STO-3G*. El orden de estabilidad de simetrías propuesto es $\mathrm{C}_{\mathrm{s}}, \mathrm{C}_{3}, \mathrm{C}_{1}$ trans, $\mathrm{C}_{1}$ cis. Sin embargo, cuando el cálculo se realiza a un nivel HF/6-31G*, el orden de estabilidad de simetrías es $C_{3}$ y $C_{s}$ para dos mínimos de energías. ${ }^{(16)}$ Esta inversión del orden relativo de energías de los mínimos con el aumento de la complejidad de la base orbital, es un efecto que también aparece en los cálculos realizados con distintas bases orbitales sobre el TMP.

Tomando el valor del momento dipolar experimental reportado en valores de alrededor de $2,8 \mathrm{D}$, se encuentra una buena estimación al considerar el valor teórico ponderado ${ }^{(17)}$ de los dos confórmeros que teóricamente coexisten para la aproximación HF/6-31G*.(16) Utilizando el procedimiento

\footnotetext{
13 Durig, J.R. and Diyorio, J.S. Vibrational spectra and structure of organophosphorus compounds. VII. IR and Raman spectra of trimethylthiophosphate. J.Mol.Struc. 3:179-190. (1969).

14 Marsault-Herail, F. Vibrational spectra of organophosphorus compounds at 293K - 15K. Molecular conformations, J. Chim. Phys. Physicochim. Biol. 68 (2): 274-286 (1971).

15 Maiants, L.S., Popov, E.M. and Kabachnik, M.I. Calculations of the caracteristics vibrations of phosphorus compounds. II. Characteristics vibrations of the $\left(\mathrm{CH}_{3} \mathrm{O}\right)_{3} \mathrm{PO}$ and $\left(\mathrm{CH}_{3} \mathrm{O}\right)_{3} \mathrm{PS}$ molecules, Opt.Spectrosc. 7 (2): 108-113 (1959).

${ }_{16}$ Katagi, T. Ab initio structures of trimethyl and dimethyl phenyl phosphorothioates. Theochem. 68, 61-67 (1990).

17 Raevsky, O.A. Conformational analysis of organophosphorus compounds by means of IR spectroscopy and dipole moments methods. J. Mol. Struct. 19, 275-287 (1973).
} 


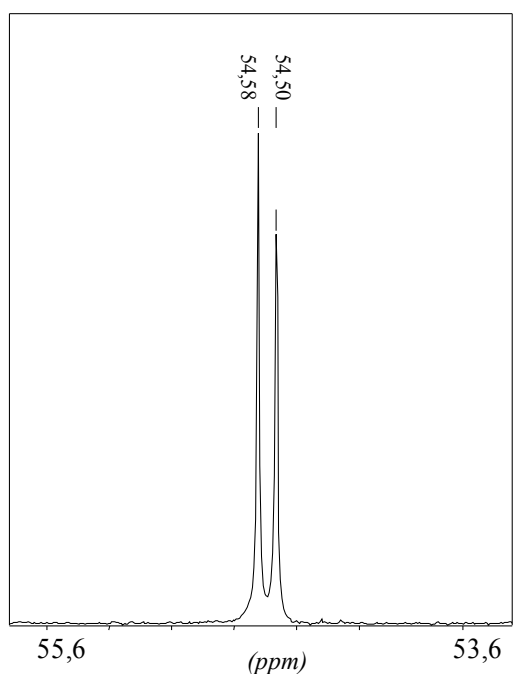

Figura 3.3: Doblete metílico ${ }^{13} \mathrm{C}$ RMN del TMSP. inverso, el momento dipolar puede evaluarse con las proporciones adecuadas de confórmeros $\mathrm{C}_{3}$ y $\mathrm{C}_{1}$, estimándose un valor de $0,8 / 0,2$ para la abundancia relativa de estos confórmeros. ${ }^{(17)}$

En las medidas de ${ }^{31} \mathrm{P}$ RMN, se han reportado cambios en el corrimiento químico de la señal del TMSP en valores de 73,4 $\mathrm{ppm},{ }^{(18)}$ por la presencia del isótopo ${ }^{34} \mathrm{~S}$ y en valores de alrededor de $20 \mathrm{ppm}$, debido a la protonación de la molécula. ${ }^{(19)}$ También se reportan valores de acoplamiento ${ }^{3} J_{\mathrm{P}-\mathrm{H}}$ de entre 15 y $20 \mathrm{~Hz}$ en compuestos del tipo $\mathrm{P}=\mathrm{S}$ de OPs pentavalentes. ${ }^{(20)}$ Finalmente, se han reportado las medidas y el análisis tanto de espectro UV visible, ${ }^{(21)}$ como del espectro fotoelectrónico ${ }^{(22)}$ del TMSP.

\section{Caracterización espectroscópica}

Nuestras medidas de ${ }^{1} \mathrm{H}$ RMN realizadas sobre el TMSP revelan una estructura fina del doblete que aparece alrededor de 3,71 ppm, correspondiente a los $\mathrm{H}$ de los grupos metilo, manifestándose cuatro máximos para cada uno de ambos picos (Figura 3.2).

Aplicando el mismo razonamiento que se utilizó en el análisis de la estructura fina del TMP, se sugiere la presencia de a lo menos un par de metilos de entornos diferentes, y que se distribuyen en una relación de áreas aproximada de 30:10:2:1 (Tabla 3.I). De esta manera, los resultados sugieren la coexistencia de metilos con distintos entornos relativos, aunque con mayor diferencia de energías que la manifestada para los confórmeros del TMP. En el espectro ${ }^{13} \mathrm{C}$ RMN del TMSP (Figura 3.3) no es posible observar las asimetrías de las señales que aparecen en el espectro ${ }^{13} \mathrm{C}$ RMN del TMP.

\footnotetext{
${ }^{18}$ Bjorholm, T. and Larsson, F.C.V. 34S isotope effects on ${ }^{31} \mathrm{P}$ chemical shifts in thiophosphate esters, Mag.Reson.Chem. 27 (2): 184-186 (1989).

${ }^{19}$ Skvortsov, N.K., Ionin, B.I. and Petrov, A.A. Change of chemical shifts of ${ }^{31} \mathrm{P}$ during protonation of phosphoryl and thiophosphoryl compounds, Zh.Obshch.Khim. 44 (1): 220-221 (1974).

20 Jones, R.A. and Katritsky, A.R. Kermagnetische Resonanzspektroskopie des Phosphors, Angew. Chem. (Internat.). 1(2): 60-68 (1962).

${ }^{21}$ Shagidullin, R.R. et al. The UV spectra of thiophosphoryl compounds, Dokl.Akad.Nauk.SSSR. 222 (4): 897-900 (1975).

22 Cowley, A.H. et al. Molecular photoelectron spectroscopic investigation of some polycyclic Group VA compounds and related acyclic species. 2. Free and coordinated phosphite, thiophosphite, and arsenite esters, Inorg. Chem. 23 (21): 3378-3383 (1984).
} 
Respecto a los espectros vibracionales, es interesante verificar que en el espectro Raman aparecen bandas que expresarían dobletes por coexistencia de confórmeros (Figura 3.4), que en el espectro infrarrojo no se pueden distinguir, ya sea por no exhibir intensidad suficiente o por estar apantalladas por bandas más intensas.

En la Tabla 3.II se listan los valores de los números de onda IR y Raman del TMSP, experimentales y teóricas, en los rangos de números de onda en los que se manifestaría la coexistencia de confórmeros, con las asignaciones propuestas. La Figura 3.6 muestra el espectro del TMSP en el rango $450-1500 \mathrm{~cm}^{-1}$, en la que se indican aquellos dobletes que se asignan a isomería conformacional. Con claridad hay al menos tres dobletes con esta asignación: los asignados al estiramiento antisimétrico del grupo $(\mathrm{C}-\mathrm{O})_{3}$, al estiramiento del enlace $\mathrm{P}=\mathrm{S}$ y a la deformación antisimétrica del grupo $\left(\mathrm{PO}_{3}\right)$.

De manera menos clara es posible argumentar acerca de la manifestación de coexistencia conformacional en los modos vibracionales combinados de estiramiento $\left(\mathrm{PO}_{3}\right)$ y deformación $\mathrm{S}=\mathrm{P}-\mathrm{O}_{3}$ y aleteo antisimétrico $(\mathrm{PO})_{3}$ y balanceo $(\mathrm{PO})_{3}$. Estas cinco manifestaciones en los espectros vibracionales, se analizan en función de las características de las bandas en las medidas respectivas y en los cálculos $a b$ initio asociados.

\section{Correlación teórica}

Para el primero de los dobletes propuestos, según los cálculos realizados, las bandas son originadas por vibraciones análogas a la banda en $1030 \mathrm{~cm}^{-1}$, que por ser muy ancha en IR, no permite la diferenciación de las dos bandas a números de onda superiores reportadas en Raman, espectro para el cual, estos modos no presentan gran intensidad y si es posible diferenciar las tres bandas.

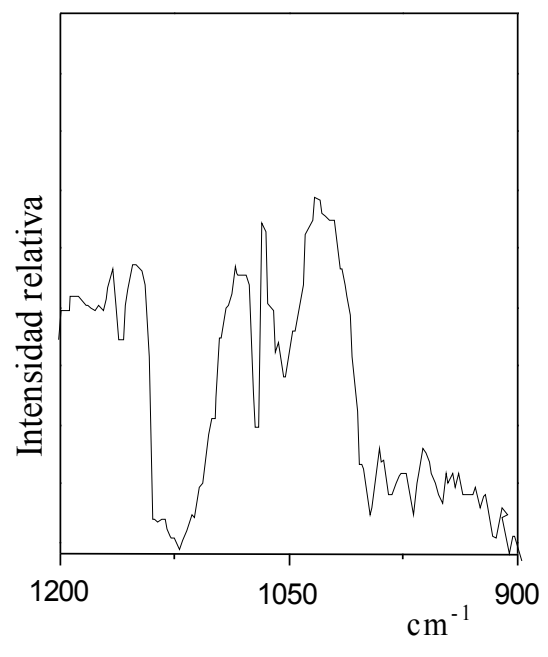

Figura 3.4: Doblete va $\mathrm{a}_{(\mathrm{C}-0) 3}$ en el espectro Raman del TMSP ubicado en 1068 y $1085 \mathrm{~cm}^{-1}$. 
Tabla 3.II: Valores experimentales, reportados previamente y calculados del espectro vibracional IR y Raman en fase líquida para el TMSP. Se muestran sólo las bandas que evidencian coexistencia de confórmeros.

\begin{tabular}{|c|c|c|c|c|c|}
\hline \multirow[t]{2}{*}{ Asignación } & \multirow[t]{2}{*}{$I R$} & \multirow[t]{2}{*}{ Raman } & \multicolumn{3}{|c|}{ Calculado (HF/6-31G**) } \\
\hline & & & $\mathrm{C}_{3}$ & $\mathrm{C}_{1}$ & Cs \\
\hline va $(\mathrm{C}-\mathrm{O})_{3} \mathrm{C}_{3}, \mathrm{C}_{\mathrm{s}}$ & $1098^{(13)}$ & $\begin{array}{l}1085^{(14)} \\
1083^{(, 12)^{(25)}} \\
1082^{(13)}\end{array}$ & 1101 & & 1100 \\
\hline$v a(C-O)_{3} \mathrm{C}_{1}$ & & $\begin{array}{l}1076^{(12)} \\
1069^{(, 12)^{(25)}} \\
1068^{(13)} \\
1065^{(14)}\end{array}$ & & 1090 & \\
\hline va $(\mathrm{C}-\mathrm{O})_{3}$ & $\begin{array}{l}1031(1,00)^{(25)} \\
1030^{(14)(13)} \\
1024^{(15)}\end{array}$ & $\begin{array}{l}1037^{(14)} \\
1035^{(13)} \\
1033_{(, 12)^{(25)}} \\
1024^{(12)}\end{array}$ & $1066(2)$ & $\begin{array}{l}1068 \\
1060\end{array}$ & 1062 \\
\hline$v(\mathrm{P}-\mathrm{O})_{3}$ & $\begin{array}{l}826(, 79)^{(25)} \\
825^{(14)(13)}\end{array}$ & $\begin{array}{l}834^{(14)} \\
831^{(13)} \\
828\left(^{(, 11)^{(25)}}\right. \\
826^{(12)}\end{array}$ & $822(2)$ & 823 & $\begin{array}{l}1049 \\
829\end{array}$ \\
\hline $\begin{array}{l}\text { va }(\mathrm{P}-\mathrm{O})_{3} \mathrm{C}_{1}, \mathrm{C}_{\mathrm{s}} \\
\delta \mathrm{s}\left(\mathrm{S}=\mathrm{P}-\mathrm{O}_{3}\right) \mathrm{C}_{3}\end{array}$ & $\begin{array}{l}813^{(14)} \\
809,6(, 67)^{(25)}\end{array}$ & & 808 & 811 & 804 \\
\hline$\delta s\left(\mathrm{~S}=\mathrm{P}-\mathrm{O}_{3}\right) \mathrm{C}_{1}, \mathrm{C}_{\mathrm{s}}$ & $775,0(, 38)^{(25)}$ & & & 792 & 790 \\
\hline$v(\mathrm{P}=\mathrm{S}) \mathrm{C}_{1}, \mathrm{C}_{\mathrm{s}}$ & $\begin{array}{l}620^{(7)} \\
619\left(^{(, 34)^{(25)}}\right. \\
619^{(14)} \\
618^{(11)(13)} \\
616^{(15)}\end{array}$ & $\begin{array}{l}621^{(13)} \\
620^{(14)} \\
617^{(, 58)^{(25)}} \\
616^{(12)}\end{array}$ & & 588 & 595 \\
\hline$v(\mathrm{P}=\mathrm{S}) \mathrm{C}_{3}$ & $\begin{array}{l}599(, 27)^{(25)} \\
598^{(7)(11)(14)} \\
597^{(13)}\end{array}$ & $\begin{array}{l}600^{(13)(14)} \\
597(1,00)^{(25)} \\
594^{(12)}\end{array}$ & 563 & & \\
\hline$\delta \mathrm{a}(\mathrm{P}-\mathrm{O}-\mathrm{C})_{3} \mathrm{C}_{1}, \mathrm{C}_{\mathrm{s}}$ & $\begin{array}{l}476^{(14)} \\
4766^{(, 24)^{(25)}} \\
472^{(13)}\end{array}$ & $\begin{array}{l}478^{(14)} \\
476^{(13)} \\
475^{(12)} \\
474(, 10)^{(25)}\end{array}$ & & 459 & 459 \\
\hline$\delta(\mathrm{P}-\mathrm{O})_{3}$ & $\begin{array}{l}448^{(14)} \\
445^{(13)}\end{array}$ & $\begin{array}{l}453^{(14)} \\
449^{(, 10)^{(25)}} \\
449^{(13)} \\
445^{(12)}\end{array}$ & $\begin{array}{l}437(2) \\
437\end{array}$ & 433 & 443 \\
\hline$\delta$ s $\mathrm{P}-(\mathrm{O}-\mathrm{C})_{3} \mathrm{C}_{3}$ & $\begin{array}{l}400^{(14)} \\
397^{(13)}\end{array}$ & $\begin{array}{l}403^{(14)} \\
398^{(13)} \\
397^{(, 10)^{(25)}} \\
388^{(12)}\end{array}$ & 407 & $\begin{array}{l}317 \\
314\end{array}$ & $\begin{array}{l}367 \\
321\end{array}$ \\
\hline$\rho w a(P-O)_{3}$ & $322^{(13)}$ & $\begin{array}{l}326^{(13)(14)} \\
324^{(12)} \\
323(12)^{(25)}\end{array}$ & $\begin{array}{l}312 \\
312\end{array}$ & & 310 \\
\hline $\operatorname{\rho r}(\mathrm{P}-\mathrm{O})_{3} \mathrm{C}_{3}$ & & $\begin{array}{l}201(, 16)^{(25)} \\
199^{(13)}\end{array}$ & 240 & & \\
\hline $\operatorname{\rho r}(\mathrm{P}-\mathrm{O})_{3} \mathrm{C}_{1}$ & $190^{(13)}$ & $\begin{array}{l}191^{(12)} \\
187^{(13)} \\
183^{(14)} \\
178(, 16)^{(25)}\end{array}$ & & 208 & 191 \\
\hline
\end{tabular}

v: vibración de estiramiento; vs: vibración de estiramiento simétrico; va: vibración de estiramiento antisimétrico; $\delta \mathrm{s}$ : vibración de deformación simétrica; $\delta a$ : vibración de deformación antisimétrica; $\rho$ r: vibración de balanceo; $\rho$ wa: vibración de aleteo antisimétrico; r: vibración de balanceo; sh: hombro. En los valores experimentales, entre paréntesis se encuentra la intensidad de cada banda. En los valores teóricos, entre paréntesis se indica el grado de degeneración energética, si existe.

Según los cálculos teóricos, de las dos bandas aparecidas en 1068 y $1085 \mathrm{~cm}^{-1}$, que corresponderían a un doblete conformacional del modo vibracional de estiramiento antisimétrico $(\mathrm{C}-\mathrm{O})_{3}$, el confórmero más estable se correspondería con la banda aparecida a números de onda más altos (Figura 3.4). 
Para el segundo de los dobletes propuestos, los cálculos sugieren una reasignación de los modos vibracionales, ubicando el doblete de isomería a 474 - 449 $\mathrm{cm}^{-1}$ para las bandas Raman asociadas al modo de deformación antisimétrica $\left(\mathrm{PO}_{3}\right)$, al igual que la banda aparecida a $397 \mathrm{~cm}^{-1}$.

Tabla 3.III: Parámetros geométricos, energéticos y fisicoquímicos de los confórmeros correspondientes a mínimos de energía del TMSP.

\begin{tabular}{|c|c|c|c|}
\hline & \multicolumn{3}{|c|}{ TMSP } \\
\hline & $\mathrm{C}_{3}(\mathrm{l})$ & $\mathrm{C}_{1}(\mathrm{II})$ & Cs (III) \\
\hline$\psi_{1}$ & 52 & 56 & -42 \\
\hline$\psi_{2}$ & 52 & 44 & 42 \\
\hline$\psi_{3}$ & 52 & 173 & 180 \\
\hline$\phi 1$ & 116 & 118 & 118 \\
\hline$\phi 2$ & 116 & 116 & 118 \\
\hline$\phi 3$ & 116 & 114 & 112 \\
\hline $\mathrm{HF} / 6-31 \mathrm{G}^{\star *}(\mathrm{kcal} / \mathrm{mol})$ & 0,00 & 1,39 & 2,69 \\
\hline$\mu \mathrm{D}$ (Müll)Calc. & 2,53 & 5,12 & 4,47 \\
\hline Prop.a $298^{\circ} \mathrm{K}$ (peso $2: 6: 3$ ) & 0,77 & 0,22 & 0,01 \\
\hline$\mu \mathrm{D}$ (Müll)Pond. (peso 2:6:3) & & 3,30 & \\
\hline Prop.a $298^{\circ} \mathrm{K}$ (peso 1:6:3) & 0,62 & 0,36 & 0,01 \\
\hline$\mu \mathrm{D}$ (Müll)Pond. (peso 1:6:3) & & 3,71 & \\
\hline Prop.a $298^{\circ} \mathrm{K}$ (peso 2:2:1) & 0,91 & 0,09 & 0,01 \\
\hline$\mu \mathrm{D}$ (Müll)Pond. (peso 2:2:1) & & 2,86 & \\
\hline MP2/6-31G** $(\mathrm{kcal} / \mathrm{mol})$ & 0,00 & 1,03 & 2,28 \\
\hline$\mu \mathrm{D}$ (Müll)Calc. & 2,37 & 4,65 & 4,05 \\
\hline Prop.a $298^{\circ} \mathrm{K}$ (peso 2:6:3) & 0,64 & 0,34 & 0,02 \\
\hline$\mu \mathrm{D}$ (Müll)Pond. (peso 2:6:3) & & 3,35 & \\
\hline Prop.a $298^{\circ} \mathrm{K}$ (peso $1: 6: 3$ ) & 0,47 & 0,50 & 0,03 \\
\hline$\mu \mathrm{D}$ (Müll)Pond. (peso 1:6:3) & & 3,73 & \\
\hline Prop.a $298^{\circ} \mathrm{K}$ (peso 2:2:1) & 0,84 & 0,15 & 0,01 \\
\hline$\mu \mathrm{D}$ (Müll)Pond. (peso 2:2:1) & & 2,84 & \\
\hline$\mu \mathrm{D}$ Exp. $298^{\circ} \mathrm{K}$ & & (23) 2,7 & \\
\hline
\end{tabular}

Según los cálculos $a b$ initio, otras bandas que aparecen como posible manifestación de coexistencia de confórmeros se encuentran en el espectro Raman a 323, 201 y $178 \mathrm{~cm}^{-1}$ correspondientes a modos de vibración de aleteo antisimétrico $(\mathrm{PO})_{3}$ y balanceo $(\mathrm{PO})_{3}$ (ver Tabla 3.II).

Alrededor de $600 \mathrm{~cm}^{-1}$ se observa un doblete característico, homólogo al encontrado en muchos fosfotioatos. Los cálculos a nivel $6-31 G^{* *}$ asignan este

\footnotetext{
${ }^{23}$ La medida se realiza en benceno. La referencia original se encuentra en Waeschke, H. Mitzner, R. Z.Chem., 17:228 (1977).

${ }^{24}$ La medida se realiza en tetracloruro de carbono y está reportada en ${ }^{(17)}$.
} 


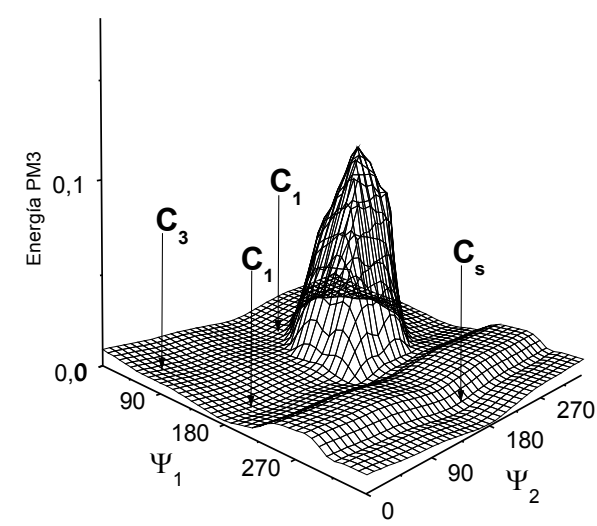

Figura 3.5: Superficies de energía conformacional del TMSP, calculadas a nivel HF/PM3 y generadas por rotación de dos ángulos diedros $\psi$ y manteniendo el otro fijo en $52^{\circ}$. doblete al estiramiento $\mathrm{P}=\mathrm{S}$ correspondiéndole la banda a 598 $\mathrm{cm}^{-1}$ al isómero de simetría $\mathrm{C}_{3}$ más estable y la banda a 618 $\mathrm{cm}^{-1}$ a una combinación de los modos correspondientes a los confórmeros menos estables $\mathrm{C}_{1}$ y $\mathrm{Cs}$, contradiciendo los resultados anteriores para la asignación de isómeros, con una relación de abundancia teórica calculada a un nivel MP2/6-31G** de 1,79 .

No se han descrito relaciones empíricas ni indicios experimentales que asocien el enlace $\mathrm{P}=\mathrm{S}$ con la banda presente en $826 \mathrm{~cm}^{-1}$, lo que aporta a su desvinculación con el modo vibracional de estiramiento del grupo $\mathrm{P}=\mathrm{S}$. Pero, a la luz de los resultados $a b$ initio descritos, esta banda se la puede asociar, junto con la banda a $775 \mathrm{~cm}^{-1}$, a una manifestación de coexistencia de los confórmeros $\mathrm{C}_{3}$ y $\mathrm{C}_{1}$ para un modo normal combinado de estiramiento $\left(\mathrm{PO}_{3}\right)$ y deformación $\mathrm{S}=\mathrm{P}-\mathrm{O}_{3}$, tal como se indica en la Tabla 3.II.

Por el mismo razonamiento ya expuesto (Capítulo 2), los máximos encontrados en el espectro ${ }^{1} \mathrm{H}$ RMN ${ }^{(25)}$ del TMSP, representarían la coexistencia de al menos cuatro metilos distintos en una relación análoga a la relación de áreas de los mismos, suponiendo además una relación respecto al fósforo en, por lo menos, dos posiciones.

Sin embargo, en este caso tampoco es sencillo justificar la medida de dos constantes de acoplamiento ${ }^{3} J_{\mathrm{P}-\mathrm{H}}$ distintas, puesto que existe libre rotación de los metilos y no pueden distinguirse cada uno de sus hidrógenos por RMN. Ya hemos mencionado que las variaciones en ${ }^{3} J_{\mathrm{P}-\mathrm{H}}$ serían dependientes del ángulo de torsión H-C-O-P. ${ }^{(26)}$

\section{Integración y justificación de resultados}

Considerando los datos precedentes, la relación teórica de picos, dadas las abundancias relativas obtenidas por los cálculos ab initio a nivel $\mathrm{HF} / 6-31 \mathrm{G}^{* *}$ para los tres confórmeros considerados, la relación teórica entre ellos sería aproximadamente de 30:15:2:1. Dada la incertidumbre experimental de la medida ${ }^{1} \mathrm{H} \mathrm{RMN}$, esta corresponde a una buena aproximación a estos valores.

\footnotetext{
${ }^{25}$ Mastrantonio, G. Della Védova, C.O. Spectroscopic and conformational comparative study of trimethyl chalcogenphosphates. J.Mol.Struc. 561:161-174 (2001).

${ }^{26}$ Gorenstein, D.G. Phosphorus-31 NMR. Principles and Applications, Academic Press, New York (1984).
} 
Mediante la aplicación de la Ecuación 1.a para los datos obtenidos a nivel HF/6-31G** y MP2/6-31G**, se obtiene un valor teórico ponderado de $2,86 \mathrm{D}$ y 2,84 para la coexistencia de tres confórmeros, como se muestra en la Tabla 3.III. Este valor es muy cercano al experimental, lo que sugiere que la aplicación de la expresión aludida sobre sólo los tres confórmeros de mínima energía hallados, es una aproximación adecuada para la justificación de los resultados experimentales y que, por lo tanto, son estas formas las que aportarían fundamentalmente a la magnitud del dipolo total.

Según los datos representados en la Figura 3.5, la descripción de la libertad conformacional referida a las rotaciones de los grupos metilo alrededor de los ángulos diedros $\psi$, se observa que cualitativamente en el TMSP la situación es similar a la del TMP. Sin embargo, cuantitativamente existe una dificultad energética relativa algo mayor en esta rotación.

Este comportamiento teórico puede asimilarse como una de las fuertes evidencias que sustentan la validez de la aplicación de la expresión de la Ecuación 1.a para el cálculo del momento dipolar total del TMSP y la razón fundamental de la congruencia de sus resultados con las medidas experimentales de este parámetro.

Se debe tomar en cuenta que en la estabilización de confórmeros las interacciones entre los hidrógenos metílicos y los pares libres del azufre no juegan un papel tan importante, dado que éstos se encuentran más deslocalizados. Por otro lado, dado el mayor tamaño del átomo de azufre los impedimentos estéricos son más importantes, aumentando las energías de las formas gauche y estabilizando las formas trans.

Estos dos efectos en conjunto se vinculan con el aumento de las energías de los estados de transición conformacional. Este efecto se manifiesta como una mayor "rugosidad" de la superficie potencial en la Figura 3.5 y como un aumento de las diferencias energéticas entre las distintas formas conformacionales estables.

Una consideración interesante es la que se puede realizar con el uso de la Ecuación 3.a para la estimación de la magnitud de la diferencia del doblete en el número de onda de estiramiento $\mathrm{P}=\mathrm{S}$, que en principio representa a los dos confórmeros más abundantes. Ya se ha indicado que esta relación se concibió para estimar el número de onda de 
estiramiento $\mathrm{P}=\mathrm{S}$ o, para algunos compuestos, el promedio de los números de onda para una serie de fosfotioatos, utilizando la carga relativa del fósforo y del azufre. ${ }^{(7)}$

Podemos calcular las cargas relativas de Mülliken para el fósforo y el azufre en cada uno de los confórmeros principales y, aplicando entonces la Ecuación 3.a, se puede estimar la separación de las bandas en el doblete $\mathrm{v}_{\mathrm{P}=\mathrm{s}}$ : para un nivel de cálculo ab initio $\mathrm{HF} / 6-31 \mathrm{G}^{* *}$, las cargas relativas (Mülliken) del fósforo son 1,38 y 1,43 para los confórmeros $\mathrm{C}_{3}$ y $\mathrm{C}_{1}$ respectivamente, mientras que la carga del azufre se estima, mediante el mismo método, en $-0,56$ y $-0,53$ para los mismos dos confórmeros de menor energía respectivamente. Esta estimación da una diferencia de $18 \mathrm{~cm}^{-1}$, lo que es un buen valor de la diferencia experimental observada en el espectro infrarrojo de $22 \mathrm{~cm}^{-1}$.

El azufre posee menos capacidad que el oxígeno para donar electrones desde orbitales no enlazantes hacia los orbitales del fósforo. ${ }^{(27)}$ Este fenómeno se asocia a contribuciones de la estabilización de estructuras. ${ }^{(28)}$ Por 10 tanto, se explicaría por estas razones una mayor inestabilidad de los confórmeros de fosfotioatos respecto a los compuestos oxofosforados.

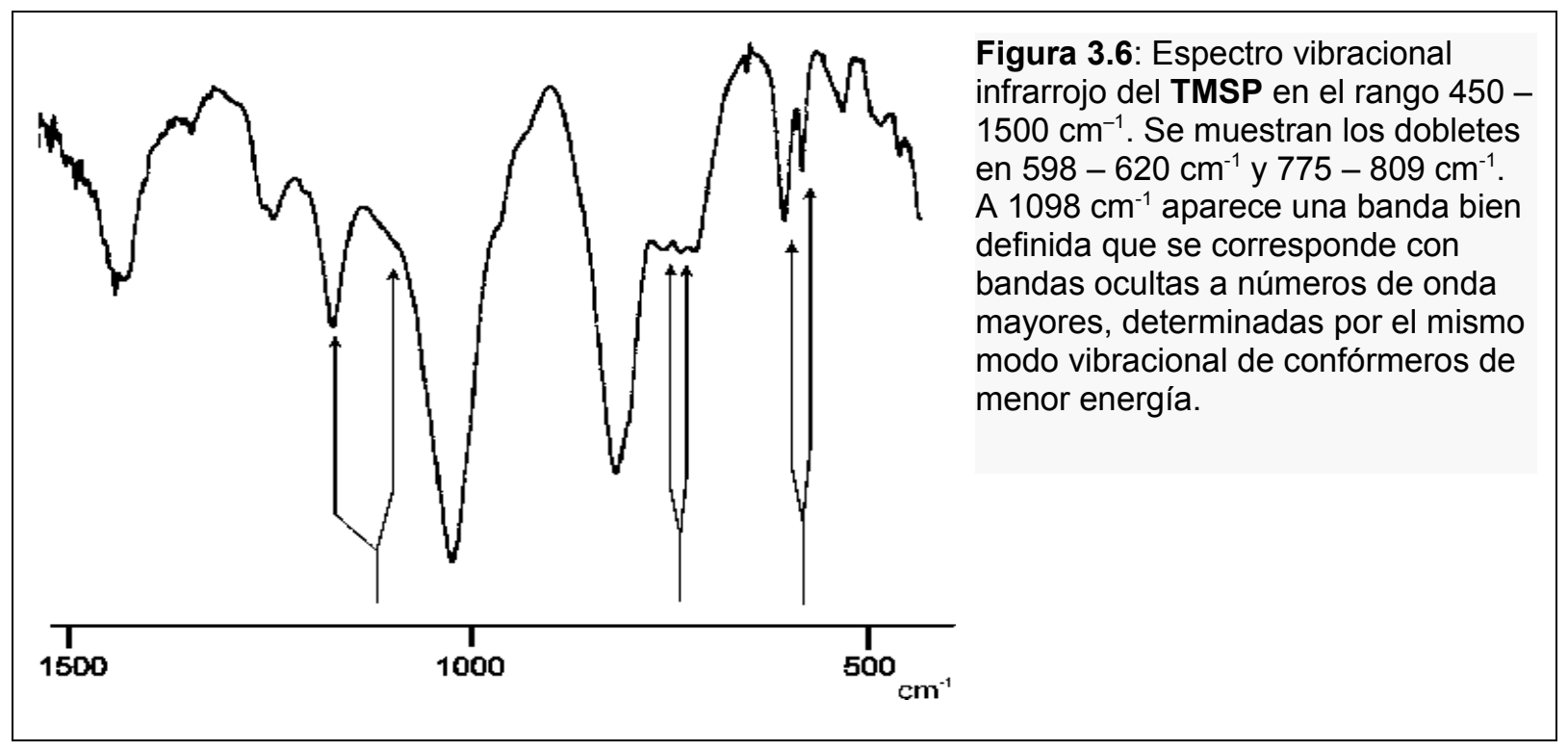

A la luz de lo expuesto, podemos afirmar que todos los resultados experimentales y teóricos son consistentes en

\footnotetext{
${ }^{27}$ Olah, G.A. and McFarland, C.W., Organophosphorus compounds. XIII. Protonation, cleavage, and alkylation of thiophosphates and thiophosphites, J.Org.Chem. 40 (18): 2582-2587 (1975).

${ }^{28}$ Kirby, A.J. The anomeric effect and related stereochemical effects at oxigen. Springer Berlin (1983).
} 
el sentido de entregar fuerte evidencia de coexistencia de confórmeros a temperatura ambiente, siendo éstos interconvertibles con una dificultad energética mayor que en el caso del compuesto oxofosforado análogo. 


\section{Comportamiento conformacional del fosfoselenoato de O,O,O-trimetilo}

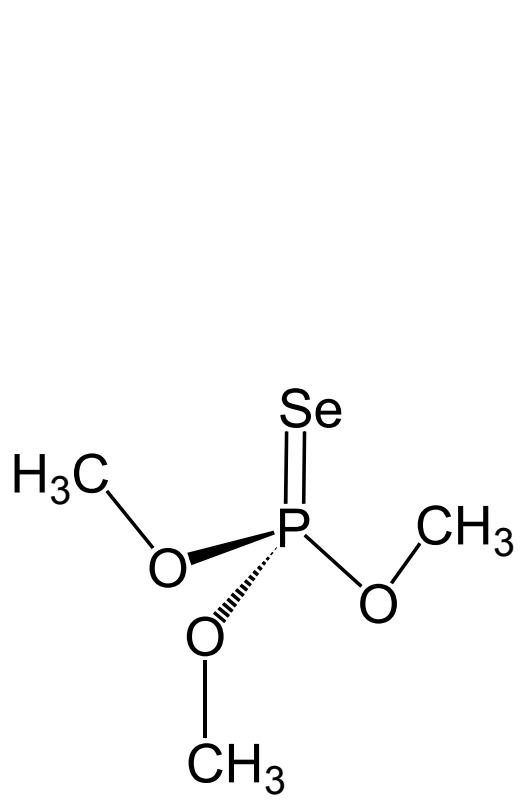

Figura 4.1: Estructura del fosforoselenoato de O,O,Otrimetilo (TMSeP)
Existen relativamente pocos estudios espectroscópicos y estructurales realizados en selenofosfatos. En comparación con otros OPs, estos no encuentran aplicaciones de importancia desde el punto de vista tecnológico. Además, el enlace $\mathrm{P}-\mathrm{Se}$ no está usualmente presente en sistemas biológicos. Probablemente la única excepción sea el selenofosfato, compuesto que ha sido propuesto como el dador de Se en organismos procariotas ${ }^{(1)}$ y eucariotas ${ }^{(2)}$ durante la síntesis de la selenocisteína (Sec), un aminoácido codificado con poca frecuencia en la estructura primaria de algunas proteínas. ${ }^{(3)}$

Por estas razones, se conoce bastante poco acerca de la actividad biológica y toxicidad de estos compuestos. Mientras que es aceptado que los fosfoselenoatos $(\mathrm{P}=\mathrm{Se})$ son pobres inhibidores enzimáticos, se ha descrito que compuestos del tipo $\mathrm{O}=\mathrm{P}-\mathrm{Se}-\mathrm{C}$, muestran una capacidad tóxica excepcional al ser administradas por vía subcutánea en ratas, ${ }^{(4)}$ incluso con una potencia mayor que los análogos de azufre. En algunas comunicaciones se ha descrito actividad

${ }^{1}$ (a) Glass, R.S. et al. Monoselenophosphate: Synthesis, characterization, and identity with the prokaryotic biological selenium donor, compound SePX, Biochem. 32 (47): 12555-12559 (1993). (b) Glass, R.S. and Stadtman, T.C. Selenophosphate, Methods.Enzymol. 252: 309-315 (1995). ${ }^{2}$ (a) Mizutani, T., Tanabe, K. and Yamada, K. A G-U base pair in the eukariotic selenocysteine tRNA is important for interaction with SePF, the putative selenocysteine - specific elongation factor, FEBS Lett. 429: 189-193 (1998). (b) Mizutani, T., Kanaya, K. and Tanabe, K. Selenophosphate as a substrate for mammalian selenocysteine syntase, its stability and toxicity, BioFactors 9: 27-36 (1999). (c) Mizutani, T., Goto, C. and Totsuka, T. Mammalian selenocysteine tRNA, its enzymes and selenophosphate, J. Heal. Sci. 46 (6): 399-404 (2000).

3 Este aminoácido forma parte, por ejemplo, del sitio activo de la selenotransferasa, enzima que cataliza la sesión del Se en la última etapa de la síntesis de la $\mathrm{Sec}$, dando lugar a una curiosa versión bioquímica de la paradoja del huevo y la gallina: ¿qué existió primero, la selenotransferasa o la selenocisteina?.

4 Åkerfeldt,S. and Fagerlind, L. Selenophosphorus compounds as powerful cholinesterase inhibitors, J.Med.Chem. 10 (1): 115-116 (1967). 
anti HIV de análogos selenofosfato de oligo desoxinucleótidos. ${ }^{(5)}$

Respecto a las características estructurales, conformacionales y de reactividad química, hay importante información que, a nuestro entender, no ha confluido en el resumen de un comportamiento generalizable a los selenofosfatos. ${ }^{(6)}$ Por comparación de las características estructurales de los oxo, tio y selenofosfatos, éstos últimos muestran una relativa menor flexibilidad conformacional con mayor energía de interconversión de confórmeros. Este comportamiento puede estar relacionado con factores estéricos y electrónicos.

El enlace $\mathrm{P}=\mathrm{Se}$ puede ser descrito como un híbrido de resonancia con dos formas canónicas: $\mathrm{P}=\mathrm{Se}$ y $\mathrm{P}^{+}-\mathrm{Se}^{-(7)}$ en forma análoga a lo descrito para los enlaces $\mathrm{P}=\mathrm{S}$, aunque con una menor contribución que en los enlaces del tipo $\mathrm{P}=\mathrm{O} \cdot{ }^{(8)} \mathrm{En}$ los fosfoselenoatos orgánicos, la estructura real y las diferencias cualitativas respecto de los análogos oxofosforados y tiofosforados, dependerán entre otros factores de la capacidad de los grupos sustituyentes para establecer interacciones $\mathrm{p} \pi-\mathrm{d} \pi$ con los orbitales del fósforo

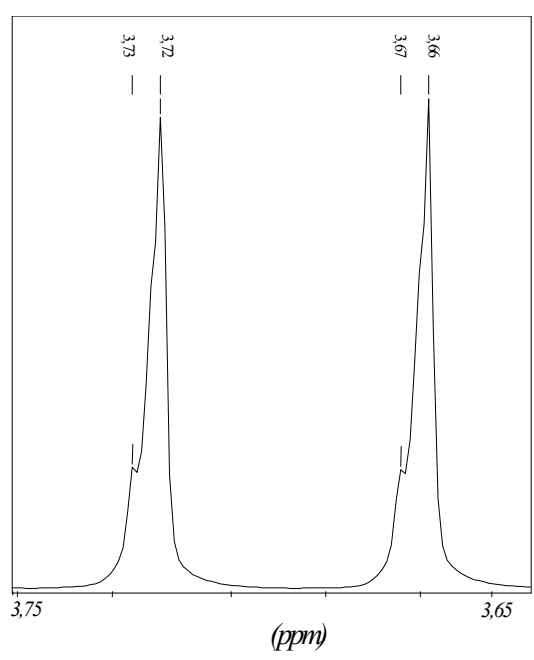

Figura 4.2: Doblete metílico ${ }^{1} \mathrm{H}$ RMN del TMSeP.

\footnotetext{
${ }^{5}$ Mori, K. et al. Phosphoroselenoate oligodeoxynucleotides: synthesis, physico- chemical characterization, anti-sense inhibitory properties and anti-HIV activity, Nucleic.Acids.Res. 17 (20): 8207-8219 (1989).

${ }^{6}$ Existen una serie de trabajos publicados en el idioma local durante las décadas del sesenta y setenta en la Unión Soviética: (a) Nuretdinov,I.A. and Grechkin, N.P. Properties of trialkyl selenophosphates, Izv.Akad.Nauk.SSSR., Ser.Khim. 12: 2831-2833 (1968). (b) Nuretdinov, I.A. et al. Acid monochlorides of phosphorus amidoseleno acids and their properties, Bull. Acad. Sciences.USSR. 7: 1423-1426 (1969). (c) Belskii, V.E. et al. Kinetics of the alkaline hydrolysis of some organoseleno phosphorus compounds, Zh. Obshch. Khim. 43 (6): 1255-1257 (1973). (d) Shagidullin, R.R. et al. Vibrational spectra and structures of trialkyl selenophosphates, Dokl. Akad. Nauk. SSSR. 225 (4): 886-889 (1975). (e) Shagidullin, R.R., Vandyukova, I.I., Nuretdinov, I.A. and Poltev, V.I. Conformation of some thio and selenophosphoryl compounds. (Mezhmol. Vzaimodeistvie Konform. Mol., Tezisy Dokl. Vses. Simp., $3^{\text {rd }}$ ), 52-53. 1976. Akad. Nauk SSSR, Nauchn. Tsentr Biol. Issled., Pushchino, USSR. (f) Turkevich, V.V. et al. Vibrations of the phosphorus selenium group in trialkyl selenophosphates, Zh. Obshch. Khim. 47 (10): 2388 (1977). (g) Shagidullin, R.R., Vandyukova, I.I. and Nuretdinov, I.A., Dipole moments of a series of selenophosphoryl compounds, Izv.Akad.Nauk.SSSR., Ser.Khim. 6: 1407-1409 (1978).

${ }^{7}$ McFarlane, W. and Rycroft, D.S., Determination of chemical shifts by heteronuclear magnetic triple resonance. Nature of the phosphorusselenium bond in organophosphorus selenides, J. Chem. Soc., Chem. Commun. 15: 902-903 (1972).

${ }^{8}$ Grim, S.O., Walton, E.D. Unsymmetrical Bis-phosphorus ligands. 12. Synthesis and nuclear magnetic resonance studies of some derivatives of bis(diphenilphosphino)methane, Inorg. Chem. 19:1982-1987 (1980).
} 
y del equilibrio de dichas interacciones con efectos estereolelectrónicos presentes en la estructura. ${ }^{(9)}$

Sin embargo, mediante el estudio comparativo mediante cristalografía de rayos $X$ de alquil $y$ aril selenofosfatos análogos, se verifican diferencias poco significativas en la longitud del enlace $\mathrm{P}=\mathrm{Se}$. Esto da cuenta que la interacción directa entre los orbitales del fósforo y el selenio juegan un papel preponderante, antes que los efectos anoméricos. ${ }^{(10)}$

En general, se reconoce menos influencia de la electronegatividad de los sustituyentes en los ésteres sobre el modo vibracional de estiramiento $\mathrm{P}=\mathrm{Se}$ que en el caso del $\mathrm{P}=\mathrm{S},{ }^{(11)}$ lo que sugiere que el efecto $\pi$ en el enlace $\mathrm{P}=\mathrm{Se}$ es menos marcado. Esto debería ser consecuencia directa de un solapamiento menos efectivo entre los orbitales correspondientes del fósforo y el selenio.

Tabla 4.I: Valores experimentales, reportados previamente y calculados del espectro vibracional IR y Raman en fase líquida para el TMSeP, expresados en $\mathrm{cm}^{-1}$. Se muestran sólo las bandas que evidencian coexistencia de confórmeros.

\begin{tabular}{|c|c|c|c|c|c|}
\hline \multirow[t]{2}{*}{ Asignación } & \multirow[t]{2}{*}{$I R$} & \multirow[t]{2}{*}{ Raman } & \multicolumn{3}{|c|}{ Calculado (HF/6-31G**) } \\
\hline & & & $\mathrm{C}_{3}$ & $\mathrm{C}_{1}$ & Cs \\
\hline va $(\mathrm{C}-\mathrm{O})_{3} \mathrm{C}_{3}, \mathrm{C}_{1}$ & $1082^{(13)}$ & 1081 & 1091 & 1096 & \\
\hline va $(\mathrm{C}-\mathrm{O})_{3} \mathrm{C}_{\mathrm{s}}$ & $1066^{(13)}$ & 1061 & & & 1085 \\
\hline $\mathrm{va}(\mathrm{C}-\mathrm{O})_{3}$ & $\begin{array}{l}1031(1,00) \\
1030\end{array}$ & 1033 & $\begin{array}{l}1058 \\
1058\end{array}$ & $\begin{array}{l}1061 \\
1055\end{array}$ & $\begin{array}{l}1052 \\
1043\end{array}$ \\
\hline $\begin{array}{l}v(\mathrm{P}-\mathrm{O})_{3} \\
\delta \mathrm{s}\left(\mathrm{Se}=\mathrm{P}-\mathrm{O}_{3}\right) \mathrm{C}_{3}\end{array}$ & $\begin{array}{l}826(, 63) \\
825^{(13)}\end{array}$ & 834 & $827(2)$ & 818 & 821 \\
\hline$\delta \mathrm{s}\left(\mathrm{Se}=\mathrm{P}-\mathrm{O}_{3}\right) \mathrm{C}_{1}, \mathrm{C}_{\mathrm{s}}$ & $791(, 70)$ & & & 804 & 801 \\
\hline$\delta \mathrm{s}\left(\mathrm{Se}=\mathrm{P}-\mathrm{O}_{3}\right) \mathrm{C}_{3}$ & $\begin{array}{l}780(, 74) \\
779\end{array}$ & & 792 & & \\
\hline & & & & 767 & 780 \\
\hline$v(\mathrm{P}=\mathrm{Se}) \mathrm{C}_{1}$ & $\begin{array}{l}559(, 41) \\
558^{(13)}\end{array}$ & 558 & & 536 & 550 \\
\hline$v(\mathrm{P}=\mathrm{Se}) \mathrm{C}_{3}$ & $504(, 43)^{(13)}$ & 505 & 486 & & \\
\hline$\delta \mathrm{s} \mathrm{P}-(\mathrm{O}-\mathrm{C})_{3}$ & & 348 & 339 & 340 & 341 \\
\hline$\rho w a(P-O)_{3}$ & & 315 & $299(2)$ & $\begin{array}{l}309 \\
293\end{array}$ & $\begin{array}{l}324 \\
290\end{array}$ \\
\hline $\operatorname{pr}(\mathrm{P}-\mathrm{O})_{3} \mathrm{C}_{3}$ & & 238 & 233 & & \\
\hline $\operatorname{\rho r}(\mathrm{P}-\mathrm{O})_{3} \mathrm{C}_{1}, \mathrm{C}_{\mathrm{s}}$ & 169 & & & 194 & 180 \\
\hline
\end{tabular}

$v$ : vibración de estiramiento; va: vibración de estiramiento antisimétrica; $\delta$ s: vibración de deformación simétrica; $\rho$ : vibración de balanceo; $\rho$ wa: vibración de aleteo antisimétrica; sh: hombro. En los valores experimentales, entre paréntesis se encuentra la intensidad de cada banda. En los valores teóricos, entre paréntesis se indica el grado de degeneración energética, si existe.

${ }^{9}$ Allen,O.W., Taylor,B.F. J. Chem. Res. (S): 392-393 (1986).

${ }^{10}$ Wolf, W. M. and Bartczak, T.J. The stereochemistry of the phosphorusselenium bond. VII. Structure of 5,5-dimethyl-2-(o-nitrophenyl)amino1,3,2- dioxaphosphorinane 2-selenide, Acta.Crystallogr. 45 ( Pt 11) (C): 1767-1770 (1989).

11 De Ketelaere, R.F. and Van der Kelen, G.P. Organophosphorus chemistry. Part IV. Vibrational spectroscopy study of the $\mathrm{P}=\mathrm{O}, \mathrm{P}=\mathrm{S}$ and $\mathrm{P}=\mathrm{Se}$ bonds in triarylphosphorus chalcogenides. J. Mol. Struct. 23: 233240 (1974). 
Por otro lado, la reactividad relativa de compuestos selenofosforados y tiofosforados, da cuenta de las diferencias entre estos enlaces. Por ejemplo, la deselenización bajo condiciones oxidativa es más eficiente que la desulfuración en compuestos del tipo fosfonatos de triarilos. ${ }^{(12)}$ Esto da cuenta de la mayor labilidad del enlace $\mathrm{P}=\mathrm{Se}$, respecto del enlace $\mathrm{P}=\mathrm{S}$, en estas condiciones.

Como ya se mencionó en el Capítulo 1, de la inversión de la constante de hidrólisis en medios apróticos respecto a la reacción medida en medios acuosos para el TMSP y el fosfoselenoato de O,O,O-trimetilo (TMSeP), se puede concluir la mayor capacidad aceptora de protones del selenio respecto al azufre en compuestos Ops. ${ }^{(6 c)}$

Un buen modelo para el estudio de las características del enlace $\mathrm{P}=\mathrm{Se}$ y el comportamiento conformacional de este tipo de OPs es el TMSeP por ser una de las moléculas más pequeñas a considerar dentro de este grupo (Figura 4.1).

En el único reporte previo detallado del espectro infrarrojo de esta molécula, ${ }^{(13)}$ no se presenta una justificación detallada de las asignaciones realizadas, excepto por su comparación con los correspondientes a TMP y TMSP. Sin duda, en los espectros vibracionales pueden ser observados dos confórmeros diferentes. Mediante una asignación a confórmeros de simetrías $\mathrm{C}_{3}$ y $\mathrm{Cs}$, se han determinado por interpretación de los espectros infrarrojos en distintos solventes la relación de abundancia entre ellos a temperatura ambiente. ${ }^{(6 d)}$

Sin embargo, los cálculos químico cuánticos anticipan tres formas posibles para esta molécula. ${ }^{(14)}$ Según estos cálculos, el confórmero principal posee simetría $\mathrm{C}_{3}$, mientras que la segunda forma presenta simetría $C_{1}$. El confórmero menos abundante, predicho teóricamente, exhibe

\footnotetext{
12 Bhardwaj, R.K. and Davidson, R.S. Oxidative desulphurisation and deselenation of pentacovalent phosphorous by photogenerated peroxidic species, Tetrahedron 43 (19): $4473-4479$ (1987).

${ }_{13}$ Marsault - Herail, F. Spectres de vibration de composes organophosphores de $293 \mathrm{~K}$ a $15 \mathrm{~K}$ conformations moleculaires. J. Chim. Phys. 68 (39):274-286 (1971)

14 Mastrantonio, G. and Della Vedova, C.O. On the Structural characteristics of the O,O,O-trimethyl selenophosphate, Journal of Molecular Structure 645: 1-8 (2003).
}

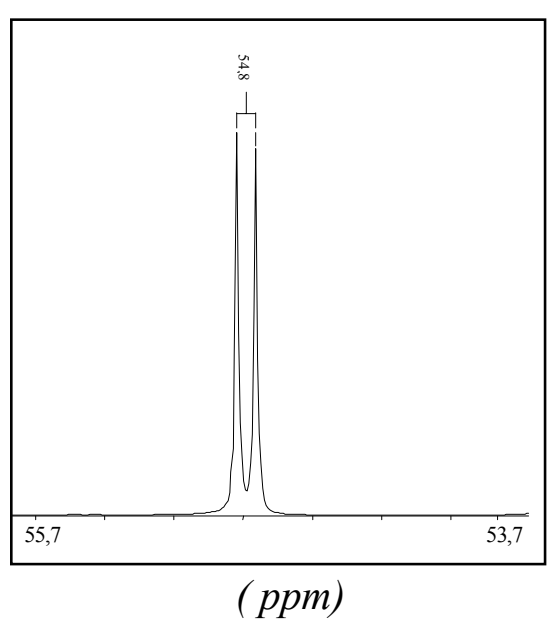

Figura 4.3: Doblete metílico ${ }^{13} \mathrm{C}$ RMN del TMSeP. 
simetría Cs. Esta situación es equivalente a la descrita ${ }^{(15)}$ para los análogos con oxígeno y azufre.

Ya se mencionó que las formas estables de los fosfoselenoatos de trialquilo y selenomonofosfonatos de trialquilo, corresponden a aquellas en las que predominan las posiciones gauche con, a lo sumo, un sustituyente en posición trans. ${ }^{(6 e)}$

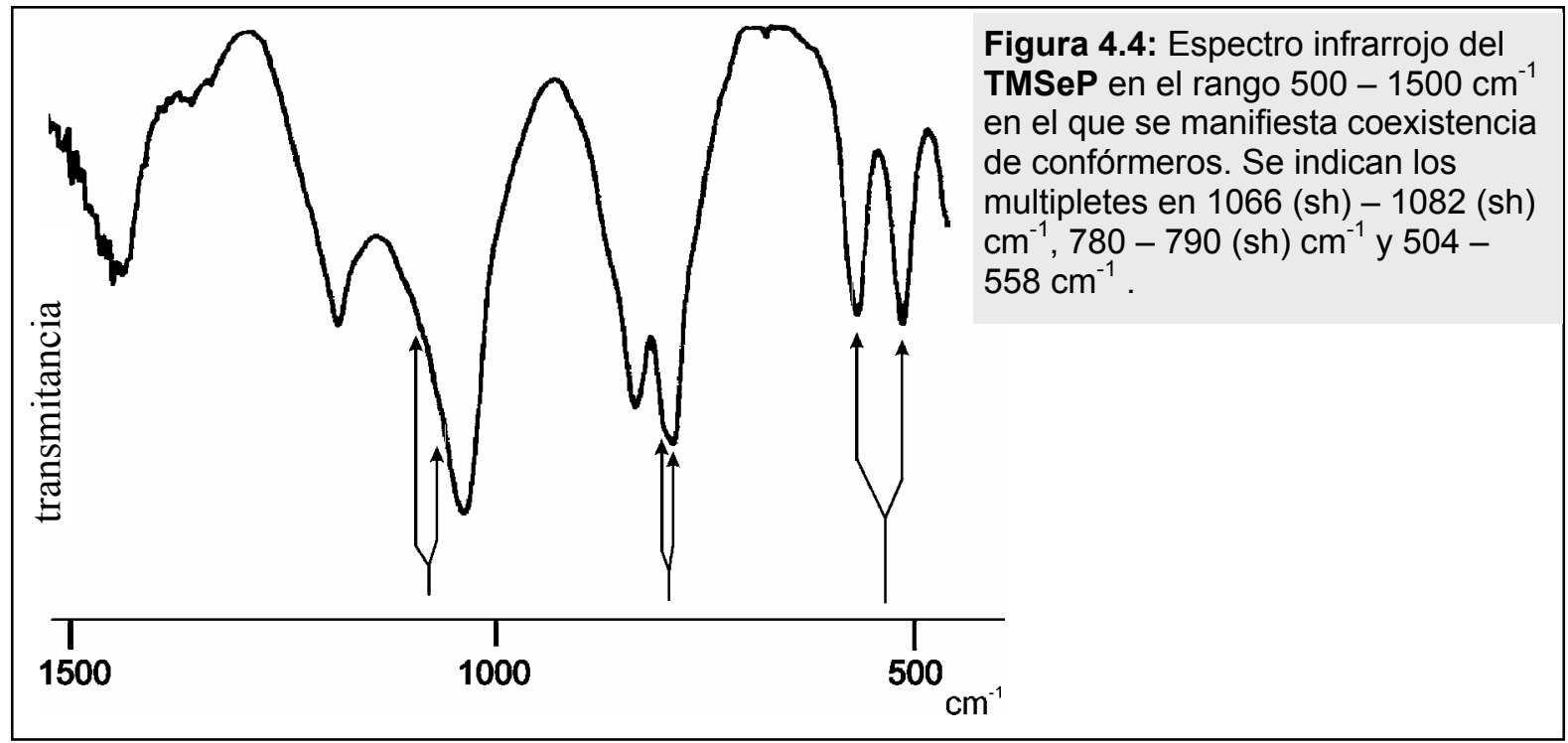

El modo vibracional de estiramiento $\mathrm{P}=\mathrm{Se}$ es esperado en la región $590-470 \mathrm{~cm}^{-1}$ para la mayoría compuestos selenofosfatos. En general, en los fosfoselenoatos de trialquilo se identifican dos bandas características en el espectro IR, ubicadas en el rango 570 $577 \mathrm{~cm}^{-1}$ y $528-575 \mathrm{~cm}^{-1}$, que para el TMSeP aparecen corridas hacia números de onda menores, exactamente a 558 $\mathrm{cm}^{-1}$ y $504 \mathrm{~cm}^{-1}$. Este par de bandas se relacionó a la manifestación de confórmeros de simetrías $C_{3}$ y $C_{3 v}$, de menor y mayor energías respectivamente ${ }^{(13)}$ asignándose al estiramiento $(\mathrm{P}=\mathrm{Se})$.

Para el TMSeP se reporta otro par de bandas en el espectro IR en fase líquida, ubicadas en $1082-1066 \mathrm{~cm}^{-1}$, que se relacionó con la existencia de equilibrio conformacional. Estas señales fueron asignadas al estiramiento $(\mathrm{C}-\mathrm{O})_{3}$, aunque para esta asignación se utiliza la posibilidad de dos isómeros de simetrías $\mathrm{C}_{3}$ y $\mathrm{C}_{3 \mathrm{v}}$, de menor y mayor energías, respectivamente. ${ }^{(13)}$

15 Mastrantonio,G. and Della Vedova,C.O. Spectroscopic and conformational comparative study of trimethyl chalcogenphosphates, Journal of Molecular Structure 561 (1-3): 161-174 (2001). 
Tabla 4.II: Valores obtenidos para el espectro de ${ }^{1} \mathrm{H}$ RMN del TMSeP (calibración respecto a TMS). Entre paréntesis se indican las intensidades relativas de los picos.

\begin{tabular}{lcc}
$\delta \mathrm{ppm}$ & $3,70(0,21)$ & $3,69(1,00)$ \\
${ }^{3} \mathrm{~J}_{\mathrm{H}-\mathrm{P}} \mathrm{Hz}$. & 14,2 & 14,2 \\
\hline
\end{tabular}

Valores obtenidos para el espectro de ${ }^{13} \mathrm{C}$ RMN del TMSeP (calibración respecto a cloroformo).

$\delta \mathrm{ppm} \quad 54,8$

${ }^{2} J_{\mathrm{C}-\mathrm{P}} \mathrm{Hz}$. $\quad 5,3$

\section{Caracterización espectroscópica}

En las medidas de ${ }^{1} \mathrm{H}$ RMN se encuentra una estructura fina del doblete correspondiente a los hidrógenos de los grupos metilo. Se encuentran dos máximos para cada uno de estos picos, distribuidos en una relación de áreas aproximada de 30:10:2:1, tal como se muestra en la Figura 4.2. Esto sustentaría la idea de coexistencia de entornos diferenciados para los metilos y la coexistencia de confórmeros, aunque en una proporción menor que para el TMP y el TMSP. El espectro ${ }^{13} \mathrm{C}$ RMN del TMSeP presenta características similares al del TMSP, es decir, aparece un doblete con una constante de acoplamiento ${ }^{2} J_{\mathrm{PC}}$ con un valor de $5,25 \mathrm{~Hz}$, presentándose ambos picos simétricos, como se muestra en la Figura 4.3.

En la Tabla 4.I se muestran los valores de los números de onda IR y Raman del TMSeP, conjuntamente con los reportados previamente, en los rangos de números de onda en las que se manifestaría la coexistencia de confórmeros. En la misma tabla se indican las asignaciones propuestas, para lo cual los cálculos teóricos fueron utilizados en forma preponderante.

No es posible identificar la presencia de bandas IR en el rango $1082-1066 \mathrm{~cm}^{-1}$, esto por la baja resolución de la medida y la cercanía de la banda en $1030 \mathrm{~cm}^{-1}$, que por su intensidad y ancho solapa con bandas menos intensas en el rango mencionado. Sí se pueden observar dos débiles señales en el espectro Raman, que según los cálculos se asignan al estiramiento asimétrico $\mathrm{C}-\mathrm{O}$. Esta situación es similar a la que se presenta para el mismo modo de vibración en el TMSP.

\section{Correlación teórica}

Además de las manifestaciones de equilibrio conformacional reportadas, según los resultados teóricos de nivel $\mathrm{HF} / 6-31 \mathrm{G}^{* *}$, es posible asociar la coexistencia de

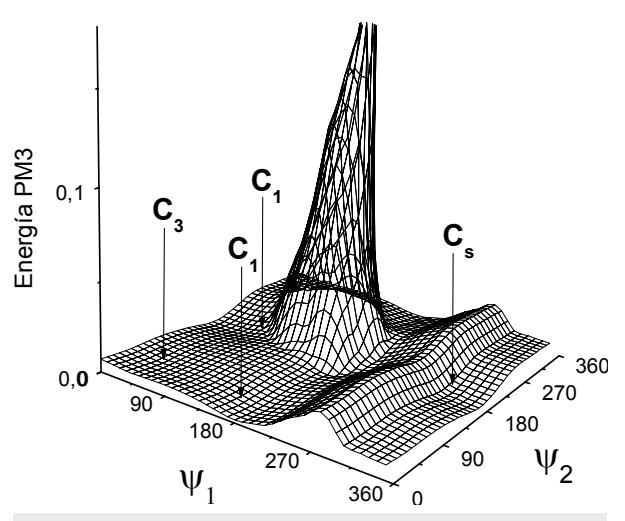

Figura 4.5: Superficie potencial del TMSeP de rotación alrededor de dos ángulos $\psi$ manteniendo el otro fijo en $42^{\circ}$ calculadas a nivel HF/PM3 
600

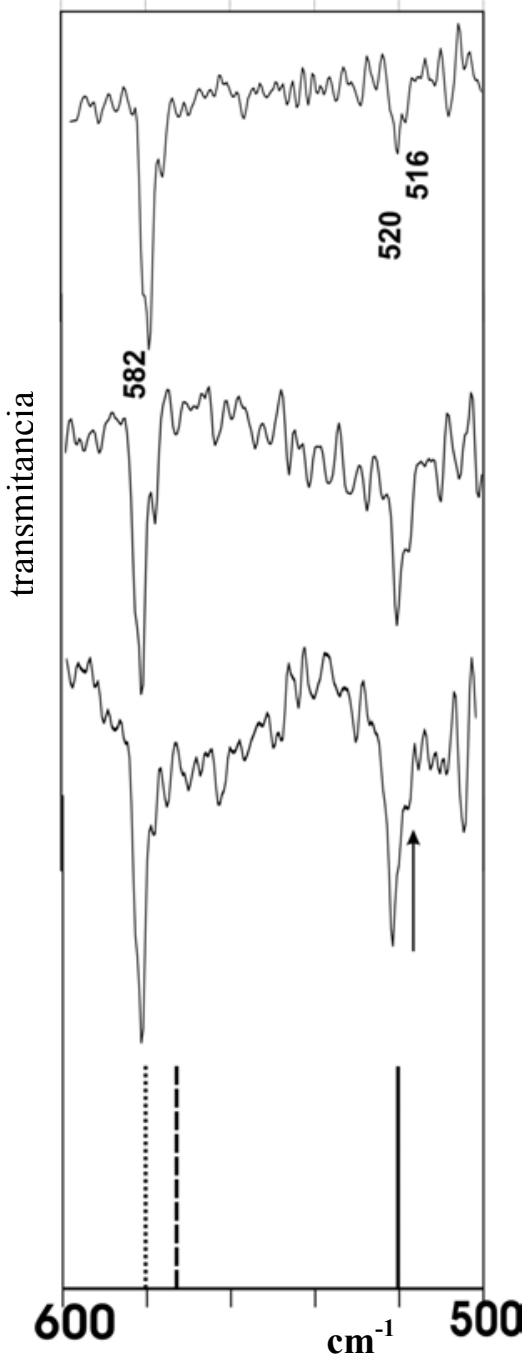

Figura 4.6: Espectro IR del TMSeP en matriz de Ar en la región $600-500 \mathrm{~cm}^{-1}$. Desde arriba hacia abajo: medida a 294K, medida a 538K, medida a $778 \mathrm{~K}$, números de onda teóricos: línea continua: confórmero $\mathrm{C}_{3}$; línea cortada: confórmero $\mathrm{C}_{1}$; línea punteada: confórmero Cs. confórmeros en las bandas de absorción a $790 \mathrm{~cm}^{-1}-780 \mathrm{~cm}^{-1}$ en el espectro IR y a $238 \mathrm{~cm}^{-1}-169 \mathrm{~cm}^{-1}$ en el espectro Raman. No es posible asignar claramente la manifestación conformacional a un tercer par de bandas, reportado en el IR y observado en nuestras medidas como dos bandas débiles en Raman a 1081 y $1061 \mathrm{~cm}^{-1}$, pues son de difícil interpretación los valores de sus absorbancia relativas a la luz de las abundancias teóricas de cada uno de ellos.

Respecto a su libertad conformacional, graficada en la Figura 4.5, se observa que en el TMSeP la situación es totalmente distinta al TMP y TMSP, manifestándose la existencia de un impedimento rotacional importante, para determinadas posiciones fijas de uno de los metilos.

Tabla 4.III: Parámetros geométricos, energéticos y fisicoquímicos de los confórmeros estables del TMSeP.

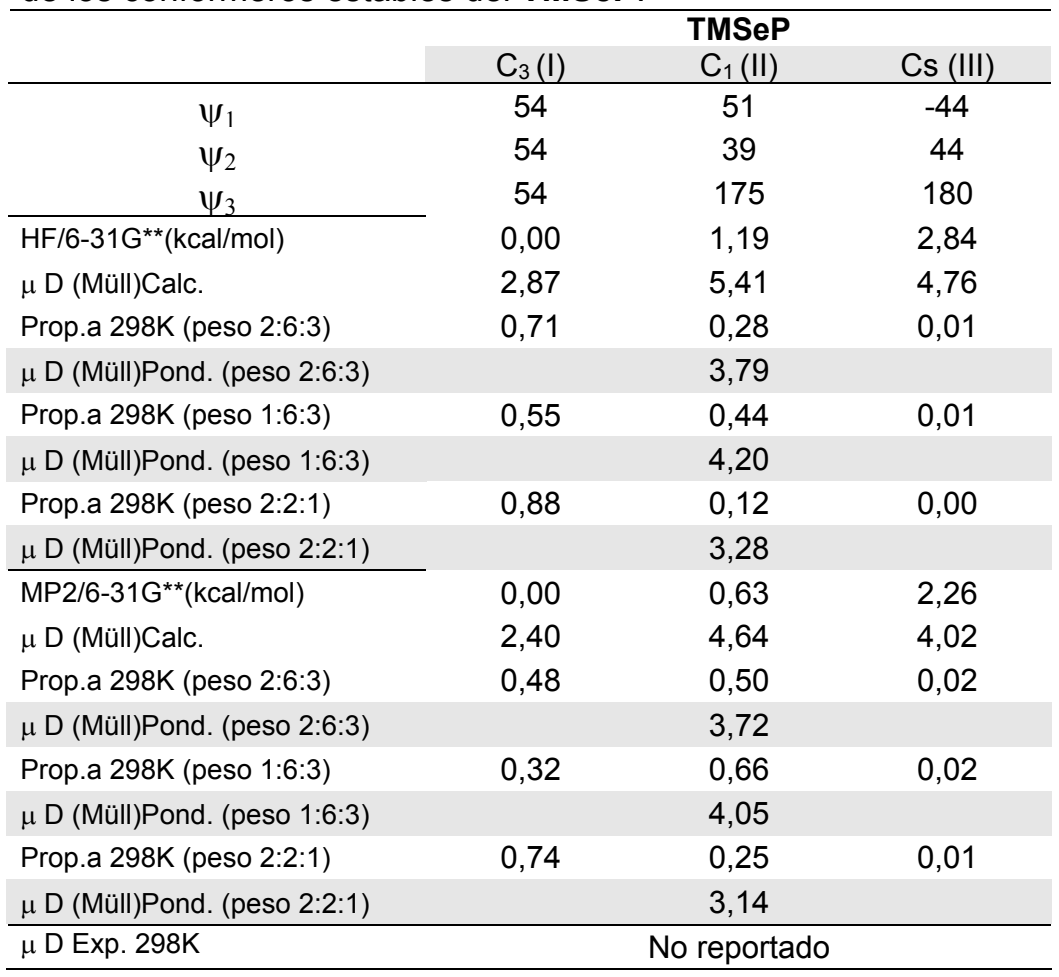

Según nuestros resultados teóricos, las diferencias de energía entre cada uno de los tres confórmeros calculados mediante la aproximación HF/6-31G**, da una abundancia, a $298 \mathrm{~K}$, de $71 \%$ para el confórmero de simetría $\mathrm{C}_{3}$. Esto resulta compatible con la asignación a dos confórmeros distintos en el modo vibracional de estiramiento $\mathrm{P}=\mathrm{Se}$, en 558 y $504 \mathrm{~cm}^{-1}$. Los cálculos realizados, confirman la asignación reportada para el modo de vibración y el correspondiente desdoblamiento para sus isómeros conformacionales, no así la correspondencia con ellos, asignándose la banda de 504 
$\mathrm{cm}^{-1}$ al confórmero más estable de simetría $\mathrm{C}_{3}$ y la banda de $558 \mathrm{~cm}^{-1}$ al confórmero $\mathrm{Cs}$, de mayor energía.

Los resultados teóricos y experimentales que evidencian una menor libertad conformacional para el TMSeP respecto al TMP y TMSP, sugieren que la aplicación de la Ecuación 2.a sobre sólo los tres confórmeros de mínima energía es una aproximación adecuada y que por lo tanto, son éstos los que fundamentalmente aportarían a la magnitud del dipolo total.

Tabla 4.IV: Parámetros geométricos para el TMSeP obtenidos mediante cálculos B3LYP.

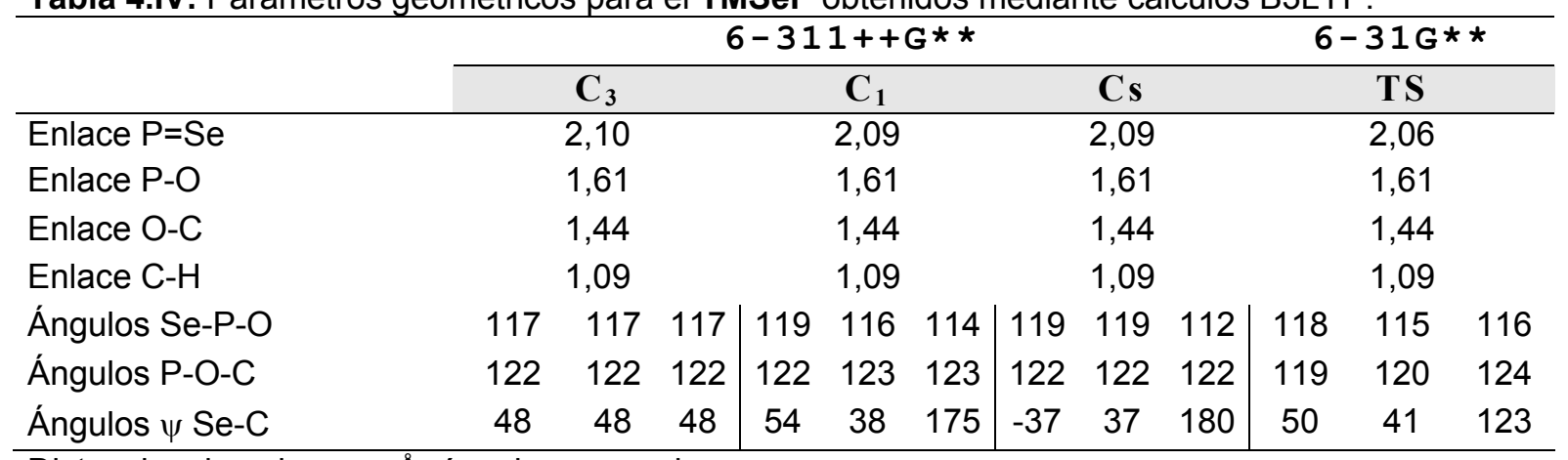

Distancias de enlace en $\AA$, ángulos en grados.

TS: forma correspondiente al estado de transición $C_{3} \rightarrow C_{1}$.

\section{Estudios espectroscópicos de alta resolución}

Los cálculos de funcionales de la densidad (DFT) con altos niveles de teoría, correlacionados con espectroscopía de alta resolución, entregan poderosas evidencias de la coexistencia de al menos tres confórmeros en equilibrio a temperatura ambiente y mayores, como se muestra en adelante.

El uso de espectroscopía de alta resolución, como lo es la espectroscopía infrarroja en matrices de $\mathrm{Ar}$ a baja temperatura con diferentes temperaturas de deposición (ver Anexo I - Metodologías), permite investigar la presencia de diferentes especies conformacionales con una sensibilidad muy alta.

Dadas las características de este tipo de medida, es adecuado correlacionarlas con resultados teóricos obtenidos con altos niveles de cálculo. En nuestro caso, se utilizaron cálculos de funcionales de la densidad (B3LYP) con base 6$311++\mathrm{G}^{* *}$ para confórmeros estables y $6-31 \mathrm{G}^{* *}$ para estados de transición conformacional (Tabla 4.IV).

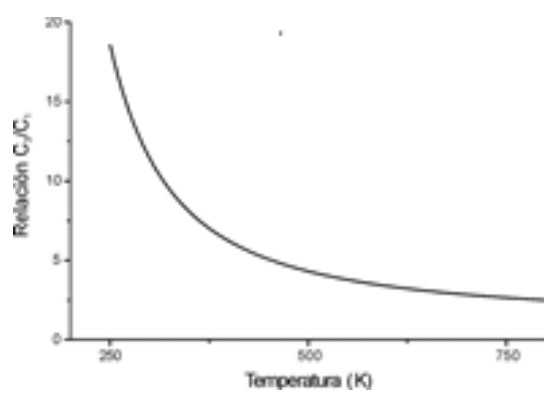

Figura 4.7: Relación teórica entre los confórmeros $\mathrm{C}_{3} / \mathrm{C}_{1}$ para el TMSeP en el rango de temperatura $250-800 \mathrm{~K}$ con un nivel de teoría B3LYP/6-

$311++G^{* *}$. 
Tabla 4.V: Cálculos B3LYP para la energía y momentos dipolares de los confórmeros del TMSeP.

\begin{tabular}{|c|c|c|c|c|}
\hline & $\mathrm{C}_{3}$ & $\mathrm{C}_{1}$ & $\mathrm{Cs}$ & TS \\
\hline & \multicolumn{3}{|c|}{$\frac{1}{6-311++G^{* *}}$} & $6-31 G * *$ \\
\hline$\mu$ & 1,58 & 3,99 & 3,30 & 3,36 \\
\hline Energía relativa & 0,00 & 1,45 & 3,26 & 2,24 \\
\hline
\end{tabular}

Momento dipolar en Debyes, energía en $\mathrm{kcal} / \mathrm{mol}$.

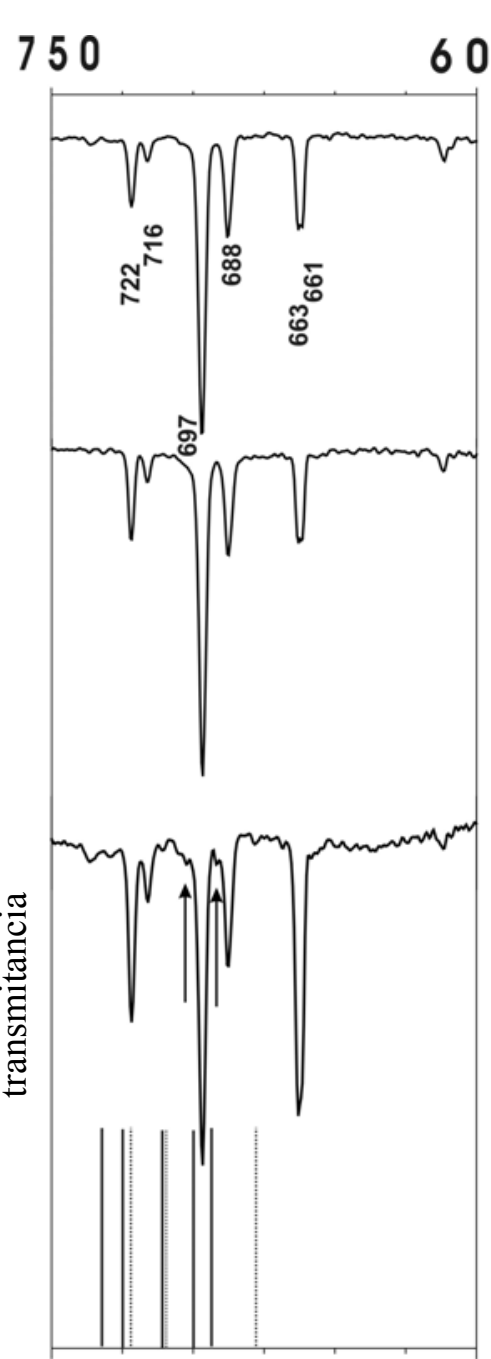

750

$\mathrm{cm}^{-1}$

600

Figura 4.8: Espectro IR del TMSeP en matriz de Ar en la región $750-600 \mathrm{~cm}^{-1}$, según patrón ya indicado.

Una de las herramientas interesantes disponibles es la 600 observación de las variaciones relativas de las intensidades de las bandas de absorción en función del aumento de la temperatura de deposición. Tomando en cuenta la Ecuación 2.b, cuando sea posible observar el aumento de intensidad de una banda que se correlaciona con el aumento de la temperatura, se podrá asignar esta banda a la manifestación de un confórmero de menor energía, que aumenta su abundancia relativa con la temperatura.

En nuestro caso, el análisis del espectro IR- matrices de Ar sobre el TMSeP se dirige sobre ciertas regiones de interés, donde la detección de distintos confórmeros es posible. Para este propósito, las regiones seleccionadas son las siguientes: $590-520 \mathrm{~cm}^{-1}, 730-640 \mathrm{~cm}^{-1}, 1050-1000$ $\mathrm{cm}^{-1}, 1300-1100 \mathrm{~cm}^{-1}$ y $1500-1440 \mathrm{~cm}^{-1}$.

Para el primero de estos rangos, en la Figura 4.6 se observa un comportamiento variable con la temperatura. La relación de intensidad de las bandas a 579 y $520 \mathrm{~cm}^{-1}$ se muestra en la Tabla 4.VI.

El comportamiento de la banda a menor longitud de onda, es decir, su aumento de intensidad relativa con la temperatura, permite asignarla al confórmero $\mathrm{C}_{1}$ menos estable. Este hecho concuerda con la ausencia de bandas en regiones alrededor de $520 \mathrm{~cm}^{-1}$ en los espectros IR de fase sólida reportados previamente ${ }^{(13)}$ y con la abundancia teórica relativa de los confórmeros $\mathrm{C}_{3}$ y $\mathrm{C}_{1}$ encontrada en este trabajo.

Es interesante notar que en este punto, los cálculos $a b$ initio de nivel $\mathrm{HF} / 6-31 \mathrm{G}^{* *}$ y los cálculos de nivel DFT B3LYP/ 6-31++G**, predicen en forma inversa los números de onda correspondientes al estiramiento $\mathrm{P}=\mathrm{Se}$ para los confórmeros $\mathrm{C}_{3} \mathrm{y}_{1}$, puesto que se prevé a un mayor número de onda el modo estiramiento para el confórmero $\mathrm{C}_{1}$, respecto a la banda asignada al confórmero $\mathrm{C}_{3}$.

La región entre $730-660 \mathrm{~cm}^{-1}$ corresponde al modo de vibración P-O estiramiento. En el espectro IR/matriz de Ar, dos grupos de picos aparecen, cada uno indicando 
Tabla 4.VI: Dependencia experimental de la relación de la intensidad de bandas del modo de estiramiento $\mathrm{P}=\mathrm{Se}$ con la temperatura.

\begin{tabular}{ccccc}
\hline Temperatura (Kelvin) & 294 & 408 & 538 & 778 \\
\hline R $581 / 520$ & 2,52 & 1,97 & 1,29 & 1,32 \\
\hline
\end{tabular}

claramente un comportamiento de coexistencia conformacional al mostrar una variación de la relación de intensidad de bandas respecto a la temperatura (Figura 4.8). Las señales que aparecen a 697 y $688 \mathrm{~cm}^{-1}$ pueden ser asignadas al confórmero $\mathrm{C}_{3}$, y aquellas a 722 y $716 \mathrm{~cm}^{-1}$ a vibraciones correspondientes al confórmero $\mathrm{C}_{1}$. Por otro lado, aunque el comportamiento de las bandas a 663 y $662 \mathrm{~cm}^{-1}$ no es completamente claro, por su solapamiento es razonable asignar al menos uno de estas vibraciones al confórmero menos estable $C_{1}$. Este conjunto de bandas es predicho a mayores números de onda en el espectro teórico, como se muestra en la Figura 4.8.

La banda intensa y ancha observada a $1030 \mathrm{~cm}^{-1}$ en el espectro FTIR del líquido (Figura 4.3), puede ser asociada con dos grupos bien definidos de bandas localizados en la región existente entre 1080 y $1000 \mathrm{~cm}^{-1}$ del espectro IR/matriz de Ar (Figura 4.9). Estas corresponden a los modos de vibración del estiramiento (P)-O-C.

Dado que la intensidad normalizada de las bandas centradas en $1043,1040,1038$ y $1034 \mathrm{~cm}^{-1}$ no muestra ningún cambio con la temperatura, y que el aumento de la intensidad relativa de las bandas centradas en 1025, 1023, $10191015 \mathrm{~cm}^{-1}$ está correlacionada con el incremento de la temperatura, el primer grupo de bandas puede ser asociado al confórmero más estable $\mathrm{C}_{3} \mathrm{y}$ el segundo grupo puede ser asignado al confórmero $\mathrm{C}_{1}$.

Durante el estudio de los espectros obtenidos mediante la técnica de matrices con deposición por chorro supersónico, para el TMP fue reportada ${ }^{(16)}$ una asignación similar en la región $1100-1000 \mathrm{~cm}^{-1}$. Sin embargo, nuestros resultados teóricos no concuerdan bien con esa asignación.

En el espectro FTIR del líquido, a $1181 \mathrm{~cm}^{-1}$ sólo una banda fue reportada. En el espectro IR de matrices de Ar a bajas temperaturas, esta ancha banda se resuelve en varias bandas muy estrechas (Figura 4.10). No se observa dependencia de la intensidad de estas bandas respecto de la temperatura.

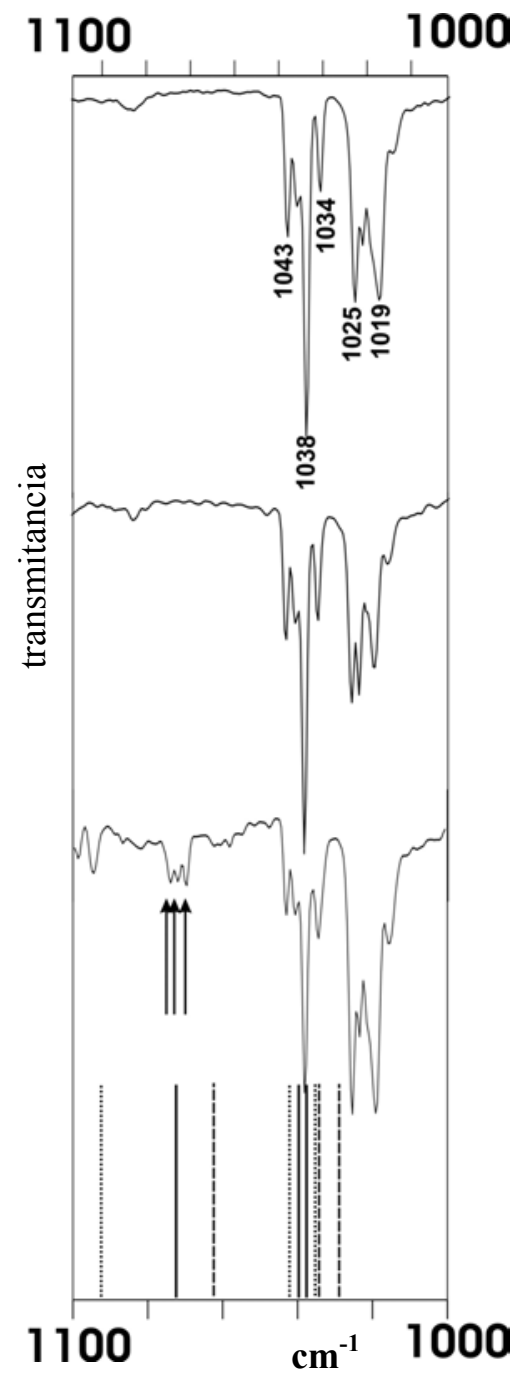

Figura 4.9: Espectro IR en matriz de Ar del TMSeP en la región $1100-1000 \mathrm{~cm}^{-1}$, según patrón ya indicado.

\footnotetext{
${ }^{16}$ Vidya, V., Sankaran, K. and Viswanathan, K. S. Matrix isolationsupersonic jet infrared spectroscopy: conformational cooling in trimethyl phosphate. Chem. Phys. Lett. 258, 113-117 (1996).
} 


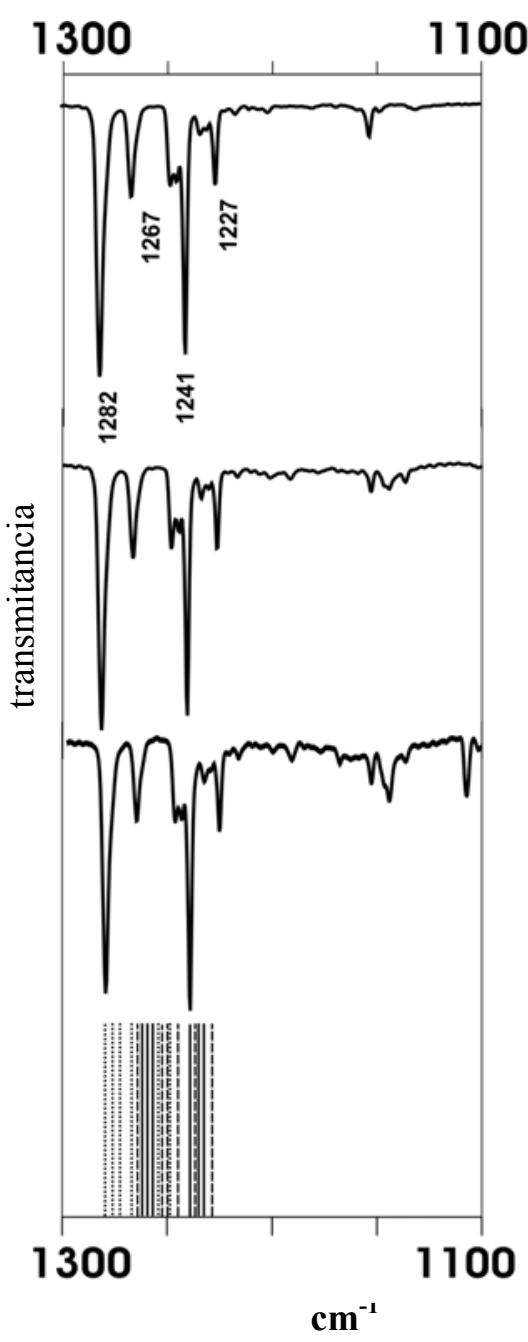

Figura 4.10: Espectro IR para el TMSeP en matriz de Ar en la región $1300-1100 \mathrm{~cm}^{-1}$, según patrón ya indicado.
Por lo tanto, los modos correspondientes a diferentes conformaciones muestran los mismos números de onda $\mathrm{y}$ ellos no pueden ser resueltos bajo nuestras condiciones experimentales. Nuestros cálculos teóricos concuerdan bien con este resultado, pues se predice un marcado solapamiento de los modos de vibración aparecidos en esta región del espectro.

Los modos de deformación de los grupos metilos se esperan en la región entre los 1500 y $1440 \mathrm{~cm}^{-1}$. Tres bandas bien definidas aparecen en el espectro IR de matrices (Figura 4.11), los que se corresponden con la ancha banda aparecida a $1454 \mathrm{~cm}^{-1}$ en el espectro FTIR del líquido.

Puesto que se observa una dependencia de la intensidad de la banda aparecida a $1498 \mathrm{~cm}^{-1}$ con la temperatura, esta puede ser originada en la segunda conformación, mientras que la banda a $1464 \mathrm{~cm}^{-1}$ será asignada al confórmero principal. El comportamiento de la intensidad relativa de la banda a $1446 \mathrm{~cm}^{-1}$ sin dependencia de la temperatura puede deberse a diferentes contribuciones conformacionales en el mismo número de onda.

Entonces, diferentes regiones del espectro vibracional muestran clara evidencia de la coexistencia de los confórmeros de simetrías $\mathrm{C}_{3}$ y $\mathrm{C}_{1}$. Pero más aún, los cálculos teóricos predicen un tercer confórmero estable con simetría Cs con una energía de alrededor de $3 \mathrm{kcal} / \mathrm{mol}$ por sobre el confórmero principal (ver Tabla 4.V). Por lo tanto, la energía relativa de este tercer confórmero, en conjunto con el comportamiento vibracional esperado, muestran que este tercer confórmero puede ser detectado experimentalmente.

El espectro de matrices de Ar a mayores temperaturas $(778 \mathrm{~K})$ es claramente la mejor condición experimental a disposición para la búsqueda de esta tercer forma de simetría Cs. A esta temperatura, las bandas que aparecen a $516 \mathrm{~cm}^{-1}$ correspondientes al modo estiramiento $\mathrm{P}=\mathrm{Se}$ (Figura 4.6), 702 y $692 \mathrm{~cm}^{-1}$ correspondiente al modo estiramiento P-O

Tabla 4.VII: Cálculos B3LYP para la abundancia relativa de los confórmeros del TMSeP.

\begin{tabular}{ccccc}
\hline Temperatura K & $\%$ & \multicolumn{2}{c}{ ponderado $^{\text {a }}$} \\
& $\mathrm{C}_{3}$ & $\mathrm{C}_{1}$ & $\mathrm{Cs}$ & Debyes \\
\hline 294 & 92 & 8 & 0 & 1,88 \\
408 & 85 & 14 & 1 & 2,11 \\
538 & 78 & 20 & 2 & 2,31 \\
653 & 73 & 24 & 3 & 2,44 \\
778 & 69 & 27 & 4 & 2,54
\end{tabular}


(Figura 4.8) y el triplete a $1080 \mathrm{~cm}^{-1}$ correspondiente a modos vibracionales de los grupos metilo (Figura 4.9), pueden ser originados por el confórmero Cs. Para mayor claridad, en las respectivas figuras estas bandas son indicadas mediante flechas.

\section{Integración y justificación de resultados}

El TMSeP muestra claramente dos conformaciones a temperatura ambiente, las que son observadas en los espectros IR/matriz de Ar, siendo sus simetrías $\mathrm{C}_{3}$, la más estable, y $\mathrm{C}_{1}$, según los resultados obtenidos mediante cálculos teóricos. Una importante predicción a partir de los datos teóricos, es el valor del momento dipolar para el TMSeP que debería encontrarse muy cerca de los valores de 3,28 D y 3,14 D correspondientes a los calculados a nivel $\mathrm{HF} / 6-31 \mathrm{G}^{* *}$ y MP2/6-31G** respectivamente.

Se sugieren evidencias de la presencia de un confórmero con mayor energía, de simetría Cs según las predicciones teóricas, asociándolo a ciertas manifestaciones en el espectro IR en matriz de $\mathrm{Ar}$ a altas temperaturas $(778 \mathrm{~K})$. Este correspondería al primer reporte experimental de esta tercer conformación para moléculas del tipo $\left(\mathrm{XP}\left(\mathrm{OCH}_{3}\right)_{3}\right)$, siendo $\mathrm{X}=\mathrm{O}, \mathrm{S}$ o Se.

Finalmente y como ya hemos indicado, los compuestos selenofosforados mostrarían menor flexibilidad conformacional comparados con sus análogos oxigenados y azufrados. Sin embargo, para el TMSeP puede ser deducida una diversidad conformacional interesante.

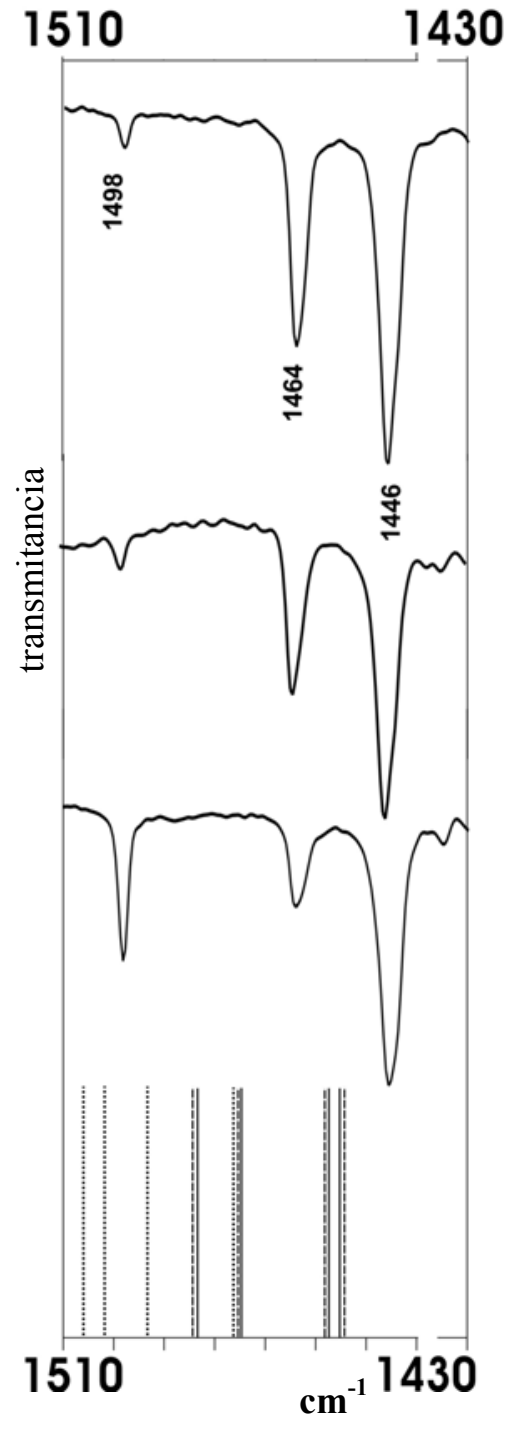

Figura 4.11: Espectro IR del TMSeP en matriz de Ar en la región $1510-1430 \mathrm{~cm}^{-1}$, según patrón ya indicado. 


\section{Acerca de la estructura de los fosfamidatos de dialquilo}
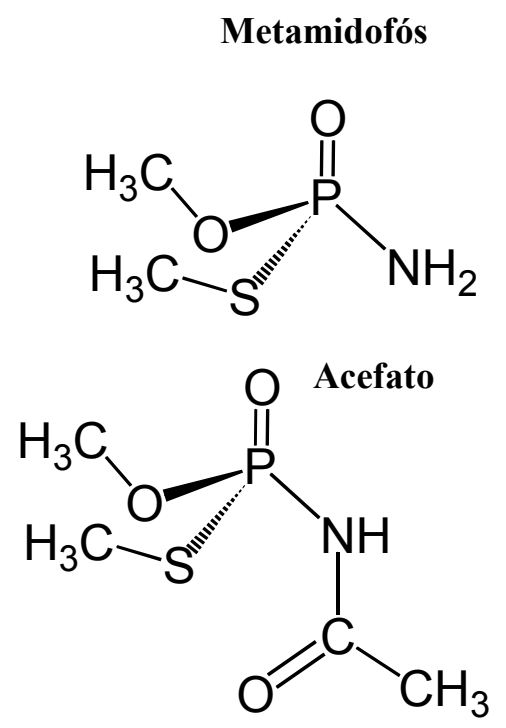

Figura 5.1: Estructuras del metamidofós (fosfamidotionato de O,S-dimetilo) y el acefato $(\mathrm{N}-$ acetil fosfamidotionato de $\mathrm{O}, \mathrm{S}$ dimetilo).
Los fosfamidatos de dialquilo exhiben innumerables aplicaciones tecnológicas, fundamentalmente como insecticidas. Los estudios originales aquí presentados servirán como base para futuros desarrollos en el área de la dilucidación estructural de este tipo de moléculas, dado que la información disponible a la fecha, aun es escasa.

Entre las moléculas más relevantes de este grupo se encuentra el metamidofós (fosfamidotionato de $\mathrm{O}, \mathrm{S}$ dimetilo) y el acefato ( $\mathrm{N}$-acetil fosfamidotionato de $\mathrm{O}, \mathrm{S}-$ dimetilo), dado el volumen en el que son utilizados para el control de ciertas plagas en arroz, tabaco y otros cultivos (Figura 5.1). Como efecto negativo se reportan miles de intoxicaciones por año entre los trabajadores dedicados a su aplicación. El gas de guerra tabún también forma parte de este grupo de sustancias.

Estos insecticidas y otros fosfamidatos, presentan abundantes datos respecto a la reactividad ${ }^{(1)}$ y al efecto biológico, ${ }^{(2)}$ con notable escasez de información respecto del comportamiento estructural. Se han usado métodos teóricos

\footnotetext{
${ }^{1}$ Nuretdinov, I.A. et al. Acid monochlorides of phosphorus amidoseleno acids and their properties, Bull.Acad.Sciences USSR. 7: 1423-1426 (1969).

${ }^{2}$ (a) Quistad, G.B., Fukuto, T.R. and Metcalf, R.L. Insecticidal, anticholinesterase, and hydrolytic properties of phosphoramidothiolates, J.Agric.Food.Chem. 18 (2): 189-194 (1970) (b) Crisp, C.E. and Look, M. Effect of esterification and side-chain alkylation on alteration of translocation characteristics of methamidophos, Xenobiotica 12 (8): 469479 (1982). (c) de Jong, L.P., Wolring, G.Z. and Benschop, H.P. Reactivation of acetylcholinesterase inhibited by methamidophos and analogous (di)methylphosphoramidates, Archives of Toxicology 49 (2): 175-183 (1982). (d) Look, M., Crisp, C.E. and Richmond, C.E. Altering translocation characteristics of methamidophos by $\mathrm{N}$-acylation with dicarboxylic half-acid esters, J.Agric.Food Chem. 30 (2): 285-289 (1982). (e) Spassova, D.P. and Singh, A.K. QSAR for acetylcholinesterase inhibition and toxicity of two classes of phosphoramidothioates, SAR.QSAR.Environ.Res. 11 (5-6): 453-471 (2001).
} 
Capítulo 5: Estructura de los calcogenofosfamidatos de dimetilo.

para el estudio de las estructuras ${ }^{(3)}$ y la reactividad ${ }^{(4)}$ de fosfamidatos.

Uno de los aspectos particulares interesantes de estas moléculas se relaciona con las propiedades del grupo amido fosfórico y, entre ellas, la eventual capacidad de sus protones para formar puentes de hidrógeno. ${ }^{(5)}$ Muchas de las características espectroscópicas, de reactividad y conformacionales estarían determinadas por la formación preferencial de puentes de hidrógeno intramoleculares (Figura 5.2).

Aunque no puedan considerarse enlaces conjugados, cálculos mecánico cuánticos indican que las nubes electrónicas de los enlaces $\mathrm{P}=\mathrm{O}$ y $\mathrm{P}-\mathrm{N}$ interaccionan apreciablemente. ${ }^{(6)}$ Este factor puede incidir, por ejemplo, en el comportamiento de estas sustancias frente a la hidrólisis mediante el ataque del enlace $\mathrm{P}-\mathrm{N}$.

En medidas comparativas realizadas mediante espectroscopía de rayos $\mathrm{X}$, se observa para cristales de estructuras del tipo $\left(\mathrm{CH}_{3} \mathrm{O}\right)_{2} \mathrm{P}(\mathrm{O}) \mathrm{NHAr}$, que los fosfamidatos prefieren la orientación cis de los grupos $\mathrm{P}=\mathrm{O}$ y $\mathrm{N}-\mathrm{H}$, con los grupos $\mathrm{N}$-arilo bisecando el ángulo $\mathrm{CH}_{3}-\mathrm{O}-\mathrm{P}-\mathrm{O}$. Otro es el comportamiento de las carboxiamidas análogas, que prefieren la configuración trans, además de disponer los anillos de sustitución con una notable rotación respecto al plano de la amida. ${ }^{(7)}$ En los mismos estudios se encontró una relación lineal entre las energías libres de los puentes de hidrógeno $\mathrm{P}=\mathrm{O} \cdots \mathrm{H}-\mathrm{N}$ y la acidez del ión $\mathrm{ArNH}_{3}{ }^{+}$. También se ha verificado la relevancia de los puentes de hidrógeno intermoleculares en la formación de cristales de fosfamidatos. ${ }^{(8)}$

\footnotetext{
${ }^{3}$ Pen'kovskii, V.V. and Boldeskul, I.E. Quantum-chemical study of dimethyl phosphoramidates, Teor.Eksp.Khim. 20 (2): 198-202 (1984).

${ }^{4}$ Klimenko, V.E., Peinel, G. and Pen'kovskii, V.V. Study of reaction centers in phosphoramidates by the molecular electrostatic potential method, Teor.Eksp.Khim. 18 (4): 477-481 (1982).

${ }^{5}$ Solov'ev, V.N., Martynov, I.V. and Zabirov, N.G. Crystal and molecular structure of two insecticides: O,S-dimethyl amidothiophosphate and O,Sdimethyl acetamidothiophosphate, Phosphorus, Sulfur Silicon.Relat.Elem. 57 (1-2): 135-141 (1991).

${ }^{6}$ Pen'kovskii, V.V. and Boldeskul, I.E. Quantum-chemical study of dimethyl phosphoramidates, Teor.Eksp.Khim. 20 (2): 198-202 (1984).

${ }^{7}$ Du, P., Modro, T.A. and Nassimbeni,L.R. Structural effects in amides. Crystal and molecular structures of phosphoric and carboxylic anilides, J.Org.Chem. 47 (12): 2313-2318 (1982).

${ }^{8}$ Du, P., Modro, T.A. and Nassimbeni, L.R. Hydrogen bonding in phosphoramides: structures of dimethyl N-phenylphosphoramidate and
} 


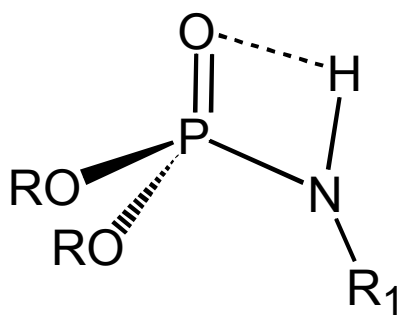

Figura 5.2: Formación del puente de hidrógeno entre el $\mathrm{H}$ amídico y el grupo fosforilo. Esta interacción determina la conformación cis en fosfamidatos primarios y no sustituidos.
En otro aspecto, se ha indicado también que la capacidad de formación de puentes de hidrógeno intermoleculares, determina las características particulares de reactividad biológica de los fosfamidatos. ${ }^{(9)}$ Este hecho es relevante dado que se ha mostrado a partir de datos de espectroscopía infrarroja, que la capacidad de formar puentes de hidrógeno por parte de fosfamidatos del tipo $(\mathrm{RO})_{2} \mathrm{P}(\mathrm{O}) \mathrm{NHCOR}_{1}$, es mayor que en las correspondientes carboxiamidas alquiladas. ${ }^{(10)}$

Otra evidencia de la relevancia del comportamiento de los hidrógenos amida en los fosfamidatos es que el grupo $\mathrm{N}$-metilamida del ácido fosfórico dimetiléster es casi treinta veces más hidrofílico que los compuestos en los cuales el grupo $-\mathrm{NH}-$ es reemplazado por $-\mathrm{O}-$ ó $-\mathrm{CH}_{2}-{ }^{(11)}$ Esta gran diferencia, mayor aún que la indicada por estudios teóricos o experimentales previos, es consistente también con el hallazgo de fuertes puentes de hidrógeno que dan lugar a la formación de complejos muy estables entre la termolisina (una enzima) y distintos inhibidores con estructura de fosfamidatos. ${ }^{(11)}$

El fosfamidotionato de O,S-dimetilo y su derivado $\mathrm{N}$-acilado, son fuertemente solubles en solución acuosa, moderadamente solubles en acetato de etilo e insolubles en nhexano. Otra evidencia importante es que la solubilidad en solución acuosa y la constante de partición con acetato de etilo de ambos compuestos es independiente del $\mathrm{pH}$ de la fase acuosa, ${ }^{(12)}$ lo que sería consecuencia directa de la preferencia de formación de puentes de hidrógeno intramoleculares, antes que con moléculas de solventes próticos.

Otra de las cuestiones de interés es que los fosfamidatos dialquílicos se han utilizado para el estudio de moléculas P-quirales, ${ }^{(13)}$ dando la posibilidad de abordar este

dimethyl N-(p-nitrophenyl)phosphoramidate, Acta.Crystallogr. B38 (5): 1504-1507 (1982).

9 Singh, A.K. Quantitative structure-activity relationships for phosphoramidothioate toxicity in housefly, Comp. Biochem. Physiol.C. Pharmacol. Toxicol. Endocrinol. 123 (3): 241-255 (1999).

${ }^{10}$ Nuzhdina, Y. et al. Structure of phosphorylated amides and properties of hydrogen bonds, Teor.Eksp.Khim. 10 (3): 396-399 (1974).

${ }^{11}$ Wolfenden, R. and Yu, L.L. Relative free energies of solvation of P-O, P-N, and P-C derivatives related to phosphoramide inhibitors of metallopeptidases. Bioorg. Chem. 19 (2): 152-156 (1991).

12 Singh,A.K. et al. Physicochemical, molecular-orbital and electronic properties of acephate and methamidophos. Comp.Biochem.Physiol. 119 (1): 107-117 (1998).

${ }^{13}$ Wozniak, L.A., Krzyzanowska, B. and Stec, W.J. A new method of phosphorus-selenium bond cleavage. Stereocontrolled synthesis of P- 
aspecto en su relación con entornos asimétricos, ${ }^{(14)}$ como los existentes en medios biológicos.

En las secciones posteriores se recorrerán las características espectroscópicas, conformacionales y de reactividad de estas moléculas en general, para luego estudiar algunos ejemplos particulares. La intención es realizar una descripción comparativa de los fosfamidatos de dimetilo, respecto de los calcogenofosfatos de trimetilo e identificar las características emergentes de las propiedades conformacionales.

\section{Espectroscopía vibracional}

Muchas de las bandas características de los fosfamidatos y compuestos relacionados corresponden a modos de vibración complejas, existiendo sólo unas pocas bandas que pueden caracterizarse esencialmente como modos vibracionales puros. ${ }^{(15)}$ Aun así, es posible identificar bandas características determinadas que eventualmente permitan el estudio de la coexistencia conformacional de estos compuestos.

En principio, se asume que el número de onda de estiramiento $\mathrm{P}=\mathrm{O}$ actuaría como un sensor de la coexistencia conformacional de los fosfamidatos O-disustituidos, por argumentos similares a los expuestos para calcogenofosfatos de trimetilo.

En ese sentido, se ha estudiado comparativamente el número de onda IR de estiramiento del grupo fosforilo en fosfamidatos de $\mathrm{N}$-arilo. En estos estudios se verifica que los efectos de los puentes de hidrógeno intramoleculares afectan fuertemente los valores de los números de onda del modo de estiramiento $\mathrm{P}=\mathrm{O}$, con la excepción de estructuras en las que

chiral phosphoric-trifluoroacetic anhydrides, J.Org.Chem. 57 (22): 6057 6060 (1992).

${ }^{14}$ (a) Miyazaki, A., Nakamura, T., Kawaradani, M., and Marumo, S. Resolution and biological activity of both enantiomenrs of methamidophos and acephate. J.Agric.Food.Chem. 36: 835-837 (1988). (b) Miyazaki, A. and Marumo, S. Stereochemistry in metabolism of achiral organophosphorus insecticides, Prog. Prospects. Organophosphorus. Agrochem. 1: 59-70 (1995).

15 Nyquist, R.A., Wass, M.N. and Muelder, W.W. Vibrational spectroscopyc study of $\mathrm{CH}_{3}-\mathrm{NH}-\mathrm{P}(=\mathrm{S}) \mathrm{Cl}_{2}$ and its deuterium analogs, Spectrochim.Acta. 25A: 611-625 (1969). 
existen grandes restricciones estéricas. ${ }^{(16)}$ Dichos efectos pueden detectarse aun en soluciones diluidas. Por otro lado, los efectos de resonancia de los sustituyentes en el grupo fosfamidato son más bajos que en las funciones del tipo carboxamida. Estudios de espectroscopía infrarroja realizados sobre amidas secundarias, muestran que estos compuestos en solución favorecen la conformación en la cual en enlace $\mathrm{N}-\mathrm{C}$ es trans respecto al enlace $\mathrm{P}=\mathrm{O}$. $^{(17)}$

A números de onda más altos, el modo de estiramiento $\mathrm{N}-\mathrm{H}$ se presenta en muchos fosfamidatos $\mathrm{y}$ fosfamidotioatos en dos bandas características que se corresponderían con dos posiciones preferenciales del hidrógeno, respecto del enlace fosforilo. De esta manera, la conformación cis (manifestada entre 3380 y $3440 \mathrm{~cm}^{-1}$ ) preferente respecto a la configuración trans (manifestada en una banda ubicada a números de onda menores de entre 3380 y $3410 \mathrm{~cm}^{-1}$ ). Sin embargo, el modo normal de vibración de balanceo $\mathrm{N}-\mathrm{H}$ se presenta sólo en una banda característica, ubicada entre los 1370 y los $1415 \mathrm{~cm}^{-1}$. ${ }^{(18)}$

Esta evidencia refuerza la idea de la importancia de la formación de puentes de hidrógeno intramoleculares respecto de las preferencias conformacionales de los fosfamidatos, dado que si ellas están determinadas por las interacciones de las nubes electrónicas del grupo $\mathrm{P}=\mathrm{X}$, siendo $\mathrm{X}$ cualquier calcógeno, y a su vez, existen fuertes efectos estereoelectrónicos por la influencia del grupo $\mathrm{N}-\mathrm{H}$ sobre $\mathrm{X}$, la fuerza de estos puentes de hidrógeno, será uno de los factores determinantes de la geometría de los confórmeros estables y su proporción.

\section{Espectroscopía de resonancia magnética nuclear}

No existen muchos trabajos que den cuenta de un comportamiento general de los fosfamidatos respecto de la espectroscopía RMN. Aún así esta herramienta se ha

\footnotetext{
${ }^{16}$ Foulds, G.A., Modro, T.A. and Rijkmans, B.P. Phosphoric amides. 4. An IR spectrophotometric study of structural effects in the phosphoramidate group, S.Afr.J.Chem. 34 (3): $72-75$ (1981).

${ }^{17}$ Xue, Ch. et al. Stereochemical dependence of ${ }^{2} \mathrm{~J}_{\mathrm{PNC}}$ coupling constants in N-dialkyloxyphosphoryl amino acids and other phosphoramidate compounds, J.Chem.Soc., Perkin Trans.2. 3: 431-434 (1990).

18 Nyquist, R.A. Correlations between infrared spectra and structure: phosphoramides and related compounds, Spectrochim.Acta. 19: 713-729 (1963).
} 
utilizado en el estudio de estas moléculas y sus derivados en distintos contextos ${ }^{(19)}$.

Como ya se ha indicado, en ocasiones determinados comportamientos observados en la espectroscopía RMN, son útiles para la interpretación de las características conformacionales de las moléculas.

En fosfamidatos, la constante de acoplamiento espín-espín de segundo orden, ${ }^{2} J_{\mathrm{PNC}}$, es dependiente de la estructura y la conformación de la función fosfamidato. De esta manera, la constante de acoplamiento de segundo orden ${ }^{2} J_{\mathrm{PNC}}$ es pequeña en amidas secundarias $(<1 \mathrm{~Hz})$, mientras que para amidas terciarias la magnitud de ${ }^{2} J_{\mathrm{PNC}}$ se incrementa hasta valores de entre 2,8 y $7,1 \mathrm{~Hz}^{(17)}$ Por otro lado, la constante ${ }^{2} J_{\mathrm{PNC}}$ en posición trans es influenciada por los cambios en la longitud por los cambios en la longitud de la cadena del grupo $\mathrm{N}$-alquilo, de manera que los valores de ${ }^{2} J_{\mathrm{PNC}}$ se incrementan sucesivamente desde $\operatorname{los} \mathrm{N}-\mathrm{CH}_{3}$ hasta los N-iso-propilo.

Las medidas de ${ }^{13} \mathrm{C}$ y ${ }^{15} \mathrm{~N}$ RMN en series de fosfamidatos dialquílicos $\mathrm{N}$-aril sustituidos, dan cuenta de la competencia que existe entre dos procesos inversos: la resonancia del par de electrones libres hacia el sistema de enlaces conjugados del anillo bencénico y la deslocalización de este mismo par de electrones hacia los orbitales del grupo $\mathrm{P}=\mathrm{O} .^{(20)}$ En estos mismos estudios, los cambios conformacionales del enlace $\mathrm{N}$-arilo fueron asociados al valor de la constante de acoplamiento espín-espín ${ }^{3} J_{\mathrm{PC}}{ }^{(20 a)}$

Para los derivados S-óxidos diasteroméricos de fosfamidotioatos se observó una gran diferencia entre los

\footnotetext{
${ }^{19}$ (a) Quin, L.D., Bourdieu, C. and Quin, G.S. Direct detection of two metaphosphoramidates in $-75^{\circ} \mathrm{C}$ THF solutions by ${ }^{31} \mathrm{P}$ NMR spectroscopy, Tetrahedron Lett. 31 (45): 6473-6476 (1990). (b) Chen, Y. et al. N-Phosphoryl amino acids and peptides. Part V. O-Alkyl substitution effects on the ${ }^{31} \mathrm{P}$ NMR spectra of phosphoramidates, Phosphorus, Sulfur Silicon.Relat.Elem. 61 (1-2): 31-39 (1991). (c) Salama, A.K. et al. Studies on the metabolism of methamidophos and acephate: synthesis and identification by proton nuclear magnetic resonance and mass spectrometry of ${ }^{14} \mathrm{C}$-acephate and some metabolites, J.Occup. Med. Toxicol. 2 (4): 399-408 (1993).

${ }^{20}$ (a) Buchanan, G.W. and Morin, F.G. Electronic and steric effects in arylphosphoramidates and phosphorimidates as monitored by ${ }^{13} \mathrm{C}$ nuclear magnetic resonance, Org.Magn.Reson. 14 (6): 517-521 (1980). (b) Buchanan, G.W., Morin, F.G. and Fraser, R.R. A ${ }^{15} \mathrm{~N}$ nuclear magnetic resonance study of $\mathrm{N}$-aryl phosphoramidates and phosphorimidates, Can.J.Chem. 58 (23): 2442-2446 (1980).
} 
corrimientos químicos para el doblete ${ }^{13} \mathrm{C}$ RMN. Esta diferencia fue atribuida a la formación de puentes de hidrógeno intramoleculares $\mathrm{S}=\mathrm{O} \cdots \mathrm{H}-\mathrm{N},{ }^{(21)}$ desplazando entonces la formación del puente de hidrógeno $\mathrm{P}=\mathrm{O} \cdots \mathrm{H}-\mathrm{N}$.

Por otro lado, se ha reportado la constante de acoplamiento espín-espín de segundo orden en el espectro de ${ }^{31} \mathrm{P}$ RMN para compuestos fosfamiditos del tipo $(\mathrm{RO})_{2} \mathrm{P}(\mathrm{O}) \mathrm{N}\left(\mathrm{CH}_{3}\right) \mathrm{P}\left(\mathrm{R}_{1}\right)_{2}$. Se observó que ${ }^{2} J_{\mathrm{PNP}}$ disminuye según la naturaleza del sustituyente $\mathrm{R}_{1}$ en el siguiente orden: $\mathrm{Cl}_{2}>$ bis(alquiltio) $>$ dialcoxi $>$ alcoxiamino. ${ }^{(22)}$

\section{Reactividad}

El enlace $\mathrm{P}-\mathrm{N}$ del metamidofós, aunque no el del acefato, se hidroliza a $\mathrm{pH} 2$. Esto significa que la presencia de una zona rica en electrones estabiliza el enlace $\mathrm{P}-\mathrm{N}$ en el caso del acefato. El enlace $\mathrm{CH}_{3} \mathrm{~S}-\mathrm{P}$ de ambas moléculas es hidrolizado a pH 11 en forma similar. Esto indica que los dos compuestos son considerablemente similares excepto que el acefato presenta un dominio rico en electrones. Por lo tanto, a

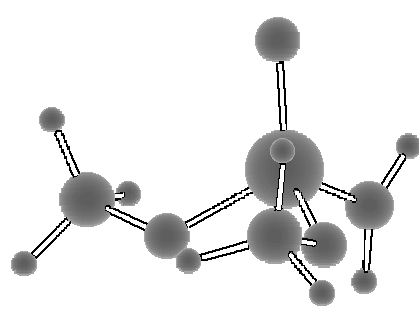

Figura 5.3: Confórmero de geometría gCs. $\mathrm{pH}$ fisiológicos las diferencias funcionales entre estas moléculas pueden asimilarse a las diferencias en las estructuras electrónicas. ${ }^{(12)}$

En medidas de determinación de las constantes cinéticas de segundo orden de la hidrólisis alcalina de fosfamidatos y fosfamidotioatos aril alquil sustituidos, se encontró una ruptura preferente de los enlaces $\mathrm{P}-\mathrm{O}$ y $\mathrm{P}-\mathrm{S}$. Esta ruptura se verificó fundamentalmente mediante un ataque nucleofílico, aunque la ruptura del enlace $\mathrm{P}-\mathrm{S}$ también ocurre, en menor medida, por un mecanismo de eliminación ${ }^{(23)}$.

Durante el estudio del desplazamiento nucleofílico de iones haluro desde fosfamidatos $\alpha$-haloetil sustituidos, se observó que fue la vía preferida de reacción la sustitución en 1,3 por el átomo $\mathrm{N}$, clivando los derivados azaridina; no

\footnotetext{
21 Thompson, C.M., Castellino, S. and Fukuto, T.R. A ${ }^{13} \mathrm{C}$ nuclear magnetic resonance study on an organophosphate. Formation and characterization of methamidophos (O,S-dimethyl phosporamido thioate) S-oxide, J.Org.Chem. 49 (10): 1696-1699 (1984).

22 Nuretdinov, I.A. et al. Mixed amides of dialkylphosphoric and phosphorous acids, Zh.Obshch.Khim. 45 (9): 1948-1953 (1975).

${ }^{23}$ Hamer, N.K. and Tack, R.D. Reappraisal of the role of the ElcB mechanism of hydrolysis of phosphoramidic derivatives, J.Chem.Soc., Perkin Trans.2. 1: 1184-1187 (1974).
} 
fueron observadas las sustituciones alternativas en 1,5, que involucraría a los grupos $\mathrm{N}$-amida o fosforilo. ${ }^{(24)}$

A diferencia de las etileniminas derivadas del fosfamidato de dimetilo, en donde ambos enlaces $\mathrm{P}-\mathrm{O}$ y $\mathrm{P}-\mathrm{N}$ son fuertemente activos a la hidrólisis básica, se observa en las derivados alquilaminas que el enlace $\mathrm{P}-\mathrm{N}$ es resistente y el enlace $\mathrm{P}-\mathrm{O}$ es desactivado después de la hidrólisis. Esto indica que la reactividad del enlace éster para los derivados alquilamina, es similar que el encontrado en el TMP. ${ }^{(25)}$ Esta evidencia es importante al evaluar los mecanismos de inhibición irreversible de los fosfamidatos, como se verá en el Capítulo 12.

Aunque se asume una mayor estabilidad del enlace $\mathrm{P}-\mathrm{N}$, respecto al enlace $\mathrm{P}-\mathrm{O},{ }^{(26)}$ en los espectros de masa característicos, se observa que la ruptura del enlace $\mathrm{P}-\mathrm{N}$ puede involucrar una fisión simple, una fisión acompañada de migración de $\mathrm{H}$ desde el grupo éster y fisión acompañada de la migración del grupo éster hasta el N saliente, ${ }^{(27)}$ revelando una reactividad importante.

\section{Conformaciones preferenciales}

Muchas de las consideraciones generales que se aplican respecto de la estructura conformacional de los calcogenofosfatos de trimetilo, son también aplicables a los fosfamidatos de dialquilo. Esto es válido por ejemplo en cuanto a la naturaleza del calcógeno unido por doble enlace al fósforo $(\mathrm{O}, \mathrm{S}$ o $\mathrm{Se})$ y a su influencia sobre las preferencias conformacionales de los restos alquilos en términos del valor preferente del ángulo $\psi$.

Se verifica que en general, los alquilos manifiestan posiciones gauche, en forma preferente respecto a las

\footnotetext{
24 Davidowitz, B. and Modro, T.A. Phosphoric amides. Part 6. Intramolecular nucleophilic displacement in $\alpha$-haloethyl-substituted phosphoramidates, S.Afr.J.Chem. 35 (2): 63-66 (1982).

${ }^{25}$ Davidowitz, B. and Modro, T.A. Phosphoric amides. Part 8. The effect of the ethylenimine substituent on the solvolytic reactivity of phosphate and phosphoramidate bonds, J.Chem.Soc., Perkin Trans.2. 2: 303-306 (1985).

${ }^{26}$ Moerat, A. and Modro, T.A. Acid-catalyzed hydrolysis of N-phenyl phosphoric amides. Kinetic implication of the reaction via the Oprotonated intermediate, Phosphorus Sulfur 14 (2): 179-183 (1983).

27 Davidowitz, B. and Modro, T.A. Phosphoric amides. Part 7. Mass spectrometry of phosphoric amido esters: fragmentation patterns and migratory aptitudes, Org.Mass Spectrom. 19 (3): 128-134 (1984).
}

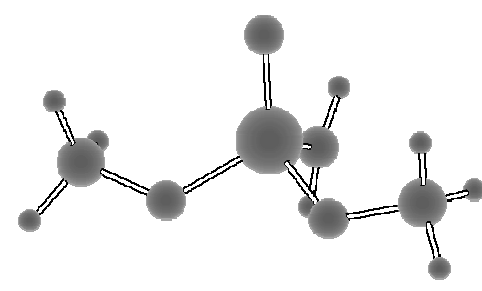

Figura 5.4: Confórmero de geometría $\mathrm{C}_{1}$. 


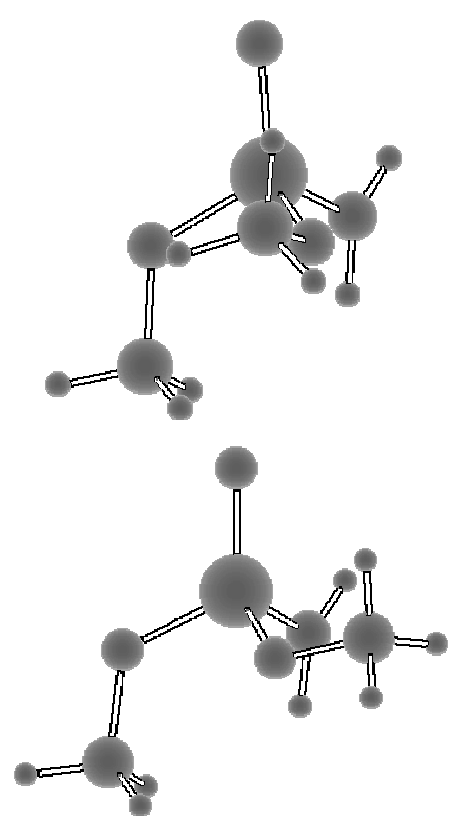

Figura 5.5: Confórmeros de geometría tCs. posiciones trans, mientras los hidrógenos del grupo amido se ubican en posiciones cis, preferentes a las trans como ya fue indicado.

Mediante cálculos químico cuánticos se observa que en el fosfamidato de dimetilo existe una formación preferencial de dímeros en conformación gauche-gauche, respecto a los dímeros lineales con conformación trans-trans, ambos estabilizados por formación de puentes de hidrógeno intermoleculares. Por resultado del mismo tipo de cálculos, en la molécula no sustituida (el derivado diácido) existe una preferencia para la formación de dímeros cíclicos. ${ }^{(28)}$ En ambos casos, la formación de los dímeros resulta primariamente de interacciones electrostáticas, antes que por transferencia de cargas entre subsistemas.

Los mapas de campos electrostáticos para moléculas del tipo $(\mathrm{RO})_{2} \mathrm{P}(\mathrm{O}) \mathrm{NH}_{2}(\mathrm{R}=\mathrm{H}, \mathrm{Me})$ en conformaciones gauche-gauche $\mathrm{y}$ trans-gauche, muestran dos mínimos conformacionales alrededor del oxígeno del fosforilo, a diferencia de la conformación trans-trans que sólo muestra un mínimo conformacional. ${ }^{(29)}$

En los fosfamidatos dialquílicos no sustituidos, dado que la rotación del ángulo $\psi_{\mathrm{N}}$ no implica grandes modificaciones estructurales ni energéticas, las conformaciones están determinadas fundamentalmente por los valores de los ángulos diedros $\psi 1$ y $\psi 2$ de cada uno de ambos ésteres metílicos. De manera que, fijando la posición de uno de los enlaces $\mathrm{N}-\mathrm{H}$ en cis respecto al enlace $\mathrm{P}=\mathrm{X}$, aparecen cuatro conformaciones preferenciales con simetrías Cs y $\mathrm{C}_{1}$. Éstas son:

Confórmero gCs: con valores $\psi 1$ y $\psi 2$ entre 10 y $90^{\circ}$. Corresponde a posiciones gauche de los dos metilos respecto al fosforilo (Figura 5.3). El confórmero -gauche, -gauche es energéticamente equivalente, por lo tanto esta conformación presenta degeneración de orden 2 .

Confórmero $\mathbf{C}_{1}$ : con valores $\psi 1$ entre 10 y $90^{\circ}$ y valores de $\psi 2$ de entre $-10 \mathrm{y}-90^{\circ}$. Corresponde a posiciones gauche, gauche (Figura 5.4). Las posiciones de los metilos correspondientes a $\psi 1$ y $\psi 2$, permiten una minimización de

\footnotetext{
${ }^{28}$ Klimenko, V.E., Peinel, G. and Pen'kovskii, V.V. Quantum-chemical study of dimeric associates of phosphoric acid amides, Teor.Eksp.Khim. 19 (5): 612-615 (1983).

29 Klimenko, V.E., Peinel, G. and Pen'kovskii, V.V. Effect of conformational states on the electrostatic field of amides of phosphoric acids, Teor.Eksp.Khim. 19 (1): 86-87 (1983).
} 
los impedimentos estéricos, pues significan un alejamiento simétrico de estos metilos respecto del eje $\mathrm{P}=\mathrm{X}$ (X:calcógeno). No hay conformaciones equivalentes, por lo tanto esta conformación presenta degeneración de orden 1.

Confórmeros tCs: Existen dos conformaciones que, aunque no son equivalentes, presentan una energía conformacional similar. En el primero de ellos los valores de $\psi 1$ se encuentran entre -10 y $-90^{\circ}$ (-gtCs) y en el otro estos valores se encuentran entre 10 y $90^{\circ}$ (gtCs). En ambos, el valor de $\psi 2$ es cercano a $180^{\circ}$. Corresponde a posiciones -gauche, trans y gauche, trans (Figura 5.5). Ambos presentan degeneración de orden 2 .

Existiría un quinto confórmero de simetría $\mathrm{C}_{1}$, con posiciones -gauche, gauche. Esta constituye una conformación de fuerte impedimento estérico. Por esta razón, no aparece en ninguno de las referencias revisadas ni en los cálculos realizados en este trabajo.

En adelante, se revisarán en detalle los comportamientos conformacionales de las moléculas fosfamidato de dimetilo (DMPA) y fosfamidotioato de O,Odimetilo (DMPTA), respecto de las evidencias experimentales y teóricas descritas. 


\section{6}

\section{La molécula del fosfamidato de dimetilo}<smiles>COP(N)(=O)OC</smiles>

Figura 6.1: Estructura del fosfamidato de dimetilo (DMAP).
El fosfoamidato de dimetilo (DMAP) corresponde al análogo oxigenado del metamidofós o fosfotionoamidato de O,S-dimetilo, un insecticida usado ampliamente un muchos cultivos y del que se reportan anualmente numerosas intoxicaciones. El DMAP puede aparecer como impureza en las formulaciones de grado técnico del metamidofós y se ha propuesto como uno de sus derivados metabólicos. ${ }^{(1)}$ Es necesario considerar que un componente importante del desarrollo de una intoxicación por pesticidas formulados puede ser responsabilidad de las impurezas que se encuentran presentes en ellos o de las sustancias derivadas por biotransformación.

Dentro de los fosfamidatos neutros, el DMAP mostrado en la Figura 6.1, es la molécula más pequeña. Esto permite estudiar con cierta precisión las características moleculares que nos interesan, sin abundancia de información en los datos experimentales que interfiera con los análisis conformacionales pertinentes.

No se ha reportado mucha información estructural desde la cual comenzar el estudio. Esta situación contrasta mucho con lo que ya fue descrito para los calcogenofosfatos de trimetilo. Aún así, es posible contar con algunos criterios generales enumerados para los fosfamidatos, que pueden aplicarse con cierta certeza para esta molécula.

Entre las propiedades más importantes de los fosfamidatos se encuentra la posibilidad de formar interacciones intramoleculares fuertes entre los hidrógenos del grupo amino y la nube electrónica del oxígeno en el grupo fosforilo. Estos puentes de hidrógeno por un lado estabilizarían en cis la posición de uno de los hidrógenos amido. El otro se ubicará en la posición correspondiente a la hibridación $\mathrm{sp}^{3}$ del nitrógeno.

\footnotetext{
${ }^{1}$ Salama, A.K. et al. Studies on the metabolism of methamidophos and acephate: synthesis and identification by proton nuclear magnetic resonance and mass spectrometry of ${ }^{14} \mathrm{C}$-acephate and some metabolites, J.Occup.Med.Toxicol. 2 (4): 399-408 (1993).
} 
Capítulo 6: La molécula de fosfamidato de dimetilo.

Tabla 6.I: Frecuencias medidas en los espectros infrarrojo en fase líquida en la zona de entre 1000 y $2000 \mathrm{~cm}^{-1}$ para la molécula DMAP. Las asignaciones propuestas se realizan en función de los valores teóricos obtenidos para cada uno de los cuatro confórmeros estables calculados a nivel $\mathrm{HF} / 6-31 \mathrm{G}^{\star *}$. Entre paréntesis, se indican las intensidades teóricas relativas.

\begin{tabular}{|c|c|c|c|c|c|}
\hline \multirow[t]{2}{*}{ Asignación } & \multirow[t]{2}{*}{ FTIR } & \multicolumn{4}{|c|}{ Calculado(HF/6-31G**) } \\
\hline & & $\mathrm{gC}_{1}$ & Cs & $-\mathrm{gtC}_{1}$ & $\mathrm{gtC}_{1}$ \\
\hline & $1844(0,03)$ & & & $1551(0,24)$ & \\
\hline \multirow[t]{5}{*}{$\delta \mathrm{a} \mathrm{NH}_{2}$} & $1653(0,30)$ & $1544(0,16)$ & & & $1543(0,19)$ \\
\hline & & & $1538(0,10)$ & & \\
\hline & & $1461(0,01)$ & & $1462(0,01)$ & \\
\hline & & $1460(0,01)$ & $1461(0,01)$ & & $1461(0,02)$ \\
\hline & & & $1459(0,01)$ & $1459(0,02)$ & $1458(0,01)$ \\
\hline \multirow[t]{6}{*}{$\delta \mathrm{a} \mathrm{CH}_{3}$} & & & $1454(0,01)$ & & $1456(0,01)$ \\
\hline & $1461(0,42)$ & $1452(0,01)$ & & $1452(0,01)$ & \\
\hline & & $1451(0,01)$ & $1452(0,00)$ & $1451(0,01)$ & $1450(0,01)$ \\
\hline & & $1439(0,01)$ & $1443(0,00)$ & $1440(0,01)$ & $1440(0,00)$ \\
\hline & $1322(0,04)$ & $1437(0,00)$ & $1437(0,00)$ & $1439(0,00)$ & $1439(0,01)$ \\
\hline & & & & & $1274(0,71)$ \\
\hline \multirow[t]{2}{*}{$v \mathrm{P}=\mathrm{O}$} & & & & $1257(0,86)$ & \\
\hline & $1197(0,66)$ & $1253(0,47)$ & $1253(0,31)$ & & \\
\hline \multirow[t]{2}{*}{ pra $\mathrm{CH}_{3}$} & & $1175(0,05)$ & $1176(0,02)$ & $1173(0,07)$ & $1176(0,09)$ \\
\hline & 1111 sh & $1175(0,07)$ & $1174(0,08)$ & $1172(0,11)$ & $1174(0,06)$ \\
\hline \multirow{3}{*}{$w \mathrm{CH}_{3}$} & & $1149(0,00)$ & $1150(0,01)$ & $1150(0,01)$ & \\
\hline & & & & $1149(0,01)$ & $1149(0,01)$ \\
\hline & & $1147(0,00)$ & $1148(0,00)$ & & $1146(0,01)$ \\
\hline \multirow{3}{*}{ vs O-C } & & & $1097(0,02)$ & & \\
\hline & & & & & $1089(0,36)$ \\
\hline & $1081 \mathrm{sh}$ & $1079(0,34)$ & & $1081(0,62)$ & \\
\hline \multirow{3}{*}{ va O-C } & & & & $1060(1,00)$ & $1061(1,00)$ \\
\hline & $1058(0,73)$ & $1050(1,00)$ & & & \\
\hline & & & $1045(1,00)$ & & \\
\hline
\end{tabular}

v: vibración de estiramiento; vs: vibración de estiramiento simétrico; va: vibración de estiramiento antisimétrico; pra: vibración de balanceo antisimétrico; w: vibración de aleteo; sh: hombro.

Por otro lado, la presencia de este puente de hidrógeno, desestabilizaría las posibles interacciones entre los hidrógenos metílicos y el grupo fosforilo. Estas interacciones se han evaluado de importancia para explicar el comportamiento conformacional de los calcogenofosfatos de trimetilo.

Además, esta molécula presenta un interés en términos de su variabilidad conformacional pues, a diferencia de los fosfatos de trialquilo, dispone de sólo dos ángulos diedros $\mathrm{O}=\mathrm{P}-\mathrm{O}-\mathrm{C}$ que tendrían libertad conformacional relevante, de manera que el número de confórmeros posibles y su degeneración energética disminuye drásticamente respecto de aquellos. Uno de los aspectos interesantes de estas moléculas se relaciona con las propiedades del grupo amido fosfórico y, entre ellas, la capacidad de sus protones para formar puentes de hidrógeno. ${ }^{(2)}$

\footnotetext{
${ }^{2}$ Solov'ev, V.N., Martynov, I.V. and Zabirov, N.G. Crystal and molecular structure of two insecticides: O,S-dimethyl amidothiophosphate and O,S-
} 
Tabla 6.II: Parámetros geométricos y fisicoquímicos del DMAP obtenidos a nivel HF/6-31G** confórmeros descritos como mínimos energéticos. Ángulos expresados en grados.

\begin{tabular}{|c|c|c|c|c|}
\hline & $\mathrm{gCs}(\mathrm{I})$ & $\mathrm{C}_{1}(\mathrm{II})$ & -gtCs (III) & gtCs (IV) \\
\hline$\psi_{1}$ & 45 & 49 & -32 & 46 \\
\hline$\psi_{2}$ & 54 & -39 & 178 & 171 \\
\hline $\mathrm{P}=\mathrm{O}(\AA)$ & 1,45 & 1,45 & 1,45 & 1,45 \\
\hline $\mathrm{P}-\mathrm{O}_{1}(\AA)$ & 1,58 & 1,58 & 1,59 & 1,58 \\
\hline $\mathrm{P}-\mathrm{O}_{2}(\AA)$ & 1,58 & 1,58 & 1,58 & 1,58 \\
\hline $\mathrm{HF} / 6-31 \mathrm{G}^{\star *}(\mathrm{kcal} / \mathrm{mol})$ & 0,00 & 1,75 & 3,00 & 3,80 \\
\hline$\mu \mathrm{D}$ (Müll)Calc. & 2,74 & 3,24 & 3,93 & 5,40 \\
\hline Prop.a $298^{\circ} \mathrm{K}$ (peso $2: 1: 2: 2$ ) & 0,97 & 0,03 & 0,01 & 0,00 \\
\hline$\mu \mathrm{D}$ (Müll)Pond. & \multicolumn{4}{|c|}{2,81} \\
\hline
\end{tabular}

Dado que ciertas características espectroscópicas, de reactividad y conformacionales están determinadas por la

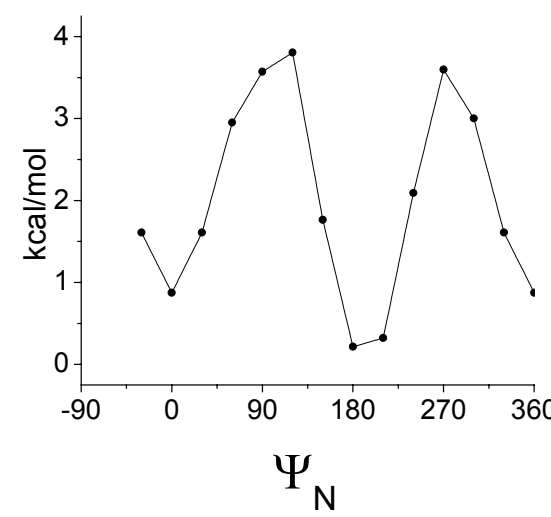

Figura 6.2: Energía de rotación del ángulo $\psi_{\mathrm{N}}$ calculada a un nivel $\mathrm{HF} / 6-31+\mathrm{G}^{*}$. formación preferencial de puentes de hidrógeno, el análisis de estas interacciones intramoleculares debería poder integrarse a la descripción del comportamiento estructural de esta molécula como componente fundamental. Ya se ha indicado que la capacidad de formación de puentes de hidrógeno intermoleculares, determina las particulares características de reactividad biológica de los fosfamidatos. ${ }^{(3)}$

Por otro lado existiría una fuerte interacción entre las nubes electrónicas de los enlaces $\mathrm{P}=\mathrm{O}$ y $\mathrm{P}-\mathrm{N}^{(4)}$. Este dato, sumado a las interacciones por puentes de hidrógeno intramoleculares, restringe apreciablemente la variabilidad del ángulo $\psi_{\mathrm{N}}$. de manera que el análisis conformacional de la molécula dependería fundamentalmente de los ángulos diedros $\psi_{1}$ y $\psi_{2}$ de rotación de los enlaces $\mathrm{P}-\mathrm{O}$.

\section{Descripción teórica}

En la Tabla 6.Il se presentan los valores de los parámetros geométricos y energéticos para cada uno de los cuatro confórmeros estables hallados a un nivel de cálculo $\mathrm{HF} / 6-31 \mathrm{G}^{* *}$.

dimethyl acetamidothiophosphate, Phosphorus, Sulfur Silicon.Relat.Elem. 57 (1-2): 135-141 (1991).

3 Singh, A.K. Quantitative structure-activity relationships for phosphoramidothioate toxicity in housefly, Comp. Biochem. Physiol.C. Pharmacol. Toxicol. Endocrinol. 123 (3): 241-255 (1999).

4 Pen'kovskii, V.V. and Boldeskul, I.E. Quantum-chemical study of dimethyl phosphoramidates, Teor.Eksp.Khim. 20 (2): 198-202 (1984). 
Capítulo 6: La molécula de fosfamidato de dimetilo.

Tabla 6.III: Frecuencias medidas en los espectros infrarrojo en fase líquida en la zona de entre 400 y $1000 \mathrm{~cm}^{-1}$ para la molécula DMAP. Las asignaciones propuestas se realizan en función de los valores teóricos obtenidos para cada uno de los cuatro confórmeros estables calculados a nivel $\mathrm{HF} / 6-31 \mathrm{G}^{* *}$. Entre paréntesis, se indican las intensidades teóricas relativas.

\begin{tabular}{|c|c|c|c|c|c|}
\hline \multirow[t]{2}{*}{ Asignación } & \multirow[t]{2}{*}{ FTIR } & \multicolumn{4}{|c|}{ Calculado(HF/6-31G**) } \\
\hline & & $\mathrm{gC}_{1}$ & Cs & $-\mathrm{gtC}_{1}$ & $\mathrm{gtC}_{1}$ \\
\hline & & & & $984(0,37)$ & \\
\hline \multirow[t]{2}{*}{ pra $\mathrm{NH}_{2}$} & $970(0,13)$ & $962(0,09)$ & & & \\
\hline & 930sh & & $940(0,07)$ & & $943(0,12)$ \\
\hline \multirow[t]{3}{*}{$v \mathrm{P}-\mathrm{N}$} & $825(0,14)$ & $880(0,25)$ & & & \\
\hline & & & $869(0,23)$ & & \\
\hline & & & & $862(0,49)$ & $859(0,39)$ \\
\hline \multirow{3}{*}{ va P-O } & $782(0,15)$ & $814(0,41)$ & $815(0,16)$ & & \\
\hline & & & & & $803(0,44)$ \\
\hline & & & & $787(0,35)$ & \\
\hline \multirow[t]{2}{*}{ vs P-O } & & & $740(0,05)$ & & \\
\hline & $703(0,03)$ & $727(0,06)$ & & $716(0,06)$ & $720(0,07)$ \\
\hline \multirow{4}{*}{$w \mathrm{NH}_{2}$} & 630sh & & & $607(0,54)$ & \\
\hline & $588(0,05)$ & $523(0,45)$ & & & \\
\hline & $561(0,03)$ & & $515(0,09)$ & & \\
\hline & $503(0,06)$ & & & & $499(0,15)$ \\
\hline \multirow{4}{*}{$\delta \mathrm{a} \mathrm{P}-\left(\mathrm{OCH}_{3}\right)_{2}$} & $495(0,06)$ & & & $505(0,06)$ & \\
\hline & $453(0,06)$ & $500(0,07)$ & & & \\
\hline & $446(0,04)$ & & $472(0,09)$ & & \\
\hline & $439(0,03)$ & & & & $455(0,11)$ \\
\hline \multirow{3}{*}{$\delta \mathrm{a}-\left(\mathrm{OCH}_{3}\right)_{2}$} & $432(0,03)$ & $458(0,10)$ & & $455(0,14)$ & \\
\hline & & & & & $423(0,29)$ \\
\hline & $424(0,04)$ & & $410(0,04)$ & & \\
\hline \multirow{3}{*}{$w \mathrm{P}\left(\mathrm{NH}_{2}\right)\left(\mathrm{OCH}_{3}\right)_{2}$} & $414(0,04)$ & & & $406(0,10)$ & $415(0,17)$ \\
\hline & $407(0,05)$ & $403(0,06)$ & & & \\
\hline & & & $382(0,00)$ & & \\
\hline
\end{tabular}

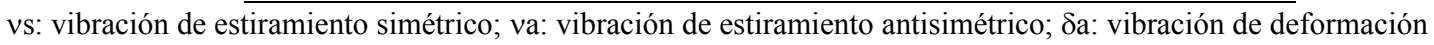
antisimétrica; sh: hombro.

Se puede observar la preferencia de los metilos en gauche, respecto al enlace $\mathrm{P}-\mathrm{O}$, de manera que los dos confórmeros de menor energía posicionan a ambos metilos en dicha situación. La superficie de energías rotacionales para ambos ángulos $\psi_{1}$ y $\psi_{2}$, presenta una complejidad mayor que en el caso de los calcógenos de trimetilo, pudiendo observarse la ubicación relativa de cada uno de los mínimos propuestos.

Según nuestros cálculos, la rotación del ángulo diedro $\psi_{\mathrm{N}}$ presenta una barrera energética de $4 \mathrm{kcal} / \mathrm{mol}$, lo que corrobora una rigidez importante (Figura 6.2). Sin embargo, se observan dos mínimos conformacionales en $0^{\circ}$ y en alrededor de $200^{\circ}$, que entre sí no presentarían diferencias de energía de más de $1 \mathrm{kcal} / \mathrm{mol}$. Dicha diferencia indica que, a temperatura ambiente, ambas posiciones resultan probables. Por otro lado, en estos mismos cálculos y para las estructuras más estables, el hidrógeno del grupo amino se ubica, respecto al oxígeno del grupo fosforilo, a una distancia de $2,7 \AA$,

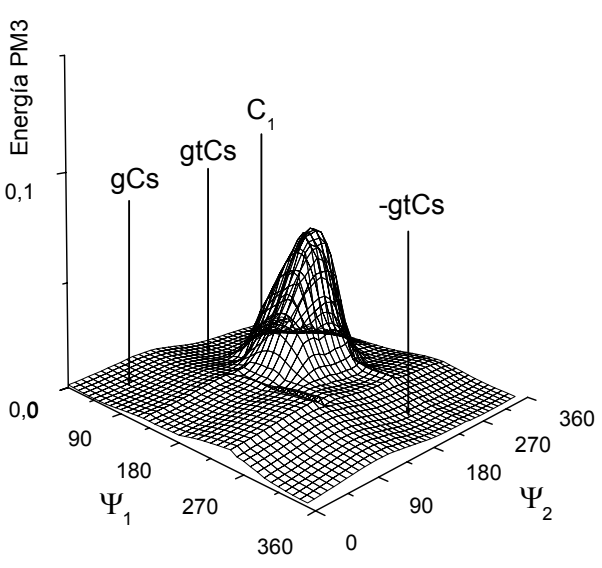

Figura 6.3: Superficie de energía potencial de rotación de los ángulos diedros $\psi 1$ y $\psi 2$, calculada a un nivel HF/PM3. Las flechas indican la ubicación de los confórmeros de mínima energía propuestos. 


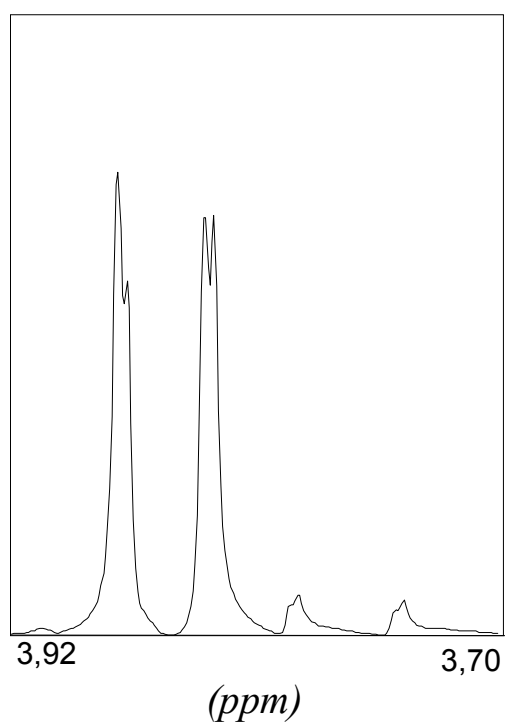

Figura 6.4: Espectro ${ }^{1} \mathrm{H}$ RMN del DMAP. Se observan dos dobletes análogos a los observados para el TMP. compatible con la presencia de un puente de hidrógeno y siempre a una distancia menor que la de los hidrógenos metílicos.

\section{Caracterización espectroscópica}

En general se disponen de escasos reportes espectroscópicos del DMAP, de manera que durante el desarrollo de este Capítulo, se incluyen la mayor parte de los datos obtenidos por medidas propias, aunque no sean importantes desde el punto de vista de la descripción conformacional de la molécula. Por la insolubilidad de esta sustancia en solventes orgánicos, las medidas de RMN se realizan en $\mathrm{D}_{2} \mathrm{O}$, a diferencia de lo realizado sobre las restantes sustancias. Esto dificulta la calibración adecuada de los espectros ${ }^{13} \mathrm{C}$ RMN.

En el espectro de ${ }^{1} \mathrm{H}$ NMR se observa claramente una estructura compleja de dobletes acoplados con dos valores diferenciados de acoplamiento espín-espín (Tabla 6.IV) que representan a dos picos independientes con una relación de 1:10 (Figura 6.4). Dichas señales corresponden a cada uno de ambos metilos. Es necesario tomar en cuenta el hecho que la medida se realiza en $\mathrm{D}_{2} \mathrm{O}$, y por lo tanto la señal correspondiente al $\mathrm{NH}_{2}$ desaparece por el rápido intercambio de dichos protones con el deuterio del solvente.

En el espectro ${ }^{13} \mathrm{C}$ RMN se observa la estructura esperada del doblete correspondiente a los carbonos metílicos con el correspondiente acoplamiento ${ }^{2} J_{\mathrm{C}-\mathrm{P}}$, como se puede apreciar en la Figura 6.5.

Tabla 6.IV: Valores de corrimiento químico (en ppm) y de acoplamientos espín-espín (en $\mathrm{Hz}$ ) para la molécula DMAP en los espectros ${ }^{1} \mathrm{H}$ RMN (calibrado respecto a la señal del HDO: 4,82 ppm) y ${ }^{13} \mathrm{C}$ RMN. Entre paréntesis se indica la relación de área de los respectivos picos. El valor reportado para las señales ${ }^{1} \mathrm{H}$ RMN es de $3,54 \mathrm{ppm}^{(1)}$.

\begin{tabular}{ccccc}
\hline \multicolumn{2}{c}{ ppm } & $\boldsymbol{J}_{\mathrm{H}}$ & ${ }^{3} \boldsymbol{J}_{\mathrm{P}-\mathrm{H}}$ & ${ }^{2} \boldsymbol{J}_{\mathrm{C}-\mathrm{P}}$ \\
\hline${ }^{1} \mathrm{H}$ RMN & $3,86(1,00)$ & 0,7 & 11,7 & \\
& $3,76(0,12)$ & 0,6 & 13,5 & 6,7 \\
\hline${ }^{13} \mathrm{C}$ RMN & 53,8 & & & \\
\hline
\end{tabular}

\section{Correlación de datos}

En los espectros infrarrojos de la fase líquida, es posible asignarlos, con cierta precisión, a los modos vibracionales correspondientes. En dicha asignación, aparecen grupos de bandas que pueden originarse en la coexistencia conformacional según las diferencias que 
arrojan los cálculos teóricos. Estos datos se resumen en las Tablas 6.I, 6.III y 6.V.

Mediante consideraciones teóricas, se puede predecir un desdoblamiento del modo vibracional de aleteo $\mathrm{PNH}_{2}$, en valores de alrededor de los $300 \mathrm{~cm}^{-1}$. Efectivamente, se predice una diferencia de $20 \mathrm{~cm}^{-1}$ para los confórmeros $\mathrm{gC}_{1} \mathrm{y} \mathrm{Cs}$, correspondiendo la banda de menor energía al confórmero más estable. De la misma manera, los modos vibracionales de balanceo antisimétrico de los grupos $\mathrm{PO}_{2}$ y $\mathrm{PO}_{2} \mathrm{~N}$, aparecen desdoblados para los cuatro confórmeros propuestos.

A la luz de lo expuesto, podemos afirmar que los resultados experimentales y teóricos son consistentes en el sentido de entregar fuerte evidencia de coexistencia de múltiples confórmeros a temperatura ambiente.

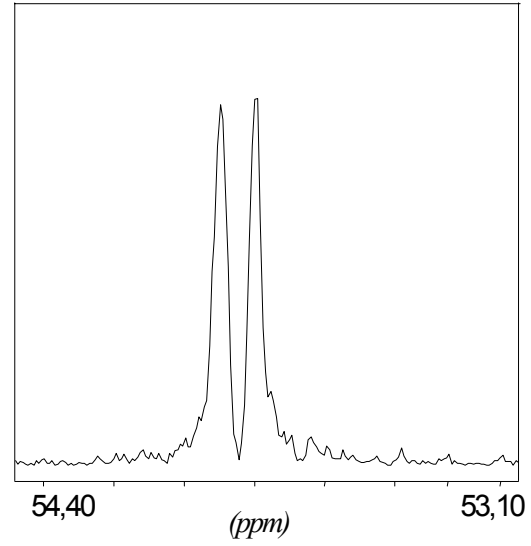

Figura 6.5: Espectro ${ }^{13} \mathrm{C}$ RMN del DMAP. Se observa el doblete análogo al observado para el TMP.

Tabla 6.V: Valores teóricos de las frecuencias obtenidas para cada uno de los cuatro confórmeros estables calculados a nivel $\mathrm{HF} / 6-31 \mathrm{G}^{* *}$ en la zona del infrarrojo cercano de entre 50 y $400 \mathrm{~cm}^{-1}$ para la molécula DMAP. Se proponen las asignaciones correspondientes. Entre paréntesis, se indican las intensidades teóricas relativas.

\begin{tabular}{|c|c|c|c|c|}
\hline \multirow[t]{2}{*}{ Asignación } & \multicolumn{4}{|c|}{ Calculado(HF/6-31G**) } \\
\hline & $\mathrm{gC}_{1}$ & Cs & $-\mathrm{gtC}_{1}$ & $\mathrm{gtC}_{1}$ \\
\hline \multirow[t]{3}{*}{$w \mathrm{P}\left(\mathrm{NH}_{2}\right)\left(\mathrm{OCH}_{3}\right)_{2}$} & $379(0,01)$ & & & \\
\hline & & $364(0,15)$ & & \\
\hline & & & $356(0,06)$ & \\
\hline & $314(0,00)$ & & & \\
\hline \multirow[t]{2}{*}{$w$ P-NH ${ }_{2}$} & & & $304(0,06)$ & $304(0,07)$ \\
\hline & & $295(0,11)$ & & \\
\hline & & $233(0,02)$ & & $236(0,00)$ \\
\hline \multirow[t]{2}{*}{$\operatorname{prs} \mathrm{PO}_{2} \mathrm{~N}$} & $221(0,04)$ & & & \\
\hline & & & $211(0,01)$ & \\
\hline \multirow{2}{*}{$w{\mathrm{P}-\mathrm{NH}_{2}}_{2}$} & & $215(0,02)$ & & \\
\hline & $208(0,01)$ & & $205(0,02)$ & $205(0,05)$ \\
\hline \multirow[t]{3}{*}{ pra $\mathrm{PO}_{2} \mathrm{~N}$} & & & & $167(0,00)$ \\
\hline & $170(0,04)$ & $159(0,01)$ & & \\
\hline & & & $151(0,09)$ & \\
\hline \multirow{6}{*}{ prs $\mathrm{PO}_{2}$} & & & & $130(0,00)$ \\
\hline & & & $123(0,02)$ & \\
\hline & $115(0,01)$ & $111(0,00)$ & & \\
\hline & $107(0,00)$ & $108(0,00)$ & & $109(0,01)$ \\
\hline & & & $94(0,00)$ & \\
\hline & $86(0,01)$ & & & \\
\hline \multirow{3}{*}{ pra $\mathrm{PO}_{2}$} & & $79(0,01)$ & & $81(0,01)$ \\
\hline & $65(0,01)$ & & $67(0,00)$ & \\
\hline & & $55(0,01)$ & $57(0,01)$ & $56(0,01)$ \\
\hline
\end{tabular}

¿a: vibración de deformación antisimétrica; prs: vibración de balanceo simétrico; pra: vibración de balanceo antisimétrico; sh: hombro. 


\section{La molécula de fosfamidotioato de dimetilo}

El fosfamidotioato de dimetilo (DMATP) corresponde a un isómero de función del metamidofós o fosfamidotionato de O,S-dimetilo, un insecticida usado ampliamente en muchos cultivos intensivos y del que se reportan anualmente numerosas intoxicaciones. El DMATP aparece como impureza en las formulaciones de grado técnico del metamidofós. Como ya se indicó en el Capítulo 6, un componente importante del desarrollo de una intoxicación por formulaciones de pesticidas puede ser responsabilidad de las impurezas que se encuentran presentes en ella.

Desde el punto de vista estructural, esta molécula es parte de la serie de calcogenofosfamidatos de dimetilo, debiéndose encontrar un comportamiento similar al descrito para el DMAP, con las particulares características aportadas por la presencia del grupo $\mathrm{P}=\mathrm{S}$.

Entre otras diferencias con su análogo oxigenado, la capacidad de formación de puentes de hidrógeno entre los nitrógenos amídicos y los pares libres del azufre estaría disminuida, fundamentalmente por la menor polarización del enlace $\mathrm{P}=\mathrm{S}$ y la mayor deslocalización de las nubes electrónicas de los pares libres del azufre. Ya hemos mencionado que uno de los aspectos particularmente interesantes de estas moléculas se relaciona con las<smiles>COP(N)(=S)OC</smiles>

Figura 7.1: Estructura de la fosfatotioamida de $\mathrm{O}, \mathrm{O}-$ dimetilo (DMATP). propiedades del grupo amido fosfórico, como la capacidad de sus protones para formar puentes de hidrógeno ${ }^{(1)}$.

Dado que ciertas características espectroscópicas, de reactividad y conformacionales están determinadas por la formación preferencial de puentes de hidrógeno, el análisis de estas interacciones intramoleculares debería poder integrarse a la descripción del comportamiento estructural de esta molécula como componente fundamental. Por otro lado, también se ha indicado que la capacidad de formación de

\footnotetext{
${ }^{1}$ Solov'ev, V.N., Martynov, I.V. and Zabirov, N.G. Crystal and molecular structure of two insecticides: O,S-dimethyl amidothiophosphate and O,Sdimethyl acetamidothiophosphate, Phosphorus, Sulfur Silicon.Relat.Elem. 57 (1-2): 135-141 (1991).
} 
puentes de hidrógeno intermoleculares, determina las características particulares de reactividad biológica en los fosfamidatos, ${ }^{(2)}$ de manera que este aspecto estructural sería importante no sólo como descriptor molecular sino como índice de actividad tóxica.

Por extensión a lo que ya se ha dicho respecto a los fosfamidatos, ${ }^{(3)}$ las nubes electrónicas de los enlaces $\mathrm{P}=\mathrm{S}$ y $\mathrm{P}-\mathrm{N}$ deberían solapar apreciablemente. Este dato, complementario a las interacciones por puentes de hidrógeno intramoleculares, restringe apreciablemente la variabilidad del ángulo $\psi_{\mathrm{N}}$. de manera que el análisis conformacional de la molécula dependería fundamentalmente de los ángulos diedros $\psi_{1}$ y $\psi_{2}$ de rotación de los enlaces $\mathrm{P}-\mathrm{O}(-\mathrm{C})$. Nuestros cálculos teóricos sobre las variantes conformacionales, dan cuenta de cuatro confórmeros estables denominados gCs, $\mathrm{C}_{1}$, -gtCs y gtCs (Tabla 7.II). Los dos primeros presentan ambos metilos en posición gauche respecto al enlace $\mathrm{P}=\mathrm{S}$ y corresponden a aquellos confórmeros que se presentarían con abundancia apreciable a temperatura ambiente. Esta situación es análoga a la que se presenta para la molécula DMAP.

En general se disponen de escasos datos espectroscópicos del DMATP, de manera que durante el desarrollo de este Capítulo, se incluirán la mayor parte de los datos obtenidos por medidas propias. En ellos se observa una buena correlación entre las bandas medidas en FTIR y las obtenidas teóricamente para las variantes conformacionales del DMATP. Las frecuencias teóricas, cada vez que fue posible, se utilizaron para la asignación de la mayor parte de los modos vibracionales (Tabla 7.III, 7.IV y 7.V).

\section{Caracterización espectroscópica}

Las medidas observadas mediante RMN dan cuenta de una estructura simple en las señales, tanto de los hidrógenos como de los carbonos (Figuras 7.2 y 7.3). Esto indicaría una restricción conformacional que, en los términos de la discusión desarrollada para los calcogenofosfatos de trimetilo, sería de magnitud suficiente como para que la coexistencia conformacional no pueda ser manifestada en las señales nucleares, como si ocurre de alguna manera en el
Tabla 7.I: Corrimientos químicos y constantes de acoplamiento para el DMATP en los espectros de ${ }^{1} \mathrm{H}$ RMN en $\mathrm{CDCl}_{3}$ (calibración respecto a TMS) y en $\mathrm{D}_{2} \mathrm{O}$ (calibración respecto a DHO 4,82 ppm), y ${ }^{13} \mathrm{C}$ RMN en $\mathrm{CDCl}_{3}$ (calibración respecto a cloroformo $77,00 \mathrm{ppm}$ ) y $\mathrm{D}_{2} \mathrm{O}$ (sin calibración interna).

\begin{tabular}{|c|c|}
\hline Núcleo & $\delta \mathrm{ppm}$ \\
\hline & ${ }^{1} \mathrm{HRMN}$ \\
\hline \multirow[t]{2}{*}{$\mathrm{CH}_{3}$} & $\begin{array}{l}3,73 \text { en } \mathrm{CDCl}_{3} \\
\left({ }^{3} J_{\mathrm{H}-\mathrm{P}}=13,8 \mathrm{~Hz}\right) \\
\left(J_{\mathrm{H}}=2.2 \mathrm{~Hz}\right)\end{array}$ \\
\hline & $\begin{array}{l}3,81 \text { en } D_{2} \mathrm{O} \\
\left({ }^{3} J_{\mathrm{H}-\mathrm{P}}=14,4 \mathrm{~Hz}\right) \\
\left(J_{\mathrm{H}}=1,5 \mathrm{~Hz}\right)\end{array}$ \\
\hline \multirow[t]{2}{*}{$\mathrm{NH}_{2}$} & 3,31 \\
\hline & ${ }^{13} \mathrm{C}$ RMN \\
\hline \multirow[t]{2}{*}{$\mathrm{CH}_{3}$} & $\begin{array}{l}53,4 \text { en } \mathrm{CDCl}_{3} \\
\left({ }^{2} J_{\text {C-P }}=4,8 \mathrm{~Hz}\right)\end{array}$ \\
\hline & $\begin{array}{l}53,8 \text { en } D_{2} \mathrm{O} \\
\left({ }^{2} J_{\text {C-P }}=6,7 \mathrm{~Hz}\right)\end{array}$ \\
\hline
\end{tabular}

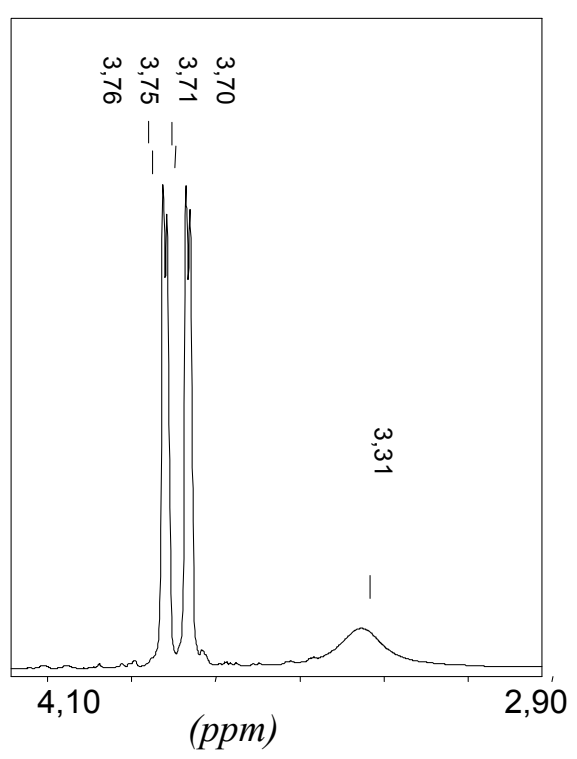

Figura 7.2: Señales ${ }^{1} \mathrm{H}$ RMN del DMATP en $\mathrm{CDCl}_{3} \mathrm{Como}$ solvente .

\footnotetext{
2 Singh, A.K. Quantitative structure-activity relationships for phosphoramidothioate toxicity in housefly, Comp. Biochem. Physiol.C. Pharmacol. Toxicol. Endocrinol. 123 (3): 241-255 (1999).

${ }^{3}$ Pen'kovskii, V.V. and Boldeskul, I.E. Quantum-chemical study of dimethyl phosphoramidates, Teor.Eksp.Khim. 20 (2): 198-202 (1984).
} 


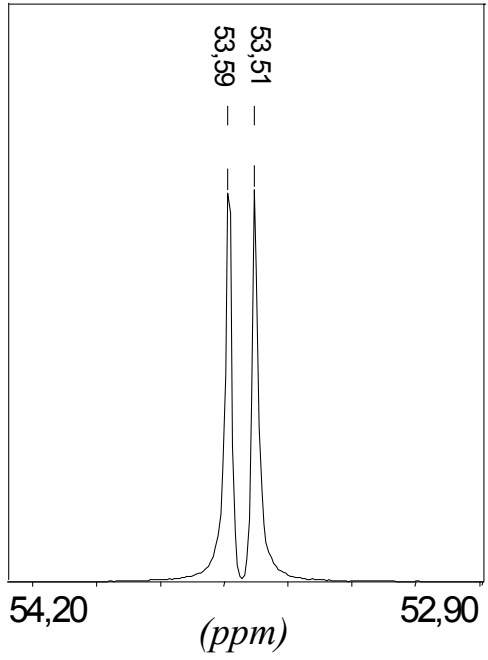

Figura 7.3: Doblete metílico en el espectro ${ }^{13} \mathrm{C}$ RMN del DMATP en $\mathrm{CDCl}_{3}$.
TMP y el TMSP. Los valores de corrimiento químico y constantes de acoplamiento obtenidos en nuestras medidas se indican en la Tabla 7.I.

Considerando los espectros FTIR y comparando las bandas obtenidas con las frecuencias predichas por los cálculos teóricos, es posible discriminar aquellos modos vibracionales en los cuales pueda expresarse coexistencia de confórmeros. Esta discriminación dependerá de que exista suficiente diferencia de las frecuencias predichas para los confórmeros más abundantes en un determinado modo vibracional. Esta característica se verifica en los modos vibracionales de estiramiento antisimétrico $\mathrm{NH}_{2}$, de deformación antisimétrica $\mathrm{NH}_{2}$, de estiramiento simétrico y antisimétrico $\mathrm{P}-\mathrm{O}(-\mathrm{C})$, de estiramiento $\mathrm{P}=\mathrm{S}$ y de aleteo $\mathrm{NH}_{2}$.

La coexpresión de confórmeros en los modos vibracionales asociados al estiramiento de los enlaces $\mathrm{P}-\mathrm{O}(-\mathrm{C})$ y de estiramiento $\mathrm{P}=\mathrm{S}$ es una característica que también se verificó en los calcogenofosfatos de trimetilo. Ambos modos vibracionales están ligados directamente a las características estructurales diferenciales de cada uno de los confórmeros posibles.

Tabla 7.II: Parámetros geométricos y fisicoquímicos del DMATP obtenidos a nivel HF/6-31G** para cada uno de los cuatro confórmeros de mínima energía hallados. Los ángulos se expresan en grados.

\begin{tabular}{|c|c|c|c|c|}
\hline & \multicolumn{4}{|c|}{ DMATP } \\
\hline & gCs (I) & $\mathrm{C}_{1}(\mathrm{II})$ & -gtCs (III) & gtCs (IV) \\
\hline$\psi_{1}$ & 51 & 53 & -50 & 51 \\
\hline$\psi_{2}$ & 59 & -48 & 186 & 170 \\
\hline$P=S(\AA)$ & 1,94 & 1,94 & 1,93 & 1,93 \\
\hline $\mathrm{P}-\mathrm{O}_{1}(\AA)$ & 1,59 & 1,58 & 1,59 & 1,59 \\
\hline $\mathrm{P}-\mathrm{O}_{2}(\AA)$ & 1,58 & 1,58 & 1,59 & 1,58 \\
\hline $\mathrm{HF} / 6-31 \mathrm{G}^{* *}(\mathrm{kcal} / \mathrm{mol})$ & 0,00 & 1,99 & 3,36 & 4,36 \\
\hline$\mu \mathrm{D}$ (Müll)Calc. & 3,93 & 4,41 & 5,33 & 6,36 \\
\hline Prop.a $298^{\circ} \mathrm{K}$ (peso 2:1:2:2) & 0,98 & 0,02 & 0,00 & 0,00 \\
\hline $\mu \mathrm{D}$ (Müll)Pond. & \multicolumn{4}{|c|}{3,95} \\
\hline Prop.a $523^{\circ} \mathrm{K}$ (peso 2:1:2:2) & 0,89 & 0,07 & 0,04 & 0,01 \\
\hline $\mu \mathrm{D}$ (Müll)Pond. & \multicolumn{4}{|c|}{4,06} \\
\hline
\end{tabular}

Por otro lado, que existan tres modos vibracionales asociados al grupo $\mathrm{NH}_{2}$, indica la fuerte dependencia de las interacciones de este grupo con el resto de la molécula y las restricciones de interconversión conformacional.

La banda que aparece a $657 \mathrm{~cm}^{-1}$ y el hombro a 638 $\mathrm{cm}^{-1}$ se pueden asignar al estiramiento $\mathrm{P}=\mathrm{S}$. No es tan claro a cual de los confórmeros se asignaría cada una de estas frecuencias. De esta manera, aunque los cálculos entregan 
Tabla 7.III: Frecuencias medidas en los espectros infrarrojo en fase líquida e infrarrojo en matrices de $\operatorname{Ar}$ (depositadas a $293^{\circ} \mathrm{K}$ ) en la zona ubicada entre 1300 y $4000 \mathrm{~cm}^{-1}$. Las asignaciones propuestas se realizan en función de los valores teóricos obtenidos para cada uno de los cuatro confórmeros estables calculados a nivel HF/6-31G**.

\begin{tabular}{|c|c|c|c|c|c|c|}
\hline \multirow[t]{2}{*}{ Asignación } & \multirow[t]{2}{*}{$I R$} & \multirow[t]{2}{*}{ IR matrices } & \multicolumn{4}{|c|}{ Calculado(HF/6-31G**) } \\
\hline & & & $g_{1}$ & $\mathrm{Cs}$ & $-\mathrm{gtC}_{1}$ & $\mathrm{gtC}_{1}$ \\
\hline \multirow[t]{2}{*}{ va $\mathrm{NH}_{2} \mathrm{gC}_{1}$} & 3579sh & $3727(0,31)$ & & $3517(0,47)$ & & \\
\hline & & & & & & $3496(0,12)$ \\
\hline \multirow[t]{2}{*}{$v a \mathrm{NH}_{2} \mathrm{Cs}$} & $3419(0,70)$ & $3711(0,51)$ & $3486(0,12)$ & & $3477(0,14)$ & \\
\hline & & & & $3398(0,04)$ & & \\
\hline \multirow[t]{9}{*}{$v s \mathrm{NH}_{2}$} & $3326(0,72)$ & $3607(0,26)$ & & & & $3386(0,10)$ \\
\hline & & & $3378(0,12)$ & & $3379(0,14)$ & \\
\hline & & $3264(0,07)$ & & & & \\
\hline & & $3229(0,04)$ & & & & \\
\hline & & $3203(0,07)$ & & & & \\
\hline & $3084(0,37)$ & $3073(0,05)$ & & & & \\
\hline & & & & $2960(0,03)$ & $2960(0,04)$ & $2962(0,04)$ \\
\hline & $2997(0,43)$ & & $2957(0,05)$ & & & \\
\hline & & & & $2958(0,03)$ & $2958(0,05)$ & \\
\hline \multirow[t]{11}{*}{ va $\mathrm{CH}_{3}$} & $2949(0,58)$ & $2967(0,09)$ & $2956(0,05)$ & & & $2957(0,05)$ \\
\hline & $2904(0,28)$ & & $2944(0,04)$ & & & \\
\hline & & & $2941(0,04)$ & & $2941(0,04)$ & $2943(0,05)$ \\
\hline & $2845(0,43)$ & & & $2939(0,03)$ & & \\
\hline & & & & & $2936(0,08)$ & \\
\hline & & & & $2932(0,04)$ & & $2928(0,07)$ \\
\hline & $2200(0,03)$ & $2352(0,08)$ & $2866(0,06)$ & & & $2865(0,08)$ \\
\hline & & $2348(0,08)$ & & & & \\
\hline & & $2347(0,08)$ & & & & \\
\hline & & $2332(0,12)$ & & & & \\
\hline & $2120(0,04)$ & $2328(0,47)$ & $2864(0,08)$ & & $2864(0,08)$ & \\
\hline \multirow[t]{8}{*}{ vs $\mathrm{CH}_{3}$} & $2038(0,07)$ & $2283(0,40)$ & & $2863(0,04)$ & $2862(0,09)$ & \\
\hline & $1960(0,07)$ & $2234(0,05)$ & & $2856(0,06)$ & & \\
\hline & & $2140(0,05)$ & & & & \\
\hline & & $2053(0,05)$ & & & & \\
\hline & $1876(0,13)$ & $1874(0,53)$ & & & & \\
\hline & & $1870(1,00)$ & & & & \\
\hline & & $1835(0,08)$ & & & & $2850(0,11)$ \\
\hline & $1626(0,18)$ & $1616(0,55)$ & & & $1547(0,21)$ & \\
\hline \multirow[t]{4}{*}{$\delta \mathrm{a} \mathrm{NH}_{2}$} & $1548(0,58)$ & $1598(0,17)$ & & & & $1541(0,18)$ \\
\hline & & $1550(0,33)$ & $1543(0,16)$ & $1534(0,11)$ & & \\
\hline & $1461(0,45)$ & $1461(0,06)$ & $1459(0,01)$ & $1458(0,01)$ & $1461(0,01)$ & $1459(0,02)$ \\
\hline & 1443sh & $1446(0,09)$ & $1458(0,01)$ & $1457(0,01)$ & $1457(0,02)$ & $1457(0,01)$ \\
\hline \multirow[t]{4}{*}{$\delta \mathrm{a} \mathrm{CH}_{3}$} & & & $1451(0,01)$ & $1452(0,01)$ & $1451(0,01)$ & $1454(0,01)$ \\
\hline & & & $1450(0,01)$ & $1451(0,00)$ & $1449(0,01)$ & $1450(0,01)$ \\
\hline & $1393(0,18)$ & $1351(0,33)$ & $1437(0,00)$ & $1440(0,00)$ & $1438(0,00)$ & $1438(0,00)$ \\
\hline & $1310(0,13)$ & & $1436(0,00)$ & $1436(0,00)$ & $1434(0,00)$ & $1436(0,00)$ \\
\hline
\end{tabular}

vs: vibración de estiramiento simétrico; va: vibración de estiramiento antisimétrico; $\delta$ a: vibración de deformación antisimétrica; sh: hombro. Entre paréntesis se indican las absorbancias relativas a la banda de mayor intensidad.

frecuencias más altas para el confórmero más estable, la banda más intensa es la que aparece a $657 \mathrm{~cm}^{-1}$.

Según los valores de las intensidades teóricas relativas obtenidas para el modo vibracional de estiramiento $\mathrm{P}=\mathrm{S}$, se observa que para los confórmeros menos estables se presentan intensidades relativas más de tres veces mayores a las formas más estables. Esto supone dos hechos: por un lado 


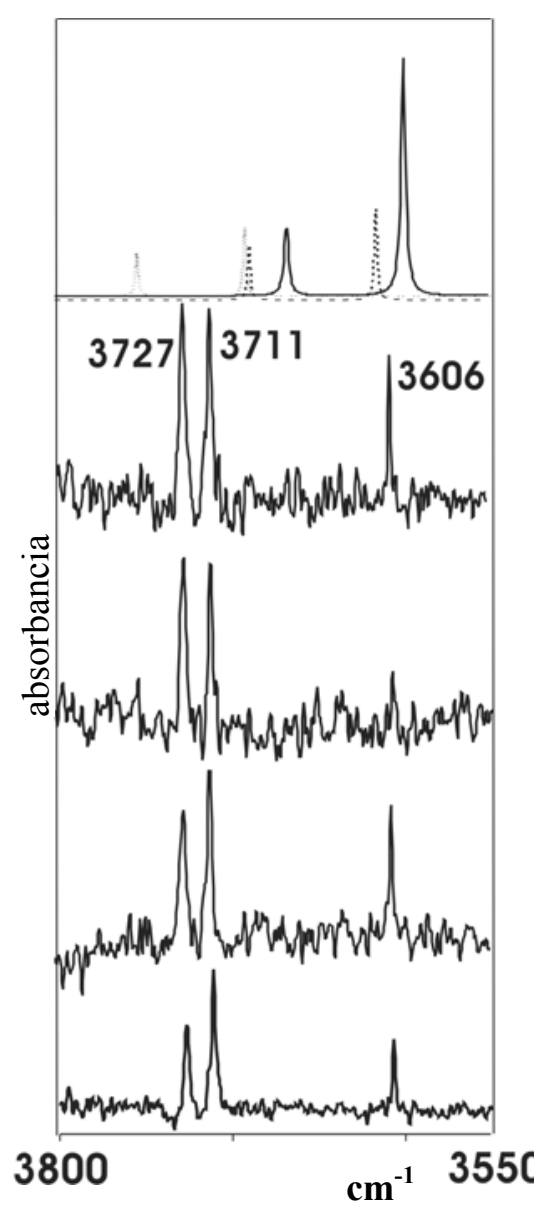

Figura 7.4: Espectros en matrices de Ar para el DMATP en la zona $3700 \mathrm{~cm}^{-1}$. Desde arriba hacia abajo se observan los espectros teóricos (línea continua para el confórmero más estable) y las medidas a $523 \mathrm{~K}, 453 \mathrm{~K}, 358 \mathrm{~K}$ y $283 \mathrm{~K}$ que estas intensidades relativas sustentan la asignación de una banda aparecida en el espectro infrarrojo a confórmeros que se encuentran en una abundancia teórica muy baja. Por otro lado, estos valores de intensidad son una indicación teórica de la gran afectación del grupo $\mathrm{P}=\mathrm{S}$ por parte de ambos metilos en posición gauche, que es lo que ocurre en ambos confórmeros de menor energía relativa.

Para el estiramiento $\mathrm{P}-\mathrm{O}(-\mathrm{C})$ se observan tres bandas bien definidas a 813, 787 y $745 \mathrm{~cm}^{-1}$. Por comparación con los valores teóricos para esta región, es posible proponer que la banda a $787 \mathrm{~cm}^{-1}$ pueda corresponder a una superposición de los modos vibracionales de estiramiento antisimétrico del confórmero Cs y de estiramiento simétrico del confórmero $-\mathrm{gtC}_{1}$. Mientras que las bandas a 813 y $745 \mathrm{~cm}^{-1}$ corresponderían a modos vibracionales puros de estiramiento $\mathrm{P}-\mathrm{O}(-\mathrm{C})$ antisimétrico y simétrico respectivamente.

En la zona de estiramiento antisimétrico $\mathrm{NH}_{2}$, se observa una banda principal a $3419 \mathrm{~cm}^{-1}$ y un hombro a 3579 $\mathrm{cm}^{-1}$. En esta zona del espectro no hay una resolución adecuada de las bandas lo que no permite una asignación segura de confórmeros. Sin embargo, la banda a $3419 \mathrm{~cm}^{-1}$ sería una expresión del confórmero Cs y el hombro a 3579 $\mathrm{cm}^{-1}$ debería asignarse al confórmero $\mathrm{gC}_{1}$.

Para el modo vibracional de deformación antisimétrica $\mathrm{NH}_{2}$ se observan dos bandas a $1548 \mathrm{~cm}^{-1}$ y a $1626 \mathrm{~cm}^{-1}$. La segunda de estas bandas podría asignarse a la expresión de confórmeros menos abundantes. Esta asignación no es clara pues no hay buena correlación con los valores teóricos predichos para estos confórmeros.

En la zona del espectro asignada el modo de aleteo $\mathrm{NH}_{2}$, se observan dos señales a 603 y $576 \mathrm{~cm}^{-1}$, asignándose la primera de ellas a una manifestación individual del confórmero $\mathrm{gC}_{1}$ y la segunda a una manifestación del confórmero Cs. Esta asignación presenta el inconveniente que la banda más intensa se asigna a un confórmero menos abundante. Pero observando las intensidades relativas en las predicciones teóricas vemos que el confórmero más abundante presenta una intensidad más de diez veces menor que el confórmero $\mathrm{gC}_{1}$. Esto aportaría a la justificación de la asignación propuesta. 
Capítulo 7: La molécula de fosfamidotioato de dimetilo

Tabla 7.IV: Frecuencias medidas en los espectros infrarrojo en fase líquida e infrarrojo en matrices de $\operatorname{Ar}\left(\right.$ depositadas a $293^{\circ} \mathrm{K}$ ) en la zona ubicada entre 500 y $1300 \mathrm{~cm}^{-1}$. Las asignaciones propuestas se realizan en función de los valores teóricos obtenidos para cada uno de los cuatro confórmeros estables calculados a nivel HF/6-31G**.

\begin{tabular}{|c|c|c|c|c|c|c|}
\hline \multirow[t]{2}{*}{ Asignación } & \multirow[t]{2}{*}{$I R$} & \multirow[t]{2}{*}{ IR matrices } & \multicolumn{4}{|c|}{ Calculado(HF/6-31G**) } \\
\hline & & & $\mathrm{gC}_{1}$ & Cs & $-\mathrm{gtC}_{1}$ & $\mathrm{gtC}_{1}$ \\
\hline & & $1279(0,10)$ & & & & \\
\hline & & $1263(0,05)$ & & & & \\
\hline & $1228(0,34)$ & $1242(0,14)$ & & & & \\
\hline & & $1228(0,04)$ & & & & \\
\hline & & $1203(0,08)$ & & & & \\
\hline \multirow{4}{*}{ pra $\mathrm{CH}_{3}$} & & & $1173(0,06)$ & $1175(0,03)$ & $1171(0,07)$ & $1172(0,07)$ \\
\hline & & & & & & \\
\hline & $1181(0,44)$ & $1183(0,05)$ & $1172(0,06)$ & $1171(0,07)$ & & $1170(0,08)$ \\
\hline & & & & & $1167(0,07)$ & \\
\hline \multirow[t]{2}{*}{$w \mathrm{CH}_{3}$} & $1153 \mathrm{sh}$ & $1156(0,08)$ & $1148(0,01)$ & $1149(0,01)$ & $1147(0,01)$ & $1148(0,01)$ \\
\hline & & $1131(0,10)$ & $1147(0,00)$ & $1147(0,00)$ & $1145(0,01)$ & $1143(0,01)$ \\
\hline \multirow{3}{*}{ vs O-C } & & & & $1097(0,01)$ & & \\
\hline & $1060(0,87)$ & $1083(0,08)$ & $1075(0,35)$ & & & $1073(0,79)$ \\
\hline & & & & & $1064(0,72)$ & \\
\hline \multirow{4}{*}{ va O-C } & & & & & & $1058(1,00)$ \\
\hline & $1027(1,00)$ & $1051(0,32)$ & & & $1054(1,00)$ & \\
\hline & & & $1049(1,00)$ & & & \\
\hline & & & & $1043(1,00)$ & & \\
\hline \multirow{2}{*}{ pra $\mathrm{NH}_{2}$} & $987(0,63)$ & $953(0,05)$ & $984(0,31)$ & & $988(0,30)$ & \\
\hline & & & & $957(0,26)$ & & $964(0,24)$ \\
\hline \multirow{3}{*}{$v \mathrm{P}-\mathrm{N}$} & & & $880(0,26)$ & & & \\
\hline & $916(0,51)$ & $903(0,10)$ & & $868(0,23)$ & $863(0,28)$ & \\
\hline & & & & & & $857(0,33)$ \\
\hline va $\mathrm{P}-\mathrm{O}$ & $813(0,83)$ & $861(0,10)$ & $796(0,30)$ & $795(0,10)$ & & \\
\hline \multirow[b]{2}{*}{$v a \mathrm{Cs}+v \mathrm{~s}-\mathrm{gtC}_{1}$} & & $841(0,06)$ & & & & $784(0,31)$ \\
\hline & 787sh & & & $781(0,24)$ & $779(0,38)$ & \\
\hline \multirow[t]{2}{*}{ vs $\mathrm{P}-\mathrm{O}$} & $745(0,32)$ & & $768(0,35)$ & & & $768(0,31)$ \\
\hline & & & & & $759(0,34)$ & \\
\hline \multirow[t]{2}{*}{$v \mathrm{P}=\mathrm{S} \mathrm{gC}_{1}+\mathrm{Cs}$} & $657(0,37)$ & $699(0,11)$ & & & $610(0,29)$ & \\
\hline & & & & & & $588(0,12)$ \\
\hline \multirow[t]{2}{*}{$v \mathrm{P}=\mathrm{S}-\mathrm{gtC}_{1}$} & 638 sh & & $570(0,08)$ & $571(0,06)$ & & \\
\hline & & & & & $552(0,35)$ & \\
\hline$w \mathrm{NH}_{2} \mathrm{gC}_{1}$ & $603(0,33)$ & & $503(0,48)$ & & & \\
\hline \multirow[t]{2}{*}{$w \mathrm{NH}_{2} \mathrm{Cs}$} & $576(0,43)$ & & & $467(0,04)$ & & $462(0,10)$ \\
\hline & & $529(0,39)$ & & & & \\
\hline
\end{tabular}

v: vibración de estiramiento; vs: vibración de estiramiento simétrico; va: vibración de estiramiento antisimétrico; pra: vibración de balanceo antisimétrico; w: vibración de aleteo; sh: hombro. Entre paréntesis se indican las absorbancias relativas a la banda de mavor intensidad.

Entonces, en estas cinco regiones se puede argumentar a favor de la manifestación de señales que indican la coexistencia de más de una forma conformacional, aunque en algunos casos no se pueda asignar con claridad cuál de éstas señales desdobladas están vinculadas a los confórmeros en particular. 


\section{Espectroscopía de alta resolución}

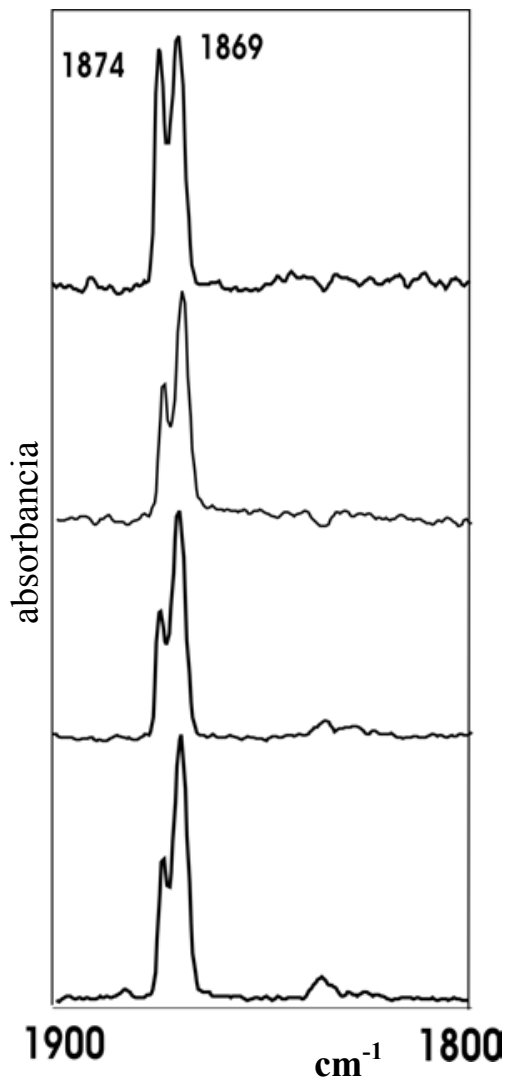

Figura 7.5: Espectros en matrices de Ar para el DMATP alrededor de 1850 $\mathrm{cm}^{-1}$. Desde arriba hacia abajo se observan las medidas a $523 \mathrm{~K}, 453 \mathrm{~K}, 358 \mathrm{~K}$ y $283 \mathrm{~K}$.
La observación de los espectros de matrices se realiza especialmente sobre las regiones ya enunciadas durante la descripción del espectro FTIR. Por otro lado, la mayor resolución del espectro permite resolver bandas que no aparecen como bandas individuales en las medidas de menor resolución. La discusión acerca del alcance de la técnica de deposición de la matriz a distintas temperaturas, ya fue expuesta en el capítulo dedicado al TMSeP.

Una de las dificultades de la obtención de estos espectros se relaciona con el hecho que, siendo esta sustancia de una volatilidad muy baja, existe dificultad para obtener espectros de calidad (ver Anexo I Metodologías). De manera que existirán regiones que presenten una relación señal / ruido muy baja, circunstancia que impide una correcta identificación de bandas o una adecuada asignación de intensidades, ambos datos necesarios para una comparación de medidas a distintas temperaturas. Por esta razón solamente es posible realizar un análisis preciso en regiones por encima de $800 \mathrm{~cm}^{-1}$, descartando modos vibracionales que, como se observó en los calcogenofosfatos de trimetilo, son interesantes para la búsqueda de manifestaciones de coexistencia de confórmeros. Por otro lado, esta dificultad también se traduce en una disminución de inteligibilidad del espectro expresado en transmitancias, como son usualmente reportadas este tipo de medidas, por lo tanto los resultados se mostrarán como una relación de absorbancias.

En la zona del modo vibracional de estiramiento $\mathrm{NH}_{2}$ (Figura 7.4) se pueden observar tres bandas muy definidas a 3727,3711 y $3606 \mathrm{~cm}^{-1}$. Se observa claramente que con el aumento de la temperatura hay un aumento de la intensidad relativa de la banda ubicada a menores frecuencias. Esta banda ubicada en $3727 \mathrm{~cm}^{-1}$, se corresponde con el hombro ubicado a $3579 \mathrm{~cm}^{-1}$ en el espectro FTIR medido para la fase líquida. En función del comportamiento observado en las medidas en matrices, se puede confirmar la asignación propuesta de correspondencia con una manifestación del modo de vibración de estiramiento antisimétrico $\mathrm{NH}_{2}$ para un confórmero de menor energía.

En la zona de modos vibracionales asociados a estiramientos de los metilos (Figura 7.5), se observa en los espectros de matrices de Ar, la resolución de dos bandas a 1869 y $1874 \mathrm{~cm}^{-1}$, correspondientes a la pequeña banda ubicada en $1879 \mathrm{~cm}^{-1}$ en el espectro FTIR de la fase líquida. 
Tabla 7.V: Valores teóricos de las frecuencias obtenidas para cada uno de los cuatro confórmeros estables calculados a nivel HF/6-31G** en la zona del infrarrojo cercano ubicada entre 50 y 460 $\mathrm{cm}^{-1}$. Se proponen las asignaciones correspondientes. Como referencia se informan los dos únicos valores de frecuencias medidas en el espectro vibracional IR en fase líquida ${ }^{(4)}$.

\begin{tabular}{|c|c|c|c|c|c|}
\hline \multirow{2}{*}{ Asignación } & \multirow[t]{2}{*}{$I R$} & \multicolumn{4}{|c|}{ Calculado(HF/6-31G**) } \\
\hline & & $\mathrm{gC}_{1}$ & Cs & $-\mathrm{gtC}_{1}$ & $\mathrm{gtC}_{1}$ \\
\hline & & $454(0,02)$ & & & \\
\hline \multirow{2}{*}{$\delta$ a $-\left(\mathrm{OCH}_{3}\right)_{2}$} & $492(0,42)$ & & & $447(0,04)$ & \\
\hline & & & & $424(0,01)$ & $427(0,21)$ \\
\hline \multirow[t]{3}{*}{$\delta \mathrm{a} P-\left(\mathrm{OCH}_{3}\right)_{2}$} & $413(0,65)$ & & & & $398(0,11)$ \\
\hline & & $395(0,07)$ & $393(0,05)$ & & \\
\hline & & $386(0,03)$ & $376(0,02)$ & & \\
\hline \multirow[t]{2}{*}{$w \mathrm{P}\left(\mathrm{NH}_{2}\right)\left(\mathrm{OCH}_{3}\right)_{2}$} & & & $371(0,01)$ & & \\
\hline & & & & $341(0,04)$ & $360(0,19)$ \\
\hline & & $330(0,01)$ & & & $332(0,04)$ \\
\hline \multirow{2}{*}{$w \mathrm{P}\left(\mathrm{NH}_{2}\right)\left(\mathrm{OCH}_{3}\right)_{2}$} & & & & $305(0,03)$ & \\
\hline & & & $286(0,07)$ & & \\
\hline \multirow[t]{3}{*}{$w \mathrm{P}^{-\mathrm{NH}_{2}}$} & & & & $269(0,02)$ & $273(0,02)$ \\
\hline & & $264(0,00)$ & & & \\
\hline & & & $249(0,16)$ & & \\
\hline \multirow[t]{2}{*}{ prs $\mathrm{PO}_{2} \mathrm{~N}$} & & $227(0,04)$ & & & $227(0,01)$ \\
\hline & & & $215(0,09)$ & & \\
\hline & & $203(0,00)$ & & & \\
\hline \multirow[t]{2}{*}{$w \mathrm{P}^{-\mathrm{NH}_{2}}$} & & & $188(0,00)$ & $195(0,01)$ & $183(0,02)$ \\
\hline & & & & $174(0,03)$ & \\
\hline \multirow[t]{3}{*}{ pra $\mathrm{PO}_{2} \mathrm{~N}$} & & & $159(0,01)$ & & $160(0,00)$ \\
\hline & & $154(0,02)$ & & $152(0,02)$ & \\
\hline & & $127(0,01)$ & & & $132(0,00)$ \\
\hline \multirow[t]{4}{*}{$\mathrm{rCH}_{3}$} & & & $122(0,00)$ & & \\
\hline & & & $119(0,00)$ & & $121(0,01)$ \\
\hline & & $115(0,00)$ & & $114(0,01)$ & \\
\hline & & & & $106(0,00)$ & \\
\hline \multirow{3}{*}{ wO- $\left(\mathrm{CH}_{3}\right)$} & & $88(0,01)$ & $81(0,00)$ & & $85(0,01)$ \\
\hline & & $70(0,01)$ & $68(0,01)$ & $73(0,00)$ & \\
\hline & & & & $53(0,02)$ & $52(0,01)$ \\
\hline
\end{tabular}

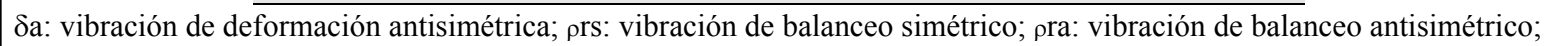
sh: hombro. Entre paréntesis se indican las absorbancias relativas a la banda de mayor intensidad.

Se observa un claro aumento relativo con la temperatura de la banda ubicada a frecuencias más bajas, asignándose entonces a esta la manifestación de un confórmero de menor energía.

En la zona de la deformación del grupo $\mathrm{NH}_{2}$ (Figura 7.6), se puede observar un grupo de bandas en las cuales hay un aumento relativo de intensidades para aquellas ubicadas a 1630,1616 y $1550 \mathrm{~cm}^{-1}$, y una disminución relativa de la banda ubicada a $1598 \mathrm{~cm}^{-1}$. La asignación más directa es indicar como manifestación del confórmero más estable a la banda a $1598 \mathrm{~cm}^{-1}$, aunque por las posiciones relativas de los valores teóricos, parece más adecuado suponer una superposición de modos vibracionales y de manifestación

\footnotetext{
${ }^{4}$ Las medidas de IR en matrices de Ar arrojan en esta zona una línea de base con mucho ruido, que impide discriminar claramente las bandas correspondientes.
} 


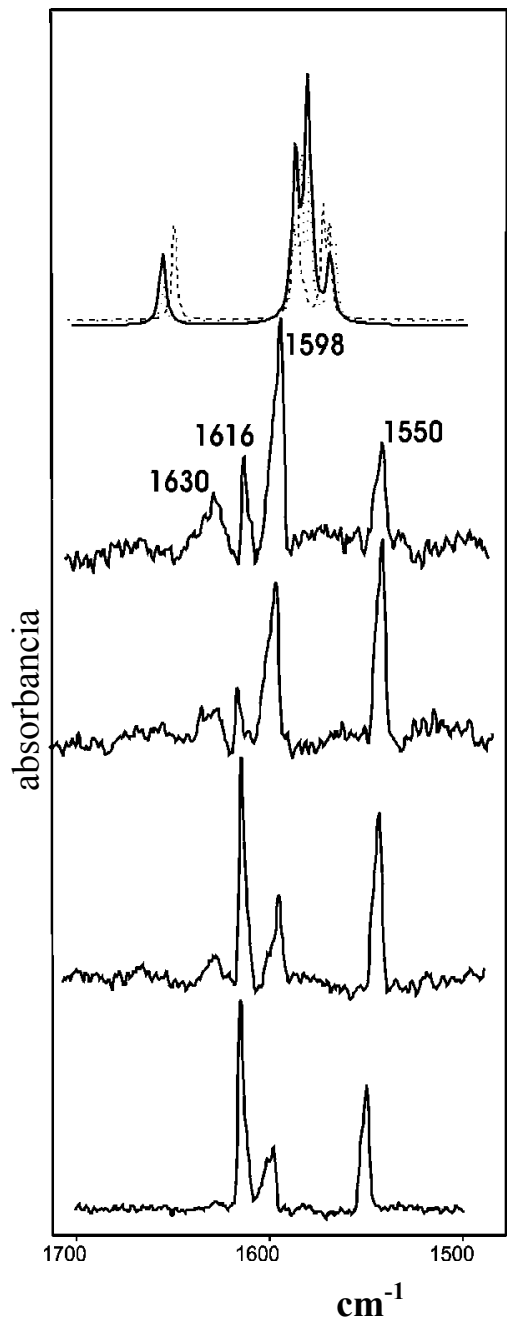

Figura 7.6: Espectros en matrices de Ar para el DMATP alrededor de $1600 \mathrm{~cm}^{-1}$. Desde arriba hacia abajo se observan los espectros teóricos (línea continua para el confórmero más estable) y las medidas a $523 \mathrm{~K}, 453 \mathrm{~K}, 358 \mathrm{~K}$ y $283 \mathrm{~K}$. conformacional, de manera que habría que asignar para la banda a $1598 \mathrm{~cm}^{-1}$, una componente mayoritaria del confórmero de mayor energía y para las otras tres bandas una componente mayoritaria de los confórmeros de menor energía.

En la zona del estiramiento $\mathrm{C}-\mathrm{O}$ (Figura 7.7), se observa un aumento relativo de dos bandas ubicadas a frecuencias más altas que la banda principal en $1051 \mathrm{~cm}^{-1}$. En función de este comportamiento y de las posiciones relativas de los valores teóricos se puede suponer que la banda a 1051 $\mathrm{cm}^{-1}$ tiene una componente principal en el estiramiento simétrico $\mathrm{C}-\mathrm{O}$ del confórmero de mayor energía. Dadas las cercanías energéticas, la banda ubicada en $1040 \mathrm{~cm}^{-1}$ tendría entonces dos componentes fundamentales, correspondiendo a una superposición del estiramiento simétrico $\mathrm{C}-\mathrm{O}$ para un confórmero de menor energía y de estiramiento antisimétrico C-O del confórmero de mayor energía. La banda que aparece sólo a temperaturas elevadas en $1036 \mathrm{~cm}^{-1}$, correspondería al estiramiento antisimétrico $\mathrm{C}-\mathrm{O}$ del confórmero de menor energía.

Se puede observar en la Figura 7.7 un comportamiento irregular en cuanto a las intensidades relativas de la banda ubicada en $952 \mathrm{~cm}^{-1}$ asignada al balanceo del grupo $\mathrm{NH}_{2}$. Esta característica esta asociada a la paulatina disminución de la relación señal / ruido que se observa hacia frecuencias más altas.

\section{Integración y justificación de resultados}

Ya se ha indicado que para los OPs, el modo vibracional de estiramiento del enlace $\mathrm{P}=\mathrm{S}$ es un buen sensor de la coexistencia conformacional. Sin embargo, ante la dificultad de poder observar el comportamiento de estas bandas en los espectros de matrices de Ar a diferentes temperaturas de deposición, es adecuado agregar más evidencia a la asignación propuesta.

Como ya se indicó, es posible derivar el número de onda de estiramiento $\mathrm{P}=\mathrm{S}$ a partir de la estructura electrónica y molecular de los compuestos tiofosforados con un buen índice predictivo, ${ }^{(5)}$ disponiéndose de una relación empírica

\footnotetext{
${ }^{5}$ Wan, J. and Zhan, C.G. Maximum bond order hybrid orbital calculations of the $\mathrm{P}=\mathrm{S}$ stretching frequencies for organophosphorus compounds. Spectrochim. Acta 51A(10):1665-1669 (1995).
} 
simplificada (Ecuación 3.a) que relaciona la carga neta del $\mathrm{P}$ $\left(Q_{\mathrm{P}}\right)$ y del azufre $\left(Q_{\mathrm{S}}\right)$ con la frecuencia de estiramiento $\mathrm{P}=\mathrm{S}$. Durante el análisis hecho sobre el TMSP mediante esta misma ecuación (Capítulo 3), se indicó la pertinencia del su uso para estimar la magnitud de la diferencia del doblete en frecuencia de estiramiento $\mathrm{P}=\mathrm{S}$, que en principio representa a los dos confórmeros más abundantes.

En la Tabla 7.VI se indican los valores de las cargas de Mülliken para el fósforo y el azufre en cada uno de los confórmeros propuestos como formas estables para un nivel de cálculo $\mathrm{HF} / 6-31 \mathrm{G}^{* *}$. Estos valores aplicados en la Ecuación 3.a arrojan una diferencia de $-6,3 \mathrm{~cm}^{-1}$ para los confórmeros gCs y -gtCs, pero esta diferencia llega a casi 18 $\mathrm{cm}^{-1}$, entre los confórmeros $\mathrm{C}_{1} \mathrm{y}-\mathrm{gtCs}$, valor que es una buena aproximación con los $-19 \mathrm{~cm}^{-1}$ de diferencia observada en las bandas asignadas al estiramiento $\mathrm{P}=\mathrm{S}$ en el espectro infrarrojo de la fase líquida (Tabla 7.IV). De esta manera, aparece una evidencia más de la correcta asignación de este doblete a la coexistencia de tres confórmeros.

En el análisis de los espectros infrarrojos en matrices de Ar, se puede indicar que en todos los casos descritos hay un comportamiento que se asocia indudablemente a la manifestación de confórmeros de diferentes energías relativas, pero en ninguno de ellos la asignación de las bandas a confórmeros particulares es completamente clara. Por esta razón, las asignaciones conformacionales propuestas se realizan con un margen de error que debería revisarse mediante medidas con técnicas alternativas. Sin embargo, es notable que sea necesario acudir por lo menos a los tres confórmeros de menor energía para explicar determinados comportamientos espectrales. A nuestro entender esta hipótesis es razonable, aun los cálculos $a b$ initio no aporten suficiente sustento para justificar la detección a temperatura ambiente del tercer confórmero de simetría - gtCs, dada su escasa abundancia relativa.

Sin embargo, y a la luz de todo expuesto, podemos afirmar que todos los resultados experimentales y teóricos son consistentes en el sentido de entregar fuerte evidencia de coexistencia de, por lo menos, tres confórmeros a temperatura ambiente, siendo interconvertibles con una dificultad energética mayor que en el caso del compuesto oxigenado análogo.

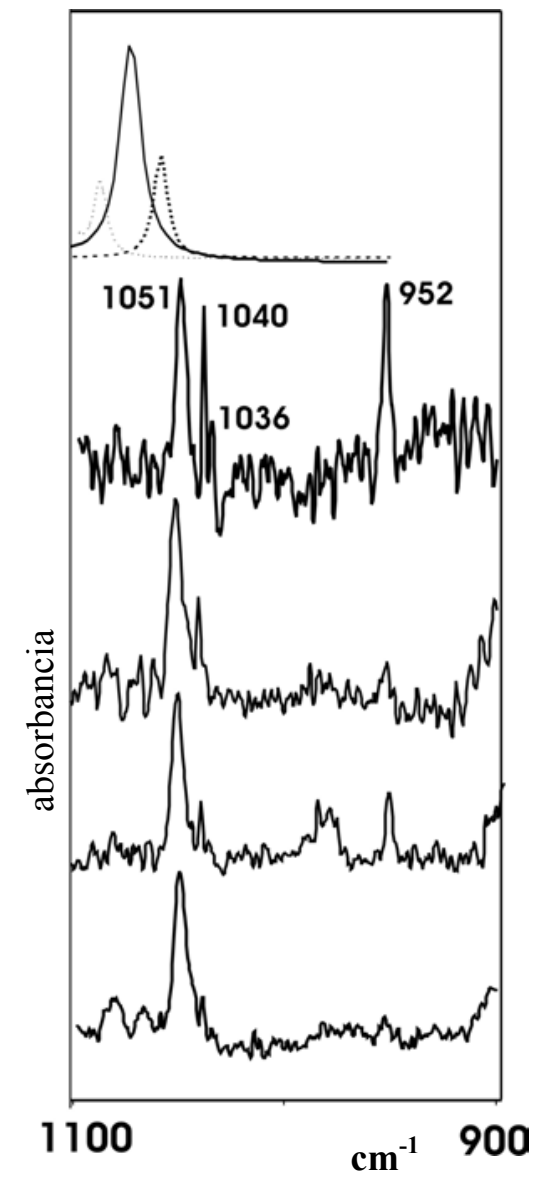

Figura 7.7: Espectros en matrices de Ar para el DMATP en la zona de $1000 \mathrm{~cm}^{-1}$, desde arriba hacia abajo según patrón ya indicado.

Tabla 7.VI: Cargas teóricas del fósforo y el azufre (según Mülliken) para cada uno de los cuatro confórmeros estables del DMATP, y diferencias estimadas para la frecuencia de estiramiento del enlace $\mathrm{P}=\mathrm{S}$ entre cada par de confórmeros.

\begin{tabular}{c|cccc}
\hline $\mathrm{HF} / 6-31 \mathrm{G}^{* *}$ & $\mathrm{gCs}$ & $\mathrm{C}_{1}$ & $-\mathrm{gtCs}$ & $\mathrm{gtCs}$ \\
\hline Carga $\mathrm{P}$ & 1,32 & 1,33 & 1,37 & 1,38 \\
\hline Carga S & $-0,54$ & $-0,56$ & $-0,52$ & $-0,52$ \\
\hline \multirow{2}{*}{$\begin{array}{c}\Delta v_{\mathrm{P}=\mathrm{S}} \\
\left(\mathrm{cm}^{-1}\right)\end{array}$} & \multicolumn{4}{|c|}{$-6,3$} \\
\cline { 2 - 5 } & \multicolumn{4}{|c|}{$-5,4$} \\
\hline
\end{tabular}




\section{8}

\section{Comportamiento conformacional: generalizaciones}

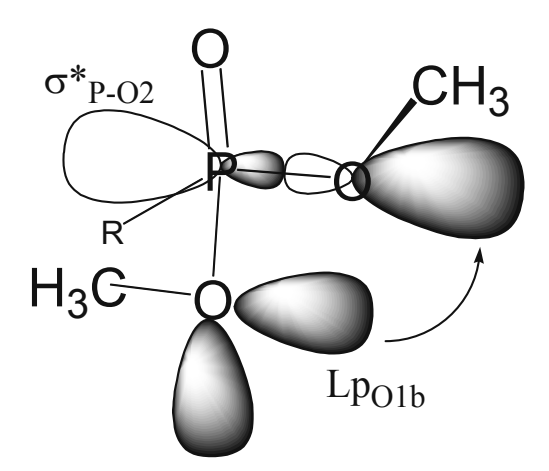

Figura 8.1: Efecto anomérico de donación de un par electrónico libre del $\mathrm{O}$ hacia el orbital antiligante $\sigma^{*}$ del enlace P-O.
Los resultados experimentales y aquellos derivados de los cálculos teóricos aplicados a la serie de calcogenofosfatos de trimetilo y fosfamidatos de dimetilo, entregan una valiosa información que se corrobora con los reportes experimentales previos y aportan elementos para interpretar con mayor profundidad los mismos.

Respecto a las características conformacionales de la serie de tres calcógenofosfatos de trimetilo se puede describir un comportamiento que, aunque cualitativamente similar, cuantitativamente se va a expresar en una progresión de valores que son manifestación de su libertad conformacional sucesivamente menor en el orden TMP, TMSP y TMSeP.

Para estas moléculas se encuentran teóricamente tres mínimos conformacionales de geometrías análogas en un orden de estabilidad de simetrías $\mathrm{C}_{3}, \mathrm{C}_{1}$ y $\mathrm{C}_{\mathrm{s}} \mathrm{y}$ existen suficientes indicios experimentales que verifican la validez de estas conclusiones. Los factores que determinan esta estabilidad se relacionarían con varios fenómenos más o menos independientes.

Por un lado es importante la minimización de momento dipolar global por la anulación de momentos dipolares locales, siendo este factor más importante en el TMP por el mayor carácter polar del enlace $\mathrm{P}=\mathrm{O}, \mathrm{y}$ decreciendo sucesivamente su peso relativo para el TMSP y TMSeP respectivamente.

Para el caso de la serie de fosfamidatos de dimetilo, se encontró un comportamiento similar desde el punto de vista experimental para el par DMAP y DMATP. En efecto, aunque en los espectros de matrices del análogo azufrado fue posible describir la coexistencia de, a lo menos, tres confórmeros, en los espectros infrarrojos de la fase líquida sólo fue posible determinar la presencia de dos de ellos en un número restringido de pares de bandas.

Como ya se expuso en los dos Capítulos anteriores, en los espectros de ${ }^{1} \mathrm{H}$ RMN se observa una mayor 
complejidad para la molécula oxigenada, que para el respectivo análogo azufrado.

Del espectro teórico calculado para la molécula fosfamidoselenoato de O,O-dimetilo (DMASeP), se puede predecir la manifestación de coexistencia conformacional en los modos vibracionales de estiramiento antisimétrico $\mathrm{NH}_{2}$ en los $3500 \mathrm{~cm}^{-1}$, de deformación $\mathrm{NH}_{2}$ en los $1530 \mathrm{~cm}^{-1}$, de estiramiento antisimétrico $\mathrm{P}-\mathrm{O}$ en los $960 \mathrm{~cm}^{-1}$ y de estiramiento $\mathrm{P}=\mathrm{Se}$ en los $350 \mathrm{~cm}^{-1}$ (Tabla 8.I). De la misma manera, los restantes resultados teóricos para esta molécula son compatibles con los datos obtenidos en los análogos oxigenado y azufrado (Tabla 8.II).

Entonces, desde el punto de vista de la estabilidad de los confórmeros, para la serie de fosfamidatos de dimetilo, es posible observar una mayor estabilización de aquellas geometrías en las que predominan los metilos en posición gauche, lo que significa una mayor abundancia de los confórmeros con geometrías gCs y $\mathrm{C}_{1}$.

Para explicar, al menos en parte, la estabilización de determinadas estructuras sobre todas las posibles, se ha argumentado respecto de la importancia de las interacciones entre el par de electrones libres del calcógeno unido al fósforo y los hidrógenos metílicos. Estas interacciones, aunque débiles, en fase gaseosa o en fase líquida y en ausencia de otros dadores de hidrógeno más fuertes, determinarían la estabilización de los ángulos $\psi$ que favorezcan el acercamiento entre los metilos y este par libre.

Aunque este fenómeno determinaría en mayor medida las conformaciones más estables en alquilfosfatos de cadena más larga, ${ }^{(1)}$ no sería despreciable su aporte para la estabilización de las conformaciones de los calcogenofosfatos de trimetilo y los fosfamidatos de dimetilo. La formación de enlaces por puentes de hidrógeno en sistemas $\mathrm{P}=\mathrm{O} \cdots \mathrm{H}-\mathrm{C}$ y $\mathrm{P}=\mathrm{S} \cdots \mathrm{H}-\mathrm{C}$ ha sido demostrada por abundante evidencia teórica y experimental. ${ }^{(2)}$

\footnotetext{
${ }^{1}$ (a) Vidya, V., Sankaran, K., Sundararajaran, K. and Viswanathan, K.S. Conformations of triethylphosphate: a supersonic jet-matrix isolation and semi-empirical (AM1) study, J.Mol.Struct. 476: 97-104 (1999). (b) George, L. et al. Matrix-isolation infrared spectroscopy of organic phosphates, Appl.Spectrosc. 48 (1): 7-12 (1994).

${ }^{2}$ Cuevas G. Hydrogen bond type contributions to the anomeric effect in S-C-P(O) and S-C-P(S) segments, J.Am.Chem.Soc. 122:692-698 (2000).
}

Tabla 8.I: Frecuencias teóricas del espectro vibracional IR de la molécula DMASeP, calculado a un nivel HF/6-31G ${ }^{* *}$ (ab initio).

\begin{tabular}{|c|c|c|c|c|}
\hline Asignación & gCs & $\mathrm{C}_{1}$ & -gtCs & gtCs \\
\hline va NH & 3487 & 3516 & 3473 & 3504 \\
\hline vs $\mathrm{NH}_{2}$ & 3375 & 3394 & 3376 & 3389 \\
\hline $\mathrm{va} \mathrm{CH}$ & $\begin{array}{l}2959 \\
2958 \\
2943 \\
2940\end{array}$ & $\begin{array}{l}2962 \\
2961 \\
2938\end{array}$ & $\begin{array}{l}2962 \\
2959 \\
2940 \\
2939\end{array}$ & $\begin{array}{l}2962 \\
2959 \\
2940\end{array}$ \\
\hline vs $\mathrm{CH}_{3}$ & $\begin{array}{l}2865 \\
2863\end{array}$ & $\begin{array}{l}2933 \\
2862 \\
2857\end{array}$ & $\begin{array}{l}2865 \\
2863\end{array}$ & $\begin{array}{l}2929 \\
2863 \\
2852\end{array}$ \\
\hline$\delta \mathrm{a} \mathrm{NH}$ & 1536 & 1527 & 1547 & 1535 \\
\hline $\mathrm{Sa} \mathrm{CH}_{3}$ & $\begin{array}{l}1461 \\
1460 \\
1451 \\
1450\end{array}$ & $\begin{array}{l}1460 \\
1458 \\
1452 \\
1450\end{array}$ & $\begin{array}{l}1462 \\
1458 \\
1451 \\
1449\end{array}$ & $\begin{array}{l}1460 \\
1458 \\
1455 \\
1449\end{array}$ \\
\hline ora $\mathrm{CH}_{3}$ & $\begin{array}{l}1437 \\
1436\end{array}$ & $\begin{array}{l}1440 \\
1436\end{array}$ & $\begin{array}{l}1438 \\
1434\end{array}$ & $\begin{array}{l}1438 \\
1436\end{array}$ \\
\hline$w \mathrm{CH}_{3}$ & $\begin{array}{l}1175 \\
1174\end{array}$ & $\begin{array}{l}1175 \\
1172\end{array}$ & $\begin{array}{l}1172 \\
1167\end{array}$ & $\begin{array}{l}1173 \\
1170\end{array}$ \\
\hline $\begin{array}{l}\text { vs } \mathrm{O}-\mathrm{C} \\
\text { va } \mathrm{O}-\mathrm{C}\end{array}$ & $\begin{array}{l}1150 \\
1148\end{array}$ & $\begin{array}{l}1151 \\
1149 \\
1092\end{array}$ & $\begin{array}{l}1149 \\
1145\end{array}$ & $\begin{array}{l}1150 \\
1143\end{array}$ \\
\hline ora $\mathrm{NH}_{2}$ & 1071 & & $\begin{array}{l}1059 \\
1050\end{array}$ & $\begin{array}{l}1067 \\
1054\end{array}$ \\
\hline$v \mathrm{P}-\mathrm{N}$ & 1044 & 1038 & & \\
\hline va $\mathrm{P}-\mathrm{O}$ & $\begin{array}{l}977 \\
879\end{array}$ & $\begin{array}{l}950 \\
868\end{array}$ & 986 & 952 \\
\hline$v \mathrm{P}-\mathrm{O}$ & 795 & 793 & $\begin{array}{l}856 \\
775\end{array}$ & $\begin{array}{l}855 \\
782\end{array}$ \\
\hline$w \mathrm{NH}_{2}$ & 750 & 476 & $\begin{array}{l}738 \\
589\end{array}$ & 519 \\
\hline$\delta a \mathrm{P}-\left(\mathrm{OCH}_{3}\right)_{2}$ & $\begin{array}{l}478 \\
440\end{array}$ & $\begin{array}{l}455 \\
376\end{array}$ & $\begin{array}{l}527 \\
436\end{array}$ & $\begin{array}{l}448 \\
395\end{array}$ \\
\hline $\begin{array}{l}w \mathrm{PNO}_{2} \\
(v \mathrm{P}=\mathrm{Se})\end{array}$ & 384 & 367 & 347 & 350 \\
\hline$v \mathrm{P}=\mathrm{Se}$ & 339 & 326 & 328 & 328 \\
\hline$w \mathrm{PNO}_{2}$ & 307 & 257 & 285 & 293 \\
\hline$w$ P-NH ${ }_{2}$ & $\begin{array}{l}238 \\
219\end{array}$ & $\begin{array}{l}238 \\
217\end{array}$ & $\begin{array}{l}242 \\
175\end{array}$ & $\begin{array}{l}259 \\
207\end{array}$ \\
\hline ors $\mathrm{PO}_{2} \mathrm{~N}$ & 187 & 162 & 158 & 161 \\
\hline pra $\mathrm{PO}_{2} \mathrm{~N}$ & $\begin{array}{l}140 \\
130\end{array}$ & 152 & 144 & $\begin{array}{l}144 \\
136\end{array}$ \\
\hline ors $\mathrm{CH}_{3}$ & 126 & $\begin{array}{l}125 \\
121\end{array}$ & $\begin{array}{l}107 \\
105\end{array}$ & 122 \\
\hline wO- $\left(\mathrm{CH}_{3}\right)$ & $\begin{array}{l}92 \\
68\end{array}$ & $\begin{array}{l}79 \\
63\end{array}$ & $\begin{array}{l}74 \\
38 \\
\end{array}$ & $\begin{array}{l}86 \\
61\end{array}$ \\
\hline
\end{tabular}

v: vibración de estiramiento; vs: vibración de estiramiento simétrico; va: vibración de estiramiento antisimétrico;

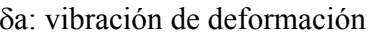
antisimétrica; vs: vibración de estiramiento simétrico; va: vibración de estiramiento antisimétrico; $\rho$ rs: vibración de balanceo simétrico pra: vibración de balanceo antisimétrico; w: vibración de aleteo. 


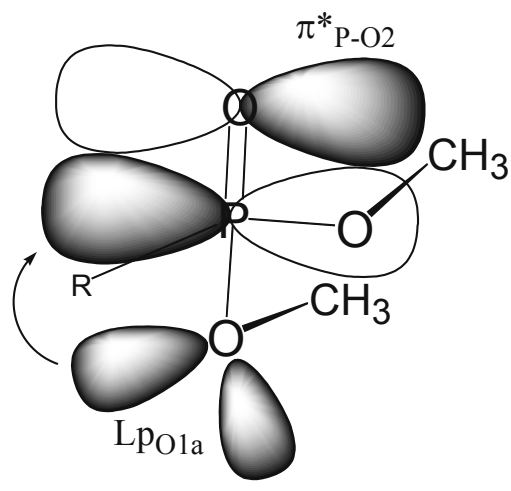

Figura 8.2: Efecto anomérico que involucra un par electrónico libre del $\mathrm{O}$ con el orbital antiligante $\pi^{*}$ del enlace $\mathrm{P}=\mathrm{O}$.
Finalmente, ciertas conformaciones van a estar fuertemente restringidas por impedimentos estéricos, fundamentalmente aquellas que implican la coexistencia de ángulos $\psi$ simultáneamente trans. Este último factor es fuertemente restrictivo y no depende de un aumento en las interacciones de los metilos entre sí, pues el valor de los ángulos $\mathrm{O}-\mathrm{P}=\mathrm{X}$, determinado mediante cálculos $a b$ initio $\mathrm{y}$ siendo $\mathrm{X}$ cualquier calcógeno, se mantiene más o menos constante para la serie de tres moléculas.

Con este esquema general, es posible justificar la posibilidad de distinguir experimentalmente la coexistencia de los confórmeros correspondientes, en la medida de la mayor o menor diferencia de energía entre ellos. Así, los cálculos permiten asignar desdoblamientos de modos vibracionales correspondientes a la coexistencia de confórmeros en cada una de ambas serie estudiadas.

Por otro lado, se puede justificar la complejidad decreciente de la estructura fina de los espectros de ${ }^{1} \mathrm{H}$ RMN para ambas series. Esta complejidad está asociada a menores valores de la constante de acoplamiento ${ }^{3} J_{\mathrm{P}-\mathrm{H}}$ para los análogos oxigenados respecto a los análogos azufrados o selenados, lo que impediría una mejor resolución de los picos individuales. Entonces, es posible argumentar respecto a que existe un aporte de la libertad conformacional de las moléculas respecto a esta complejidad.

Tabla 8.II: Parámetros geométricos y fisicoquímicos, calculados a un nivel HF/6-31G** (ab initio), para la molécula DMASeP. Longitudes en amstrong, ángulos en grados.

\begin{tabular}{|c|c|c|c|c|}
\hline & gCs & $\mathrm{C}_{1}$ & -gtCs & gtCs \\
\hline$\psi_{1}$ & 48 & 49 & -44 & 48 \\
\hline$\psi_{2}$ & 55 & -44 & 183 & 173 \\
\hline $\mathrm{P}=\mathrm{Se}(\AA)$ & 2,08 & 2,08 & 2,07 & 2,07 \\
\hline $\mathrm{P}-\mathrm{O}_{1}(\AA)$ & 1,59 & 1,58 & 1,59 & 1,59 \\
\hline $\mathrm{P}-\mathrm{O}_{2}(\AA)$ & 1,58 & 1,58 & 1,59 & 1,58 \\
\hline $\mathrm{HF} / 6-31 \mathrm{G}^{* *}(\mathrm{kcal} / \mathrm{mol})$ & 0,00 & 1,88 & 3,64 & 4,65 \\
\hline$\mu$ D (Müll)Calc. & 3,67 & 4,12 & 5,10 & 6,18 \\
\hline Prop.a $298^{\circ} \mathrm{K}$ (peso 2:1:2:2) & 0,98 & 0,02 & 0,00 & 0,00 \\
\hline $\mu$ D (Müll)Pond. & & & & \\
\hline
\end{tabular}

En forma general, el hecho de la mayor estabilización de los confórmeros con metilos en gauche, ha sido descrito como el llamado "efecto gauche" (3) manifestado en las estructuras conformacionales de los OPs, al favorecerse el

3 Durig,J.R. Structures and conformations of organophosphorus molecules. J.Mol.Struc.113 (1984) 127-140. 
mayor número de interacciones gauche entre pares de electrones y/o enlaces polares adyacentes.

Aunque en todos los casos los confórmeros más estables son los de menor momento dipolar, dado que los sistemas experimentales se realizan con entornos no polares y los cálculos se efectúan en el vacío, es posible esperar que en entornos polares las relaciones se inviertan y aumente la abundancia de las conformaciones de momento dipolar más alto, en donde estén favorecidas a la vez las interacciones con el solvente y las intramoleculares, como ocurre con la conformación de simetría $\mathrm{C}_{1}$. Sin embargo, el entorno no polar, supuesto en los cálculos ab initio, es suficiente para explicar los espectros vibracionales obtenidos en fase líquida de la sustancia pura, que es polar.

\section{Análisis NBO}

El análisis de enlaces por orbitales naturales (Natural Bond Orbital - NBO) permite realizar una descripción cualitativa y cuantitativa de la naturaleza de los enlaces covalentes y de las interacciones de estabilización de los pares libres, en función de la población de las nubes orbitales de valencia y los orbitales antiligantes de baja energía de una molécula.

En la medida que esta descripción pueda realizarse mediante un esquema relativamente simple y permita identificar diferencias entre confórmeros de una misma molécula, será posible argumentar acerca de los factores estereoelectrónicos que estabilizan cada forma conformacional y, si es posible, adjudicar las diferencias poblacionales de cada confórmero a estas interacciones electrónicas. Los efectos mesoméricos y anoméricos (4) pueden ser estudiados mediante este tipo de cálculos.

Para la molécula TMP, y según los resultados NBO a un nivel HF/6-31G* (ver Tabla 8.III), existen tres efectos predominantes en los distintos confórmeros estables. Para los metilos en gauche se impondrán los efectos anoméricos $\mathrm{Lp}_{\mathrm{O}} \rightarrow \sigma^{*}{ }_{\mathrm{P}-\mathrm{O}}$ (Figura 8.1), además de una donación $\mathrm{Lp} \mathrm{p}_{\mathrm{O}} \rightarrow \pi^{*} \mathrm{O}=\mathrm{P}$ (Figura 8.2), mientras que para los metilos en trans se impondrá un efecto de donación $\mathrm{L} \mathrm{p}_{\mathrm{O}} \rightarrow \sigma^{*} \mathrm{P}=\mathrm{O}$ (Figura 8.4).

\footnotetext{
${ }^{4}$ Kirby,A.J. The anomeric effect and related stereochemical effects at oxigen. Springer Berlin (1983).
}

Tabla 8.III: Análisis NBO para los dos confórmeros más estables del TMP. Entre paréntesis se indican las energías respectivas en $\mathrm{kcal} / \mathrm{mol}$.

\begin{tabular}{|c|c|}
\hline $\mathrm{C}_{3}$ & $\mathrm{C}_{1}$ \\
\hline & $\begin{array}{l}\mathrm{LpO}_{\mathrm{P}} \rightarrow \pi^{*}{ }_{(3)} \mathrm{P}=\mathrm{O}^{(1,29)} \\
\mathrm{LpO}_{\mathrm{P}} \rightarrow \sigma^{*} \mathrm{P}-\mathrm{O}_{3}{ }^{(1,82)}\end{array}$ \\
\hline $\begin{array}{l}\mathrm{LpO}_{1}{ }^{\mathrm{a}} \rightarrow \pi^{*}{ }_{(3)} \mathrm{P}=\mathrm{O}^{(3,72)} \\
\mathrm{LpO}_{1}{ }^{\mathrm{a}} \rightarrow \sigma^{*} \mathrm{P}-\mathrm{O}_{2}{ }^{(3,81)} \\
\mathrm{LpO}_{1}{ }^{\mathrm{b}} \rightarrow \sigma^{*} \mathrm{P}=\mathrm{O}^{(6,04)} \\
\mathrm{LpO}_{1}{ }^{\mathrm{a}} \rightarrow \sigma^{*} \mathrm{P}=\mathrm{O}^{(6,86)} \\
\mathrm{LpO}_{1}{ }^{\mathrm{b}} \rightarrow \sigma^{*} \mathrm{P}-\mathrm{O}_{3}{ }^{(6,98)} \\
\mathrm{LpO}_{1}{ }^{\mathrm{b}} \rightarrow \pi^{*}{ }_{(3)} \mathrm{P}=\mathrm{O}^{(8,36)}\end{array}$ & $\begin{array}{l}\mathrm{LpO}_{1}{ }^{\mathrm{b}} \rightarrow \sigma^{*} \mathrm{P}_{-} \mathrm{O}_{2}{ }^{(1,47)} \\
\mathrm{LpO}_{1}{ }^{\mathrm{a}} \rightarrow \sigma^{*} \mathrm{P}-\mathrm{O}_{2}{ }^{(3,34)} \\
\mathrm{LpO}_{1}{ }^{\mathrm{b}} \rightarrow \pi^{*}{ }_{(3)} \mathrm{P}=\mathrm{O}^{(3,37)} \\
\mathrm{LpO}_{1}{ }^{\mathrm{a}} \rightarrow \sigma^{*} \mathrm{P}=\mathrm{O}^{(3,59)} \\
\mathrm{LpO}_{1}{ }^{\mathrm{a}} \rightarrow \pi^{*}{ }_{(3)} \mathrm{P}=\mathrm{O}^{(6,39)} \\
\mathrm{LpO}_{1}{ }^{\mathrm{b}} \rightarrow \sigma^{*} \mathrm{P}=\mathrm{O}^{(7,32)} \\
\mathrm{LpO}_{1}{ }^{\mathrm{b}} \rightarrow \sigma^{*} \mathrm{P}-\mathrm{O}_{3}\end{array}$ \\
\hline $\begin{array}{l}\mathrm{LpO}_{2}{ }^{\mathrm{a}} \rightarrow \sigma^{*} \mathrm{P}-\mathrm{O}_{3}{ }^{(3,81)} \\
\mathrm{LpO}_{2}{ }^{\mathrm{a}} \rightarrow \pi^{*}{ }_{(2)} \mathrm{P}=\mathrm{O}^{(4,16)} \\
\mathrm{LpO}_{2}{ }^{\mathrm{b}} \rightarrow \sigma^{*} \mathrm{P}=\mathrm{O}^{(6,07)} \\
\mathrm{LpO}_{2}{ }^{\mathrm{a}} \rightarrow \sigma^{*} \mathrm{P}=\mathrm{O}^{(6,84)} \\
\mathrm{LpO}_{2}{ }^{\mathrm{b}} \rightarrow \pi^{*}{ }_{(2)} \mathrm{P}=\mathrm{O}^{(6,94)} \\
\mathrm{LpO}_{2}{ }^{\mathrm{b}} \rightarrow \sigma^{*} \mathrm{P}-\mathrm{O}_{1}{ }^{(6,98)} \\
\mathrm{LpO}_{3}{ }^{\mathrm{a}} \rightarrow \sigma^{*} \mathrm{P}-\mathrm{O}_{1}{ }^{(3,81)} \\
\mathrm{LpO}_{3}{ }^{\mathrm{a}} \rightarrow \sigma^{*} \mathrm{P}=\mathrm{O}^{(6,87)} \\
\mathrm{LpO}_{3}{ }^{\mathrm{b}} \rightarrow \pi^{*}{ }_{(3)} \mathrm{P}=\mathrm{O}^{(2,79)} \\
\mathrm{LpO}_{3}{ }^{\mathrm{b}} \rightarrow \pi^{*}{ }_{(2)} \mathrm{P}=\mathrm{O}^{(5,65)} \\
\mathrm{LpO}_{3}{ }^{\mathrm{b}} \rightarrow \sigma^{*} \mathrm{P}=\mathrm{O}^{(6,03)} \\
\mathrm{LpO}_{3}{ }^{\mathrm{b}} \rightarrow \sigma^{*} \mathrm{P}-\mathrm{O}_{2}{ }^{(6,98)}\end{array}$ & $\begin{array}{l}\mathrm{LpO}_{2}{ }^{\mathrm{a}} \rightarrow \pi^{*}{ }_{(2)} \mathrm{P}=\mathrm{O}^{(4,56)} \\
\mathrm{LpO}_{2}{ }^{\mathrm{a}} \rightarrow \sigma^{*} \mathrm{P}-\mathrm{O}_{3}{ }^{(5,11)} \\
\mathrm{LpO}_{2}{ }^{\mathrm{a}} \rightarrow \sigma^{*} \mathrm{P}=\mathrm{O}^{(5,27)} \\
\mathrm{LpO}_{2}{ }^{\mathrm{b}} \rightarrow \pi^{*}{ }_{(3)} \mathrm{P}=\mathrm{O}^{(2,85)} \\
\mathrm{LpO}_{2}{ }^{\mathrm{b}} \rightarrow \sigma^{*} \mathrm{P}=\mathrm{O}^{(5,34)} \\
\mathrm{LpO}_{2}{ }^{\mathrm{b}} \rightarrow \sigma^{*} \mathrm{P}-\mathrm{O}_{1}{ }^{(6,37)} \\
\mathrm{LpO}_{2}{ }^{\mathrm{b}} \rightarrow \pi^{*}{ }_{(2)} \mathrm{P}=\mathrm{O}^{(7,20)} \\
\mathrm{LpO}_{3}{ }^{\mathrm{b}} \rightarrow \sigma^{*} \mathrm{P}-\mathrm{O}_{2}{ }^{(4,74)} \\
\mathrm{LpO}_{3}{ }^{\mathrm{b}} \rightarrow \sigma^{*} \mathrm{P}-\mathrm{O}_{1}{ }^{(6,61)} \\
\mathrm{LpO}_{3}{ }^{\mathrm{b}} \rightarrow \pi^{*}{ }_{(2)} \mathrm{P}=\mathrm{O}^{(9,89)} \\
\mathrm{LpO}_{3}{ }^{\mathrm{a}} \rightarrow \pi^{*}{ }_{(3)} \mathrm{P}=\mathrm{O}^{(18,32)}\end{array}$ \\
\hline
\end{tabular}


En el confórmero de simetría $\mathrm{C}_{3}$, correspondiente a la forma más estable, se encuentra que existe una situación de regularidad para cada uno de los tres grupos P-O-C, en cuanto a los efectos predominantes en la constitución de estas estructuras. Por un lado se observa una donación de carga de cada uno de ambos pares electrónicos de los tres oxígenos del éster al orbital $\sigma^{*}$ del enlace $\mathrm{P}=\mathrm{O}$ que suma más de 13 $\mathrm{kcal} / \mathrm{mol}$ en cada uno de ellos. Por otro lado se observa una donación de carga por parte del par electrónico adyacente hacia el orbital $\sigma^{*}$ P-O, con un valor de cerca de $7 \mathrm{kcal} / \mathrm{mol}$ en cada uno de los tres enlaces. Esta donación puede considerarse una estabilización por efecto anomérico (Figura 8.1).

El balance global de las energías de estabilización por vía de donación electrónica desde pares libres hasta

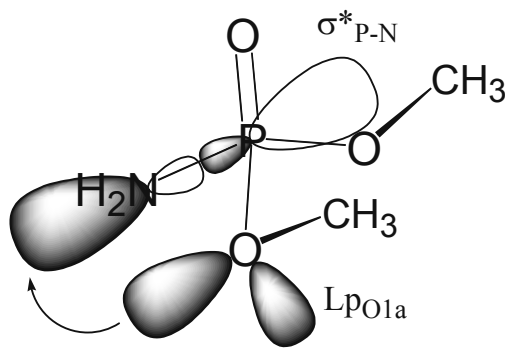

Figura 8.3: Efecto anomérico que involucra un par electrónico libre del $\mathrm{O}$ con el orbital antiligante $\sigma^{*}$ del enlace P-N. orbitales antiligantes, arroja un valor de $0,2 \mathrm{kcal} / \mathrm{mol}$ a favor del confórmero de simetría $C_{1}$. De esta manera, los efectos estereoelectrónicos no sólo no explican por si mismos la abundancia relativa de los confórmeros $C_{3}$ y $C_{1}$, sino que es necesario argumentar mediante restricciones estéricas la diferencia de $2,2 \mathrm{kcal} / \mathrm{mol}$ a favor del confórmero $\mathrm{C}_{3}$. Estas restricciones estéricas, para el confórmero $\mathrm{C}_{1}$, estarían dadas por las interacciones desestabilizantes de los pares libres del oxígeno en trans con orbitales $\pi$ del enlace $\mathrm{P}=\mathrm{O}$. Considerando el balance de las interacciones NBO y las diferencias energéticas entre ambos confórmeros, es posible atribuir un valor de alrededor de $2,5 \mathrm{kcal} / \mathrm{mol}$ para la energía de estabilización por vía de interacciones estéricas.

Dado que según los datos experimentales y teóricos presentados, los órdenes relativos de los confórmeros de los tres análogos calcogenofosfatos de trimetilo son similares, cualitativamente serán los mismos efectos descritos los que expliquen el comportamiento conformacional de las moléculas TMSP y TMSeP.

Para la molécula DAMP, y según los resultados $\mathrm{NBO}$ a un nivel HF/6-31G*, existen cuatro efectos que predominan en los distintos confórmeros estables (ver Tabla 8.IV). Para los metilos en gauche se impondrán un efecto de donación $\mathrm{Lp}_{\mathrm{O}} \rightarrow \pi_{\mathrm{O}=\mathrm{P}}^{*}$ (Figura 8.2) y los efectos anoméricos $\mathrm{Lp}_{\mathrm{O}} \rightarrow \sigma^{*}{ }_{\mathrm{P}-\mathrm{N}}$ (Figura 8.3), $\mathrm{Lp}_{\mathrm{O}} \rightarrow \sigma^{*}{ }_{\mathrm{P}-\mathrm{O}}$ (Figura 8.1), mientras que para los metilos en trans se impondrá el efecto de donación $\mathrm{Lp} \rightarrow \sigma^{*} \mathrm{P}=\mathrm{O}$ (Figura 8.4). Esta es una situación análoga a la que explica la estabilidad relativa de los calcogenofosfatos de trimetilo. 
Los confórmeros de menor energía, gCs y $\mathrm{C}_{1}$, presentan ambos metilos en gauche, respecto al enlace $\mathrm{P}=\mathrm{O}$. Por esta razón, el efecto de donación de uno de los pares libres de cada oxígeno de los enlaces éster al sistema $\pi^{*}$ del enlace $\mathrm{P}=\mathrm{O}$ determina una estabilización de ambos confórmeros respecto a aquellos con metilos en posición trans. Sumado a este efecto, la estabilización de las estructuras por efectos anoméricos que involucran a los orbitales $\sigma^{*}$ de los enlaces $\mathrm{P}-\mathrm{O}$ y $\mathrm{P}-\mathrm{N}$, determinan una menor energía relativa de estos.

Por otro lado, la simetría se rompe en el confórmero gCs, para favorecer la donación de electrones desde uno de los pares libres del oxígeno 1 (éster fosfórico) al orbital sigma antiligante del enlace fósforo-oxígeno $2\left(\sigma_{\mathrm{P}-\mathrm{O} 2}\right)$, antes que al orbital sigma antiligante del enlace fósforo nitrógeno $\left(\sigma^{*}{ }_{\mathrm{P}-\mathrm{N}}\right)$, favoreciendo el efecto anomérico en el sistema $\mathrm{Lp}_{\mathrm{O} 1} \rightarrow \sigma^{*} \mathrm{P}-\mathrm{O} 2$, antes que en los pares $\mathrm{Lp}_{\mathrm{O} 1} \rightarrow \sigma^{*} \mathrm{P}-\mathrm{N}$.

Por otro lado, en el confórmero $\mathrm{C}_{1}$, está favorecida la donación de los pares de electrones libres a los sistemas $\sigma^{*}{ }_{\mathrm{P}-\mathrm{O}}$ en forma recíproca. De hecho, la coplanaridad de los enlaces $\mathrm{P}-\mathrm{O}(-\mathrm{C})$ es evidencia adecuada: los ángulos diedros de los enlaces $\mathrm{C}-\mathrm{O}-\mathrm{P}-\mathrm{O}$ en el confórmero gCs son de $164^{\circ} \mathrm{y}$ $63^{\circ}$ respectivamente, mientras que en el confórmero $\mathrm{C}_{1}$ son de $162^{\circ} \mathrm{y}-173^{\circ}$ (calculados a un nivel HF/6-31G*).

Es decir, aunque el efecto anomérico es el que explica la estabilidad de los metilos en gauche, es el efecto por donación de uno de los pares libres del oxígeno hacia el orbital $\sigma^{*}$-N el que determina la mayor estabilidad del confórmero gCs por sobre el confórmero $\mathrm{C}_{1}$. En este punto es posible valorar la mayor capacidad del orbital $\sigma^{*}{ }_{\mathrm{P}-\mathrm{N}}$ de ser mejor aceptor de electrones respecto al orbital $\sigma^{*} \mathrm{P}-\mathrm{O}$, para esta geometría molecular.

El balance global de las energías de estabilización por vía de donación electrónica desde pares libres hasta orbitales antiligantes, arroja un valor de $2,2 \mathrm{kcal} / \mathrm{mol}$ a favor del confórmero de simetría $\mathrm{C}_{1}$. De esta manera, los efectos electrónicos no sólo no explican por si mismos la abundancia relativa de los confórmeros gCs y $\mathrm{C}_{1}$, sino que es necesario argumentar mediante restricciones estéricas la diferencia de $2,2 \mathrm{kcal} / \mathrm{mol}$ a favor del confórmero gCs.

Estas restricciones estéricas, para el confórmero $\mathrm{C}_{1}$, estarían dadas por las interacciones desestabilizantes de los pares libres del oxígeno en cis con orbitales ligantes de los

Tabla 8.IV: Análisis NBO para los dos confórmeros más estables del DMAP. Entre paréntesis se indican las energías respectivas en $\mathrm{kcal} / \mathrm{mol}$.

\begin{tabular}{|c|c|}
\hline $\mathrm{gCs}$ & $\mathrm{C}_{1}$ \\
\hline $\mathrm{Lp}_{\mathrm{N}} \rightarrow \sigma_{\mathrm{P}-\mathrm{O} 1}^{*}(6,05)$ & $\mathrm{Lp}_{\mathrm{N}} \rightarrow \sigma_{\mathrm{P}-\mathrm{O} 1}{ }^{(6,52)}$ \\
\hline $\mathrm{Lp}_{\mathrm{N}} \rightarrow \pi^{*}{ }_{(2) \mathrm{O}=\mathrm{P}}{ }^{(7,81)}$ & $\mathrm{Lp}_{\mathrm{N}} \rightarrow \pi^{*}{ }_{(2) \mathrm{O}=\mathrm{P}}{ }^{(8,69)}$ \\
\hline $\mathrm{Lp}_{\mathrm{O} 1}{ }^{\mathrm{a}} \rightarrow \pi^{*}{ }_{(3) \mathrm{O}=\mathrm{P}}{ }^{(1,38)}$ & $\mathrm{Lp}_{\mathrm{O} 1}{ }^{\mathrm{a}} \rightarrow \pi^{*}{ }_{(3) \mathrm{O}=\mathrm{P}}{ }^{(9,48)}$ \\
\hline $\mathrm{Lp}_{\mathrm{O} 1}{ }^{\mathrm{a}} \rightarrow \sigma^{*}{ }_{\mathrm{P}-\mathrm{N}}(3,86)$ & $\mathrm{Lp}_{\mathrm{O} 1}{ }^{\mathrm{b}} \rightarrow \sigma^{*}{ }_{\mathrm{P}-\mathrm{N}}(8,76)$ \\
\hline $\mathrm{Lp}_{\mathrm{O} 1}{ }^{\mathrm{b}} \rightarrow \pi^{*}{ }_{(2) \mathrm{O}=\mathrm{P}}{ }^{(7,96)}$ & $\mathrm{Lp}_{\mathrm{O} 1}{ }^{\mathrm{b}} \rightarrow \pi^{*}{ }_{(3) \mathrm{O}=\mathrm{P}}{ }^{(1,11)}$ \\
\hline $\mathrm{Lp}_{\mathrm{O} 1}{ }^{\mathrm{b}} \rightarrow \sigma^{*}{ }_{\mathrm{O}=\mathrm{P}}{ }^{(6,32)}$ & $\mathrm{Lp}_{\mathrm{O} 1}{ }^{\mathrm{b}} \rightarrow \sigma_{\mathrm{O}=\mathrm{P}}^{*}{ }^{(9,93)}$ \\
\hline $\mathrm{Lp}_{\mathrm{O} 1}{ }^{\mathrm{b}} \rightarrow \sigma^{*}{ }_{\mathrm{P}-\mathrm{O} 2}{ }^{(5,95)}$ & $\mathrm{Lp}_{\mathrm{O} 1}{ }^{\mathrm{a}} \rightarrow \sigma_{\mathrm{P}-\mathrm{O} 2}^{*}{ }^{(3,69)}$ \\
\hline $\mathrm{Lp}_{\mathrm{O} 2}{ }^{\mathrm{a}} \rightarrow \pi^{*}{ }_{(3) \mathrm{O}=\mathrm{P}}{ }^{(7,25)}$ & $\mathrm{Lp}_{\mathrm{O} 2}{ }^{\mathrm{a}} \rightarrow \pi^{*}{ }_{(3) \mathrm{O}=\mathrm{P}}{ }^{(8,36)}$ \\
\hline $\mathrm{Lp}_{\mathrm{O} 2}{ }^{\mathrm{b}} \rightarrow \sigma_{\mathrm{P}-\mathrm{N}}^{*}{ }^{(7,62)}$ & $\mathrm{Lp}_{\mathrm{O} 2}{ }^{\mathrm{b}} \rightarrow \sigma_{\mathrm{P}-\mathrm{N}}^{*}$ \\
\hline $\mathrm{Lp}_{\mathrm{O} 2}{ }^{\mathrm{b}} \rightarrow \sigma^{*}{ }_{\mathrm{O}=\mathrm{P}}(11,17)$ & $\mathrm{Lp}_{\mathrm{O} 2}{ }^{\mathrm{b}} \rightarrow \sigma_{\mathrm{O}=\mathrm{P}}{ }^{(13,29)}$ \\
\hline
\end{tabular}


enlaces $\mathrm{P}-\mathrm{N}$ y $\mathrm{P}=\mathrm{O}$. Considerando el balance de las interacciones NBO y las diferencias energéticas entre ambos confórmeros, es posible atribuir un valor de alrededor de 4 $\mathrm{kcal} / \mathrm{mol}$ para la energía de estabilización por vía de interacciones estéricas. Según los datos experimentales y teóricos presentados, los órdenes relativos de los confórmeros de los tres análogos calcogenofosfamidatos de dimetilo son similares, entonces, serían los mismos efectos descritos los que expliquen el comportamiento conformacional de las moléculas DMATP y DMASeP.

En general, las descripciones NBO de todas las moléculas estudiadas, son coincidentes en explicar la existencia del "efecto gauche" mediante la estabilización aportada por la donación de electrones desde los pares libres

Figura 8.4: Interacción de un par de electrones libres del $\mathrm{O}$ con el orbital $\sigma^{*}$ del grupo fosforilo. de los $\mathrm{O}$ de los enlaces éster hacia los orbitales $\sigma^{*}$ de los enlaces $\mathrm{P}-\mathrm{O}$ adyacentes. En este mismo sentido, es interesante la proposición que indica que las interacciones por puentes de hidrógeno del tipo $\mathrm{P}=\mathrm{X} \cdots \mathrm{H}-\mathrm{C}(\mathrm{X}=\mathrm{O}, \mathrm{S})$, serían factores estabilizantes de los efectos anoméricos ${ }^{(2)}$.

Sin embargo, este fenómeno podría no es suficiente para explicar las abundancias relativas de cada confórmero, ni por lo tanto la predominancia de este tipo de geometrías en los confórmeros estables de los OPs. Habrá que acudir entonces a un aporte de factores estéricos para completar el análisis. Desde este punto vista, es interesante observar la estabilización del confórmero gauche,-gauche en la serie de calcogenofosfamidatos de dimetilo respecto al confórmero análogo de geometría $\mathrm{C}_{1}$ en la serie de calcogenofosfatos de dimetilo.

Dicha estabilización puede explicarse considerando el impedimento estérico aportado por las nubes electrónicas de los pares libres del tercer oxígeno del éster en el confórmero $\mathrm{C}_{1}$ de los calcogenofosfatos de trimetilo, que en el confórmero $\mathrm{C}_{1}$ de la serie de fosfamidatos está reducido sólo a un par electrónico libre del grupo $\mathrm{NH}_{2}$. Más aún, para la serie de calcogenofosfatos de trimetilo ni siquiera teóricamente fue posible encontrar un confórmero estable con geometría gauche, gauche, -gauche. Esto no es difícil de justificar mediante la observación que en esta geometría existe un fuerte impedimento estérico por la superposición de las nubes electrónicas de metilos adyacentes.

La generalización del comportamiento conformacional descrito para las moléculas estudiadas, depende de la posibilidad de especificar el mismo tipo de 
fenómenos comunes en sistemas en los que aparezcan otro tipo de interacciones o determinantes moleculares particulares y verificar que, aunque aparezcan características secundarias, son aquellas primeras características las que permiten explicar el comportamiento general de cualquier OP.

\section{Sistemas con grandes restricciones}

Una vez que se determinan los factores predominantes en el comportamiento conformacional de las moléculas modelo, es necesario contrastar estas conclusiones con sistemas más complejos. En este sentido, es interesante verificar las hipótesis con moléculas en las cuales estos factores presenten restricciones. En función de ello, dos tipos de sustancias son interesantes; por una lado aquellas moléculas con grandes restricciones estéricas, y por otro lado moléculas con importantes restricciones estereoelectrónicas.

Dentro del primer caso se encuentran aquellos ésteres fosfóricos con grandes sustituyentes, como los fosfatos de triarilo. En estas moléculas, habría grandes impedimentos estéricos por parte de los anillos aromáticos que interferirían con las posiciones gauche adyacentes. De manera los efectos electrónicos que aparezcan por donación de electrones desde los pares libres de los oxígenos del éster hacia orbitales $\sigma^{*}$ de los enlaces $\mathrm{P}-\mathrm{O}$ adyacentes estarían fuertemente restringidos.

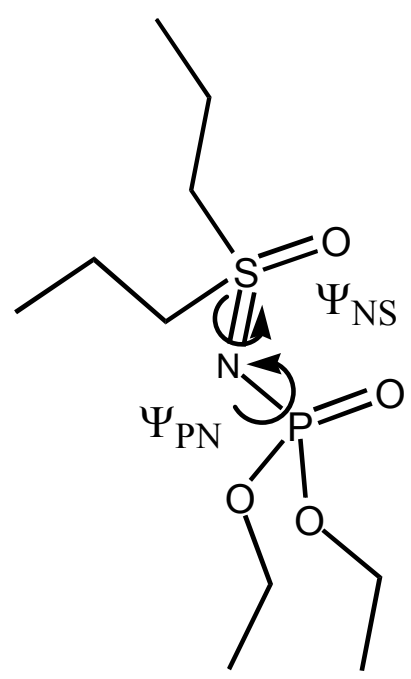

Figura 8.5: Estructura del fosfoiminato de dietil $\mathrm{N}$ dipropilsulfoxido. Se indican los ángulos diedros característicos $\Psi_{\mathrm{PN}}$ y $\Psi_{\mathrm{NS}}$.

Tabla 8.V: Valores energéticos y geométricos para los confórmeros estables de la molécula fosfoiminato de dietil $\mathrm{N}$-dipropil sulfóxido, obtenidas a un nivel HF/6-31G* (ab initio). Los ángulos se expresan en grados.

\begin{tabular}{lcccccccc}
\hline Confórmero & $\begin{array}{c}\text { Energía } \\
\mathrm{kcal} / \mathrm{mol}\end{array}$ & $\begin{array}{c}\text { Abundancia } \\
298 \mathrm{~K}\end{array}$ & $\begin{array}{c}v_{\mathrm{P}=\mathrm{O}} \\
\left(\mathrm{cm}^{-1}\right)\end{array}$ & $\begin{array}{c}v_{\mathrm{S}=\mathrm{O}} \\
\left(\mathrm{cm}^{-1}\right)\end{array}$ & $\Psi_{1}$ & $\Psi_{2}$ & $\Psi_{\mathrm{PN}}$ & $\Psi_{\mathrm{NS}}$ \\
\hline gg-g/-g & 0,00 & 0,69 & 1203 & 1196 & 56 & 46 & -22 & -66 \\
tg-g/-g & 0,48 & 0,31 & 1225 & 1189 & 177 & 49 & -23 & -68 \\
$\mathrm{ggg} / \mathrm{g}$ & 2,88 & 0,01 & \multicolumn{2}{c}{1213} & 41 & 47 & 47 & 44 \\
$\mathrm{gtc} / \mathrm{t}$ & 3,49 & 0,00 & 1208 & 1234 & 46 & 177 & 13 & 150 \\
\hline
\end{tabular}

Puede predecirse que estas moléculas tendrán una estabilización de confórmeros en los que las posiciones trans o gauche fuertemente deformadas son las preferidas. Por ejemplo, para el fosfato de trifenilo se ha descrito isomería conformacional, ${ }^{(5)}$ manifestada en dos bandas de estiramiento $\mathrm{P}=\mathrm{O}$ en fase líquida, ubicadas en 1314 y $1298 \mathrm{~cm}^{-1}$.

\footnotetext{
${ }^{5}$ Mortimer, F.S. Vibrational assignment and rotational isomerism in some simple organic phosphates, Spectrochim.Acta. 9: 270-281 (1957).
} 


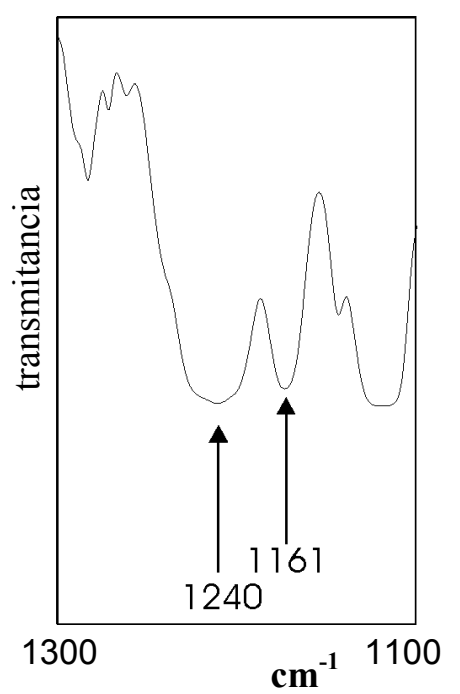

Figura 8.6: Espectro vibracional infrarrojo del fosfoiminato de dietil Ndipropilsulfóxido alrededor de $1200 \mathrm{~cm}^{-1}$, indicando las bandas asignadas al estiramiento $\mathrm{P}=\mathrm{O}$ y $\mathrm{S}=\mathrm{O}$.
En el segundo caso, es interesante el enfoque de moléculas en las que existan sistemas orbitales que impongan restricciones de rotación de ángulos diedros alrededor del átomo de fósforo. En este sentido, hemos realizado algunos estudios preliminares sobre derivados sulfóxido de fosfoiminatos. ${ }^{(6)}$ Se puede observar en estas moléculas un sistema de electrones $\pi$, ubicados sobre el grupo $\mathrm{N}=\mathrm{S}=\mathrm{O}$, que pueden tener un cierto grado de deslocalización hacia el sistema $\pi$ del enlace $\mathrm{P}=\mathrm{X}$, siendo $\mathrm{X}$ cualquier calcógeno (Figura 8.5). Esta estructura impondría una restricción importante a la rotación de los ángulos diedros $\Psi_{\mathrm{PN}} \mathrm{y} \Psi_{\mathrm{NS}}$. En efecto, el resultado de los cálculos ab initio, a un nivel HF/6-31G* (Tabla 8.V), permite describir la existencia de dos confórmeros predominantes y dos confórmeros que, a temperatura ambiente, existen en una muy baja proporción. En los tres confórmeros más abundantes, se observa la presencia de posiciones gauche para ambos diedros analizados.

Más aún, para los dos confórmeros más abundantes, la estructura se rigidiza en valores de $\Psi_{\mathrm{PN}} \sim 20^{\circ}$ y $\Psi_{\mathrm{NS}} \sim 66^{\circ}$, existiendo variaciones sólo en los ángulos diedros de los sustituyentes éster etílico en posiciones gauche y trans, tal como ya fue descrito para las moléculas estudiadas en los Capítulos anteriores. En el espectro vibracional infrarrojo se observa una ancha banda alrededor de $1240 \mathrm{~cm}^{-1}$ y una banda más angosta en $1161 \mathrm{~cm}^{-1}$ (Figura 8.6) que corresponderían respectivamente a los modos vibracionales de estiramiento $\mathrm{P}=\mathrm{O}$ y $\mathrm{S}=\mathrm{O}$. Estas bandas se correlacionan numéricamente bien con los valores teóricos para estos dos modos vibracionales.

Esta correlación se puede extender al hecho que las diferencias entre los números de onda para los dos confórmeros mas abundantes asignados al modo vibracional $v_{\mathrm{P}=\mathrm{O}}$ es de más de $20 \mathrm{~cm}^{-1}$ (banda ancha), mientras que la misma característica para el modo vibracional $v_{\mathrm{O}=\mathrm{S}}$ es de menos de $10 \mathrm{~cm}^{-1}$, lo que se corresponde con una banda más angosta (ver Tabla 8.V). Medidas del espectro vibracional en matrices a distintas temperaturas de deposición, probablemente resuelvan un número de bandas compatible con la coexistencia de, al menos, dos confórmeros.

\footnotetext{
${ }^{6}$ Estas sustancias forman parte de una serie de fosfatoiminas N-sulfóxido sustituidas cuyas síntesis se encuentran en el marco de un estudio, realizado en la Universidad Nacional de la Pampa (Argentina), inédito a la fecha de culminación de este trabajo.
} 
Sin duda, es necesario profundizar el análisis de este tipo de estructuras, pero con los datos preliminares presentados es posible concluir que la variabilidad conformacional de este tipo de moléculas se manifiesta fundamentalmente en las posiciones gauche y trans de los dos restos alquilo, revelándose una relativa rigidez del enlace $\mathrm{P}-\mathrm{N}$.

\section{Conclusiones generales}

Se puede afirmar la preferencia de las posiciones gauche por sobre las trans y de ambas sobre cualquier otra. Esto se ha verificado tanto para las moléculas descritas aquí como en la mayor parte de las referencias consultadas. Como segunda cuestión importante se puede asumir que, en general, son los efectos electrónicos (de atracción) los que determinarían las geometrías estables, mientras que los efectos estéricos (de repulsión) son los que determinarían las abundancias relativas de aquellas conformaciones.

La alineación de los dipolos internos, argumentados como un factor importante para explicar la estabilidad conformacional, pueden asimilarse a un aspecto particular de los efectos estereolectrónicos aquí descritos. Es interesante la presencia de ciertos modos vibracionales que actuarían como sensores de la coexistencia de confórmeros, entre los que se encuentran el modo de estiramiento $\mathrm{P}=\mathrm{X}(\mathrm{X}=\mathrm{O}, \mathrm{S}, \mathrm{Se})$ y el modo de estiramiento $\mathrm{P}-\mathrm{O}$.

Otra generalización importante es el aumento de las barreras de interconversión conformacional en la medida que aumenta el peso atómico del calcógeno del grupo fosforilo. Este hecho se expresa, por ejemplo, en la mayor cantidad de pares de bandas de coexistencia conformacional en los espectros vibracionales de los análogos oxigenados respecto a los análogos de azufre y selenio. Durante este trabajo se ha especulado además acerca de la correlación entre esta característica y el aumento de la complejidad de los espectros ${ }^{1} \mathrm{H}$ RMN.

El paso siguiente en todo este análisis se relaciona con la posibilidad de asociar estos comportamientos generales, con el aporte de elementos para la explicación de los mecanismos de inhibición de las esterasas por parte de los oxofosfatos y los respectivos análogos de azufre y selenio. 
Segunda Parte: Estudios CONFORMACIONALES EN ENTORNOS BIOLÓGICOS Y DETERMINACIONES CINÉTICAS

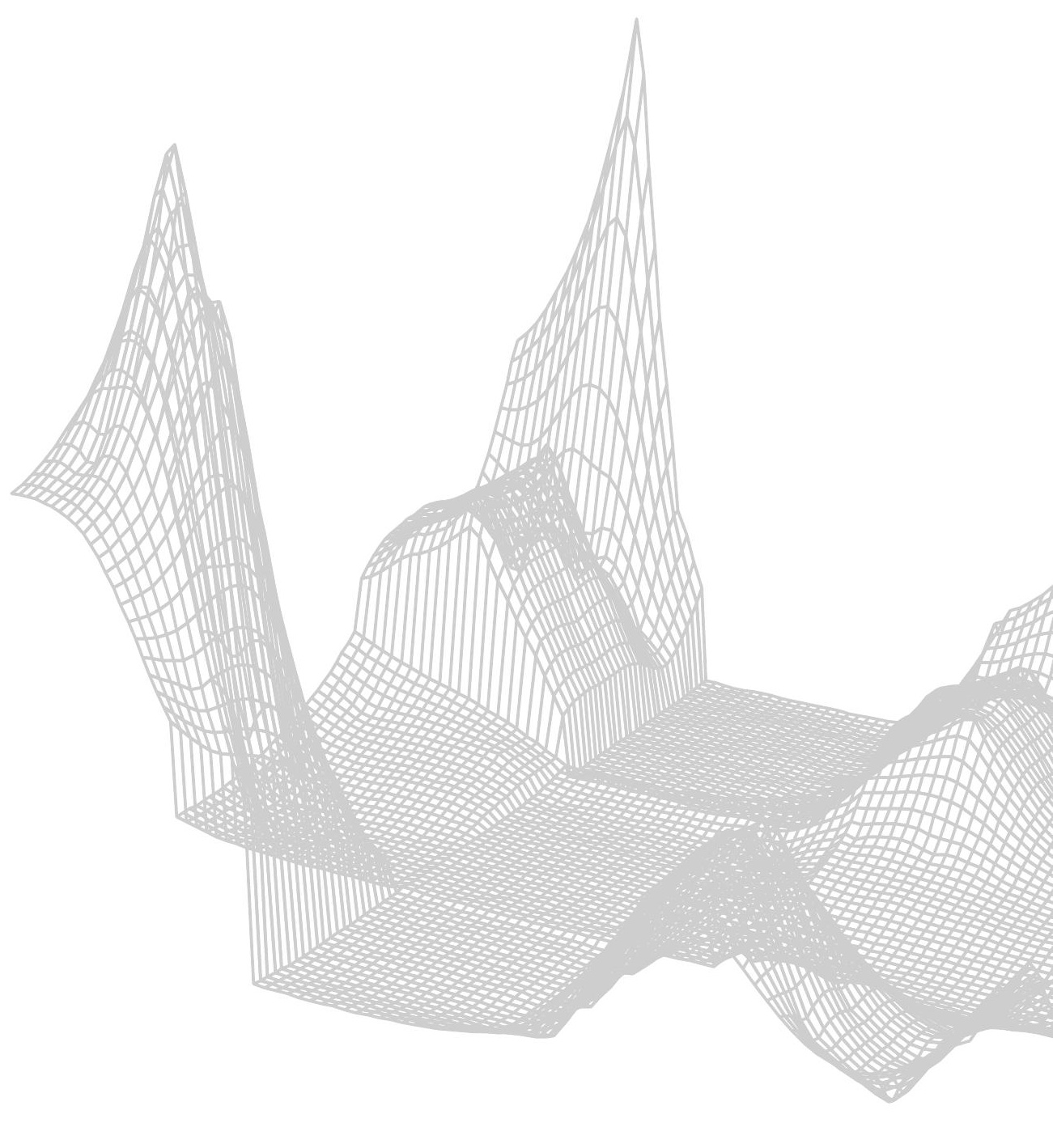





\section{Reseña de la toxicología de los compuestos organofosforados}

Tabla 9.I: Propiedades toxicológicas de algunos OPs, expresadas en la $\mathrm{DL}_{50}(\mathrm{mg} / \mathrm{Kg})$ para ratas macho.

\begin{tabular}{lrr} 
& \multicolumn{2}{c}{$\mathrm{DL}_{50}(1)$} \\
\cline { 2 - 3 } TEPP & Oral & Dérmica \\
Mevinfós & 1,1 & 2,4 \\
Disulfoton & 6,1 & 4,7 \\
Azinfosmetil & 6,8 & 15 \\
Paratión & 13 & 220 \\
Metilparatión & 13 & 21 \\
Diclorvós & 14 & 67 \\
Diazinón & 80 & 107 \\
Dimetoato & 108 & 200 \\
Triclorfón & 215 & 260 \\
Clorotión & 630 & $>2000$ \\
Malatión & 880 & 3000 \\
Ronel & 1375 & $>4444$ \\
& 1250 & $>5000$ \\
\hline
\end{tabular}

La aplicación tecnológica más relevante de los compuestos OPs, como ya lo hemos indicado, está relacionada con su gran capacidad de inhibir la actividad de la AChE de invertebrados y, por lo tanto, de inhabilitar sus funciones neurológicas. Se utilizan entonces ampliamente como plaguicidas. Así mismo, son importantes tóxicos de vertebrados por inhibir la misma enzima y de aquí su uso como sustancias de agresión bélica. Por esta razón, tanto una como otra aplicación encuentran un campo de investigación común. En este sentido, se dispone de un vasto conocimiento de los mecanismos de acción de estos compuestos en los distintos niveles biológicos (molecular, celular, tisular, orgánico y sobre ecosistemas) y para una gran variedad de organismos, fundamentalmente insectos y mamíferos.

Existen distintas clases de colinesterasa: se encuentran presentes en diferentes tejidos y en distintos organismos. En realidad constituyen una familia de enzimas que, catalizando la misma reacción, presentan diferente localización tisular, afinidad de sustratos y función fisiológica. Sin embargo, para muchas de ellas el papel que cumplen no está muy claro. ${ }^{(2)}$ En mamíferos, las principales enzimas son la AChE, con localización en tejido nervioso y eritrocitos, y la colinesterasa plasmática (PChE) o pseudocolinesterasa, que corresponde a un grupo de enzimas que también se encuentran en las células gliales, el plasma y el hígado.

Se muestran en las Tablas 9.1 y $\mathbf{9 . I I}$ las características toxicológicas de una serie de OPs seleccionados. ${ }^{(3)}$ En la actualidad se han dejado de utilizar

\footnotetext{
${ }^{1} \mathrm{DL}_{50}$ o Dosis letal 50\%: corresponde a la dosis de la sustancia que aplicada a una cantidad de individuos, provoca la muerte de la mitad de los mismos. Está expresado en $\mathrm{mg} / \mathrm{Kg}$ para ratas macho.

${ }^{2}$ Ver referencias correspondientes en Introducción.

${ }^{3}$ (a) Heath, D.F. Organophosphorus poisons. Anticholinesterases and related compounds. Part II Biochemistry. Pergamon Press (1961) (b) Murphy, S.D., Lauwerys, R.R. and Cheever, K.L. Comparative acetilcholinesterase action of organophosphorus insecticides in vertebrates, Toxicol. Appl. Pharmacol. 12 :22-35 (1968).
} 
muchos de estos compuestos por su selectividad deficiente sobre plagas y su alta toxicidad para vertebrados.

\section{Mecanismos de acción tóxica}

Bajo condiciones fisiológicas, la $\mathrm{AChE}$ hidroliza a la acetilcolina (ACh), un mediador químico de la transmisión de impulsos nerviosos, en el resto colina y en ácido acético. Un OP inhibidor se puede describir como un grupo fosforilo $(\mathrm{P}=\mathrm{O})$ unido a tres sustituyentes, de los cuales uno es hidrolizable en condiciones biológicas. En presencia de OPs, la AChE es fosforilada y como consecuencia de ello es inhibida. ${ }^{(4)}$ Esto significará una acumulación de ACh en las terminales sinápticas de los nervios parasimpáticos (acción muscarínica), en la placa neuromotora (acción nicotínica) y en el sistema nervioso central (SNC), hecho que es el responsable de los síntomas típicos del envenenamiento agudo por OPs. ${ }^{(5)}$

Se ha avanzado en la descripción de nuevas funciones de la $\mathrm{AChE}{ }^{(6)}$ que, eventualmente, pueden participar de mecanismos alternativos de toxicidad de los OPs. Adicionalmente, se ha sugerido que la fosforilación de otras estructuras celulares puede mediar en mecanismos complementarios de toxicidad que pueden ser relevantes para algunos tipos de OPs. ${ }^{(7)}$

\footnotetext{
4 Aldridge, W.N. The nature of the reaction of organophosphorus compounds and carbamates with esterases, Bull.World.Health.Organ. 44 (1): 25-30 (1971).

5 (a) DuBois, K.P. The toxicity of organophosphorus compounds to mammals, Bull.World.Health.Organ. 44: 233-240 (1971). (b) Namba, T. Cholinesterase inhibition by organophosphorus compounds and its clinical effects, Bull.World.Health.Organ. 44: 289-307 (1971).

${ }^{6}$ Soreq, H. and Seidman, S. Acetylcholinesterase: new roles for an old actor, Nat.Rev.Neurosci. 2 (4): 294-302 (2001).

${ }^{7}$ (a) Pope, C.N. Organophosphorus pesticides: do they all have the same mechanism of toxicity?, J.Toxicol.Environ.Health.B.Crit.Rev. 2 (2): 161181 (1999). (b) Richards, P.G., Johnson, M.K. and Ray, D.E. Identification of acylpeptide hydrolase as a sensitive site for reaction with organophosphorus compounds and a potential target for cognitive enhancing drugs, Mol.Pharmacol. 58 (3): 577-583 (2000). (c) Duysen, E.G. et al. Evidence for nonacetylcholinesterase targets of organophosphorus nerve agent: supersensitivity of acetylcholinesterase knockout mouse to VX lethality, J Pharmacol Exp Ther 299 (2): 528-535 (2001). (d) Li, Q. et al. Organophosphorus pesticides markedly inhibit the activities of natural killer, cytotoxic $\mathrm{T}$ lymphocyte and lymphokineactivated killer: a proposed inhibiting mechanism via granzyme inhibition, Toxicology 172 (3): 181-190 (2002).
} 
Figura 9.1: ${ }^{(8)}$ Cada una de las fases del proceso de acción tóxica, determina cómo finalmente se expresarán los efectos tóxicos. Se discrimina entre la interacción con dianas relevantes (T1) e interacciones sin cambios (T2).

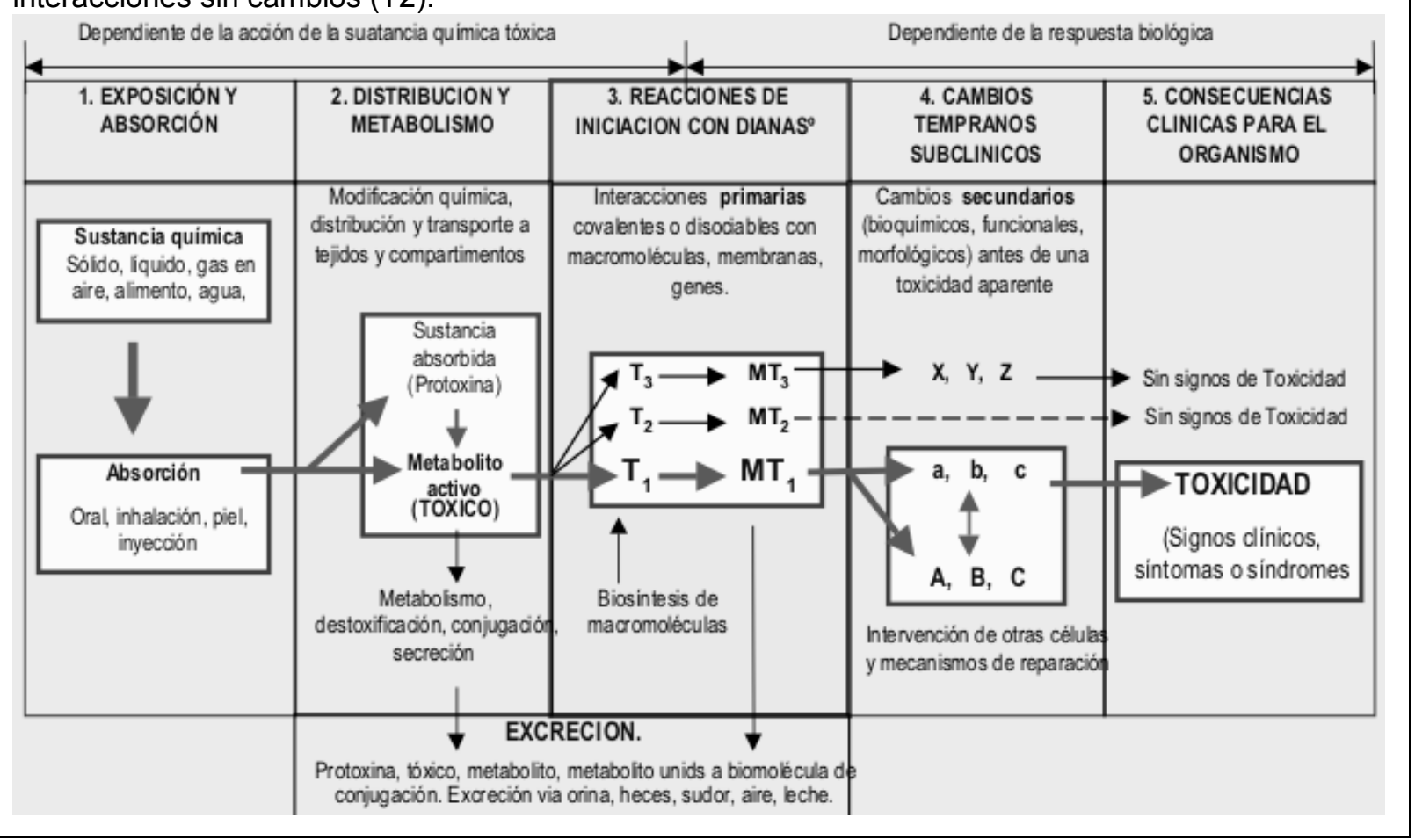

En el mecanismo de acción tóxica de los OPs es necesario distinguir cada una de las fases del proceso de manera de poder evaluar el contexto en el que se estudiará cada uno de los aspectos relacionados a él. Es decir, aunque en forma relevante el mecanismo está determinado por la inhibición de la AChE, cómo es que finalmente ocurrirá una intoxicación, o no, dependerá de todos los aspectos en los que está involucrado el OP, en relación con el organismo expuesto y las circunstancias en las que ocurre la exposición.

Es por ello que la forma de absorción, la distribución tisular, las modificaciones metabólicas, los procesos de destoxicación y eliminación de la sustancia en cuestión deben ser tenidas en cuenta en la discusión global acerca de la manera en que una sustancia ejerce su acción deletérea sobre un organismo (Figura 9.1).

Entonces, aunque el presente trabajo presta atención solamente a algunos de los eventos relacionados a la interacción del los OPs con la AChE, debemos al menos

\footnotetext{
8 Extraido de Vilanova et al. Curso de Experto Universitario en Toxicologia, adaptado del esquema de Aldridge. En: Toxicología de Postgrado. M. Repetto. ed. Área de Toxicología. Universidad de Sevilla. CD-ROM. Sevilla (2003).
} 
mencionar algunos de los otros procesos relevantes involucrados en la toxicidad de estos compuestos.

Las primeras investigaciones sobre el paratión y su derivado oxigenado, el paraoxón, mostraron que aparentemente además de conferir mayor estabilidad a la hidrólisis no enzimática, la sustitución $\mathrm{de}=\mathrm{S}$ por $=\mathrm{O}$ en el compuesto fosforado altera sus propiedades tóxicas. ${ }^{(5 a)} \mathrm{El}$ paratión mostró ser menos tóxico que el paraoxón para los animales y algunos factores que afectan la toxicidad en ratas del paratión no afectan la del paraoxón, aunque ambos compuestos inhiben la ACh y producen signos colinérgicos agudos similares.

Se demuestra entonces que existe una marcada relación entre la toxicidad y la biotransformación de los OPs. Muchos de ellos necesitan de una previa transformación in vivo para ejercer su acción. Un caso típico es el de los ésteres tiofosfóricos, que necesitan una oxidación primaria para adquirir poder inhibitorio. En el caso de los fosfamidatos se han propuesto una variedad de mecanismos de bioactivación que puedan explicar las diferencias entre los resultados de las pruebas in vitro y los ensayos in vivo. ${ }^{(11)}$

Por ello existe una clasificación de OPs tóxicos que distingue a los inhibidores indirectos de los inhibidores directos de la $\mathrm{AChE}$, según necesite o no de una transformación in vivo antes de actuar sobre las enzimas. Esta clasificación es útil en la práctica, pues frente a una exposición a inhibidores directos, los signos y síntomas de la intoxicación aparecen durante la misma, mientras que se puede manifestar la intoxicación por un inhibidor indirecto sólo tiempo después de haber cesado la exposición.

\footnotetext{
${ }^{9}$ NNE o Nivel de No Efecto: corresponde a la máxima dosis de la sustancia que aplicada a un animal, no provoca signos ni síntomas de intoxicación. Está expresada en $\mathrm{mg} / \mathrm{Kg}$ /día para ratas.

${ }^{10}$ IDA o Ingesta Diaria Aceptable: corresponde a la máxima cantidad de sustancia que, ingerida diariamente, no provoca signos ni síntomas de intoxicación en humanos. Está expresada en $\mathrm{mg} / \mathrm{Kg}$.

${ }^{11}$ (a) Mahajna, M., Quistad, G.B. and Casida, J.E. Acephate insecticide toxicity: safety conferred by inhibition of the bioactivating carboxyamidase by the metabolite methamidophos, Chem.Res.Toxicol. 10 (1): 64-69 (1997). (b) Mahajna, M. and Casida, J.E. Oxidative bioactivation of methamidophos insecticide: synthesis of $\mathrm{N}$ hydroxymethamidophos (a candidate metabolite) and its proposed alternative reactions involving $\mathrm{N} \rightarrow \mathrm{O}$ rearrangement or fragmentation through a metaphosphate analogue, Chem.Res.Toxicol. 11 (1): 26-34 (1998). (c) Miyamoto, T. et al. A Novel Bioactivation Mechanism of Phosphoramidate Insecticides, Pestic.Biochem.Physiol. 63 (3): 151-162 (1999).
}

Tabla 9.II ${ }^{(3)}$ : Propiedades toxicológicas de algunos OPs.

\begin{tabular}{lll}
\hline & $N^{(9)}$ & IDA $^{(70)}$ \\
\hline Azinfosmetil & 0,125 & 0,0025 \\
Paratión & 0,05 & 0,005 \\
Cholfenvinfós & 0,05 & 0,002 \\
Diclorvós & 0,5 & 0,004 \\
Diazinón & 0,1 & 0,002 \\
Dimetoato & 0,4 & 0,02 \\
Triclorfón & 2,5 & 0,01 \\
Malatión & 0,5 & 0,02 \\
Ronel & 0,5 & 0,01 \\
\hline
\end{tabular}


Para la mayoría de los OPs, la exposición dérmica y la subsiguiente absorción a través de la piel intacta representa la ruta de ingreso más importante, en el caso de la exposición ocupacional. La vía oral es importante en el caso de las intoxicaciones accidentales o en intentos de suicidio. La intoxicación por vía oral puede también ocurrir como resultado de practicas de higiene deficientes o procedimientos inseguros de trabajo. La vía inhalatoria es la menos importante, y depende en gran medida de la volatilidad del compuesto, el tipo de formulación y la técnica de aplicación.

Aunque el efecto neurotóxico más frecuentemente observado corresponde al colinérgico agudo, se describen mecanismos de toxicidad retardada con dos variantes: el síndrome intermedio y la polineuropatía retardada. Además se ha reportado un síndrome extrapiramidal ${ }^{(12)}$ aun no muy bien descrito.

Algunos OPs pueden producir neuropatía retardada en animales de experimentación y se conoce su correlato en reportes en humanos aunque sólo por exposición aguda y nunca por exposición crónica. ${ }^{(13)}$ Los síntomas de neuropatía retardada aparecen una a tres semanas luego de la exposición, con temblores y calambres musculares que comienzan en las piernas y pueden eventualmente involucrar a los brazos. El examen de la electromiografía muestra un patrón de denervación parcial de los músculos afectados y la electroneurografía muestra un marcado retardo del tiempo de latencia de la terminal motora con una conducción máxima motora normal o muy poco reducida en la mayor parte de los casos.

Se describe la existencia de una esterasa neurotóxica (NTE), que corresponde a una proteína de membrana presente en las terminales axonales, aunque no se conoce su función en este tejido. Su inhibición sería la responsable de los síntomas de la neuropatía retardada, ${ }^{(14)}$ aunque hay datos

12 Hsieh, B.H. et al. Acetylcholinesterase inhibition and the extrapyramidal syndrome: a review of the neurotoxicity of organophosphate, Neurotoxicol. 22 (4): 423-427 (2001).

${ }_{13}$ (a) Aldridge, W.N. and Johnson, M.K. Side effects of organophosphorus compounds: delayed neurotoxicity, Bull .World. Health. Organ. 44: 259-263 (1971). (b) Abou-Donia, M.B. Organophosphorus ester-induced delayed neurotoxicity, Ann. Rev. Pharmacol. Toxicol. 21: 511-548 (1981). (c) Eray, D., Kamer, U.M. and Ozden, A. Organophosphate-induced delayed polyneuropathy, J. Trop. Pediatr. 41 (3): 189 (1995).

${ }^{14}$ Johnson, M.K. Organophosphates and delayed neuropathy-is NTE alive and well?, Toxicol.Appl.Pharmacol. 102 (3): 385-399 (1990). 
en contrario ${ }^{(15)}$ y se sugieren mecanismos alternativos como el de la fosforilación generalizada del citoesqueleto neuronal. En base a resultados in vitro, se han desarrollado diversos procedimientos para caracterizar a los OPs AChE inhibidores y a los NTE inhibidores. ${ }^{(16)}$

Respecto a los efectos sobre el sistema inmunitario, se han descrito procesos de inmunotoxicidad en exposiciones crónicas a OPs ${ }^{(17)}$ y por parte de OPs que pueden encontrarse como impurezas en los formulados de pesticidas frecuentemente usados. ${ }^{(18)}$

Existen datos incompletos respecto a la capacidad de los OPs de interaccionar directamente con el material genético, aunque estas interacciones son dependientes de la naturaleza de los grupos sustituyentes, antes que del grupo fosfato en sí. Sin embargo, se ha demostrado que los OPs están involucrados en la producción de especies oxigenadas reactivas que participan de la destrucción del DNA, pudiendo desencadenar, de esta manera, mecanismos tóxicos de acción retardada. Así mismo se ha demostrado su inducción de la actividad de la proteinquinasa $C{ }^{(19)}$ enzima que cumple un importante papel mediador en procesos intracelulares.

Respecto de los efectos a largo plazo, se han realizado pruebas de mutagenicidad, ${ }^{(20)}$ genotoxicidad ${ }^{(21)} \mathrm{y}$

15 Davis, S.L. et al. Organophosphorus-induced neurotoxicity in the absence of neuropathy target esterase inhibition: the effects of triphenyl phosphine in the European ferret, Toxicol.Sci. 49 (1): 78-85 (1999).

${ }^{16}$ (a) Ehrich, M., Correll, L. and Veronesi, B. Acetylcholinesterase and neuropathy target esterase inhibitions in neuroblastoma cells to distinguish organophosphorus compounds causing acute and delayed neurotoxicity, Fundam.Appl.Toxicol. 38 (1): 55-63 (1997). (b) McConnell, R. et al. Organophosphate neuropathy due to methamidophos: biochemical and neurophysiological markers, Arch.Toxicol. 73 (6): 296-300 (1999).

17 (a) Luster, M.I. and Blank, J.A. Molecular and cellular basis of chemically induced inmunotoxicity, Ann.Rev.Pharmacol.Toxicol. 27: 2349 (1987). (b) Ray, D.E. and Richards, P.G. The potential effects of chronic, low-dose exposure to organophosphates, Toxicol.Lett. 120: 343351 (2001).

18 Rodgers, K.E., Imamura, T. and Devens, B.H. Organophosphorus pesticide immunotoxicity: effects of O,O,S-trimethyl phosphorothioate on cellular and humoral immune response systems, Immunopharmacology 12 (3): 193-202 (1986).

${ }^{19}$ Bagchi, D. et al. Comparative in vitro and in vivo protein kinase C activation by selected pesticides and transition metal salts, Toxicol.Lett. 91 (1): 31-37 (1997).

${ }^{20}$ Moriya, M. et al. Further mutagenicity studies on pesticides in bacterial reversion assay systems, Mutat.Res. 116 (3-4): 185-216 (1983).

${ }^{21}$ (a) Osaba, L. et al. Genotoxicity testing of six insecticides in two crosses of the Drosophila wing spot test, Mutat.Res. 439 (1): 49-61 
carcinogenicidad ${ }^{(22)}$ de OPs en distintos modelos. En la mayoría de los casos se han observado resultados negativos, aunque se ha descrito que el TMP ${ }^{(23)}$ y algunos otros OPs ${ }^{(21 b)}$ son mutagénicos.

\section{Clínica de la intoxicación por OP ${ }^{(24)}$}

Los efectos agudos de una intoxicación por OPs aparecen inmediatamente luego de una corta exposición a estos compuestos por vía dérmica, usualmente dentro de las dos a tres horas de iniciada la misma, sin embargo, es posible observar los efectos de la intoxicación dentro de la hora siguiente a la exposición, dependiendo de las circunstancias del caso. Algunos OPs pueden ser retenidos en pequeñas concentraciones en el tejido adiposo y esto determina la aparición de síntomas después de las veinticuatro horas de la exposición aguda.

Los signos y síntomas se distinguen según afecten al SNC o al sistema nervioso periférico (SNP). Entre los síntomas y signos manifestados a partir de la afectación del SNP se diferencian efectos de tipo muscarínico y efectos de tipo nicotínico.

Los efectos muscarínicos incluyen el incremento de la sudoración, salivación y lacrimación, broncoconstricción, e incremento de la secreción de las glándulas bronquiales, espasmos abdominales con vómitos, diarrea y bradicardia. Los efectos nicotínicos incluyen taquicardia y fasciculación muscular o temblor de los músculos finos. En casos más graves se afectan el diafragma y los músculos respiratorios que se constituyen en la causa primaria de la muerte.

(1999). (b) Das, P. and John, G. Induction of sister chromatid exchanges and chromosome aberrations in vivo in Etroplus suratensis (Bloch) following exposure to organophosphorus pesticides, Toxicol.Lett. 104 (12): 111-116 (1999).

${ }^{22}$ (a) Kato, T. et al. Lack of promoting activity of four pesticides on induction of preneoplastic liver cell foci in rats, Teratog. Carcinog.Mutagen. 15 (5): 251-257 (1995). (b) Bomhard, E.M. et al. Trimethylphosphate: a 30-month chronic toxicity/carcinogenicity study in Wistar rats with administration in drinking water, Fundam.Appl.Toxicol. 40 (1): 75-89 (1997).

${ }^{23}$ Connor, T.H. The mutagenicity of trimethylphosphate, Mutat.Res. 65 (2): 121-131 (1979).

${ }^{24}$ Aunque existen descripciones completas en la mayoría de los textos de toxicología clínica, se encuentran muy buenas revisones en: (a) Marrs, T.C. Organophosphate poisoning, Pharmacol.Ther. 58 (1): 51-66 (1993). (b) Jeyaratnam, J. and Maroni, M. Organophosphorous compounds, Toxicology 91 (1): 15-27 (1994). 
Los síntomas del SNC incluyen dolor de cabeza, ligero cansancio, vértigos, ansiedad, confusión, además de signos como convulsiones, depresión del centro respiratorio y coma. La duración e intensidad de los signos y síntomas dependen del compuesto OP involucrado y de la ruta y nivel de exposición.

Los primeros síntomas usualmente son náuseas, dolor de cabeza, vértigo, cansancio y visión borrosa. Dependiendo de la severidad de la intoxicación, los efectos pueden manifestarse en vómitos, dolor abdominal, diarrea, salivación y sudoración abundante. El empeoramiento progresivo continúa con temblores musculares que generalmente comienza en los párpados y en la lengua, siguiendo con convulsiones generalizadas y finalmente parálisis. En los estados finales, hay vasoconstricción, broncoconstricción e hipersecreción bronquial para finalmente observarse parálisis, convulsiones, depresión respiratoria y coma. En una intoxicación aguda por OP la inmediata causa de la muerte es la asfixia resultante de la depresión respiratoria.

El diagnóstico de la intoxicación por OPs se basa generalmente en dos aspectos: por un lado una historia de exposición a compuestos OPs en las seis horas previas a la aparición de los signos o síntomas de intoxicación y por otro lado la disminución de los niveles de actividad de la AChE eritrocitaria o de la colinesterasa sérica.

Existe una buena correlación entre los niveles de inhibición de la AChE y la progresión de la intoxicación. Esta correlación puede ser deficiente o directamente no existir luego de una exposición aguda con niveles de exposición crónica previas. Así, los síntomas de intoxicación pueden aparecer recién luego de una inhibición de $85-90 \%$ de los niveles de AChE de preexposición, mientras que con un 60$70 \%$ de inhibición, en un evento único y agudo de exposición, es suficiente para que aparezcan las primeras manifestaciones de una intoxicación.

Se han intentado encontrar correlaciones entre la exposición a OPs y otros marcadores biológicos de naturaleza cínica ${ }^{(25)}$ o bioquímica. ${ }^{(26)}$ En general, estos no han mostrado

\footnotetext{
${ }^{25}$ (a) Santander, J.L. et al. Hallazgos electrofisiologicos en trabajadores expuestos a organofosforados, Rev.Cubana.Hig.Epidemiol. 26 (2): 74-84 (1988). (b) Srivastava, A.K. et al. Clinical, biochemical and neurobehavioural studies of workers engaged in the manufacture of quinalphos, Food.Chem.Toxicol. 38 (1): 65-69 (2000).
} 
la sensibilidad y sencillez de la determinación de la AChE eritrocitaria. $^{(27)}$

Sin embargo, recientemente se ha sugerido que la egasina, una proteína accesoria de la $\beta$-glucuronidasa microsomal hepática, puede ser un marcador plasmático mucho más sensible y específico para evaluar la exposición a OPs, debido a su marcado incremento pocas horas luego de la exposición a estos compuestos. ${ }^{(28)}$ No se han diseñado aún sistemas validados, con la suficiente practicidad y desempeño necesarios para que este marcador pueda ser utilizado en la toxicología clínica de rutina.

\section{Consideraciones finales}

Cuando se realiza una descripción de la toxicidad de los compuestos OPs, nos estamos refiriendo a una cantidad de eventos que involucran a distintos compartimentos orgánicos, toxicocinéticas parciales características, biotransformaciones e interacciones con moléculas que comprenden o no a la enzima diana y que, en conjunto, son las que determinan la capacidad toxica de estas sustancias.

Esto implica que las características de la interacción con la molécula diana, refiriéndonos en particular a la AChE, no es el único factor determinante de cuan tóxico es un $\mathrm{OP} u$ otro. De hecho, otro tipo de interacciones y procesos pueden ser igualmente importantes para el transcurso de una intoxicación en un determinado organismo. No es menor, por ejemplo, la importancia de la actividad de las enzimas que

${ }^{26}$ (a) Panemangalore, M., Dowla, H.A. and Byers, M.E. Occupational exposure to agricultural chemicals: effect on the activities of some enzymes in the blood of farm workers, Int.Arch.Occup.Environ.Health $\mathbf{7 2}$ (2): 84-88 (1999). (b) Azaroff, L.S. Biomarkers of exposure to organophosphorous insecticides among farmers' families in rural El Salvador: factors associated with exposure, Environ.Res. 80 (2 Pt 1): 138147 (1999).

27 (a) Podolak, M. and Panasiuk, L. Biological indicators for the assessment of human exposure to organophosphorous compounds, Przegl.Lek. 54 (10): 719-722 (1997). (b) Mason, H.J. The recovery of plasma cholinesterase and erythrocyte acetylcholinesterase activity in workers after over-exposure to dichlorvos, Occup.Med.(Lond.). 50 (5): 343-347 (2000). (c) Nigg, H.N. and Knaak, J.B. Blood cholinesterases as human biomarkers of organophosphorus pesticide exposure, Rev.Environ.Contam.Toxicol. 163: 29-111 (2000).

${ }^{28}$ Satoh, T. et al. Toxicological significance in the cleavage of esterase- $\beta$ glucuronidase complex in liver microsomes by organophosphorus compounds, Chem.Biol.Interact. 119-120 : 471-478 (1999). 
son capaces de hidrolizar a los OPs antes que estos lleguen a interaccionar con la $\mathrm{AChE} .{ }^{(29)}$

Pero si es cierto que en el proceso de intoxicación por OPs, la inhibición de la AChE es un proceso central. Sin embargo, no debemos perder de vista la diferencia entre la capacidad inhibitoria de un OP y su toxicidad. En este trabajo sólo nos estamos preocupando de algunos aspectos asociados directamente al primero de estos factores.

\footnotetext{
${ }^{29}$ (a) Sogorb, M.A., Pla, A. and Vilanova, E. The esterases hydrolising organophosphorus compounds: an efficient detoxification mechanism, Rev.Toxicol. 13: $43-48$ (1996). (b) Vilanova, E. and Sogorb, M.A. The role of phosphotriesterases in the detoxication of organophosphorus compounds, Crit.Rev.Toxicol. 29 (1): 21-57 (1999).
} 


\section{0 \\ Acetilcolinesterasa: caracterización del sitio activo}

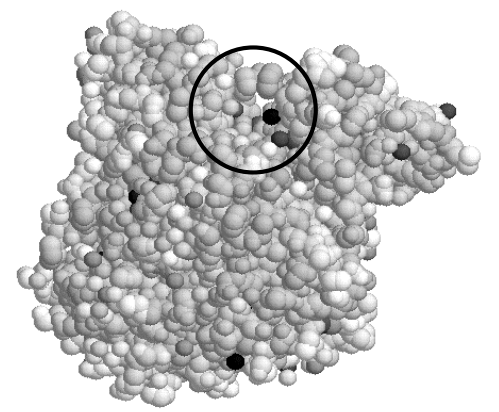

Figura 10.1: Modelo tridimensional de la AChE de anguila. ${ }^{(10 b)}$ Se indica la posición del bolsillo hidrofóbico.
La inhibición de la acetilcolinesterasa (AChE) es un proceso complejo que se puede describir desde dos puntos de vista: el cinético y el mecánico - molecular. En el caso de los OPs, la descripción cinética implica la elaboración de modelos que den cuenta del carácter irreversible de la reacción. Esta cuestión se abordará en el Capitulo 12.

La descripción desde el punto de vista de la mecánica de la inhibición, necesita de una correcta interpretación de las estructuras involucradas en el proceso. Esto implica, por un lado, un conocimiento de la arquitectura del sitio activo de la AChE, es decir, no sólo de las estructuras moleculares, sino también de los procesos dinámicos que se suceden en el transcurso de la interacción con sustratos e inhibidores. ${ }^{(1)}$

Pero por otro lado, es necesario reconocer el papel del comportamiento de las moléculas que interaccionan con este sitio activo. Ya hemos visto que existe una importante riqueza conformacional en moléculas del tipo de los OPs.

El problema está en identificar si estas características, descritas para entornos simétricos (en el vacío o en solución), resultan también en variabilidad conformacional en un sitio restringido o asimétrico. Ambas características, la de asimetría y la de restricción estérica, están presentes en el sitio activo de la AChE y nuestro objetivo es evaluar la magnitud con la que estos efectos conformacionales influyen en los mecanismos de inhibición.

En esta sección se hará una descripción de los avances realizados en el conocimiento de los mecanismos moleculares de catálisis e inhibición y se intentará aportar

\footnotetext{
${ }^{1}$ Ordentlich, A. et al. The architecture of human acetylcholinesterase active center probed by interactions with selected organophosphate inhibitors, J.Biol.Chem. 271 (20): 11953-11962 (1996).
} 
elementos al problema conformacional en este entorno biológico.

\section{Dinámica del sitio activo de la $\mathrm{AChE}$}

La hidrólisis del sustrato natural por parte de la AChE, la acetilcolina (ACh), ocurre mediante un mecanismo catalítico que involucra tres etapas: ${ }^{(2)}$ (a) interacción inicial del sitio activo con el éster y formación del complejo activado que involucra a un hidroxilo serínico del sitio activo de la proteína, (b) hidrólisis del enlace éster con formación de un enlace covalente entre el sustrato y el oxígeno serínico y (c) hidrólisis final con restitución de la actividad enzimática, en la que participarían moléculas de agua fijas en los entornos del hidroxilo serínico. De la misma manera, se han descrito varios otros subsitios en el sitio activo que participan en mayor o menor medida en cada una de estas etapas ${ }^{(3)}$ y que tienen individualmente sus características cinéticas definidas.

En el proceso de inhibición de la enzima con OPs, se describen etapas similares, ${ }^{(4)}$ pero la última de ellas tiene una cinética tal que, en condiciones fisiológicas, la unión del fosforado con la enzima implica la formación de un enlace covalente estable que da lugar a una inhibición irreversible. Sin embargo, existe evidencia que el proceso puede ser más complejo, dado que existirían interacciones con los sitios periféricos alternativos al sitio principal. ${ }^{(5)} \mathrm{La}$

2 (a) Rosenberry, T.L. Acetylcholinesterase, Adv. Enzymol. Relat. Areas. Mol. Biol. 43: 103-218 (1975). (b) Barnett, P. and Rosenberry, T.L. Catalysis by acetylcholinesterase. Acceleration of the hydrolysis of neutral acetic acid esters by certain aromatic cations, J.Biol.Chem. 252 (20): 7200-7206 (1977). (c) Rosenberry, T.L., Barnett, P. and Mays, C. Acetylcholinesterase, Methods.Enzymol. 82 Pt A: 325-339 (1982).

3 Barnett, P. and Rosenberry, T.L. Functional identity of catalytic subunits of acetylcholinesterase, Biochim.Biophys.Acta 567 (1): 154 160 (1979).

${ }^{4}$ (a) Rosenberry, T.L. and Bernhard, S.A. Studies of catalysis by acetylcholinesterase. Synergistic effects of inhibitors during the hydrolysis of acetic acid esters, Biochem. 11 (23): 4308-4321 (1972). (b) Andersen, R.A. et al. Inhibition of acetylcholinesterase from different species by organophosphorus compounds, carmabates and methylsulphonylfluoride, Gen.Pharmacol. 8: $331-334$ (1977). (c) Fukuto, T.R. Mechanism of action of organophosphorus and carbamate insecticides, Environ.Health Perspect. 87: 245-254 (1990).

5 (a) Friboulet, A. et al. Interaction of an organophosphate with a peripheral site on acetylcholinesterase, Biochem. 29 (4): 914-920 (1990). (b) Radic, Z., Reiner, E. and Taylor, P. Role of the peripheral anionic site on acetylcholinesterase: inhibition by substrates and 
presencia de un sitio de ligando periférico es el que determina la interacción de ciertos inhibidores no competitivos ${ }^{(6)}$ y se ha sugerido que es crítico en el mecanismo de catálisis principal, participando en un primer paso de interacción del sustrato con la enzima ${ }^{(7)}$. Este sitio periférico principal tiene su centro en el resto $\operatorname{Asp}^{74}$. (8)

¿Por qué la interacción con la ACh y sustratos similares regenera al enzima y la interacción con OPs da lugar a la formación de un enlace covalente estable en condiciones fisiológicas? Esta interrogante ha sido respondida con mayor o menor suerte mediante el uso de distintas metodologías que corroboran distintas hipótesis. Cada una de ellas le asigna importancia alternativamente a las características electrónicas, polares, estéricas y/o energéticas de las moléculas involucradas.

Sólo en los últimos años se han descrito con cierta precisión las características estructurales generales de la $\mathrm{AChE}^{\left({ }^{(9)}\right.}$ y se ha dilucidado la estructura tridimensional

coumarin derivatives, Mol.Pharmacol. 39 (1): 98-104 (1991). (c) Selwood, T. et al. Parallel mechanisms in acetylcholinesterasecatalyzed hydrolysis choline esters, J.Am.Chem.Soc. 115: 10477-10482 (1993). (d) Barak, D. et al. Acetylcholinesterase peripheral anionic site degeneracy conferred by amino acid arrays sharing a common core, J.Biol.Chem. 269 (9): 6296-6305 (1994). (e) Lin, G., Lai, C.Y. and Liao, W.C. Molecular recognition by acetylcholinesterase at the peripheral anionic site: structure-activity relationships for inhibitions by aryl carbamates, Bioorg.Med.Chem. 7 (12): 2683-2689 (1999).

6 Mallender, W.D., Szegletes, T. and Rosenberry, T.L. Organophosphorylation of acetylcholinesterase in the presence of peripheral site ligands. Distinct effects of propidium and fasciculin, J.Biol.Chem. 274 (13): 8491-8499 (1999).

7 (a) Szegletes, T. et al. Substrate binding to the peripheral site of acetylcholinesterase initiates enzymatic catalysis. Substrate inhibition arises as a secondary effect, Biochem. 38 (1): 122-133 (1999). (b) De, F. et al. Thioflavin $\mathrm{T}$ is a fluorescent probe of the acetylcholinesterase peripheral site that reveals conformational interactions between the peripheral and acylation sites, J.Biol.Chem. 276 (26): 23282-23287 (2001).

8 Mallender, W.D., Szegletes, T. and Rosenberry, T.L. Acetylthiocholine binds to $\mathrm{Asp}^{74}$ at the peripheral site of human acetylcholinesterase as the first step in the catalytic pathway, Biochem. 39 (26): 7753-7763 (2000).

${ }^{9}$ (a) Schumacher, M. et al. Primary structure of torpedo californica acetylcholinesterase deduced from its cDNA sequence, Nat. 319: 407 (1986). (b) Aslanian, D. et al. Raman spectroscopic study on the conformation of $11 \mathrm{~S}$ form acetylcholinesterase from Torpedo californica, FEBS Lett. 219 (1): 202-206 (1987). (c) Gnagey, A.L., Forte, M. and Rosenberry, T.L. Isolation and characterization of acetylcholinesterase from Drosophila, J.Biol.Chem. 262 (27): 1329013298 (1987). 
tanto de la proteína completa, como de su sitio activo ${ }^{(10)}$ y de las estructuras de los complejos formados con sustratos e inhibidores. ${ }^{(11)} \mathrm{Se}$ han reconocido los restos aminoacídicos que participan directa o indirectamente del mecanismo de catálisis del sustrato natural ${ }^{(12)}$ y de inhibición por OPs y otras sustancias. ${ }^{(13)}$ Esta descripción se ha hecho mediante el auxilio de distintas técnicas fisicoquímicas y metodologías de biología molecular.

Así mismo, se han podido descifrar algunas de las razones que determinan los requerimientos moleculares (estéricos, electrónicos o energéticos) que definen a un buen o mal inhibidor.

El sitio activo está descrito por distintos grupos moleculares ${ }^{(14)}$ que dan cuenta de cada uno de los procesos

${ }^{10}$ (a) Sussman, J.L. et al. Purification and crystallization of a dimeric form of acetylcholinesterase from Torpedo californica subsequent to solubilisation with phosphatidylinositol-specific phospholipase C. J.Mol.Biol. 203:821-823 (1988). (b) Sussman, J.L. et al. Atomic structure of acetylcholinesterase from Torpedo californica: a prototypic acetylcholine- binding protein, Scien. 253 (5022): 872-879 (1991).

${ }^{11}$ (a) Vilanova, E., Johnson, M.K. and Vicedo, J.L. Interaction of some unsubstitued phosphoramidate analogs of methamidophos (O,Sdimethyl phosphorothioamidate) with acetylcholinesterase and neuropathy target esterase of hen brain, Pestic.Biochem.Physiol. 28: 224-238 (1987). (b) Kovach, I.M. Structure and dynamics of serine hydrolase-organophosphate adducts, J.Enzym.Inhib. 2 (3): 199-208 (1988).

${ }^{12}$ (a) Rosenberry, T.L. and Bernhard, S.A. Studies of catalysis by acetylcholinesterase. I. Fluorescent titration with a carbamoylating agent, Biochem. 10 (22): $4114-4120$ (1971). (b) Hosea, N.A. et al. $\mathrm{Asp}^{74}$ as a primary determinant in acetylcholinesterase giverning specificity to cationic organophosphates, Biochem. 35: 10995-11004 (1996).

${ }^{13}$ (a) Aldridge, W.N. The nature of the reaction of organophosphorus compounds and carbamates with esterases, Bull.World.Health.Organ. 44 (1): 25-30 (1971). (b) Rosenberry, T.L. and Neumann, E. Interaction of ligands with acetylcholinesterase. Use of temperaturejump relaxation kinetics in the binding of specific fluorescent ligands, Biochem. 16 (17): 3870-3878 (1977). (c) Segall, Y. et al. Direct observation and elucidation of the structures of aged and nonaged phosphorylated cholinesterases by ${ }^{31} \mathrm{P}$ NMR spectroscopy, Biochem. 32 (49): 13441-13450 (1993).

${ }^{14}$ (a) Shafferman, A. et al. Mutagenesis of human acetylcholinesterase. Identification of residues involved in catalytic activity and in polypeptide folding, J.Biol.Chem. 267 (25): 17640-17648 (1992). (b) Shafferman, A. et al. Substrate inhibition of acetylcholinesterase: residues affecting signal transduction from the surface to the catalytic center, EMBO J. 11 (10): 3561-3568 (1992). (c) Ordentlich, A. et al. Dissection of the human acetylcholinesterase active center determinants of substrate specificity. Identification of residues 
verificados durante la catálisis (Tabla 10.I). Aunque en general las distintas variantes de la AChE presentan parámetros cinéticos diferentes, ${ }^{(15)}$ el mecanismo molecular de la catálisis básicamente es el mismo, lo que es consecuencia de las similitudes en las estructuras de los sitios activos correspondientes.

La AChE es una enzima peculiar en muchos sentidos. Como máquina catalítica es muy eficiente, actuando cerca del límite biológico de catálisis, ${ }^{(2 a)}$ de manera que las reacciones con sustratos naturales e inhibidores están limitadas casi fundamentalmente por procesos de difusión. Además, es una proteína que tiene un momento dipolar muy elevado, estimado en valores de entre 1100 y $1600 \mathrm{D}$ para distintos tipos de colinesterasa (16) y medido en $1000 \mathrm{D}$ para AChE de serpiente (Bungarus fasciculatus). ${ }^{(17)}$ Esta característica es poco común para una enzima de este tipo y sería un fenómeno asociado al aumento de la energía de interacción con moléculas cargadas. ${ }^{(18)}$

Se describen además fluctuaciones del campo eléctrico alrededor del enzima que podrían contribuir al direccionamiento de moléculas cargadas positivamente hacia el sitio activo, ${ }^{(19)}$ fenómeno que es compatible con el hecho que el vector del momento dipolar presenta un ángulo de entre 18 y 35 grados con el eje de las estructuras del sitio activo. ${ }^{(16)}$ Sin embargo, algunos autores estiman que esta distribución asimétrica de cargas no contribuye ni a la eficiencia catalítica ni a la estabilización de los estados

constituting the anionic site, the hydrophobic site, and the acyl pocket, J.Biol.Chem. 268 (23): 17083-17095 (1993).

${ }^{15}$ Dave, K.R., Syal, A.R. and Katyare, S.S. Tissue cholinesterases. A comparative study of their kinetic properties, Z.Naturforsch.[C.]. 55 (1-2): 100-108 (2000).

${ }^{16}$ Felder, C.E. et al. External and internal electrostatic potentials of cholinesterase models, J.Mol.Graphics.Mod. 15: 318-327 (1997).

${ }^{17}$ Porschke, D. et al. Electrooptical measurements demonstrate a large permanent dipole moment associated with acetylcholinesterase, Biophys.J. 70: 1603-1608 (1996).

18 Selzer, T. and Schreiber, G. Predicting the rate enhancement of protein complex formation from the electrostatic energy of interaction, J.Mol.Biol. 287 (2): 409-419 (1999).

${ }^{19}$ Wlodek, S.T., Shen, T. and McCammon, J.A. Electrostatic steering of substrate to acetylcholinesterase: analysis of field fluctuations, Biopolymers. 53 (3): 265-271 (2000). 
de transición (20) durante el desarrollo del mecanismo catalítico.

\section{Estructura del sitio activo de la $\mathrm{AChE}$}

Alrededor del 4\% del volumen total de la proteína es hueco y cerca de la mitad de este vacío se encuentra en el centro de la estructura proteica, ${ }^{(21)}$ que es donde se ubica el sitio activo. Varios grupos aromáticos, que participan durante el proceso dinámico de interacción de sustratos e inhibidores con el sitio activo, ${ }^{(2)}$ constituyen un profundo canal o garganta de $20 \AA$, en el fondo del cual se encuentra la $\operatorname{Ser}^{200}$ reactiva. ${ }^{(23)}$ Las dimensiones del canal y el fuerte campo eléctrico generado por la enzima son consistentes con el elevado número de recambio. ${ }^{(24)}$

El llamado subsitio aniónico, se constituye con los restos $\mathrm{Glu}^{327}$ y Glu ${ }^{199}$, y tiene como función el guiar a las moléculas cargadas positivamente a lo largo del canal hidrofóbico, acelerando la interacción con el sitio activo y la catálisis misma. ${ }^{(14 c)}$ Esta subestructura más la $\operatorname{Ser}^{200}$, corresponden a los grupos funcionales del sitio activo de la AChE que se conocen desde hace más tiempo. ${ }^{(25)}$

La llamada tríada catalítica involucra al resto $\mathrm{Hys}^{440}$ y $\mathrm{Glu}^{327}$, quienes durante la formación del estado activado durante la catálisis, formarían fuertes puentes de

\footnotetext{
${ }^{20}$ Shafferman, A. et al. Electrostatic attraction by surface charge does not contribute to the catalytic efficiency of acetylcholinesterase, EMBO J. 13 (15): 3448-3455 (1994).

${ }^{21}$ Kovach, I.M., Qian, N. and Bencsura, A. Efficient product clearance through exit channels in substrate hydrolysis by acetylcholinesterase, FEBS Lett. 349 (1): 60-64 (1994).

${ }^{22}$ (a) Harel, M. et al. Quaternary ligand binding to aromatic residues in the active- site gorge of acetylcholinesterase, Proc. Natl. Acad. Sci. U.S.A. 90: $9031-9035$ (1993). (b) Ariel, N. et al. The 'aromatic patch' of three proximal residues in the human acetylcholinesterase active centre allows for versatile interaction modes with inhibitors, Biochem.J. 335 ( Pt 1): 95-102 (1998).

${ }^{23}$ Eastman, J. et al. Fasciculin 2 binds to the peripheral site on acetylcholinesterase and inhibits substrate hydrolysis by slowing a step involving proton transfer during enzyme acylation, J.Biol.Chem. 270 (34): 19694-19701 (1995).

${ }^{24}$ Kronman, C. et al. The back door hypothesis for product clearance in acetylcholinesterase challenged by site-directed mutagenesis, J.Biol.Chem. 269 (45): 27819-27822 (1994).

${ }_{25}$ Augustinsson, K. The nature of an "anionic" site in butyryl cholinesterase compared with that of a similar site in acetylcholinesterase, Biochim. Biophys. Acta 128: 351-362 (1966).
} 
hidrógeno ${ }^{(26)}$ que facilitarían la migración del hidrógeno de la $\mathrm{Ser}^{200}$, hacia el resto ácido de Glu ${ }^{327}$. Por otro lado, el ingreso de la molécula huésped queda claramente limitada ${ }^{(27)}$ por la cantidad de grupos aromáticos (hidrofóbicos) que rodean al grupo reactivo, ${ }^{(28)}$ sobre todo por el llamado bolsillo acilo. Es decir, el tamaño del bolsillo acilo y las limitaciones estéricas impuestas por éste, determina la especificidad de la enzima. ${ }^{(29)}$

Tabla 10.I: Grupos funcionales que describen la estructura del sitio activo de la AChE. Los números en superíndice indican la posición del resto aminoacídico en la estructura primaria de la proteína.

Sitio activo: ubicado dentro de una "garganta" o canal hidrofóbico en el que se distinguen varios subsitios:

$\checkmark \quad$ Subsitio esterásico $\left(\right.$ Ser $^{200}$ ) que participa directamente en el mecanismo de hidrólisis.

$\checkmark$ Tríada catalítica completada por los restos de $\mathrm{Hys}^{440}$ y $\mathrm{Glu}^{327}$

$\checkmark$ Subsitio aniónico (Glu ${ }^{327,199}$ ) que guía a substratos e inhibidores catiónicos a lo largo del canal, acelerando la interacción con el sitio activo.

$\checkmark$ Subsitios de retención de moléculas de agua (Glu ${ }^{202,450}$ ) en el entorno inmediato del sitio esterásico.

$\checkmark$ Cavidad oxianiónica (Gly ${ }^{121,122}$, Ala $^{204}$ ) que mediante hidrógenos amídicos estabiliza grupos

$\mathrm{P}=\mathrm{O}$ ○ $\mathrm{C}=\mathrm{O}$ en posición catalítica.

$\checkmark$ Bolsillo acilo (Phe ${ }^{295,297}$ ) que limita estéricamente al sitio activo.

$\checkmark$ Subsitios aniónicos periféricos $\left(A s D^{74} \cdot \operatorname{TrD}^{66}\right)$ aue barticinan de la interacción con inhibidores

Aunque la AChE es capaz de catalizar la hidrólisis de su sustrato natural en un amplio rango de concentraciones, la ACh también actúa como un inhibidor de la enzima a altas concentraciones. Este hecho pone de manifiesto el carácter alostérico de la enzima. Aunque se ha recurrido a varios modelos cinéticos, el que mejor logra describir este fenómeno, es el que sugiere una activación de la desacetilación de la enzima acetilada. ${ }^{(30)}$

El mecanismo alostérico operaría mediante una transición entre conformaciones activas y no activas del subsitio aniónico ubicado en $\operatorname{Trp}^{86(31)}$ sugiriéndose además

\footnotetext{
${ }^{26}$ Massiah, M.A. et al. Short, strong hydrogen bonds at the active site of human acetylcholinesterase: proton NMR studies, Biochem. 40 (19): 5682-5690 (2001).

27 Zahavi, M., Tahori, A.S. and Klimer, F. Insensivity of acetylcholinesterases to organophosphorus compounds as related to size of esteratic site, Mol.Pharm. 7: 611-619 (1971).

28 Bracha, P. and O'brien, R.D. Trialkyl phosphate and phosphorothiolate anticholinesterases. II. Effects of chain length on potency, Biochem. 7 (4): 1555-1559 (1968).

${ }_{29}$ Taylor, P. et al. Structural bases for the specificity of cholinesterase catalysis and inhibition, Tox. Lett. 82/83: 453-458 (1995).

30 Brochier, L. et al. Involvement of deacylation in activation of substrate hydrolysis by Drosophila acetylcholinesterase, J.Biol.Chem. 276 (21): 18296-18302 (2001).

${ }^{31}$ Ordentlich, A. et al. Contribution of aromatic moieties of $\operatorname{Tyr}^{133}$ and of the anionic subsite $\operatorname{Trp}^{86}$ to catalytic efficiency and allosteric
} 
que en la $\mathrm{AChE}$ humana esta transición estaría asociada a un movimiento del lazo $\mathrm{Cys}^{69}{ }^{-} \mathrm{Cys}^{96}$ que sería inherente a la dinámica de la enzima nativa. ${ }^{(32)}$ Sin embargo, un análisis cinético realizado considerando un proceso de no equilibrio, da cuenta que los cambio conformacionales pueden no ser necesarios para explicar el papel de los sitios periféricos en las inhibiciones reversibles de la $\mathrm{AChE}{ }^{(33)}$

Aunque en general se asume que, dentro de los OPs sólo los triésteres fosfóricos son inhibidores de la AChE, el reconocimiento de la actividad anticolinesterasa por parte de ciertos fosfatos orgánicos monoácidos ha servido para dilucidar algunos de los aspectos del mecanismo de inhibición. ${ }^{(34)}$ Se conocen además otros tipos de inhibidores que actuarían a distintos niveles del sitio activo de la AChE como los carbamatos, con un mecanismo similar al de los OPs, y otras moléculas como la rivastigmina, ${ }^{(35)}$ la galantamina, ${ }^{(36)}$ derivados del polientilenglicol, ${ }^{(37)}$ derivados de la aminopiridazina, ${ }^{(38)}$ derivados catiónicos del anilinio, ${ }^{(39)}$ derivados de la tacrina ${ }^{(40)}$ y de las cumarinas ${ }^{(41)}$ quienes, como ya se indicó, se

modulation of acetylcholinesterase, J.Biol.Chem. 270 (5): 2082-2091 (1995).

32 Barak, D. et al. Allosteric modulation of acetylcholinesterase activity by peripheral ligands involves a conformational transition of the anionic subsite, Biochem. 34 (47): 15444-15452 (1995).

33 Szegletes, T., Mallender, W.D. and Rosenberry, T.L. Nonequilibrium analysis alters the mechanistic interpretation of inhibition of acetylcholinesterase by peripheral site ligands, Biochem. 37 (12): 4206-4216 (1998).

34 Aharoni, A.H. and O'brien, R.D. The inhibition of acetylcholinesterse by anionic organophosphorus compounds, Biochem. 7 (4): 1538-1545 (1968).

35 Ramirez, E. et al. Acetylcholinesterase inhibitor SDZ ENA 713 (Rivastigmine) increases brain pyrrolidone carboxyl peptidase activity, Eur.Neuropsychopharmacol. 11 (5): 381-383 (2001).

${ }^{36}$ (a) Luttmann, E., Linnemann, E. and Fels, G. Galanthamine as bisfunctional ligand for the acetylcholinesterase, J.Mol.Model. 8 (6): 208216 (2002).

${ }^{37}$ Koellner, G. et al. A Neutral Molecule in a Cation-binding Site: Specific Binding of a PEG-SH to Acetylcholinesterase from Torpedo californica, J.Mol.Bio. 320 (4): 721-725 (2002).

${ }^{38}$ (a) Sippl, W. et al. Structure-based 3D QSAR and design of novel acetylcholinesterase inhibitors, J.Comput.Aided Mol.Des. 15 (5): 395 410 (2001). (b) Contreras, J.M. et al. Design, synthesis, and structureactivity relationships of a series of 3-[2-(1-benzylpiperidin-4-yl) ethylamino] pyridazine derivatives as acetylcholinesterase inhibitors, $J$ Med Chem 44 (17): 2707-2718 (2001).

${ }^{39}$ Grove, S.J. et al. Oxyaniliniums as acetylcholinesterase inhibitors for the reversal of neuromuscular block, Bioorg Med Chem Lett 12 (2): 193-196 (2002).

40 (a) Marco, J.L. et al. Synthesis and acetylcholinesterase / butyrylcholinesterase inhibition activity of new tacrine-like analogues, 


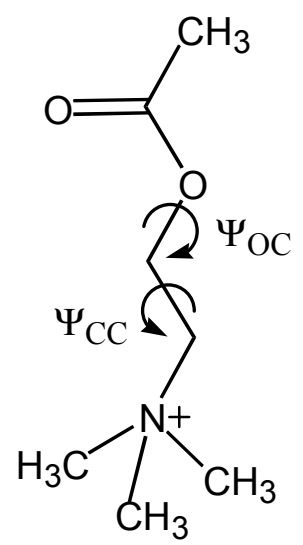

Figura 10.2: Ángulos diedros $\Psi_{\text {oc }}$ y $\Psi_{\text {CC }}$ que, por rotación, determinan las variantes conformacionales de la ACh. unen a sitios periféricos y en general actúan como inhibidores reversibles. De otros inhibidores de la $\mathrm{AChE},{ }^{(42)}$ no se conocen los mecanismos de acción precisos. Algunos de estos inhibidores reversibles de la AChE han encontrado aplicación en el tratamiento de patologías del sistema nervioso. ${ }^{(43)}$ Existen unos pocos fármacos $\mathrm{OPs}$, inhibidores de la $\mathrm{AChE}$, utilizados en el tratamiento tópico de dolencias oftalmológicas, entre los que podemos citar al ecotiofato ${ }^{(44)} \mathrm{y}$ al isofluorofato. ${ }^{(44 b)}$

Se ha reconocido que las interacciones dipolodipolo son relevantes durante la interacción de ciertos inhibidores con el sitio activo de la $\mathrm{AChE}$, hecho que ha sido puesto de manifiesto mediante medidas de relajación de RMN. ${ }^{(45)}$ También se ha sugerido que la presencia de moléculas de agua de hidratación son importantes en el mecanismo de unión de sustratos y productos a lo largo del canal hidrofóbico. ${ }^{(46)}$

Se necesita de una eliminación efectiva de los productos de hidrólisis de la $\mathrm{AChE}$, factor del que depende

Bioorg. Med. Chem. 9 (3): 727-732 (2001). (b) Ros, E. et al. The pharmacology of novel acetylcholinesterase inhibitors, (+/-)-huprines $\mathrm{Y}$ and X, on the Torpedo electric organ, Eur.J.Pharmacol. 421 (2): 77 84 (2001).

${ }^{41}$ (a) Bruhlmann, C. et al. Coumarins derivatives as dual inhibitors of acetylcholinesterase and monoamine oxidase, J.Med.Chem. 44 (19): 3195-3198 (2001). (b) Kang, S.Y. et al. Coumarins isolated from Angelica gigas inhibit acetylcholinesterase: structure-activity relationships, J.Nat.Prod. 64 (5): 683-685 (2001).

42 den Hartog, G.J.M. et al. Hypochlorous acid is a potent inhibitor of acetylcholinesterase, Toxicol.Appl.Pharmacol. 181 (3): 228-232 (2002).

${ }^{43}$ (a) Bartolucci, C. et al. Three-dimensional structure of a complex of galanthamine (Nivalin) with acetylcholinesterase from Torpedo californica: implications for the design of new anti-Alzheimer drugs, Proteins 42 (2): 182-191 (2001). (b) Gobburu, J.V. et al. Pharmacokinetic-pharmacodynamic modeling of rivastigmine, a cholinesterase inhibitor, in patients with Alzheimer's disease, $J$ Clin Pharmacol 41 (10): 1082-1090 (2001). (c) Talesa, V.N. Acetylcholinesterase in Alzheimer's disease, Mechanism of Aging and Development (2001).

${ }^{44}$ (a) Murray, D.D. and Mc Gavi, M.B. Depressed levels of serumpseudocholinesterase with ecothiophate-iodide eyedrops, The Lancet 286 (7406): 272-273 (1965). (b) Bustamante, S.E. y Morales, M.A. Fármacos Colinérgicos. Apuntes Docentes de Obstetricia, Facultad de Medicina U. de Chile (2002).

${ }^{45}$ Delfini, M. et al. ${ }^{1} \mathrm{H}$ NMR Relaxation Investigation of Inhibitors Interacting with Torpedo californica Acetylcholinesterase, J.Magn.Reson. 144 (1): 129-133 (2000).

${ }^{46}$ Koellner, G. et al. Active-site gorge and buried water molecules in crystal structures of acetylcholinesterase from Torpedo californica, J.Mol.Biol. 296 (2): 713-735 (2000). 
en forma crítica el tiempo de recambio del sitio activo. Y ya se ha comentado que en la AChE este tiempo es muy pequeño. Se ha mapeado la estructura terciaria de la AChE mediante técnicas de modelado molecular y se han encontrado rutas potenciales para la salida de productos de hidrólisis cargados positiva y negativamente. ${ }^{(21)}$

Para fragmentos cargados negativamente se identificó un ancho canal ubicado por debajo del canal de entrada. Este canal posee una relativa abundancia de restos cargados negativamente. Por otro lado para la salida de productos cargados positivamente se propone una pequeña cámara ubicada por encima de los restos $\operatorname{Trp}^{84} \mathrm{y} \mathrm{Met}^{83}$. Otros autores sin embargo, han argumentado que la "puerta de salida" descrita no es la ruta de los productos ${ }^{(24)}$ y por lo tanto probablemente no tenga importancia en la actividad catalítica.

Respecto a los cambios conformacionales globales de la estructura proteica, se ha reportado que durante el pasaje del sustrato u otras pequeñas moléculas a lo largo del canal hidrofóbico, se verificarían al menos dos estados conformacionales de la proteína expresados en fluctuaciones estructurales, ${ }^{(47)}$ lo que sugeriría un mecanismo de apertura y cierre de canal durante este proceso. $^{(48)}$

Finalmente, y dada la naturaleza asimétrica de la interacción con el sitio activo, ${ }^{(49)}$ que se manifiesta por ejemplo en la diferente reactividad frente a isómeros ópticos ${ }^{(50)}$, es importante realizar las consideraciones

\footnotetext{
${ }^{47}$ Shen, T.Y., Tai, K. and McCammon, J.A. Statistical analysis of the fractal gating motions of the enzyme acetylcholinesterase, Phys. Rev. E. Stat. Phys. Plasmas. Fluids. Relat. Interdiscip. Topics. 63 (4 Pt 1): 041902 (2001).

${ }^{48}$ Tai, K. et al. Analysis of a 10-ns molecular dynamics simulation of mouse acetylcholinesterase, Biophys.J. 81 (2): 715-724 (2001).

${ }^{49}$ Jarv, J.L. et al. The arrangement of substrate and organophosphorusinhibitor leaving groups in acetylcholinesterase active site, Biochem.J. 167: 823-825 (1977).

50 (a) Chiu, Y.C. and Dauterman, W.C. The affinity and phosphorylation constants of the optical isomers of O,O-diethyl malaoxon and the geometrics isomers of phosdrin with acetylcholinesterase, Biochem.Pharmacol. 18: 359 (1969). (b) Thompson, C.M., Ryu, S. and Berkman, C.E. Consequence of phosphorus stereochemistry upon the postinhibitory reaction kinetics of acetylcholinesterase poisoned by phosphorothiolates, J.Am.Chem.Soc. 114 (27): 10710-10715 (1992). (c) Ordentlich, A. et al. Exploring the active center of human acetylcholinesterase with stereomers of an organophosphorus inhibitor with two chiral centers, Biochem. 38 (10): 3055-3066 (1999).
} 
Tabla 10.II: Momento dipolar y ángulos de torsión (en grados) del confórmero más estable de la ACh calculado a nivel $\mathrm{HF} / 6-$ $31 \mathrm{G}^{* *}$.

\begin{tabular}{ll}
\hline$\mu$ (Debyes) & 6,9 \\
$\Psi_{\mathrm{CC}}$ & 156 \\
$\Psi_{\mathrm{CO}}$ & 280 \\
\hline
\end{tabular}

necesarias en el momento de analizar la asimetría molecular o conformacional de las moléculas que se modelan en el interior de este sitio activo.

Por ejemplo, tales características se expresarán en variaciones cinéticas de las interacciones de uno $u$ otro isómero. ${ }^{(51)}$ En general, la asimetría estructural del sistema estudiado es crítica en todos los procesos que involucran interacciones biológicas y no corresponde a una excepción, sino más bien a la regla en el contexto de los sistemas moleculares bioquímicos. Durante el presente análisis se pondrá acento en las variaciones conformacionales que expresen asimetría espacial. Más adelante profundizaremos este aspecto de la interacción.

En resumen, el proceso de ingreso de moléculas al seno del sitio activo de la enzima se revela complejo. En este contexto, nuestra elección de compuestos pequeños para el modelado de las interacciones con el sitio activo se relaciona con dos aspectos.

Por un lado, facilita el estudio estructural y espectroscópico mediante metodologías experimentales y teóricas, pues al disminuir el número de átomos y enlaces, se puede lograr una asignación más precisa de bandas en el espectro IR con modos vibracionales o rotacionales, de bandas del espectro UV-visible con niveles electrónicos y de descripciones adecuadas de los espectros RMN, además de contar con la posibilidad de recurrir a cálculos teóricos con un menor número de aproximaciones. Esto es lo que hemos descrito en los primeros nueve Capítulos de este trabajo.

Por otro lado, se modela sobre una interacción enzima-inhibidor en donde se eliminan algunos puntos de contacto del sitio activo con el inhibidor de cierta relevancia para moléculas de mayor tamaño; fundamentalmente no se toma en cuenta el papel del sitio aniónico que interacciona con cargas positivas del sustrato natural y de una gran cantidad de inhibidores pues éste se encuentra a cuatro átomos de distancia del sitio de ataque nucleofílico sobre el grupo carbonilo (en el sustrato e inhibidores carbamato) o fosforilo (en inhibidores fosfato).

\footnotetext{
${ }^{51}$ Jianmongkol, S. et al. Kinetic evidence for different mechanisms of acetylcholinesterase inhibition by (1R)- and (1S)- stereoisomers of isomalathion, Toxicol.Appl.Pharmacol. 155 (1): 43-53 (1999).
} 
Aunque no permite describir la totalidad de los factores que determinan la formación del complejo inicial enzima-sustrato, esta última característica de los modelos utilizados permite aislar factores que condicionarían la posterior hidrólisis del enlace éster.

\section{Comportamiento conformacional de la $\mathrm{ACh}$}

Como ya se mencionó, la ACh corresponde al sustrato natural de la AChE. Esta molécula presenta una variabilidad conformacional que está determinada fundamentalmente por dos ángulos diedros que denominaremos $\Psi_{\mathrm{OC}}$ y $\Psi_{\mathrm{CC}}$ (Figura 10.2). Es interesante revisar los aspectos estudiados respecto al comportamiento conformacional de esta molécula, tomando en cuenta los diversos entornos en los que han sido hechos. Se ha recurrido con cierta frecuencia al análisis de las espectroscopías vibracionales, ${ }^{(52)}$ tanto para la AChE como para sus análogos.

Cálculos teóricos asignan mayor estabilidad para el confórmero gauche- $\Psi_{\mathrm{OC}}$, trans $-\Psi_{\mathrm{CC}}$, tanto en vacío como en solución acuosa, aunque se estima que en solución acuosa, el confórmero de geometría trans- $-\Psi_{\mathrm{OC}}$, trans $-\Psi_{\mathrm{CC}}$ tiene una abundancia apreciable. Ambos son datos obtenidos por dinámica molecular (AMBER). ${ }^{(53)}$ Los cálculos $a b$ initio realizados en este trabajo, también encuentran un confórmero de geometría gauche- $\Psi_{\mathrm{OC}}$, trans $-\Psi_{\mathrm{CC}}$ que correspondería al de mayor estabilidad y cuyos parámetros se muestran en la Tabla 10.II.

Dado que la hidrólisis de la ACh catalizada por la $\mathrm{AChE}$ es muy eficiente, aislar los intermediarios de la reacción constituye un problema experimental muy importante. Por ello, para dilucidar las estructuras de los transientes moleculares, se ha recurrido a la formación de los intermediarios in situ a partir de moléculas fotolábiles (54) o al modelado molecular. A pesar de estas dificultades,

\footnotetext{
52 Aslanian, D. Vibrational spectroscopic approach to the study of acetylcholine and related compounds, Life.Sci. 32 (25): 2809-2825 (1983).

${ }^{53}$ Edvardsen, O. and Dahl, S.G. Molecular structure and dynamics of acetylcholine, J.Neural.Transm.Gen.Sect. 83 (3): 157-170 (1991).

54 Peng, L. et al. Biochemical evaluation of photolabile precursors of choline and of carbamylcholine for potential time-resolved crystallographic studies on cholinesterases, Biochem. 35 (33): 10854 10861 (1996).
}

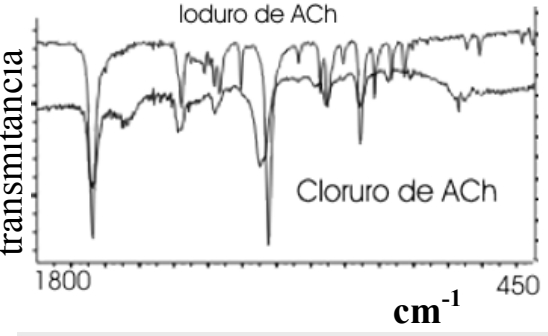

Figura 10.3: Espectros infrarrojos de ioduro y cloruro de ACh en fase sólida. Los valores de número de onda se expresan en $\mathrm{cm}^{-1}$ y la intensidad de las bandas en transmitancia porcentual. 


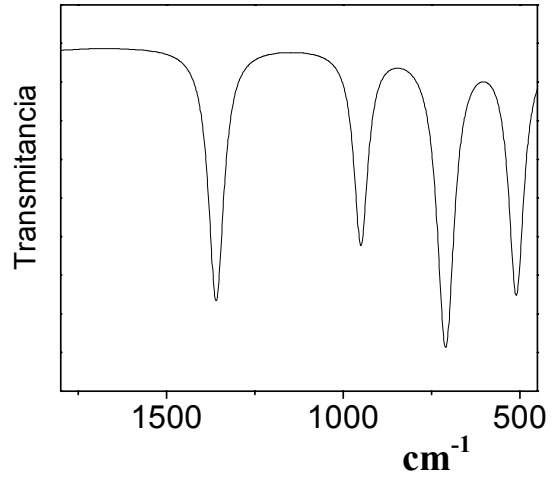

Figura 10.4: Espectro infrarrojo predicho para la AChE según un cálculo ab initio a un nivel HF/6$31 \mathrm{G}^{* *}$, entre 450 y $2000 \mathrm{~cm}^{-1}$. se ha reportado que el centro activo de la $\mathrm{AChE}$ reconoce a la conformación trans $-\Psi_{\mathrm{OC}}$, trans $-\Psi_{\mathrm{CC}}{ }^{(5 c)}$ que corresponde a la a la forma menos abundante de la $\mathrm{ACh}$, de manera que en este sitio hay una estabilización de la forma menos estable en solución.

Nosotros hemos medido del espectro vibracional IR de las dos formas salificadas de la ACh en fase sólida, como primer paso en la identificación de isomerías conformacionales (Figura 10.3). Estas medidas se pueden comparar con los espectros predichos mediante cálculos $a b$ initio para la misma estructura (Figura 10.4). En ellas, no es posible discriminar la presencia de isomería conformacional, aunque es necesario advertir que nos referimos a una técnica de baja resolución, como lo son las medidas del espectro infrarrojo en fase líquida y sólida.

Considerando la energía libre necesaria para estabilizar la conformación menos estable de la ACh, se puede comparar con las energías libres de cambio conformacional de los ésteres de fosfato. Es necesario tomar en cuenta que, existiendo varias conformaciones cercanamente estables, los valores pueden relacionarse con distintas formas de reconocimiento de los fosfatos al sitio activo.

Respecto a ese reconocimiento conformacional, se puede homologar la respuesta del centro activo de la AChE a los OPs, identificando cuales estructuras de aquellos se encuentran en solución y cuales de ellas prevalecen en los entornos del sitio activo de la enzima. Es necesario un estudio que permita caracterizar la coexistencia de confórmeros de la $\mathrm{ACh}$ en los entornos del sitio activo, de manera de complementar la información ya existente.

\section{Dinámica del sitio activo de la AChE y sus aductos con OPs}

Como ya se mencionó, es reciente la descripción precisa de la estructura y dinámica del sitio activo. El estudio de la dinámica de las interacciones de catálisis e inhibición se han abordado mediante técnicas, entre las que sobresalen las técnicas de difracción de $\mathrm{RX}^{(55)}$ la

\footnotetext{
55 Axelsen, P.H. et al. Structure and dynamics of the active center gorge of acetylcholinesterase: Synergistic use of molecular dynamics simulation and X-ray crystallography, Protein.Sci. 3 (2): 188-197 (1994).
} 
construcción de modelos reducidos ${ }^{(56)}$ y las metodologías de cálculo de sistemas simulados. ${ }^{(57)}$ Un capítulo aparte corresponde a las técnicas que involucran mutagénesis dirigidas de la AChE, tema sobre el que volveremos en el Capítulo 13.

Para que transcurra eficientemente la inhibición y previamente a la formación del intermediario pentavalente (Figura 10.5), es necesario que ocurran tres eventos simultáneos: $^{(58)}$ (a) superposición del fosfato con el oxígeno- $\gamma$ de la $\mathrm{Ser}^{200}$, (b) polarización del enlace $\mathrm{P}=\mathrm{O}$ por su posición en el agujero axianiónico y (c) posicionamiento del grupo saliente $X_{1}$ hacia la salida de la garganta o canal del sitio activo. Este comportamiento general puede sufrir variaciones drásticas en la medida que otros grupos químicos intervengan durante la interacción de las moléculas con el entorno del sitio activo.

\footnotetext{
${ }^{56}$ Thompson, C.M., Suarez, A.I. and Rodriguez, O.P. Synthesis and ${ }^{31} \mathrm{P}$ chemical shift identification of tripeptide active site models that represent human serum acetylcholinesterase covalently modified at serine by certain organophosphates, Chem.Res.Toxicol. 9 (8): 13251332 (1996).

57 (a) Qian, N. and Kovach, I.M. Key active site residues in the inhibition of acetylcholinesterases by soman, FEBS Lett. 336 (2): 263266 (1993). (b) Bencsura, A. et al. 1994. Phosphonate ester active site probes of acetylcholinesterase, trypsin and chymotrypsin. In Enzymes Cholinesterase Fam. Edited by D. M. Quinn. N.Y.: Plenum. (c) Bencsura, A., Enyedy, I. and Kovach, I.M. Probing the active site of acetylcholinesterase by molecular dynamics of its phosphonate Ester Adducts, J.Am.Chem.Soc. 118 (36): 8531-8541 (1996). (d) Bernard, P.P. et al. 3D Model of the acetylcholinesterase catalytic cavity probed by stereospecific organophosphorus inhibitors, J.Mod.Mol. 4: 323-334 (1998). (e) Elcock, A.H. et al. Computer simulation of protein-protein association kinetics: acetylcholinesterase-fasciculin, J.Mol.Bio. 291 (1): 149-162 (1999). (f) Golbraikh, A., Bernard, P.P. and Chretien, J.R. Validation of protein-based alignment in $3 \mathrm{D}$ quantitative structureactivity relationships with CoMFA models, Eur.J.Med.Chem. 35 (1): 123-136 (2000). (g) Hirashima, A., Kuwano, E. and Eto, M. Docking study of enantiomeric fonofos oxon bound to the active site of Torpedo californica acetylcholinesterase, Bioorg.Med.Chem. 8 (3): 653-656 (2000). (h) Pilger, C. et al. Accurate prediction of the bound conformation of galanthamine in the active site of Torpedo californica acetylcholinesterase using molecular docking, J.Mol.Graph.Model. 19 (3-4): 288 (2001). (i) Enyedy, I.J., Kovach, I.M. and Bencsura, A. Molecular dynamics study of active-site interactions with tetracoordinate transients in acetylcholinesterase and its mutants, Biochem.J. 353 (Pt 3): 645-653 (2001).

58 Taylor, P. et al. Determining ligand orientation and trans phosphonylation mechanisms on acetylcholinesterase by $\mathrm{Rp}, \mathrm{Sp}$ enantiomer selectivity and site-specific mutagenesis, Enantiomer. 2 (34): 249-260 (1997).
} 


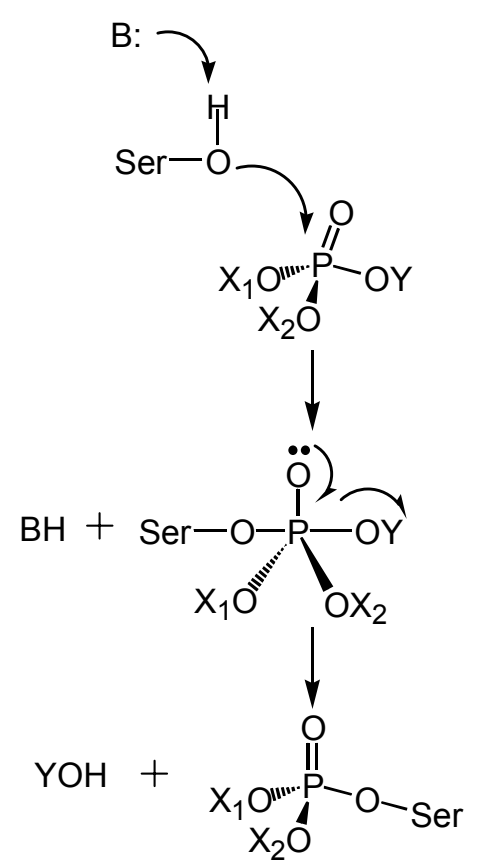

Figura 10.5: Formación del complejo pentacoordinado E$P$, consecuente al ataque nucleofílico por parte del hidroxilo $\mathrm{Ser}^{200}$.
Por ejemplo, se sugiere que el sitio reactivo puede interaccionar con sustratos e inhibidores de manera que no se favorezca la hidrólisis respectiva. En este sentido, se ha propuesto que, en forma análoga a similares modelos planteados para inhibidores catiónicos de la AChE, la molécula fosfamidotionato de O,S-dimetilo, o metamidofós, el grupo $\mathrm{P}=\mathrm{O}$ del forma puentes de hidrógeno dentro el agujero oxianiónico causando que el grupo saliente $\left(-\mathrm{SCH}_{3}\right)$ se oriente a través de la apertura de la garganta. Esta orientación permitiría que el átomo de fósforo del metamidofós interaccione con la $\mathrm{Se}^{200}$, resultando en la fosforilación de la enzima. Sin embargo, en el acefato (que corresponde al metamidofós $\mathrm{N}$ acetilado), tanto el grupo $\mathrm{P}=\mathrm{O}$, como el grupo $\mathrm{C}=\mathrm{O}$ del resto acetilo, aunque no ambos, interaccionan con el agujero oxianiónico, lo que desestabiliza la unión del acefato con el sitio activo, resultando en una fosforilación reducida de la $\mathrm{AChE}{ }^{(59)}$

Se han estudiado muchas correlaciones entre de distintas propiedades moleculares con indicadores de esta actividad biológica. A partir de estas correlaciones se han logrado encontrar ciertos parámetros o "descriptores" necesarios para cuantificar la capacidad de inhibición por parte de OPs. ${ }^{(60)}$ Sobre este punto profundizaremos en el Capítulo 13.

Los pequeños ésteres de fosfatos propuestos en este trabajo, permiten una aproximación al problema a través de modelos relativamente simplificados de esta interacción con el sitio activo de la enzima y las propiedades moleculares relacionadas con el entorno inmediato al átomo de fósforo. Existen evidencias que la afinidad de los grupos del sitio activo de la AChE hacia las moléculas entrantes es más importante que la actividad nucleofílica (1) durante el proceso de formación del intermediario pentacoordinado. Estos datos sustentan la importancia del estudio de los sucesos que se suceden durante el acercamiento de sustratos e inhibidores hacia el grupo reactivo $\mathrm{Ser}^{200}$.

Se ha examinado la posición dentro del sitio activo de la AChE del grupo planar del carboxilo y

\footnotetext{
${ }^{59}$ Singh,A.K. et al. Physicochemical, molecular-orbital and electronic properties of acephate and methamidophos. Comp.Biochem.Physiol. 119 (1): 107-117 (1998).

${ }^{60}$ Sobre este punto volveremos con mayor profundidad en el Capítulo 12 .
} 
tetraédrico de los OPs, ${ }^{(61)}$ dando cuenta que el grupo saliente tiene una posición específica hacia afuera de la garganta hidrofóbica, en forma análoga para ambos tipos de sustancias. Éste fenómeno es el que determinaría la enantioselectividad de los fosfatos asimétricos.

Es a partir de estos datos que se pretende construir un modelo que represente el sitio activo de la AChE y sus aductos con compuestos OPs pequeños con el fin de, por un lado, reconocer características geométricas, electrónicas y energéticas en la interacción de estos compuestos con el sitio activo $\mathrm{y}$, por otro lado, encontrar valores termodinámicos teóricos de esta interacción, que puedan utilizarse luego para su comparación con medidas cinéticas de inhibición enzimática y así verificar las conclusiones extraídas del modelo.

El estímulo para abordar los problemas asociados a los eventos moleculares durante el desarrollo de la catálisis o inhibición del enzima mediante metodologías de modelado molecular es la idea que la simulación del entorno molecular bajo estudio, con el actual nivel de conocimientos de la arquitectura de éste, debería entregar una cantidad y calidad de datos suficientes para una interpretación de estos eventos con una muy elevada correlación con datos experimentales.

61 Hosea, N.A., Berman, H.A. and Taylor, P. Specificity and orientation of trigonal carboxyl esters and tetrahedral alkylphosphonyl esters in cholinesterases, Biochem. 34 (36): 11528-11536 (1995). 


\section{Acetilcolinesterasa: modelado del sitio activo y sus aductos con organofosforados}

El estudio de las características de la interacción entre los OPs y el sitio activo de la AChE presenta una faz teórica y una faz experimental. En ambos sentidos se ha aportado abundante información, como ya se describió en el Capítulo precedente. A medio camino se encuentran las metodologías del modelado y la simulación. En la última porción del siglo pasado, el gran avance de las metodologías de modelado molecular y de las tecnologías de cálculo, ha posibilitado la construcción de modelos que permiten una mejor interpretación de los resultados experimentales en función de las bases teóricas existentes, modificar conceptos teóricos de manera de adecuarlos a los resultados experimentales o permitir el diseño de nuevas variantes experimentales de manera de probar hipótesis que surgen del modelado molecular. Es abundante el trabajo realizado, pero lejos se está de agotarse el aporte desde estas áreas.

Para construir un modelo teórico del sitio de interacción de la $\mathrm{AChE}$, es necesario tener claridad en dos tipos de consideraciones: Por un lado aquellas que se relacionan con el conocimiento de las estructuras y procesos dinámicos que ocurren durante la interacción y por otro lado la jerarquía de estos procesos y estructuras en relación con el tipo de fenómeno que se pretende estudiar.

De esta manera el modelo será adecuado para simular aquellas cuestiones que se pretenden modelar para que, con alguna precisión, se ajusten a lo que ocurre en la realidad y por otro lado no será excesivo ni demasiado complejo de manera de permitir una interpretación relativamente sencilla de los procesos que se pretenden describir.

En el presente caso, estamos interesados en los primeros pasos del proceso de inhibición. Básicamente, este proceso se puede describir mediante una cinética en dos etapas, donde un resto de serina del sitio activo realiza un 
Tabla 11.I: Estructuras funcionales constituyentes del modelo y función de cada uno de los restos aminoacídicos involucrados en ellas.

\begin{tabular}{|c|c|c|}
\hline Denominación & Función & Restos aminoacídicos \\
\hline $\begin{array}{l}\text { Agujero } \\
\text { oxianiónico }\end{array}$ & $\begin{array}{l}\text { estabiliza el grupo }=0 \text { del sustrato/inhibidor mediante tres } \\
\text { hidrógenos imida }\end{array}$ & Gly $^{118}$-Gly ${ }^{119}$, Ala $^{201}$ \\
\hline $\begin{array}{l}\text { Tríada } \\
\text { catalítica }\end{array}$ & $\begin{array}{l}\text { mediante transferencia de un protón, forma el complejo } \\
\text { enzima-sustrato/inhibidor con el oxhidrilo de la serina }\end{array}$ & $\begin{array}{l}\text { Ser }^{200} \\
\text { Hys }^{440} \text { Glu }^{327}\end{array}$ \\
\hline Bolsillo acilo & retiene restos hidrofóbicos del sutrato/inhibidor & $\begin{array}{l}\text { Phe }{ }^{288}, \text { Phe }^{290} \\
\text { Phe }^{330}-\text { Phe }^{331} \\
\text { Trp }^{233}\end{array}$ \\
\hline $\begin{array}{l}\text { Subsitio } \\
\text { aniónico }\end{array}$ & unión a cargas positivas de sustratos e inhibidores & $\operatorname{Trp}^{84}$ \\
\hline
\end{tabular}

ataque nucleofílico al átomo de fósforo, participando de éste ataque un grupo básico que le otorga la suficiente densidad electrónica al oxígeno serínico.

Se forma entonces un intermediario pentacoordinado que, como ya se mencionó, luego de la hidrólisis de un grupo saliente que se produce por un reordenamiento de los electrones ubicados en orbitales enlazantes $\mathrm{P}-\mathrm{O}$, forma el complejo pentacoordinado que corresponde a la primera forma estable de la enzima inhibida.

\section{Constitución del modelo}

Se utiliza como estructura de partida las coordenadas moleculares obtenidas de los espectros de difracción de rayos $\mathrm{X}$ de la $\mathrm{AChE}^{(1)}$ extraída del órgano eléctrico del pez anguila (T. Californica) ${ }^{(2)}$.

Tabla 11.II: Los siete péptidos constituyentes del modelo del sitio activo, que incluyen a cada uno de los restos aminoacídicos involucrados en los mecanismos de catálisis e inhibición. Las relaciones tridimensionales entre estos péptidos, son las que surgen de la estructura terciaria de la enzima nativa.
(a) $\mathrm{Met}^{83}-\mathrm{Trp}^{84}-\mathrm{Asn}^{85}$
(b) Gly ${ }^{117}-$ Gly $^{118}-$ Gly $^{119}-$ Phe $^{120}$
(c) Glu ${ }^{199}-\mathrm{Ser}^{200}-\mathrm{Ala}^{201}-\mathrm{Gly}^{202}$
(d) $\mathrm{Pro}^{232}-\mathrm{Trp}^{233}-\mathrm{Ala}^{234}$
(e) $11 e^{287}-\mathrm{Phe}^{288}-\mathrm{Arg}^{289}-\mathrm{Phe}^{290}-\mathrm{Ser}^{291}$
(f) $11 e^{439}-\mathrm{Hys}^{440}-\mathrm{Gly} \mathrm{y}^{441}$
(g) Asp ${ }^{326}-$ Glu $^{327}-\mathrm{Gly}^{328}-\mathrm{Ser}^{329}-\mathrm{Phe}^{330}-\mathrm{Phe}^{331}-\mathrm{Leu}^{332}$

\footnotetext{
${ }^{1}$ Sussman, J.L. et al. Atomic structure of acetylcholinesterase from Torpedo californica: a prototypic acetylcholine- binding protein, Scien. 253 (5022): 872-879 (1991).

${ }^{2}$ Sussman, J.L. et al. Purification and crystallization of a dimeric form of acetylcholinesterase from Torpedo californica subsequent to solubilisation with phosphatidylinositol-specific phospholipase C. J.Mol.Biol. 203:821-823 (1988).
} 
Tabla 11.III: Parámetros geométricos encontrados para conformaciones estables del complejo AChE(sa) con el TMP, a un nivel de cálculo DREIDING (MM). La energía se expresa en $\mathrm{kcal} / \mathrm{mol}$, los ángulos en grados y las distancias en $\AA$. Los valores presentados en la última columna, corresponden a los parámetros obtenidos por RX para AChE(sa) libre (2).

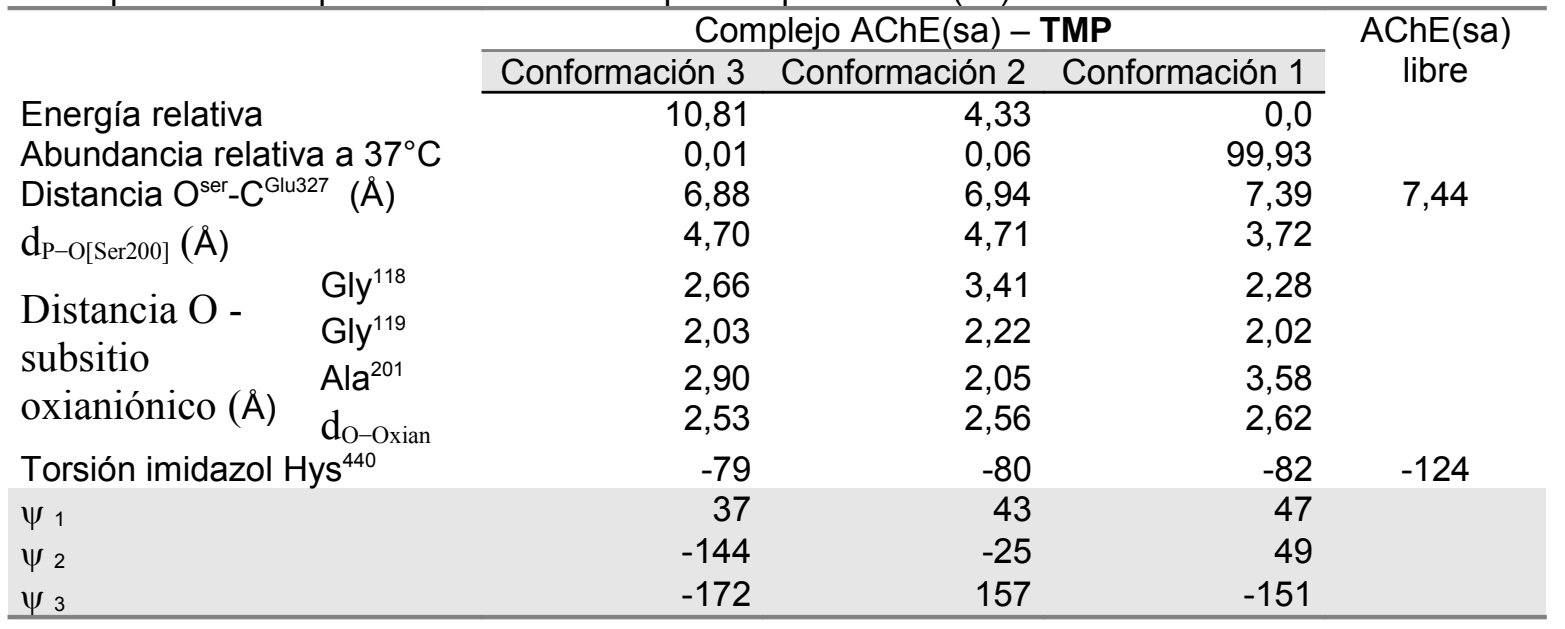

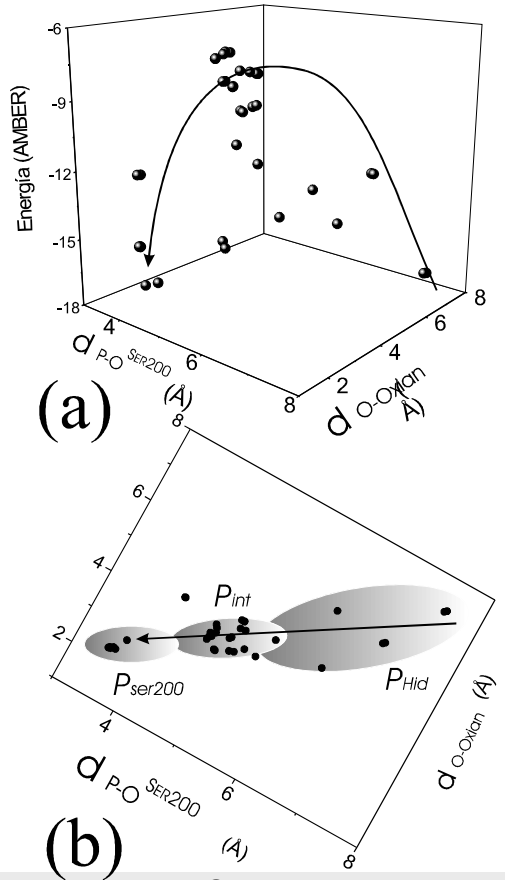

Figura 11.1: Complejos estables del TMP (AMBER) que, a lo largo del canal hidrofóbico de la AChE, definen las tres posiciones $\boldsymbol{P}_{\text {Hid }}$, $\boldsymbol{P}_{\text {Int }}$ y $\boldsymbol{P}_{\text {ser20o, }}$, para el modelo descrito. (a) Distribución de los complejos estables según su energía relativa. La flecha representa el recorrido teórico del inhibidor desde el exterior hasta el sitio reactivo $\mathrm{Ser}^{200}$. (b) Proyección en el plano de las distancias $(\AA)$ del inhibidor con $\mathrm{Ser}^{200}$ y con el agujero oxianiónico (ver texto).
El modelo estructural del sitio activo surgirá por remoción de las porciones de la cadena aminoacídica de la AChE que no participan del entorno del sitio de catálisis, involucrando a todos los restos aminoacídicos con algún papel en el mismo y a los restos aminoacídicos marginales a éstos, agregando grupos - $\mathrm{OH}$ y $-\mathrm{H}$ que neutralicen carga en los extremos de las cadenas resultantes.

En la Tabla 11.I se describen los sitios funcionales y restos aminoacídicos relevantes para los mecanismos de catálisis e inhibición. A estos restos se le incluyen los restos marginales a los reactivos incluyéndose grupos -OH y $-\mathrm{H}$ que neutralizan la carga en los extremos de las cadenas, constituyendo un modelo de sitio activo con los siete péptidos que conforman el modelo, enumerados en la Tabla 11.II. A este modelo lo denominaremos AChE(sa).

Como modelo de inhibidor, en una primera aproximación, se elige al TMP, por sus características de simetría y simplicidad. Para estudiar la influencia de los grupos fosfotionilo y selenotionilo, en una segunda etapa se modela la interacción de las moléculas TMSP y TMSeP.

Existen tres categorías en las metodologías de cálculo para el modelado molecular (Ver Anexo I Metodologías). Estas tres categorías se han utilizado en distintas etapas del planteo y resolución del modelo. Así se acude a una resolución multicapas, en donde el entorno se modela mediante aproximaciones mayores que el núcleo del mismo. 
Figura 11.2: Curvas de energía potencial del TMP ubicado en $\boldsymbol{P}_{\text {ser20o, }}$, para las libres rotaciones de los ángulos $\Psi_{1}, \Psi_{2}$ y $\Psi_{3}$. La flecha indica la posición del confórmero -gauche, -gauche, -gauche de menor energía. Se indica también el elemento que determina la restricción conformacional representada en las regiones de alta energía.
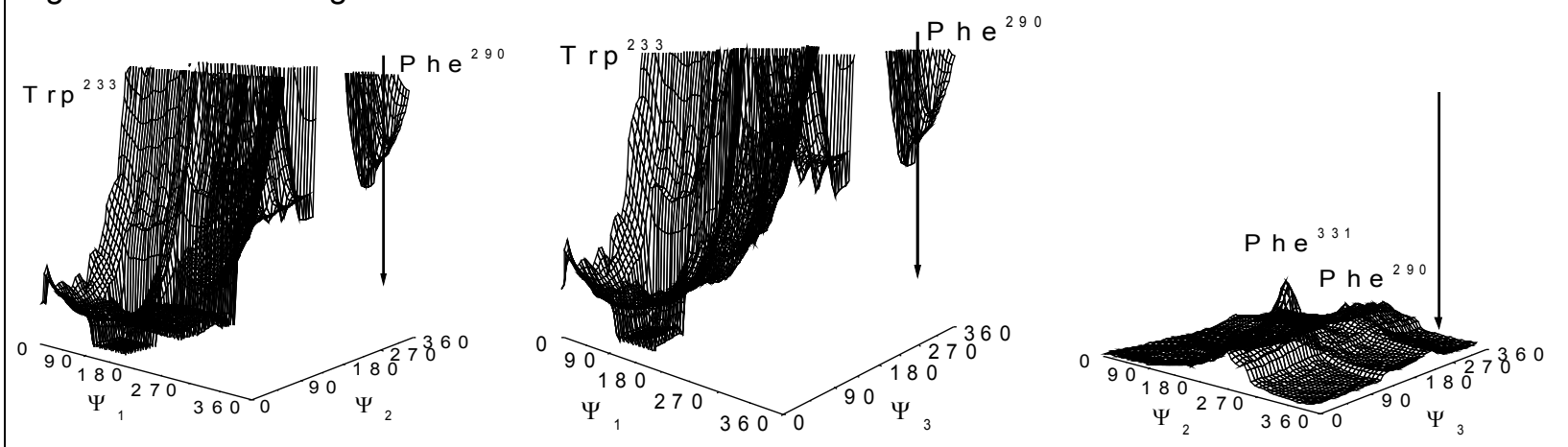

A partir del modelo formado y la introducción en posición adecuada del inhibidor propuesto, se optimizaron geometrías, determinado estructuras más estables. Los parámetros de referencia corresponden a los que se obtienen de los cálculos realizados sobre el modelo de sitio activo libre, sin interacción con el inhibidor.

\section{Resultados}

Distintos autores han propuesto dos o tres mínimos conformacionales para el TMP. Cada uno de ellos difiere en el ángulo $\psi$ de rotación alrededor de los tres enlaces P-O. Nuestros estudios confirman tres confórmeros estables y dos de ellos predominantes. Estos mínimos conformacionales son todos verificados por cálculos $a b$ initio, obteniéndose energías relativas comparables (ver Capítulo 2).

En forma análoga se realiza una búsqueda de mínimos conformacionales del TMP ubicado en el entorno del resto $\operatorname{Ser}^{200}$, mediante la minimización energética de la geometría para el complejo TMP-AChE(sa). Este cálculo se realiza a través de cálculos de mecánica molecular (MM), relajando todos los restos aminoacídicos exceptuando a los marginales de manera de sostener un anclaje respecto a la estructura nativa obtenida de RX.

Se encuentran tres estados del complejo que corresponde a tres conformaciones posibles para el TMP, 
algunos de cuyos valores energéticos y geométricos se muestran en la Tabla 11.III ${ }^{(3)}$.

En una segunda aproximación se realiza una recorrida dentro del sitio activo, buscando otras ubicaciones del TMP que correspondan a ubicaciones estables. El objeto de esta búsqueda es poder de reconocer distintas posiciones de baja energía que se correspondan con el recorrido de las moléculas desde el exterior hacia el resto $\operatorname{Ser}^{200}$ y a lo largo del canal ya descrito ${ }^{(4)}$. Dicha búsqueda se realiza suponiendo una estructura proteica rígida.

Efectivamente, además de la posición ubicada en el entorno inmediato al sitio de catálisis, se encuentran otras dos posiciones respectivamente definidas por la distancia desde el átomo de fósforo hasta el oxígeno reactivo de la $\mathrm{Ser}^{200}$ $\left(\mathrm{d}_{\mathrm{P}-\mathrm{O}[\mathrm{Ser} 200]}\right)$, y por la distancia desde el oxígeno del fosforilo hasta el agujero oxianiónico $\left(\mathrm{d}_{\mathrm{O}-\mathrm{Oxian}}\right)$, tal como se muestra en la Figura 11.1. El valor de $\mathrm{d}_{\mathrm{O}-\mathrm{Oxian}} \mathrm{se}$ obtiene de promediar las distancias desde el oxígeno del grupo fosforilo, hasta cada uno de los tres hidrógenos que forman parte del subsitio o agujero oxianiónico.

Figura 11.3: Curvas de energía potencial del TMP ubicado en $\boldsymbol{P}_{\text {int }}$, para las libres rotaciones de los ángulos $\Psi_{1}, \Psi_{2}$ y $\Psi_{3}$. La flecha indica la posición del confórmero trans, gauche, -gauche de menor energía. Se indica también el elemento que determina la restricción conformacional representada en las regiones de alta energía.
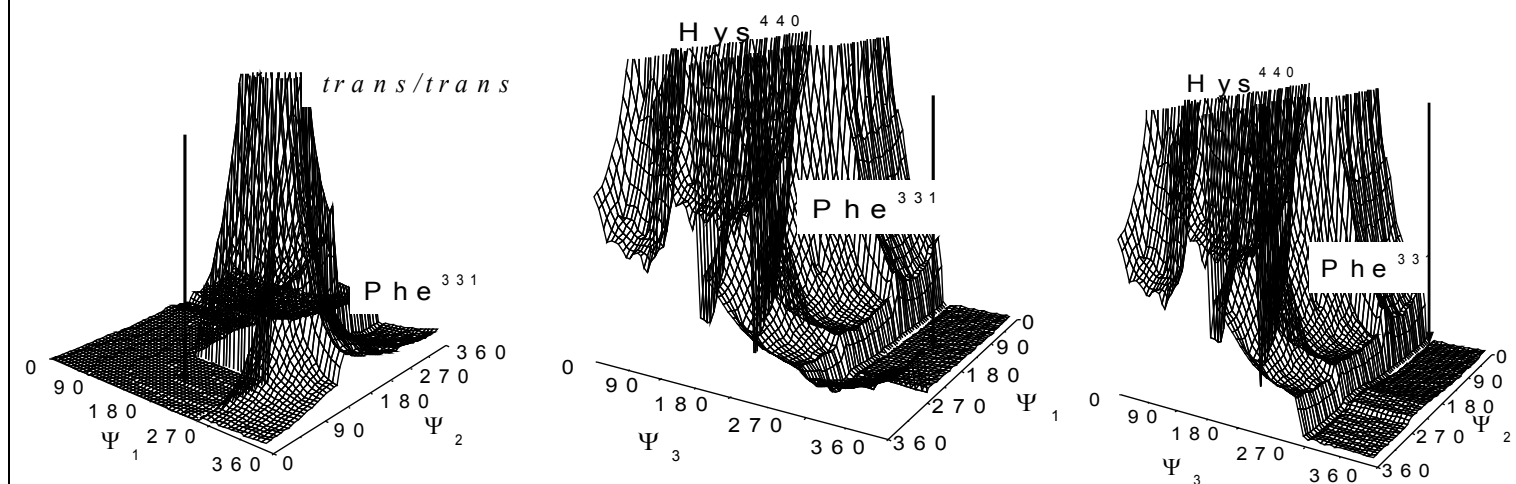

\footnotetext{
${ }^{3}$ Esta tabla es un resumen de los datos ya presentados en: Mastrantonio, G., Della Vedova, C.O., Giannuzzi,L. Cálculo de un modelo teórico para estudios cinéticos de la inhibición de la acetilcolinesterasa por OPs. Comunicación Libre en Póster al XI Congreso Argentino de Toxicología y XIX Jornadas Interdisciplinarias de Toxicología. La Plata, Argentina (1999).

${ }^{4}$ Kovach, I.M., Qian, N. and Bencsura, A. Efficient product clearance through exit channels in substrate hydrolysis by acetylcholinesterase, FEBS Lett. 349 (1): 60-64 (1994).
} 
Se reconocen entonces tres zonas estables a lo largo del recorrido de ingreso del TMP, cada una determinada por un rango de variación de los parámetros $\mathrm{d}_{\mathrm{P}-\mathrm{O}[\mathrm{Ser200]}} \mathrm{y}_{\mathrm{O}-\mathrm{Oxian}}$. Por relacionarse teóricamente con mínimos energéticos a lo largo del recorrido en la garganta o canal hidrofóbico desde el exterior de la proteína hasta el sitio reactivo, a cada uno de estas posiciones las denominaremos Pausa Serínica o $\boldsymbol{P}_{\text {Ser200, }}$ que corresponde a la posición inmediata anterior al ataque nucleofílico, Pausa Intermedia o $\boldsymbol{P}_{\text {int }}$ y Pausa Hidrofóbica o $\mathbf{P}_{\text {Hid, }}$ por su proximidad a los grupos hidrofóbicos de los restos de Phe en el subsitio hidrofóbico.

Tomando estos tres lugares como referencia, se realiza una búsqueda de conformaciones de mínima energía (Tablas 11.IV y 11.V) y, a partir de ellas, la construcción de las superficies de energía potencial por rotación de cada uno de los tres ángulos diedros $\Psi$, tanto para el TMP (Figuras 11.2, 11.3 y 11.4) como para los análogos TMSP y TMSeP (Figuras 11.5, 11.6 y 11.7), con el fin de realizar una comparación de la libertad conformacional de estas moléculas en cada una de las tres Pausas propuestas.

En estas representaciones de las curvas de energía conformacional relativa, los valores de energía se reescalan en cada serie para permitir una mejor descripción cualitativa de la situación particular. Es necesario tomar en cuenta que, al nivel de cálculo utilizado, los valores absolutos de las energías no tienen interés cuantitativo, sino sólo en térmimos de las relaciones cualitativas entre ellos.

Figura 11.4: Curvas de energía potencial del TMP ubicado en $\boldsymbol{P}_{\text {Hid, }}$ para las libres rotaciones de los ángulos $\Psi_{1}, \Psi_{2}$ y $\Psi_{3}$. La flecha indica la posición del confórmero gauche, -gauche, -gauche de menor energía. Se indica también el elemento que determina la restricción conformacional representada en las regiones de alta energía.
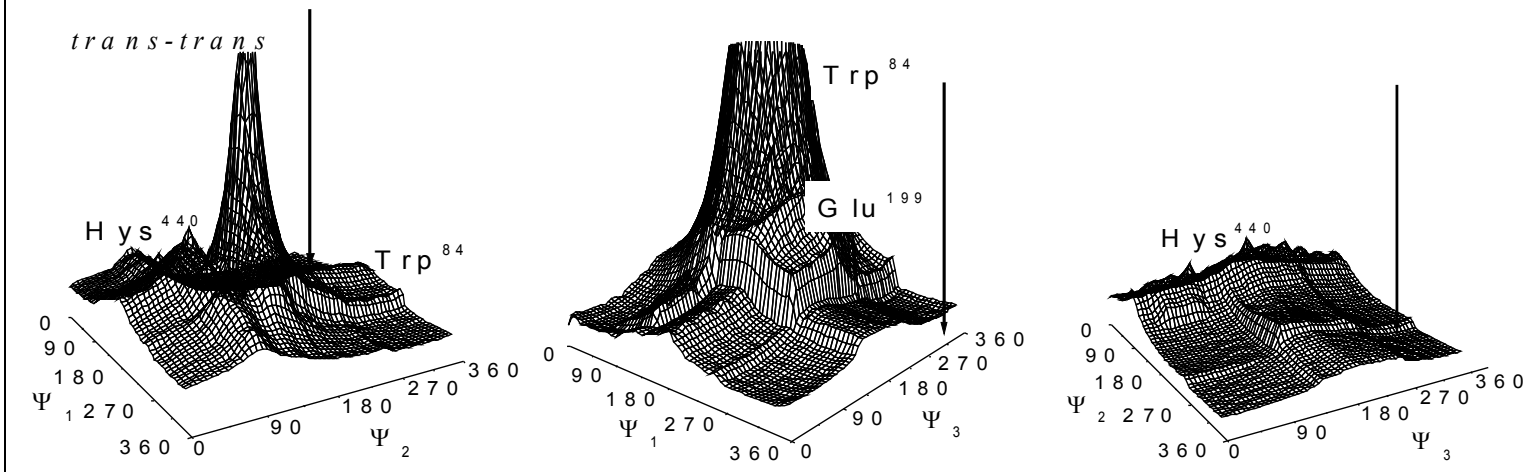

Tabla 11.IV: Valores energéticos y geométricos para las ubicaciones de mínima energía (PM3) para TMP en AChE(sa) 


\begin{tabular}{ccccc}
\hline & & $\mathrm{P}_{\text {Ser200 }}$ & $\mathrm{P}_{\text {int }}$ & $\mathrm{P}_{\text {Hid }}$ \\
\hline & $\mathrm{d}_{\text {O-Oxian }} \AA$ & 2,4 & 2,9 & 3,8 \\
& $\mathrm{~d}_{\mathrm{P}-\mathrm{O}[\mathrm{Ser200]}} \AA$ & 2,3 & 4,3 & 5,7 \\
TMP & Energía (rel) & 5,1 & 5,1 & 5,1 \\
& $\Psi_{1}$ & -gauche & trans & gauche \\
& $\Psi_{2}$ & -gauche & gauche & -gauche \\
& $\Psi_{3}$ & -gauche & -gauche & -gauche \\
\hline
\end{tabular}

\section{Conclusiones obtenidas a partir del modelo}

De nuestros resultados ya presentados (3), se concluyen tres cuestiones importantes: (a) las interacciones de los pequeños fosfatos con el modelo reducido del enzima, no implican variaciones importantes en los restos aminoacídicos considerados ${ }^{(5)}$, son menores para las conformaciones de menor energía y son comparables a las encontradas en bibliografía ${ }^{(6)}$; (b) existe más de un confórmero estable en el sitio activo de la enzima y en general la conformación más estable en el vacío no es necesariamente la más estable en el entorno de este sitio; (c) las conformaciones del TMP en los distintos complejos son comparables a las conformaciones presentes en la superficie potencial obtenida para el vacío ${ }^{(7)}$.

Comparando los resultados mostrados en la Tabla 11.III con aquellos existentes en la Tabla 11.IV, es posible indicar que el análisis preliminar de la primera de las aproximaciones, se refiere a la ubicación del TMP en $\boldsymbol{P}_{\boldsymbol{I n t} \text {. }}$ Sin embargo, las conclusiones descritas se pueden generalizar para cada una de las tres ubicaciones.

Caracterizando comparativamente $\boldsymbol{P}_{\text {Hid }}, \boldsymbol{P}_{\text {Int }}$ y $\boldsymbol{P}_{\text {Ser20o, }}$ es posible indicar que, respecto a la permisividad conformacional, aquellas se ordenan según $\boldsymbol{P}_{\text {Hid }}>\boldsymbol{P}_{\text {ser200 }}>$ $\boldsymbol{P}_{\boldsymbol{I n t}}$. Es decir, en $\boldsymbol{P}_{\text {Hid }}$ el TMP se ubicará con una libertad conformacional similar a aquella que posee en el vacío, de

\footnotetext{
${ }^{5}$ La variación más importante, y esto coincide con lo encontrado en bibliografía, es respecto al ángulo de rotación del anillo imidazol de la $\mathrm{Hys}^{440}$ que forma parte de la tríada catalítica.

${ }^{6}$ Enyedy, I.J., Kovach, I.M. and Bencsura, A. Molecular dynamics study of active-site interactions with tetracoordinate transients in acetylcholinesterase and its mutants, Biochem.J. 353 (Pt 3): 645-653 (2001).

${ }^{7}$ Esto es coherente con el criterio aplicado por muchos autores, en el sentido de que las simulaciones de entornos hidrofóbicos, como lo es la estructura estudiada, son homologables con los cálculos o mediciones hechas en el vacío.
} 
forma que las características de las superficies de energías relativas en una y otra situación, son comparables entre sí.

Figura 11.5: Curvas de energía potencial del TMSP y TMSeP ubicados en $\boldsymbol{P}_{\text {Ser }}$, para las libres rotaciones de los ángulos $\Psi_{1}, \Psi_{2}$ y $\Psi_{3}$. La flecha indica la posición de los confórmeros de menor energía. Se indican también los restos aminoacídicos que determinan la restricción conformacional representada en las regiones de alta energía.

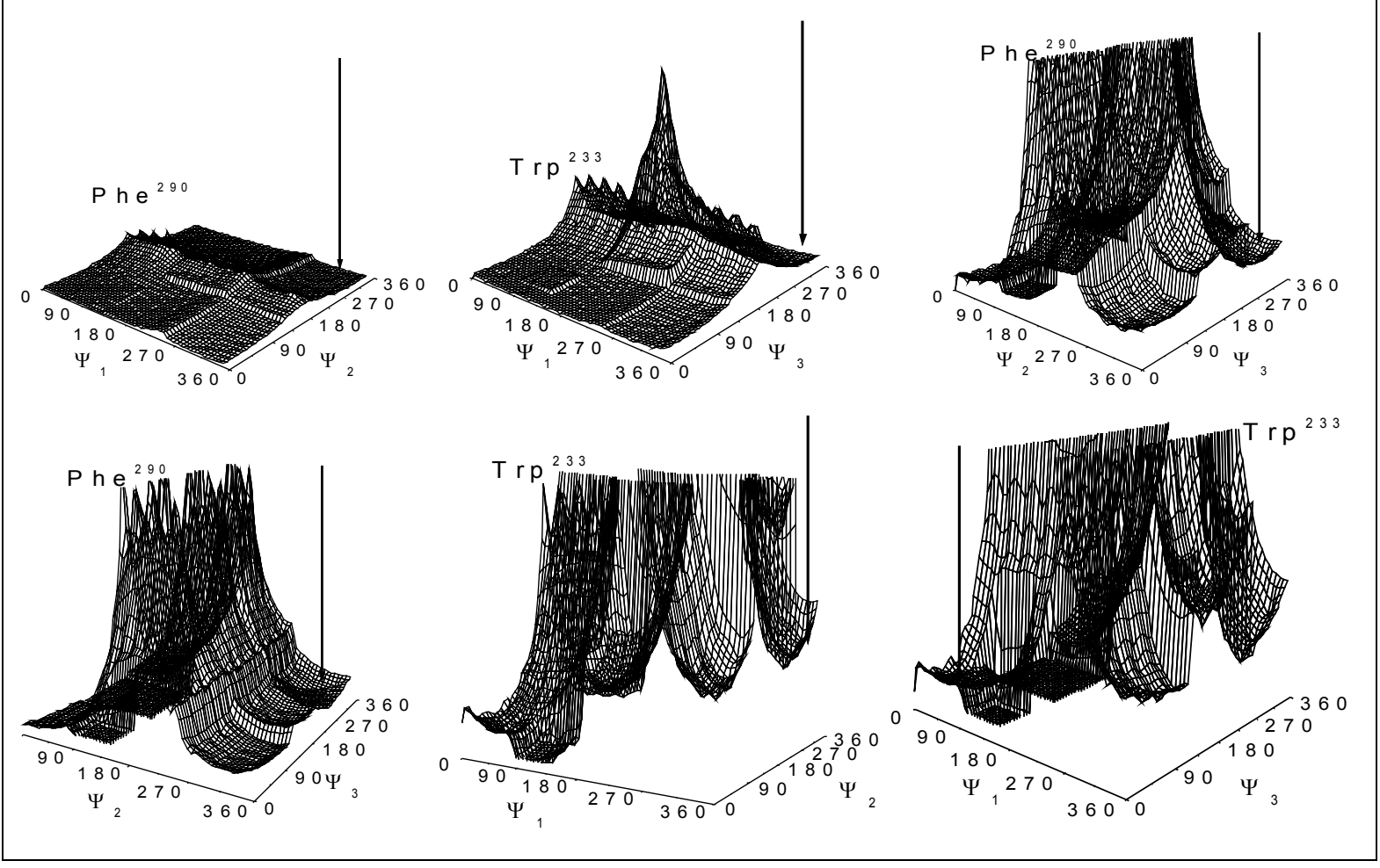

Por otro lado en $\boldsymbol{P}_{\text {Ser20o, el TMP debe ubicarse según }}$ las condiciones conformacionales ya descritas ${ }^{(8)}$, de manera de iniciar el proceso de interacción con $\operatorname{Ser}^{200}$ y proceder a la hidrólisis del grupo saliente. En ese sentido, existe una restricción conformacional que orienta los sustituyentes del fósforo en la medida de cumplir las condiciones conformacionales previos al ataque nucleofílico. Esto se ve reflejado en las superficies de energías relativas respectivas.

Finalmente, $\boldsymbol{P}_{\text {Int }}$ correspondería a un "cuello de botella" del canal de entrada del sitio activo, existiendo una importante restricción conformacional representada en la forma de las superficies de energías relativas y en una restricción estérica que da lugar a la dificultad para ubicar un confórmero estable para el TMSeP.

\footnotetext{
8 Taylor, P. et al. Determining ligand orientation and transphosphonylation mechanisms on acetylcholinesterase by $\mathrm{Rp}, \mathrm{Sp}$ enantiomer selectivity and site-specific mutagenesis, Enantiomer. 2 (3-4): 249-260 (1997).
} 
Mediante la observación de las diferencias cualitativas de las superficies conformacionales para los diferentes OPs, se puede concluir que la restricción conformacional existe en dos sentidos diferentes: por un lado hay una superposición de las restricciones inherentes a cada molécula de inhibidor con los impedimentos estéricos de los restos cercanos, de manera que en las curvas aparece una superposición de esta "libertad intrínseca" con las restricciones impuestas por restos aminoacídicos que superponen sus estructuras con los metilos en rotación. Tal situación es válida tanto para $\boldsymbol{P}_{\boldsymbol{I n t}}$ como para $\boldsymbol{P}_{\text {Ser20o y }} \boldsymbol{P}_{\text {Hid. }}$. Esto aparece claramente cuando existe la restricción por cercanía de restos de la estructura polipeptídica es menor, al punto de homologar parte de estas curvas con aquellas de libre rotación de las moléculas (ver Figuras 2.5, 3.4 y 4.5).

Tabla 11.V: Valores energéticos y geométricos para las ubicaciones de mínima energía (PM3) para TMSP y TMSeP en $\mathrm{AChE}(\mathrm{sa})$

\begin{tabular}{|c|c|c|c|c|}
\hline & & $P_{\text {Ser200 }}$ & $P_{\text {int }}$ & $\mathrm{P}_{\text {Hid }}$ \\
\hline \multirow{6}{*}{ TMSP } & $\mathrm{d}_{\mathrm{O}-\text { - Xxian }} \AA$ & 3,8 & 3,3 & 4,1 \\
\hline & $\mathrm{d}_{\mathrm{P}-\mathrm{O}[\mathrm{Ser} 200]} \AA$ & 2,9 & 4,8 & 5,7 \\
\hline & Energía (rel) & 8,8 & 6,3 & 6,0 \\
\hline & $\Psi_{1}$ & -gauche & -gauche & gauche \\
\hline & $\Psi_{2}$ & -gauche & gauche & -gauche \\
\hline & $\Psi_{3}$ & -gauche & -gauche & -gauche \\
\hline \multirow{6}{*}{ TMSeP } & $\mathrm{d}_{\mathrm{O}-\text { Oxian }} \AA$ & 2,4 & - & 6,2 \\
\hline & $\mathrm{d}_{\mathrm{P}-\mathrm{O}[\mathrm{Ser} 200]} \AA$ & 2,9 & - & 6,1 \\
\hline & Energía (rel) & 9,1 & - & 5,3 \\
\hline & $\Psi_{1}$ & -gauche & - & gauche \\
\hline & $\Psi_{2}$ & -gauche & - & -gauche \\
\hline & $\Psi_{3}$ & -gauche & - & gauche \\
\hline
\end{tabular}

Pero además es necesario considerar otro tipo de restricción y es la que aparece por el desplazamiento de los grupos metilos en la medida que el tamaño del calcógeno unido al átomo de fósforo aumenta. Se puede observar que en la medida que los espacios restringidos del canal son ocupados por grupos fosforilo, tionilo y selenilo, sucesivamente más grandes, los restos metilo son desplazados hacia entornos diferentes. Esto se verifica en una modificación cualitativa de las superficies de energía conformacional en la medida que nos movemos desde el TMP hasta el TMSP y el TMSeP. Esta modificación del entorno de los metilos se hace más importante en $\boldsymbol{P}_{\boldsymbol{I n t}}$, donde la molécula debe ocupar un espacio relativamente más pequeño. 
Como ya es descrito en publicaciones anteriores, los restos $\operatorname{Trp}^{89}$ y $\mathrm{Phe}^{331}$ juegan un papel importante en la conformación del sitio de interacción hidrofóbica. En este modelo interactúan en mayor o menor medida con los metilos en $\boldsymbol{P}_{\text {int }}$ y en $\boldsymbol{P}_{\text {Hid. }}$. Es interesante el papel restrictivo que, según el modelo, juegan los restos aminoacídicos $\mathrm{His}^{440}$ y Glu ${ }^{199}$. Dicha funcionalidad no es descrita ni reportada previamente en ninguno de los trabajos previos de modelado del sitio activo.

Se han logrado obtener correlaciones entre las propiedades conformacionales de inhibidores reversibles, del tipo trimetilamonio polimetileno sustituidos, y su actividad respecto a la $\mathrm{AChE}$ eritrocitaria. En estos estudios se han descrito diferencias en los mecanismos de interacción, respecto a las variabilidades conformacionales ${ }^{(9)}$. Se verifica entonces la importancia de las restricciones conformacionales en las interacciones con el sitio activo.

Figura 11.6: Curvas de energía potencial del TMSP ubicado en $\boldsymbol{P}_{\text {int }}$, para las libres rotaciones de los ángulos $\Psi_{1}, \Psi_{2}$ y $\Psi_{3}$. La flecha indica la posición del confórmero de menor energía. Se indica también el resto aminoacídico que determina la restricción conformacional representada en las regiones de alta energía.
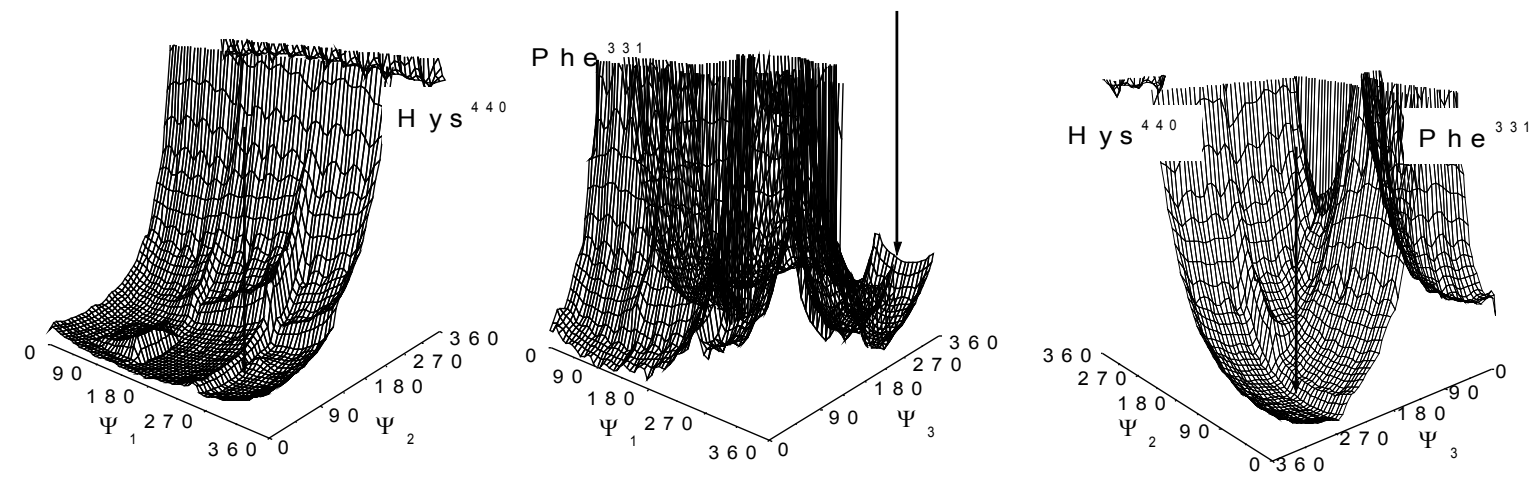

\section{Consideraciones respecto a la actividad anti colinesterasa de los OPs}

\footnotetext{
${ }^{9}$ Rozengart, E.V. et al. The conformational aspects of the interaction of the cholinesterases of Pacific Ocean squid and vertebrates with derivatives of polymethylene bis (trimethylammonium), $Z$ h. Evol. Biokhim. Fiziol. 30 (2): 168-176 (1994).
} 


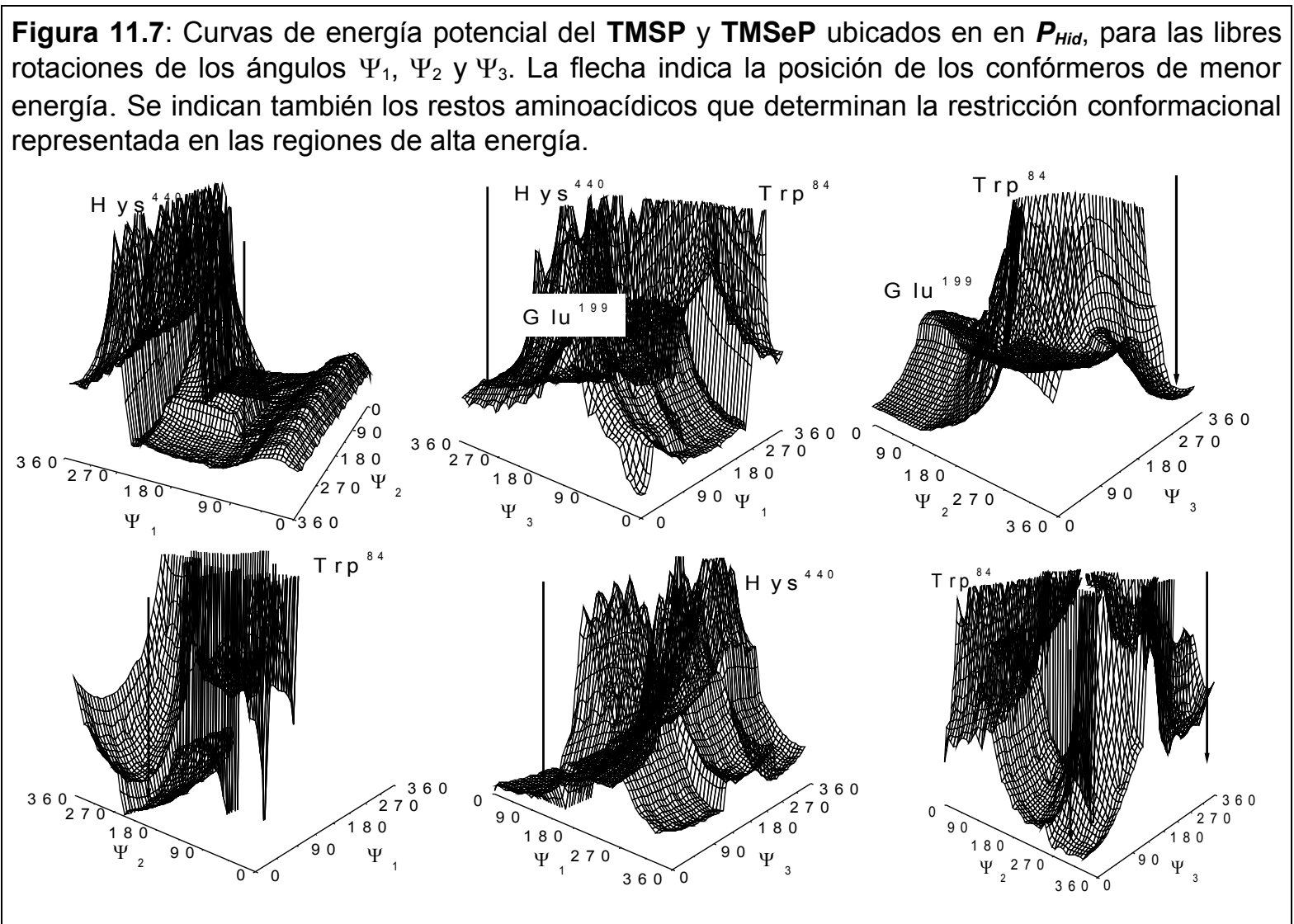

Por antecedentes ya publicados y descritos en el Capítulo anterior, el centro activo de la AChE reconoce a la conformación menos común de la $\mathrm{ACh}$ en solución que corresponde a la forma trans, trans, de manera que en el sitio activo hay una estabilización de la forma menos estable en solución. Respecto a ese reconocimiento de conformación, se puede extender la diferente respuesta del centro activo de la $\mathrm{AChE}$ a los OPs identificando cuales estructuras de aquel se encuentran en solución y cuales de ellas prevalecen. Se puede eventualmente reconocer el sitio de catálisis por medición del producto de hidrólisis y efectuar consideraciones acerca de la forma de reconocimiento de los fosforados.

Considerando la energía libre necesaria para estabilizar la conformación menos estable de la ACh, se puede comparar con las energías libres de cambio conformacional de los ésteres de fosfato. Es necesario tomar en cuenta que, existiendo varias configuraciones cercanamente estables, los valores pueden relacionarse con distintas formas de reconocimiento de los fosfatos al sitio activo.

Una cuestión interesante a discutir se relaciona con la ruptura de la degeneración energética de formas conformacionales equivalentes en el vacío, según se muestra en la Figura 11.8. Éstas, enfrentadas a las estructuras 
asimétricas del sitio activo, son seleccionadas de manera tal que confórmeros de igual energía en el vació (o en solución), pasan a tener energías muy diferentes en el entorno del sitio activo. Por ejemplo, la forma -gauche, -gauche, -gauche corresponde al confórmero de menor energía en $\boldsymbol{P}_{\text {ser20o, pero }}$ su confórmero enantiomérico gauche, gauche, gauche, presenta grandes restricciones estéricas y tiene una energía muy alta que no corresponde a una estructura estable del complejo enzima-inhibidor. Ambas conformaciones en el vacío presentan la misma energía conformacional y son estructuras estables.

Este hecho es válido también para $\boldsymbol{P}_{\text {Int }}$ y $\boldsymbol{P}_{\text {Hid, }}$, puesto que ambos lugares se estructuran en configuraciones asimétricas respecto a la molécula entrante. Esto se relaciona con el comportamiento de moléculas que presentan isomería óptica, por ejemplo, se ha reportado que la hidrólisis catalizada de la forma $\mathrm{Rp}$ de la cicloheptil metilfosfonil tiocolina es alrededor de doscientas veces más rápida que la hidrólisis de la forma $\mathrm{Sp}{ }^{(10)}$. Considerando que el grupo con características enantioméricas se ubica en el bolsillo acilo, este hecho da cuenta de la enantioselectividad de este subsitio de la AChE.

Esta ruptura de degeneración energética conformacional significa que los grados de degeneración utilizados para el cálculo de las abundancias relativas de los confórmeros en el vacío $(2: 2: 1)^{(11)}$, pierden validez para el cálculo de estas abundancias conformacionales en el entorno de la proteína de naturaleza asimétrica. Las consecuencias de este hecho se rediscutirán en el Capítulo 14.

Se describió a $\boldsymbol{P}_{\text {int }}$ como un "cuello de botella" del canal hacia el sitio activo del enzima. Esta característica del canal da lugar a especular acerca de la función de esta estructura. Recordemos que la búsqueda de estas Pausas se realiza con un modelo rígido. Por otro lado está descrita la gran eficiencia catalítica de la AChE y la gran afinidad de los inhibidores por su sitio activo.

Entonces, la estabilización de inhibidores y sustratos a lo largo del canal debe entenderse como parte de los eventos que favorecen los procesos de inhibición o catálisis y no como parte de su restricción. De alguna manera, el cálculo de las energías de los confórmeros más estables en $\boldsymbol{P}_{\text {Hid }}, \boldsymbol{P}_{\text {Int }} \mathrm{y}$

\footnotetext{
${ }^{10}$ Hosea, N.A., Berman, H.A. and Taylor, P. Specificity and orientation of trigonal carboxyl esters and tetrahedral alkylphosphonyl esters in cholinesterases, Biochem. 34 (36): 11528-11536 (1995).

11 Ver Capítulos 1 y 2 para una discusión más detallada de estas asignaciones.
}

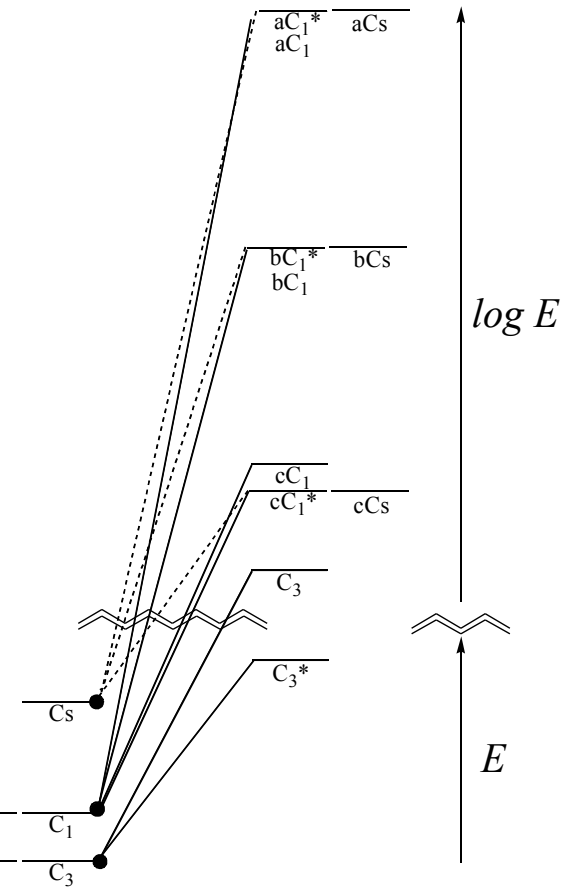

Figura 11.8: Ruptura de la degeneración conformacional energética para las opciones conformacionales del TMP en el sitio activo de la AChE. Las conformaciones $a, b$ y $c$ corresponden a las rotaciones de $120^{\circ}$ alrededor del eje determinado por el enlace $\mathrm{P}=\mathrm{O}$. Las conformaciones denotadas con * representan a las imágenes especulares del correspondiente confórmero. 
$\boldsymbol{P}_{\text {ser200 }}$ puede interpretarse como el cálculo de las energías de estabilización del complejo enzima-inhibidor, asociadas a modificaciones de la estructura terciaria (tridimensional) de la proteína, más que al cálculo de barreras energéticas.

Según esta interpretación, el paso de moléculas como el TMP a lo largo del canal, genera tensiones en la estructura terciaria de la proteína, que forzaría modificaciones que favorecerían los eventos subsiguientes del proceso. Así, en $\boldsymbol{P}_{\text {Int }}$ se generarían las tensiones más grandes, y la ubicación de las moléculas en este lugar, daría lugar a las modificaciones conformacionales más importantes de la proteína. La hipótesis que surge de este razonamiento es que $\boldsymbol{P}_{\text {Int }}$ sería una estructura relevante en el contexto de un modelo de "encaje inducido" de la actividad de la AChE, actuando como una "cerradura dinámica" del sitio activo ${ }^{(12)}$.

Sin embargo, reconocemos que existe una aparente contradicción entre la hipótesis planteada y el hecho que en la sucesión $\mathrm{P}=\mathrm{O}, \mathrm{P}=\mathrm{S}$ y $\mathrm{P}=\mathrm{Se}$, existe una mayor dificultad torsional en el vacío que se correlaciona con la dificultad torsional en $\boldsymbol{P}_{\boldsymbol{I n t}}$. Interpretando directamente el enunciado propuesto, una mayor dificultad torsional en $\boldsymbol{P}_{\boldsymbol{I n t}}$ promovería un mayor cambio conformacional en la estructura proteica que a su vez se relacionaría con una mayor eficiencia en la interacción.

Pero la potencia inhibitoria, por lo menos en esta serie propuesta, decrece en el sentido que disminuye la libertad conformacional del fosfato. Recordemos que se describe en general que los tionofosfatos tienen una capacidad inhibitoria que es, por lo menos, dos órdenes de magnitud menor que los oxofosfatos análogos.

Pero la inhibición comienza con el pasaje de las moléculas a través del canal y continúa, como ya hemos descrito, con el ataque nucleofílico al átomo del fósforo. En esta sucesión entonces, el factor relevante sería este segundo evento puesto que las diferencias de electronegatividad de los pares de átomos indican que los oxofosfatos exponen un fósforo más desprovisto de electrones que sus análogos tio y selenofosfatos.

\footnotetext{
${ }^{12}$ El concepto de "cerradura dinámica" aquí utilizado no debe entenderse en homología al concepto de "llave-cerradura" usado como modelo clásico antitético al de "encaje inducido". Ver textos de enzimología para más detalles.
} 
Sobre la diferencia entre estos eventos (pasaje a través del canal y fosforilación propiamente tal) y la relevancia del comportamiento conformacional en cada uno de ellos, volveremos en el Capítulo 13.

Entonces, la hipótesis propuesta debería estar acotada por dos cuestiones independientes: o el fenómeno de cerradura es relevante sólo para explicar la mayor o menor dificultad para el pasaje de moléculas a través del canal y poco influye en los eventos asociados a la fosforilación, o estas modificaciones conformacionales de la proteína sólo son eficientes en la medida que ocurran dentro de los márgenes que determina el pasaje de un oxofosfato a su través, mientras que el pasaje de tiofosforados o selenofosforados promueve un cambio conformacional en exceso, contraproducente respecto al efecto buscado.

Una o otra posibilidad no son excluyentes entre sí y con peso relativo aportarían en resolver la aparente contradicción de la hipótesis. 


\section{2}

\section{Acetilcolinesterasa: Cinética de la inhibición}

El estudio de las características cinéticas de la catálisis y la inhibición de la $\mathrm{AChE}$, es el complemento necesario para el total entendimiento de la mecánica de estos procesos. Como ya se describió en Capítulos anteriores, para describir la interacción de los OP con el sitio activo de la enzima, clásicamente se utilizó un modelo que considera cuatro puntos críticos (Figura 12.1). B es un grupo básico representado en un nitrógeno imidazol de la histidina, $\mathbf{O H}$ corresponde al grupo hidroxilo de la serina, HA es un grupo acídico (hidroxilo de tirosina) y $\mathbf{S}$ es un sitio aniónico, organizados en un "bolsillo" determinado por la estructura terciaria del enzima.

Una vez formado, este complejo experimenta una rápida sustitución nucleofílica dentro de la enzima liberando un resto sustituyente de la tercera posición del éster fosfórico. Respecto de los carbamatos, se ha descrito que presentan un mecanismo cualitativamente equivalente de inhibición, pero cuantitativamente muy distinto, lo que condiciona sus diferencias en capacidades inhibitorias. Ya se ha dicho que la catálisis opera en el límite difusional, de manera que la actividad enzimática es muy sensible a la interacción con inhibidores de distinta naturaleza.

Este proceso de inhibición tiene varios pasos que pueden esquematizarse según el esquema de la Figura 12.2. En el primer paso, la relación entre $k_{-1}$ y $k_{1}$ es lo que determina la afinidad del OP por la $\mathrm{AChE}$, en un proceso que ocurre a través de un estado de transición con un fósforo pentacoordinado. ${ }^{(1)}$ Estudios del efecto de la temperatura sobre estas constantes cinéticas, dan cuenta que $\mathrm{Ka}$ es una expresión de la asociación-disociación de la enzima con el inhibidor. Dicha relación $k_{-1} / k_{1}$ se denomina constante de

\footnotetext{
${ }^{1}$ Singh, A.K. Kinetic analysis of inhibition of brain and red blood cell acetylcholinesterase and plasma cholinesterase by acephate or methamidophos, Toxicol.Appl.Pharmacol. 81 (2): 302-309 (1985).
} 
afinidad (Ka) y determina la inhibición reversible de la enzima. ${ }^{(2)}$

En un paso siguiente, la constante $k_{p}$ o constante de fosforilación, es la que determina la velocidad de formación de un enlace covalente estable, con la consecuencia de la inhibición permanente de la enzima y la liberación de un grupo saliente $\mathbf{Y H}{ }^{(3)}$. En general, las constantes de inhibición están influidas por una cierta cantidad de factores como el $\mathrm{pH}$ (4), la presencia de detergentes aniónicos ${ }^{(5)} \mathrm{o}$ de ciertas sustancias orgánicas como aquellas derivadas de la fisostigmina y la acridina. ${ }^{(6)}$ Determinados modelos de interacción con el sustrato, implican considerar relaciones cinéticas más complejas que la simple formación de intermedios de activación como el descrito. ${ }^{(7)}$

Aunque se considera que la inhibición de los OP es irreversible, en un esquema general se debe considerar la reacción de hidrólisis del resto fosfato unido al centro activo en un mecanismo análogo a la hidrólisis del sustrato, con la restitución del centro activo y la liberación de un dialquil fosfato como producto final del proceso. Esta es una reacción de hidrólisis, con su constante cinética asociada, $k_{4}$. La desfosforilación de la $\mathrm{AChE}$ es un proceso espontáneo, pero tiene una cinética lenta, de manera que la irreversibilidad de la inhibición esta determinada por la pequeña magnitud de la constante de hidrólisis del complejo enzima-OP ${ }^{(8)}$.

\footnotetext{
${ }^{2}$ Brestkin, A.P. et al. The mechanism of anticholinesterase action of acetylene organophosphorus inhibitors, Bioorg.Khim. 18 (8): 1067-1072 (1992).

3 Langel, U.L. and Jarv, J.L. Leaving group effects in butyrylcholinesterase reaction with organophosphorus inhibitors, Biochim.Biophys.Acta 525 (1): 122-133 (1978).

${ }^{4}$ Brestkin, A.P. et al. [The mechanism of anticholinesterase action of acetylene organophosphorus inhibitors], Bioorg.Khim. 18 (8): 1067-1072 (1992).

5 Singh, A.K. Molecular properties and inhibition kinetics of acetylcholinesterase obtained from rat brain and cockroach ganglion, Toxicol.Ind.Health. 6 (6): 551-570 (1990).

6 Galli, A. et al. In vitro protection of acetylcholinesterase and butyrylcholinesterase by tetrahydroaminoacridine. Comparison with physostigmine, Biochem.Pharmacol. 43 (11): 2427-2433 (1992).

Dunn, S.M. and Raftery, M.A. Agonist binding to the Torpedo acetylcholine receptor. 1. Complexities revealed by dissociation kinetics, Biochem. 36 (13): 3846-3853 (1997).

${ }^{8}$ (a) de Jong, L.P., Wolring, G.Z. and Benschop, H.P. Reactivation of acetylcholinesterase inhibited by methamidophos and analogous (di)methylphosphoramidates, Arch.Toxicol. 49 (2): 175-183 (1982). (b) Langenberg, J.P. et al. Spontaneous and oxime-induced reactivation of acetylcholinesterase inhibited by phosphoramidates, Arch.Toxicol. 62 (4): 305-310 (1988). (c) Kovach, I.M. Competitive irreversible inhibition of
}

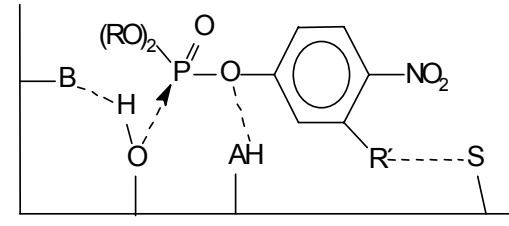

Figura 12.1: Modelo elemental clásico de la interacción de OPs con el sitio activo del enzima AChE. 


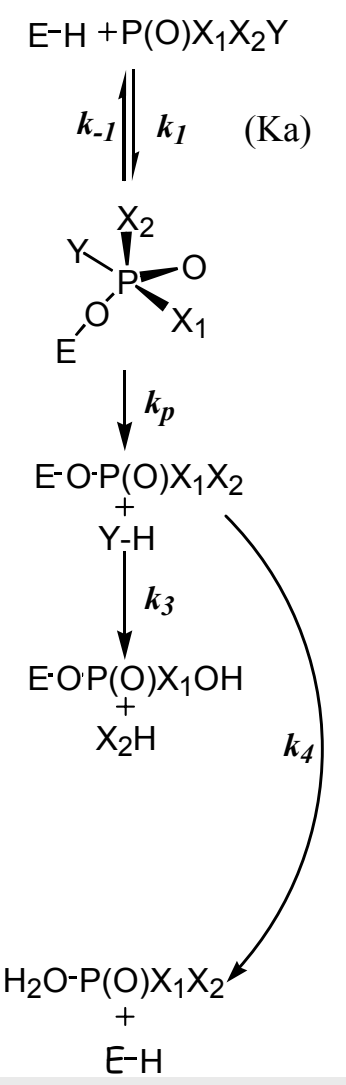

Figura 12.2: Procesos implicados en la inhibición de la AChE por parte de un inhibidor OP.
Una prueba directa del mecanismo de inhibición descrito para los OPs, se obtiene midiendo las constantes de reactivación $k_{4}$ de la AChE inhibida con ésteres dialquilfosfóricos, que se demuestra dependiente de los grupos $-\mathbf{X}_{\mathbf{1}}$ y $-\mathbf{X}_{\mathbf{2}}$ e independiente del grupo saliente $-\mathbf{Y} .^{(9)}$

Este proceso de reactivación puede ser favorecido por sustancias capaces de interaccionar con el complejo enzima-fosfato dialquilo, denominadas agentes de reactivación, liberando el sitio activo para dar lugar nuevamente a la enzima funcional. En estos procesos es posible estudiar la cinética de reactivación. ${ }^{(10)}$ Entre dichas sustancias se encuentran las oximas, sustancias que son consideradas como agentes terapéuticos adecuados para los primeros estadíos de una intoxicación por OPs. ${ }^{(11)}$

El último proceso a considerar, es la hidrólisis de uno de los restos alquilo del grupo fosfato unido al centro activo, dando lugar a un complejo muy estable $\mathrm{y}$ caracterizado por la presencia de un grupo ácido en el centro fosfórico. Este proceso se denomina "envejecimiento" de la fosforilación. La irreversibilidad de la reacción está establecida por la magnitud de la constante cinética $k_{3}{ }^{(12)}$ y en condiciones fisiológicas implica la imposibilidad de remoción del grupo fosfato mediante agentes de reactivación.

enzymes in the presence of a substrate: scope and limitations, J.Enzyme.Inhib. 4 (3): 201-212 (1991).

9 Aldridge, W.N. and Davison, A.N. The mechanism of inhibition of cholinesterases by organophosphorus compuonds, Biochem.J. 55:763-766 (1953).

${ }^{10} \mathrm{Su}$, C.T. et al. Kinetic studies and structure-activity relationships of bispyridinium oximes as reactivators of acetylcholinesterase inhibited by organophosphorus compounds, Fundam.Appl.Toxicol. 6 (3): 506-514

11 Worek, F., Diepold, C. and Eyer, P. Dimethylphosphoryl-inhibited human cholinesterases: inhibition, reactivation, and aging kinetics, Arch.Toxicol. 73 (1): 7-14 (1999).

12 (a) Clothier, B., Johnson, M.K. and Reiner, E. Interaction of some trialkyl phosphorothiolates with acetylcholinesterase. Characterization of inhibition, aging and reactivation, Biochim.Biophys.Acta 660 (2): 306-316 (1981). (b) Glickman, A.H., Wing, K.D. and Casida, J.E. Profenofos insecticide bioactivation in relation to antidote action and the stereospecificity of acetylcholinesterase inhibition, reactivation, and aging, Toxicol.Appl.Pharmacol. 73 (1): 16-22 (1984). (c) Wing, K.D., Glickman, A.H. and Casida, J.E. Phosphorothiolate pesticides and related compounds: oxidative bioactivation and aging of the inhibited acetylcholinesterase, Pestic.Biochem.Physiol. 21 (1): 22-30 (1984). (d) Berman, H.A. and Decker, M.M. Kinetic, equilibrium and spectroscopic studies on dealkylation ("aging") of alkyl organophosphonyl acetylcholinesterase, J.Biol.Chem. 261: 10646-10652 (1986). (e) Ordentlich, A. et al. Engineering resistance to 'aging' of phosphylated human acetylcholinesterase. Role of hydrogen bond network in the active center, FEBS Lett. 334 (2): 215-220 (1993). 
Se sugiere que el proceso de envejecimiento depende de un mecanismo que involucra a los residuos $\mathrm{Glu}^{202}$ y $\mathrm{Phe}^{338}$ quienes estabilizan la tríada catalítica $\mathrm{His}^{440}$ mediante interacciones carga-carga y carga- $\pi$, previo a la hidrólisis del grupo $\mathbf{X}_{\mathbf{1}}{ }^{(13)}$

Aunque no es importante para el comportamiento global del enlace enzima-fosfato, existe un proceso que da lugar a un complejo enzima-inhibidor aun más estable, que consiste en la hidrólisis y liberación de un tercer grupo saliente $\mathbf{X}_{\mathbf{2}} \mathbf{H}$, dando lugar a una fosforilación por un fosfato inorgánico del resto serina.

En la bibliografía clásica, se definen como determinantes de tipo estérico y de tipo energético del poder inhibidor de los OPs: (a) el tamaño de los grupos $-\mathbf{X}_{\mathbf{1}}$ y $-\mathbf{X}_{\mathbf{2}} \mathbf{y}$ el grupo saliente principal $-\mathbf{Y},{ }^{(14)}$ que permitan el ingreso al sitio activo sin impedimentos estéricos; (b) la lipofilicidad de la molécula entrante, ${ }^{(15)}$ que permite una mejor interacción en el entorno del sitio activo; (c) la naturaleza funcional los sustituyentes del fosfato ${ }^{(16)}$ que determina la eficiencia de la interacción con los correspondientes subsitios del sitio activo; (d) la labilidad del enlace P-Y, ${ }^{(17)}$ que tiene implicancia directa en la cinética del proceso y finalmente (e) la accesibilidad del átomo de fósforo al ataque nucleofílico por parte del hidroxilo del enzima, que está determinada por el tamaño de los sustituyentes del fosfato. Según nuestras hipótesis, un factor que también influiría en el comportamiento cinético, es la libertad conformacional de la molécula entrante. Sobre este tema profundizaremos en el siguiente Capítulo.

13 Shafferman, A. et al. Aging of phosphorylated human acetyl cholinesterase: catalytic processes mediated by aromatic and polar residues of the active centre, Biochem.J. 318 ( Pt 3): 833-840 (1996).

14 (a) Berman, H.A. and Leonard, K. Ligand exclusion on acetylcholinesterase, Biochem. 29 (47): 10640-10649 (1990). (b) Wallace, K.B. and Kemp, J.R. Species specificity in the chemical mechanisms of organophosphorus anticholinesterase activity, Chem.Res.Toxicol. 4 (1): 41-49 (1991).

15 Antosiewicz, J., Wlodek, S.T. and McCammon, J.A. Acetylcholinesterase: Role of the enzymes charge distribution in stering charged ligands toward the active site, Biopolymers. 39 (1): 85-94 (1996).

${ }^{16}$ Nolte, H.J., Rosenberry, T.L. and Neumann, E. Effective charge on acetylcholinesterase active sites determined from the ionic strength dependence of association rate constants with cationic ligands, Biochem. 19 (16): 3705-3711 (1980).

${ }^{17}$ Kemp, J.R. and Wallace, K.B. Molecular determinants of the speciesselective inhibition of brain acetylcholinesterase, Toxicol. Appl. Pharmacol. 104: 246-258 (1990). 
Tabla 12.I: Criterios cinéticos que definen a un inhibidor de la AChE como un buen pesticida. $^{(22)}$

\begin{tabular}{ll}
\hline Afinidad por la colinesterasa & $\mathrm{K}_{\mathrm{a}}$ (peste) $<\mathrm{K}_{\mathrm{a}}$ (mamífero) \\
Conversión de E-inhibida a E-acilada & $\mathrm{kp}$ (peste) $>\mathrm{kp}$ (mamífero) \\
Envejecimiento & $\mathrm{k}_{3}$ (peste) $>\mathrm{k}_{3}$ (mamífero) \\
Estabilidad del enzima acilada & $\mathrm{k}_{4}$ (peste) $<\mathrm{k}_{4}$ (mamífero) \\
\hline
\end{tabular}

Cuando se diseña un $\mathrm{OP}$, con el propósito de obtener productos de mayor poder pesticida $\mathrm{y}$, al mismo tiempo, grandes márgenes de seguridad para los mamíferos, se procura que se cumplan relaciones adecuadas entre las distintas constantes cinéticas (Tabla 12.I).

Otros factores a considerar incluyen los mecanismos de destrucción in vivo del inhibidor por hidrólisis o procesos oxidativos. Los datos publicados, indican que entre los factores más importantes que determinan las diferencias entre cepas resistentes y cepas sensibles dentro de una misma especie, se encuentra la capacidad de destruir al inhibidor mediante reacciones de oxidación ${ }^{(18)}$ o por la presencia de esterasas específicas que dan lugar a la hidrólisis de los xenobióticos. ${ }^{(19)}$ De igual manera, se han descrito cepas de insectos que presentan resistencia por modificaciones estructurales ${ }^{(20)}$ o de las concentraciones in vivo ${ }^{(21)}$ de la enzima diana de la inhibición.

18 (a) Yu, S.J. and Nguyen, S.N. Detection and biochemical characterization of insecticide resistance in the diamondback moth, Pestic. Biochem. Physiol. 44 (1): 74-81 (1992). (b) Rufingier, C. et al. Mechanisms of insecticide resistance in the aphid Nasonovia ribisnigri (Mosley) (Homoptera: Aphididae) from France, Insect Biochem.Mol.Biol. 29 (4): 385-391 (1999).

${ }^{19}$ (a) Newcomb, R.D. et al. A single amino acid substitution converts a carboxylesterase to an organophosphorus hydrolase and confers insecticide resistance on a blowfly, Proc.Natl.Acad.Sci.U.S.A. 94 (14): 7464-7468 (1997). (b) Bracco, J.E., Barata, J.M. and Marinotti, O. Evaluation of insecticide resistance and biochemical mechanisms in a population of Culex quinquefasciatus (Diptera: Culicidae) from Sao Paulo, Brazil, Mem.Inst.Oswaldo.Cruz. 94 (1): 115-120 (1999). (c) Szlendak, E. et al. Pirimiphos-methyl resistance in two stored product mites, Acarus siro and Acarus farris, as detected by impregnated paper bioassay and esterase activity assays, Exp.Appl.Acarol. 24 (1): 45-54 (2000).

20 (a) Gunning, R.V., Moores, G.D. and Devonshire, A.L. Insensitive acetylcholinesterase and resistance to organophosphates in australian Helicoverpa armigera, Pestic.Biochem.Physiol. 62 (3): 147-151 (1998). (b) (c) Villatte, F. et al. A high number of mutations in insect acetylcholinesterase may provide insecticide resistance, Pestic. Biochem. Physiol. 67 (2): 95-102 (2000). (d) Byrne, F.J. and Toscano, N.C. An insensitive acetylcholinesterase confers resistance to methomyl in the beet armyworm Spodoptera exigua (Lepidoptera: Noctuidae), J.Econ.Entomol. 94 (2): 524-528 (2001). (e) Mori, A. et al. Comparative linkage map development and identification of an autosomal locus for insensitive 
Finalmente, en el estudio de las propiedades cinéticas de la inhibición, un factor muy difícil de evaluar, es la influencia de la concentración local del enzima. Para la mayoría de los estudios in vitro, las concentraciones de trabajo de la enzima son del orden de $10^{-10} \mathrm{M}$ o menores, siendo las concentraciones locales in vivo mucho mayores, ${ }^{(22)}$ por ejemplo la existente en el entorno de las terminaciones nerviosas.

\section{Formalismo cinético}

La reacción de fosforilación irreversible de la $\mathrm{AChE}$ es dependiente de la temperatura y puede ser descrita según la Ecuación 12.a, donde EH es la enzima, IY el OP inhibidor, EI la enzima inhibida mientras que $\mathbf{Y H}$ es el producto de hidrólisis del inhibidor, es decir, corresponde al grupo saliente. Respecto del esquema presentado en la Figura 12.1, el grupo I corresponde al resto -POX $\mathbf{X}_{\mathbf{2}}$. Esta relación define una constante de inhibición bimolecular, $\mathrm{Ki}$, que es tanto mayor para los buenos inhibidores y suele expresarse en unidades de $\mathrm{M}^{-1} \mathrm{~min}^{-1}$.

Esta reacción bimolecular muestra en la práctica una cinética de primer orden pues las concentraciones del inhibidor son diez o más veces las de la enzima. La potencia inhibidora de un OP en particular siempre se expresa como una relación de constantes definidas por las condiciones experimentales. Esto es así pues la adición de sustrato disminuye rápidamente el rango de reacción de los $\mathrm{OP}$ con la $\mathrm{AChE}$, y se asume entonces que el OP reacciona con el centro activo del enzima. El modelo se ve corroborado por las similitudes estructurales en los requerimientos tanto para sustratos como para inhibidores. Es natural, por esta razón, considerar un intermediario durante la inhibición, similar al complejo de Michaelis con el sustrato (Ecuación 12.b), donde EHIY representa al complejo intermediario enzimainhibidor.

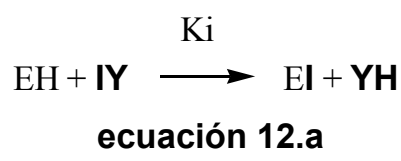

\footnotetext{
acetylcholinesterase-mediated insecticide resistance in Culex tritaeniorhynchus, Insect Mol.Biol. 10 (3): 197-203 (2001).

${ }^{21}$ Charpentier, A. and Fournier, D. Levels of total acetylcholinesterase in Drosophila melanogaster in relation to insecticide resistance, Pestic.Biochem.Physiol. 70 (2): 100-107 (2001).

${ }^{22}$ Aldridge, W.N. The nature of the reaction of organophosphorus compounds and carbamates with esterases, Bull.World.Health.Organ. 44 (1): 25-30 (1971).
}

$$
\frac{[\mathrm{IY}]}{K i}=\frac{[\mathrm{IY}]}{k_{p}}-\frac{K \mathrm{a}}{k_{p}}
$$

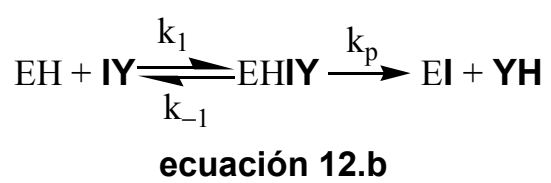




$$
K i=\frac{k p}{K a}
$$

ecuación 12.d

$$
\begin{gathered}
\ln v_{t}=\ln v_{o}-\mathrm{Ki} t \\
\text { ecuación 12.e }
\end{gathered}
$$

$$
\mathrm{Ki}=\frac{1}{\left([\mathbf{I Y}]_{0}-[\mathrm{EH}]_{0}\right) t} \ln \frac{[\mathrm{EH}]_{0}[\mathbf{I Y}]_{\mathrm{t}}}{[\mathbf{I Y}]_{0}[\mathrm{EH}]_{\mathrm{t}}}
$$$$
[\mathrm{EH}]=\frac{v}{a_{m}}
$$

ecuación 12.g
Ya mencionamos que a la relación entre $\mathrm{k}_{1} \mathrm{y} \mathrm{k}_{-1}$ se la denomina usualmente constante de afinidad $\left(\mathrm{Ka}=\mathrm{k}_{-1} / \mathrm{k}_{+1}\right)$, pues representa la capacidad del OP de ingresar al sitio activo $\mathrm{y}$ formar el complejo intermedio de inhibición. Se argumenta que Ka es una medida directa de la afinidad del inhibidor por la enzima. La constante de afinidad es tanto menor para aquellos OP que actúan como buenos inhibidores y suele expresarse en unidades de moles (M).

El segundo paso de fosforilación irreversible, es considerado el determinante principal en la capacidad de inhibición. Esta definido por la constante de equilibrio $k_{\mathrm{p}}$ correspondiente, de manera en que los buenos inhibidores este parámetro presentan valores altos, expresándose normalmente en unidades de $\min ^{-1}$. Dado que esta etapa de la interacción es bastante rápida, se necesitan de muchos controles experimentales para tener resultados fiables durante su determinación.

A partir de este modelo se plantea la Ecuación 12.c, en donde Ki es la constante de primer orden en la formación de la enzima fosforilada con una concentración dada del inhibidor y Ka queda planteada como una constante análoga a la constante de Michaelis, correspondiendo a la concentración del inhibidor que inhibe a la mitad de la enzima. En esta expresión está implícita la definición de cada constante respecto de las demás Ecuación 12.d.

Las relaciones que permiten obtener experimentalmente los distintos parámetros cinéticos, son dependientes de las condiciones bajo las cuales son hechas estas medidas experimentales. Bajo condiciones en las que $[\mathrm{EH}]<<[\mathrm{IY}]$, la constante de inhibición $\mathrm{Ki}$, se denomina constante de pseudoprimer orden y puede calcularse mediante la Ecuación 12.e, ${ }^{(23)}$ donde $v_{o}$ es la actividad de la enzima a tiempo cero y $v_{t}$ es la actividad enzimática a tiempo t.

Pero bajo condiciones de cinética de segundo orden, es decir cuando $[\mathbf{E H}] \approx[\mathbf{I Y}]$, la constante $\mathrm{Ki}$ se calcula mediante la Ecuación 12.f, donde $[\mathbf{E H}]_{0}$ y $[\mathbf{I Y}]_{0}$ corresponden a las concentraciones de enzima e inhibidor a tiempo cero, mientras que $[\mathbf{E H}]_{\mathrm{t}}$ y $[\mathbf{I Y}]_{\mathrm{t}}$ corresponden a las respectivas concentraciones a tiempo t. Las concentraciones iniciales de enzima, $[\mathbf{E H}]_{0}$, se obtienen por cálculo a partir de

\footnotetext{
${ }^{23}$ Sharp, T.R. and Rosenberry, T.L. A pseudo-first-order kinetic approach to measurement of acetylcholine hydrolysis by acetylcholinesterase, J.Biochem.Biophys.Methods. 6 (2): 159-172
} 
la velocidad inicial de catálisis, conociendo la actividad molecular $a_{m}$, para este sustrato según la Ecuación 12.g.

Bajo condiciones de pseudoprimer orden, cuando $[\mathbf{E H}]<<[\mathbf{I Y}]$, la constante aparente de inhibición Ki depende de la concentración del inhibidor (Ecuación 12.h), que puede ser escrita en forma de doble inversa para la obtención de una función lineal (Ecuación 12.i). Evaluando ambas constantes, kp y Ka, se obtendrá el valor de Ki.

Mediante un formalismo diferente, ${ }^{(24)}$ se define el parámetro $1 / \rho=\Delta \ln v / \Delta t$, en donde $v$ es la velocidad de catálisis de la enzima no inhibida. En condiciones tales que $[\mathbf{E H}]>$ [IY], se utiliza la ecuación de inhibición (ecuación 12.j) y su transformación lineal (ecuación 12.k).

Para la obtención experimental del valor de $\rho$, que es el valor dependiente de ambas constantes cinéticas, en nuestro caso recurrimos al siguiente planteo matemático: Considerando que la velocidad de la reacción catalizada puede ser expresada como un diferencial de la absorbancia en función del tiempo (ecuación 12.I), en donde $\mathbf{A}$ corresponde a la absorbancia del medio de reacción, el valor $\rho$ puede ser expresado según la ecuación 12.m.

Esta expresión constituye una ecuación diferencial de segundo orden, que se resuelve adecuadamente considerado el comportamiento de las curvas experimentales. Dicho comportamiento está definido por una absorbancia creciente y la existencia de una absorbancia máxima a tiempos infinitos. Esto implica imponer a una solución del tipo $\mathbf{A}=f(t)$, las condiciones $\delta \mathrm{A} / \mathrm{d} t>0$ y $\delta^{2} \mathrm{~A} / \mathrm{d} t^{2}<0$. La función que cumple ambas condiciones está representada en la ecuación 12.n.

De esta manera, el valor de $\rho$ aparece de los ajustes de los valores experimentales a esta función. En principio, $\rho$ es la única constante significativa en $f(t)$, a los efectos de la determinación de las constantes cinéticas, siendo los valores de $\mathbf{A}_{\mathbf{0}}$ y $\mathbf{A}_{\mathbf{1}}$ dependientes de las condiciones iniciales de cada ensayo ${ }^{(25)}$ y cumplen el papel de permitir un control experimental interno.

\footnotetext{
${ }^{24}$ Main, A.R. Affinity and phosphorylation constants for the inhibition of esterase by organophosphates, Science 144: 992-993 (1964).

${ }^{25}$ Para $f(\mathrm{t})$ existen analíticamente infinitas soluciones, siendo los valores de $\mathbf{A}_{\mathbf{0}}$ y $\mathbf{A}_{\mathbf{1}}$ dependientes entre sí y experimentalmente se debería verificar que para $\mathrm{t}_{\infty} \rightarrow \mathbf{A}=\mathbf{A}_{\mathbf{0}}$, y para $\mathrm{t}_{0} \rightarrow \mathbf{A}=\mathbf{A}_{\mathbf{0}}-\mathbf{A}_{\mathbf{1}}$. Por otro lado, $\boldsymbol{\rho}$ corresponde a un valor independiente.
}

$$
K i=\frac{k p[\mathbf{I Y}]}{K \mathrm{a}+[\mathbf{I Y}]}
$$

ecuación 12.h

$$
\frac{1}{K i}=\underset{\substack{\text { ecuación 12.i }}}{\frac{1}{k p}}+\frac{1}{k p}
$$

$$
\frac{1}{\rho}=\frac{k p[\mathrm{IY}]}{K a+[\mathrm{IY}]}
$$

$$
\frac{1}{[\mathrm{IY}]}=-\frac{1}{K a}+\frac{k p}{K a} \rho
$$

$v=\frac{\delta A}{\delta t}$

ecuación 12.1 


$$
\rho=\frac{\delta \ln v}{\delta t}=\frac{1}{\delta A / \delta t} \frac{\delta^{2} A}{\delta t^{2}}
$$$$
f(t)=A_{0}-A_{1} e^{-t / \rho}
$$

ecuación 12.n
Una de las limitaciones importantes en la aplicación de este procedimiento, tiene relación con el encontrar las condiciones experimentales bajo las cuales, en el lapso de los tiempos de medida, exista una concentración apreciable del complejo EH-IY, de manera que sea posible manifestar sus concentraciones en las variaciones de la actividad, ${ }^{(26)}$ es decir, en el factor $\rho$ asociado a la curvatura de la cinética de reacción. El ajuste de los datos experimentales a la ecuación 12.n permite disponer de un margen de trabajo más amplio que los posibles según el procedimiento usual de la aplicación de la expresión $1 / \rho=\Delta \ln v / \Delta t$ a las curvas experimentales.

Se han desarrollado otras metodologías para la determinación simultánea de las constantes de afinidad y de fosforilación, con manejos alternativos de las relaciones cinéticas, ${ }^{(27)}$ pero el método de Main aparenta ser el más generalizable y es sobre el cual hemos desarrollado nuestras medidas.

Se ha examinado el comportamiento de la inhibición en presencia de sustrato. El caso más simple descrito es la inhibición de la colinesterasa plasmática por DFP (fosfofluoridato de diisopropilo) en presencia de fenilacetato. La expresión de la constante de fosforilación sufre modificaciones bajo dos situaciones: que una razonable proporción de la enzima se encuentre como complejo de Michaelis u otro complejo o que un exceso de sustrato cause una inhibición de la actividad enzimática. Ambas situaciones dan cuenta de las características complejas de la cinética de los procesos involucrados en las interacciones de la $\mathrm{AChE}$ con sus sustratos e inhibidores y que los formalismos aquí detallados, corresponden sólo a uno de los posibles acercamientos al problema.

Con la metodología propuesta se evaluaron las constantes cinéticas de inhibición del TMP de la actividad AChE en tres sistemas distintos: extracto de Drosophila $s p$. $\left(\mathrm{AChE}_{\mathrm{D}}\right)$, AChE de Drosophila melanogaster recombinante

\footnotetext{
26 (a) Huang, C.T. and Dauterman, W.C. Inhibition of fly head acethylcholinesterase by dimethoxon analogs, Pest.Biochem.Phys. 4: 249253 (1974). (b) Eto, M. ChapterIV: Biochemistry. En: Organophosphorus pesticides: organic and biological chemistry. $1^{\circ}$ ed. Editado por Zweig, G. Boca Raton, Florida: CRC Press Inc (1980).

${ }^{27}$ Hart, G.J. and O'brien, R.D. Recording spectrophotometric method for determination of dissociation and phosphorilation constants for the inhibition of acethylcholinesterase by organophosphates in the presence of substrate, Biochem. 12 (15): 2940-2945 (1973).
} 
variante $\mathrm{E} 69 \mathrm{~W} / \mathrm{F}_{370 \mathrm{~A}^{(28)}}\left(\mathrm{AChE}_{\mathrm{R}}\right)$ y extracto de cerebro de rata $\left(\mathrm{AChE}_{\mathrm{C}}\right)$. Los resultados se muestran en la Tabla 12.II.

\section{Análisis de las constantes cinéticas para OPs modelo}

Los valores hallados indican que el contexto de tres enzimas de distintos orígenes $\mathrm{y}$, por lo tanto diferentes entornos mediatos al sitio activo de catálisis, no implican variaciones importantes en la actividad inhibitoria de una molécula de pequeño tamaño. Este no es un resultado inesperado, dado que se ha indicado que la estructura elemental de los sitios activos de colinesterasas de distintos orígenes es conservada y las diferencias aparecen en grupos químicos de distancias medianas o grandes.

Sin embargo, dentro de los rangos de error de la medida, se puede indicar que $\mathrm{AChE}_{\mathrm{C}}$ presenta menor afinidad por el TMP que $\mathrm{AChE}_{\mathrm{D}}$, mientras que esta molécula fosforila con mayor eficiencia a esta última enzima que a la de mamífero. $\mathrm{AChE}_{\mathrm{R}}$ se encuentra en una situación intermedia para ambos parámetros cinéticos.

De la misma manera, se evaluaron las constantes cinéticas de inhibición de la actividad $\mathrm{AChE}_{\mathrm{C}}$ para el resto de la serie de los OPs estudiados y descritos en los capítulos precedentes, además de metamidofós y DDVP como inhibidores de referencia. Los resultados se muestran en la Tabla 12.III.

El cálculo de los Ki para cada uno de los isómeros del metamidofós se realiza mediante la estimación que $1 / 2 \mathrm{Ki}$ $(\mathrm{Rp})+1 / 2 \mathrm{Ki}(\mathrm{Sp})=\mathrm{Ki}$ (racemato) y suponiendo una relación estimada desde datos de bibliografía ${ }^{(29 a)} \mathrm{Ki}(\mathrm{Rp}) / \mathrm{Ki}(\mathrm{Sp})=$ 22,5. Es notable la diferencia en los valores de referencia para el metamidofós medidos en $\mathrm{AChE}$ de distinta

Tabla 12.II: Constantes cinéticas de inhibición para TMP obtenidos sobre distintos sistemas con actividad acetilcolinesterasa a $\mathrm{pH} 7,80$ y $24^{\circ} \mathrm{C}$ de temperatura.

\begin{tabular}{lccc}
\hline & $\mathrm{Ka}(\mathrm{M})$ & $\mathrm{kp}\left(\mathrm{min}^{-1}\right)$ & $\mathrm{Ki}\left(\mathrm{M}^{-1} \mathrm{~min}^{-1}\right)$ \\
${$\cline { 2 - 2 }$} } }$ & 1,70 & $5,48 \times 10^{-2}$ & $3,23 \times 10^{-2}$ \\
$\mathrm{AChE}_{\mathrm{R}}$ & 1,91 & $6,49 \times 10^{-2}$ & $3,38 \times 10^{-2}$ \\
$\mathrm{AChE}_{\mathrm{C}}$ & 2,35 & $7,38 \times 10^{-2}$ & $3,14 \times 10^{-2}$ \\
\hline
\end{tabular}

\footnotetext{
${ }^{28}$ Esta nomenclatura indica que en la posición 69 se reemplazó el resto $\mathrm{E}$ presente en la enzima nativa, por el resto $\mathrm{W}$ en la enzima recombinante $\mathrm{y}$ en la posición 370 se reemplazó el resto $\mathrm{F}$ por el A. Se debe considerar
} 
Tabla 12.III: Constantes cinéticas para distintos $\mathrm{OPs}$ obtenidos para actividad $\mathrm{AChE}_{\mathrm{C}}$ a pH 7,80 y $24^{\circ} \mathrm{C}$ de temperatura. Los valores marcados "*" son considerados constantes cinéticas aparentes.

\begin{tabular}{|c|c|c|c|}
\hline & $\mathrm{Ka}(\mathrm{M})$ & $\mathrm{kp}\left(\min ^{-1}\right)$ & $\mathrm{Ki}\left(\mathrm{M}^{-1} \min ^{-1}\right)$ \\
\hline TMSP & ${ }^{*} 4,74 \times 10^{-2}$ & ${ }^{\star} 5,26 \times 10^{-1}$ & *11,12 \\
\hline TMSeP & ${ }^{*} 9,45 \times 10^{-2}$ & *7,57x $10^{-1}$ & *8,01 \\
\hline DMAP & $2,46 \times 10^{-3}$ & $5,94 \times 10^{-2}$ & 24,18 \\
\hline DMATP & $1,37 \times 10^{-1}$ & $6,72 \times 10^{-3}$ & $4,91 \times 10^{-2}$ \\
\hline Metamidofós ${ }^{(29)}$ & $4,56 \times 10^{-6}$ & $1,90 \times 10^{-1}$ & $4,16 \times 10^{4}$ \\
\hline Metamidofós (R) & - & - & $7,97 \times 10^{4}$ \\
\hline Metamidofós (S) & - & - & $3,54 \times 10^{3}$ \\
\hline DDVP ${ }^{(30)}$ & $4,68 \times 10^{-6}$ & $1,24 \times 10^{-1}$ & $2,64 \times 10^{4}$ \\
\hline Acefato $^{(29 b)}$ & $2,38 \times 10^{-2}$ & 14,3 & $6 \times 10^{4}$ \\
\hline
\end{tabular}

naturaleza; dichas diferencias pueden ser adjudicadas a las distintas condiciones de ensayo. Para el acefato se incluyen sólo resultados de bibliografía.

Se ha reportado ${ }^{(31)}$ que las diferencias de las constantes de inhibición para OPs análogos oxigenados y azufrados implican una variación de hasta dos órdenes de magnitud para la constante de afinidad $\mathrm{Ka}$ y de un orden de magnitud para $\mathrm{kp}$. El balance arroja una variación de entre dos y tres órdenes de magnitud para las diferencias entre $\mathrm{Ki}$ de compuestos OPs oxigenados respecto de sus derivados azufrados.

que tanto el resto 69 como el resto 370 , se encuentran a varios $\AA$ de distancia del sitio activo.

${ }^{29}$ (a) Ki reportado en $\mathrm{AChE}$ de cerebro humano $240\left(\mathrm{M}^{-1} \mathrm{~min}^{-1}\right)$ para el isómero $\mathrm{D}(+)$ metamidofós y $5,7 \times 10^{3}\left(\mathrm{M}^{-1} \mathrm{~min}^{-1}\right)$ para el isómero $\mathrm{L}(-)$ metamidofós. En AChE de cerebro de gallina $130\left(\mathrm{M}^{-1} \mathrm{~min}^{-1}\right)$ para el isómero $\mathrm{D}(+)$ metamidofós y $2,8 \times 10^{3}\left(\mathrm{M}^{-1} \mathrm{~min}^{-1}\right)$ para el isómero $\mathrm{L}(-)$ metamidofós. En: Bertolazzi, M. et al. Interaction of methamidophos with hen and human acetylcholinesterase and neuropathy target esterase, Arch.Toxicol. 65 (7): 580-585 (1991).

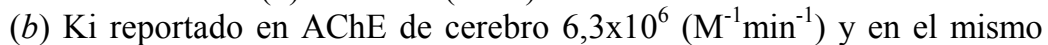
sistema se reporta un $\mathrm{kp}$ de $0,13\left(\mathrm{~min}^{-1}\right)$ para metamidofós racémico. En: Singh, A.K. Kinetic analysis of acetylcholinesterase inhibition by combinations of acephate and methamidophos, Toxicology 42 (2-3): 143156 (1986).

(c) Ki reportado en $\mathrm{AChE}$ de cerebro de rata $6,6 \times 10^{3}\left(\mathrm{M}^{-1} \mathrm{~min}^{-1}\right)$ para metamidofós racémico. Condiciones de los ensayos cinéticos $\mathrm{pH}=7,4$ y $37^{\circ} \mathrm{C}$. En Robinson, C.P. and Beiergrohslein, D. Cholinesterase inhibition by methamidophos and its subsequent reactivation, Pest.Biochem.Phys. 13:267-273 (1980).

${ }^{30} \mathrm{Ki}$ reportado en $\mathrm{AChE}$ de eritrocito bovino 2,77 $\times 10^{4}\left(\mathrm{M}^{-1} \mathrm{~min}^{-1}\right)$. Las condiciones de los ensayos cinéticos fueron $\mathrm{pH}=7,4$ y $25^{\circ} \mathrm{C}$. En: Aharoni, A.H. and O'brien, R.D. The inhibition of acetylcholinesterse by anionic organophosphorus compounds, Biochem. 7 (4): 1538-1545 (1968).

${ }_{31}$ Maxwell, D.M. and Brecht, K.M. Quantitative structure-activity analysis of acetylcholinesterase inhibition by oxono and thiono analogues of organophosphorus compounds, Chem.Res.Toxicol. 5 (1): 66-71 (1992). 
Los resultados obtenidos para el par DMATP y DMAP son consistentes con aquellos resultados previos, encontrándose dos órdenes de magnitud en las diferencias de $\mathrm{Ka}$ y un orden de magnitud en las diferencias de $\mathrm{kp}$, lo que implica una diferencia de tres órdenes de magnitud para los respectivos Ki.

\section{Comportamiento anómalo del TMSP y TMSeP}

En la Tabla 12.III se puede observar que comparativamente los resultados obtenidos para el par TMSP y TMP no son los esperados, en los términos de la mayor capacidad inhibitoria teórica de los oxofosforados respecto a los fosfotioatos. La constante de afinidad medida es de dos ordenes de magnitud mayor para el TMSP respecto al TMP, mientras que la constante cinética de fosforilación es un orden de magnitud mayor para el TMSP respecto al TMP, lo que implica una constante de inhibición de tres órdenes de magnitud por encima del TMSP respecto al TMP.

Para la correcta interpretación de estos valores, es necesario considerar las condiciones en las que se desarrolló el ensayo de inhibición y las características de las curvas colorimétricas de los ensayos blanco. ${ }^{(32)}$ En ellas se evidencia la aparición de una especie de naturaleza química no identificada que es capaz de reaccionar con el reactivo de Ellman (DTNB) y dar lugar a un complejo coloreado que absorbe a $412 \mathrm{~nm}$ de la misma manera que el resto tiol resultante de la hidrólisis de la acetiltiocolina (ATC). Es decir, en forma independiente de la presencia de sustrato y de enzima, el compuesto OP es capaz de dar lugar a una especie que presentaría un resto tiol adecuado para la formación del complejo coloreado. Dicho complejo aparece, dentro de los rangos del ensayo, a velocidad constante y aparentemente de manera independiente de las concentraciones de enzima y de ATC.

La manera más sencilla de compatibilizar estos resultados es el considerar la formación en medio acuoso de una especie derivada del TMSP que sea capaz de hidrolizarse para dar lugar a la formación de un derivado tiol, capaz de formar un complejo coloreado con el DTNB y al mismo tiempo presente capacidad anticolinesterasa mayor al mismo TMSP. Previamente se ha reportado la isomerización del

\footnotetext{
${ }^{32}$ Para mayor detalle de estos ensayos y los ajustes realizados para la estimación de las constantes cinéticas correspondientes al TMSP, ver Anexo I-Metodología.
}<smiles>COP(=S)(OC)OC</smiles>

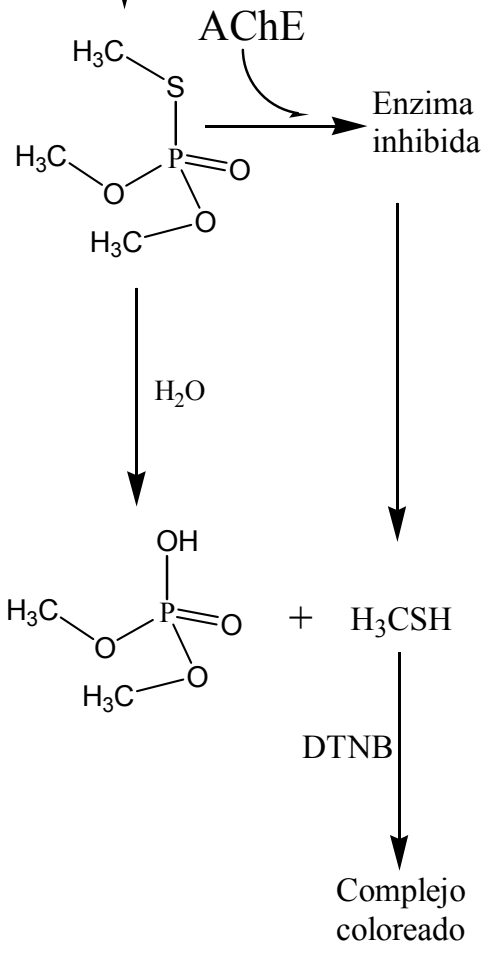

Figura 12.3: Hipótesis de formación del fosfotioato de O,O,S-trimetilo en medio acuoso. 
TMSP a fosfotionato de O,O,S-trimetilo en presencia de electrófilos fuertes. ${ }^{(33)}$ En contraste con estos resultados, se ha reportado que la vida media del TMSP a $40^{\circ} \mathrm{C}$ y pH 7,2 es de 1300 horas. $^{(34)}$ Sin embargo, bajo determinadas condiciones pueden aparecer intermediarios de oxidación o isomerización, de vida media relativamente larga, que pueden intervenir en los procesos biológicos desencadenados por fosfotioatos. ${ }^{(35)}$ Es de suponer entonces que el DTNB puede jugar un papel importante durante la formación del intermediario reactivo en el sistema utilizado.

Comparando los valores de las constantes cinéticas correspondientes a las moléculas DMAP y metamidofós, se desprende que los resultados para el TMSP son compatibles con valores hipotéticos de la molécula fosfotionato de O,O,Strimetilo, y que esta sea la especie que se forme in situ en la mezcla de reacción. En efecto, la constante de afinidad medida para el metamidofós es de tres órdenes de magnitud mayor respecto al DMAP, mientras que la constante cinética de fosforilación es un orden de magnitud mayor para el metamidofós respecto al DMAP, lo que implica una constante de inhibición de dos órdenes de magnitud por encima del metamidofós respecto al DMAP.

Más aún, la presencia de una molécula como el fosfotionato de O,O,S-trimetilo, explicaría la formación de restos tiol por hidrólisis directa del metanotiol en las condiciones del ensayo (Figura 12.3). Es interesante contrastar esta hipótesis con el reporte de las capacidades alquilante comparables del TMSP y la molécula de fosfotionato de O,O,S-trimetilo, ${ }^{(36)}$ actividad que difiere de las mayores capacidades de otros fosfatos orgánicos azufrados como el fosfoditionato de O,S,S-trimetilo, el isomalatión y el malatión.

\footnotetext{
33 Elegant, L., Gal, J.F., Azzaro, M. Enthalpies of isomerization of trimethyl thiophosphate and trimethyl selenophosphate in the presence of $\mathrm{BF}_{3}$, Bull.Soc.Chim.Fr. 12: 4273-4274 (1969).

${ }^{34}$ Kuan, Y.W. et al. Studies of organophosphorus pollutants in well water near a dimethoate plant. III. Hydrolysis rates of O,O,S-trimethyl dithiophosphate, O,O,O-trimethyl thiophosphate, DDVP, dimethoate and its ester, Huan.Ching.K.'o.Hsueh. 2: 4-9 (1979).

35 Swinson J. et al. Thiono compounds. X. Intermediates from the oxidation of phosphorothioates, Phosphorus,Sulfur.Silicon.Relat.Elem. 44 (1-2): 39-52 (1989).

36 Imamura, T., Talcott, R.E. Mutagenic and alkylating activities of organophosphate impurities of commercial malathion, Mutat.Res. 155 (12): 1-6 (1985).
} 
Los resultados de las constantes cinéticas obtenidos para el TMSeP son comparables que aquellas del TMSP. En el caso de la molécula selenada, no se observaron las variaciones de la línea de base tal como se describió en los blancos de TMSP. Pero estas constantes cinéticas no son compatibles con la capacidad inhibitoria teórica de esta molécula dado el pobre poder electrofílico del fósforo en el grupo $\mathrm{P}=\mathrm{Se}$, propiedad necesaria para iniciar el proceso de fosforilación.

Dado que se ha reportado un proceso de isomerización análogo al del TMSP, ${ }^{(33)}$ podemos suponer la formación in situ de una molécula del tipo fosfoselenoato de $\mathrm{O}, \mathrm{O}$, Se-trimetilo, de mayor poder inhibitorio que el respectivo TMSeP. Para explicar la ausencia de variación de absorbancia a $412 \mathrm{~nm}$ en los blancos, es necesario recordar que el DTNB reacciona específicamente con grupos tiol y no con grupos del tipo $\mathrm{R}-\mathrm{SeH}$ o análogos.

Existen dos evidencias experimentales que son compatibles con las observaciones aquí expuestas y con las hipótesis relacionadas: en primer lugar, es un dato confirmado el hecho de la mayor actividad antiocolinesterásica de las moléculas $\mathrm{O}=\mathrm{P}-\mathrm{S}-\mathrm{R}$ respecto de los respectivos análogos $\mathrm{O}=\mathrm{P}-\mathrm{O}-\mathrm{R}$ expresadas en diferencias de entre uno y cinco órdenes de magnitud para la $\mathrm{I}_{50}$ de unas y otras sustancias. ${ }^{(40)}$ Por otro lado, existen reportes respecto de la actividad in vivo $\mathrm{e}$ in vitro de moléculas selenofosforadas del tipo $\mathrm{O}=\mathrm{P}-\mathrm{Se}-\mathrm{R}$, que dan cuenta de una elevada toxicidad de este tipo de sustancias. ${ }^{(37)}$

Desde el punto de vista de la actividad in vivo, tanto del TMSP como del TMSeP y moléculas análogas, es interesante especular acerca de la coactividad tanto de las moléculas del tipo $\mathrm{P}=\mathrm{X}$, siendo $\mathrm{X}$ un átomo de azufre $\mathrm{o}$ selenio, como de los productos de su isomerización o intermediarios de su hidrólisis. En este sentido, cobran relevancia las observaciones del papel antagónico del TMSP, respecto de la actividad de inhibición de colinesterasas y carboxilesterasas séricas en rata tratadas con fosfotionato de $\mathrm{O}, \mathrm{O}, \mathrm{S}$-trimetilo, ${ }^{(38)}$ que no se manifiesta como toxicidad aguda, sino como una toxicidad retardada.

\footnotetext{
37 Åkerfeldt,S. and Fagerlind, L. Selenophosphorus compounds as powerful cholinesterase inhibitors, J.Med.Chem. 10 (1): 115-116 (1971).

${ }^{38}$ Umetsu, N. et al. Toxicological properties of phosphorothioate and related esters present as impurities in technical organophosphorus insecticides, J.Toxicol.Environ.Health 7 (3-4): 481-497 (1981).
} 
Otra observación interesante es la que da cuenta de la inhibición específica de la isoforma del citocromo Cyt $\mathrm{P}_{450}$-2BI del parénquima pulmonar, tanto por parte del TMSP como por el fosfoditionato de O,O,S-trimetilo y no por otros OPs, aunque no se ha probado la actividad del fosfotionato de O,O,S-trimetilo. ${ }^{(39)}$

Por las razones expuestas, las constantes cinéticas medidas para las moléculas TMSP y TMSeP son consideradas constantes cinéticas aparentes. En ambos casos los resultados correspondientes presentados en la Tabla 12.III se marcan con un asterisco.

\section{Correspondencia del comportamiento cinético}

Para las restantes sustancias, TMP, DMP, DMTP, metamidofós y DDVP, se encuentra que la constante de inhibición varía en un rango de seis ordenes de magnitud, entre $3 \times 10^{-2}\left(\mathrm{M}^{-1} \mathrm{~min}^{-1}\right)$ para el TMP y $4 \times 10^{4}\left(\mathrm{M}^{-1} \mathrm{~min}^{-1}\right)$ para el metamidofós. En dicha variación, los factores más importantes están representados por el amplio rango de variación de la constante de afinidad $\mathrm{Ka}$, que se encuentra entre valores de 2,35 (M) para el TMP y $4 \times 10^{-6}(\mathrm{M})$ para el metamidofós y DDVP. La variación de $\mathrm{kp}$ se manifiesta en un rango de algo más de un orden de magnitud.

Los modelos clásicos de interacción entre compuestos OPs y la AChE, han considerado que los valores de $\mathrm{Ka}$ se encuentran determinados fundamentalmente por factores de tipo hidrofóbico ${ }^{(3)}$ y estérico, en tanto que $\mathrm{kp}$ depende de factores electrónicos, que son las características que gobiernan el ataque nucleofílico al fósforo por parte de la $\mathrm{Ser}^{200}$ reactiva. ${ }^{(40)}$ En nuestro caso, dado que la serie de moléculas no presentan grandes variaciones de tipo estérico, puesto que cada una de ellas se caracterizan por ser pequeñas estructuras, no aparece tan directa la interpretación del gran rango de variación de la constante Ka.

En principio, es notable que aunque el valor de $\mathrm{Ka}$ de DMATP sea mayor que el de su respectivo análogo oxigenado, al mismo tiempo sea un valor menor que al

\footnotetext{
${ }^{39}$ Verschoyle, R.D., Dinsdale, D., Wolf, C.R. Inhibition and induction of cytochrome P 450 isoenzymes in rat lung, J.Pharmacol.Exp.Ther. 265 (1): 386-391 (1993).

40 Eto, M. Biochemistry. In: Organophosphorus pesticides: organic and biological chemistry (Zweig, $\mathrm{G}$, ed), $1^{\circ}$ edn. Boca Raton, Florida: CRC Press Inc., 123-231.(1980)
} 
correspondiente al TMP, que aunque pobre inhibidor, es un oxofosforado. Se pude especular respecto del gran error de medida de estos valores de inhibidores pobres por la eventual presencia de impurezas que, en muy baja concentración, alteren grandemente los valores de $\mathrm{Ki}$ y por lo tanto de sus dos componentes, Ka y kp.

Sin embargo, metodológicamente este error fue considerado (ver Anexo I - Metodología), de manera que podemos suponer que, al menos en el rango de ordenes de magnitud, estos valores son correctos y es posible asegurar que la afinidad del sitio activo de la AChE de cerebro de rata es mayor para un fosfotioato, el DMATP, que para el TMP, un oxofosforado.

El hecho que Ki para estos dos compuestos sea similar se explica observando que $\mathrm{kp}$ es diez veces mayor para el TPM que para el DMATP, lo que es lógico en términos de la influencia del grupo $\mathrm{P}=\mathrm{S}$ en la disminución de la electroafinidad del fósforo. De la misma manera se puede observar que los valores de kp medidos para TMP y DMAP son similares, siendo ambos oxofosforados, de manera que el mayor poder inhibitorio de DMAP respecto al TMP está determinado fundamentalmente por los factores que determinan su afinidad.

Para sortear la dificultad de interpretar en forma directa estos resultados, es necesario involucrar en el análisis más datos relacionados con las estructuras químicas, tanto de los inhibidores como del sitio activo de la AChE. 
Tercera Parte: Correlación de Resultados respecto al MECANISMO DE ACCIÓN TÓXICA 



\section{3}

\section{Relaciones estructura - función}

Aunque hay una importante cantidad de pasos farmacocinéticos involucrados en la disposición, y metabolismo de los OP, y son diferentes para las distintas especies, la sensibilidad de los organismos a estos compuestos está determinada, fundamentalmente, por la sensibilidad de la AChE.

Los primeros OP anticolinesterasa fueron triésteres fosfato (Figura 13.1), con una fuerte acción inhibidora tanto in vivo como in vitro. El desarrollo del paratión introdujo a los fosfotioatos. Actualmente, la mayoría de los compuestos utilizados como insecticidas contienen al grupo tioato $\left(\mathrm{R}_{3} \mathrm{P}=\mathrm{S}\right)$ o ditionato $\left(\mathrm{RS}-\mathrm{PR}_{2}=\mathrm{S}\right)$, es decir, corresponden a inhibidores indirectos (Figura 13.2), tal como se definió en el Capítulo 9. Considerando las estructuras de los compuestos inhibidores, se puede demostrar que entre las relaciones que determinan su actividad anticolinesterasa, se distinguen factores estéricos por un lado y energéticos por otro.

Desde otro punto de vista, uno de los aspectos más relevantes del estudio de la actividad anticolinesterasa de los OPs consiste en determinar los factores que condicionan su actividad especie-específica, es decir, de que manera se puede contar con OPs que tengan una marcada acción plaguicida y un mínimo riesgo toxicológico para los vertebrados en general y los humanos en particular. Se ha sugerido por ejemplo, que la resistencia de ciertos insectos y peces a determinados OP está determinada por las pequeñas dimensiones relativas del bolsillo del sitio activo de la AChE, 
y por lo tanto la exclusión estérica del OP del mismo ${ }^{(1)} \mathrm{o}$ variables estéricas similares ${ }^{(2)}$.

Se ha trabajado mucho para desentrañar las propiedades moleculares de los OP que determinan sus propiedades inhibitorias de las esterasas. Se ha encontrado a grandes rasgos que la capacidad inhibitoria está relacionada directamente con la labilidad del enlace P-O-R ${ }^{(3)}$. Esta labilidad se puede evaluar teóricamente mediante el cálculo de la constante de Hammett $\sigma$, que provee una estimación de las propiedades de donación de electrones de los sustituyentes $\mathrm{R}, \quad \mathrm{o}$ experimentalmente observando las frecuencias de vibración del grupo P-O-R o las constantes de hidrólisis alcalinas del enlace éster fosfórico ${ }^{(4)}$. Aunque la labilidad del enlace éster es, por mucho, el factor más importante que determina la actividad anticolinesterasa, las propiedades estéricas de la moléculas en ciertas ocasiones afectan significativamente las constantes de inhibición.

La importancia de la similitud entre el sustrato y el inhibidor se demuestra en la elevada actividad demostrada para el fosfato dietil, m-dimetilamino y el fosfato de dietil, m-tertbutilfenilo, dos compuestos relacionados entre sí y con la $\mathrm{ACh}$, en el sentido que la distancia entre el átomo central del sustituyente y el fósforo es similar a la distancia entre el átomo de carbono carbonílico y el amonio cuaternario de la $\mathrm{ACh}{ }^{(5)}$. Dicha actividad elevada está muy por encima de los esperado, considerando la labilidad relativa del enlace éster fosfórico en estos compuestos.

Un ejemplo de la estereoespecificidad en la interacción de los OP con el sitio activo de la AChE es la

1 (a) Cheymol, J. et al. Comparative toxicity of phosphorylated aminoethanethiol derivatives in mammals, insects, and acarians, C.R.Acad.Sci., Paris., Ser.D. 268 (16): 2150-2153 (1969). (b) Darlington, W.A., Partos, R.D. and Ratts, K.W. Correlation of cholinesterase inhibition and toxicology in insects and mammals. I. Ethylphosphonates, Toxicol. Appl. Pharmacol. 18 (3): $542-547$ (1971).

${ }^{2}$ Smissaert, H.R. and Jansen, A.A. On the variation of toxic effects over species, its cause, and analysis by "structure-selectivity relations", Ecotoxicol.Environ Saf 8 (3): 294-302 (1984).

${ }^{3}$ Fukuto, T.R. Relationships between the structure of organophosphorus compounds and their activity as acetylcholinesterase inhibitors, Bull. World. Health. Organ. 44 (1): 31-42 (1971).

${ }^{4}$ Fukuto, T.R. and Metcalf, R.L. Structure and insecticidal activity of some diethyl subtituted phenil phosphates, J.Agric.Food.Chem. 4 (11): 930-935 (1956).

${ }^{5}$ Hansch, C. and Deutsch, E.W. The use of substituents constants in the study of structure-activity relationships in cholinesterase inhibitors, Biochim. Biophys. Acta 126:117-128 (1966).<smiles>CCOP(=O)(OCC)OC(C)CC(=O)OC</smiles><smiles>CCOP(=O)(OC=C(Cl)Cl)OCC</smiles>

Diclorvós (DDVP)

Figura 13.1: OPs inhibidores directos 
<smiles>CCOP(=S)(OCC)Oc1cc(C)nc(C(C)C)n1</smiles><smiles>CCOP(=S)(OCC)Oc1cc(Cl)c(Cl)cc1Cl</smiles><smiles>CCOP(=S)(OCC)Oc1ccc([N+](=O)[O-])c(Cl)c1</smiles>

Figura 13.2: OPs inhibidores indirectos. diferencia cuantitativa en las actividades anticolinesterasa del cis-crotonato y trans-crotonato, mostrados en la Figura 13.3. Se observa que en la AChE de mosca, la forma trans es cerca de diez veces más activo inhibidor que el compuesto cis. Esta estereoespecificidad está dada por la mayor reactividad intrínseca del éster fosfórico del compuesto trans, puesto que aunque Ka para ambos compuestos varía en un factor de 2, $\mathrm{kp}$ varía en un orden de magnitud ${ }^{(6)}$.

Un caso interesante ocurre con el metamidofós y su derivado $\mathrm{N}$-acetilado, el acefato. Ambos son insecticidas solubles en agua que tienen potencia insecticida similar, pero difieren en su toxicidad para mamíferos. El metamidofós en un potente inhibidor mientras que el acefato es un pobre inhibidor de $\mathrm{AChE}$ de mamíferos. A pH fisiológico, ambos insecticidas se acumulan en eritrocitos en forma similar, aunque el acefato se une más fuertemente a las proteínas del plasma que el metamidofós ${ }^{(7)}$. Ambos son muy solubles en solución acuosa, moderadamente solubles en acetato de etilo e insolubles en n-hexano.

Estos comportamientos disímiles se atribuyen a las diferencias en sus propiedades fisicoquímicas, estructuras moleculares y orbitales y sus propiedades electrónicas. ${ }^{(7)} \mathrm{El}$ acefato presenta dos átomos $\mathrm{O}$ con pares de electrones no enlazantes $(\mathrm{P}=\mathrm{O} \delta$ - y $\mathrm{C}=\mathrm{O} \delta$-) donde $\mathrm{P}=\mathrm{O}$ y $\mathrm{C}=\mathrm{O}$ están en direcciones opuestas. El metamidofós presenta un solo átomo de $\mathrm{O}$ con pares de electrones no enlazantes $(\mathrm{P}=\mathrm{O} \delta$-). Sin embargo, dado que existe translocación de cargas, el grupo $\mathrm{C}=\mathrm{O}$ del acefato existe como $\mathrm{C}-\mathrm{O}^{-}$y el grupo $\mathrm{P}-\mathrm{NH}_{3}{ }^{+}$del metamidofós existe como $\mathrm{P}=\mathrm{NH}_{2}{ }^{+}$a $\mathrm{pH}$ menores que su pKa. Dichas estructuras resonantes tienen capacidad de verificar interacciones dipolo-dipolo con el sitio activo de la AChE, eventualmente responsables de las diferencias de reactividad entre una y otra molécula.

\section{Bioactivación de los OPs}

En la evaluación de la toxicidad de los OPs sobre los organismos, es necesario considerar las sustancias resultantes

\footnotetext{
6 Fukuto, T.R., Hornig, E.O., Metcalf, R.L. and Winton, M.Y. Configuration of the alfa-and beta isomers of methyl 3(dimethoxyphosphinyloxy)- crotonate (phosdrin) J.Org.Chem. 26:46204623 (1961).

7 Singh, A.K. et al. Physicochemical, molecular-orbital and electronic properties of acephate and methamidophos. Comp.Biochem.Physiol. 119 (1): 107-117 (1998).
} 
de las modificaciones metabólicas. Los estudios iniciales sobre el paratión altamente purificado, mostraron que no produce inhibición de la colinesterasa in vitro, y que la actividad anticolinesterasa de muestras menos purificadas podían ser atribuidas a la contaminación con los isómeros del paratión S-etil y S-fenil o por su análogo oxigenado. Posteriormente se ha demostrado que el compuesto metabólicamente activo en la inhibición de la colinesterasa es el paraoxón, formado a partir del paratión en ratas. La activación de paratión y otros insecticidas tiofosforados sin actividad anticolinesterasa directa, puede ser realizada por incubación aeróbica con extractos hepáticos.

Se ha establecido que la conversión de fosfotioatos y fosfoditionatos a sus correspondientes análogos oxigenados, es un requisito necesario para su efectiva acción anticolinesterasa (Figura 13.4, reacción I). Esta reacción se verifica en sistemas enzimáticos hepáticos constituidos por el grupo de oxidasas microsomales de función mixta dependientes de NADPH. En este punto es necesario aclarar que los inhibidores indirectos pueden ser inhibidores de la colinesterasa in vitro, aunque con menos potencia que su respectivo producto metabólico.

Existen algunos casos inversos, por ejemplo el fosfotioato de trietilo es un inhibidor más potente que el respectivo oxofosfato. Como hemos planteado durante el desarrollo del Capítulo 12, este hecho puede considerarse análogo a lo que se verifica para el par TMP/TMSP. De esta manera, no deberían considerarse tanto al TMSP como al fosfotioato de trietilo como inhibidores directos, sino que en fase acuosa o en el entorno del sitio activo habría un reordenamiento molecular que de lugar a un tipo de inhibidor más fuerte.

Adicionalmente a la presencia del grupo oxón $(\mathrm{P}=\mathrm{O})$ para la existencia de actividad anticolinesterasa, las modificaciones metabólicas de los sustituyentes arílicos o alquílicos pueden influir sobre la actividad anticolinesterasa. Las reacciones II, III, IV, y V (Figura 13.4), son detoxificaciones enzimáticas que pueden dar lugar a productos sin actividad anticolinesterasa. Para el paraoxón se ha descrito a la reacción $\mathrm{V}$, como la más importante para la destoxicación mediante una actividad enzimática ampliamente distribuida en distintos tejidos en ratas y otros mamíferos. Esta reacción es catalizada por una enzima microsomal que requiere NADPH y oxígeno y parece ser la misma que verifica la reacción III. Además, distintas<smiles>COC(=O)/C=C(/C)OP(=O)([O-])OC(=O)/C=C(\C)OP(=O)(OC)OC</smiles>

Trans-crotonato

Figura 13.3: Cis-crotonato y trans-crotonato, dos isómertos geométricos con diferente actividad anticolinesterásica. 
evidencias han mostrado que el intermediario de la reacción $\mathrm{V}$, es común a la reacción I. La dealquilación de las reacciones II y IV no ocurren u ocurren en muy pequeña extensión para el paratión, sin embargo, la desalquilación oxidativa dependiente de NADPH se ha demostrado para otros compuestos y parece determinar la toxicidad del clorfenvinfós.

Existen otras reacciones de activación que involucran a unos pocos compuestos, en las que los insecticidas son precursores de agentes anticolinesterasa más potentes. Estas incluyen la N-desmetilación de fosfamidatos, como en el shradán, la oxidación de tioéteres, como en el disulfotón y sus análogos oxigenados, a sus correspondientes solfóxidos y sulfonas, el reemplazo de restos halógenos, alquil o aril ésteres por hidroxilos mediado por esterasas de plasma e hígado y la posible introducción de hidroxilos en fosfatos de difenilo.

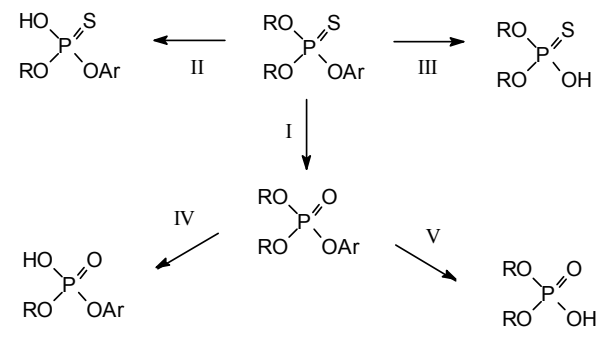

Un caso interesante es el de las acetofenonas oximas sustituidas que contienen sustituyentes donantes de electrones que se reorganizan, según el rearreglo de Beckman, al correspondiente enol fosfato tal como se muestra en la Figura 13.5. Cuando el grupo $\mathrm{X}$ es un atractor de electrones, он по se favorece este reordenamiento molecular. Ya que el producto del rearreglo da lugar a un éster muy reactivo, es

Figura 13.4: Modificaciones metabólicas por reacciones hidrolíticas y oxidativas de los OPs indirectos. probable que la alta actividad colinesterasa aparezca por un rearreglo in vivo. ${ }^{(8)}$ La selectividad característica de estos compuestos, es decir, son altamente tóxicos para invertebrados y muy poco tóxicos para vertebrados, sugiere que este rearreglo molecular esta mediado por algún sistema enzimático presente solamente en los primeros.

Para el metamidofós se han sugerido distintas alternativas de bioactivación, dado que es un mejor inhibidor in vivo que in vitro. Existe evidencia que el sistema de oxidadasas que determina esta modificación es diferente a la que actúa sobre el paratión. ${ }^{(9)}$ Tanto la S-oxidación como la N-hidroxilación son propuestos como metabolitos activos según las rutas mostradas en la Figura 13.6. Respecto al

\footnotetext{
${ }^{8}$ Fukuto, T.R., Metcalf, R.L., Jones, R.L. and Myers, R.O. Structure, reactivity, and biological activity of $\mathrm{O}$-(diethyl phosphoryl) oximes and O-(methylcarbamoyl) Oximes of substituted acetophenones and alfasubstituted benzaldehydes J.Agr.Food Chem. 17(5): 923-930 (1969).

${ }^{9}$ Mahajna, M. and Casida, J.E. Oxidative bioactivation of methamidophos insecticide: synthesis of N-hydroxymethamidophos (a candidate metabolite) and its proposed alternative reactions involving $\mathrm{N} \rightarrow \mathrm{O}$ rearrangement or fragmentation through a metaphosphate analogue, Chem.Res.Toxicol. 11 (1): 26-34 (1998).
} 
derivado N-hidroxilado, corresponde a una sustancia inestable a pH 7,4 $\left(\mathrm{t}_{1 / 2}=10\right.$ minutos a $\left.37^{\circ} \mathrm{C}\right)$ con descomposición a través de dos mecanismos a intermediarios activos. $^{(9)}$

Sin embargo, existe contundente evidencia que es el mismo metamidofós el que se ubica en el sitio activo de la $\mathrm{AChE}$, de manera tal que el grupo $-\mathrm{SCH}_{3}$ se posiciona como grupo saliente. ${ }^{(10)}$ Por otro lado su constante cinética de activación $\mathrm{k}_{+3}$ es alta ${ }^{(11)}$ por el carácter aceptor de protones del grupo $-\mathrm{NH}_{2}$, a diferencia de otras fosfatoamidas como el tabún que tienden al envejecimiento de la fosforilación por escisión del enlace P-N. ${ }^{(10 b)}$ Estas cuestiones son importantes a la hora de decidir cuál es el grupo saliente y, por lo tanto, cuales son las variables estéricas o electrónicas a evaluar.

Como queda brevemente expuesto, aunque existen procesos de modificación metabólica in vivo, existe una marcada relación entre las propiedades íntimas de los OPs y su capacidad inhibitoria. Entonces, del estudio de estas propiedades moleculares, debería extraerse abundante información útil para la comprensión de los procesos biológicos involucrados.

\section{Cuantificación de las relaciones estructura- actividad}

En la inmediata época de postguerra a fines de la década de los cuarenta, la toxicología predictiva tuvo entre sus primeros desarrollos el estudio de las relaciones entre la estructura y la actividad de los compuestos OPs como inhibidores de la $\mathrm{AChE}$, en la búsqueda de herramientas que aportaran al diseño racional de nuevos insecticidas. El paso siguiente fue la cuantificación de estas relaciones (QSARs) en términos de algoritmos de correlación, generalmente en forma de un polinomio.

\footnotetext{
${ }^{10}$ (a) de Jong, L.P., Wolring, G.Z. and Benschop, H.P. Reactivation of acetylcholinesterase inhibited by methamidophos and analogous (di)methylphosphoramidates, Arch.Toxicol. 49 (2): 175-183 (1982). (b) Elhanany, E. et al. Resolving Pathways of Interaction of Covalent Inhibitors with the Active Site of Acetylcholinesterases: MALDITOF/MS Analysis of Various Nerve Agent Phosphyl Adducts, Chem.Res.Toxicol. 14 (7): 912-918 (2001).

${ }^{11}$ (a) Bertolazzi, M. et al. Interaction of methamidophos with hen and human acetylcholinesterase and neuropathy target esterase, Arch.Toxicol. 65 (7): 580-585 (1991). (b) Barak, D. et al. Evidence for P-N bond scission in phosphoramidate nerve agent adducts of human acetylcholinesterase, Biochem. 39:1156-1161 (2000).
}

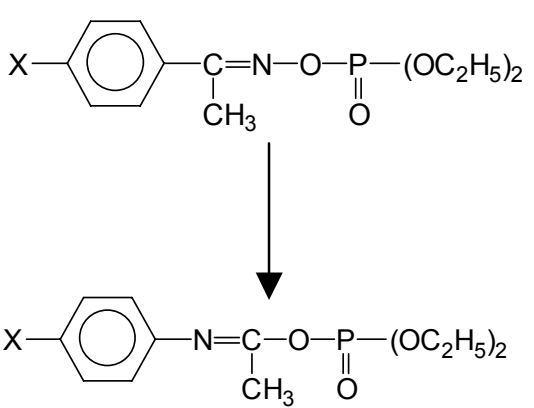

Figura 13.5: Bioactivación de las acetofenonas oximas sustituidas. 


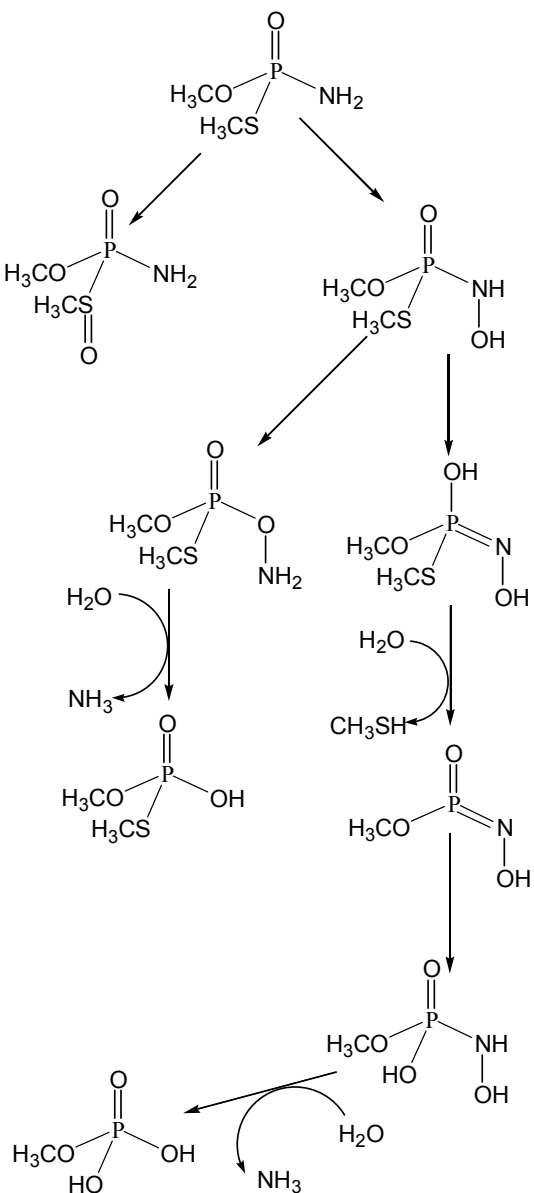

Figura 13.6: Rutas hipotéticas de bioactivación del metamidofós.
El primer acercamiento sistemático al problema de la cuantificación de las relaciones estructura actividad ${ }^{(12)}$ está expuesto en los trabajos de Hansch ${ }^{(5)}$ a principios de la década del sesenta. La idea central consistía en, luego de conocidas las actividades biológicas de una serie de moléculas y de obtener un número adecuado de descriptores de sus estructuras, encontrar correlaciones numéricas entre actividades y descriptores que sirvieran para interpolar o, en el mejor de los casos, extrapolar sus resultados mediante el planteo de moléculas hipotéticas que eventualmente tendrían una actividad biológica predicha a partir de estas correlaciones.

Desde este punto en adelante, las metodologías utilizadas para la búsqueda de estas correlaciones se fue haciendo paulatinamente más compleja, sobre todo por el aporte realizado desde las matemáticas con los algoritmos de correlaciones no lineales y de la química básica por el desarrollo de las metodologías de cálculo de las propiedades estructurales de las moléculas orgánicas.

Por otra parte, el estudio de la actividad antilcolinesterásica de los OPs rápidamente se convirtió en sólo uno de los objetos de estudio de QSARs buscando aplicaciones en el estudio de otras propiedades biológicas (metabolización, interacción con receptores, transporte a través de membranas biológicas, degradabilidad ambiental, etc.) para un sinnúmero de moléculas orgánicas de distinta naturaleza. Todas las técnicas desarrolladas fueron aplicadas entonces a diversos grupos de sustancias. Hoy en día, el análisis QSAR es un paso obligado en el diseño racional de tóxicos y fármacos en todas sus variantes.

Son interesantes los esfuerzos de la EPA (Environmmental Protection Agency - EE.UU.) para aplicar los QSAR para contaminantes ambientales en el marco de la normativa de habilitación de sustancias nuevas. ${ }^{(13)} \mathrm{Se}$ pretende minimizar los costos de las pruebas experimentales y los tiempos requeridos para la verificación de los márgenes de seguridad de los nuevos productos, no sólo en cuanto a toxicidad crónica o aguda, sino también para estimar valores

12 Debnath, A.K. Quantitative structure-activity relationship (QSAR) paradigm: Hansch era to new millennium, Mini. Rev. Med. Chem. 1 (2): 187-195 (2001).

${ }^{13}$ (a) Lange A.W. and Vormann K. Experiences with the application of QSAR in the routine of the notification procedure, SAR. QSAR. Environ. Res. 3 (3): 171-177 (1995). (b) Zeeman, M. et al. U.S. EPA regulatory perspectives on the use of QSAR for new and existing chemical evaluations, SAR. QSAR. Environ. Res. 3 (3): 179-201 (1995). 
de comportamiento ambiental como persistencia en aguas o absorción en suelos. ${ }^{(18 b)}$

\section{Definiciones}

Dado un cierto mecanismo de acción y una cierta estructura química correspondiente, sería posible plantear un algoritmo de correlación entre ciertos parámetros que surgen de la estructura molecular y la magnitud del efecto descrito. Estos parámetros serán representación de la estructura dinámica de la molécula y su relación con el entorno, como longitudes de enlace, polaridad, potenciales de ionización, características conformacionales, etcétera y serán denominados descriptores moleculares. Clásicamente, los descriptores moleculares se diferenciaron en tres tipos: aquellos que cuantifican propiedades electrónicas, descriptores de hidrofobicidad y descriptores estéricos.

En un abordaje general, dada una cierta molécula que tenga una dada permeabilidad a membranas biológicas, descrita cuantitativamente por $\boldsymbol{p}$, una acción farmacológica determinada, mensurable por un $\boldsymbol{f}$, una actividad tóxica, mensurable por un $\boldsymbol{t}$ y una cierta vía de metabolización, cuya velocidad sea cuantificable por $\boldsymbol{m}$, que describen su comportamiento in vivo y contando al mismo tiempo con una serie de parámetros moleculares mensurables $(\mathbf{a}, \mathbf{b}, \mathbf{c}, \mathbf{d}, \ldots)$, llamados descriptores moleculares, sería posible encontrar funciones o más generalmente algoritmos $\mathscr{P}, \mathcal{F}, \mathcal{T}, \mathcal{M}$, que aplicados sobre estos descriptores, arroje la magnitud de cada parámetro de actividad biológica de cada cierta molécula (ecuación 13.a).

Tal descripción puede ser ampliada a cada uno de aquellos aspectos de su actividad biológica que, por depender de su comportamiento molecular en entornos particulares diferenciados, esté condicionada por distintos factores, por ejemplo la existencia de una acción farmacológica determinada por el impacto sobre varias proteínas diana, la metabolización por distintas enzimas, distintos mecanismos de acción tóxica, etcétera. Cada una de estas funciones o algoritmos constituyen, en forma independiente, una relación $\mathscr{P}(\mathbf{a}, \mathbf{b}, \mathbf{c}, \mathbf{d}, \ldots)=\boldsymbol{p}$ $F(\mathbf{a}, \mathbf{b}, \mathbf{c}, \mathbf{d}, \ldots)=\boldsymbol{f}$ $\mathcal{T}(\mathbf{a}, \mathbf{b}, \mathbf{c}, \mathbf{d}, \ldots)=\boldsymbol{t}$ $\mathcal{M}($ a. b. c. d. ...) $=m$ cuantitativa entre la estructura y la actividad de la molécula ecuación 13.a en sus distintos aspectos (Quantitative Structure Property Relationship, QSPR), que se interrelacionan en distintas dimensiones conceptuales. 


\section{$C(\mathcal{F}, \mathcal{T}, \mathcal{M})=\boldsymbol{c}$} ecuación 13.b

Tabla 13.I: Denominación de los OPs sobre los que se realiza en análisis de correlación.

\begin{tabular}{lc}
\hline Sustancia & Denominación \\
\hline TMP & I \\
TMSP & II \\
TMSeP & III \\
STMSP & IV \\
SeTMSeP & V \\
DMAP & VI \\
DMATP & VII \\
Meth (R) & VIII(R) \\
Meth (S) & VIII(S) \\
DDVP & IX \\
Acefato & X \\
\hline
\end{tabular}

Encontrándose el QSPR para cada una de los comportamientos diferenciados, en un paso siguiente sería posible establecer un algoritmo multidimensional $C$ que describa un parámetro vectorial $\boldsymbol{c}$, que represente cuantitativamente el comportamiento de un grupo de moléculas en un contexto determinado (ecuación 13.b). Las dos hipótesis subyacentes a este planteo son, por un lado, que las propiedades y el comportamiento de una molécula están implícitos en su estructura, y por otro, muy relacionada con la anterior, que las sustancias pertenecientes a una misma "clase química" deben comportarse de una manera similar. ${ }^{(14)}$

Por lo tanto, con las bases del conocimiento para entender el mecanismo de un determinado proceso molecular, $\mathrm{y}$ reconociendo los grupos químicos críticos $\mathrm{y}$ conformaciones que gobiernan la acción del mecanismo específico, se estaría en condiciones de realizar generalizaciones respecto a una "clase química" y su mecanismo de acción $\mathrm{y}$, por lo tanto, formular predicciones respecto a moléculas hipotéticas o de aquellas de las que no se cuente con datos experimentales suficientes. Este planteo general es válido para describir la acción tóxica, ${ }^{(15)}$ farmacológica o fisiológica, ${ }^{(16)}$ biodegración ambiental, ${ }^{(17)}$ ecotoxicidad (18) o metabolismo, ${ }^{(19)}$ tanto de moléculas naturales, sintéticas como hipotéticas.

14 Barratt, M.D. Integration of QSAR and in vitro toxicology, Environ.Health.Perspect. 106 (Suppl 2):459-465 (1998).

15 (a) Mekenyan, O.G., Veith, G.D. Relationships between descriptors for hydrophobicity and soft electrophilicity in predicting toxicity, SAR. QSAR. Environ. Res. 1 (4): 335-344 (1993). (b) Coats, J.R. Mechanisms of toxic action and structure-activity relationships for organochlorine and synthetic pyrethroid insecticides, Environ.Health.Perspect. 87:255-262 (1990). (c) Dunn, W.J. QSAR approaches to predicting toxicity, Toxicol.Lett. 43 (1-3): 277-283 (1988).

${ }^{16}$ (a) Viswanadhan V.N. et al. An Approach to Rapid Estimation of Relative Binding Affinities of Enzyme Inhibitors: Application to Peptidomimetic Inhibitors of the Human Immunodeficiency Virus Type 1 Protease, J.Med.Chem. 39 (3): 705-712 (1996). (b) Sato K., Nakagawa J. An improvement of neural networks applied to pharmaceutical problems, Chem.Pharm.Bull.(Tokyo.). 45 (1): 107-115 (1997).

17 Rorije E., Van Wezel, M.C., Peijnenburg, W.J. On the use of backpropagation neural networks in modeling environmental degradation, SAR.QSAR.Environ.Res. 4 (4): 219-235 (1995).

18 (a) Veith, G.D., De Foe, D., Knuth, M. Structure-activity relationships for screening organic chemicals for potential ecotoxicity effects, Drug.Metab.Rev. 15 (7): 1295-1303 (1984). (b) Zeeman, M. et al. U.S. EPA regulatory perspectives on the use of QSAR for new and existing chemical evaluations, SAR.QSAR.Environ.Res. 3 (3): 179-201 (1995).

${ }^{19}$ Krarup L.H. et al. Predicting drug absorption from molecular surface properties based on molecular dynamics simulations, Pharm.Res. 15 (7): 972-978 (1998). 
Un problema de mucha significación práctica que puede ser abordado con estas metodologías, basadas en la similaridad de modos de acción, consiste en determinar la validez de la extrapolación de resultados de una especie a otra y la posibilidad de aplicar resultados experimentales obtenidos con modelos in vitro a sistemas vivos. ${ }^{(20)}$ En el campo de la toxicología, con una apropiada base de conocimiento de químicos específicos y los respectivos modos de acción, pueden ser desarrollados sistemas predictivos que asignen a especies químicas no probadas un modo de acción tóxica.

La asignación de una categoría de "clase química" no está libre de incertezas. En los últimos años se ha modificado la noción de que la similaridad en los modos de acción está relacionada necesariamente con los esquemas clásicos de clasificación química $\mathrm{y}$ ha tendido hacia una clasificación más consistente con suposiciones que tienen en cuenta sus modos de acción. ${ }^{(15 c)}$ Esto aparenta una contradicción implícita, pues manifiesta una incapacidad para una clasificación que se base exclusivamente en las propiedades estructurales, lo que constituye una de las hipótesis de partida.

Aunque la aproximación general es correcta, el paso inicial crítico es definir claramente el modo de acción para una aplicación y que existan criterios válidos para la asignación de un QSAR apropiado. Aplicando los conceptos anteriores, el dominio del modo de acción tóxica está determinado por tres dimensiones descritas por variables continuas o discretas: (a) el régimen de exposición, (b) el modelo biológico y punto final o "end point" y (c) las propiedades químicas de las sustancias consideradas.

El régimen de exposición es fundamental para decidir cual es el mecanismo de acción tóxica que estamos estudiando. Son muchos los ejemplos de sustancias que administradas en dosis únicas y altas determinan cuadros de intoxicación diferentes a aquellos que se manifiestan por exposiciones de larga duración con dosis bajas. El punto final corresponde al efecto a observar, en el sistema biológico utilizado, como respuesta a la acción de la sustancia química estudiada, es decir, la inhibición enzimática, aparición de mutaciones, muerte celular, muerte del organismo, falla reproductiva, desarrollo de cáncer, etc.

\footnotetext{
20 Bradbury S.P. Predicting modes of toxic action from chemical structure: an overview, SAR.QSAR.Environ.Res. 2 (1-2): 89-104 (1994).
}

Tabla 13.II: Descriptores de naturaleza electrónica: carga neta del fósforo ${ }^{\dagger} \mathbf{P}$ y momento cuadrupolar $\mathbf{Q p}$ del confórmero más estable a un nivel ab initio HF/STO-3G.

\begin{tabular}{ccc}
\hline Sust. & ${ }^{+} \mathbf{P}$ & $\mathbf{Q p}$ \\
\hline $\mathbf{I}$ & 1,242 & $-45,9$ \\
II & 0,998 & $-52,5$ \\
III & 1,021 & $-74,2$ \\
IV & 1,005 & $-52,6$ \\
V & 1,039 & $-61,4$ \\
VI & 1,232 & $-42,4$ \\
VII & 0,993 & $-48,9$ \\
VIII(R) & 0,996 & $-49,0$ \\
VIII(S) & 0,996 & $-49,0$ \\
IX & 1,260 & $-81,0$ \\
X & 1,030 & $-65,5$ \\
\hline
\end{tabular}


Tabla 13.III: Descriptores de naturaleza electrónica: momento octapolar Op y momento hexadecapolar Hdp del confórmero más estable a un nivel $a b$ initio HF/STO-3G.

\begin{tabular}{ccc}
\hline Sust. & Op & Hdp \\
\hline I & $-2,8$ & $-560,9$ \\
II & 7,9 & $-567,1$ \\
III & $-29,1$ & $-580,7$ \\
IV & $-7,6$ & $-849,0$ \\
V & $-27,3$ & $-853,0$ \\
VI & 2,0 & $-548,9$ \\
VII & $-2,5$ & $-555,6$ \\
VIII(R) & $-5,1$ & $-840,1$ \\
VIII(S) & $-5,1$ & $-840,1$ \\
IX & 73,4 & $-2932,7$ \\
X & 7,2 & $-1558,5$ \\
\hline & &
\end{tabular}

Esto significa que la asignación de un modo de acción a una determinada estructura tipo es un procedimiento contextoespecífico y depende de la duración y dosis asociada con la exposición, el punto final considerado ${ }^{(14)}$ y del modelo biológico considerado (subcelular, celular, tisular, organismo, población, ecosistema, etcétera). ${ }^{(21)}$

Las extrapolaciones, por lo tanto, tendrán sentido en la medida del ajuste a este marco. Por ejemplo, la toxicidad aguda de un inhibidor de la AChE tiene un modo de acción común en invertebrados y vertebrados asumiendo que utilicen a la ACh como neurotransmisor. Sin embargo, este modo de acción es enteramente inapropiado para predecir la toxicidad de estos compuestos, por ejemplo, sobre algas. ${ }^{(20)}$ Se pueden cometer errores significativos al asumir la clasificación de un punto final sin un modelo biológico apropiado para diferentes regímenes de exposición. ${ }^{(20)}$

Se define como serie de entrenamiento o "training set" al conjunto de sustancias con valores de actividad conocidos, a partir del cual se construye un determinado QSAR, por elección de los algoritmos y de los descriptores asociados de la forma más apropiada posible, es decir, de la manera que se obtengan las correlaciones más altas.

Las metodologías aplicadas durante la búsqueda de los algoritmos de correlación, deben asegurar que las correlaciones encontradas no sean dependientes de las características no químicas de la serie de entrenamiento. Este constituye un problema respecto a la naturaleza del algoritmo, que puede ser resuelto mediante criterios puramente matemáticos. Por ejemplo, si queremos buscar una ecuación de correlación y utilizamos para ellos dos sustancias con magnitudes de acción tóxica conocidas y descriptores moleculares calculados, siempre será posible encontrar una recta que pase por los dos puntos dibujados en el plano [descriptor v/s magnitud de acción tóxica], cualesquiera que sean los descriptores moleculares utilizados. Más en general, siempre habrá un polinomio de grado " $n$ " que describa exactamente el comportamiento en el plano de un grupo de " $n+1$ " sustancias, cualesquiera sea el o los descriptores utilizados. Estos constituyen ejemplos de "artefactos" de las correlaciones, es decir, ellas no están describiendo el comportamiento biológico del grupo de sustancias, sino que se construyen a partir de las propiedades matemáticas de los

${ }^{21}$ Benigni R. and Richard A.M. Quantitative structure-based modeling applied to characterization and prediction of chemical toxicity, Methods 14 (3): 264-276 (1998). 
algoritmos utilizados. $Y$ aunque efectivamente sean excelentes correlaciones, carecerán de poder predictivo e interpretativo.

Para poder distinguir entonces cuándo una correlación, además de ser buena, está además describiendo adecuadamente el correspondiente fenómeno biológico, debemos probar su poder predictivo. Más aún, podemos describir correlaciones que sin ser buenas, estadísticamente hablando, posean un poder predictivo que las haga adecuadas para su uso en determinados contextos.

Para ensayar el poder predictivo de un QSAR particular se utiliza una serie de prueba o "data set". Estas corresponden a un conjunto de sustancias con valores de actividad conocidos, sobre las cuales se aplica el QSAR, antes diseñado, para evaluar la capacidad de correlación y predicción del mismo. También de alguna manera sirve para delimitar la "clase química" sobre la cual se podrá aplicar el QSAR. La capacidad del QSAR de predecir la actividad biológica de esta serie de prueba, de alguna manera determinará la validación del mismo, dentro de los márgenes definidos previamente por la metodología aplicada para su construcción y por la "clase química" definida empíricamente por la naturaleza de la variabilidad estructural delimitadas por la serie de entrenamiento y la serie de prueba.

Las reglas cuantitativas usadas en el proceso de clasificación se corresponden con modelos matemáticos derivados de la información de las estructuras químicas de los compuestos. ${ }^{(15 c, 23)}$ Existen distintas metodologías para asignar un mecanismo determinado a una sustancia química definida. Básicamente se distinguen es sistemas clásicos basados en reglas y sistemas de clasificación no basados en reglas. Ambos implican algoritmos de decisión en los que subyace en menor o mayor grado un factor subjetivo. La validez de los resultados de unos y otros están determinados por los grados de correlación obtenidos. ${ }^{(14)}$

En un sistema experto clásico basado en reglas, se prueba una estructura química por subestructuras especificas que, si son identificadas, se asigna un modo de acción

\footnotetext{
${ }^{22}$ El método de fragmentación utilizado no tiene parametrización para el grupo $\mathrm{P}=\mathrm{Se}$, de manera que no es posible estimar $\log \mathbf{P}$ ni $\mathbf{R M}$ para el TMSeP, según esta metodología.

${ }^{23}$ Lipnick R.L. Outliers: their origin and use in the classification of molecular mechanisms of toxicity, Sci.Total.Environ. 109-110:131-153 (1991).
}

Tabla 13.IV: Descriptores de propiedades hidrofóbicas: momento dipolar $\boldsymbol{\mu}$, en Debyes y logaritmo del coeficiente de partición octanol/agua logP (desviación estandar de 0,49).

\begin{tabular}{ccc}
\hline Sust. & $\boldsymbol{\mu}$ & logP \\
\hline $\mathbf{I}$ & 0,1153 & 0,55 \\
II & 0,8569 & 1,29 \\
III & 2,3963 & $(22)$ \\
IV & 0,7850 & 0,90 \\
V & 1,3993 & 0,75 \\
VI & 1,3993 & 0,02 \\
VII & 2,0766 & 0,76 \\
VIII(R) & 1,8679 & 0,37 \\
VIII(S) & 1,8679 & 0,37 \\
IX & 4,1533 & 1,84 \\
X & 2,9320 & 0,00 \\
\hline
\end{tabular}


Tabla 13.V: Descriptores de propiedades estéricas: Volumen molar VM, en $\mathrm{cm}^{3} / \mathrm{mol}$, radio de bohr ao, en $\AA$ y refractividad molar RM,en $\mathrm{cm}^{3} / \mathrm{mol}$ (desviación estándar de 0,77 ).

\begin{tabular}{cccc}
\hline Sust. & VM & ao & RM \\
\hline I & 80,70 & 3,99 & 28,1 \\
II & 101,49 & 4,27 & 36,09 \\
III & 84,64 & 4,05 & $(22)$ \\
IV & 99,09 & 4,24 & 34,54 \\
V & 116,76 & 4,45 & 37,74 \\
VI & 75,05 & 3,91 & 26,34 \\
VII & 85,50 & 4,06 & 34,33 \\
VIII(R) & 91,69 & 4,15 & 32,78 \\
VIII(S) & 91,69 & 4,15 & 32,78 \\
IX & 125,81 & 4,55 & 42,28 \\
X & 117,38 & 4,46 & 41,16 \\
\hline
\end{tabular}

determinado. Para cada mecanismo se asigna un QSAR. Si el fragmento satisface más de un modo de acción, aquel que registre una más alta predicción de potencia, se elige por defecto, identificando, sin embargo, los otros. De esta manera se constituye un sistema intermedio entre los conceptos de asignación de "clase química" y de asignación de modo de acción. ${ }^{(14)}$ Un concepto aplicado recientemente es el de retrometabolismo, con el cual, mediante sistemas automatizados, se predice la actividad biológica de una molécula considerando las posibles modificaciones bioquímicas in vivo que puedan producirse. ${ }^{(24)}$ Es inherente en las reglas predefinidas la necesidad de especificar los subfragmentos estructurales asociados con determinado modo de acción, lo que está determinado por el criterio subjetivo del experto que los define.

Se ha acumulado una gran cantidad de experiencia en la búsqueda de algoritmos de correlación, y junto con las aplicaciones concretas de algoritmos con capacidad predictiva, se ha recurrido a ramas avanzadas de la computación y de las matemáticas, en una relación en ambos sentidos productiva. Dado que, en esencia, la resolución de un QSPR es un problema de regresión no lineal, ${ }^{(25)}$ ha sido abordada con diferentes grados de éxito mediante modelos de redes neuronales. ${ }^{(26)}$

\footnotetext{
${ }^{24}$ Bodor N., Buchwald P., Huang M.J. Computer-assisted design of new drugs based on retrometabolic concepts, SAR.QSAR.Environ.Res. 8 (1-2): 41-92 (1998).

${ }^{25}$ Duprat A.F., Huynh T., Dreyfus G. Toward a principled methodology for neural network design and performance evaluation in QSAR. Application to the prediction of $\log \mathrm{P}$, J.Chem.Inf.Comput.Sci. 38 (4): 586594 (1998).

${ }^{26}$ (a) Aoyama T. Neural network applied to quantitative structure-activity relationship, J.Med.Chem. 33 ( 905-908 (1990). (b) Domine D. et al. Estimating pesticide field half-lives from a back-propagation neural network, SAR.QSAR.Environ.Res. 1 ( $211-219$ (1993). (c) Ichikawa H. and Aoyama T. How to see characteristics of structural parameters in QSAR analysis: descriptor mapping using neural networks, SAR. QSAR. Environ. Res. 1 (2-3): 115-130 (1993). (d) Wiese M. and Schaper K.J. Application of neural networks in the QSAR analysis of percent effect biological data: comparison with adaptive least squares and nonlinear regression analysis, SAR. QSAR. Environ. Res. 1 (2-3): 137-152 (1993). (e) Zakarya D. et al. Analysis of structure-toxicity relationships for a series of amide herbicides using statistical methods and neural network, SAR. QSAR. Environ. Res. 5 (4): 269-279 (1996). (f) Huuskonen J., Salo M. and Taskinen J. Aqueous solubility prediction of drugs based on molecular topology and neural network modeling, J. Chem. Inf. Comput. Sci. 38 (3): 450-456 (1998). (g) Kireev D.B. et al. Application of Kohonen Neural Networks in classification of biologically active compounds, SAR.QSAR.Environ.Res. 8 (1-2): 93-107 (1998).
} 
Cada vez que se avanza en la complejidad de los sistemas predictivos, para los usuarios éstos se transforman finalmente en "cajas negras", de las que se conocen los parámetros de entrada y los resultados de salida, sin acceder a los procedimientos que vinculan a unos con otros. En la actualidad es posible acceder a algoritmos predictivos con aplicaciones en gran cantidad de áreas del conocimiento en donde la toxicología es una de las más exploradas.

En el caso de los OPs se asume que los mecanismos de acción tóxica de estos compuestos, es una variable determinada por múltiples factores asociados con el transporte, la bioconcentración, el metabolismo y la acción sobre diferentes enzimas. De esta manera, es sabido que encontrar QSAR adecuados para describir la toxicidad sobre los organismos para los OPs en general no es una tarea sencilla. ${ }^{(27)}$ Sin embargo, sí es posible encontrar QSAR con buenas correlaciones para la actividad sobre la $\mathrm{AChE}^{(28)} \mathrm{u}$ otras actividades moleculares ${ }^{(29)}$ para grupos restringidos de OPs.

Puesto que el evento determinante de la inhibición es un ataque nucleofílico al átomo de fósforo, existe una marcada correlación entre las constantes cinéticas y varios indicadores fisicoquímicos de las propiedades electrónicas del fósforo. ${ }^{(30)}$ No obstante, para la mayoría de los

\footnotetext{
${ }^{27}$ de Bruijn J., Hermens J. Qualitative and quantitative modelling of toxic effects of organophosphorous compounds to fish, Sci.Total.Environ. 109110: $441-455$ (1991).

${ }^{28}$ El número de trabajos en este tópico es muy importante. Ver por ejemplo: (a) Sanborn, J.R. and Fukuto, T.R. Insecticidal, anticholinesterase and hydrolytic properties of S-aryl phosphoramido thioates, J.Agric.Food.Chem. 20 (5): $926-930$ (1972). (b) Pillai, M.K. et al. Structure-activity relationships of phosphorus amides in Aedes aegypti and Culex pipiens fatigans (Diptera: Culicidae), J. Med. Entomol. 12 (5): 497-502 (1975). (c) Mager, P.P. Branching in structure-toxicity relationships applied to organophosphorus pesticides of the DDVP (dichlorvos) type: a novel phenomenon in QSAR, Pharmazie 36 (6): 448449 (1981). (d) Kuusk, V.V. et al. [Inhibition of cholinesterase activity with fluorine-containing derivatives of alpha-aminophosphonic acid], Bioorg.Khim. 16 (11): 1500-1508 (1990). (e) Makhaeva, G.F. et al. Comparative studies of O,O-dialkyl-O-chloromethylchloroformimino phosphates: interaction with neuropathy target esterase and acetylcholinesterase, Neurotoxicol. 19 (4-5): 623-628 (1998). (f) Spassova, D.P. and Singh, A.K. QSAR for acetylcholinesterase inhibition and toxicity of two classes of phosphoramidothioates, SAR. QSAR. Environ. Res. 11 (5-6): 453-471 (2001).

${ }^{29} \mathrm{Li}, \mathrm{S}$. et al. Structure-activity relationship analysis of mutagenicity of organophosphorus pesticides and their molecular mechanism, Zhongguo Yaolixue Yu Dulixue Zazhi 7 (2): 93-99 (1993).

${ }^{30}$ (a) Pillai, M.K. et al. Structure-activity relationships of phosphorus amides in Aedes aegypti and Culex pipiens fatigans (Diptera: Culicidae),
}

(a)

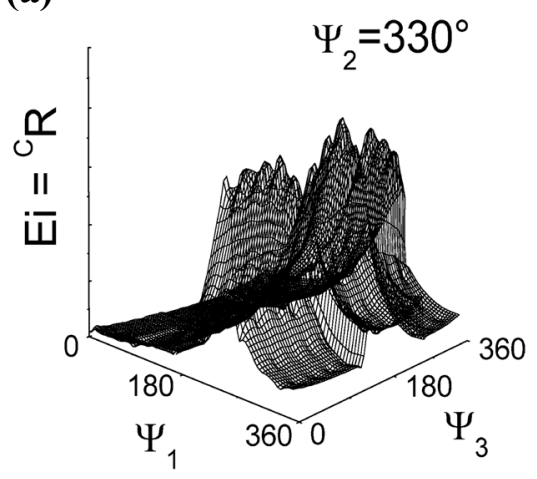

(b)

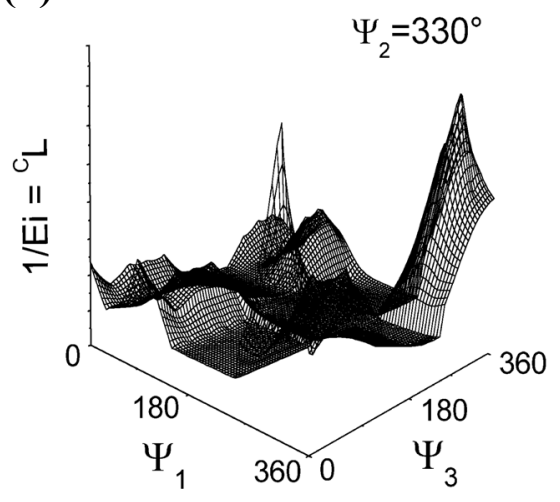

Figura 13.7: Representación de las superficies de restricción conformacional (a) y libertad conformacional (b), para el TMP en $\boldsymbol{P}_{\text {ser } 200}$, fijando $\boldsymbol{\Psi}_{2}$ en $-30^{\circ}$. 
Tabla 13.VI: Descriptores conformacionales intrínsecos: ${ }^{\mathrm{I}} \mathbf{R}_{\mathbf{A}}$, ${ }^{\mathrm{I}} \mathbf{L}_{\mathbf{B}}$ e ${ }^{\mathrm{I}} \mathbf{R}_{\mathbf{C}}$.

\begin{tabular}{crcc}
\hline & \multicolumn{1}{c}{${ }^{\mathbf{I}} \mathbf{R}_{\mathbf{A}}$} & ${ }^{\mathbf{I}} \mathbf{L}_{\mathbf{B}}$ & ${ }^{\mathbf{I}} \mathbf{R}_{\mathbf{C}}$ \\
\hline $\mathbf{I}$ & 126,92388 & 0,16359 & 2,12035 \\
$\mathbf{I I}$ & 121,67803 & 0,12549 & 2,45052 \\
$\mathbf{I I I}$ & 9542,52667 & 0,15055 & 2,70747 \\
$\mathbf{I V}$ & 2531,66982 & 0,17682 & 2,00095 \\
$\mathbf{V}$ & 2526,83166 & 0,18163 & 1,92306 \\
VI & 768,30201 & 0,18094 & 1,91205 \\
VII & 26327,01197 & 0,17766 & 2,02838 \\
VIII(R) & 5,11225 & 0,19564 & 1,63156 \\
VIII(S) & 5,11225 & 0,19564 & 1,63155 \\
$\mathbf{I X}$ & 6,26484 & 0,16399 & 1,81630 \\
$\mathbf{X}$ & ND & ND & ND \\
\hline \multicolumn{4}{c}{} \\
\hline
\end{tabular}

compuestos OPs son más importantes los descriptores que involucran propiedades de los sustituyentes, fundamentalmente del grupo saliente. ${ }^{(3)}$

Aunque los estudios QSAR encuentran un interesante campo en la investigación de los descriptores 3D, en contadas ocasiones se han aplicado en el estudio de la actividad anticolinesterasa ${ }^{(31)}$ y existen pocos antecedentes en este tipo de QSAR aplicados a los compuestos OPs. ${ }^{(32)}$ Los descriptores $4 \mathrm{D},{ }^{(12)}$ se han utilizado con cierto éxito en el desarrollo de sustancias con actividad farmacológica. ${ }^{(33)}$ Estos descriptores se determinan a partir del comportamiento de las distintas estructuras conformacionales de una misma molécula enfrentada a un entorno determinado.

Finalmente, muy recientemente se ha introducido el concepto de 5D-QSAR ${ }^{(34)}$, asociado a la consideración tanto de las variaciones de las estructuras espaciales de las moléculas de las que se pretende estimar su actividad, como de las estructuras que se constituyen en la diana molecular de esta actividad. No se encontraron antecedentes ni de desarrollos 4D-QSAR ni de aplicaciones 5D-QSAR para inhibidores de la la AChE.

J.Med.Entomol. 12 (5): 497-502 (1975). (b) Barbato, F. et al. Inhibition of butyrylcholinesterase by organophosphorus compounds: a quantitative structure- activity analysis, Farmaco [Sci] 37 (8): 519-536 (1982).

31 (a) Golbraikh, A., Bernard, P.P. and Chretien, J.R. Validation of protein-based alignment in 3D quantitative structure-activity relationships with CoMFA models, Eur.J.Med.Chem. 35 (1): 123-136 (2000). (b) Kaur, J. and Zhang, M.Q. Molecular modelling and QSAR of reversible acetylcholinesterase inhibitors, Curr.Med.Chem. 7 (3): 273-294 (2000). (c) Recanatini, M. et al. SAR of 9-amino-1,2,3,4-tetrahydroacridine-based acetylcholinesterase inhibitors: synthesis, enzyme inhibitory activity, QSAR, and structure-based CoMFA of tacrine analogues, J.Med.Chem. 43 (10): 2007-2018 (2000). (d) Sippl, W. et al. Structure-based 3D QSAR and design of novel acetylcholinesterase inhibitors, J.Comput.Aided Mol.Des. 15 (5): 395-410 (2001).

32 El Yazal, J. et al. Prediction of organophosphorus acetylcholinesterase inhibition using three-dimensional quantitative structure-activity relationship (3D-QSAR) methods, Toxicol Sci 63 (2): 223-232 (2001).

${ }^{33}$ Ver por ejemplo: (a) Krasowski, M.D. et al. 4D-QSAR analysis of a set of propofol analogues: mapping binding sites for an anesthetic phenol on the GABA(A) receptor, J. Med. Chem. 45 (15): 3210-3221 (2002). (b) Kuz'min, V.E. et al. The analysis of structure-anticancer and antiviral activity relationships for macrocyclic pyridinophanes and their analogues on the basis of 4D QSAR models (simplex representation of molecular structure), Acta. Biochim. Pol. 49 (1): 157-168 (2002).

${ }^{34}$ Vedani, A., Dobler, M. 5D-QSAR: the key for simulating induced fit?, J. Med. Chem. 45 (11): 2139-2149 (2002). 


\section{Definición de descriptores moleculares}

El intento de construir un QSAR para la serie de moléculas, requiere por un lado ampliar el número de sustancias para el análisis de las correlaciones y por otro lado definir el tipo de propiedades que determinan a estas sustancias como un grupo con características comunes, aun asumiendo que todas actúan como inhibidores de la AChE.

Respecto a la primera de las cuestiones, las sustancias sobre las cuales se realiza el análisis, se enumeran en la Tabla 13.I. Se observa que están incluidos el fosfotionato de S,O,O-trimetilo (STMSP) y el fosfoselenoato de Se,O,O-trimetilo (SeTMSeP), moléculas que podrían corresponder a las especies que interaccionan con el sitio activo de la AChE durante las medidas de inhibición por parte del fosfotioato de O,O,O-trimetilo (TMSP) y el fosfoselenoato de O,O,O-trimetilo (TMSeP) respectivamente, según el planteo expuesto en el Capítulo 12.

Respecto a la segunda de las cuestiones, es posible identificar al menos una propiedad importante para la serie de moléculas analizadas: el tamaño. Efectivamente, todas las sustancias son derivados dimetilo de oxo, tio y selenofosforados, con respectivos sustituyentes $-\mathrm{CH}_{3},-\mathrm{NH}_{2}$, $-\mathrm{NHC}(\mathrm{O}) \mathrm{CH}_{3} \mathrm{y}-\mathrm{CHCCl}_{2}$, de pequeño tamaño.

Se calcularon distintos descriptores moleculares para la serie de sustancias en las que se determinaron las constantes cinéticas $\mathrm{Ka} \mathrm{y} \mathrm{kp}$ (Capítulo 12). Es necesario considerar que para la determinación de las constantes cinéticas del metamidofós se utilizó una mezcla racémica. En principio, se discrimina a los descriptores en tanto sean electrónicos, estéricos o de hidrofobicidad, es decir, según el esquema clásico. En un cuarto grupo, se ubican los descriptores asociados con el comportamiento conformacional.

Entonces se calcularon por un lado descriptores que dieran cuenta de las propiedades electrónicas de las moléculas. Estos fueron: la carga neta del fósforo $\left({ }^{+} \mathbf{P}\right)$, calculada según la distribución de Mülliken de cargas, momento cuadrupolar (Qp) (ambos valores mostrados en la Tabla 13.III), momento octapolar (Op) y momento hexadecapolar (Hdp) (ambos valores mostrados en la Tabla 13.IV), todos parámetros calculados a un nivel $a b$ initio HF/STO-3G.
Tablas 13.VII: Descriptores contextuales de restricción conformacional: ${ }^{{ }^{C}} \mathbf{R}_{A},{ }^{C}{ }_{B},{ }^{C} \mathbf{R}_{\mathrm{J}}$ y ${ }^{\mathrm{C}} \mathbf{R}_{\mathbf{K}}$.

(a)

\begin{tabular}{crr}
\hline & ${ }^{\mathbf{C}} \mathbf{R}_{\mathbf{A}}\left(\times 10^{-6}\right)$ & $\mathbf{C}_{\mathbf{B}}\left(\times 10^{-6}\right)$ \\
\hline I & 1,49734 & 1,49721 \\
II & 250,69700 & 250,69700 \\
III & 4,97595 & 4,96641 \\
IV & 196,9090 & 196,907 \\
V & 89295,3 & 89295,3 \\
VI & 4,52234 & 4,52158 \\
VII & 18,27820 & 18,25190 \\
VIII(R) & 14,1399 & 14,1374 \\
VIII(S) & 174,0980 & 174,0980 \\
IX & 269,8800 & 269,8800 \\
$\mathbf{X}$ & $\mathrm{ND}$ & $\mathrm{ND}$ \\
\hline
\end{tabular}

(b)

\begin{tabular}{crc}
\hline & \multicolumn{1}{c}{${ }^{\mathbf{C}} \mathbf{R}_{\mathbf{J}}$} & ${ }^{\mathbf{C}} \mathbf{R}_{\mathbf{K}}$ \\
\hline $\mathbf{I}$ & 9,57764 & 5,08771 \\
II & 9,78608 & 4,50286 \\
III & 9,52146 & 4,73989 \\
IV & 10,55633 & 5,95725 \\
V & 12,10833 & 6,98923 \\
VI & 8,58512 & 4,90414 \\
VII & 8,46396 & 4,71871 \\
VIIII(R) & 8,83479 & 5,41351 \\
VIII(S) & 9,23574 & 5,65963 \\
IX & 9,64501 & 5,32307 \\
$\mathbf{X}$ & ND & ND \\
\hline
\end{tabular}


Tablas 13.VIII: Descriptores contextuales de libertad conformacional: ${ }^{{ }^{C}} \mathbf{L}_{\mathbf{C}},{ }^{C} \mathbf{L}_{\mathbf{D}}$, ${ }^{\mathrm{C}} \mathbf{L}_{\mathbf{E}},{ }^{\mathrm{C}} \mathbf{L}_{\mathbf{F}},{ }^{\mathrm{C}} \mathbf{L}_{\mathbf{H}}$ y ${ }^{\mathrm{C}} \mathbf{L}_{\mathbf{I}}$.

\section{(a)}

\begin{tabular}{|c|c|c|c|}
\hline & ${ }^{\mathbf{C}} \mathbf{L}_{\mathbf{C}}$ & ${ }^{{ }^{C}} \mathbf{L}_{D}$ & ${ }^{\mathrm{C}} \mathbf{L}_{\mathbf{E}}$ \\
\hline I & 0,00347 & 0,02202 & 0,00412 \\
\hline II & 00485 & 0,05045 & 0,00823 \\
\hline III & 00508 & 2,00705 & 0,00910 \\
\hline IV & 00304 & 0,02680 & 0,00365 \\
\hline V & 00300 & 0,02524 & 0,00359 \\
\hline VI & 0475 & 0,03674 & 0,00546 \\
\hline VII & 0882 & 0,06002 & 0,01400 \\
\hline I(R & 00694 & 0,03536 & 0,00820 \\
\hline I(S & 0,00717 & 0,03652 & 0,00879 \\
\hline IX & 0,00312 & 0,01895 & 0,00361 \\
\hline$x$ & חה & ND & ND \\
\hline \multicolumn{4}{|l|}{ (b) } \\
\hline & ${ }^{\mathrm{C}} \mathbf{L}_{\mathbf{F}}$ & $\mathbf{L}_{\mathbf{H}}$ & ${ }^{\mathrm{C}} \mathbf{L}_{\mathbf{I}}$ \\
\hline I & 0,16012 & 1,01290 & 0,02810 \\
\hline II & 12064 & 1,07453 & 0,0745 \\
\hline III & 0,14546 & 1,1 & 0,1005 \\
\hline IV & 0,17377 & 81 & 0,4098 \\
\hline V & 0,17863 & 871 & 0,4387 \\
\hline VI & 0,17619 & 1,02886 & 0,02886 \\
\hline VII & 0,16884 & 1,64158 & 0,64158 \\
\hline VIII(R) & 0,18870 & 1,04178 & 0,04178 \\
\hline VIII(S) & 0,18847 & 1,04477 & 0,04477 \\
\hline IX & 0,16086 & 1,02190 & 0,02190 \\
\hline $\mathbf{X}$ & ND & ND & ND \\
\hline
\end{tabular}

Por otro lado, se determinaron descriptores que dieran cuenta de las propiedades estéricas de las moléculas. Estos fueron: refractividad molar (RM), calculada por el método de fragmentación de Viswanadhan, ${ }^{(35)}$ volumen molar (VM, expresado en $\mathrm{cm}^{3} / \mathrm{mol}$ ) y radio de Bohr (ao, expresado en $\AA$ ), estos últimos calculados a partir del confórmero de mínima energía a un nivel ab initio HF/STO-3G. Dichos valores se muestran en la Tabla 13.V.

Para considerar propiedades hidrofóbicas se calcularon los siguientes parámetros: momento dipolar del confórmero más estable ( $\mu$ expresado en Debyes) calculado a un nivel $a b$ initio HF/STO-3G y logaritmo de la constante de partición octanol/agua ( $\log \mathbf{P})$, calculado por el método de fragmentación de Viswanadhan. ${ }^{(35)}$

Finalmente se procedió a la definición de descriptores moleculares que dieran cuenta del comportamiento conformacional de las moléculas estudiadas tanto en el vacío como en el contexto del sitio activo, calculadas a partir de las energías de interacción obtenidas por mecánica molecular mediante el método DREIDING. La definición de cada descriptor se describe en el Anexo I.

Se construyen dos tipos de descriptores conformacionales: intrínsecos (denominados con el superíndice "I") y contextuales (denominados mediante el superíndice "C"). También se discriminan a los descriptores que presentan una correlación positiva con las energías de interacción (denominados "R", de restricción conformacional), de aquellos que son indirectamente proporcionales a las energías de interacción (denominados "L", de libertad conformacional). Mientras la restricción conformacional se puede visualizar sin dificultad en los gráficos de las superficies de energías de interacción, la libertad conformacional puede visualizarse en un gráfico que represente una superficie construida por las inversas de las energías de interacción, como se puede observar en la Figura 13.7.

35 (a) Ghose, A.K., Pritchett, A. and Crippen, G.M. Atomic physicochemical parameters for three dimensional structure directed QSAR. 3: Modeling hydrophobic interactions, J.Comput.Chem. 9:80-90 (1988). (b) Viswanadhan, V.N. et al. Atomic physicochemical parameters for three dimensional structure directed QSAR. 4. Additional parameters for hydrophobic and dispersive interactions and their application for an automated superposition of certain naturally occurring nucleoside antibiotics, J.Chem.Inf.Comput.Sci. 29:163-172 (1989). 
Los descriptores conformacionales intrínsecos no dependen de las relaciones de interacción con el sitio activo, sino que se calculan a partir de la molécula libre. Esto significa que se definen en forma independiente de su actividad anticolinesterasa o de la relación de estas sustancias con el sitio activo de la AChE. Dichos descriptores se muestran en la Tabla 13.VI.

En el cálculo de los descriptores conformacionales contextuales ${ }^{(36)}$ se consideraron las energías de interacción de cada uno de los confórmeros posibles de la molécula ubicada en la posición de mínima energía en $\boldsymbol{P}_{\text {ser200 }}$ calculada a un nivel PM3 (cálculo semiempírico). Por esta razón, estos descriptores deben considerarse como

Tabla 13.IX: Correlaciones propuestas para cada una de las constantes cinéticas en la serie de moléculas planteada. Entre paréntesis se indica el error analítico de las constantes de ajuste de cada correlación. Se indica además el valor de $\mathrm{r}^{2}$ como índice de correlación.

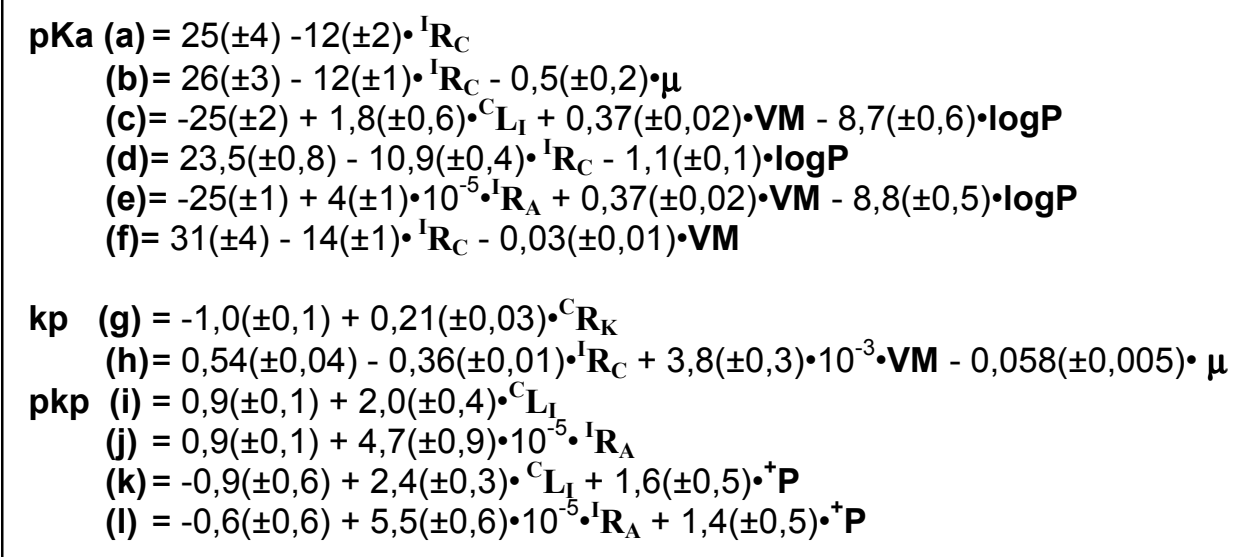

pKi $(\mathbf{m})=-27( \pm 5)+14( \pm 2) \cdot \mathbf{I}_{\mathbf{C}}$

$(\mathbf{n})=-24( \pm 3)+16( \pm 1) \cdot{ }^{\cdot} \mathbf{R}_{\mathbf{C}}-7( \pm 2) \cdot{ }^{+} \mathbf{P}$

(o) $=25( \pm 4)+0,8( \pm 1,1) \cdot{ }^{\mathbf{C}} \mathbf{R}_{\mathrm{C}}-0,37( \pm 0,03) \cdot \mathrm{VM}+8,7( \pm 0,8) \cdot \log \mathbf{P}$

$\left[r^{2}=0,92961\right]$

$\left[r^{2}=0,99748\right]$

$\left[r^{2}=0,83617\right]$

$\left[r^{2}=0,86893\right]$

$\left[r^{2}=0,95723\right]$

$\left[r^{2}=0,96295\right]$

$(\mathbf{p})=27,2( \pm 0,6)+0,4( \pm 0,2) \cdot{ }^{C} \mathbf{R}_{\mathbf{K}}-0,408( \pm 0,009) \cdot \mathbf{V M}+9,6( \pm 0,2) \cdot \log \mathbf{P}$

$(q)=28,5( \pm 0,5)-0,3( \pm 0,2) \cdot{ }^{C} \mathbf{L}_{I}-0,400( \pm 0,006) \cdot \mathbf{V M}+9,5( \pm 0,2) \cdot \log P$

\footnotetext{
${ }^{36}$ La idea de descriptor contextual no es nueva. De hecho, el parámetro logP depende del comportamiento de la sustancia en el sistema binario octanol/agua. Si con algoritmos adecuados puede estimarse el parámetro $\log \mathbf{P}$ a partir de descriptores moleculares no contextuales (Katagi, T. et al. Theoretical estimation of octanol-water partition coefficient for organophosphorus pesticides, ACS.Symp.Ser. 606:48-61 (1995)), no es difícil suponer que los descriptores conformacionales puedan estimarse de similar manera. De aquí surge una interesante discusión acerca de la validez de las correlaciones entre descriptores contextuales, respecto al concepto original de las QSAR, que indica que todas las propiedades pueden ser descritas exclusivamente a partir de la estructura molecular, sin supuestos ajenos a ésta.
} 
contextuales específicos. ${ }^{(37)}$ Los descriptores contextuales de restricción conformacional calculados se listan en la Tablas VII, en tanto los de libertad conformacional se muestran en las Tablas VIII.

Estos últimos, son los únicos descriptores que permiten discriminar entre las formas $R \quad y \quad S$ del metamidofós. Esta propiedad da cuenta del hecho de ser descriptores que incluyen al dominio 3D. Aun más, dado que cuantifican la variabilidad conformacional, es decir, el desempeño energético de las múltiples estructuras en el espacio para una misma molécula, no es arriesgado definir a estos descriptores como pertenecientes al dominio 4D.

Las sustancias I, VI, VII, VIII, IX y $\mathbf{X}$ se utilizan para la construcción de las correlaciones y pueden considerarse nuestra serie de entrenamiento. A partir de cada serie de los descriptores enumerados fue posible encontrar correlaciones con un alto índice de ajuste para cada una de las tres constantes cinéticas estudiadas: Ka, kp y Ki. Estas correlaciones se muestran en la Tabla 13.IX.

Estas correlaciones constituyen por una lado una herramienta con poder predictivo, es decir, sería posible estimar las constantes cinéticas de moléculas de las cuales sólo se contaran los respectivos descriptores. Por otro lado, en la medida que una correlación derive directa o indirectamente de los eventos que vinculan las causas con los efectos medidos, es posible asignar a la estructura de la correlación un poder interpretativo respecto de los fenómenos que determinan el efecto biológico descrito. Ambas propiedades son ensayadas en el contexto de la serie completa de moléculas.

\section{Análisis de las correlaciones encontradas}

Aunque se trabajó solamente en la búsqueda de correlaciones lineales, ${ }^{(38)}$ se puede observar que existe buen ajuste para funciones dependientes de uno, dos y tres

\footnotetext{
${ }^{37}$ Es decir, ${ }^{{ }^{C}} \mathbf{L}$ o ${ }^{\mathbf{C}} \mathbf{R}$ son además descriptores tóxico específicos, pues dependen del comportamiento de la sustancia en el entorno del grupo reactivo de la diana de acción tóxica, mientras que $\log \mathbf{P}$ sería un descriptor contextual no específico.

${ }^{38}$ En rigor, se realizó la búsqueda tanto de correlaciones lineales como exponenciales, puesto que se utilizaron como variables de ajuste tanto a las constantes cinéticas como a sus logaritmos.
} 
descriptores. ${ }^{(39)}$ En general, es posible indicar que los descriptores electrónicos elegidos no resultaron adecuados a los fines perseguidos. Esto no es de extrañar para los momentos multipolares, puesto que no se tienen antecedentes de su uso exitoso como descriptores moleculares. Respecto a la carga del fósforo como descriptor electrónico, sólo se lo encuentra en las correlaciones que determinan la estimación de pkp y $\mathrm{pKi}$, lo que es lógico puesto que son las dos constantes cinéticas que involucran al ataque nucleofílico al átomo de fósforo por parte de la $\mathrm{Ser}^{200}$. Sin embargo, esta misma carga varía en función de los ángulos $\Psi$, de manera que no es de extrañar que aparezca en las correlaciones de menor ajuste.

Tabla 13.X: Estimación de las constantes cinéticas Ka, kp y Ki para el metamidofós(R), VIII(R) y el metamidofós(S), VIII(S).

\begin{tabular}{|c|c|c|c|c|c|c|}
\hline & \multirow{2}{*}{$\frac{\mathrm{Ka}}{\operatorname{QSAR}^{\mathrm{C}}(\mathbf{c})}$} & \multicolumn{3}{|c|}{$\mathrm{kp}$} & \multicolumn{2}{|c|}{$\mathrm{Ki}$} \\
\hline & & $\operatorname{QSAR}^{\mathrm{C}}(\mathbf{g})$ & $\operatorname{QSAR}_{(\mathbf{i})}$ & $\operatorname{QSAR}^{\mathrm{C}}(\mathbf{k})$ & $\operatorname{QSAR}^{\mathrm{C}}(\mathbf{p})$ & $\operatorname{QSAR}^{\mathrm{C}}(\mathbf{q})$ \\
\hline VIII(R) & $1,654 \times 10^{-6}$ & 0,137 & 0,104 & 0,161 & $3,11 \times 10^{4}$ & $4,72 \times 10^{4}$ \\
\hline VIII(S) & $1,633 \times 10^{-6}$ & 0,189 & 0,102 & 0,158 & $2,48 \times 10^{4}$ & $4,73 \times 10^{4}$ \\
\hline
\end{tabular}

Como ya se ha expuesto en Capítulos anteriores, el momento dipolar también varía con la conformación. Por ello, aparece sólo en dos de las correlaciones y éstas no están entre las de mejor ajuste. En términos de descriptores estéricos e hidrofóbicos, VM y $\log \mathbf{P}$ respectivamente aparecen como descriptores óptimos en el marco de la serie de sustancias estudiada. No es tan clara la razón por la cual RM no aparece como un buen descriptor, mientras que VM sí lo es.

Es notable el predominio de los descriptores conformacionales en todas las correlaciones encontradas. Más aún, ${ }^{\mathbf{I}} \mathbf{R}_{\mathbf{C}}$ presenta un alto grado de correlación individual con pKi y pKa (Figura 13.8), en tanto ${ }^{C} \mathbf{R}_{\mathbf{K}}$ y ${ }^{C_{\mathbf{L}}}$ lo presentan con kp. Esto no ocurre con ninguno de los demás descriptores utilizados en el análisis. Por esta razón, a todas éstas las consideramos como correlaciones entre las estructura conformacional y la actividad o QSAR $^{\mathrm{C}}$ (la denominación de cada una surge de la lista mostrada en la Tabla 13.IX). Considerando el comportamiento generalizado de estos QSAR $^{\mathrm{C}}$, una primera aproximación posible es el considerar a los descriptores conformacionales como buenos sustituyentes de los descriptores electrónicos. Esto queda en evidencia sobre todo en las correlaciones triples.

\footnotetext{
${ }^{39}$ En la práctica, se definió de buen ajuste a aquellas correlaciones con $r>0,82, r>0,95$ y $r>0,99$ para uno, dos y tres descriptores respectivamente.
} 


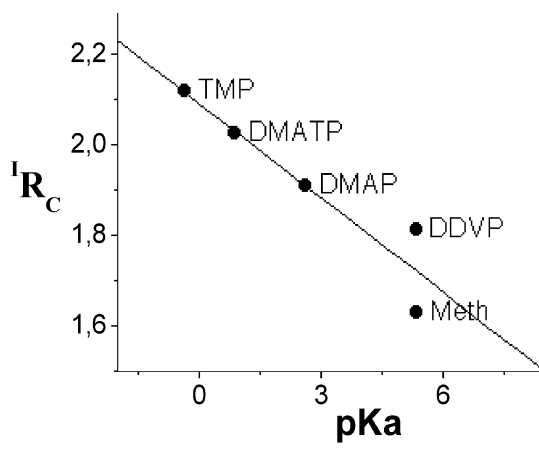

Figura 13.8: Correlación lineal entre pKa e ${ }^{\mathbf{I}} \mathbf{R}_{\mathbf{C}}$ para la serie de entrenamiento.
Sin embargo, tomando en cuenta las características de la serie utilizada, esto es, el pequeño tamaño de las moléculas y la escasa cantidad de congéneres, es de gran riesgo suponer una extrapolación exitosa hacia moléculas con grupos laterales más voluminosos. Más aún, se puede observar en la correlación individual $\mathrm{pKa} \mathrm{v} / \mathrm{s}{ }^{\mathbf{I}} \mathbf{R}_{\mathbf{C}}$ (Figura 13.8), que la dispersión de los datos aumenta con el valor de $\mathrm{Ka}$, es decir, el ajuste es mejor con las sustancias de menor poder inhibitorio. Esto de alguna manera puede ser indicio que las correlaciones perderían validez para inhibidores fuertes, aunque permitan su aplicación en moléculas fosforadas de bajo poder inhibitorio o de muy pequeño tamaño, es decir, en aquellas sustancias con características moleculares cada vez más adecuadas para actuar como inhibidores, las restricciones del entorno del sitio activo serían cada vez menos predominantes, aunque sin perder totalmente su importancia.

\section{Análisis del poder predictivo de los $Q S A R^{C}$}

Si a las sustancias I, VI, VII, VIII, IX y $\mathbf{X}$ se las puede considerar como la serie de entrenamiento, las respectivas II, III, IV , V, VIII(R) y VIII(S) pueden utilizarse como serie de datos de prueba. Aunque no dispongamos de las constantes cinéticas de estas sustancias en el contexto de las medidas realizadas, sí podemos estimarlas para contrastar con los resultados obtenidos con las correlaciones halladas. Los valores estimados por estos $\mathrm{QSAR}^{\mathrm{C}}$, se muestran en las Tablas 13.X y 13.XI.

Respecto a la estimación de las actividades de ambos isómeros ópticos del metamidofós, se observa que en general, los $\mathrm{QSAR}^{\mathrm{C}}$ son más adecuados para el cálculo de las diferencias en $\mathrm{kp} \mathrm{y} \mathrm{Ki}$, puesto que en los $\mathrm{QSAR}^{\mathrm{C}}$ para la estimación de $\mathrm{Ka}$ no predominan los descriptores conformacionales contextuales, que son los que pueden verificar diferencias para este tipo de estructuras moleculares. Observando las diferencias numéricas de los descriptores contextuales, en las Tablas $\mathbf{1 3 . V I I}$ se verifica una mayor restricción para el isómero $(\mathrm{S})$, sin embargo no aparece claramente una diferencia en los descriptores de libertad conformacional (Tablas 13.VIII), siendo algunos mayores para el isómero (S) y otros mayores para el isómero (R). Tal como fue expuesto en el Capítulo anterior, es la forma (R) del metamidofós la de mayor actividad inhibitoria. La estimación de las constantes cinéticas se realiza mediante los 
QSAR $^{\mathrm{C}}$ que contienen descriptores conformacionales contextuales, de manera que se manifiesten diferencias en los valores, posibles de ser justipreciadas. Dichas estimaciones se muestran en la Tabla 13.X. Se observa que ambas estimaciones de $\mathrm{Ki}$ van en el sentido de los datos experimentales, es decir, para el metamidofós (R) se estima un valor algo mayor que para el metamidofós (S). Sin embargo la diferencia estimada es mucho menor que la experimental (Tabla 12.III), reportándose un valor de Ki para metamidofós (R) más de veinte veces mayor que para metamidofós (S). Es decir, habría una buena estimación del valor para metamidofós (R) y una sobreestimación para el metamidofós (S).

Tabla 13.XI: Estimación de las constantes cinéticas Ka, kp y Ki para TMSP (II), TMSeP (III), STMSP (IV) y SeTMSeP (V).

$\mathrm{Ka} \quad \mathrm{kp} \quad \mathrm{Ki}$

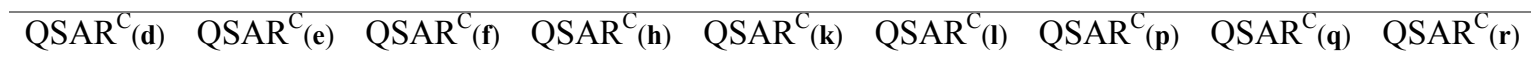

\begin{tabular}{lrrrrrrrrr}
\hline II & $4,26 \times 10^{4}$ & $6,31 \times 10^{-2}$ & $2,25 \times 10^{6}$ & $-6,23 \times 10^{-3}$ & 0,133 & 0,157 & 1,054 & 0,730 & 0,697 \\
III & ND & ND & $2,78 \times 10^{9}$ & 0,252 & 0,106 & 0,044 & ND & ND & ND \\
IV & 0,120 & $4,52 \times 10^{-4}$ & 0,968 & 0,151 & 0,020 & 0,113 & 160,63 & 511,61 & 403,04 \\
V & $1,93 \times 10^{-2}$ & $1,98 \times 10^{-12}$ & 0,266 & 0,210 & 0,015 & 0,101 & $2,77 \times 10^{10}$ & $1,62 \times 10^{11}$ & $1,31 \times 10^{11}$ \\
\hline
\end{tabular}

Para considerar cual es la componente más importante en la determinación de estas diferencias, se observa que en los $\mathrm{QSAR}^{\mathrm{C}}$ de mejor correlación, el valor de kp es mayor para metamidofós $(\mathbf{R})$, no obstante, también se observa un valor mayor para Ka. El cálculo de Ki a partir de los valores estimados de $\mathrm{kp} \mathrm{y} \mathrm{Ka}$, deriva en valores con la misma tendencia que los valores de $\mathrm{Ki}$ estimados directamente por QSAR $^{\mathrm{C}}: 9,73 \times 10^{5}$ y $9,68 \times 10^{5}$ para metamidofós (R) y metamidofós $(\mathbf{S})$, respectivamente.

En general, entonces, aunque en sus tendencias los valores predicen adecuadamente el sentido de las diferencias de actividad de los isómeros ópticos del metamidofós, pero no en la magnitud adecuada. Respecto a este punto, notamos que los descriptores ${ }^{\mathbf{C}_{\mathbf{R}}} \mathbf{R}_{\mathbf{A}}$ y ${ }^{\mathbf{C}_{\mathbf{R}}} \mathbf{R}_{\mathbf{B}}$ no pudieron incluirse en correlaciones de buen ajuste. Ambos descriptores conformacionales presentan diferencias importantes para los isómeros del metamidofós. Tal vez encontrando los descriptores no conformacionales adecuados sea posible estimar con más exactitud las diferencias de actividad para estos isómeros ópticos.

Para el contraste de resultados, respecto al TMSP, TMSeP y sus isómeros, se utilizaron las correlaciones (d), (e) y (f), para Ka, (h), (k) y (I) para kp y (p), (q) y (r) para Ki que son correspondientemente las de mayor $\mathrm{r}^{2}$, para cada una de 


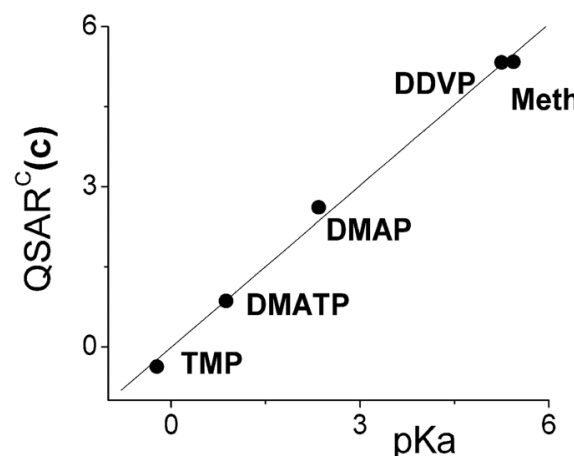

Figura 13.9: Correlación lineal entre pKa y $\operatorname{QSAR}^{\mathrm{C}}(\mathbf{c})$, para la serie de entrenamiento. las tres constantes cinéticas consideradas. Se observa una cierta continuidad de los valores estimados para cada una de las constantes en todas las sustancias, excepto en la estimación de $\mathrm{Ka}$, fundamentalmente mediante la expresión $\operatorname{QSAR}^{\mathrm{C}}(\mathbf{e})$. Sobre las demás, aparentemente es posible realizar alguna interpretación de cierta confiabilidad.

Dado que existen limitaciones metodológicas ${ }^{(22)}$, no se cuenta con la estimación del logP para el TMSeP, de manera que no es posible estimar los valores de Ki mediante el uso de los QSAR $^{\mathrm{C}}$ de mayor correlación. Sin embargo, los valores estimados para esta constante son de $1,25 \times 10^{-11} \mathrm{y}$ $6,72 \times 10^{-13} \mathrm{M}^{-1} \min ^{-1}$ según $\operatorname{QSAR}^{\mathrm{C}}(\mathbf{m})$ y $\operatorname{QSAR}^{\mathrm{C}}(\mathbf{n})$ respectivamente. A modo comparativo, la estimación de esta constante mediante las mismas correlaciones para el TMSP son $4,93 \times 10^{-8}$ y $5,99 \times 10^{-9} \mathrm{M}^{-1} \mathrm{~min}^{-1}$ respectivamente.

Aunque éstos aparentan ser valores muy sobreestimados, son coherentes entre sí y asigna una diferencia de entre tres y cuatro ordenes de magnitud para los Ki del TMSP y TMSeP respectivamente. Extrapolando esta valoración para las correlaciones más altas, los mostrados en la Tabla 13.X, se estimaría un Ki que se encontraría en valores de entre $10^{-3}$ y $10^{-4} \mathrm{M}^{-1} \mathrm{~min}^{-1}$ para el TMSeP. Todos los valores estimados son incompatibles con los $\mathrm{Ki}^{*}$ medidos para estas sustancias en los ensayos cinéticos. Esto apunta a sostener que existe algún evento de modificación molecular in vitro que determina que la estructura molecular que finalmente interacciona en $\boldsymbol{P}_{\text {Ser200, no es el respectivo }}$ calcogenofosfato de trimetilo sino otra, tal como argumentamos en el Capítulo 12.

Observando los valores estimados para el STMSP y SeTMSeP existe una cierta compatibilidad entre los valores de $\mathrm{kp}$ y los valores de $\mathrm{kp}^{*}$ medidos para TMSP y TMSeP, estando en los mismos órdenes de magnitud. También son comparables los valores estimados de $\mathrm{Ka}$ respecto a los $\mathrm{Ka}^{*}$ medidos. Sin embargo, los valores de Ki estimados son altos respecto a los medidos para TMSP y muy altos respecto a los medidos para TMSeP. No obstante, los valores estimados de Ki para SeTMSeP podrían ser compatibles con la constante de hidrólisis alcalina del enlace $\mathrm{P}-\mathrm{Se}$.

En resumen, aunque sobre la base de los valores estimados para los derivados O,O,O-trimetilos, se puede descartar el hecho que sean éstas las especies que interaccionan directamente con la $\mathrm{AChE}$, no es posible concluir que sean sus isómeros $\mathrm{P}-\mathrm{X}-\mathrm{CH}_{3}$, los que 
efectivamente actúen como inhibidores, aunque no hay completa incompatibilidad con ello.

\section{Análisis del poder interpretativo de $\operatorname{los} \operatorname{QSAR}^{\mathrm{C}}$}

Ya se mencionó el hecho que todos los QSAR de correlaciones altas involucran a alguno de los descriptores conformacionales tal como fueron definidos. Aun más, contamos con correlaciones individuales de descriptores conformacionales con las distintas constantes cinéticas ensayadas. Esto sugiere un importante papel en la capacidad de inhibición tanto del comportamiento conformacional de las moléculas individuales como de las restricciones estéricas del sitio activo que imponen dificultades de interconversión conformacional de las moléculas entrantes a él, que es lo que representan respectivamente los descriptores conformacionales intrínsecos y contextuales respectivamente.

Observando la estructura de los QSAR ${ }^{\mathrm{C}}$ hallados, se encuentra un mayor peso los descriptores intrínsecos para la estimación de $\mathrm{Ka}$, mientras que esto se modifica en las funciones de estimación de $\mathrm{kp} \mathrm{y} \mathrm{Ki}$. A partir de ello, es posible argüir que las libertades conformacionales de las moléculas individuales son de mayor importancia durante el ingreso de la misma a lo largo del canal hidrofóbico, hacia el centro reactivo del enzima, mientras que son las restricciones conformacionales impuestas por las estructuras del sitio activo, las que pesan durante los eventos que implican la formación del transiente pentacoordinado.

Esto implicaría, por ejemplo, que el poder inhibitorio disminuido por parte de fosforados del tipo $\mathrm{P}=\mathrm{S}$ y $\mathrm{P}=\mathrm{Se}$ puede explicarse en parte por un aumento de Ka en función del aumento de las restricciones conformacionales intrínsecas de estas moléculas. No se pierde de vista que la explicación clásica más directa es la que sigue predominando en este comportamiento, es decir, que la menor electronegatividad del $\mathrm{S}$ y Se no predispone de la mejor manera al OP para un ataque nucleofílico al átomo de fósforo, de manera que $\mathrm{kp}$ disminuye drásticamente. Sin embargo, el comportamiento conformacional jugaría algún papel en la determinación de la magnitud de kp.

En general, los $\mathrm{QSAR}^{\mathrm{C}}$ son coherentes con ambas argumentaciones. Por ejemplo, el factor que en general acompaña a $\mathbf{I} \mathbf{R}$ en la estimación de $\mathrm{Ka}$ es negativo, lo que implica que un aumento en las restricciones

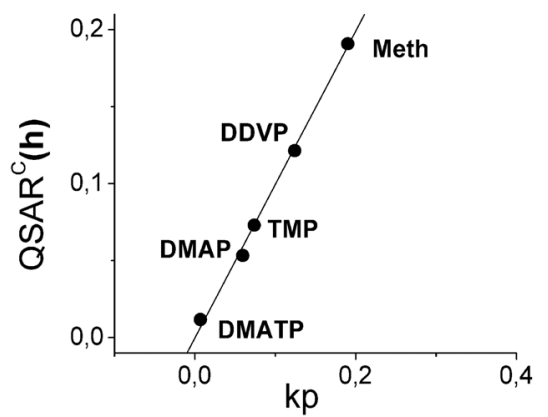

Figura 13.10: Correlación lineal entre kp y $\operatorname{QSAR}^{\mathrm{C}}(\mathbf{h})$, para la serie de entrenamiento. 
conformacionales intrínsecas del inhibidor aumenta Ka y por lo tanto disminuye su capacidad de inhibición. De la misma manera, el factor que acompaña a ${ }^{\mathbf{C}} \mathbf{R}$ en la estimación de $\mathrm{kp}$ es positivo, mientras que ${ }^{\mathbf{C}} \mathbf{L}$ en la estimación de pkp también es positivo, lo que implica que un aumento en las restricciones conformacionales contextuales de los OPs, aumentaría el valor de $\mathrm{kp}$ y, por lo tanto, la velocidad de fosforilación.

Nuevamente se vuelve sobre una hipótesis que hemos planteado en los inicios de este trabajo y es que las restricciones conformacionales sobre la molécula entrante le imponen una cierta dirección en la determinación de los confórmeros más estables, de manera que éstos no son equivalentes a los estables en el vacío o en solución, pero son los que predisponen de la mejor manera para la formación del complejo pentacoordinado que determina el curso de la fosforilación. Es decir, la arquitectura del sitio activo poseería como propiedad intrínseca la capacidad de imponer las restricciones estéricas necesarias para que la reacción con la $\operatorname{Ser}^{200}$ ocurra de la manera más eficiente posible. No olvidemos que en tanto enzima, la $\mathrm{AChE}$ trabaja en el límite difusional de la catálisis y los OPs son inhibidores muy eficientes, por lo tanto, cualquier fenómeno emergente en los fenómenos de inhibición o de catálisis debe entenderse como un factor que aporta positivamente durante estos eventos.

Observando la estructura de los $\operatorname{QSAR}^{\mathrm{C}}$ respecto a los descriptores no conformacionales, para nuestra serie de moléculas existe un peso importante del valor de logP en la estimación tanto de $\mathrm{Ka}$ como de $\mathrm{Ki}$, no así en la estimación de $\mathrm{kp}$. Se ha mencionado que los descriptores de las propiedades hidrofóbicas son los que menos determinan en las correlaciones de $\mathrm{Ki}$ para fosfamidatos, en experiencias realizadas con AChE de mosca, lo que implicaría un rol menor de las interacciones hidrofóbicas durante la formación del complejo enzima-inhibidor. ${ }^{(40)}$

Pero según nuestros datos que incluyen fosfamidatos, se puede afirmar que, por lo menos para la serie estudiada, en general con bajos valores de Ki y pequeño tamaño, las interacciones hidrofóbicas cobran mayor relevancia. Más aún, dado el signo negativo del factor de correlación que acompaña a $\log P$ en todas $\operatorname{los} \mathrm{QSAR}^{\mathrm{C}}$ de

40 Singh, A.K. Quantitative structure-activity relationships for phosphoramidothioate toxicity in housefly, Comp. Biochem. Physiol. C. Pharmacol. Toxicol. Endocrinol. 123 (3): 241-255 (1999). 
estimación de $\mathrm{Ka}$, se puede especular respecto a que el aumento de las interacciones hidrofóbicas favorecería un aumento del valor de Ka y una mayor dificultad para ubicar a la molécula en el sitio activo de manera adecuada.

Para decirlo de alguna manera: en esta serie, el mayor peso de las interacciones hidrofóbicas "molesta" en el mecanismo de inhibición. Esto es así sobre todo en un entorno hidrofóbico como lo es el canal de ingreso al sitio activo, haciendo mas lento el traslado desde la interfase solvente-proteína y a través del canal hacia los entornos de la $\mathrm{Ser}^{200}$. En la medida que puedan predominar las interacciones por dipolos o electrostáticas por sobre las interacciones hidrofóbicas, aumentaría la capacidad inhibitoria de la molécula entrante. Sobre la misma argumentación, la pérdida del momento dipolar local en la sucesión $\mathrm{P}=\mathrm{O}>\mathrm{P}=\mathrm{S}>\mathrm{P}=\mathrm{Se}$, implicaría por si solo un factor de aumento del $\mathrm{Ka}$ y por lo tanto una disminución del Ki para esta serie.

Sin duda, la capacidad respecto a la posible interpretación de los mecanismos de la fosforilación de la AChE aparece como la propiedad más interesante de los QSAR $^{\mathrm{C}}$ descritos. Sin embargo, el trabajo con series de entrenamiento más extensas, probablemente mejore en mucho la posibilidad de constituir QSAR $^{\mathrm{C}}$ con capacidades predictivas superiores y dispongamos entonces de herramientas poderosas para la estimación de constantes cinéticas de moléculas que por alguna razón no permitan la medida experimental de estos parámetros. Dos áreas posibles de aplicación inmediata serían, por un lado, la determinación de las constante cinéticas de distintas variantes moleculares del metamidofós (Figura 13.6), lo que permitiría contrastar las diferentes hipótesis planteadas, algunas contradictorias entre sí, respecto a cual es el mecanismo de acción in vitro e in vivo de este inhibidor.

Por otro lado, resta interpretar adecuadamente el hecho que las constantes cinéticas aparentes del TMSP y TMSeP, descritas en el Capítulo anterior, y del fosfotioato de $\mathrm{O}, \mathrm{O}, \mathrm{O}$-trietilo, reportado previamente, muestran un poder inhibitorio anormalmente alto respecto de sus estructuras. Estudios más completos de las dinámicas en solución acuosa de estas sustancias y QSAR $^{\mathrm{C}}$ suficientemente potentes podrían auxiliar en dilucidar cuales son las estructuras que efectivamente interaccionan con el sitio activo.

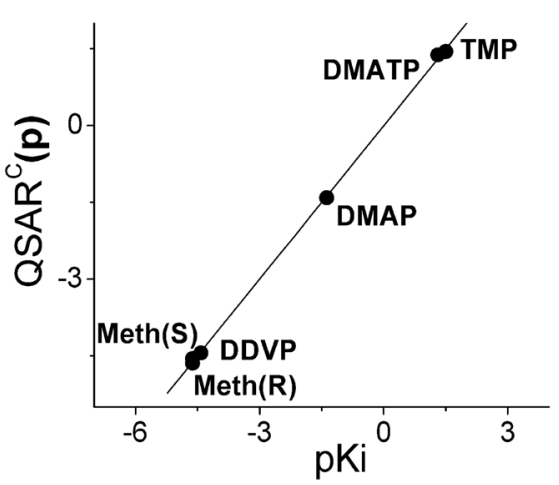

Figura 13.11: Correlación lineal entre pKi y $\operatorname{QSAR}^{\mathrm{C}}(\mathbf{p})$, para la serie de entrenamiento. 


\section{4}

\section{A modo de recapitulación...}

A lo largo de los Capítulos precedentes se ha recorrido una serie de aspectos que relacionan la estructura de pequeños OPs, y en particular su comportamiento conformacional, con las manifestaciones fenomenológicas de éstas en entornos biológicos y no biológicos.

Durante esta exploración se han contrastado los datos obtenidos con la abundante información disponible en la literatura $\mathrm{y}$, en general, estos datos no encuentran contradicción con aquella, salvo en algunos aspectos en donde surgen diferencias de interpretación, que no pueden definirse como incongruencias definitivas. Estas discordancias aparecen por ejemplo en la asignación de algunos modos vibracionales en los que incluso dentro de los datos previamente existentes son comunes estas diferencias. En nuestro caso hemos puesto el acento en aquellos modos asociados a la manifestación de confórmeros y, en este aspecto, fundamentalmente hemos complementado la información preexistente.

Con relación a la dinámica de interacción de los compuestos OPs con el sitio activo de la AChE, nuestros datos aportan a un plano de estudio que actualmente no se puede suponer siquiera como medianamente claro. De manera que estos resultados, y las conclusiones que surgen de ellos, sólo pueden considerarse como preliminares y, en todo caso, como hipótesis de partida de futuros trabajos.

$\mathrm{Ni}$ en este trabajo, ni en ningún otro, podemos asignar a los datos obtenidos del modelado molecular el carácter de conclusiones absolutas, en el sentido de aportar explicación definitiva a los mecanismos de las interacciones dinámicas entre las moléculas. Sí encontramos de cierto interés el estudio de la problemática conformacional en entornos biológicos como herramienta de generación de hipótesis con un alto nivel interpretativo de los fenómenos. En nuestro caso, la definición de parámetros que describen la libertad conformacional como descriptores cuantitativos de la interacción enzima-inhibidor, nos parece uno de los aportes al 
área que puede suponer algunas derivaciones de cierta importancia.

De todas formas, nos permitimos integrar los resultados en forma de conclusiones esbozadas y de hipótesis de trabajo que proponen la continuidad de este estudio mediante el uso de nuevas series de sustancias, el desarrollo de nuevas metodologías experimentales y de técnicas de modelado molecular más potentes.

\section{Generalizaciones respecto del comportamiento conformacional de pequeños OPs}

Un hecho ya previamente reportado, y verificado durante el estudio de las moléculas abordadas en este trabajo, es la estabilización de las posiciones gauche por sobre las trans para los ángulos $\Psi$ en los ésteres fosfóricos. Este es el llamado "efecto gauche". Esto determina por ejemplo la mayor estabilidad del confórmero $\mathrm{C}_{3}$ para los calcogenofosfatos de trimetilo y del confórmero gCs para los calcogenofosfatoamidas de dimetilo.

El otro resultado de relevancia se refiere a la cuantificación del aumento de las barreras energéticas conformacionales en la medida del aumento del peso atómico de $\mathrm{X}$ en el grupo $\mathrm{P}=\mathrm{X}$. En tal sentido, son ilustrativos los gráficos de las superficies energéticas conformacionales para la serie de moléculas TMP (Figura 2.5), TMSP (Figura 3.4) y TMSeP (Figura 4.5) y para la serie equivalente de fosfamidatos de dimetilo.

Ya se ha descrito cómo cada uno de los confórmeros estables de los pequeños fosfatos orgánicos estudiados presenta una variación importante de sus momentos dipolares. Esta variación está determinada por el hecho que el vector del dipolo local para el enlace $\mathrm{O}-\mathrm{C}$ de los ésteres fosfóricos se encuentra en oposición al vector correspondiente al enlace $\mathrm{P}=\mathrm{X}$ en posición gauche $\mathrm{y}$ en la misma dirección de este vector cuando se encuentra en posición trans, siendo $\mathrm{X}$ un calcógeno. Una de las explicaciones que se ha dado para la minimización de las energías conformacionales es la de la anulación de los momentos dipolares locales, ${ }^{(1)}$ favoreciendo de esta manera el

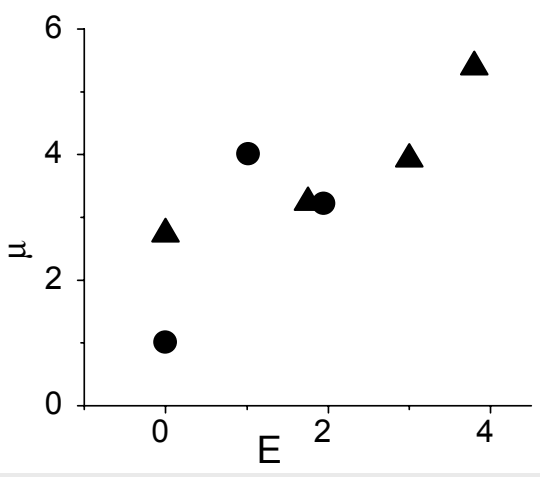

Figura 14.1: Correlación entre las energías conformacionales (en $\mathrm{kcal} / \mathrm{mol}$ calculadas a un nivel $\mathrm{HF} / 6-31 \mathrm{G}^{* *}$ ) y el momento dipolar (en Debyes) para los confórmeros del TMP $(\bullet)$ y del DMAP $(\boldsymbol{\Delta})$.

\footnotetext{
${ }^{1}$ Van Wazer, J.R. and Ewig, C.S. Ab initio structures of phosphorus acids and esters. II. Methyl Phosphinate, dimethyl phosphonate, and trimethyl phosphate. J. Am. Chem. Soc. 108: 4354-4360 (1986).
} 
aumento del momento dipolar con el aumento de las energías conformacionales (Figura 14.1).

El análisis NBO expuesto en el Capítulo 8 sugiere que, al menos para la serie de moléculas estudiadas, son las interacciones de orbitales de los pares libres del oxígeno en el grupo $\mathrm{P}-\mathrm{O}-\mathrm{C}$ con los orbitales antiligantes de enlaces $\mathrm{P}=\mathrm{X}$ ó $\mathrm{P}-\mathrm{O}$ las que explicarían la estabilización de los distintos confórmeros, mientras que las de repulsión entre pares libres y los orbitales $\pi$ del enlace $\mathrm{P}=\mathrm{X}$, las que determinarían las energías relativas de cada uno de ellos.

\section{Manifestaciones espectroscópicas de la variedad conformacional}

En las distintas espectroscopías utilizadas, se encontraron características que pudieron relacionarse directa o indirectamente a la comanifestación de confórmeros. Es indudable que la espectroscopía infrarroja en matrices de baja temperatura, medidas a distintas temperaturas de deposición, corresponde una metodología muy potente en este sentido. Mediante esta técnica y con el apoyo de la química teórica, es posible afirmar que se puede resolver completamente el problema conformacional para el tipo de moléculas estudiadas.

Sin embargo, en la literatura se encuentra abundante información respecto a la coexistencia conformacional, aun antes de la invención de la técnica de medida de espectros infrarrojos en matrices. Por esta razón es interesante un análisis de las características espectroscópicas que en otras técnicas den cuenta del fenómeno. En la espectroscopía vibracional, Raman e infrarrojo, pueden identificarse determinados modos vibracionales que actúan como "sensores" conformacionales, es decir, cuyo desdoblamiento es fuerte evidencia de coexistencia conformacional.

Entre estos sensores, los más relevantes serían el modo de estiramiento $\mathrm{P}=\mathrm{X}$, de alrededor de $1280 \mathrm{~cm}^{-1}$ para oxofosforados, $650 \mathrm{~cm}^{-1}$ para fosfotioatos y $500 \mathrm{~cm}^{-1}$ para fosfoselenoatos, el modo de estiramiento simétrico $(\mathrm{P}-\mathrm{O})_{3}$ ubicado en la región alrededor de $745 \mathrm{~cm}^{-1}$, el modo de estiramiento antisimétrico $(\mathrm{P}-\mathrm{O})_{3}$ ubicado en la región alrededor de $840 \mathrm{~cm}^{-1}$ y el modo de estiramiento (P)-O-C ubicado en los $1040 \mathrm{~cm}^{-1}$. 
Durante este trabajo se ha descrito una correlación entre el aumento de la complejidad de la estructura fina de las señales ${ }^{1} \mathrm{H}$ RMN con el aumento de la libertad conformacional de los OPs estudiados. Aunque hemos insistido con el hecho que la técnica de medida es muy "lenta" para "ver" cada confórmero en forma individual, la correlación es notable. No obstante, es necesario también evaluar la influencia del calcógeno en el grupo $\mathrm{P}=\mathrm{X}$, sobre las constantes de acoplamiento espín-espín de largo alcance, que implique una disminución de su magnitud en la medida del aumento del peso atómico de $\mathrm{X}$ y por lo tanto una menor resolución de las bandas en el espectro correspondiente.

\section{Descripción de propiedades emergentes del sitio activo de la AChE con relación a pequeños OPs}

Uno de los fenómenos interesantes que surge durante la interacción de ésteres fosfóricos con cierto grado de simetría, que implique algún grado de degeneración energética conformacional, es precisamente el hecho de la ruptura de los grados de degeneración energética durante la interacción específica de los inhibidores con el sitio activo (Figura 11.7). Sin embargo, esta ruptura implica también que la interpretación de las abundancias relativas de los confórmeros en los entornos inmediatos del sitio activo se modifica, esto es en la interfase entre el solvente y el sitio activo, cuando las interacciones aún no son específicas.

La variación más notable ocurre en el caso de moléculas con un alto grado de simetría, como lo son los calcogenofosfatos de trimetilo. En estas moléculas habrá una diferenciación energética en términos tanto de la posición relativa de los ángulos $\Psi$ en gauche o -gauche como de la rotación alrededor del eje determinado por el enlace $\mathrm{P}=\mathrm{X}$. De esta manera, aunque el confórmero $\mathrm{C}_{3}$ mantiene sus grados de degeneración, los confórmeros $\mathrm{C}_{1}$ y Cs los aumentan al doble (ver Tabla 2.II). Este hecho tiene como consecuencia un aumento en el momento dipolar ponderado que surge de la aplicación de la ecuación 1.a.

Un aumento en el momento dipolar neto (ponderado) en la interfase solvente/sitio activo podría funcionar como una fuerza impulsora que guíe a la molécula entrante, en un entorno hidrofóbico, hacia determinadas posiciones en donde las interacciones dipolo-dipolo sean relevantes y promuevan la formación de las interacciones específicas que den lugar a la formación del complejo

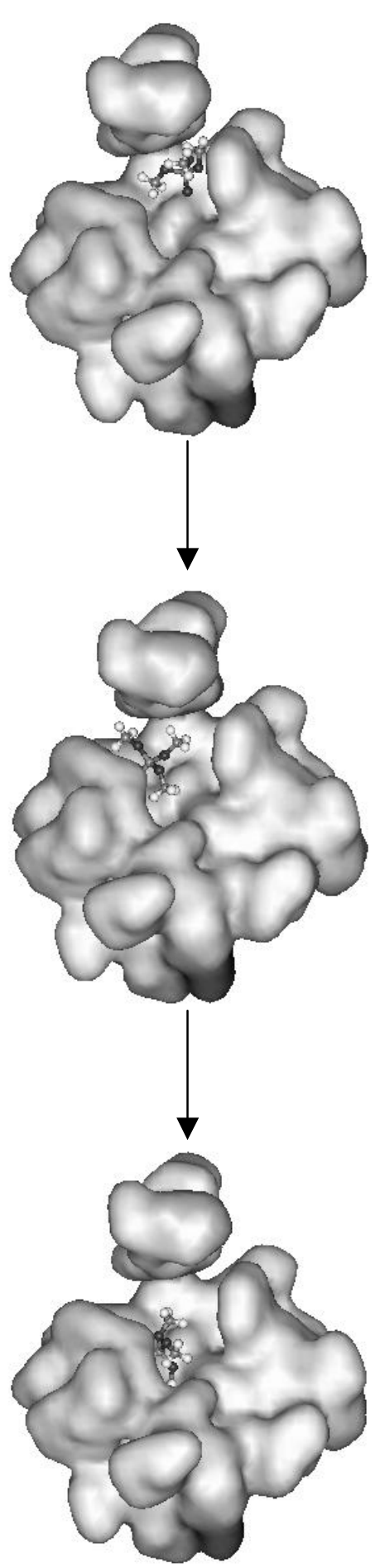

Figura 14.2: Ingreso del TMP a lo largo del canal hidrofóbico de la AChE, ubicándose sucesivamente en $\boldsymbol{P}_{\text {Hid }}, \boldsymbol{P}_{\text {Int }}$ y $\boldsymbol{P}_{\text {Ser200, }}$, según el modelo propuesto. 
activado. Ya se ha reconocido que las interacciones de tipo dipolo-dipolo son relevantes en la interacción de ciertos inhibidores con la $\mathrm{AChE}^{(2)}$

Aunque en esta reinterpretación de los grados de degeneración energética hay un razonamiento forzado, en el sentido de otorgarle al acercamiento a la interfase solvente/proteína un papel de "relectura" de las estructuras dinámicas de los ésteres fosfóricos, lo que si es cierto es que los fenómenos de interfase suelen ser de gran complejidad. En nuestro caso, acudimos a un modelo simplificado de estos fenómenos, que permite justificar un aumento de la afinidad de la molécula entrante por un aumento aparente del momento dipolar resultante, tanto mayor para moléculas con alto grado de simetría.

En un entorno no polar, como el del sitio activo de la AChE descrito como un "bolsillo hidrofóbico", la magnitud del momento dipolar de una molécula entrante debería cobrar relevancia y es necesario valorar la influencia de la variación del momento dipolar de la molécula del inhibidor durante su interacción con los restos del sitio activo. En el caso del TMP, en la medida que las restricciones estéricas lo permitan, es interesante verificar que la forma $\mathrm{C}_{3}$, de menor momento dipolar, es la que estabiliza en $\boldsymbol{P}_{\text {Ser200. De alguna }}$ manera, las restricciones estéricas no impedirían la minimización de las interacciones dipolo-dipolo.

En el sentido inverso, un aumento de las interacciones de este tipo, estabilizantes, tendería a favorecer la estabilización de confórmeros con momentos dipolares altos, propendiendo a la ubicación de los grupos salientes en la posición adecuada para la reacción de hidrólisis subsiguiente. En este sentido, el entorno no polar favorecería la estabilización de confórmeros que en solución acuosa no lo son y son estas formas las que a la vez permitirían una geometría más adecuada para el ataque nucleofílico. No obstante, en moléculas cuyas estructuras no favorecen los eventos de interacción que determinan la inhibición, el entorno no polar actuaría aumentando las interacciones hidrofóbicas que a su vez tenderían a aumentar la constante de afinidad y por ende, a disminuir aun más el poder inhibitorio de la molécula, tal como queda en evidencia en las correlaciones con descriptores de tipo hidrofóbico para moléculas de bajo poder inhibitorio.

${ }^{2}$ Delfini, M. et al. ${ }^{1} \mathrm{H}$ NMR Relaxation Investigation of Inhibitors Interacting with Torpedo californica Acetylcholinesterase, J.Magn.Reson. 144 (1): 129-133 (2000). 
En el transcurso del modelado del complejo enzimainhibidor, aparece otro resultado interesante: la evidencia de interacciones entre el fosfato entrante y ciertos restos aminoacídicos que determinarían restricciones estéricas (conformacionales) de las cadenas laterales. Dichos restos son la $\mathrm{Hys}^{440}$ (Figuras 11.4, 11.5, 11.7 y 11.8), que desempeña un papel en la tríada catalítica durante la transferencia de un protón desde $\mathrm{Ser}^{200}$ hasta $\mathrm{Glu}^{327}$, y la Glu $^{199}$ (Figuras 11.5 y 11.8), que corresponde al resto aminoacídico N-marginal a la $\operatorname{Ser}^{200}$, centro catalítico de la AChE. En la bibliografía que describe el papel de las estructuras del sitio activo de la AChE, no aparece mencionada la relevancia de estos restos aminoacídicos en la restricción estérica de sustratos e inhibidores. En este mismo sentido, la descripción de dos subsitios adicionales de estabilización energética, denominados aquí como $\boldsymbol{P}_{\text {int }}$ y $\boldsymbol{P}_{\text {Hid }}$, tampoco encuentran antecedentes en bibliografía. Resta bastante por verificar la real influencia de estos restos adicionales y la relevancia relativa de los dos subsitios accesorios, en términos de la dinámica de la inhibición o de catálisis de la AChE.

Tanto los grupos que verificarían interacciones dipolo-dipolo con sustratos e inhibidores, como los restos aminoacídicos y los subsitios adicionales propuestos, corresponden a propiedades estructurales intrínsecas del sitio activo. Estas surgen durante la interacción con moléculas entrantes. Todos estos elementos apuntan en el sentido de la hipótesis esbozada durante el comienzo de este trabajo que indicaba la "inversión" conformacional como una propiedad intrínseca del sitio activo de la AChE respecto de sus sustratos e inhibidores.

\section{Papel de la restricción conformacional en la disminuida capacidad inhibitoria de tio y selenofosforados}

Durante las primeras etapas del desarrollo de este trabajo, nos hemos empeñado en encontrar alguna relación directa entre la dispar libertad conformacional de los compuestos del tipo $\mathrm{P}=\mathrm{O}$ respecto a los compuestos $\mathrm{P}=\mathrm{S}$ o $\mathrm{P}=\mathrm{Se}$ con su capacidad como inhibidores. En este sentido, la correlación existente entre el aumento de las energías de interconversión conformacional de las moléculas libres en el orden $\mathrm{P}=\mathrm{O}<\mathrm{P}=\mathrm{S}<\mathrm{P}=\mathrm{Se}$, respecto de la disminución de la capacidad inhibitoria de la $\mathrm{AChE}$ en el orden $\mathrm{P}=\mathrm{O}>\mathrm{P}=\mathrm{S} \geq$ $\mathrm{P}=\mathrm{Se}$, aparece como una hipótesis de trabajo interesante que 
estimula la búsqueda de alguna relación causa-efecto en dicha correlación. ${ }^{(3)}$

Los datos obtenidos del modelado molecular no entregan ninguna evidencia contundente de una relación en ese sentido. Aún más, es notable el aumento de las energías calculadas de interacción enzima-inhibidor, que se encuentran muy por encima de las energías conformacionales de las moléculas libres. Por esta razón, se esperaría una pequeña influencia de las barreras rotacionales de los OPs calculadas en el vacío, sobre las restricciones conformacionales relevantes durante la interacción enzimainhibidor.

Sin embargo, son interesantes las correlaciones individuales existentes entre los descriptores conformacionales intrínsecos y las distintas constantes cinéticas estudiadas. Ellas indicarían que el aumento de la libertad conformacional de las moléculas individuales favorece una disminución de $\mathrm{Ka}$ y un aumento de $\mathrm{kp}$ y $\mathrm{Ki}$ (ver Tabla 13.IX), es decir, un aumento de las capacidades como inhibidor de un OP.

Es posible asignar otra relación para la serie de moléculas, que surge de los estudios de interacción: hemos descrito que la posición de mayor estabilidad para cada una de las tres moléculas simétricas utilizadas como modelo, TMP, TMSP y TMSeP, se encuentra desplazada en la medida que el respectivo grupo $\mathrm{P}=\mathrm{X}$, sucesivamente más voluminoso, busca la posición de mínima interacción con el entorno inmediato. Esto es asi tanto para la ubicación de estabilidad definida como $\boldsymbol{P}_{\boldsymbol{S} e r \mathbf{2 0 0}}$, como para las posiciones $\boldsymbol{P}_{\text {int }}$ y $\boldsymbol{P}_{\text {Hid. }}$. Este desplazamiento implica que las estructuras que esterifican al fosfato se ubicarán en entornos levemente diferentes $\mathrm{y}$ por lo tanto con restricciones estéricas (conformacionales) distintas. De esta manera, existe la posibilidad que el enfrentamiento diferencial de las cadenas laterales de un OP, que dependería del ser oxofosforado, tiofosforado o selenofosforado, condicione la interacción, desfavoreciendo en los dos últimos casos la formación del complejo intermediario y del adecuado ataque nucleofílico por parte de la $\operatorname{Ser}^{200}$ al átomo de fósforo.

\footnotetext{
${ }^{3}$ Esta hipótesis se encuentra apenas esbozada durante la discusión de los resultados del estudio conformacional comparativo del TMP, TMSP y TMSeP: Mastrantonio,G. and Della Védova, C.O. Spectroscopic and conformational comparative study of trimethyl chalcogenphosphates. J.Mol.Struc. 561:161-174 (2001).
} 
En forma generalizada, para inhibidores directos e indirectos, existirían entonces interacciones diferentes tanto de los restos del tipo metilo o etilo, como de los grupos salientes, que podrían ser importantes en determinar las diferencias de magnitud en su $\mathrm{Ki}$, fundamentalmente por influencias sobre Ka. En forma clásica, se asigna a la disminución de la electrofilicidad de fosfotioatos y fosfoselenoatos la causa de la menor capacidad inhibitoria de éstos. Las hipótesis alternativas propuestas bien puede corresponder explicaciones complementarias, más no contradictorias.

Para corroborar o descartar estas conjeturas, se necesitan estudios de dinámica de proteínas, además de la adecuada resolución estructural, mediante difracción de RX por ejemplo, de complejos enzima-inhibidor para inhibidores del tipo $\mathrm{P}=\mathrm{S}$ y $\mathrm{P}=\mathrm{Se}$. Hasta el día de hoy sólo se han resuelto estructuras para inhibidores fuertes del tipo $-\mathrm{O}-\mathrm{P}=\mathrm{O}$ y $-\mathrm{S}-$ $\mathrm{P}=\mathrm{O}$.

\section{Comportamiento conformacional del TMP como justificación de su pobre poder inhibitorio}

La medida de las constantes de inhibición del TMP para AChE da cuenta de una reducida capacidad de inhibición, aun siendo un oxofosforado. Más aún, se determinó que sus propiedades como inhibidor son comparables a un fosfotioato, el DMATP. La posibilidad de justificar tal comportamiento puede abordarse mediante dos hipótesis medianamente esbozadas durante la exposición de estos fenómenos. Estas hipótesis no son excluyentes, de manera que sin riesgo a contradicción, ambas pueden ser distintos aspectos de la explicación de la magnitud de dichos valores cinéticos.

Según los resultados de modelado del TMP en $\boldsymbol{P}_{\text {Ser200, hemos descrito que la forma conformacional más }}$ estable para esta molécula corresponde a una de las posiciones -gauche, -gauche, -gauche (Tabla 11.IV) y lo mismo ocurre para los análogos azufrado y selenado (Tabla 11.V). Por efecto de la ruptura de la degeneración energética conformacional (Figura 11.7), el confórmero enantiomérico del TMP ubicado en $\boldsymbol{P}_{\text {Ser200, se encuentra en un nivel de }}$ energía muy superior.

Este comportamiento es diferente a lo descrito para fuertes inhibidores, en los cuales el grupo saliente se ubica en 
posición trans respecto al grupo fosforilo. Esto parece ser válido tanto para grupos salientes voluminosos ${ }^{(4)}$ como para pequeñas cadenas laterales. ${ }^{(5)}$

Aunque se puede relacionar el pobre poder de inhibición del TMP en términos de la constante de hidrólisis alcalina del grupo saliente $-\mathrm{O}-\mathrm{CH}_{3}$, este argumento sólo es suficiente para justificar adecuadamente el valor de la constante de fosforilación $\mathrm{kp}$, calculada en $6 \times 10^{-2}$, uno o dos órdenes de magnitud por debajo de un inhibidor fuerte y algo mayor a los valores típicos de los inhibidores indirectos. Parece evidente la relación entre esta estabilización conformacional, con la magnitud de Ka. Una de las cuestiones que puede aportar en este sentido es el hecho que las posiciones trans y gauche predisponen a la nube electrónica alrededor del átomo de fósforo de una manera distinta para eventuales reacciones de la molécula.

El efecto gauche, como caso particular del efecto anomérico, ha sido descrito como una redistribución de los orbitales sobre el grupo involucrado que determina una mayor o menor reactividad de las moléculas. Se ha propuesto para la ribonucleasa A, que cataliza la degradación del RNA por hidrólisis de los enlaces éster fosfórico, un mecanismo en el cual el sitio activo fija la conformación trans-gauche de los dos sustituyentes alrededor del fósforo, excluyendo la posibilidad del confórmero gauche-gauche, aumentando de esta manera la basicidad del grupo saliente. ${ }^{(6)}$ Se ha dicho que la enzima obtiene grandes ventajas de las consecuencias estereolectrónicas de las conformaciones en trans de los ésteres fosfóricos.

Conociendo acerca de la lógica de la naturaleza de los procesos bioquímicos, mediante la cual se establecen una variedad enorme de procesos a partir de unos pocos principios iniciales, es natural asociar un mecanismo similar para la inhibición de la AChE por parte de los OPs: la ubicación en posición trans del grupo saliente,

\footnotetext{
${ }^{4}$ Hirashima, A., Kuwano, E. and Eto, M. Docking study of enantiomeric fonofos oxon bound to the active site of Torpedo californica acetylcholinesterase, Bioorg.Med.Chem. 8(3):653-656 (2000).

${ }^{5}$ En nuestros estudios preliminares sobre la interacción del DDVP y el metamidofós con el sitio activo de la $\mathrm{AChE}$ de anguila, los respectivos ángulos del grupo saliente son y .

${ }^{6}$ Gorenstein, D.G. et al. Stereolectronic control in carbon-oxygen and phosphorus-oxygen bond breaking processes. Ab initio calculations and speculations on the mechanism of action of ribonuclease A, staphylococcal nuclease, and lysozyme, J.Am.Chem.Soc. 99:3473-3479 (1977).
} 
correspondería, respecto a la posición gauche, a la mejor ubicación de los orbitales electrónicos alrededor del átomo de fósforo para el consecuente ataque nucleofílico por parte del oxígeno serínico. En este tópico es interesante notar la gran capacidad inhibitoria de los compuestos del tipo P-S-R, en donde $-\mathrm{S}-\mathrm{R}$ se constituye en el grupo saliente.

De hecho, los dos órdenes de magnitud de diferencia entre los $\mathrm{kp}$ del DMAP respecto al metamidofós están determinados fundamentalmente por los tres órdenes de diferencia es sus respectivos Ka. Ya hemos mencionado que cualquiera sea el grupo saliente $-\mathrm{O}-\mathrm{CH}_{3}$ del DMAP, presentan ambos una posición preferencial en gauche (Capítulo 6), mientras que el grupo $-\mathrm{S}-\mathrm{CH}_{3}$, propuesto como grupo saliente para el metamidofós presenta una posición preferencial en trans. $^{(7)}$

El TMP presenta tres sustituyentes de pequeño tamaño y cada uno con un pk de hidrólisis alcalina muy bajo. De esta manera, las restricciones estéricas que aparecen en el reducido espacio en el entorno de la $\mathrm{Ser}^{200}$, no son suficientes para forzar a alguno de ellos, el ubicado hacia "afuera" del bolsillo hidrofóbico, hasta la posición trans. Por esta razón, la molécula se estabiliza en posición -gauche,-gauche,-gauche que no sería las más apropiada para el ataque nucleofílico, estabilizándose en un estado previo a la constitución del transiente pentacoordinado. De allí su muy baja capacidad de inhibición de la AChE y otras esterasas.

En la Tabla 11.IV es interesante verificar que el confórmero más estable para el TMP en posición $\boldsymbol{P}_{\boldsymbol{i n t}}$, sea trans, gauche, -gauche, es decir, correspondiente al confórmero Cs, estable de mayor energía según los cálculos en el vacío (Tabla 2.III). Dicha conformación podría corresponder entonces a un estado activado conformacional previo a la ubicación del grupo saliente en trans en posición $\boldsymbol{P}_{\text {Ser200 }}$ para inhibidores del tipo $\mathrm{P}=\mathrm{O}$. Sin embargo, el ingreso al entorno inmediato a $\operatorname{Ser}^{200}$, aumentará su libertad conformacional y tenderá a volver a una conformación gauche,-gauche, -gauche, menos reactiva.

Ya hemos descrito a $\boldsymbol{P}_{\text {int }}$ como un "cuello de botella" por la existencia de una mayor restricción conformacional y la imposibilidad de estabilizar teóricamente

\footnotetext{
7 Solov'ev V.N., Martynov I.V., Zabirov N.G. Crystal and molecular structure of two insecticides: O,S-dimethyl amidothiophosphate and O,Sdimethyl acetamidothiophosphate, Phosphorus, Sulfur Silicon.Relat.Elem. 57 (1-2): 135-141 (1991).
} 
a moléculas $\mathrm{P}=\mathrm{Se}$ en la estructura de la enzima nativa, debiendo sufrir entonces, en este punto, algún tipo de desplazamiento estructural que a su vez podría ser funcional a los siguientes eventos de interacción molecular.

También es notable que la estabilización de TMSP en $\boldsymbol{P}_{\text {int }}$ ocurra en una conformación -gauche, gauche, -gauche (Tabla 11.V). Esto significa que el voluminoso grupo $\mathrm{P}=\mathrm{S}$ desplaza a la molécula hacia posiciones tales que los grupos metilo no sufren las mismas restricciones conformacionales de su análogo oxigenado. De manera que ni el paso siguiente hacia la formación del complejo activado enzima-inhibidor, ni los hipotéticos cambios conformacionales de la proteína ocurrirán de la misma manera y eventualmente son hechos que también determinan el aumento de $\mathrm{Ka}$ y la disminución de kp para moléculas del tipo $\mathrm{P}=\mathrm{S}$ y $\mathrm{P}=\mathrm{Se}$, respecto a los respectivas moléculas del tipo $\mathrm{P}=\mathrm{O}$.

En este razonamiento, es necesario considerar que tanto el pk del grupo saliente como la posición en trans del mismo son influencias concurrentes y de cierta independencia. En este sentido, la actividad anticolinesterasa de moléculas del tipo $\left(\mathrm{CH}_{3} \mathrm{O}\right)\left(\mathrm{CH}_{3-\mathrm{n}} \mathrm{F}_{\mathrm{n}} \mathrm{O}\right) \mathrm{P}=\mathrm{O}$, de constantes de hidrólisis alcalinas crecientes con $n$, pero con libertades conformacionales similares al TMP, deberían presentar $\mathrm{kp}$ crecientes pero Ka similares entre sí y al mismo TMP.

\section{Activación in situ de pequeños tiofosforados}

Ya hemos descrito como durante las medidas de inhibición de la actividad AChE por parte de dos moléculas: TMSP y TMSeP, se obtuvieron valores cinéticos calificados como constantes cinéticas "aparentes". Dichos valores, se caracterizan por corresponder a actividades inhibitorias mucho mayores a las esperadas para estas sustancias, respecto a la medida para TMP. También se ha reportado que el fosfotioato de O,O,O-trietilo presenta una constante de inhibición anormalmente alta, respecto a su análogo oxigenado.

La explicación más directa de este comportamiento surge de la posibilidad de un reordenamiento molecular que de lugar a la aparición de estructuras del tipo $-\mathrm{X}-\mathrm{P}=\mathrm{O}$. Este proceso podría ocurrir en el seno del solvente acuoso, en la interfase solvente proteína o en el entorno inmediato al sitio activo. Dicho reordenamiento se puede concebir sencillamente como una isomerización o, como ya se ha 
propuesto para fosfotioatos, como un proceso complejo de oxidación parcial que implica a estructuras intermediarias del tipo $\mathrm{P}=\mathrm{S}_{\mathrm{n}} \cdot{ }^{(8)}$

En este sentido, el uso de los $\mathrm{QSAR}^{\mathrm{C}}$ para estimar las correspondientes constantes cinéticas del TMSP como del TMSeP y de dos de sus isómeros propuestos como las estructuras de interacción, sugieren que las constantes cinéticas experimentales "aparentes" son compatibles con estos últimos, antes que con las estructuras O,O,O-trimetil sustituidas. Considerando que la interacción estudiada ocurre con pequeñas moléculas, no queda descartada la hipótesis, muy atractiva, que indicaría que en el entorno del sitio activo ocurran procesos de reordenamiento molecular que den lugar a la aparición in situ de estructuras de mayor poder inhibitorio.

Más aún, el hecho que el TMP y el DMATP presenten valores cinéticos de inhibición comparables, puede deberse a que el mismo DMATP sufra algún proceso análogo de manera que los valores medidos para esta sustancia también sean "aparentes", por lo tanto algo mayores que los esperados y comparables a los medidos para TMP. Pero en este caso no hay sustento en los $\mathrm{QSAR}^{\mathrm{C}}$, puesto que tanto el TMP como el DMATP mantienen los patrones de regularidad de los mismos.

En realidad, esta descripción de hechos no vincula necesariamente los resultados entregados con una hipotética activación in situ de los inhibidores indirectos. Tal como se presenta, correspondería a un fenómeno anómalo en el contexto de lo ya conocido de los procesos dinámicos de la interacción de la AChE con sus sustratos e inhibidores. Pero no cabe duda que la cuestión no puede responderse con la cantidad de información actualmente acumulada ni con los datos que se presentan a lo largo de este trabajo.

De hecho, tampoco esta claro que el fenómeno descrito para el TMSP y el TMSeP ocurra con la participación de las estructuras proteicas de la AChE. El diseño experimental propuesto en las páginas precedentes no apunta a resolver esta cuestión. Aun no hay suficiente información acerca del comportamiento de estas $u$ otras sustancias similares en soluciones acuosas.

\footnotetext{
${ }^{8}$ Swinson J. et al. Thiono compounds. X. Intermediates from the oxidation of phosphorothioates, Phosphorus,Sulfur.Silicon.Relat.Elem. 44 (1-2): 39-52 (1989).
} 
Pero la complejidad de las estructuras participantes del bolsillo hodrofóbico y la propuesta de sitios de unión estable alternativos, $\boldsymbol{P}_{\text {int }}$ y $\mathbf{P}_{\text {Hid }}$, estimulan las conjeturas respecto del papel funcional que puedan tener estos $\mathrm{u}$ otros sitios del entorno del resto reactivo de la $\mathrm{Ser}^{200}$, en diferentes procesos dinámicos previos a la formación del complejo pentacoordinado.

\section{Aportes al diseño de variantes de la $\mathrm{AChE}$}

Hay ciertos elementos que surgen del estudio, tanto de los inhibidores en forma independiente, como en el contexto de la interacción con las estructuras, que pueden utilizarse en un camino inverso, es decir, en el diseño racional de nuevas enzimas.

Una de las enzimas utilizadas en las determinaciones de las constantes cinéticas del TMP corresponde a una variante estructural de la $\mathrm{AChE}$ nativa de mosca drosófila. Dicha variante es parte de la gran colección de mutantes de AChE que se han sintetizado en la búsqueda de enzimas con propiedades de hipersensibilidad a la inhibición por distintos OPs ${ }^{(9)}$ y corresponde a uno de los intentos de contar con una variante hipersensible al metamidofós, esfuerzo que aún no cuenta con logros importantes. ${ }^{(10)}$ Estas variantes hipersensibles permiten el desarrollo de biosensores de mayor sensibilidad respecto a los construidos con enzimas nativas o con sensibilidades normales a los OPs. ${ }^{(11)}$

Algunas de las dificultades en el diseño de mutantes hipersensibles surgen en parte por el hecho que el mecanismo de interacción íntimo de este pesticida con la $\mathrm{AChE}$ aún no se encuentra totalmente dilucidado y, por otro lado, siendo una molécula de pequeño tamaño, existen menos opciones de variación de la estructura de la proteína respecto a los restos

9 (a) Villatte, F. et al. Engineering sensitive acetylcholinesterase for detection of organophosphate and carbamate insecticides, Biosens. Bioelectron. 13 (2): 157-164 (1998). (b) Boublik, Y. et al. Acetylcholinesterase engineering for detection of insecticide residues, Protein.Eng. 15 (1): 101-108 (2001).

${ }^{10}$ Comunicación personal de Didie Fournier, director del grupo de trabajo de síntesis de biomoléculas, Universidad de Toulousse.

11 (a) Bachmann, T.T. et al. Improved multianalyte detection of organophosphates and carbamates with disposable multielectrode biosensors using recombinant mutants of Drosophila acetylcholinesterase and artificial neural networks, Biosens.Bioelectron. 15 (3-4): 193-201 (2000). (b) Danet, A.F. et al. Flow analysis for determination of paraoxon with use of immobilized acetylcholinesterase reactor and new type of chemiluminescent reaction, Biopolymers. 57 (1): 37-42 (2000). 
que tomen contacto con el inhibidor de manera de promover un mecanismo de interacción más eficiente. La introducción de $\mathbf{L}$ como parámetro de estimación de las constantes cinéticas de inhibición, que aparentemente tendría una cierta importancia por lo menos para inhibidores de pequeño tamaño, podría aportar en el diseño racional de variantes de AChE, minimizando los caminos posibles a adoptar en la búsqueda de enzimas hipersensibles a este tipo de sustancias.

Nosotros hemos abordado apenas tangencialmente el problema y se han hecho algunos comentarios respecto a las diferencias cinéticas entre variantes nativas y mutantes observadas para el TMP, pero sin duda, este es una vía abierta de futuros estudios con frutos promisorios.

\section{Propiedades conformacionales como descriptores "4D" en la constitución de QSAR}

La libertad conformacional ${ }^{\mathbf{C}} \mathbf{L}$ y su contraparte, la restricción conformacional ${ }^{\mathbf{C}} \mathbf{R}$, han sido definidas aquí como descriptores moleculares contextuales "4D". Contextuales, porque no surgen como una propiedad molecular intrínseca, sino que son calculados a partir de las distintas posibilidades de interacción de los confórmeros en el contexto de ciertas posiciones en el sitio activo de una proteína, en este caso AChE de anguila eléctrica. $Y$ puede ser definida perfectamente como descriptor “4D" porque se está cuantificando una propiedad molecular que no depende solamente de una cierta estructura tridimensional, sino que además de las distintas posibilidades de interacción en función de la variabilidad conformacional de la molécula.

Una propiedad relevante es la capacidad de estos descriptores de poder distinguir perfectamente a los isómeros ópticos entre sí, propiedad invalorable a la hora de construir correlaciones cuantitativas estructura-actividad. Esto es así dado que los eventos que determinan la actividad biológica se dan en contextos en donde las dianas moleculares son asimétricas (sitios activos, receptores, canales de membrana, etc.). En nuestro caso ejemplificamos esta capacidad en los descriptores para las formas $\mathrm{R}$ y S del metamidofós (Tabla 13.).

Sin embargo, una dificultad propia de la definición de los parámetros conformacionales contextuales es la necesidad de conocer perfectamente la estructura tridimensional de la diana molecular que determina el 
mecanismos de acción del grupo de moléculas sobre las que se pretende construir el QSAR. Pero, al mismo tiempo, dado que ${ }^{{ }^{C}} \mathbf{L}$ es un descriptor que surge desde el contexto de la interacción que determina el mecanismo de actividad biológica, aparece interesante en términos de su potencia para cuantificar muy adecuadamente dicha actividad.

Por otro lado, en nuestro caso, nos limitamos a la construcción de $\mathbf{L}$ en función de tres grados de libertad, cada uno de los ángulos $\Psi$. El uso de más grados de libertad necesita de una capacidad de cómputo muy importante. De hecho, el cálculo del parámetro $\mathbf{L}$ para el acefato, con cuatro grados de libertad no nos fue posible con la capacidad de cálculo disponible y no pudo ser incluida en la estimación de la correlación propuesta.

Nuestro intento de construir QSARs que correlacionaran con un cierto ajuste las distintas constantes cinéticas de la serie de OPs propuestos, tiene su mayor deficiencia en el número de moléculas de la serie. Uno de los criterios para otorgar a un QSAR un cierto carácter de generalizable es el de construirse a partir de un mínimo de ocho moléculas. ${ }^{(12)}$ En este trabajo utilizamos cinco. Será necesario el trabajo con series de OPs más grandes o ampliar la ya propuesta para validar o modificar las correlaciones encontradas.

Sin embargo, una de las propiedades interesantes que sugiere el análisis $\mathrm{QSAR}^{\mathrm{C}}$ a partir de la serie de OPs es que tanto la variabilidad conformacional de las moléculas individuales como las restricciones conformacionales impuestas por el estrecho bolsillo hidrofóbico del sitio activo serían factores críticos en el sistema. Este, sin duda, es un aporte importante a la discusión acerca de los mecanismos de interacción de OPs con esterasas.

Finalmente, existe la posibilidad de ampliar el campo de aplicación de los descriptores conformacionales, tal como han sido aquí descritos. Otros sistemas de interacción biológica en donde ${ }^{\mathbf{I}} \mathbf{L}$ o ${ }^{\mathbf{C}_{\mathbf{L}}}$ puedan corresponder a descriptores críticos en la estimación de las correlaciones estructura actividad, deberían cumplir con dos propiedades: (a) suficiente variabilidad conformacional y (b) que esta variabilidad determine directa $o$ indirectamente sus propiedades de acción biológica. Además existen dos

\footnotetext{
${ }^{12}$ Hansch,C. and Fujita,T. Status of QSAR at the end of the twentieth century. Chapter 1:1-12. On: Classical and three-dimensional QSAR in agrochemistry. American Chemical Society eds (1995).
} 
limitantes prácticas para esta aplicación: (c) se necesita de cierto conocimiento de la estructura molecular de la diana molecular y (d) es necesaria una capacidad de cómputo suficiente para el cálculo de $\mathbf{L}$, en función de los grados de libertad necesarios.

Algunos grupos de sustancias que puede cumplir en mayor o menor medida las condiciones enumeradas son los hidrocarburos alifáticos halógeno sustituidos y su capacidad de producir inhibición de la función nerviosa por su acción sobre células neuronales, las sustancias peptidomiméticas de Phe-Ala-Pro que actúan como inhibidores de la enzima conversora de angiotensina (ECA), para la cual no ha sido descrita aún su estructura tridimensional ni las características del sitio activo, ${ }^{(13)}$ y los carbamatos que, aunque con menos libertad conformacional, también actúan como inhibidores de la AChE. Se han realizado interesantes intentos de relacionar las variantes estructurales en todo el espacio conformacional con descriptores estéricos clásicos para sustancias con actividad $\beta$-bloqueante; ${ }^{(14)}$ este grupo de sustancias también aparece como una interesante oportunidad de aplicar el concepto de descriptor conformacional, respecto de sus propiedades farmacológicas.

Para todos estos grupos de sustancias puede resultar interesante la búsqueda de $\mathrm{QSAR}^{\mathrm{C}}$, como forma de validar a los descriptores conformacionales como herramientas generales de análisis de la actividad biológica $\mathrm{y}$, al mismo tiempo, perfeccionar las ecuaciones con las que aquí los hemos definido. Pensamos que, en la medida que este tipo de descriptores pueda ser utilizado con cierto éxito en distintos sistemas, puede llegar a constituirse en el aporte más significativo de este trabajo.

\footnotetext{
13 Zamarbide, G.N. Isomerismo cis-trans de enlaces peptídicos en pequeños compuestos, modelos de péptido miméticos conformacionalmente flexibles, usados como inhibidores de la ECA. Tesis Doctoral, Universidad Nacional de San Luis, Fac. Quim. Bioq. y Farm. (1999).

14 Krarup, L.H., Christensen, I.T., Hovgaard, L. and Frokjaer, S. Predicting drug absorption from molecular surface properties based on molecular dynamic simulations, Pharm. Res. 15(7): 972-978 (1998).
} 


\title{
Material y métodos
}

El desarrollo del trabajo expuesto se realizó, durante el transcurso de cuatro años. La mayoría de las metodologías utilizadas en los aspectos teóricos y experimentales durante este período dependieron fundamentalmente de la instrumentación disponible que además fuese adecuada al tipo de sustancias objeto de estudio.

Aunque se trabajó con distintos procedimientos de síntesis y purificación, se seleccionaron finalmente aquellas con las cuales se obtuvieron sustancias en suficiente cantidad y pureza. Las mayores dificultades encontradas en este aspecto se debieron a la baja presión de vapor de las sustancias obtenidas y la tendencia de las mismas a la oxidación, isomerización y polimerización. De manera que, aunque los estudios se iniciaron con la previsión de algo más de una decena de sustancias, finalmente se trabajó con solamente siete.

Se dispuso de distintas técnicas espectroscópicas, tanto para el análisis estructural, tanto como para el seguimiento de procedimientos de síntesis y verificación de identidad. Distintas metodologías cromatográficas se utilizaron con fines preparativos y separativos, útiles además para la verificación de identidad y pureza.

El aspecto biológico se abordó mediante la aplicación de distintas metodologías cinéticas. Muchas de ellas finalmente no se adecuaron a los fines perseguidos, siendo necesario el desarrollo de una metodología que corresponde a una modificación de un modelo ya reportado. Esta modificación se describe en el Capítulo 12, pero el desempeño de la metodología se describe en la presente sección.

Finalmente, los cálculos teóricos adquirieron un papel vinculante de cada uno de los aspectos químicos y biológicos del planteo general. El software utilizado tanto en este aspecto como en el modelado gráfico de las moléculas se describe adecuadamente. Se describe el software complementario de cálculo y gráfica utilizado.

Los equipos utilizados en las diferentes medidas fueron los siguientes:

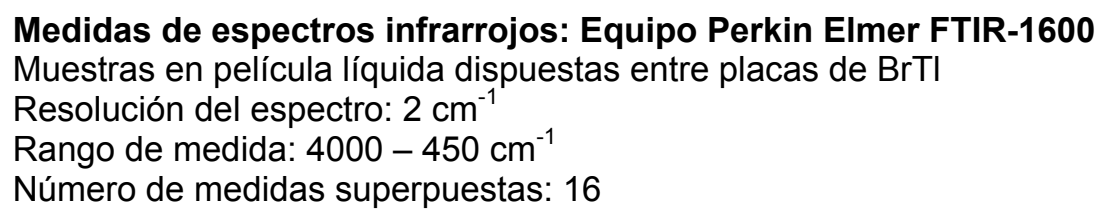

Medidas de espectros infrarrojos: Equipo Perkin Elmer FTIR-1600

Muestras en película líquida dispuestas entre placas de $\mathrm{BrTI}$

Resolución del espectro: $2 \mathrm{~cm}^{-1}$

Rango de medida: $4000-450 \mathrm{~cm}^{-1}$

Número de medidas superpuestas: 16

Medidas de espectros infrarrojos: Equipo FTIR Bruker IFS 66

Muestras en película líquida dispuestas entre placas de $\mathrm{BrTI}$

Resolución del espectro: $1 \mathrm{~cm}^{-1}$

Rango de medida: $4000-450 \mathrm{~cm}^{-1}$

Número de medidas superpuestas: 16

\author{
Medidas de espectros Raman \\ Muestras en capilares de vidrio sellados al vacío \\ Láser monocromático de 457,9 nm \\ Potencia en salida: $10 \mathrm{~mW}$ \\ Tiempo de integración: 0,5 segundos \\ Resolución de espectro: $5 \mathrm{~cm}^{-1}$ \\ Rango de medida: $4000-250 \mathrm{~cm}^{-1}$
}




\title{
Medidas de espectros infrarrojo en matrices: Espectrómetro Bruker IFS 66v \\ Fourier
}

Rango de medida: 4000 y $400 \mathrm{~cm}^{-1}$

Resolución del espectro: $1 \mathrm{~cm}^{-1}$

Proporción a la matriz de Ar: 1:1000

Temperatura de la matriz: $15 \mathrm{~K}$

Medidas de espectros RMN:Equipo Bruker AC 250-E

Solvente: $\quad \mathrm{CCID}_{3}$ (Aldrich Chemical Co. Ltda.) más 0,03\% v/v tetrametilsilano $\mathrm{OD}_{2}$ sin patrón de referencia

Temperatura de medida: constante a $21^{\circ} \mathrm{C}$

\author{
Medidas de Cromatografía Gaseosa: Equipo Perkin Elmer GC Autosystem \\ Carrier aire seco a $200 \mathrm{kPa}, 100 \mathrm{ml} / \mathrm{min}$ \\ Hidrógeno $200 \mathrm{kPa}, 1,5 \mathrm{ml} / \mathrm{min}$ \\ Columna BD5, $30 \mathrm{mts}$
}

Medidas de Espectroscopía Visible: Equipo Perkin Elmer Lambda 11/Bio

Espectrofotómetro UV/visible

Lámpara halógena $900-326 \mathrm{~nm}$

Temperatura constante a $24 \pm 1^{\circ} \mathrm{C}$

\section{Métodos espectroscópicos}

Como metodologías de identificación se cuenta con la espectroscopía FT-IR, rápida, muy específica, pero con inconvenientes para constituirse en técnica de cuantificación en el caso del tipo de sustancias de este estudio. En muchos casos también resulta de interés la espectroscopía Raman de la que existen también muchas aplicaciones tecnológicas.

Con fines identificatorios complementarios se recurre a la espectroscopía de resonancia magnética nuclear $(\mathrm{RMN})$, que tiene especial utilidad cuando se trata de la síntesis de nuevos compuestos, de los cuales no se cuenta con referencias previas. Las identidades y grados de pureza fueron evaluados con cierta sensibilidad mediante resonancia magnética nuclear de hidrógenos $\left({ }^{1} \mathrm{H} \mathrm{RMN}\right)$ y de carbonos $\left({ }^{13} \mathrm{C} \mathrm{RMN}\right)$.

El seguimiento de las reacciones se realiza mediante la determinación de los espectros IR de la mezcla de reacción en distintos tiempos, comparando con el espectro IR del reactivo de partida y procurando identificar el momento de aparición de bandas típicas de los compuestos que se quiere obtener.

En las medidas de los espectros de matrices se encontraron dificultades para la obtención de espectros adecuados en los fosfamidatos por su baja presión de vapor, con la consecuencia de espectros con una baja relación señal/ruido. Las matrices de Ar fueron realizadas mediante un sistema criogénico. La mezcla fue depositada en un espejo metálico enfriado a $15 \mathrm{~K}$ aplicando la técnica de deposición continua.

Para la cuantificación y evaluación de pureza, las distintas metodologías cromatográficas constituyen los métodos de elección por su sencillez y exactitud. Se utilizaron fundamentalmente cromatografías planar (TLC) y gaseosa (CG). En el primero de los casos con límites de detección bastante altos pero sumamente útil para el seguimiento de reacciones y procedimientos separativos de sustancias puras.

La TLC se constituyó en una técnica muy versátil y sencilla para el seguimiento de reacciones de síntesis y cromatografías preparativas. Para la obtención de un sistema adecuado de revelado se recurrió a distintas metodologías reportadas para encontrar la que mejor se ajustara a los requerimientos del trabajo. Las TLC se realizaron con el uso de cromatofolios de silicagel en soporte de aluminio sin indicador fluorescente. Como revelador universal se utilizó $\mathrm{I}_{2}$ por sublimación a $30^{\circ} \mathrm{C}$. 
En el caso de la CG, se contó con la valiosa ayuda de un detector específico para compuestos OPs y nitrogenados (detector nitrógeno fósforo, DNP), lo que significó la disposición de una poderosa herramienta para el desarrollo de técnicas extremadamente sensibles a los compuestos objetos de estudio. La CG/ DNP entregó un rango de variación en los tiempos de retención de $\pm 0,054$ segundos, a un nivel del $99 \%$ de confianza, lo que la hizo muy adecuada para la evaluación de la identidad.

\section{$\underline{\text { Síntesis, purificación y caracterización de sustancias }}$}

\section{Caracterización del fosfato de trimetilo (TMP).}

Se dispuso de TMP comercial que se utiliza sin purificación previa. El control de la pureza se realiza mediante medidas en CG/DNP. Las condiciones utilizadas fueron: inyección de $1 \mu \mathrm{l}$ de solución al $10 \%$ en diclorometano, temperatura inyección $250^{\circ} \mathrm{C}$, columna a $50^{\circ} \mathrm{C}$ con rampa $8^{\circ} \mathrm{C} /$ minuto, temperatura inyector $300^{\circ} \mathrm{C}$.

La identidad y pureza del producto final se investiga por cromatografía planar en silicagel (solvente hexano/acetona (7:3), revelado con vapores de yodo), CG/DNP y ${ }^{1} \mathrm{H}$ NMR.

\section{Síntesis y purificación de fostatotioato de O,O,O-trimetilo (TMSP).}

El TMSP se obtiene mediante una reacción entre azufre elemental y fosfito de trimetilo ${ }^{(1)}$. El procedimiento implica la adición de 4 gramos de azufre sólido (Merck técnico) desecado $100^{\circ} \mathrm{C}$ durante una hora, se disuelven en $20 \mathrm{ml}$ de disulfuro de carbono en un balón de $100 \mathrm{ml}$. Se agregan lentamente 16 gramos de fosfito de trimetilo (Fluka art. $9273097 \%$ GC). La reacción ocurre primero muy rápidamente con intensa ebullición, para después proseguir sin ebullición. La mezcla se calienta luego hasta $50-60^{\circ} \mathrm{C}$ procurando la disolución completa del azufre y luego hasta $150^{\circ} \mathrm{C}$ en baño de aceite hasta desaparición de los sedimentos de azufre. Se mantiene la mezcla a temperatura ambiente durante 72 horas. Se destila a $20 \mathrm{mmHg}$ para la obtención de una fracción a $34-36^{\circ} \mathrm{C}$.

La identidad y pureza del producto final se investiga por cromatografía planar en silicagel (solvente hexano/acetona, 7:3), CG/DNP y ${ }^{1} \mathrm{H}$ NMR.

Condiciones CG/NPD: inyección de $1 \mu \mathrm{l}$ de solución al $1 \%$ en acetona, temperatura inyección $250^{\circ} \mathrm{C}$, a $50^{\circ} \mathrm{C} 3$ minutos, luego rampa $5^{\circ} \mathrm{C} /$ minuto hasta $270^{\circ} \mathrm{C}$, temperatura inyector $300^{\circ} \mathrm{C}$. Tiempo de retención en columna de 30 metros $6,1 \pm 0,1$ minutos.

\section{Síntesis y purificación de fosofoselenoato de O,O,O-trimetilo (TMSeP)}

El TMSeP se obtiene mediante reacción de adición de selenio ${ }^{(2)}$ al fosfito de trimetilo. Se obtiene por lenta adición a temperatura ambiente, dado que la reacción es enérgica, de 1,2 gramos de selenio metálico (Carlo Erba-N25 $>99 \%$ ), desecado a $150^{\circ} \mathrm{C}$ durante 30 minutos, a 1,6 mililitros de fosfito de trimetilo, eliminándose los residuos sólidos remanentes mediante filtrado con papel en lecho de florisil.

La purificación se realiza mediante dos procedimientos: mediante columna de silicagel (Merk G-9385) con el sistema solvente hexano/acetona (7:3) de la que se separa la primera fracción de 20 mililitros de un lecho total de $100 \mathrm{ml}$. Se elimina el solvente mediante rotavapor. En un segundo procedimiento se desarrolla una destilación fraccionada a baja presión ( 100 mbar). Ambas formas resultan ser adecuadas a los fines perseguidos.

La identidad y pureza del producto final se investiga por cromatografía planar en silicagel (solvente hexano/acetona; 7:3), GC/NPD y ${ }^{1} \mathrm{H}$ NMR.

\footnotetext{
${ }_{1}^{1}$ Kosolapoff, G. Organophosphorus compounds. John Wiley and Sons, INC. New York (1958).

${ }^{2}$ Corbridge,D.E.C. Phosphorus. An outline of its chemistry, biochemistry and technology. $3^{\text {rd }}$ ed. Elsevier Science Publishers B.V.1985.
} 


\section{Síntesis y caracterización del fosfamidato de O,O-dimetilo (DMAP).}

El compuesto se obtuvo por oxidación selectiva del fosfamidato de O,O-dimetilo (DMATP) en un exceso de peróxido de hidrógeno y posterior separación mediante cromatografía en columna de silicagel. La purificación del compuesto se realizó mediante destilación a presión reducida.

En el procedimiento utilizado se dispusieron 6,6 gramos de DMATP grado técnico (Bayer) en un balón de 100 mililitros. Luego se agregaron, gota a gota, 5,5 mililitros de peróxido de hidrógeno (100 volúmenes) con agitación magnética a temperatura ambiente. En un primer momento la solución permanece límpida hasta la aparición, luego de unos minutos, de una marcada turbidez. En ese momento se adiciona un refrigerante vertical y se sumerge el balón en un baño de hielo, continuándose con la agitación durante veinticuatro horas. En ausencia del baño de hielo, la reacción prosigue con una violenta ebullición.

La mezcla resultante se eluyó en una columna de silicagel (30 mililitros de volumen muerto) utilizando metanol como solvente, y separándose los primeros 10 mililitros, luego de la reducción del volumen en rotavapor, se procedió a una destilación fraccionada en vacío. El producto destilado es de aspecto acuoso, incoloro e inodoro.

El seguimiento de la reacción, así como de las distintas fracciones se realiza mediante cromatografía planar en silicagel (solvente acetonitrilo/hidróxido de amonio/agua; $83: 3: 12)^{(3)}$. La identidad y pureza del producto final se investiga por espectroscopía FTIR y ${ }^{1} \mathrm{H}$ NMR.

\section{Caracterización del fosfamidotioato de O,O-dimetilo (DMATP).}

El DMATP utilizado en los ensayos de inhibición enzimática se obtuvo por destilación fraccionada en vacío de la sustancia en grado técnico (Bayer). El producto destilado presenta baja viscosidad, es incoloro y de olor suave.

La identidad y pureza del producto final se investiga por cromatografía planar en silicagel (solvente acetonitrilo/hidróxido de amonio/agua $(83: 3: 12)^{(3)}$, revelado con vapores de yodo), espectroscopía FTIR y ${ }^{1} \mathrm{H}$ NMR.

\section{Caracterización del fosfamidotionato de O,S-dimetilo.}

El fosfamidotionato de O,S-dimetilo o metamidofós utilizado en los ensayos de inhibición enzimática se obtuvo por destilación fraccionada en vacío del producto en grado técnico (Bayer). El producto destilado tiene aspecto oleoso, incoloro y de olor fuerte.

La identidad y pureza del producto final se investiga por cromatografía planar en silicagel (solvente acetonitrilo/hidróxido de amonio/agua; 83:3:12) ${ }^{(3)}$, espectroscopía FTIR y ${ }^{1} \mathrm{H}$ NMR.

\section{Caracterización del fosfato de dimetilclorovinilo.}

El fosfato de dimetilclorovinilo o DDVP utilizado en los ensayos de inhibición enzimática se obtuvo por destilación fraccionada en vacío de la sustancia en grado técnico $97 \% \mathrm{p} / \mathrm{p}$. El producto destilado tiene el aspecto de un líquido de baja viscosidad, incoloro y de olor dulce.

\section{Determinaciones de cinéticas de inhibición enzimática}

\section{Soluciones y reactivos:}

- Buffer fosfato de sodio $100 \mathrm{mM}\left(\mathrm{PO}_{4} \mathrm{HNa}_{2} \cdot 7 \mathrm{H}_{2} \mathrm{O}+\mathrm{PO}_{4} \mathrm{H}_{2} \mathrm{Na} \cdot \mathrm{H}_{2} \mathrm{O}\right.$ en las proporciones adecuadas), cloruro de magnesio $1 \mathrm{mM}$, ajustado en 8 unidades de $\mathrm{pH}$.

\footnotetext{
${ }^{3}$ Salama, A.K. et al. Studies on the metabolism of methamidophos and acephate: synthesis and identification by proton nuclear magnetic resonance and mass spectrometry of $14 \mathrm{C}$-acephate and some metabolites, J.Occup.Med.Toxicol. 2 (4): 399-408 (1993).
} 
- Cloruro de acetiltiocolina (ATC) (Sigma Chemical Co. Lote 99C-0071, PM: 197,7) disuelto hasta $12,5 \mathrm{mM}$ en agua bidestilada.

- Ácido 5,5'-ditio-bis-(2-nitrobenzoico) (DTNB PM: 396,2) en solución 12,5 mM preparada en el buffer (se envasa protegido de la luz).

Fuente de enzima (Las diluciones enzimáticas se preparan en el buffer):

Homogenizado de cerebro de rata $\left(\mathrm{AChE}_{\mathrm{C}}\right)$

Homogenizado de Drosophila sp. ( $\left.\mathrm{AChE}_{\mathrm{D}}\right)$

Suspensión de recombinante Drosophila melanogaster $\left(\mathrm{AChE}_{\mathrm{R}}\right)$

\section{Curva de tiempo:}

Se agregan sucesivamente al tubo: $0,1 \mathrm{ml}$ de ATC, $0,1 \mathrm{ml}$ de DTNB, $1,1 \mathrm{ml}$ de buffer, $0,1 \mathrm{ml}$ de enzima, manteniendo la mezcla de reacción a $24 \pm 1^{\circ} \mathrm{C}$. Se agita en vórtex y se lee en el espectrofotómetro a $412 \mathrm{~nm}$. La primera lectura se la considera tiempo cero. Se grafica $\mathrm{A}_{412}$ en función del tiempo. Se buscan las concentraciones del enzima que varíen la $\mathrm{A}_{412}$ en $0,8-1,1$ unidades en el lapso de 20 minutos.

\section{Curvas de inhibición:}

Se agregan sucesivamente al tubo: $0,1 \mathrm{ml}$ de DTNB, 1,0 $\mathrm{ml}$ de buffer, $0,1 \mathrm{ml}$ de enzima, $0,1 \mathrm{ml}$ de la solución del inhibidor. Se agita en vórtex y se lee en el espectrofotómetro. A esta lectura se la considera tiempo cero. Se agrega luego de 1 a 4 minutos (dependiendo del inhibidor utilizado) $0,1 \mathrm{ml}$ de ATC. Se agita en vórtex y se lee en el espectrofotómetro durante un tiempo de entre quince minutos (para buenos inhibidores) y hasta veinticuatro horas (para inhibidores pobres), manteniendo permanentemente la mezcla de reacción a $24 \pm 1^{\circ} \mathrm{C}$. Se grafica $\mathrm{A}_{412}$ en función del tiempo. Se trabaja con entre cinco y siete concentraciones de inhibidor y cada ensayo se realiza por duplicado.

Para cada concentración se ajusta una curva referida a la ecuación 12.n, calculándose el valor de $\mathrm{Chi}^{2}$, el ajuste de la regresión (r) y el valor de cada parámetro $\mathrm{A}_{0}, \mathrm{~A}_{1}$ y $\rho$ con los errores analíticos asociados. Una curva típica de inhibición está ejemplificada en el siguiente gráfico en el que se describe la variación de la $\mathrm{A}_{412}$ a lo largo del tiempo para la reacción de Ellman respecto de AChEb en presencia de TMP como inhibidor en una concentración de 2,609 M. Se muestra la respectiva curva de ajuste:

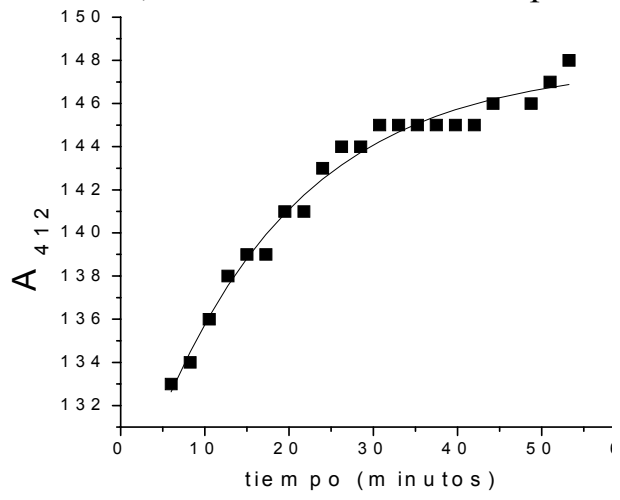

En el caso de las medidas de inhibición por TMSP y TMSeP, se observa en los blancos de ATC, la aparición de color, es decir, el DTNB se hidroliza en presencia de grupos R-SH (o R R-SeH), dando lugar a la formación de un complejo coloreado en ausencia de ATC. Estas curvas presentan una cinética lineal dependiente de la concentración inicial del inhibidor, e independiente de la presencia de AChE. Sin especular acerca del mecanismo de hidrólisis del inhibidor que se verificaría en las condiciones del ensayo, es necesario considerar dicho fenómeno para ajustar adecuadamente los valores de $\rho$. De esta manera, se realiza una modificación en la curva de ajuste, que denominaremos ecuación 12.n', en la que se incluye un término $\mathbf{H}$ que representa la constante de primer orden de formación del complejo coloreado en ausencia de actividad enzimática sobre la ATC. 


$$
A_{t}=t \cdot H+A_{o}+A_{1} e^{-t / p} \quad \text { ecuación 12.n' }
$$

\section{Control de pureza biológica:}

Para una adecuada interpretación de los resultados cinéticos, es necesario evaluar la presencia de impurezas, fundamentalmente en las sustancias para las cuales se obtienen constantes de inhibición bajas. Se entiende que los criterios cromatográficos y espectroscópicos entregan un dato importante, pero la existencia de impurezas que presenten una capacidad de inhibición de varios órdenes de magnitud por encima que la de la sustancia que se pretende evaluar, puede deformar los resultados cinéticos. Para evitar este inconveniente, se aplican dos criterios experimentales adicionales que permiten una mayor confiabilidad en estas medidas.

El primer criterio es el de equivalencia inhibitoria, y corresponde a la medida de las curvas de inhibición para un extracto final y para el mismo extracto sometido nuevamente al último paso de purificación. Si existe una variabilidad que se puede asignar a las incertezas de la medida cinética, se supone que o no existen impurezas que interfieran con el análisis cinético o no es posible remover una mayor cantidad de impurezas interferentes mediante la metodología utilizada. En nuestro caso, no se encontraron diferencias significativas para los ensayos con distintos fracciones de purificación.

El segundo criterio es el de secuestro enzimático in situ y consiste en proceder para los inhibidores pobres a ensayos cinéticos durante un lapso prolongado de tiempo. Se utilizaron medidas de hasta veinticuatro horas para sustancias como el DMATP y el TMP. Si existen impurezas con un poder inhibitorio importante, éstas reaccionarán con la enzima durante un primer y corto tiempo de la experiencia. Si la curva obtenida presenta el mismo ajuste tomando todos los puntos o descartando los primeros, se asume que no hay interferencias en el ensayo. Este criterio permite además realizar igualmente un ajuste adecuado, mediante el descarte de los primeros puntos. En nuestro caso, no se encontraron diferencias significativas para ambos tipos de ajuste.

\section{Obtención y caracterización de extractos enzimáticos}

Las AChEs constituyen una familia muy extendida de enzimas que cumplen funciones en el sistema nervioso de animales superiores y de artrópodos. Esta variedad de orígenes sugiere que el estudio del comportamiento cinético de los OPs debería realizarse en extractos con actividad $\mathrm{AChE}$ de diversas fuente, procurando recorrer un rango amplio en especies. En un principio, se trabajó con fuentes de AChE obtenidas comercialmente (eel acetylcholinesterase - Sigma Aldrich). Pero los precios prohibitivos obligaron a la búsqueda de una fuente alternativa de $\mathrm{ChE}$ correspondiente a un organismo superior.

Para este trabajo se obtuvieron extractos de cerebro de rata Wister y de mosca de la fruta (Drosophila sp.). Además se contó con AChE variante de Drosophila sp. obtenida por modificación mediante técnicas de ingeniería genética. Dicha enzima fue cedida por el Dr. Didie Fournier del Laboratorio de Síntesis y fisicoquímica de Moléculas de Interés Biológico de la Universidad Paul Sabatier (Francia).

\section{Caracterización de AChE variante de drosófila}

La mutante a elección consistió en la denominada E69W/F370A que presenta la característica de ser hipersensible a metamidofós, un fosfamidato. El Ki medido en buffer fosfato $25 \mathrm{mM}$ de $\mathrm{pH} 7$ es $10 \mathrm{mM}^{-1} \mathrm{~min}^{-1}$. En las mismas condiciones, el Ki para la AChE de anguila es de $1 \mathrm{mM}^{-1} \mathrm{~min}^{-1}$.

Al momento del trabajo, esta corresponde a la enzima con mayor sensibilidad a este tipo de OP. La actividad específica de esta mutante es de $2,5 \mathrm{pmol} / \mathrm{ml}$, para obtener una velocidad de $1 \mathrm{DO} / \mathrm{min}$ con $1 \mathrm{mM}$ ATC en $25 \mathrm{mM}$ de buffer fosfato a $\mathrm{pH} 7$ a $25^{\circ} \mathrm{C}$. La 
AChE de anguila es más activa en las mismas condiciones, necesitándose $0,38 \mathrm{pmol} / \mathrm{ml}$ para una actividad de $1 \mathrm{DO} / \mathrm{min}$.

\section{Obtención de AChE de mosca de la fruta (Drosophila sp.)}

Uno de los aspectos interesantes que es sometido permanentemente a estudio es el hecho de la selectividad en la actividad inhibitoria de los OPs utilizados como agentes de agresión química. En general, se buscarán sustancias con una gran actividad inhibitoria sobre AChEs de insectos y muy baja para AChEs de mamíferos para su uso como pesticidas o insecticidas, pero se procurará gran actividad sobre AChEs de mamíferos para el caso de las sustancias utilizadas como agentes de guerra química.

En nuestro caso, la búsqueda de fuentes alternativas de enzimas significó el abordaje de metodologías de obtención de actividad AChE de Drosophila sp. (mosca de la fruta) para tener una referencia de centro activo correspondiente a un artrópodo. Por otro lado, la enzima recombinante aportadas por el Doctor Marty (ver agradecimientos), en una variedad hipersensible a la inhibición por parte de fosfamidatos, corresponde a una estructura modificada obtenida a partir de Drosophila melanogaster, por lo que resulta pertinente contar con la variedad salvaje del enzima.

Fue necesario desarrollar metodologías de cultivo de la mosca de la fruta para poder obtener una cantidad de material de partida suficiente para la obtención de cantidades apreciables de extractos con actividad enzimática. La drosófila es un organismo adecuado pues es de crianza sencilla y crecimiento rápido. Es un insecto con metamorfosis completa (huevo, larva, ninfa, ímago o adulto) y su desarrollo transcurre en un lapso de 20 a 30 días. Cada hembra adulta coloca más de cincuenta huevos, de manera que en un lapso de dos meses podemos aumentar una población, si no existen limitaciones de espacio y alimento en condiciones favorables, en tres órdenes de magnitud.

Para el cultivo de los ejemplares se prepara una papilla de cultivo de larvas con la siguiente composición:

- $200 \mathrm{ml} \mathrm{H} 2 \mathrm{O}$

- 1 gr gelatina

- 10 gr levadura

- 10 gr azúcar

- $0,5 \mathrm{ml}$ ácido acético

Dicha mezcla se dispone en Erlenmeyers de $250 \mathrm{ml}$ de capacidad, tapados mediante torunda de algodón. En su interior se coloca una tira de cartón, sobre la que se adherirán las ninfas una vez que las larvas completen su desarrollo. La siembra de huevos se realiza mediante captura de ejemplares adultos vivos, la adecuada caracterización de los especímenes y su disposición en los Erlenmeyers así preparados.

Una vez que se obtienen los ejemplares adultos luego de cada ciclo de crecimiento, y aquellos depositen una cierta cantidad de huevos, se trasvasan a frascos vacíos. Estos insectos presentan fototropismo positivo, de manera que la forma mas sencilla de trasvase es mediante la comunicación de ambos Erlenmeyers, de crianza y vacío, oscureciendo luego el que corresponde al de crianza e iluminando el otro. En unos pocos minutos, los insectos se encontrarán en el frasco iluminado. Estos frascos se depositan en frío a $0^{\circ} \mathrm{C}$ hasta la muerte de los ejemplares.

Se dispone de 5,487 gr de moscas enteras (alrededor de 2370 ejemplares) en $15 \mathrm{ml}$ de $\mathrm{KCl} 0,1 \mathrm{M}$, procediéndose a la licuación en homogenizador celular. El resultante se filtra mediante torunda de algodón para eliminar fragmentos de exoesqueleto. El filtrado resultante se centrifuga a $4^{\circ} \mathrm{C}$ a $13,300 \mathrm{G}$ durante una hora obteniéndose un sobrenadante y un pellet que se resuspende en $1 \mathrm{ml}$ de $\mathrm{KCl} 0,1 \mathrm{M}$ para sus usos como extractos enzimáticos.

Se procede a la medida de las actividades de los extractos enzimáticos según la técnica de Ellman tal como se describe en la sección de cinética enzimática. La medida de la 
actividad enzimática se realizó con diluciones de los extractos, obteniéndose los siguiente resultados para la dilución con un rango de medida más adecuado:

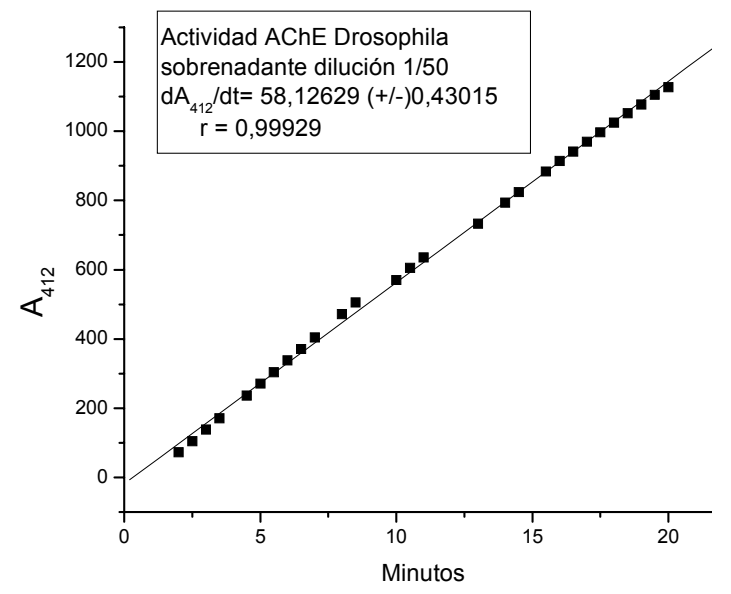

Se observa una cinética de primer orden, hasta por lo menos 1,200 unidades de densidad óptica (DO), con un ajuste de regresión lineal muy elevado $(\mathrm{r}=0,99929)$. El extracto obtenido es adecuado para los ensayos previstos, obteniéndose un homogenato con una actividad de $2,9 \times 10^{3} \mathrm{DO} / \mathrm{min} \mathrm{ml}$.

\section{Obtención de AChE de cerebro de rata.}

Se optó por la obtención de extractos con actividad enzimática de cerebro de rata Wistar, por la disponibilidad de especimenes y por contar con un procedimiento de extracción relativamente sencillo.

Se dispone de un ejemplar, sacrificado por trauma cefálico, en el que se procede a la ablación del tejido cerebral completo. Se pesa, obteniéndose un valor de 1,37 gramos. Sobre hielo, se procede a su disgregación manual mediante tijeras y pinzas de disección, para sumergirlo luego en $6 \mathrm{ml}$ de buffer HEPES (pH 7,5). Posteriormente se licua en un homogenizador celular obteniéndose un extracto que se filtra mediante una torunda de algodón, para eliminar restos de tejido fibroso. El filtrado resultante se centrifuga a $4^{\circ} \mathrm{C}$ a $13,300 \mathrm{G}$ durante una hora obteniéndose un sobrenadante y un pellet que se resuspende en 1 $\mathrm{ml}$ de HEPES para sus usos como extractos enzimáticos.

Se procede a la medida de las actividades de los extractos enzimáticos según la técnica de Ellman tal como se describe en la sección de cinética enzimática.

La medida de la actividad enzimática se realizó con diluciones de los extractos, obteniéndose los siguiente resultados para la dilución de 1/50:

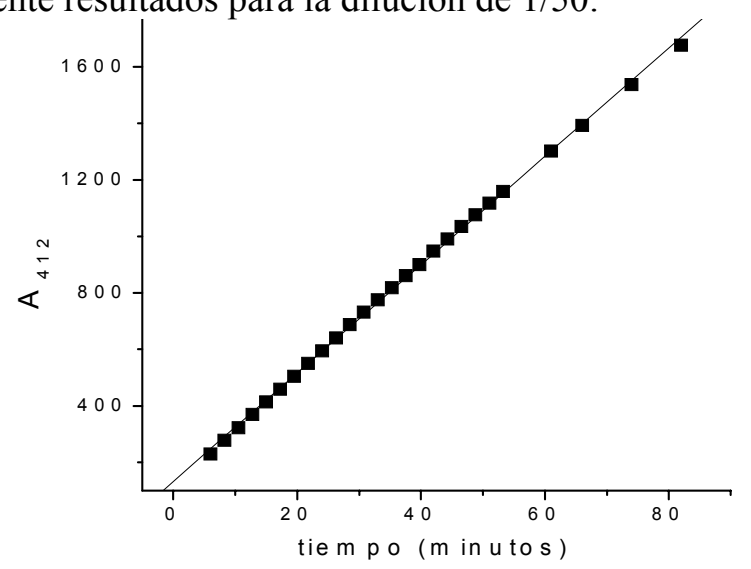

Se observa una cinética de primer orden, hasta por lo menos 1,500 unidades de densidad óptica (DO), con un ajuste de regresión muy elevado $(\mathrm{r}=0,9996)$. Se concluye que 
el extracto obtenido es adecuado para los ensayos previstos, obteniéndose un homogenato con una actividad de $953 \mathrm{DO} / \mathrm{min} \mathrm{ml}$.

\section{Monitoreo de la seguridad en el manejo de OPs}

Una de las técnicas más utilizadas en toxicología clínica para la evaluación de la intoxicación por OPs es el método peachimétrico de Michel (para AChE eritrocitaria) que se fundamenta en el cambio de $\mathrm{pH}$ por la liberación de protones durante la reacción hidrolítica de la ACh. Este procedimiento se utilizó para seguir la exposición del operador a los OPs durante las síntesis que se realizaron durante el presente trabajo. Los bajos niveles de exposición, evaluados por la mínima variación de los valores de colinesterasa eritrocitaria, dan cuenta de la eficacia de las medidas de prevención de la exposición a OPs durante la manipulación de estos compuestos.

Se trabaja con aproximadamente $8 \mathrm{ml}$ de sangre heparinizada, obtenida por venipunción (evitando la hemólisis), se transfiere luego a un tubo heparinizado (con una gota de heparina). Luego de tapar, se agita cuidadosamente por inversión.

\section{Reactivos:}

- Solución buffer para eritrocitos: barbital sódico 0,2 N (4,1236 gr), fosfato de potasio diácido, $0,004 \mathrm{~N}(0,5444$ gr) y cloruro de potasio $0,6 \mathrm{~N}(44,736$ gr). Se disuelven en $950 \mathrm{ml}$ de agua y se ajusta el $\mathrm{pH}$ en 8,10 exactamente mediante el agregado de $\mathrm{ClH} 0,1 \mathrm{~N}$, medidos a $25^{\circ} \mathrm{C}$ (se requieren alrededor de $28 \mathrm{ml}$ ). Se agregan gotas de tolueno y se guarda en refrigerador. Se comprueba el $\mathrm{pH}$ ya que tiende a variar con el tiempo.

- Ioduro de acetilcolina: Se disuelven 3,004 gr de Ioduro de acetilcolina en agua y se llevan a $100 \mathrm{ml}$. Se agregan unas gotas de tolueno y se guarda en heladera.

- Saponina al 0,01\%: Se disuelven $10 \mathrm{mg}$ de saponina en agua y se llevan a $100 \mathrm{ml}$.

- Heparina: $10000 \mathrm{U} / \mathrm{ml}$.

Se transfieren $0,04 \mathrm{ml}$ exactos de glóbulos rojos sedimentados (se trabaja con la muestra a temperatura ambiente) a un matraz de $5,0 \mathrm{ml}$ conteniendo $2,0 \mathrm{ml}$ de saponina, enjuagando la pipeta con la mezcla de saponina y glóbulos rojos, hasta que todas las células se desprenden de la pipeta. Se mezcla mediante vórtex. Una vez preparadas las muestras, se añade a cada tubo $2,0 \mathrm{ml}$ de solución buffer, esperando $10 \mathrm{~min}$. Se determina el $\mathrm{pH}$ de la solución hasta 0,01 de unidad y se anota como $\mathrm{pH}_{1}$, (si la lectura es menor de $\mathrm{pH} 7,97$ se desecha la prueba y se comienza otro ensayo con una solución buffer recién preparada). Se añaden $0,4 \mathrm{ml}$ de solución de ioduro de acetilcolina, mezclando con vórtex y anotando como $\mathrm{T}_{1}$ el momento en que se añadió el ioduro, dejando reaccionar durante 1 hora a temperatura ambiente. Cumplido este tiempo se determina el $\mathrm{pH}$ de la mezcla, y se toma como $\mathrm{pH}_{2}$ y el momento que se mide como $\mathrm{T}_{2}$. $\mathrm{El} \Delta \mathrm{pH} /$ hora se determina siguiendo el siguiente esquema:

$$
\frac{\Delta p H}{h r .}=\frac{\left(p H_{1}-p H_{2}-b\right) f}{t}
$$

$b:$ corrección para la hidrólisis no enzimática (ver tabla)

$f:$ corrección para la variación de $\Delta \mathrm{pH} /$ hora con el $\mathrm{pH}$.

$t: \mathrm{T}_{2}-\mathrm{T}_{1}$, expresado en horas.

Los factores de corrección para la hidrólisis no enzimática, no son lineales con respecto al tiempo, y dependen del $\mathrm{pH}$ : 


\begin{tabular}{|ccc|ccc|ccc|}
\hline $\mathrm{pH}$ & $\mathrm{b}$ & $\mathrm{f}$ & $\mathrm{pH}$ & $\mathrm{b}$ & $\mathrm{f}$ & $\mathrm{pH}$ & $\mathrm{b}$ & $\mathrm{f}$ \\
\hline 7,90 & 0,03 & 0,94 & 7,40 & 0,00 & 0,99 & 6,90 & 0,00 & 0,99 \\
7,80 & 0,02 & 0,95 & 7,30 & 0,00 & 1,00 & 6,80 & 0,00 & 0,98 \\
7,70 & 0,01 & 0,96 & 7,20 & 0,00 & 1,00 & 6,70 & 0,00 & 0,97 \\
7,60 & 0,00 & 0,97 & 7,10 & 0,00 & 1,00 & 6,60 & 0,00 & 0,97 \\
7,50 & 0,00 & 0,98 & 7,00 & 0,00 & 1,00 & 6,50 & 0,00 & 0,97 \\
\hline
\end{tabular}

La cifra promedio de la actividad de la colinesterasa en un grupo de individuos normales de acuerdo a Michel, es de $\Delta \mathrm{pH} /$ hora: 0,75 , el que se constituye entonces en valor de referencia. Los valores medidos para el operador, en distintas oportunidades durante el período de manipulación de los compuestos OPs, presentaron un promedio de 0,80 $\Delta \mathrm{pH} /$ hora, sin variaciones significativas.

\section{Química computacional}

La química computacional abarca las técnicas de cálculo sobre estructuras moleculares que, contando con el auxilio de sistemas de computación automática, entregan resultados teóricos acerca de las características geométricas y energéticas de las mismas, y por extensión describe resultados espectroscópicos, comportamientos en diferentes entornos, mecanismos de reacción, etc., sobre la base de los resultados iniciales. Básicamente las utilizaremos para el cálculo de propiedades conformacionales y espectroscópicas, para el modelado de la interacción de la enzima con los inhibidores y para la estimación de descriptores moleculares para el análisis QSAR.

Existen tres categorías en las metodologías utilizadas para la obtención de resultados teóricos, y cada una se distingue por la cantidad de supuestos teóricos utilizados. La elección de la metodología a aplicar en cada caso depende del objetivo perseguido, es decir, de cual es la interrogante que estimula el cálculo, y de la complejidad del sistema a analizar, además de la disponibilidad de cálculo a disposición.

Respecto a esto último, junto con el rápido desarrollo del hardware de los sistemas de computación, siendo accesibles a bajo costo equipos de uso personal con capacidad comparable a equipos que hace sólo una década eran disponibles sólo en centros de cierta magnitud, la evolución de las metodologías de procesamiento ha resultado en la aparición de software de mucha potencia, como los sistemas operativos de alto desempeño (como LINUX) y los algoritmos que permiten la optimización del procesamiento paralelo (como el sistema LINDA).

Las metodologías de Mecánica Molecular recurren a las leyes de la mecánica clásica que describen las fuerzas de atracción y repulsión considerando partículas puntuales. Estos métodos utilizan ecuaciones de ajuste a los datos experimentales muy simples, minimizando la cantidad de cálculos a realizar. Por ello se pueden aplicar a moléculas muy grandes, incluidas macromoléculas; ejemplos son el método SYBYL aplicable a moléculas con cualquier elemento de la tabla periódica y el MMFF desarrollado para moléculas orgánicas y biopolímeros. Se han desarrollado varios esquemas de cálculo que se encuentran parametrizados para distintos entornos. En general, requieren de un bajo poder computacional y, dependiendo de lo complejo de los sistemas y del ajuste de las parametrizaciones utilizadas, los resultados entregan aproximaciones relativamente buenas. En nuestro trabajo hemos utilizado DREIDING para el cálculo de las energías de interacción de las variantes conformacionales de los inhibidores en el sitio activo y, a partir de ellas, el cálculo de los descriptores de libertad y restricción conformacional.

Los métodos semiempíricos utilizan ecuaciones fundamentales con introducción de parámetros empíricos, es decir, también trabajan en un contexto de mecánica cuántica pero introduciendo datos experimentales que simplifican las expresiones del cálculo. Esto agrega un cierto grado de incertidumbre en los resultados, pero disminuye enormemente los requerimientos computacionales. Mejoran los cálculos respecto a las anteriores, pero son 
aplicables a moléculas de hasta algunas centenas de átomos; ejemplos son los métodos AM1, AM1-SM2 para cálculos en solución acuosa, PM3, MOPAC, CNDO. En este trabajo se utilizó PM3, para los cálculos semiempíricos de hipersuperficies por su buen tratamiento de enlaces del tipo $\mathrm{P}=\mathrm{O}$ y $\mathrm{P}-\mathrm{O}$. Para la búsqueda de estructuras estables en el complejo enzima inhibidor también se utilizó la misma metodología.

Los métodos ab initio consisten en la resolución, mediante distintos métodos matemáticos de aproximación, de la ecuación de Schrödinger sin el auxilio de parámetros empíricos, es decir, son métodos que trabajan en el contexto de la mecánica cuántica. Aunque pueden entregar resultados de extrema precisión, en la práctica son aplicables en la mayoría de los casos, a moléculas de unas pocas decenas de átomos., aunque existen algunas experiencias de aplicación de metodologías ab initio a sistemas complejos como proteínas con resultados prometedores. En este trabajo se utilizaron los métodos SCF de Hartree-Fock (HF) y la teoría de perturbación de Møller-Pleset de orden dos (MP2), con distintos niveles de complejidad.

Las metodologías de cálculo por funcionales de la densidad (DFT) se encuentran al mismo nivel de complejidad que los cálculos ab initio, pero utilizan como fundamento del cálculo la determinación de las distribuciones de probabilidad de densidad electrónica en el contexto de la mecánica cuántica. Fueron utilizados el funcional Becke's Three Parameter Hybrid con la correlación LYP (B3LYP), para el análisis de las estructuras conformacionales y el comportamiento de los espectros de matrices a distintas temperaturas para el TMSeP y el DMATP.

Los cálculos teóricos entregan resultados que difícilmente puedan accederse mediante metodologías experimentales pero deben correlacionarse de alguna manera con éstos para confirmar la validez de los datos obtenidos y corroborar la efectividad de la metodología teórica utilizada.

En principio, sólo los cálculos ab initio o DFT son capaces de aproximar mejor el modelado de sistemas moleculares, pero cuando la complejidad del sistema implica enormes dificultades por las capacidades computacionales requeridas, y dado que en sistemas biológicos fácilmente se superan las capacidades de equipos tipo PC, se recurre al desarrollo de metodologías de parametrización empírica, ya sea métodos Semiempíricos o de Mecánica Molecular. Para el modelo del sitio activo desarrollado y con la capacidad computacional disponible, no fue posible implementar adecuadamente métodos ab initio, lo que de alguna manera limita la calidad de los resultados obtenidos. Sin embargo, y como ya se ha mencionado para el desarrollo de cálculos semiempíricos en moléculas aisladas, las limitaciones son cuantitativas, siendo el aspecto cualitativo del problema, adecuadamente resuelto en los términos utilizados.

Todos los cálculos expuestos en este trabajo, ab initio, Semiempíricos y de Mecánica Molecular, se realizaron mediante software Gaussian 98 en sistema operativo Linux con entorno Linda ${ }^{(4)}$.

Las geometrías iniciales para TMP ${ }^{(5)}$ y TMSP $^{(6)}$ se obtuvieron de reportes previos, en tanto para TMSeP se trabajó con parámetros geométricos iniciales correspondientes a TMP y TMSP utilizando referencias de longitudes de enlace $\mathrm{P}=\mathrm{Se}$ de otros compuestos selenofosforados. Dichas estructuras se tomaron como referencia para el cálculo de las geometrías de energía mínima de DMAP, DMASP y DMASeP. Todos los puntos estacionarios fueron probados mediante el desarrollo de cálculos vibracionales.

\footnotetext{
${ }^{4}$ Gaussian 98, Revision A.7, Frisch M.J. et al. Gaussian, Inc., Pittsburgh PA, 1998.

${ }^{5}$ Van Wazer, J. R. and Ewig, C.S. Ab initio structures of phosphorus acids and esters. II. Methyl Phosphinate, dimethyl phosphonate, and trimethyl phosphate. J. Am. Chem. Soc. 108, 4354-4360 (1986).

${ }^{6}$ Katagi, T. Ab initio structures of trimethyl and dimethyl phenyl phosphorothioates. Theochem. 68, 61-67 (1990).
} 
Las geometrías de transición conformacional se calcularon con el método Synchronous Transit-Guided Quasi-Newton (STQN) mediante la opción QST2, es decir, con el ingreso de los estados iniciales y finales de la transición.

Los resultados teóricos vibracionales, obtenidos con SCF-HF, se reescalaron mediante un factor de 0,89 para compensar la sobreestimación dada por los cálculos ab initio que no consideran la correlación de orbitales. Para el caso de los cálculos vibracionales con un alto nivel de teoría, como son los cálculos DFT para el análisis de los datos vibracionales de espectroscopías de alta resolución, el factor de corrección utilizado fue de 0,98 , por comparación de los resultados con el pico de mayor intensidad en los datos experimentales del espectro IR en matrices (1030 $\mathrm{cm}^{-1}$ para el TMSeP y $1027 \mathrm{~cm}^{-1}$ para el DMASP).

En el modelado del sitio activo se utilizó el método PM3 para la determinación de estructuras estables y, a partir de ellas, las energías de interacción de cada una de las variantes conformacionales se determinaron mediante el método de mecánica molecular DREIDING.

\section{$\underline{\text { Definición de descriptores conformacionales }}$}

El cálculo de los descriptores moleculares conformacionales se realiza considerando cada uno de los valores de energía de interacción $\mathbf{E}\left(\Psi_{1}, \Psi_{2}, \Psi_{3}\right)$ para cada una de las variantes conformacionales, con saltos de $30^{\circ}$ para los parámetros $\Psi$. En aquellas moléculas con sustituyentes diferentes se considera además la rotación en valores de $120^{\circ}$ alrededor del eje $\mathrm{P}=\mathrm{X}$, siendo $\mathrm{X}$ un calcógeno. Éste es el caso de los fosfamidatos sustituidos y el DDVP.

La denominación de los descriptores conformacionales se realiza considerando a cada una de las energías de interacción de la molécula libre $\boldsymbol{E}_{\boldsymbol{i}}^{\boldsymbol{L}}$, con el superíndice "II" de intrínseco, y de las energías de interacción del inhibidor con el sitio activo $\boldsymbol{E}_{\boldsymbol{i}}^{\boldsymbol{S}}$, usando el superíndice "C", de contextual. Finalmente, si la función que define al descriptor es creciente respecto a la energía de interacción, el descriptor es denominado "R", de restricción conformacional o "L", de libertad conformacional. Las funciones que definen a cada descriptor conformacional son las siguientes:

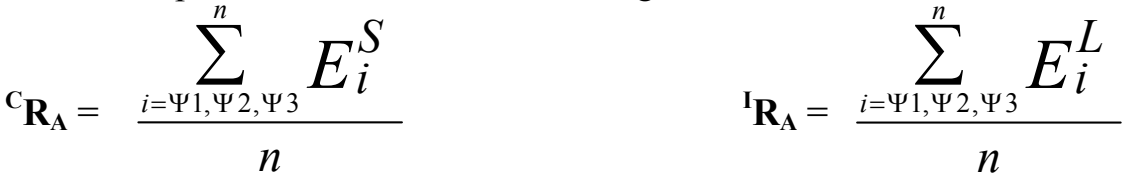

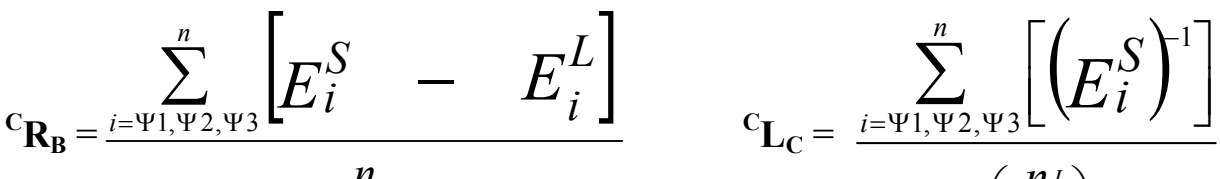

$$
\begin{aligned}
& { }^{\mathbf{I}} \mathbf{L}_{\mathbf{B}}=\frac{\sum_{i=\Psi 1, \Psi 2, \Psi 3}^{n}\left(E_{i}^{L}\right)^{-1}}{n} \quad \mathbf{C}_{\mathbf{L}_{\mathbf{D}}=\frac{\sum_{i=\Psi 1, \Psi 2, \Psi 3}^{n}\left(\frac{E_{i}^{L}}{E_{i}^{S}}\right)}{n}}^{n}
\end{aligned}
$$$$
{ }_{\mathbf{C}_{\mathbf{L}}}=\frac{\sum_{i=\Psi 1, \Psi 2, \Psi 3}^{n}\left[\left(E_{i}^{S}-E_{i}^{L}\right)^{-1}\right]}{n}
$$ 


$$
\begin{aligned}
{ }_{\mathbf{C}_{\mathbf{F}}}=\frac{\sum_{i=\Psi 1, \Psi 2, \Psi 3}^{n}\left[\left(E_{i}^{L}\right)^{-1}-\left(E_{i}^{S}\right)^{-1}\right]}{n} \\
\mathrm{C}_{\mathbf{L}_{\mathbf{G}}}=\frac{\sum_{i=\Psi 1, \Psi 2, \Psi 3}^{n}\left[E_{i}^{L} /\left(E_{i}^{L}-E_{i}^{S}\right)\right]}{n} \\
{ }_{\mathrm{C}_{\mathbf{L}_{\mathbf{H}}}}=\frac{\left.\sum_{i=\Psi 1, \Psi 2, \Psi 3}^{n}\left[\left(E_{i}^{L}\right)^{-1} /\left(E_{i}^{L}\right)^{-1}-\left(E_{i}^{S}\right)^{-1}\right]\right]}{n}
\end{aligned}
$$

$$
\mathbf{C}_{\mathbf{L}_{\mathbf{I}}=}=\frac{\sum_{i=\Psi 1, \Psi 2, \Psi 3}^{n}\left[\left(E_{i}^{S}\right)^{-1} /\left[\left(E_{i}^{L}\right)^{-1}-\left(E_{i}^{S}\right)^{-1}\right]\right]}{n}
$$

${ }^{\mathbf{I}} \mathbf{R}_{\mathbf{C}}=\frac{\sum_{i=\Psi 1, \Psi 2, \Psi_{3}}^{n}\left[\ln \left(E_{i}^{L}\right)\right]}{n}$

$$
{ }^{\mathbf{C}_{\mathbf{J}}}=\frac{\sum_{i=\Psi 1, \Psi 2, \Psi 3}^{n}\left[\ln \left(E_{i}^{s}\right)\right]}{n}
$$

${ }^{\mathbf{C}_{\mathbf{R}}}=\frac{\sum_{i=\Psi 1, \Psi 2, \Psi 3}^{n}\left[\frac{\ln \left(E_{i}^{s}\right)}{\ln \left(E_{i}^{L}\right)}\right]}{n}$

Según estas definiciones, ${ }^{\mathrm{C}} \mathbf{L}_{\mathbf{G}}$ y ${ }^{\mathbf{C}} \mathbf{L}_{\mathbf{I}}$ son numéricamente iguales. Por otro lado para

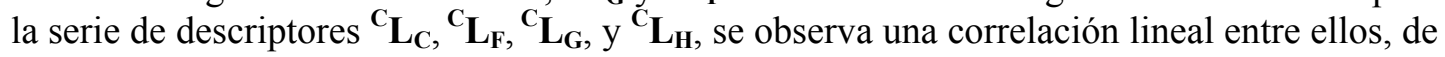
manera que en términos de descriptores de comportamiento conformacional son equivalentes y para la búsqueda de correlaciones entre éstos con las constantes cinéticas, se utiliza finalmente sólo ${ }^{{ }^{C}} \mathbf{L}_{\mathbf{I}}$. 


\title{
Anexo II
}

\section{Constantes físicas y biológicas de referencia}

\section{Constantes físicas}

\author{
Fosfato de trimetilo (TMP). CAS [512-56-1] \\ Peso molecular $=140,08$ \\ Fórmula molecular $=\mathrm{C}_{3} \mathrm{H}_{9} \mathrm{O}_{4} \mathrm{P}$ \\ Punto de fusión $=-46^{\circ} \mathrm{C}$ \\ Punto de ebullición $=197,2^{\circ} \mathrm{C}$ \\ Solubilidad en agua a $20^{\circ} \mathrm{C}=500 \mathrm{~g} / 1^{(7)}$ \\ Presión de vapor a $25^{\circ} \mathrm{C}=0,85 \mathrm{~mm} \mathrm{Hg}{ }^{(8)}$ \\ Coeficiente de partición ciclohexano/agua $(\operatorname{logKcw})=-2,220^{(9)}$ \\ Calor de formación a $25^{\circ} \mathrm{C}=-265,00 \mathrm{kcal} / \mathrm{mol}$ (estimado) ${ }^{(10)}$ \\ Fostatotioato de O,O,O-trimetilo (TMSP). CAS [152-18-1] \\ Peso molecular $=156,14$ \\ Fórmula molecular $=\mathrm{C}_{3} \mathrm{H}_{9} \mathrm{O}_{3} \mathrm{PS}$ \\ Calor de formación a $25^{\circ} \mathrm{C}=-183,00 \mathrm{kcal} / \mathrm{mol}$ (estimado) ${ }^{(10)}$ \\ Densidad $($ estimada $)=1,365 \pm 0,133$ \\ Fosfoselenoato de O,O,O-trimetilo (TMSeP) \\ Peso molecular $=203,04$ \\ Fórmula molecular $=\mathrm{C}_{3} \mathrm{H}_{9} \mathrm{O}_{3} \mathrm{PSe}$ \\ Punto de ebullición $(11 \mathrm{mbar})=73-4^{\circ} \mathrm{C}^{(11)}$ \\ Densidad $20^{\circ} \mathrm{C}=1,5112 \mathrm{~g} / \mathrm{ml}^{(11)}$ \\ Densidad $($ estimada $)=1,443 \pm 0,023 \mathrm{~g} / \mathrm{ml}$ \\ $\mathrm{n}_{20} \mathrm{D}=1,4887^{(11)}$ \\ Fosfamidato de O,O-dimetilo (DMAP). \\ Peso molecular $=125$ \\ Fórmula molecular $=\mathrm{C}_{2} \mathrm{H}_{8} \mathrm{O}_{3} \mathrm{NP}$ \\ Fosfamidotioato de O,O-dimetilo (DMATP). \\ Peso molecular $=141,131$ \\ Fórmula molecular $=\mathrm{C}_{2} \mathrm{H}_{8} \mathrm{O}_{2} \mathrm{NPS}$ \\ Densidad (estimada) $=1,308 \pm 0,023 \mathrm{~g} / \mathrm{ml}$
}

\footnotetext{
${ }^{7}$ (a) Dannenfelser, R.M. and Yalkowsky, S.H. Database for aqueous solubility of nonelectrolytes. Computer Applications in the Biosciences 5(3): 235-236 (1989). (b) Yalkowsky, S.H. and Dannenfelser, R.M. Aquasol Database of Aqueous Solubility. Version 5. College of Pharmacy, University of Arizona - Tucson, AZ PC Version (1992).

8 Daubert,T.E. and Danner,R.P. Physical and thermodynamic properties of pure chemicals, Supplement 1. Hemisphere Pub (1991).

${ }^{9}$ Wolfenden,R. and Williams,R., Affinities of phosphoric acids, esters and amides for solvent water J.Am.Chem.Soc. 105:1028-1031 (1983).

${ }^{10}$ Liebman,J.F. in "Gas Phase Ion and Neutral Thermochemistry"J. Phys. Chem. Ref. Data, Vol. 17, Suppl. 1 (1988).

${ }_{11}$ Nuretdinov, I. A. and Grechkin, N.P.N. Properties of trialkyl selenophosphates, Izv. Akad. Nauk. SSSR., Ser.Khim. 12: 2831-2833 (1968).
} 
Fosfamidotionato de O,S-dimetilo. CAS [10265-92-6]

Peso molecular $=141,131$

Fórmula molecular $=\mathrm{C}_{2} \mathrm{H}_{8} \mathrm{O}_{2} \mathrm{NPS}$

Punto de fusión $=46^{\circ} \mathrm{C}$

Solubilidad en agua a $20^{\circ} \mathrm{C}=1000 \mathrm{~g} / 1^{(7)}$

Coeficiente de partición n-octanol/agua $=-0,660^{(12)}$

Densidad estimada $=1,214 \pm 0,031 \mathrm{~g} / \mathrm{ml}$

${ }^{1} \mathrm{H}$ NMR: $2,33 \mathrm{~d}\left(\mathrm{CH}_{3} \mathrm{~S}\right) ; 3,79 \mathrm{~d}\left(\mathrm{CH}_{3} \mathrm{O}\right) ; 3,24 \mathrm{~s}\left(\mathrm{NH}_{2}\right)$

Fosfato de dimetilclorovinilo CAS [62-73-7]

Peso molecular $=220,976$

Fórmula molecular $=\mathrm{C}_{4} \mathrm{H}_{7} \mathrm{Cl}_{2} \mathrm{O}_{4} \mathrm{P}$

Punto de ebullición $=234,1^{\circ} \mathrm{C}$

Punto de fusión $<25^{\circ} \mathrm{C}$

Presión de vapor a $25^{\circ} \mathrm{C}=0,721 \mathrm{~Pa}{ }^{(13)}$

Solubilidad en agua a $25^{\circ} \mathrm{C}=1000,0 \mathrm{mg} / \mathrm{l}^{(14)}$

Solubilidad en agua a $20^{\circ} \mathrm{C}=8000 \mathrm{mg} / 1^{(15)}$

Densidad estimada $=1,411 \pm 0,016 \mathrm{~g} / \mathrm{ml}$

\section{$\underline{\text { Referencias espectroscópicas }}$}

La aplicación de espectroscopía IR (correlacionada con Raman), entrega una valiosa herramienta para la identificación de sustancias. Los OPs presentan varias regiones de absorción interesantes en su aplicación específica.

De gran importancia son las bandas más intensas de vibración P-O y las específicas $\mathrm{P}=\mathrm{X}$, en donde $\mathrm{X}$ representa un calcógeno. En adelante se tabulan los valores utilizados para la identificación de grupos funcionales y agrupamientos atómicos mediante espectroscopía FT-IR, espectroscopía Raman y espectroscopía de resonancia magnética nuclear de hidrógenos.

\begin{tabular}{lcc}
\hline \multicolumn{1}{c}{ Asignación } & Frecuencia $\left(\mathrm{cm}^{-1}\right)$ & Descripción \\
\hline P-OH & $2750-2550^{(16)}$ & intensa ancha \\
estiramiento & $2700-2650^{(17)}$ & \\
P-H estiramiento & $2300-2250^{(17)}$ & media \\
& $2450-2240^{(16)}$ & (w to m) \\
P=N & $2440-2275^{(17)}$ & (vs) \\
P=O estiramiento & $1439-1120^{(16)}$ & (vs) \\
& $1320-1200^{(16)}$ & suave \\
$\mathrm{R}_{3} \mathrm{P}=\mathrm{O}$ & $1300-960^{(17)}$ & \\
$\mathrm{R}_{2}\left(\mathrm{R}^{\prime} \mathrm{O}\right) \mathrm{P}=\mathrm{O}$ & $1190-1150^{(17)}$ & \\
$\mathrm{R}\left(\mathrm{R}^{\prime} \mathrm{O}\right)_{2} \mathrm{P}=\mathrm{O}$ & $1265-1200^{(17)}$ & \\
$\left.\mathrm{R}^{\prime} \mathrm{O}\right)_{3} \mathrm{P}=\mathrm{O}$ & $1280-1240^{(17)}$ & \\
$\mathrm{TEP}^{(17)}$ & \\
$\mathrm{R}(\mathrm{OH})_{2} \mathrm{P}=\mathrm{O}$ & $1300-1260^{(17)}$ & \\
$\mathrm{R}_{2}(\mathrm{OH}) \mathrm{P}=\mathrm{O}$ & $1270,5,1305^{(18)}$ & \\
& $1220-1150^{(17)}$ & \\
\hline
\end{tabular}

${ }^{12}$ Hussain M., Fukuto T.R., Reynolds H.T. Physical and chemical basis for systemic movement of organophosphorus esters in the cotton plant, J.Agric.Food.Chem. 22 (2): 225-230 (1974).

${ }^{13}$ Kim Y.H., Woodrow J.E., Seiber J.N. Evaluation of a gas chromatographic method calculating vapor pressures with organophosphorus pesticides, J.Chromatogr. 314:37-53 (1984).

${ }^{14}$ Albanese, V., Milano, J.C. and Vernet, J.L. A study of the evaporation of trace concentrations of low molecular weight halogenated hydrocarbons dissolved in water Environ.Technol.Lett. 8(12):65768 (1987).

${ }^{15}$ USDA Pesticide Properties Database

${ }^{16}$ Smith,D.J.H. Introduction at phosphorus compounds. In: Comprehensive organic chemistry, The syntesis and reactions of organic compounds. Barton and Ollis ed. 1979 Pergamon Press Ltd.

${ }^{17}$ Pretsch, E., Clerc, T., Seibl, J. and Simon, W. Tables of spectral data for structure determinations of organic compounds. Second edition, 1989. Springler-Verlag. 


\begin{tabular}{|c|c|c|}
\hline $\begin{array}{l}\mathrm{RO}(\mathrm{OH})_{2} \mathrm{P}=\mathrm{O} \\
(\mathrm{RO})_{2}(\mathrm{OH}) \mathrm{P}=\mathrm{O} \\
\mathrm{R}(\mathrm{RO})(\mathrm{OH}) \mathrm{P}=\mathrm{O} \\
\left(\mathrm{R}{ }_{2} \mathrm{P}=\mathrm{O}\right)_{2} \mathrm{O} \\
(\mathrm{R}(\mathrm{OH}) \mathrm{P}=\mathrm{O})_{2} \mathrm{O} \\
(\mathrm{R}(\mathrm{RO}) \mathrm{P}=\mathrm{O})_{2} \mathrm{O} \\
(\mathrm{RO}(\mathrm{OH}) \mathrm{P}=\mathrm{O})_{2} \mathrm{O} \\
\left((\mathrm{RO})_{2} \mathrm{P}=\mathrm{O}\right)_{2} \mathrm{O} \\
\left(\mathrm{RO}\left(\mathrm{R}_{2} \mathrm{~N}\right) \mathrm{P}=\mathrm{O}\right)_{2} \mathrm{O} \\
\left.\left((\mathrm{RO})_{2} \mathrm{P}=\mathrm{O}\right) \mathrm{O}\left(\mathrm{R}_{2} \mathrm{~N}\right)_{2} \mathrm{P}=\mathrm{O}\right) \\
\left.\left((\mathrm{R})_{2} \mathrm{~N}\right)_{2} \mathrm{P}=\mathrm{O}\right)_{2} \mathrm{O} \\
\mathrm{R}(\mathrm{X}) \mathrm{P}=\mathrm{O} \\
\mathrm{R}(\mathrm{X})_{2} \mathrm{P}=\mathrm{O} \\
(\mathrm{RO})_{2}(\mathrm{X}) \mathrm{P}=\mathrm{O} \\
\mathrm{RO}(\mathrm{X})_{2} \mathrm{P}=\mathrm{O}\end{array}$ & $\begin{array}{l}\sim 1250^{(17)} \\
1250-1210^{(17)} \\
1220-1170^{(17)} \\
1240-1205^{(17)} \\
\sim 1195^{(17)} \\
1265-1250^{(17)} \\
\sim 1250^{(17)} \\
1310-1260^{(17)} \\
\sim 1275^{(17)} \\
\sim 1300^{(17)} \sim^{(124)} \\
\sim 1235^{(17)} \\
1265-1240^{(17)} \\
1365-1260^{(17)} \\
1330-1280^{(17)} \\
1365-1260^{(17)}\end{array}$ & \\
\hline$(\mathrm{P}-) \mathrm{CH}_{3}$ deformación & $1310-1280$ & \\
\hline $\mathrm{P}=\mathrm{C}$ & $1230-1180^{(16)}$ & media \\
\hline P-OH deformación & $\sim 1280^{(17)}$ & intensa \\
\hline $\begin{array}{l}\text { P-O estiramiento } \\
\text { P-OC } \\
\text { P-OH } \\
\text { TEP } \\
\text { TEP } \\
\text { TEP } \\
\text { P-OP }\end{array}$ & $\begin{array}{l}1260-855^{(17)} \\
1050-970^{(17)} \\
1100-940^{(17)} \\
975^{(17)} \\
991-988^{(17)} \\
976^{(17)} \\
980-900^{(17)}\end{array}$ & $\begin{array}{l}\text { suave } \\
\text { ancha } \\
\text { intensa } \\
(\mathrm{w}, \mathrm{w}) \\
(\mathrm{vw})\end{array}$ \\
\hline $\mathrm{P}-\mathrm{N}-\mathrm{C}$ estiramiento & $\begin{array}{l}1110-930 \\
770-680^{(17)}\end{array}$ & \\
\hline $\begin{array}{l}\text { P-(O-C) estiramiento } \\
\text { TEP } \\
\text { TEP }\end{array}$ & $\begin{array}{c}1060-905^{[\mathrm{a}]} \\
1042,5 \\
1047,1 \\
1044,9^{(17)} \\
1029,5^{(17)}\end{array}$ & $\begin{array}{l}\text { (vs) } \\
\text { (vs,s,m) } \\
\text { intensa }\end{array}$ \\
\hline P-H deformación & $1090-910^{(17)}$ & suave \\
\hline P-F estiramiento & $905-760^{(17)}$ & \\
\hline $\begin{array}{l}\text { (P-O)-C estiramiento } \\
\text { TEP } \\
\text { TEP }\end{array}$ & $\begin{array}{c}875-730^{[\mathrm{a}]} \\
830-740^{(17)} \\
820^{(17)} \\
820,7^{(18)} \\
826,5^{(18)}\end{array}$ & suave \\
\hline P-C estiramiento & $800-700^{(17)}$ & \\
\hline $\mathrm{P}=\mathrm{S}$ estiramiento & $\begin{array}{l}750-550^{[\mathrm{a}]} \\
750-580^{(17)}\end{array}$ & suave \\
\hline P-S estiramiento & $<600$ & \\
\hline $\mathrm{P}-\mathrm{Cl}$ & $590-420^{[a]}$ & suave \\
\hline P-P & $510-390^{[a]}$ & intensa \\
\hline $\begin{array}{l}\mathrm{PO}_{3} \text { deformación antisimétrica } \\
\text { TEP }\end{array}$ & $\begin{array}{l}551,2 \\
543,4^{(18)}\end{array}$ & \\
\hline $\begin{array}{l}\mathrm{PO}_{3} \text { deformación simétrica } \\
\text { TEP }\end{array}$ & $460^{(18)}$ & \\
\hline
\end{tabular}

En el caso de la espectroscopía de resonancia magnética nuclear, se entrega valiosa información estructural. En este trabajo se contó con la posibilidad de obtención de espectros ${ }^{1} \mathrm{H}$ RMN y ${ }^{13} \mathrm{C}$ RMN.

Para los estudios de compuestos OPs, la existencia de acoplamientos espín-espín $\mathrm{P}-\mathrm{H}$ significa información adicional acerca de la ubicación relativa de los $\mathrm{H}$ respecto al o los átomos de $\mathrm{P}$ presentes en las moléculas estudiadas. Para resonancia magnética nuclear de hidrógeno se reportan las siguientes constantes de acoplamiento de fósforo-hidrógeno:

\begin{tabular}{cc}
\hline Grupo & $\begin{array}{c}\text { Constantes de } \\
\text { acoplamiento }(\mathrm{hz} .)^{(16)}\end{array}$ \\
\hline$>\mathrm{P}-\mathrm{H}$ & $150-225$ \\
$>\mathrm{P}(\mathrm{O}) \mathrm{H}$ & $450-725$ \\
$>\mathrm{P}(\mathrm{O}) \mathrm{CH}_{3}$ & $8-18$ \\
$>\mathrm{P}-\mathrm{C}-\mathrm{C} \underline{\mathrm{H}}_{3}$ & $10-30$
\end{tabular}

\footnotetext{
${ }^{18}$ Vidya,V., Sankaran, K., Sundararajan, K. and Viswanathan, K.S. Conformations of triethyl phosphate: a supersonic jet-matrix isolation and semi-empirical (AM1) study. J.Mol.Struct. 476:97104(1999).
} 


\begin{tabular}{cc}
$>\mathrm{POCH}_{2} \mathrm{CH}_{3}$ & $0-4$ \\
$>\mathrm{P}-\mathrm{O}-\mathrm{C}_{3}$ & $7-15$ \\
\hline
\end{tabular}

En las mediciones de ${ }^{31} \mathrm{P}$ RMN se encuentran los valores de corrimiento químico que se muestran en la siguiente tabla. Se observa que estos valores decrecen según la siguiente serie: fosfatos, fosfamidatos, fosfonatos, fosfotioatos, fosfamidotioatos, fosfotionatos y fosfoditioatos.

\begin{tabular}{|c|c|c|c|c|c|}
\hline \multicolumn{6}{|c|}{ Valores de corrimiento químico para $\mathrm{RMN}^{31} P$} \\
\hline \multicolumn{3}{|c|}{ Naturaleza de los átomos unidos al P } & & & \multirow[t]{2}{*}{ ppm } \\
\hline Clase de OP & $\mathbf{W}$ & $X$ & & $\boldsymbol{Z}$ & \\
\hline Fosfatos & $\mathrm{O}$ & $\mathrm{O}$ & $\mathrm{O}$ & $\mathrm{O}$ & $\begin{array}{c}116,1-120,5^{(16)} \\
0,012^{(17)} \\
-9,45^{(17)} \\
5,45^{(17)}\end{array}$ \\
\hline Fosfotioatos & $\mathrm{O}$ & $\mathrm{O}$ & 0 & $\mathrm{~S}$ & $45,9-52,2^{(16)}$ \\
\hline Fosfoditionatos & $\mathrm{O}$ & 0 & $\mathrm{~S}$ & $\mathrm{~S}$ & $15,3-21,3^{(16)}$ \\
\hline Fosfamidotioatos & $\mathrm{O}$ & $\mathrm{O}$ & $\mathrm{N}$ & $\mathrm{S}$ & $43,6-56,2^{(16)}$ \\
\hline Fosfamidatos & $\mathrm{O}$ & $\mathrm{O}$ & $\mathrm{N}$ & $\mathrm{O}$ & $104,9^{(16)}$ \\
\hline Fosfonotioatos & $\mathrm{O}$ & $\mathrm{O}$ & $\mathrm{C}$ & $S$ & $28,0^{(16)}$ \\
\hline Fosfonatos & $\mathrm{O}$ & $\mathrm{O}$ & $\mathrm{C}$ & 0 & $95,3^{(16)}$ \\
\hline Fosfitos & $\mathrm{O}$ & $\mathrm{O}$ & $\mathrm{O}$ & - & $-27,1^{(16)}$ \\
\hline Fosfotritioitos & $\mathrm{S}$ & $\mathrm{S}$ & $\mathrm{S}$ & - & $104,9^{(16)}$ \\
\hline
\end{tabular}

\section{Estudios QSAR}

Se presenta una compilación de este tipo de estudios de este tipo publicados para la inhibición de la $\mathrm{AChE}$ de diferentes orígenes por parte de OPs de distintas clases. Se detallan las relaciones propuestas para cada uno de los sistemas.

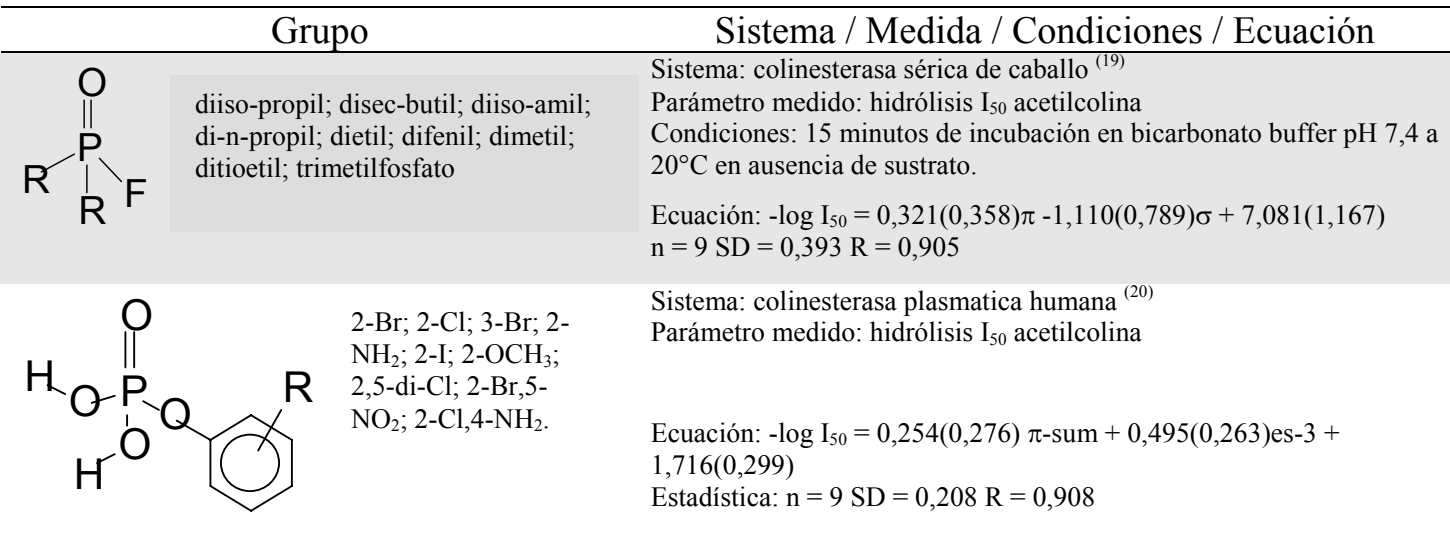

\footnotetext{
${ }^{19}$ Mackworth J.F. and Webb E.C. The inhibition of serum cholinesterase by alkyl fluorophosphonates, Biochem.J. 42:91-95 (1948).

${ }^{20}$ Freedman L.D. et al. The preparation of some organophosphorus compounds possessing anticholinesterase activity, J.Am.Chem.Soc. 75 :1379-1381 (1953).
} 


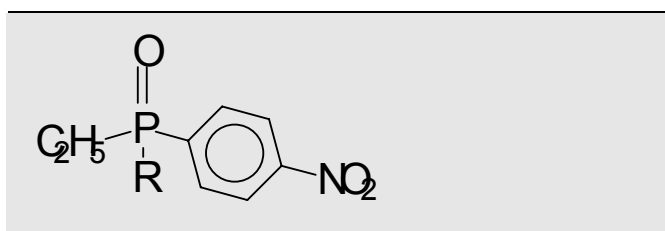

Sistema: colinesterasa de cabeza de mosca
Parámetro medido: Inhibición de la hidrólisis de acetilcolina

Ecuación: $\log \mathrm{ki}=2,705(0,536) \mathrm{esc}+8,118(0,525)$

etoxi, etil, propil, alil, metil, isopentil, butil, $\mathrm{ClCH}_{2}$, fenil, Estadística: $\mathrm{n}=17 \mathrm{SD}=0,524 \mathrm{R}=0,945$ isohexil, pentil, hexil, isobutil, benzil, isopropil, ciclohexil, t-butil.<smiles>[R]N([R])P(=O)(OC)Oc1cc(Cl)c(C)cc1Cl</smiles>

$\mathrm{H}, \mathrm{H} ; \mathrm{H}, \mathrm{CH}_{3} ; \mathrm{H}$ $\mathrm{C}_{2} \mathrm{H}_{7} ; \mathrm{H}, \mathrm{n}$-propil; $\mathrm{H}$, n-butil; $\mathrm{CH}_{3}$, $\mathrm{CH}_{3} ; \mathrm{H}$, iso-propil; $\mathrm{H}$, t-butil.
Sistema:: colinesterasa de cabeza de mosca ${ }^{(22)}$

Parámetro medido: inhibición de la hidrólisis de acetilcolina

Ecuación: $-\log \mathrm{ki}=1,249(0,340)$ esc $+6,056(0,313)$

Estadística: $\mathrm{n}=8 \mathrm{SD}=0,292 \mathrm{R}=0,973$<smiles>[R][R]OP(=O)(O[R])S(=O)O[R]</smiles>

(a) $\left(\mathrm{CH}_{2}\right)_{2} \mathrm{CH}\left(\mathrm{C}_{2} \mathrm{H}_{7}\right)_{2}, \mathrm{CH}\left(\mathrm{C}_{2} \mathrm{H}_{7}\right)_{2}$, $\mathrm{CH}_{2} \mathrm{CH}\left(\mathrm{C}_{2} \mathrm{H}_{7}\right)_{2},\left(\mathrm{CH}_{2}\right)_{3} \mathrm{CH}\left(\mathrm{C}_{2} \mathrm{H}_{7}\right)_{2}$, $\left(\mathrm{CH}_{2}\right)_{4} \mathrm{CH}\left(\mathrm{C}_{2} \mathrm{H}_{7}\right)_{2}$, $\mathrm{R}\left(\mathrm{CH}_{2}\right)_{5} \mathrm{CH}\left(\mathrm{C}_{2} \mathrm{H}_{7}\right)_{2},\left(\mathrm{CH}_{2}\right)_{2} \mathrm{C}\left(\mathrm{CH}_{3}\right)_{3}$ ( $\left.\mathrm{CH}_{2}\right)_{3} \mathrm{C}_{(}\left(\mathrm{CH}_{3}\right)_{3},\left(\mathrm{CH}_{2}\right)_{4} \mathrm{C}\left(\mathrm{CH}_{3}\right)_{3}$ (b) di- $\mathrm{C}_{2} \mathrm{H}_{7}-\mathrm{CH}_{3}, 3,3-\mathrm{di}-\mathrm{C}_{2} \mathrm{H}_{7}-$ propil, 4,4-di- $\mathrm{C}_{2} \mathrm{H}_{7}$-butil, 5,5-di$\mathrm{C}_{2} \mathrm{H}_{7}$-fenil, 6,6-di- $\mathrm{C}_{2} \mathrm{H}_{7}$-hexil, tbutil, $\mathrm{CH}_{2} \mathrm{C}\left(\mathrm{CH}_{3}\right)_{3},\left(\mathrm{CH}_{2}\right)_{2} \mathrm{C}\left(\mathrm{CH}_{3}\right)_{3}$ $\left(\mathrm{CH}_{2}\right)_{3} \mathrm{C}\left(\mathrm{CH}_{3}\right)_{3},\left(\mathrm{CH}_{2}\right) 4 \mathrm{c}\left(\mathrm{CH}_{3}\right)_{3}$.

\section{$\mathrm{CH}_{3}, \mathrm{H} ; \mathrm{CH}_{3}, 4-\mathrm{Cl} ; \mathrm{C}_{2} \mathrm{H}_{7}, \mathrm{H}$;} $\mathrm{C}_{2} \mathrm{H}_{7}, 4-\mathrm{Cl} ; \mathrm{C}_{2} \mathrm{H}_{7}, 2-\mathrm{Cl}$;

2 T,2,4,5-tri-Cl; $\mathrm{C}_{2} \mathrm{H}_{7}, 4-\mathrm{Br}$; $\mathrm{C}_{2} \mathrm{H}_{7}, 4-\mathrm{F} ; \mathrm{C}_{2} \mathrm{H}_{7}, 4-\mathrm{CH}_{3}$; $\mathrm{C}_{2} \mathrm{H}_{7}, 4-\mathrm{NO}_{2}$; iso-propil, $\mathrm{H}$; iso-propil,4-Cl; isopropilPr,2,4,5-tri-Cl<smiles>[R]c1cccc(OP(=O)(OC)OC)c1</smiles>

4-t-Butil; 4-Cl; 4-SCH 3 ; 4-COOH; 4- $\mathrm{SO}_{2} \mathrm{CH}_{3} ; 4-\mathrm{CHO} ; 4$ $\mathrm{CN} ; 4-\mathrm{NO}_{2} ; 3-\mathrm{OCH}_{3} ; 3$-t-Butil; 3-SF 5 ; 3-NO $\mathrm{NO}_{2} ; 3-$

$\mathrm{N}\left(\mathrm{CH}_{3}\right)_{3}+$.
Sistema: colinesterasa de eritrocito bovino ${ }^{(23)}$

Parámetro medido: $\mathrm{I}_{50}$ de la inhibición de hidrólisis acetilcolina

Ecuaciones:

(a) $\log 1 / \mathrm{c}=3,616(1,401) \log p-0,388(0,177) \log p * * 2-1,574(2,686)$

Estadística: $\mathrm{n}=9 \mathrm{SD}=0,127 \mathrm{R}=0,978$

(b) $\log 1 / \mathrm{c}=1,040(0,394) \operatorname{clogp}-1,074(0,978)$ bilin(clogp $)+$

3,069(0,829)

Estadística: $\mathrm{n}=10 \mathrm{SD}=0,223 \mathrm{R}=0,973$

Sistema: colinesterasa de cabeza de mosca ${ }^{(24)}$

Parámetro medido: $\mathrm{I}_{50}$ de la hidrólisis de acetilcolina

Ecuación: $-\log \mathrm{I}_{50}=1,412(0,526) \mathrm{i}_{2}+1,632(0,773) \mathrm{MR}_{4}+$

$5,896(0,488)$

$\mathrm{i}_{1}=1$ para $\mathrm{CH}_{3}, \mathrm{i}_{2}=1$ para $\mathrm{C}_{2} \mathrm{H}_{5}$ e $\mathrm{i}_{3}=1$ para isopropil $\mathrm{i}_{-0}=1$ para $\mathrm{S}$-aril y 0 para $\mathrm{O}$-aril

Estadística: $\mathrm{n}=13 \mathrm{SD}=0,397 \mathrm{r}=0,936$

Sistema: colinesterasa de cabeza de mosca ${ }^{(25)}$

Parámetro medido: $\mathrm{I}_{50}$ de la hidrólisis de acetilcolina

Ecuación: $\log \mathrm{I}_{50}=-0,556(0,20) \mathrm{Es}+2,452(0,54) \sigma^{-}+4,818(0,41)$

Estadística: $\mathrm{n}=13 \mathrm{SD}=0,408 \mathrm{r}=0,962$

21 Fukuto T.R., Metcalf R.L., Winton M. Alkylphosphonic acid esterases insecticides, J.Econ.Entomol. 52:1121-1127 (1959).

22 Fukuto T.R. et al. Structure and insecticidal activity of alkyl 2,4,5-trichlorophenyl Nalkylphosphoramidates, J.Econ.Entomol. 56 (6): 808-810 (1963).

${ }^{23}$ Bracha P., O'brien R.D. Trialkyl phosphate and phosphorothiolate anticholinesterases. I. Amiton analogs, Biochem. 7 (4): 1545-1554 (1968).

${ }^{24}$ Murdock, L.L. and Hopkins T.L. Insecticidal, anticolinesterase and hydrolytic properties of O,Odialkyl S aryl phosphorothiolates in relation to structure, J.Agric.Food.Chem. 16(6): 954-958 (1968).

${ }_{25}$ Hansch, C. Steric parameters in structure activity correlations. Cholinesterase inhibitors, J.Org.Chem. 35:620 (1970). 


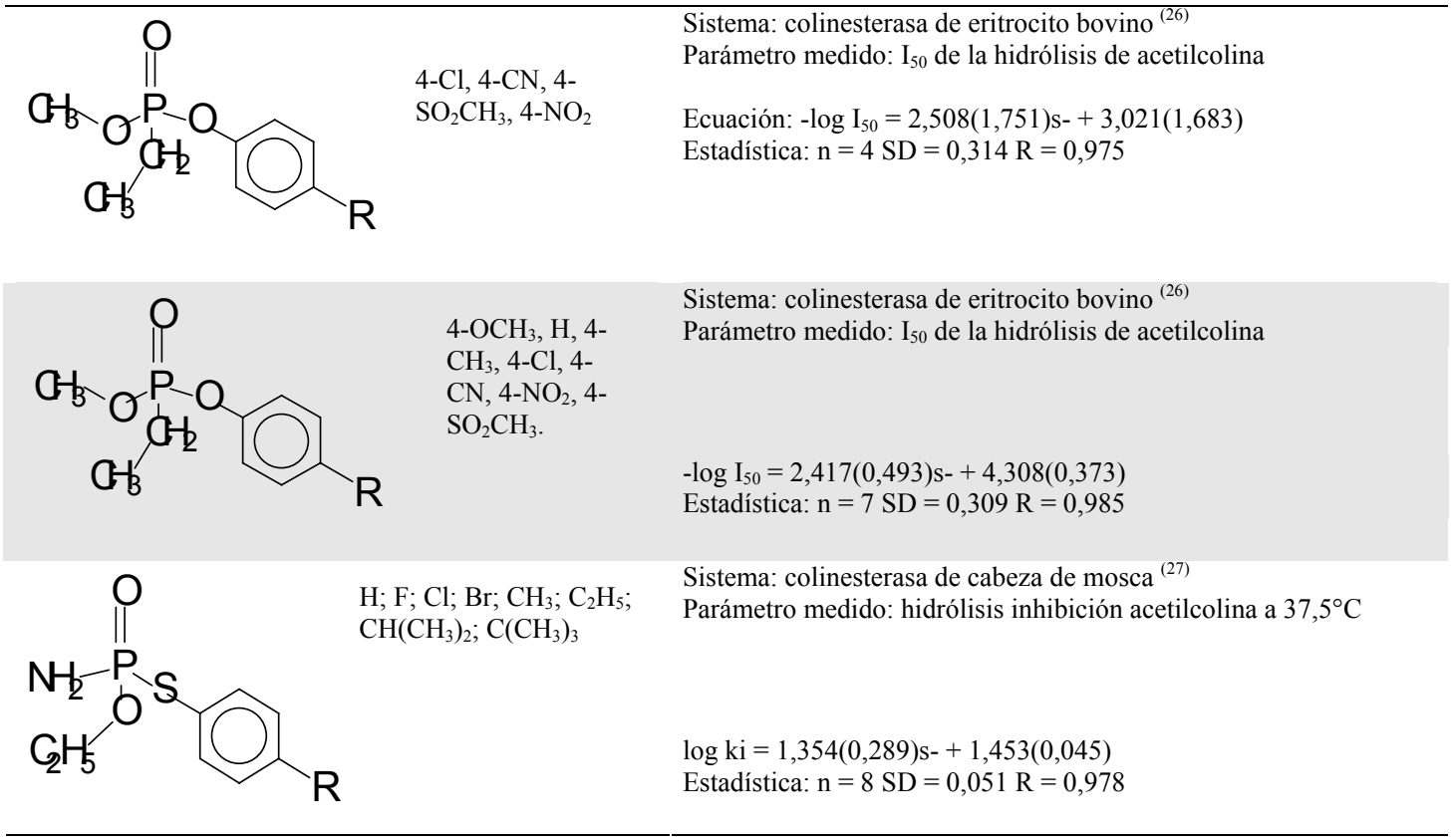

${ }^{26}$ Darlington, W.A., Partos, R.D. and Ratts K.W. Correlation of cholinesterase inhibition and toxicology in insects and mammals. I. Ethylphosphonates, Toxicol.Appl.Pharmacol. 18 (3): 542-547 (1971).

27 Sanborn J.R., Fukuto T.R. Insecticidal, anticholinesterase and hydrolytic properties of S-aryl phosphoramidothioates, J.Agric.Food.Chem. 20 (5): 926-930 (1972). 


\section{Anexo III}

\section{Índice de Figuras}

Figura 0.1: Pirofosfatos orgánicos inhibidores de la AChE sintetizados durante los primeros desarrollos de estas sustancias.

Figura 0.2: Gases nerviosos del grupo de los "Agentes G".

Figura 0.3: El DF8 originalmente fue concebido como gas de guerra.

Hoy se lo conoce como DFP y se utiliza frecuentemente como modelo de inhibidor enzimático.

Figura 0.4: Estructura del agente VX, también llamado gas-V.

$\begin{array}{ll}\text { Figura 0.5: Derivados tiofosforados utilizados como plaguicidas. } & 14\end{array}$

Figura 0.6: Estructura terciaria de la AChE. Se indican algunos constituyentes funcionales de la enzima.

Figura 0.7: Disposición gauche de los derivados éster del ácido fosfórico.

Figura 0.8: Disposición trans de los derivados éster del ácido fosfórico.

Figura 0.9: "Las leyes físicas subyacentes necesarias para la teoría matemática de una gran parte de la física y la totalidad de la química se conocen completamente y la única dificultad, es que la aplicación exacta de estas leyes conduce a ecuaciones demasiado complejas para ser solubles" (Paul Andrien Maurice Dirac, 1902-1984).

Figura 0.10: Estructura de la acetilcolina, neurotransmisor involucrado en los mecanismos de acción tóxica de los OPs.

Figura 1.1: Ángulos diedros correspondientes a la rotación de enlaces PO y O-C.

Figura 1.2: Confórmero de geometría $\mathrm{C}_{3}$.

Figura 1.3: Confórmero de geometría Cs.

Figura 1.4: Confórmeros de geometría $\mathrm{C}_{1} \mathrm{y}_{\mathrm{C}_{\mathrm{l}}}$.

Figura 1.5: Confórmeros de geometría $\mathrm{C}_{3 \mathrm{v}}$. 32

Figura 1.6a: Modos normales de vibración.

Figura 1.6b: Modos normales de vibración. $\quad 36$

Figura 2.1: Estructura del fosfato de trimetilo (TMP). 39

Figura 2.2: Doblete metílico ${ }^{1} \mathrm{H}$ RMN del TMP. $\quad 43$

Figura 2.3: Doblete metílico ${ }^{13} \mathrm{C}$ RMN del TMP. $\quad \mathbf{4 4}$

Figura 2.4: Espectro infrarrojo del TMP en el rango $500-1500 \mathrm{~cm}^{-1}$ en

el que se manifiesta coexistencia de confórmeros. Se indican los

multipletes en $1273(\mathrm{sh})-1290 \mathrm{~cm}^{-1}, 845-852(\mathrm{sh}) \mathrm{cm}^{-1}, 740-755 \mathrm{~cm}^{-1}$, $500-503-520(\mathrm{sh}) \mathrm{cm}^{-1}$.

Figura 2.5: Doblete $\mathrm{P}=\mathrm{O}$ en el espectro Raman del TMP ubicado en 736 $\mathrm{cm}^{-1}$ y $750 \mathrm{~cm}^{-1}$.

Figura 2.6: Superficie potencial del TMP, obtenida por rotación alrededor de dos ángulos $\psi$ manteniendo el otro fijo en $42^{\circ}$ calculadas a nivel HF/PM3.

Figura 2.7: Representación de planos sucesivos respecto del ángulo diedro $\psi_{1}$ de las energías conformacionales del TMP en función de la rotación de ángulos $\psi_{2} \mathrm{y} \psi_{3}$. 
Figura 2.8: Espacio conformacional que representa la total rotación de cada uno de los ángulos $\psi$ en el TMP. Los puntos se ubican en las superficies de energía equipotencial de $10 \mathrm{kcal} / \mathrm{mol}$ por encima del confórmero más estable.

Figura 3.1: Estructura del fosfotioato de O,O,O-trimetilo (TMSP).

Figura 3.4: Doblete $v \mathrm{a}_{(\mathrm{C}-\mathrm{O}) 3}$ en el espectro Raman del TMSP ubicado en 1068 y $1085 \mathrm{~cm}^{-1}$.

Figura 3.5: Superficies de energía conformacional del TMSP, calculadas a nivel HF/PM3 y generadas por rotación de dos ángulos diedros $\psi$ y manteniendo el otro fijo en $52^{\circ}$.

Figura 3.6: Espectro vibracional infrarrojo del TMSP en el rango 450 $1500 \mathrm{~cm}^{-1}$. Se muestran los dobletes en $598-620 \mathrm{~cm}^{-1}$ y $775-809 \mathrm{~cm}^{-1}$. A $1098 \mathrm{~cm}^{-1}$ aparece una banda bien definida que se corresponde con bandas ocultas a números de onda mayores, determinadas por el mismo modo vibracional de confórmeros de menor energía.

Figura 4.1: Estructura del fosfoselenoato de O,O,O-trimetilo (TMSeP).

Figura 4.2: Doblete metílico ${ }^{1} \mathrm{H}$ RMN del TMSeP.

Figura 4.3: Doblete metílico ${ }^{13} \mathrm{C}$ RMN del TMSeP.

Figura 4.4: Espectro infrarrojo del TMSeP en el rango $500-1500 \mathrm{~cm}^{-1}$ en el que se manifiesta coexistencia de confórmeros. Se indican los multipletes en $1066(\mathrm{sh})-1082(\mathrm{sh}) \mathrm{cm}^{-1}, 780-790(\mathrm{sh}) \mathrm{cm}^{-1}, 504-558$ $\mathrm{cm}^{-1}$.

Figura 4.5: Superficie potencial del TMSeP para la rotación alrededor de dos ángulos $\psi$ manteniendo el otro fijo en $42^{\circ}$, calculadas a nivel HF/PM3.

Figura 4.6: Espectro IR del TMSeP en matriz de Ar en la región $600-$ $500 \mathrm{~cm}^{-1}$. Desde arriba hacia abajo: medida a $294 \mathrm{~K}$, medida a $538 \mathrm{~K}$, medida a $778 \mathrm{~K}$, números de onda teóricos: línea continua: confórmero $\mathrm{C}_{3}$; línea cortada: confórmero $\mathrm{C}_{1}$; línea punteada: confórmero Cs.

Figura 4.7: Relación teórica entre los confórmeros $\mathrm{C}_{3} / \mathrm{C}_{1}$ del TMSeP en el rango de temperatura 250 - 800K con un nivel de teoría B3LYP/6$311++\mathrm{G}^{* *}$.

Figura 4.8: Espectro IR del TMSeP en matriz de Ar en la región 750 $600 \mathrm{~cm}^{-1}$, según patrón ya indicado.

Figura 4.9: Espectro IR del TMSeP en matriz de Ar en la región $1100-$ $1000 \mathrm{~cm}^{-1}$, según patrón ya indicado.

Figura 4.10: Espectro IR del TMSeP en matriz de Ar en la región 1300 $-1100 \mathrm{~cm}^{-1}$, según patrón ya indicado.

Figura 4.11: Espectro IR del TMSeP en matriz de Ar en la región 1510 $-1430 \mathrm{~cm}^{-1}$, según patrón ya indicado.

Figura 5.1: Estructuras del metamidofós (fosfamidotionato de O,Sdimetilo) y el acefato ( $\mathrm{N}$-acetil fosfamidotionato de $\mathrm{O}, \mathrm{S}$-dimetilo).

Figura 5.2: Formación del puente de hidrógeno entre el $\mathrm{H}$ amídico y el grupo fosforilo. Esta interacción determina la conformación cis en fosfamidatos primarios y no sustituidos.

Figura 5.3: Confórmero de geometría gCs.

Figura 5.4: Confórmero de geometría $\mathrm{C}_{1}$.

Figura 5.5: Confórmeros de geometría tCs.

Figura 6.1: Estructura del fosfamidato de dimetilo (DMAP). 
Figura 6.2: Energía de rotación del ángulo $\psi_{\mathrm{N}}$ calculada a un nivel $\mathrm{HF} / 6-31+\mathrm{G}^{*}$.

Figura 6.3: Superficie de energía potencial de rotación de los ángulos diedros $\psi 1$ y $\psi 2$, calculada a un nivel HF/PM3. Las flechas indican la ubicación de los confórmeros de mínima energía propuestos.

Figura 6.4: Espectro ${ }^{1} \mathrm{H}$ RMN del DMAP. Se observan dos dobletes análogos a los observados para el TMP.

Figura 6.5: Espectro ${ }^{13} \mathrm{C}$ RMN del DMAP. Se observa el doblete análogo al observado para el TMP.

Figura 7.1: Estructura del fosfamidato de O,S-dimetilo (DMATP).

Figura 7.2: Señales ${ }^{1} \mathrm{H}$ RMN del DMATP.

Figura 7.3: Doblete metílico en el espectro ${ }^{13} \mathrm{C}$ RMN del DMATP.

Figura 7.4: Espectros en matrices de Ar para el DMATP en la zona $3700 \mathrm{~cm}^{-1}$. Desde arriba hacia abajo se observan los espectros teóricos (línea continua para el confórmero más estable) y las medidas a $523 \mathrm{~K}$, $453 \mathrm{~K}, 358 \mathrm{~K}$ y $283 \mathrm{~K}$.

Figura 7.5: Espectros en matrices de Ar para el DMATP alrededor de $1850 \mathrm{~cm}^{-1}$. Desde arriba hacia abajo se observan las medidas a 523K, $453 \mathrm{~K}, 358 \mathrm{~K}$ y $283 \mathrm{~K}$.

Figura 7.6: Espectros en matrices de Ar para el DMATP alrededor de $1600 \mathrm{~cm}^{-1}$. Desde arriba hacia abajo se observan los espectros teóricos (línea continua para el confórmero más estable) y las medidas a $523 \mathrm{~K}$, $453 \mathrm{~K}, 358 \mathrm{~K}$ y $283 \mathrm{~K}$.

Figura 7.7: Espectros en matrices de Ar para el DMATP en la zona de $1000 \mathrm{~cm}^{-1}$, según patrón ya indicado.

Figura 8.1: Efecto anomérico de donación de un par electrónico libre del $\mathrm{O}$ hacia el orbital antiligante $\sigma^{*}$ del enlace P-O.

Figura 8.2: Efecto anomérico que involucra un par electrónico libre del $\mathrm{O}$ con el orbital antiligante $\pi^{*}$ del enlace $\mathrm{P}=\mathrm{O}$.

Figura 8.3: Efecto anomérico que involucra un par electrónico libre del $\mathrm{O}$ con el orbital antiligante $\sigma^{*}$ del enlace P-N.

Figura 8.4: Interacción de un par de electrones libres del O con el orbital $\sigma^{*}$ del grupo fosforilo.

Figura 8.5: Estructura del fosfoiminato de dietil N-dipropilsulfoxido. Se indican los ángulos diedros característicos $\Psi_{\mathrm{PN}} \mathrm{y} \Psi_{\mathrm{NS}}$.

Figura 8.6: Espectro vibracional infrarrojo de la fosfoiminato de dietil $\mathrm{N}$-dipropilsulfoxido alrededor de $1200 \mathrm{~cm}^{-1}$, indicando las bandas asignadas al estiramiento $\mathrm{P}=\mathrm{O}$ y $\mathrm{S}=\mathrm{O}$.

Figura 9.1: Cada una de las fases del proceso de acción tóxica, determina cómo finalmente se expresarán los efectos tóxicos. Se discrimina entre la interacción con dianas relevantes $\left(T_{1}\right)$ e interacciones sin cambios $\left(\mathrm{T}_{2}\right)$.

Figura 10.1: Modelo tridimensional de la $\mathrm{AChE}$ de anguila. Se indica la posición del bolsillo hidrofóbico.

Figura 10.2: Ángulos diedros $\Psi_{\mathrm{OC}}$ y $\Psi_{\mathrm{CC}}$ que, por rotación, determinan las variantes conformacionales de la ACh.

Figura 10.3: Espectros infrarrojos de ioduro y cloruro de ACh en fase sólida. Los valores de número de onda se expresan en $\mathrm{cm}^{-1}$ y la intensidad de las bandas en transmitancia porcentual.

Figura 10.4: Espectro infrarrojo predicho para la AChE según un cálculo ab initio a un nivel $\mathrm{HF} / 6-31 \mathrm{G}^{* *}$, entre 450 y $2000 \mathrm{~cm}^{-1}$.

Figura 10.5: Formación del complejo pentacoordinado E-P, consecuente 
al ataque nucleofílico por parte del hidroxilo $\operatorname{Ser}^{200}$.

Figura 11.1: Complejos estables del TMP (AMBER) que, a lo largo del canal hidrofóbico de la AChE, definen las tres posiciones $\boldsymbol{P}_{\text {Hid }}, \boldsymbol{P}_{\text {Int }} \mathrm{y}$

$\boldsymbol{P}_{\text {Ser20o, }}$ para el modelo descrito. (a) Distribución de los complejos estables según su energía relativa. La flecha representa el recorrido teórico del inhibidor desde el exterior hasta el sitio reactivo $\mathrm{Ser}^{200}$. (b) Proyección en el plano de las distancias $(\AA)$ del inhibidor con $\operatorname{Ser}^{200}$ y con el agujero oxianiónico (ver texto).

Figura 11.2: Curvas de energía potencial del TMP ubicado en $\boldsymbol{P}_{\text {Ser200, }}$ para las libres rotaciones de los ángulos $\Psi_{1}, \Psi_{2}$ y $\Psi_{3}$. La flecha indica la posición del confórmero -gauche, -gauche, -gauche de menor energía. Se indica también el elemento que determina la restricción conformacional representada en las regiones de alta energía.

Figura 11.3: Curvas de energía potencial del TMP ubicado en $\boldsymbol{P}_{\text {int }}$, para las libres rotaciones de los ángulos $\Psi_{1}, \Psi_{2}$ y $\Psi_{3}$. La flecha indica la posición del confórmero trans, gauche, -gauche de menor energía. Se indica también el elemento que determina la restricción conformacional representada en las regiones de alta energía.

Figura 11.4: Curvas de energía potencial del TMP ubicado en $\boldsymbol{P}_{\text {Hid, }}$, para las libres rotaciones de los ángulos $\Psi_{1}, \Psi_{2}$ y $\Psi_{3}$. La flecha indica la posición del confórmero gauche, -gauche, -gauche de menor energía. Se indica también el elemento que determina la restricción conformacional representada en las regiones de alta energía.

Figura 11.5: Curvas de energía potencial del TMSP y TMSeP ubicados en $\boldsymbol{P}_{\text {Ser }}$, para las libres rotaciones de los ángulos $\Psi_{1}, \Psi_{2}$ y $\Psi_{3}$. La flecha indica la posición de los confórmeros de menor energía. Se indican también los restos aminoacídicos que determinan la restricción conformacional representada en las regiones de alta energía.

Figura 11.6: Curvas de energía potencial del TMSP ubicado en $\boldsymbol{P}_{\text {int }}$, para las libres rotaciones de los ángulos $\Psi_{1}, \Psi_{2}$ y $\Psi_{3}$. La flecha indica la posición del confórmero de menor energía. Se indica también el resto aminoacídico que determina la restricción conformacional representada en las regiones de alta energía.

Figura 11.7: Curvas de energía potencial del TMSP y TMSeP ubicados en $\boldsymbol{P}_{\text {Hid, }}$, para las libres rotaciones de los ángulos $\Psi_{1}, \Psi_{2}$ y $\Psi_{3}$. La flecha indica la posición de los confórmeros de menor energía. Se indican también los restos aminoacídicos que determinan la restricción conformacional representada en las regiones de alta energía.

Figura 11.8: Ruptura de la degeneración conformacional energética para las opciones conformacionales del TMP en el sitio activo de la AChE. Las conformaciones a, b y c corresponden a las rotaciones de $120^{\circ}$ alrededor del eje determinado por el enlace $\mathrm{P}=\mathrm{O}$. Las conformaciones denotadas con “*” representan a las imágenes especulares del correspondiente confórmero.

Figura 12.1: Modelo elemental clásico de la interacción de OPs con el sitio activo del enzima AChE.

Figura 12.2: Procesos implicados en la inhibición de la $\mathrm{AChE}$ por parte de un inhibidor OP.

Figura 12.3: Hipótesis de formación del fosfotioato de O,O,S-trimetilo en medio acuoso.

Figura 13.1: OPs inhibidores directos.

Figura 13.2: OPs inhibidores indirectos. 
Figura 13.3: cis-crotonato y trans-crotonato, dos isómeros geométricos con diferente actividad anticolinesterásica.

Figura 13.4: Modificaciones metabólicas por reacciones hidrolíticas y oxidativas de los OPs indirectos.

Figura 13.5: Bioactivación de las acetofenonas oximas sustituidas.

Figura 13.6: Rutas hipotéticas de bioactivación del metamidofós.

Figura 13.7: Representación de las superficies de restricción conformacional (a) y libertad conformacional (b), para el TMP en $\boldsymbol{P}_{\text {ser200, fijando }} \Psi_{2}$ en $-30^{\circ}$.

Figura 13.8: Correlación lineal entre pKa e ${ }^{\mathrm{I}} \mathbf{R}_{\mathbf{C}}$ para la serie de entrenamiento.

Figura 13.9: Correlación lineal entre pKa y $\operatorname{QSAR}^{\mathrm{C}}(\mathbf{c})$, para la serie de entrenamiento.

Figura 13.10: Correlación lineal entre $\mathrm{kp} \mathrm{y}_{\operatorname{QSAR}}{ }^{\mathrm{C}}(\mathbf{h})$, para la serie de entrenamiento.

Figura 13.11: Correlación lineal entre $\mathrm{pKi}$ y $\operatorname{QSAR}^{\mathrm{C}}(\mathbf{p})$, para la serie de entrenamiento.

Figura 14.1: Correlación entre las energías conformacionales (en $\mathrm{kcal} / \mathrm{mol}$ calculadas a un nivel HF/6-31G**) y el momento dipolar (en Debyes) para los confórmeros del TMP $(\bullet)$ y del DMAP $(\boldsymbol{\Delta})$.

Figura 14.2: Ingreso del TMP a lo largo del canal hidrofóbico de la AChE, ubicándose sucesivamente en $\boldsymbol{P}_{\text {Hid }}, \boldsymbol{P}_{\boldsymbol{I n t}}$ y $\boldsymbol{P}_{\text {Ser200, según el modelo }}$ propuesto.

\section{Índice de Tablas}

Tabla 2.I: Valores experimentales, reportados previamente y calculados del espectro vibracional IR y Raman en fase líquida para el TMP. Se muestran sólo las bandas que evidencian coexistencia de confórmeros.

Tabla 2.II: Corrimientos químicos y constantes de acoplamiento en los espectros RMN de ${ }^{1} \mathrm{H}$ y ${ }^{13} \mathrm{C}$ para el TMP.

Tabla 2.III: Parámetros geométricos, energéticos y fisicoquímicos de los confórmeros correspondientes a mínimos de energía del TMP.

Tabla 2.IV: Parámetros energéticos y geométricos de las formas correspondientes a estados de transición entre confórmeros estables.

Tabla 3.I: Corrimientos químicos y constantes de acoplamiento en los espectros de ${ }^{1} \mathrm{H}$ RMN y ${ }^{13} \mathrm{C}$ RMN de TMSP. Entre paréntesis se indican las alturas relativas de los picos.

Tabla 3.II: Valores experimentales, reportados previamente y calculados del espectro vibracional IR y Raman en fase líquida para el TMSP. Se muestran sólo las bandas que evidencian coexistencia de confórmeros.

Tabla 3.III: Parámetros geométricos, energéticos y fisicoquímicos de los confórmeros correspondientes a mínimos de energía del TMSP.

Tabla 4.I: Valores experimentales, reportados previamente y calculados del espectro vibracional IR y Raman en fase líquida para el TMSeP. Se muestran sólo las bandas que evidencian coexistencia de confórmeros. Tabla 4.II: Valores obtenidos para el espectro de ${ }^{1} \mathrm{H}$ RMN del TMSeP (calibración respecto a TMS).

Tabla 4.III: Parámetros geométricos, energéticos y fisicoquímicos de los confórmeros estables del TMSeP.

Tabla 4.IV: Parámetros geométricos para el TMSeP, obtenidos mediante cálculos B3LYP 
Tabla 4.V: Cálculos B3LYP para la energía y momentos dipolares de los confórmeros del TMSeP.

Tabla 4.VI: Dependencia experimental de la relación de la intensidad de banda del modo de estiramiento $\mathrm{P}=\mathrm{Se}$ con la temperatura.

Tabla 4.VII: Cálculos B3LYP para la abundancia relativa de los confórmeros del TMSeP.

Tabla 6.I: Frecuencias medidas en los espectros infrarrojo en fase líquida en la zona de entre 1000 y $2000 \mathrm{~cm}^{-1}$ para la molécula DMAP. Las asignaciones propuestas se realizan en función de los valores teóricos obtenidos para cada uno de los cuatro confórmeros estables calculados a nivel $\mathrm{HF} / 6-31 \mathrm{G}^{* *}$. Entre paréntesis, se indican las intensidades teóricas relativas.

Tabla 6.II: Parámetros geométricos y físicoquímicos del DMAP obtenidos a nivel HF/6-31G** para cada uno de los cuatro confórmeros descritos como mínimos energéticos. Ángulos expresados en grados.

Tabla 6.III: Frecuencias medidas en los espectros infrarrojo en fase líquida en la zona de entre 400 y $1000 \mathrm{~cm}^{-1}$ para la molécula DMAP. Las asignaciones propuestas se realizan en función de los valores teóricos obtenidos para cada uno de los cuatro confórmeros estables calculados a nivel $\mathrm{HF} / 6-31 \mathrm{G}^{* *}$. Entre paréntesis, se indican las intensidades teóricas relativas.

Tabla 6.IV: Valores de corrimiento químico (en ppm) y de acoplamientos espín-espín (en Hz) para la molécula DMAP en los espectros ${ }^{1} \mathrm{H}$ RMN (calibrado respecto a la señal del HDO: 4,82 ppm) y

${ }^{13} \mathrm{C}$ RMN. Entre paréntesis se indica la relación de área de los respectivos picos. El valor reportado para las señales ${ }^{1} \mathrm{H}$ RMN es de 3,54 ppm.

Tabla 6.V: Valores teóricos de las frecuencias obtenidas para cada uno de los cuatro confórmeros estables calculados a nivel HF/6-31G** en la zona del infrarrojo cercano de entre 50 y $400 \mathrm{~cm}^{-1}$ para la molécula DMAP. Se proponen las asignaciones correspondientes. Entre paréntesis, se indican las intensidades teóricas relativas.

Tabla 7.I: Corrimientos químicos y constantes de acoplamiento en los espectros de ${ }^{1} \mathrm{H}$ RMN (calibración respecto a TMS) y ${ }^{13} \mathrm{C}$ RMN (calibración respecto a cloroformo 77,00 ppm) para el DMATP.

Tabla 7.II: Parámetros geométricos y fisicoquímicos del DMATP obtenidos a nivel HF/6-31G** para cada uno de los cuatro confórmeros de mínima energía hallados.

Tabla 7.III: Frecuencias medidas en los espectros infrarrojo en fase líquida e infrarrojo en matrices de $\operatorname{Ar}$ (depositadas a $293^{\circ} \mathrm{K}$ ) en la zona ubicada entre 1300 y $4000 \mathrm{~cm}^{-1}$. Las asignaciones propuestas se realizan en función de los valores teóricos obtenidos para cada uno de los cuatro confórmeros estables calculados a nivel HF/6-31G**.

Tabla 7.IV: Frecuencias medidas en los espectros infrarrojo en fase líquida e infrarrojo en matrices de $\mathrm{Ar}$ (depositadas a $293^{\circ} \mathrm{K}$ ) en la zona ubicada entre 500 y $1300 \mathrm{~cm}^{-1}$. Las asignaciones propuestas se realizan en función de los valores teóricos obtenidos para cada uno de los cuatro confórmeros estables calculados a nivel HF/6-31G**.

Tabla 7.V: Valores teóricos de las frecuencias obtenidas para cada uno de los cuatro confórmeros estables calculados a nivel $\mathrm{HF} / 6-31 \mathrm{G}^{* *}$ en la

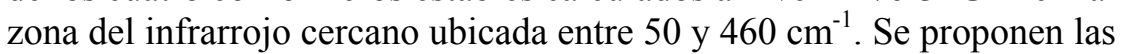
asignaciones correspondientes. Como referencia, se informan los dos únicos valores de frecuencias medidas en el espectro vibracional IR en fase líquida. 
Tabla 7.VI: Cargas teóricas del fósforo y el azufre (según Mülliken) para cada uno de los cuatro confórmeros estables del DMATP, y diferencias estimadas para la frecuencia de estiramiento del enlace $\mathrm{P}=\mathrm{S}$ entre cada par de confórmeros.

Tabla 8.I: Frecuencias teóricas del espectro vibracional IR de la molécula DMASeP, calculado a un nivel HF/6-31G** (ab initio).

Tabla 8.II: Parámetros geométricos y fisicoquímicos, calculados a un nivel HF/6-31G** (ab initio), para la molécula DMASeP.

Tabla 8.III: Análisis NBO para los dos confórmeros más estables del TMP. Entre paréntesis se indican las energías respectivas en $\mathrm{kcal} / \mathrm{mol}$.

Tabla 8.IV: Análisis NBO para los dos confórmeros más estables del DMAP. Entre paréntesis se indican las energías respectivas en $\mathrm{kcal} / \mathrm{mol}$. Tabla 8.V: Valores energéticos y geométricos para los confórmeros estables de la molécula fosfoiminato de dietil N-dipropil sulfóxido, obtenidas a un nivel HF/6-31G* (ab initio). Los ángulos se expresan en grados.

Tabla 9.I: Propiedades toxicológicas de algunos OPs, manifestadas en la $\mathrm{DL}_{50}(\mathrm{mg} / \mathrm{Kg})$ para ratas macho.

Tabla 9.II: Propiedades toxicológicas de algunos OPs.

Tabla 10.I: Grupos funcionales que describen la estructura del sitio activo de la AChE. Los números en superíndice indican la posición del resto aminoacídico en la estructura primaria de la proteína.

Tabla 10.II: Momento dipolar y ángulos de torsión (en grados) del confórmero más estable de la ACh calculado a nivel HF/6-31G**.

Tabla 11.I: Estructuras funcionales constituyentes del modelo y función de cada uno de los restos aminoacídicos involucrados en ellas.

Tabla 11.II: Los siete péptidos constituyentes del modelo del sitio activo, que incluyen a cada uno de los restos aminoacídicos involucrados en los mecanismos de catálisis e inhibición. Las relaciones tridimensionales entre estos péptidos, son las que surgen de la estructura terciaria de la enzima nativa.

Tabla 11.III: Parámetros geométricos encontrados para conformaciones estables del complejo AChE(sa) con el TMP, a un nivel de cálculo DREIDING (MM). La energía se expresa en $\mathrm{kcal} / \mathrm{mol}$ y las distancias se expresan en $\AA$. Los valores presentados para $\mathrm{AChE(sa)} \mathrm{libre,}$ corresponden a los parámetros obtenidos por RX.

Tabla 11.IV: Valores energéticos y geométricos para las ubicaciones de mínima energía (PM3) para TMP en AChE(sa)

Tabla 11.V: Valores energéticos y geométricos para las ubicaciones de mínima energía (PM3) para TMSP y TMSeP en AChE(sa)

Tabla 12.I: Criterios cinéticos que definen a un inhibidor de la AChE como un buen pesticida.

Tabla 12.II: Constantes cinéticas de inhibición para TMP obtenidos sobre distintos sistemas con actividad acetilcolinesterasa a $\mathrm{pH}$ 7,80 y $24^{\circ} \mathrm{C}$ de temperatura.

Tabla 12.III:Constantes cinéticas para distintos OPs obtenidos para actividad $\mathrm{AChE}_{\mathrm{C}}$ a $\mathrm{pH} 7,80$ y $24^{\circ} \mathrm{C}$ de temperatura. Los valores marcados “*” son considerados constantes cinéticas aparentes.

Tabla 13.I: Denominación de los OPs sobre los que se realiza en análisis de correlación.

Tabla 13.II: Descriptores de naturaleza electrónica: carga neta del fósforo ${ }^{+} \mathbf{P}$ y momento cuadrupolar $\mathbf{Q p}$ del confórmero más estable a un 
nivel ab initio HF/STO-3G.

Tabla 13.III: Descriptores de naturaleza electrónica: momento octapolar Op y momento hexadecapolar Hdp del confórmero más estable a un nivel ab initio HF/STO-3G.

Tabla 13.IV: Descriptores de propiedades hidrofóbicas: momento

dipolar $\mu$, en Debyes y logaritmo del coeficiente de partición octanol/agua $\log P$ (desviación estandar de 0,49).

Tabla 13.V: Descriptores de propiedades estéricas: Volumen molar $\mathbf{V M}$, en $\mathrm{cm}^{3} / \mathrm{mol}$, radio de bohr ao, en $\AA$ y refractividad molar RM,en $\mathrm{cm}^{3} / \mathrm{mol}$ (desviación estándar de 0,77 ).

Tabla 13.VI: Descriptores conformacionales intrínsecos: ${ }^{\mathrm{I}} \mathbf{R}_{\mathbf{A}},{ }^{\mathrm{I}} \mathbf{L}_{\mathbf{B}}$ e ${ }^{\mathrm{I}} \mathbf{R}_{\mathbf{C}}$.

Tablas 13.VIII: Descriptores contextuales de libertad conformacional:

${ }^{C_{L_{C}}},{ }^{{ }^{C}} \mathbf{L}_{\mathbf{D}},{ }^{C_{L_{E}}},{ }^{C_{\mathbf{F}}},{ }^{C_{L_{H}}}$ y ${ }^{C_{L_{I}}}$.

Tabla 13.IX: Correlaciones propuestas para cada una de las constantes cinéticas en la serie de moléculas planteada. Entre paréntesis se indica el error analítico de las constantes de ajuste de cada correlación. Se indica además el valor de $\mathrm{r}^{2}$ como índice de correlación.

Tabla 13.X: Estimación de las constantes cinéticas Ka, kp y Ki para el metamidofós(R), VIII(R) y el metamidofós(S), VIII(S).

Tabla 13.XI: Estimación de las constantes cinéticas Ka, kp y Ki para TMSP (II), TMSeP (III), STMSP (IV) y SeTMSeP (V). 